Technische Universität München

Lehrstuhl für Betriebswirtschaftslehre - Finanzmanagement und Kapitalmärkte Univ.-Prof. Dr. Chr. Kaserer

\title{
The Equity Volatility Smile and Default Risk
}

\section{An Empirical Analysis of Companies of the German DAX-Index}

\author{
Dipl.-Ing. Bernd Walter
}

Vollständiger Abdruck der von der Fakultät für Wirtschaftswissenschaften der Technischen Universität München zur Erlangung des akademischen Grades eines

Doktors der Wirtschaftswissenschaften (Dr. rer. pol.) genehmigten Dissertation

Vorsitzender:

Univ.-Prof. Dr. G. Friedl

Prüfer der Dissertation:

1. Univ.-Prof. Dr. Chr. Kaserer

2. Univ.-Prof. Dr. R. K. von Weizsäcker

Die Dissertation wurde am 03.07.2007 bei der Technischen Universität München eingereicht und durch die Fakultät für Wirtschaftswissenschaften am 07.05.2008 angenommen. 



\section{Acknowledgments}

First and foremost I would like to thank my dear wife for all her patience and support throughout the last year and beyond, especially in times I was too absorbed to fully assume my role as father and husband.

I am also very thankful for the backing of my parents who among other things contributed to the continuity of my family income during the last few months.

Further, I would like to thank my employer, Siemens Financial Services, and especially my boss, Peter Rathgeb, also for contributing to the continuity of my family income and particularly for granting me the flexibility of a sabbatical agreement in order to accomplish this dissertation.

Very importantly, I wish to thank my professor, Christoph Kaserer, for accepting and welcoming me as an external doctoral candidate at his chair, for his pragmatic and uncomplicated mentoring style and finally for the possibility to work in an undisturbed manner in the rooms of his chair which would not have been possible for me at home.

But my thanks also apply to the chair's staff and my fellow doctorands for their organizational and technical support as well as their companionship during the last months.

Moreover, I feel obliged to thank Boris Leblanc from the Global Equity and Derivatives Quantitative R\&D Team at BNP Paribas for a very fruitful discussion and his view as expert and practitioner.

Finally I would like to thank Alexander Thomas for his very valuable LaTeX hints and last but not least my deputy at Siemens Financial Services, Thomas Meurer, for managing my team during the time of my absence. 



\section{Contents}

Contents I

\begin{tabular}{ll}
\hline List of Figures & XI
\end{tabular}

\begin{tabular}{ll}
\hline List of Tables & XIII
\end{tabular}

Symbols and Abbreviations $\quad$ XXI

1 Introduction 1

1.1 Motivation and Objectives $\ldots \ldots \ldots$. . . . . . . . . . 1

1.2 Structure of the Analysis $\ldots \ldots \ldots$. . . . . . . . . . . 4

1.3 Summary of the Main Results of the Analysis . . . . . . . . . . . 6

2 Financial Valuation Theory 9

2.1 Risk-Neutral Valuation . . . . . . . . . . . . . . . . . . 9

2.1 .1 The Concept of Risk-Neutral Valuation . . . . . . . . . . 9

2.1 .2 Risk-Neutral Valuation and Market Completeness . . . . . . . 10

2.2 The Role of Risk Aversion as a Link between Physical and RiskNeutral Probability Density . . . . . . . . . . . . . . . . . . 12

3 Option Prices, Volatility Smile, and Risk-Neutral Density $\quad 15$

3.1 The Volatility Smile Phenomenon . . . . . . . . . . . . . 15

3.1.1 The Black-Scholes Model . . . . . . . . . . . . . . . . 15

3.1.2 The Shortcomings of the Black-Scholes Model and the Volatility Smile Phenomenon . . . . . . . . . . . . . . . 17

3.1.3 The Relationship between Option Prices, Volatility Smile and Risk-Neutral Density . . . . . . . . . . . . . . . 20

3.1.3.1 The Discounted Risk-Neutral Density as the Second Derivative of a European Call . . . . . . . . . . . 20

3.1.3.2 The Relationship between the Shape of the Smile and the Form of the Risk-Neutral Density . . . . . . 22 
3.1.4 The Role of Risk Aversion in an Idealized Black-Scholes Economy . . . . . . . . . . . . . . . . . 23

$3.2 \quad$ Estimation of the Volatility Smile and the Risk-Neutral Density . . . . 24

3.2 .1 Introduction . . . . . . . . . . . . . . . . . . . . . 24

3.2 .2 Non-Parametric Techniques . . . . . . . . . . . 25

3.2.2.1 Polynomial Smoothing of the Volatility Smile. . . . 25

$3.2 .2 .2 \quad$ Kernel Regression . . . . . . . . . . . . . 26

3.2.2.3 The Estimation of the Moments of the Risk-Neutral Density with the Bakshi-Madan Method. . . . . . . 27

3.2 .3 Parametric Techniques . . . . . . . . . . . . . . . . . . 29

3.2.3.1 Mixture of Lognormal Distributions . . . . . . . . . 29

3.2.3.2 Expansion Techniques . . . . . . . . . . . 30

3.2.4 Other Non-Parametric and Parametric Methods . . . . . . . . 31

3.2 .5 Summary and Appraisal of the Available Methods . . . . . . . 32

4 Explanations of the Equity Volatility Smile Excluding Default Risk 35

4.1 Introduction . . . . . . . . . . . . . . . . . . 35

4.2 Phenomenological Explanations of the Equity Volatility Smile . . . . 36

$4.2 .1 \quad$ Stylized Facts of Stock Returns . . . . . . . . . . . . . . . . . 36

$\begin{array}{lll}4.2 .2 & \text { Deterministically Changing Volatility in the Stock Price Process } 37\end{array}$

4.2.2.1 The CEV Model . . . . . . . . . . . . . . . . 37

4.2.2.2 The Concept of Local Volatility . . . . . . . . . . . 38

4.2.3 Stochastically Changing Volatility in the Stock Price Process . 40

4.2.3.1 A General Representation of a Stochastic Volatility Model. . . . . . . . . . . . . . . . 41

4.2.3.2 The Ability of Stochastic Volatility Models to Replicate the Equity Volatility Smile . . . . . . . . . . . 43

4.2 .4 Allowing for Jumps in the Stock Price Process . . . . . . . . . 43

$4.2 .4 .1 \quad$ Jump-Diffusion Processes . . . . . . . . . . . . . 44

4.2.4.2 Infinite Activity Processes . . . . . . . . . . . . . 45

4.2.4.3 The Ability of Models Incorporating Jumps to Replicate the Equity Volatility Smile . . . . . . . . . 47

4.2 .5 Stochastic Interest Rates . . . . . . . . . . . . . . . 48

4.3 Causal Explanations of the Equity Volatility Smile . . . . . . . . . . . 49

4.3.1 Market Microstructure and Efficiency Related Explanations. . 50

4.3 .2 The Role of Risk Aversion . . . . . . . . . . . . . . . . 52

4.3.2.1 The Manifestation of Risk Aversion in the Real World 52 
4.3.2.2 Fundamental Explanations of the Real World Manifestation of Risk Aversion . . . . . . . . . . . . . 54

4.4 Summary and Conclusions $\ldots \ldots \ldots$. . . . . . . . . . . . 56

5 Default Risk as an Explanation of the Equity Volatility Smile 59

5.1 The Nature of Default Risk and its Theoretical and Empirical Connection with the Equity Volatility Smile . . . . . . . . . . . . . . . . 59

5.1 .1 Default Risk in Theory . . . . . . . . . . . . . . . . . . 60

5.1.1.1 Structural Credit Risk Models . . . . . . . . . . . 60

5.1.1.2 Reduced-Form Credit Risk Models . . . . . . . . . 62

5.1 .1 .3 The Leverage Effect . . . . . . . . . . . . . . . . . 64

5.1.2 Theoretical Connection between Default Risk and the Implied Volatility Smile . . . . . . . . . . . . . . . . . 65

5.1.2.1 Theoretical Connection between Default Risk and the Level of Equity Volatility $\ldots$. . . . . . . . . 65

5.1.2.2 Theoretical Connection between Default Risk and the Shape of the Smile . . . . . . . . . . . . . 67

5.1 .3 Existing Empirical Evidence of the Relationship between Default Risk and the Equity Volatility Smile . . . . . . . . . 68

5.1.3.1 The Leverage Effect . . . . . . . . . . . . . . 68

5.1.3.2 Default Risk and the Level of Equity Volatility . . . 70

5.1.3.3 Default Risk and the Shape of the Equity Volatility Smile . . . . . . . . . . . . . . . 71

5.1 .4 Review of the Smile Explanations which are not Related to Default Risk . . . . . . . . . . . . . . . . . . . . . . 74

$5.1 .4 .1 \quad$ Introduction . . . . . . . . . . . . . 74

5.1.4.2 Continuous Process Models in the Light of Default Risk. . . . . . . . . . . . . . . . . 74

5.1.4.3 Discontinuous Process Models in the Light of Default Risk . . . . . . . . . . . . . . . . . 76

5.2 Enhanced Credit Risk Models Incorporating the Implied Volatility Smile 77

$5.2 .1 \quad$ Introduction . . . . . . . . . . . . . . . . . 77

5.2.2 Structural Credit Risk Models Incorporating the Implied Volatility Smile. . . . . . . . . . . . . . . . . . . 78

5.2.2.1 Options on a European Call on the Firm's Assets . . 79

5.2.2.2 Options on a Down-And-Out Barrier Call on the Firm's Assets . . . . . . . . . . . . . 80 
5.2 .2 .2 .1 Toft/Prucyk Model . . . . . . . . . . . . . 81

5.2 .2 .2 .2 EBIT-Based Model . . . . . . . . . . . . 82

5.2.2.2.3 Extension of the CreditGrades Model . . . 83

5.2 .2 .3 Option-Implied Density Approach . . . . . . . . . . 84

5.2.3 Reduced Form Credit Risk Models Incorporating the Implied Volatility Smile . . . . . . . . . . . . . . . . . . . 85

$5.2 .3 .1 \quad$ Introduction and Overview . . . . . . . . . . . 85

5.2 .3 .2 Model Mechanics . . . . . . . . . . . . . . . 87

5.2.3.2.1 Models with a Deterministic Stock Price Diffusion Volatility. . . . . . . . . . . . 88

5.2.3.2.2 Models with a Stochastic Stock Price Diffusion Volatility . . . . . . . . . . 90

5.3 Summary and Conclusions $\ldots \ldots \ldots \ldots \ldots$

6 Empirical Analysis of the Relationship between the Equity Volatility Smile and Default Risk 95

6.1 Introduction . . . . . . . . . . . . . . . . . . 95

6.2 Hypotheses . . . . . . . . . . . . . . . . . . . . 96

6.3 Statistical Methods Used $\ldots \ldots \ldots \ldots$

6.3 .1 Introduction . . . . . . . . . . . . . . . . . . . . . . 98

6.3 .2 Ordinary Least Squares Regression. . . . . . . . . . . . . . . 98

6.3.2.1 General Assumptions and Properties of the Ordinary Least Squares Regression . . . . . . . . . . . . . 98

6.3.2.2 Application of the Ordinary Least Squares Regression in the Time-Series Domain . . . . . . . . . . . 99

6.3.3 Autocorrelation and Heteroscedasticity in the Residuals and their Treatment . . . . . . . . . . . . . . . . . . . 100

6.3.3.1 Causes and Effects of Autocorrelation and Heteroscedasticity . . . . . . . . . . . . . 100

6.3.3.2 Detection of Autocorrelation and Heteroscedasticity 101

6.3.3.3 Treatment of Autocorrelation and Heteroscedasticity 102

6.3 .4 Non-Stationarity . . . . . . . . . . . . . . . . . . . 103

$6.3 .4 .1 \quad$ Non-Stationarity and its Causes . . . . . . . . . . 103

6.3.4.2 Detection and Treatment of Non-Stationarity . . . . 104

6.3.4.2.1 Detection and Treatment of a Time Trend . 104

6.3.4.2.2 Detection and Treatment of a Stochastic Trend . . . . . . . . . . . . . . . . . 104 
6.4 Data Processing and Conditioning . . . . . . . . . . . . . 107

6.4 .1 Introduction . . . . . . . . . . . . . . . 107

6.4 .2 Data Sources . . . . . . . . . . . . . . . . 107

6.4 .3 Computation of Historical Moments . . . . . . . . . . . . 111

6.4 .3 .1 Observation Period and Frequency. . . . . . . . . 111

6.4 .3 .2 Generation of the Return Series . . . . . . . . . . 112

6.4 .3 .3 Computation of Moments . . . . . . . . . . 112

6.4 .4 Computation of Implied Volatilities . . . . . . . . . . . . 113

6.4 .4 .1 Introduction . . . . . . . . . . . . . 113

6.4.4.2 Input Variables for the Option Evaluation Function . 113

6.4 .4 .3 Data Quality Requirements . . . . . . . . . . . 114

6.4.4.4 Option Price Evaluation with the Cox-Ross-Rubinstein Binomial Tree . . . . . . . . . . 115

6.4 .4 .5 Root-finding Algorithm . . . . . . . . . . 115

6.4.5 The Smoothing of the Implied Volatility Smile . . . . . . . . 116

6.4.5.1 Assessment of the Available Implied Volatilities . . 116

6.4.5.2 Quadratic Polynomial as the Method of Choice for the Smoothing of the Implied Volatility Smile. . . . 119

6.4 .5 .3 Regression Model . . . . . . . . . . . . . . . 121

6.4 .5 .4 Regression Results . . . . . . . . . . . . . . . . 122

6.4.5.4.1 Regression Results and Regression Statistics 122

6.4.5.4.2 Interpretation of the Regression Results . . 125

6.4.6 Computation of Risk-Neutral Skewness and Kurtosis . . . . . 127

6.4.6.1 Qualitative Assessment of the Risk-Neutral Return Density . . . . . . . . . . . . . . . 127

6.4.6.2 Computation of the Moments of the Risk-Neutral Return Density . . . . . . . . . . . . . . . 129

6.4.6.2.1 Practical Issues and Problems Concerning the Implementation of the Bakshi-Madan Method . . . . . . . . . . . 129

6.4.6.2.2 Modification of the Bakshi-Madan Method . . . . . . . . . . . 130

6.4 .7 The Variables in the Regression Analysis . . . . . . . . . . . 133

6.4.7.1 Generation of Constant Maturity Smile, Skewness and Kurtosis . . . . . . . . . . . . . . . . . . . 133

6.4 .7 .2 Synopsis of the Variables . . . . . . . . . . 133

6.4.7.3 Summary Statistics of the Variables . . . . . . . 136 
6.4.7.3.1 Summary Statistics of All Variables . . . . 136

6.4.7.3.2 Summary Statistics of Specific Variables Deserving a Closer Examination. . . . . . 137

6.4.7.4 Assessment of the Stationarity of the Variables . . . 140

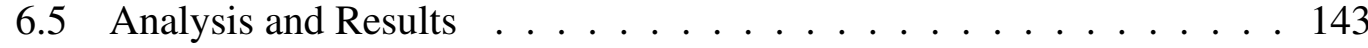

6.5 .1 Introduction . . . . . . . . . . . . . . . . . . . 143

6.5 .1 .1 Generalities. . . . . . . . . . . . . . . . . 143

6.5 .1 .2 Robustness Checks . . . . . . . . . . . . . . . . . 144

6.5.1.2.1 The Issue of Possible Non-Stationarity . . 144

6.5.1.2.2 Robustness in the Presence of Additional Explanatory Variables . . . . . . . . . 144

6.5 .1 .3 Structure of the Empirical Analysis . . . . . . . . 146

6.5 .2 The Leverage Effect . . . . . . . . . . . . . . . . . 146

6.5.2.1 Derivation of the Regression Model . . . . . . . . 147

6.5 .2 .2 Regression Results . . . . . . . . . . . . . . . . . 148

6.5.2.2.1 Examination in the Time Series Dimension 148

6.5.2.2.2 Examination in the Framework of a Pooled Series Regression . . . . . . . . . 151

6.5 .2 .3 Robustness of the Regression Results . . . . . . . . 152

6.5.2.3.1 Robustness Check of the Time Series Regression Results . . . . . . . . . . 152

6.5.2.3.2 Robustness of the Pooled Series Regression Results $\ldots \ldots \ldots \ldots \ldots$

6.5.2.4 Summary and Discussion of the Results in the Light of Hitherto Existing Findings $\ldots \ldots \ldots$

6.5.2.4.1 Summary of the Results . . . . . . . . . 157

6.5.2.4.2 Discussion of the Results in the Light of Hitherto Existing Findings . . . . . . . 157

6.5.3 The Credit Spread Level and the Implied Volatility Level . . . 159

6.5 .3 .1 Derivation of the Regression Models . . . . . . . 159

6.5 .3 .2 Regression Results . . . . . . . . . . . . . . . . . . 159

6.5.3.2.1 Examination in the Time-Series Dimension 159

6.5.3.2.2 Examination in the Framework of a Pooled Series Regression . . . . . . . . . . 163

6.5 .3 .3 Robustness of the Regression Results . . . . . . . . 164 6.5.3.3.1 Examination in the Time-Series Dimension 164 
6.5.3.3.2 Examination in the Framework of a Pooled

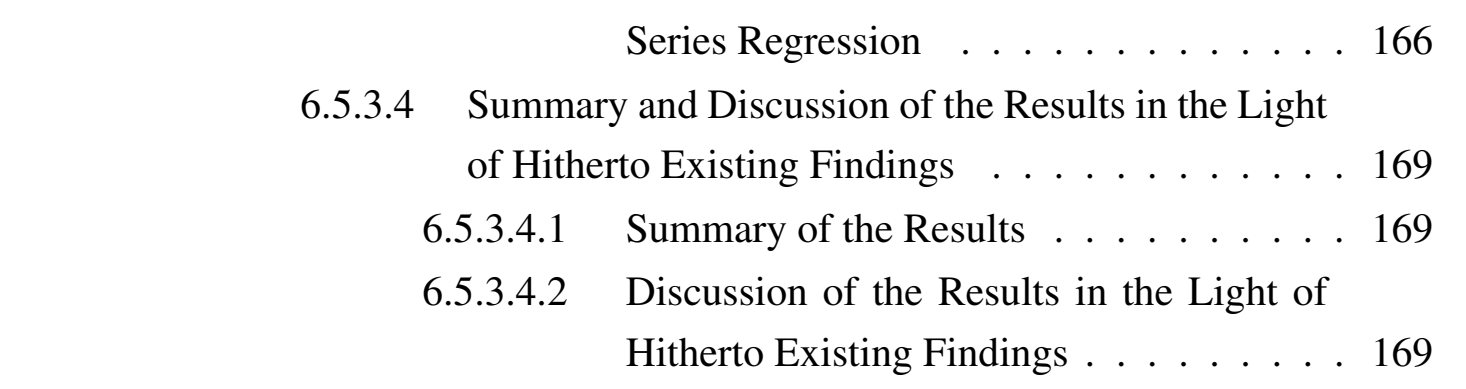

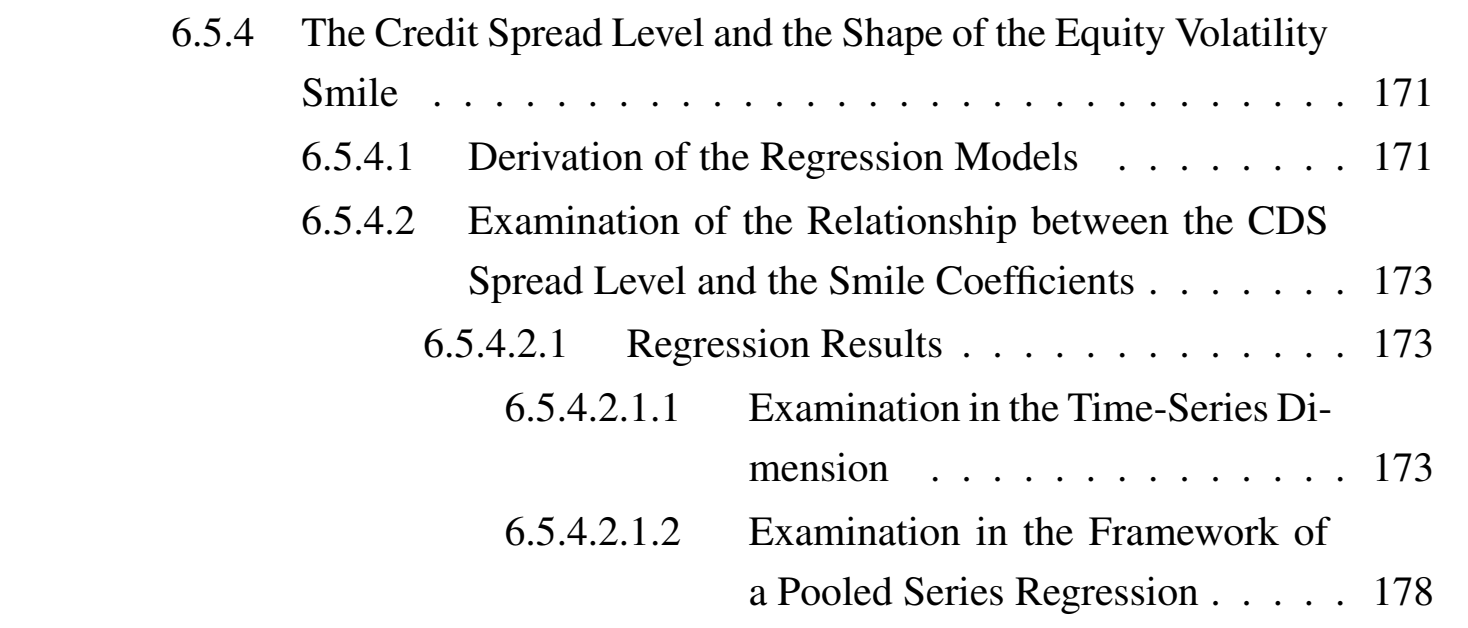

6.5.4.2.2 Robustness of the Regression Results . . . 179

6.5.4.2.2.1 Examination in the Time-Series Dimension . . . . . . . . 179

6.5.4.2.2.2 Examination in the Framework of a Pooled Series Regression. . . . . 182

6.5.4.3 Examination of the Relationship between the CDS Spread Level and the Slope of the Smile. . . . . . . 184

$6.5 .4 .3 .1 \quad$ Regression Results . . . . . . . . . . . . . 184

6.5.4.3.1.1 Examination in the Time-Series Dimension . . . . . . . . 184

6.5.4.3.1.2 Examination in the Framework of a Pooled Series Regression. . . . . 191

6.5.4.3.2 Robustness of the Regression Results . . . 193

6.5.4.3.2.1 Examination in the Time-Series Dimension . . . . . . . . 193

6.5.4.3.2.2 Examination in the Framework of a Pooled Series Regression. . . . 197

6.5.4.3.2.3 Disentangling the Volatility-Only from the Default-Risk-Only-Effect . . . 199 
6.5.4.4 Summary and Discussion of the Results in the Light of Hitherto Existing Findings . . . . . . . . . 202

6.5.4.4.1 Summary of the Results . . . . . . . . . 202

6.5.4.4.2 Discussion of the Results in the Light of Hitherto Existing Findings . . . . . . . . 203

6.5.5 The Credit Spread Level and the Moments of the Risk-Neutral Equity Return Density . . . . . . . . . . . . . . . . 204

6.5.5.1 Derivation of the Regression Models . . . . . . . . 204

$6.5 .5 .2 \quad$ Regression Results . . . . . . . . . . . . . . . . 206

6.5.5.2.1 Examination in the Time-Series Dimension 206

6.5.5.2.2 Examination in the Framework of a Pooled Series Regression . . . . . . . . . . . . . 209

6.5.5.3 Robustness of the Regression Results . . . . . . . . 210

6.5.5.3.1 Examination in the Time-Series Dimension 210

6.5.5.3.2 Examination in the Framework of a Pooled Series Regression . . . . . . . . . . 213

6.5.5.3.3 Disentangling the Volatility-Only from the Default-Risk-Only Effect . . . . . . . . 215

6.5.5.4 Summary and Discussion of the Results in the Light of Hitherto Existing Findings . . . . . . . . . . 218

$6.5 .5 .4 .1 \quad$ Summary of the Results . . . . . . . . . 218

6.5.5.4.2 Discussion of the Results in the Light of Hitherto Existing Findings . . . . . . . . 219

6.6 Summary and Conclusions . . . . . . . . . . . . . . . . 221

7 Conclusions and Implications $\quad 227$

7.1 Conclusions . . . . . . . . . . . . . . . . . . . 227

7.1.1 Summary and Conclusions of the Theoretical Part . . . . . . . 227

7.1.2 Summary and Conclusions of the Empirical Part. . . . . . . . 229

7.2 Implications . . . . . . . . . . . . . . . . 233

$7.2 .1 \quad$ Implications for Future Research . . . . . . . . . . . . . . . 233

7.2 .2 Implications for Practice . . . . . . . . . . . . 235

\section{Appendices}

\begin{tabular}{|l|l|}
\hline A C++ Code Extracts & 237
\end{tabular}

A.1 Cox-Ross-Rubinstein Binomial Tree Allowing Multiple Dividends . . 237

A.2 Quadratic Polynomial Smoothing of the Implied Volatility Smile . . . 239 
A.3 Implementation of the Modified Bakshi-Madan Method . . . . . . . . 242

\begin{tabular}{ll}
\hline B Summary Statistics & $\mathbf{2 4 5}$
\end{tabular}

B.1 Quadratic Polynomial Smoothing of the Implied Volatility Smile . . . 245

B.2 Smile Dependent Regression Variables on an Individual Constant Maturity Basis - All Cross-Sections Pooled Together . . . . . . . . . . . 248

B.3 All Regression Variables on an Individual Cross-Sectional and an Individual Constant Maturity Basis . . . . . . . . . . . . . 251

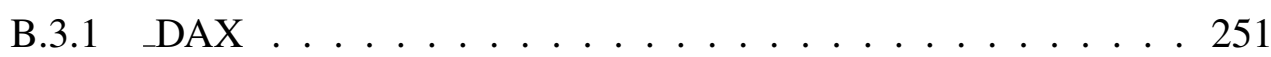

B.3.2_ALL . . . . . . . . . . . . . . . . 252

B.3.3 SIE . . . . . . . . . . . . . . . . 253

B.3.4_DTE . . . . . . . . . . . . . . . . . 254

B.3.5 DAIM . . . . . . . . . . . . . . 255

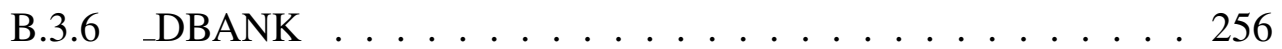

B.3.7_MRE . . . . . . . . . . . . . . . . 257

B.3.8 BAY . . . . . . . . . . . . . . . . 258

B.3.9_VW . . . . . . . . . . . . . . . . . . . 259

B.3.10_BASF. . . . . . . . . . . . . . . 260

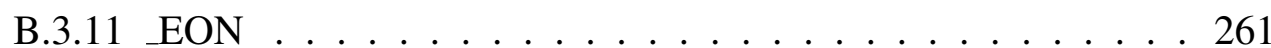

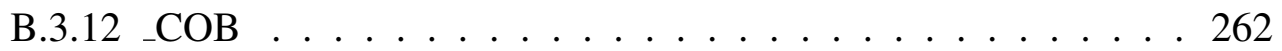

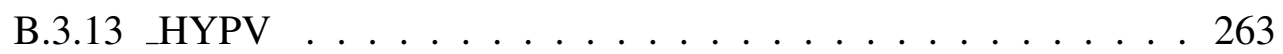

B.3.14 RWE . . . . . . . . . . . . . . . . . . . . 264

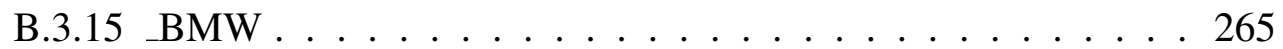

B.3.16_LH . . . . . . . . . . . . . 266

B.3.17_TYSS . . . . . . . . . . . . . . 267

B.3.18_METRO . . . . . . . . . . . . . . . . . 268

B.3.19_TUI . . . . . . . . . . . . . . . . . . . . . 269

B.3.20 LIN . . . . . . . . . . . . . . . . . 270

B.3.21_HEN . . . . . . . . . . . . . . . 271

B.3.22_CONT . . . . . . . . . . . . . . 272

B.3.23 DP . . . . . . . . . . . . . 273

B.3.24_DEG . . . . . . . . . . . . . . . . 274

C Details of the Results of the Empirical Analysis 275

C.1 The Leverage Effect . . . . . . . . . . . . . . . . . . . . . . . 275

C.2 The Credit Spread Level and the Implied Volatility Level . . . . . . . 280

C.3 The Credit Spread Level and the Shape of the Equity Volatility Smile. 285 
C.3.1 Examination of the Relationship between the CDS Spread Level and the Smile Coefficients . . . . . . . . . . . . . 285

C.3.2 Examination of the Relationship between the CDS Spread Level and the Slope of the Smile . . . . . . . . . . . . . . . 295

C.4 The Credit Spread Level and the Moments of the Risk-Neutral Equity Return Density . . . . . . . . . . . . . . . . . . . . . 314

Bibliography 


\section{List of Figures}

$3.1 \quad$ DAX index implied volatility surface . . . . . . . . . . . . . . 19

5.1 Schematic representation of the local volatility surface $\ldots . . . .76$

$6.1 \quad$ Implied volatilities for a high CDS spread and a low CDS spread day for the 6 most important stocks . . . . . . . . . . . . . . . . 118

6.2 Implied volatility smile approximation with polynomials of order 1, 2, 3 and 6 for a typical short term maturity of 26 days . . . . . . . . . 120

6.3 Implied volatility smiles approximated with quadratic polynomials for a high CDS spread and a low CDS spread day for the 6 most important stocks . . . . . . . . . . . . . . . . . 126

$6.4 \quad$ Standardized risk-neutral log return densities for a high CDS spread and a low CDS spread day for the 6 most important stocks . . . . . . 128 



\section{List of Tables}

5.1 Extract of the literature analyzing the causes of the asymmetric volatility phenomenon .................. 70

5.2 Extract of the literature analyzing the relationship between default risk and equity volatility . . . . . . . . . . . . . . . 71

5.3 Compilation of the literature analyzing the relationship between default risk and the slope of the equity volatility smile . . . . . . . 72

5.4 Overview of enhanced reduced form credit risk models incorporating the implied volatility smile . . . . . . . . . . . . . . 87

6.1 Companies considered in the analysis . . . . . . . . . . . . . 109

6.2 Median regression results and statistics for the smoothing of the smiles which have been used for the construction of the 36 days constant maturity variables . . . . . . . . . . . . . . . . . . . . . . 124

$6.3 \quad$ Average minimum and maximum $\frac{X}{S}$ and corresponding $\tilde{R}$ resulting from actual option prices . . . . . . . . . . . . . 132

6.4 Variables in the regression analysis . . . . . . . . . . . . . . 135

6.5 Summary statistics of all cross-sections and all constant maturities pooled together . . . . . . . . . . . . . . 136

6.6 Median smile coefficients of all stock cross-sections and of the DAX index . . . . . . . . . . . . . . . . . . . 137

6.7 Median smile slopes of all stock cross-sections and the DAX index . . 138

6.8 Median smile risk-neutral moments of all stock cross-sections and the DAX index . . . . . . . . . . . . . . . . . . . . . . . 139

6.9 Rounded ADF probabilities of error for the rejection of the null hypothesis of a unit root $\ldots \ldots \ldots$

6.10 Time-series results for the leverage effect hypothesis for a constant maturity of 36 days . . . . . . . . . . . . . . . . . . . . . 149

6.11 Time-series regression results for the leverage effect hypothesis for a constant maturity of 60 days $\ldots \ldots \ldots$ 
6.12 Pooled series regression results for the leverage effect hypothesis for constant maturities of 36 and 60 days . . . . . . . . . . . . . . 151

6.13 Robustness check of the time-series regression results for the leverage

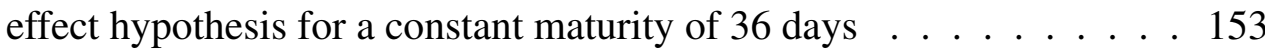

6.14 Robustness check of the time-series regression results for the leverage effect hypothesis for a constant maturity of 60 days . . . . . . . . . 154

6.15 Robustness check of the pooled series regression results for the leverage effect hypothesis for constant maturities of 36 and 60 days . . . . 156

6.16 Time-series regression results for the test of the relationship between default risk and implied volatility for a constant maturity of 36 days . 161

6.17 Cross-sectional averages of time-series regression results for the test of the relationship between default risk and implied volatility . . . . . 162

6.18 Pooled series regression results for the test of the relationship between default risk and implied volatility for constant maturities of 36, 60 and 180 days . . . . . . . . . . . . . . . . . 163

6.19 Robustness check of the time-series regression results for the test of the relationship between default risk and implied volatility for a constant maturity of 36 days . . . . . . . . . . . . . . . 165

6.20 Robustness check of the pooled series regression results for the test of the relationship between default risk and implied volatility . . . . . . 166

6.21 Results of the panel unit root tests for the robustness check of the pooled series regression results for the test of the relationship between default risk and implied volatility . . . . . . . . . . . . . . . 168

6.22 Time-series regression results for the test of the relationship between \begin{tabular}{|c|}
\hline default risk and the smile coefficient $B 0$ for a constant maturity of 36 \\
\hline
\end{tabular}

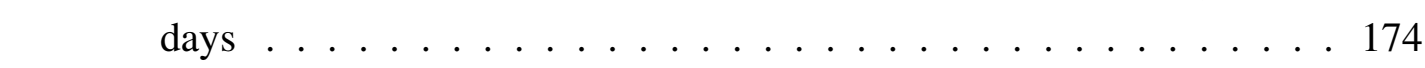

6.23 Time-series regression results for the test of the relationship between \begin{tabular}{|c|}
\hline default risk and the smile coefficient $B 1$ for a constant maturity of 36 \\
\hline
\end{tabular}

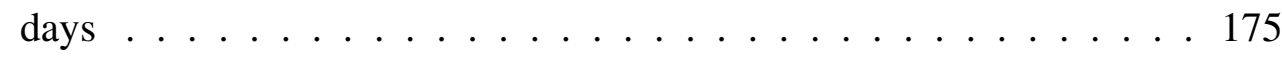

6.24 Time-series regression results for the test of the relationship between default risk and the smile coefficient $B 2$ for a constant maturity of 36

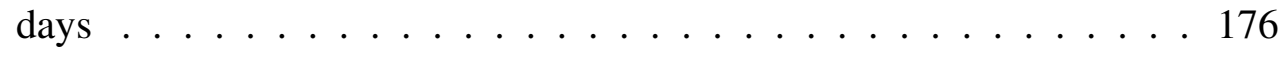

6.25 Cross-sectional averages of the time-series regression results for the test of the relationship between default risk and the smile coefficients. 177

6.26 Pooled series regression results for the test of the relationship between default risk and the smile coefficients . . . . . . . . . . . . . . 178 
6.27 Robustness check of the time-series regression results for the test of the relationship between default risk and the smile coefficients for a

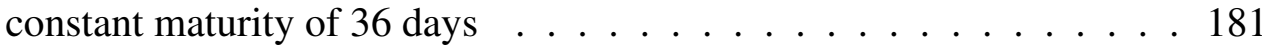

6.28 Robustness check of the pooled series regression results for the test of the relationship between default risk and the smile coefficients . . . . 182

6.29 Time-series regression results for the test of the relationship between default risk and the slope variable $S L P 70$ for a constant maturity of 36 days . . . . . . . . . . . . . . . . . . 185

6.30 Time-series regression results for the test of the relationship between default risk and the slope variable $S L P 90$ for a constant maturity of 36 days . . . . . . . . . . . . . . . 186

6.31 Time-series regression results for the test of the relationship between default risk and the slope variable $S L P 100$ for a constant maturity of 36 days . . . . . . . . . . . . . . . . . . 187

6.32 Time-series regression results for the test of the relationship between default risk and the slope variable $S L P 110$ for a constant maturity of 36 days . . . . . . . . . . . . . . . . . 188

6.33 Time-series regression results for the test of the relationship between default risk and the slope variable $S L P 130$ for a constant maturity of 36 days . . . . . . . . . . . . . . . . . 189

6.34 Time-series regression results for the test of the relationship between default risk and the horizontal coordinate $X S M I N$ for a constant maturity of 36 days $\ldots \ldots \ldots$. . . . . . . . . . . . . . . . . 189

6.35 Cross-sectional averages of time-series regression results for the test of the relationship between default risk and the slope of the smile . . . 190

6.36 Pooled series regression results for the test of the relationship between default risk and slope variables . . . . . . . . . . . . . . 191

6.37 Robustness check of the time-series regression results for the test of the relationship between default risk and the slope of the smile for a constant maturity of 36 days - Part I . . . . . . . . . . . . . . 195

6.38 Robustness check of the time-series regression results for the test of the relationship between default risk and the slope of the smile for a constant maturity of 36 days - Part II . . . . . . . . . . . . 196

6.39 Robustness check of the pooled series regression results for the test of the relationship between default risk and slope variables . . . . . . . . 198 
6.40 Dissecting the default-risk-only effect by eliminating the volatilityonly effect in the test of the relationship between default risk and slope variables . . . . . . . . . . . . . . . . . . . . . . . . . . . . . 199

6.41 Dissecting the volatility-only effect by eliminating the default-riskonly effect in the test of the relationship between equity volatility and slope variables . . . . . . . . . . . . . . . . . . . 201

6.42 Time-series regression results for the test of the relationship between default risk and risk-neutral skewness for a constant maturity of 36 days 206

6.43 Time-series regression results for the test of the relationship between \begin{tabular}{|l|l|}
\hline default risk and risk-neutral kurtosis for a constant maturity of 36 days 207 \\
\hline
\end{tabular}

6.44 Cross-sectional averages of time-series regression results for the test

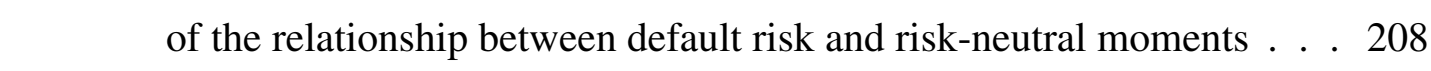

6.45 Pooled series regression results for the test of the relationship between

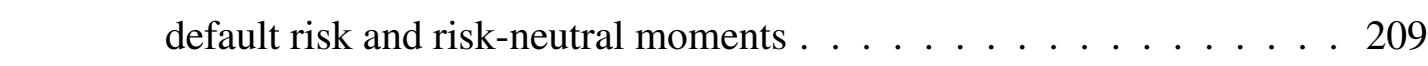

6.46 Robustness check of the time-series regression results for the test of \begin{tabular}{|c|}
\hline the relationship between default risk and the risk-neutral moments for \\
\hline a
\end{tabular} a constant maturity of 36 days . . . . . . . . . . . . . . 212

6.47 Robustness check of the pooled series regression results for the test of

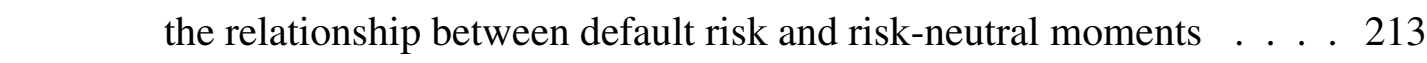

6.48 Dissecting the default-risk-only effect by eliminating the volatilityonly effect in the test of the relationship between default risk and the

\begin{tabular}{|l|l}
\hline only effect in the test of the relationship between default risk and the \\
\hline risk-neutral moments $\ldots \ldots \ldots \ldots \ldots \ldots \ldots \ldots \ldots \ldots \ldots \ldots \ldots \ldots \ldots$
\end{tabular}

6.49 Dissecting the volatility-only effect by eliminating the default-riskonly effect in the test of the relationship between equity volatility and the risk-neutral moments . . . . . . . . . . . . 216

B.1 Median regression results and statistics for the smoothing of the smiles

\begin{tabular}{|c|}
\hline which have been used for the construction of the 60 days constant \\
\hline
\end{tabular}
maturity variables . . . . . . . . . . . . . . . 245

B.2 Median regression results and statistics for the smoothing of the smiles which have been used for the construction of the 180 days constant maturity variables . . . . . . . . . . . . . . . 246

B.3 Median regression results and statistics for the smoothing of the smiles \begin{tabular}{|l|l}
\hline which have been used for the construction of the 240 days constant \\
\hline maturity variables . . . . . . . . . . . . . . . 247
\end{tabular}

B.4 Summary statistics for smile dependent variables with constant maturities of 36 and 60 days (all cross sections pooled together. . . . . . . 249 
B.5 Summary statistics for smile dependent variables with constant maturities of 180 and 240 days (all cross sections pooled together . . . . . 250

B.6 $\quad$ Summary statistics for cross section _DAX . . . . . . . . . . . . 251

B.7 $\quad$ Summary statistics for cross section _ALL . . . . . . . . . . . . 252

B.8 $\quad$ Summary statistics for cross section_SIE. . . . . . . . . . . . . 253

B.9 $\quad$ Summary statistics for cross section _DTE . . . . . . . . . . . . 254

B.10 Summary statistics for cross section_DAIM . . . . . . . . . . . 255

B.11 Summary statistics for cross section DBANK . . . . . . . . . . 256

B.12 Summary statistics for cross section _MRE . . . . . . . . . . . . . 257

B.13 Summary statistics for cross section _BAY . . . . . . . . . . . . 258

B.14 Summary statistics for cross section _VW . . . . . . . . . . . . 259

B.15 Summary statistics for cross section_BASF . . . . . . . . . . . 260

B.16 Summary statistics for cross section EON . . . . . . . . . . . . 261

B.17 Summary statistics for cross section_COB . . . . . . . . . . . . 262

B.18 Summary statistics for cross section_HYPV . . . . . . . . . . . 263

B.19 Summary statistics for cross section _RWE . . . . . . . . . . . . . 264

B.20 Summary statistics for cross section_BMW . . . . . . . . . . 265

B.21 Summary statistics for cross section _LH . . . . . . . . . . . . 266

B.22 Summary statistics for cross section_TYSS . . . . . . . . . . . . 267

B.23 Summary statistics for cross section_METRO . . . . . . . . . . . . . 268

B.24 Summary statistics for cross section _TUI . . . . . . . . . . . . 269

B.25 Summary statistics for cross section _LIN . . . . . . . . . . . 270

B.26 Summary statistics for cross section _HEN . . . . . . . . . . . . . 271

B.27 Summary statistics for cross section_CONT . . . . . . . . . . . . 272

B.28 Summary statistics for cross section_DP . . . . . . . . . . . . 273

B.29 Summary statistics for cross section _DEG . . . . . . . . . . . . . . 274

C.1 Time-series regression results for the leverage effect hypothesis for a constant maturity of 36 days . . . . . . . . . . . . . 276

C.2 Time-series regression results for the leverage effect hypothesis for a constant maturity of 60 days . . . . . . . . . . . . . . . . . . 277

C.3 Robustness check of the time-series regression results for the leverage effect hypothesis for a constant maturity of 36 days . . . . . . . . 278

C.4 Robustness check of the time-series regression results for the leverage effect hypothesis for a constant maturity of 60 days . . . . . . . . . 279

C.5 Time-series regression results for the test of the relationship between default risk and implied volatility for a constant maturity of 36 days . 281 
C.6 Time-series regression results for the test of the relationship between default risk and implied volatility for a constant maturity of 60 days . 282

C.7 Time-series regression results for the test of the relationship between default risk and implied volatility for a constant maturity of 180 days . 283

C.8 Time-series regression results for the test of the relationship between default risk and implied volatility for a constant maturity of 240 days . 284

C.9 Time-series regression results for the test of the relationship between default risk and the smile coefficient $B 0$ for a constant maturity of 36 days . . . . . . . . . . . . . . . . . 286

C.10 Time-series regression results for the test of the relationship between \begin{tabular}{|c|}
\hline default risk and the smile coefficient $B 1$ for a constant maturity of 36 \\
\hline
\end{tabular}

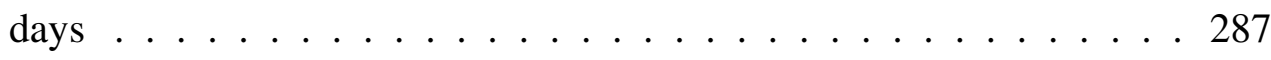

C.11 Time-series regression results for the test of the relationship between default risk and the smile coefficient $B 2$ for a constant maturity of 36 days . . . . . . . . . . . . . . . . . . 288

C.12 Time-series regression results for the test of the relationship between default risk and the smile coefficient $B 0$ for a constant maturity of 60 days . . . . . . . . . . . . . . . . . . . 289

C.13 Time-series regression results for the test of the relationship between \begin{tabular}{|c|}
\hline default risk and the smile coefficient $B 1$ for a constant maturity of 60 \\
\hline
\end{tabular} days . . . . . . . . . . . . . . . . . . 290

C.14 Time-series regression results for the test of the relationship between \begin{tabular}{|c|}
\hline default risk and the smile coefficient $B 2$ for a constant maturity of 60 \\
\hline
\end{tabular} days . . . . . . . . . . . . . . . . . 291

C.15 Time-series regression results for the test of the relationship between default risk and the smile coefficient $B 0$ for a constant maturity of 180

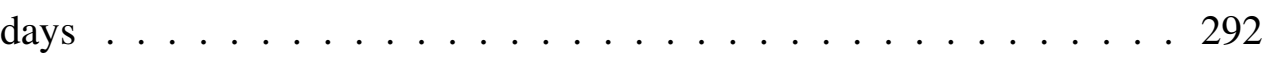

C.16 Time-series regression results for the test of the relationship between default risk and the smile coefficient $B 1$ for a constant maturity of 180 days . . . . . . . . . . . . . . . . . . . 293

C.17 Time-series regression results for the test of the relationship between default risk and the smile coefficient $B 2$ for a constant maturity of 180 days ............................ 294

C.18 Time-series regression results for the test of the relationship between default risk and the slope variable $S L P 70$ for a constant maturity of 36 days . . . . . . . . . . . . . . . . 296 
C.19 Time-series regression results for the test of the relationship between default risk and the slope variable $S L P 90$ for a constant maturity of 36 days . . . . . . . . . . . . . . . . . . . . 297

C.20 Time-series regression results for the test of the relationship between default risk and the slope variable $S L P 100$ for a constant maturity of 36 days . . . . . . . . . . . . . . . . . . . . . . . 298

C.21 Time-series regression results for the test of the relationship between default risk and the slope variable $S L P 110$ for a constant maturity of 36 days . . . . . . . . . . . . . . . . . . 299

C.22 Time-series regression results for the test of the relationship between default risk and the slope variable $S L P 130$ for a constant maturity of 36 days . . . . . . . . . . . . . . . . 300

C.23 Time-series regression results for the test of the relationship between default risk and the horizontal coordinate $X S M I N$ for a constant maturity of 36 days . . . . . . . . . . . . . . . . . . . . 301

C.24 Time-series regression results for the test of the relationship between default risk and the slope variable $S L P 70$ for a constant maturity of 60 days . . . . . . . . . . . . . . . . . . . 302

C.25 Time-series regression results for the test of the relationship between default risk and the slope variable $S L P 90$ for a constant maturity of 60 days . . . . . . . . . . . . . . . . . . 303

C.26 Time-series regression results for the test of the relationship between default risk and the slope variable $S L P 100$ for a constant maturity of 60 days . . . . . . . . . . . . . . . . . . 304

C.27 Time-series regression results for the test of the relationship between default risk and the slope variable $S L P 110$ for a constant maturity of 60 days . . . . . . . . . . . . . . 305

C.28 Time-series regression results for the test of the relationship between default risk and the slope variable $S L P 130$ for a constant maturity of 60 days . . . . . . . . . . . . . . . 306

C.29 Time-series regression results for the test of the relationship between default risk and the horizontal coordinate $X S M I N$ for a constant maturity of 60 days . . . . . . . . . . . . . . . . . . . 307

C.30 Time-series regression results for the test of the relationship between default risk and the slope variable $S L P 70$ for a constant maturity of 180 days . . . . . . . . . . . . . . . . . . . . 308 
C.31 Time-series regression results for the test of the relationship between default risk and the slope variable $S L P 90$ for a constant maturity of 180 days . . . . . . . . . . . . . . . . . . . . . . 309

C.32 Time-series regression results for the test of the relationship between default risk and the slope variable $S L P 100$ for a constant maturity of 180 days . . . . . . . . . . . . . . . . . . 310

C.33 Time-series regression results for the test of the relationship between default risk and the slope variable $S L P 110$ for a constant maturity of 180 days . . . . . . . . . . . . . . . . . . 311

C.34 Time-series regression results for the test of the relationship between \begin{tabular}{|c|}
\hline default risk and the slope variable $S L P 130$ for a constant maturity of \\
\hline
\end{tabular}

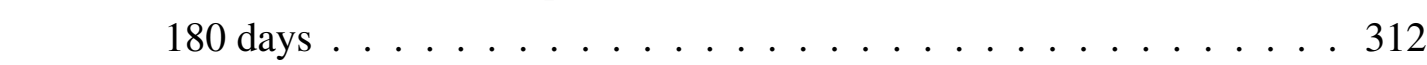

C.35 Time-series regression results for the test of the relationship between default risk and the horizontal coordinate $X S M I N$ for a constant ma-

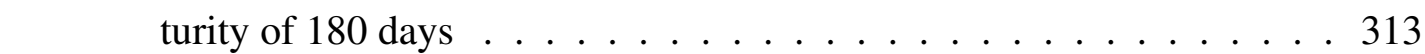

C.36 Time-series regression results for the test of the relationship between default risk and risk-neutral skewness for a constant maturity of 36 days 315

C.37 Time-series regression results for the test of the relationship between default risk and risk-neutral kurtosis for a constant maturity of 36 days 316

C.38 Time-series regression results for the test of the relationship between default risk and risk-neutral skewness for a constant maturity of 60 days 317

C.39 Time-series regression results for the test of the relationship between

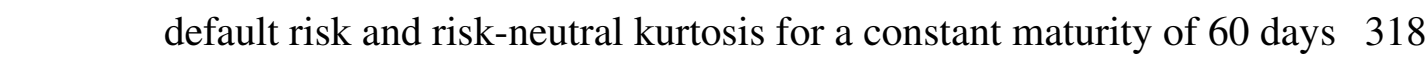

C.40 Time-series regression results for the test of the relationship between default risk and risk-neutral skewness for a constant maturity of 180 days . . . . . . . . . . . . . . . . . . . . . 319

C.41 Time-series regression results for the test of the relationship between default risk and risk-neutral kurtosis for a constant maturity of 180 days 320

C.42 Time-series regression results for the test of the relationship between default risk and risk-neutral skewness for a constant maturity of 240

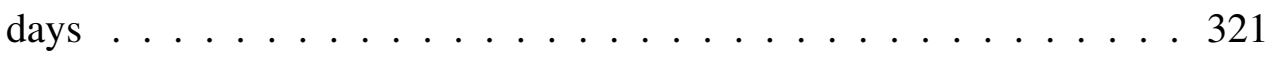

C.43 Time-series regression results for the test of the relationship between default risk and risk-neutral kurtosis for a constant maturity of 240 days 322 


\section{Symbols and Abbreviations}

$\mathrm{ADF}$

$a(t)$

$\alpha$

ATM

$B$

$\hat{B}$

$b$

b

$b(\cdot)$

$\beta$

$\boldsymbol{\beta}$

$c_{t}$

$c_{t}^{B S}$

$\hat{c}_{t}$

$c$

CDS

CEV

$D$
Augemented Dickey-Fuller test for the presence of a unit root

Deterministic function in the specification of the hazard rate $h_{t}$ in an enhanced reduced form model with deterministically changing variance

Speed of mean reversion

At-the-money

Present value of $D$

Present value of $D$, if $D$ were a non-defaultable government bond

Estimate of the coefficient $\beta$ of an independent variable in a regression model

(k x 1)-estimate of the coefficient vector $\boldsymbol{\beta}$ of the independent variables in a regression model

Brownian motion

Coefficient of an independent variable in a regression model

(k x 1)-coefficient vector of the independent variables in a regression model

Value of a call option

Value of a call option according to the Black-Scholes model

Actual market price of a traded call option

Constant in a regression model

Credit default swap

Constant elasticity of variance

Face value of a zero coupon bond 
$d(t) \quad$ Deterministic function in the specification of the hazard rate $h_{t}$ in an enhanced reduced form model with deterministically changing variance

$d_{1} \quad$ Argument of $N(\cdot)$ in the Black-Scholes option pricing formula

$d_{2} \quad$ Argument of $N(\cdot)$ in the Black-Scholss option pricing formula

$\delta \quad$ Exponent in a CEV-type specification of the variance

$D \operatorname{Exp}(\cdot) \quad$ Double-exponential distribution

$D_{H} \quad$ Dummy variable indicating a high level of a certain variable

$D_{L} \quad$ Dummy variable indicating a low level of a certain variable

$d q \quad$ Innovations of a Poisson process

$d w \quad$ Innovations of a Wiener process

$d z \quad$ Innovations of a Wiener process

DW $\quad$ Durbin-Watson test statistic

E Entropy

EDS $\quad$ Equity default swap

$E_{t}^{P}[\cdot] \quad$ Expectation under the physical measure $P$ conditional on the information available up to time $\mathrm{t}$

$E_{t}^{Q}[\cdot] \quad$ Expectation under the risk-neutral measure $Q$ conditional on the information available up to time $\mathrm{t}$

$\epsilon \quad$ Elasticity of the stock return volatility with regard to the stock price

$\eta_{t} \quad$ EBIT-process in Genser's EBIT-model

$\eta_{B} \quad$ EBIT default boundary in Genser's EBIT-model

$\eta_{R} \quad$ Elasticity of the pricing kernel $\Lambda$ with respect to the level of wealth

$F \quad$ F-test statistic in a regression analysis

$\mathcal{F} \quad \sigma$-field

$f^{P} \quad$ Physical stock price or firm value probability density function

$f^{Q} \quad$ Risk-neutral stock price or firm value probability density function

$F^{Q} \quad$ Cumulative risk-neutral probability density function: $F^{Q}=\int d f^{Q}$

$F_{t} \quad$ Derivative value

$\gamma(\cdot) \quad$ Gamma process 
GARCH Generalized autoregressive conditional heteroscedasticity

GLS Generalized least squares method

$\hat{H}(\mathbf{Z}) \quad$ Nadaraya-Watson estimator in a kernel regression

$H(\mathbf{Z}) \quad$ Function to be estimated in a kernel regression

$H_{i} \quad$ Vector of outputs in a kernel regression

$h_{t} \quad$ Jump intensity or hazard rate process

$H(\cdot) \quad$ Payoff function of a derivative

$I(0) \quad$ Stationary process

$I(1) \quad$ Difference-stationary process

i.i.d. Identically, independently distributed

$J_{t} \quad$ Jump size

$K \quad$ kernel weighting function

$k \quad$ Number of independent variables in a regression analysis

$\kappa_{i} \quad$ i-th cumulant of a probability distribution

$\lambda \quad$ Market price of risk

$\Lambda \quad$ Pricing kernel

$L N(\cdot) \quad$ Lognormal probability distribution

$L_{t} \quad$ Leverage ratio

$l$

Argument of $N(\cdot)$ and $N_{2}(\cdot)$ in Geske's compound option pricing formula

$m \quad$ Argument of $N(\cdot)$ and $N_{2}(\cdot)$ in Geske's compound option pricing formula

$\mu \quad$ Drift parameter

$\mu_{b} \quad$ Drift parameter of a Brownian motion in the VG model

$\mu_{\gamma} \quad$ Drift parameter of gamma process in the VG model

$n \quad$ Number of observations in a regression analysis

$N(\cdot) \quad$ Cumulative standard normal density function

$n(\cdot) \quad$ Standard normal density function

$N_{2}(\cdot) \quad$ Bivariate, cumulative standard normal density function 
$N_{t} \quad$ Counting process driven by the jump intensity or hazard rate process $h_{t}$

$\nu \quad$ Variance rate of the gamma process in the VG model

OLS Ordinary least squares method

$\Omega \quad$ Sample space

$\omega \quad$ Constant in the specification of the hazard rate $h_{t}$ in an enhanced reduced-form model

OTM Out-of-the-money

OTC Over-the-counter

$p_{t} \quad$ Value of a put option

$p_{t}^{B S} \quad$ Value of a put option according to the Black-Scholes model

$\hat{p}_{t} \quad$ Actual market price of a traded put option

$\phi^{Q} \quad$ Risk-neutral stock return probability density function

$p_{\tau_{i}} \quad$ Series of regularly spaced price observations

$p_{\tau_{j}} \quad$ Series of actual, irregularly spaced price observations

$\pi_{t_{0}, T}^{W} \quad$ State price, the price in time $t_{0}$ of an Arrow-Debreu security paying 1 unit of payment in time $T$ and in state $W$ and zero otherwise

$p_{t_{0}, T}^{W} \quad$ Physical probability to reach state $W$ in time span between $t_{0}$ and $T$

$P \quad$ Physical probability measure or probability

$q_{t_{0}, T}^{W} \quad$ Risk-neutral probability to reach state $W$ in time span between $t_{0}$ and $T$

$Q \quad$ Risk-neutral probability measure or probability

$Q_{d} \quad$ Risk-neutral default probability

$R \quad$ Log-return

$\bar{R} \quad$ Sample mean of log-return

$R_{t_{0}, T} \quad \tau$-period log-return

$\tilde{R} \quad$ Standardized log-return

$\hat{R} \quad$ Log-return of the DAX-index whose standard deviation has been replaced by that of one of his constituents

$R_{\tau_{i}} \quad$ Series of regularly spaced log-return observations

$r \quad$ Risk-free rate 


\begin{tabular}{|c|c|}
\hline$\rho$ & Correlation coefficient \\
\hline RND & Risk-neutral probability density \\
\hline$S_{t}$ & Stock price \\
\hline$\tilde{S}$ & Median of $S_{t}$ in a data sample \\
\hline$\tilde{S}_{t^{*}}$ & $\begin{array}{l}\text { Value of } S_{t} \text { in Geske's compound option model for which: } \\
\tilde{S}_{t^{*}}(\tilde{V})=X\end{array}$ \\
\hline$s$ & Risk premium \\
\hline$\hat{s}$ & Population standard deviation \\
\hline$\sigma$ & Volatility parameter \\
\hline$\sigma^{*}$ & Absolute, time horizon adjusted volatility: $\sigma^{*}=\sigma \sqrt{\tau}$ \\
\hline$\hat{\sigma}$ & Implied volatility function \\
\hline$\tilde{\sigma}$ & Black-Scholes implied volatility \\
\hline$\sigma_{S_{t_{0}}, t_{0}}(X, T)$ & Local volatility \\
\hline$\Sigma$ & $(\mathrm{kx} \mathrm{k})$-covariance matrix of $\mathbf{b}$ \\
\hline$t$ & time index or t-test statistic \\
\hline$t_{0}$ & Reference point in time \\
\hline$T$ & Maturity point in time \\
\hline$t^{*}$ & Compound option expiry \\
\hline$t_{d}$ & Time of default \\
\hline$\tau$ & Distance between two points in time \\
\hline$\theta$ & Long term mean reversion level \\
\hline$U(\cdot)$ & Utility function \\
\hline$U^{\prime}(\cdot)$ & Marginal utility function \\
\hline$u$ & Error term in a regression model \\
\hline$v(t)$ & $\begin{array}{l}\text { Deterministic function in the specification of the variance in an en- } \\
\text { hanced reduced form model with deterministically changing vari- } \\
\text { ance }\end{array}$ \\
\hline$V_{t}$ & Firm value \\
\hline$V_{B}$ & Default barrier in a structural credit risk model \\
\hline
\end{tabular}


$\tilde{V} \quad$ Value of $V_{t}$ in Geske's compound option model for which: $\tilde{S}_{t^{*}}(\tilde{V})=X$

$\mathbf{V}(\mathrm{n} \times \mathrm{n})$-covariance matrix of the residuals in a regression analysis

$v_{t} \quad$ Variance, $v_{t}=\sigma_{t}^{2}$

$\varsigma \quad$ Volatility of variance in a stochastic volatility specification

$V G(\cdot) \quad$ Variance-gamma process

$V_{t_{0}, T}$ Volatility contract of the Bakshi-Madan method: $V_{t_{0}, T} \equiv E_{t_{0}}^{Q}\left[e^{-r \tau} R_{t_{0}, T}^{2}\right]$

$W \quad$ Particular state of the world

$W_{t_{0}, T}$ Cubic contract of the Bakshi-Madan method: $W_{t_{0}, T} \equiv E_{t_{0}}^{Q}\left[e^{-r \tau} R_{t_{0}, T}^{3}\right]$

$w \quad$ Scaling factor

X $\quad$ Strike price

$\mathbf{X} \quad\left(\right.$ n x 1)-matrix of $\mathbf{x}_{i}^{\prime}$

$\mathbf{x}_{i} \quad(\mathrm{k} \mathrm{x}$ 1)-vector of independent variables in a regression analysis

$x_{t} \quad$ Independent risk factor which is not correlated with $h_{t}$ in the CarrWu enhanced reduced-form model

$\xi \quad$ First jump time of a counting process

$y_{i} \quad$ Dependent variable in a regression analysis

$\mathbf{y} \quad(\mathrm{n} \times 1)$-vector of observations of the dependent variable

Z Particular state of the world

$\zeta \quad$ Parameter in the in the specification of $h_{t}$ in the Carr-Wu enhanced reduced form model

$\mathbf{Z}_{i} \quad$ Vector of inputs in a kernel regression

$Z_{t_{0}, T} \quad$ Quartic contract of the Bakshi-Madan method: $Z_{t_{0}, T} \equiv E_{t_{0}}^{Q}\left[e^{-r \tau} R_{t_{0}, T}^{4}\right]$ 


\section{Introduction}

\subsection{Motivation and Objectives}

Since the introduction of the celebrated Black-Scholes option pricing formula, practitioners and academics alike are concerned with so-called Black-Scholes pricing biases. One widely known manifestation of these pricing biases is the volatility smile phenomenon, which is owed to the fact that the volatility implied in the market prices of options is not constant. Instead - plotted above the option's strike price - the implied volatility describes a more or less U-shaped curve resembling a "smile". The observation of this smile phenomenon challenges the rather restrictive assumptions of the Black-Scholes model and has spurred numerous attempts to explain its existence $\mathrm{T}^{1}$ However, the fundamental causes of the implied volatility smile in general and the individual stock smile in particular are still not well understood.

This lack of comprehension contrasts sharply with the significance of the smile for the valuation of options and also with the ever-growing importance of equity options trading $2^{2}$ Although this impressive growth is accompanied by more and more sophisticated and intricate option pricing models capable of perfectly fitting the implied volatility smile and its term structure, the question as to the true, fundamental determinants of the individual stock smile still remains largely unanswered.

Theoretically, default risk could qualify as a possible, fundamental explanation of the individual stock option smile. Such a connection is assumed to be the consequence of the leverage effect, which links the firm's indebtedness with the volatility of its stock return. In theory, the leverage effect can be shown to produce a convex, monotonically downward sloping volatility smile, which is all the more steeper the higher the level

$1 \quad$ For a survey of the numerous and diverse attempts to explain the equity volatility smile phenomenon, see for example JACKWERTH (2004), pp. 6-9.

2 This growing importance can be read off the growth rate of the number of equity option contracts on individual stocks and on the DAX index traded at EUREX, the Swiss-German derivatives exchange: On average the yearly growth rate in the period from 1998 through to 2006 equaled $26 \%$ (see Derivatives Market Statistics Factbook from Deutsche Börse at www.deutsche-boerse.com) 
of default risk. However, a monotonically downward sloping smile pattern contradicts the evident characteristic U-shaped curve of the individual stock option smile. Already this contradiction calls into question the theoretical prediction of a default risk induced downward sloping smile. Moreover, the empirical substantiation of the existence of the leverage effect is still subject of much debate. And more importantly, empirical evidence of a default risk induced steepening of the smile is extremely scarce. And if existing, extant studies are limited to a very reduced angle of view only focusing on the slope of the smile at or around the at-the-money strike level which reveals the need for further, extensive research in this field.

The leverage effect, suspected of exerting an influence on the individual stock option smile, forms an integral part of Merton's famous firm value model. The firm value setting of this model allows to bridge the gap between the market of the company's debt and that of its equity, and inferences between the respective markets based on Merton's model and its derivatives have meanwhile entered common practice. Recently, the growing interdependence between the market of the firm's equity and that of its debt has been stressed by the advent of a new generation of enhanced credit risk models derived from the class of reduced form credit risk models and conceived for the integrated pricing of credit and equity derivatives. This new model generation has become very popular for the valuation of long standing hybrid products like convertible bonds, which can basically be regarded as the combination of a bond with an equity option. But also the emergence of the very recent, hybrid $\operatorname{EDS}^{3}$ contract exhibiting features of both a credit derivative and an equity option - emphasizes that the market of the company's equity and equity options and that of its debt become growingly intertwined.

But despite this increasing integration of the different markets, their connection is in parts still largely unexplored empirically. While the positive link between the level of implied volatility and that of default risk is well documented, empirical evidence of a connection between the shape of the implied volatility smile and the level of default risk is extremely scarce or even missing at all, as already noted before. This scarcity or even lack of empirical insights contrasts with the increasing abundance of new, enhanced models of the class mentioned above. In accordance with the theoret-

3 EDS stands for equity default swap. An EDS contract is similarly structured as a CDS, except that the default event is defined as a substantial drop (usually 70\%) of the equity price with regard to the equity price level at the initiation of the contract. In exchange for payments in installments the buyer of the contract is entitled to the reception of a prespecified compensation in the event of the equity default. The EDS can be considered to be a mixture of a digital American put and a credit default swap. Cf. e.g. AlBANESE/CHEN (2005) or MEDOVA/SMITH (2006). 
ical implications of the leverage effect, these models generate a default risk induced monotonically downward sloping implied volatility smile. But at the same time, most of them ignore the evident characteristic U-shape of the individual stock option smile and also fail to offer any new theoretical insights beyond the leverage effect on the smile. All this stresses again the necessity and also primacy of further empirical research on the link between the level of default risk and the smile.

The scarcity of extant empirical evidence as well as the weaknesses of even the most recent enhanced models represent a discrepancy as to the potential gain of inferences from the company's equity options to its debt market. If the link between equity options and the smile on the one hand and default risk on the other were better scrutinized, a new quality of inferences would be feasible thanks to the improved exploitation of the rich data source of the organized equity options market. Further, the lead-lag relationship between the equity and the equity options market on the one hand and the debt market on the other ${ }^{4}$ would make high-quality inferences very rewarding, all the more so that the $\mathrm{CDS}_{5}^{5}$ market is currently boosted by average annual growth rates of more than $100 \% 6$

In summary, the motivation for the examination of the link between the equity volatility smile and the level of default risk can be outlined in the following way:

- The ever-growing importance of equity options trading contrasts with the fact that the individual stock option smile still awaits a convincing, fundamental explanation. In fact, financial theory suggests that default risk in the guise of the leverage effect could partly contribute to its explanation and should lead to a convex, monotonically downward sloping smile, which is all the more steeper the higher the level of default risk. However, this theoretical prediction is belied by the evident characteristic U-shaped curve of the smile. And more importantly, empirical evidence concerning the link between the level of default risk

$4 \quad$ Recent studies find evidence of a lead-lag relationship between the equity and the credit default swap market (see CONSIGLI (2004), BYSTRÖM (2005) or NORDEN/WEBER (2004)) and also between the equity and the credit default swap market on the one hand and the bond market on the other (see LONGSTAFF ET AL. (2003) and LONGSTAFF ET AL. (2005)). It is reasonable to assume that the lead-lag relationship does not only exist between the equity and the debt market, but likewise between the equity options and the debt market, since the evidence produced by CONSIGLI (2004) is based on option implied volatilities and since the organized equity options market on the electronic trading platform of the Swiss-German derivatives exchange EUREX is closely connected to the underlying stock market, featuring instantaneous option price reactions to changes of the underlying stock price.

$5 \quad$ Credit default swap

6 The worldwide outstanding credit default swap volume on average more than doubled each year during 2001 and 2006 (see Market Survey Historical Data from the International Swaps and Derivatives Association at www.isda.org). 
Introduction

and the shape of the individual stock option smile is extremely scarce or even lacking at all.

- The growing integration of the company's debt and its equity and equity options market is paralleled by an increasing potential gain thanks to prospective high-quality inferences from one market to the other. However, extant models bridging the gab between the equity options and the debt market are all based on the theoretical, yet apparently simplistic and poorly evidenced prediction of a default risk induced downward sloping smile. Without a deepened understanding of the relationship between the volatility smile and the level of default risk extant models cannot be developed further and the potential gain of inferences from one market to the other cannot be fully unlocked.

These findings directly lead to the two central questions of this thesis: Is there really a connection between the individual stock option smile and the level of default risk? And if such a relationship is existent, what exactly is the nature of this link? How these questions will be addressed is the subject of the next section.

\subsection{Structure of the Analysis}

The analysis of the above questions is partitioned in a theoretical, primarily literaturebased part, comprising the chapters 2 to 5 , and an empirical part, coinciding with chapter 6

The theoretical part approaches the above research questions by compiling and connecting existing theoretical views and extant empirical evidence regarding the relationship between the implied volatility smile and the level of default risk. Whereas the empirical part derives and empirically tests hypotheses as to this relationship by building on the insights gained in the theoretical part.

Chapter 2 starts out with a discussion of some theoretical fundamentals which will be essential throughout the whole thesis. These fundamentals are the ubiquitous principle of risk-neutral valuation and the role of risk aversion as a link between the physical and the option-implied, risk-neutral probability density.

Chapter 3 then introduces the concept of the implied volatility smile and describes the static features of the smile phenomenon. Chapter 3 further examines the relationship between option prices, the implied volatility smile and the option-implied, risk-neutral density. The subsequent survey of the available statistical techniques for 
the smoothing of the implied volatility smile and the extraction of the risk-neutral density concludes with their comparative, critical appraisal, whose results will still be helpful in the empirical part later on.

Based on the description of the volatility smile phenomenon in chapter 3 , chapter 4 examines its most common explanations which are not explicitly related to default risk. These can be distinguished into phenomenological and causal approaches. While the first center on option pricing models trying to statically replicate the volatility smile and its term structure, the latter advance rather fundamental explanations of the smile phenomenon. Both approaches will be analyzed as to their capacity to offer a convincing and preferably also fundamental explanation of the implied volatility smile in general and the individual stock option smile in particular.

While chapter 4 concentrates on explanations of the smile which are not related to default risk, chapter 5 explicitly treats the link between default risk and the individual stock option smile. Section 5.1 as the first part of chapter 5 explains the theoretical relationship between default risk and the risk-neutral return density as well as between the former and the implied volatility smile by recurring to the fundamentals of credit risk modeling as well as to the leverage effect relationship. These theoretical implications of default risk for the individual stock option smile are subsequently confronted with the static features of the smile phenomenon as described in chapter 3 . The theoretical examination of the impact of default risk on the smile is complemented by a survey and an assessment of the corresponding extant empirical research. Section 5.2 as the second part of chapter 5 presents the class of enhanced credit risk models which are able to incorporate equity options and to replicate the volatility smile phenomenon. Similar to traditional credit risk models, this class of models can be partitioned into exponents based on the structural firm value setting on the one hand and into the more recent strand of models derived from the reduced form approach on the other. Finally, it will be examined in how far these enhanced models can contribute any new, relevant insights beyond the theoretical connection between default risk and the smile as already illustrated in the first part of chapter 5 .

The empirical part, coinciding with chapter 6 , is devoted to the analysis of the relationship between default risk and the smile and starts with the derivation of 4 hypotheses grounded on the theoretical and empirical insights gained in the literature-based first part of this thesis. These hypotheses involve the leverage effect relationship, further the link between the level of default risk and that of implied volatility, moreover the relationship between the level of default risk and the smile and finally the connection between the level of default risk and the shape of the risk-neutral density. It ensues 
a sketch of the statistical techniques applied throughout the empirical analysis, which is followed by a description of the data processing from the raw data sources to the ready to use regression variables. The data sample stretches across the 3 year period from the beginning of 2002 to the end of 2004 and comprises those 23 DAX companies for whom sufficient data is available during the 3 year period covered by the analysis. While the level of default risk is proxied by the CDS spread level, variables describing the smile and the risk-neutral density are derived based on the techniques recommended in chapter 3 . The section illustrating the data processing concludes with a discussion of the summary statistics of the regression variables, allowing a first challenge of the 4 hypotheses. The statistical test of the hypotheses is treated in section 6.5 .

Chapter 7 finally concludes with a summary of the various results of the theoretical and the empirical part of this thesis and discusses possible implications for practice and further research.

Before progressing further, some technicalities need to be clarified: First, for ease of notation a possible role of dividends is completely ignored in all firm value or stock price process specifications of the theoretical part. However, this is not true for the empirical part. There, dividends are certainly considered in the context of the computation of implied volatilities. Secondly, the existence of a probability space $(\Omega, \mathcal{F}, P)$ and $(\Omega, \mathcal{F}, Q)$ consisting of a sample space $\Omega$, a $\sigma$-field $\mathcal{F}$ of subsets of $\Omega$ and a physical probability measure $P$ and a risk-neutral measure $Q$, respectively, is presupposed everywhere throughout this document in the context of stochastic process specifications. Further, every stochastic variable is assumed to be adapted to a filtration $\left(\mathcal{F}_{t}\right)_{t>0}$ on its particular probability space. However, the repeated mentioning of these premises is likewise omitted for ease of notation. And thirdly, symbols are only explained when mentioned the first time, otherwise the reader may refer to the list of symbols and abbreviations.

\subsection{Summary of the Main Results of the Analysis}

The main finding of the literature-based part consists of the theoretical prediction of a default risk induced left-skewness of the risk-neutral stock return density. Carried over to the smile, this left-skewness is supposed to manifests itself in a convex, monotonically downward sloping implied volatility smile, which is assumed to be all the more steeper, the higher the level of default risk. However, it is also shown that this theo- 
retical prediction contrasts with the observation of a generally $U$-shaped smile pattern for individual stock options and that empirical evidence supporting this prediction is extremely scarce, if not missing at all.

The cardinal contribution of the empirical part comprises the insight that the impact of rising default risk on the risk-neutral stock return density can be decomposed into a default-risk-only effect on the one hand and a volatility-only effect on the other. The default-risk-only effect on the risk-neutral density is that of a rising level of default risk, as measured by the CDS spread level, if the level of equity volatility is held constant. Whereas the volatility-only effect on the risk-neutral density is that of a rising level of equity volatility, if the CDS spread level is kept unchanged. While the default-risk-only effect leads to increasing left-skewness and increasing kurtosis of the risk-neutral equity return density, the volatility-only effect entails decreasing risk-neutral kurtosis. Both effects together imply a negative relationship between the level of default risk on the one hand and the level of risk-neutral return skewness and kurtosis on the other. This shows that with regard to the level of risk-neutral kurtosis, the default-risk-only effect seems to be dominated by the volatility-only effect.

Carried over to the smile behavior, the default-risk-only effect implies a steepening of both wings of the U-shaped smile, whereas the volatility-only effect involves a flattening and right-translation of the smile. Again, the volatility-only effect dominates the default-risk-only effect in that both effects in union lead to a default risk induced flattening and right-translation of the smile.

While the default-risk-only effect has been partly predicted by the literature, the volatilityonly effect and its interplay with the default-risk-only effect seem to be completely unknown. But also the default-risk-only effect of increasing risk-neutral kurtosis and of a corresponding steepening of the right wing of the smile is largely ignored in the literature.

Comparable empirical studies are extremely scarce and are all limited to the link between the level of default risk and the slope of the smile at the at-the-money strike level. Although they all find weak evidence of a default risk induced, local steepening of the smile and from this observation deduce a global steepening, the empirical analysis of this thesis shows something else: While the smile horizontally shifts to the right, its steeper descending left wing passes through the at-the-money point, thus creating the local impression of a steepening of the smile, whereas in reality it globally flattens, thanks to the dominating volatility-only-effect. 



\section{Financial Valuation Theory}

The present chapter focuses on key concepts of the financial valuation theory which are relevant for the understanding of the literature-based and the empirical part of this thesis. This is first and foremost the principle of risk-neutral valuation, which is important for option pricing, the description of the stock price dynamics, the explanation of the volatility smile and the derivation of the moments of the risk-neutral equity price density in the empirical part. The second domain of interest of this section is the role of risk aversion as a link between the physical and the risk-neutral probability density. This relationship will still be relevant for the explanation of the volatility smile phenomenon and the interpretation of the results of the empirical part.

\subsection{Risk-Neutral Valuation}

In the following, the general principle of risk-neutral valuation will be introduced briefly. It will then be discussed shortly, under which circumstances markets are complete or incomplete and what this entails for the concept of risk-neutral valuation.

\subsubsection{The Concept of Risk-Neutral Valuation}

If the physical probability measure governing the process and distribution of an asset price as well as the corresponding risk premium were known, the valuation of a claim contingent on this asset would be straightforward. Be $t_{0}, T$ two points in time with $T>t_{0}$, be further $S_{t}$ the underlying asset, $F_{t}$ the value of a claim contingent on $S_{t}$, let $H\left(S_{T}\right)$ specify a certain payoff-function, be $P$ the physical probability measure related to $S_{T}, r$ the continuously compounded risk-free rate, $s$ the risk premium 
and finally $E^{P} t_{0}[\cdot]$ the expectation with respect to $P$ conditional on the information available until time $t$; then $F_{t_{0}}$ can be valued in the following form 7 :

$$
F_{t_{0}}=e^{-(r+s)\left(T-t_{0}\right)} E_{t_{0}}^{P}\left[H\left(S_{T}\right)\right]
$$

Expression 2.1 states that the value of the claim equals the expectation of the future payoff under the physical probability measure discounted at the risk-adjusted rate.

Often however, the risk premium is unknown and therefore the above expression cannot be evaluated. Then it can be convenient to use a probability measure $Q$, equivalent to $P^{8}$, which makes the discounting with the risk-adjusted rate $r+s$ dispensable. Instead, the risk-free rate can be used and risk-aversion is now encapsulated in the equivalent measure $Q$, which leads to the following expression for $F_{t_{\mathrm{d}}}$ ?;

$$
F_{t_{0}}=e^{-r\left(T-t_{0}\right)} E_{t_{0}}^{Q}\left[H\left(S_{T}\right)\right]
$$

The approach of expression 2.2 is called risk-neutral valuation, since discounting at the risk-free rate amounts to assuming that market participants are risk-neutral, although in reality they are not. The change from the physical measure $P$ to the equivalent measure $Q$ ensures that discounting at the risk-free rate still yields the correct value for $F_{t_{0}}$.

An equivalent probability measure $Q$ is also called a martingale or risk-neutral measure $^{10}$. If and only if such a risk-neutral measure $Q$ exists, the market is free of arbitrage ${ }^{11}$. This equivalence is called the First Fundamental Theorem of Finance ${ }^{12}$.

\subsubsection{Risk-Neutral Valuation and Market Completeness}

The financial market is said to be complete, if there exist as many traded assets as there are corresponding random sources ${ }^{13}$. If and only if the market is complete, there

Cf. ZIMMERMANN (1998), p. 162.

8 The probability measure $Q$ is said to be equivalent to the measure $P$, if and only if for each measurable event $A$ : $Q(A)=0 \Leftrightarrow P(A)=0$ (cf. for example CONT/TANKOV (2004), p. 297 or FÖLLMER/SCHIED (2002), p. 6).

Cf. ZIMMERMANN (1998), p. 162

See BJÖRK (2004), p. 8

See HARRISON/KREPS (1979).

See BJÖRK (2004), p. 29.

See MUSIELA/RUTKOWSKI (2005), Proposition 8.2.1., p. 300. In the case of discrete states of the world, completeness is characterized by the existence of as many linearly independent assets as there are states of the world, which is illustrated by KASERER (1993) for example, pp. 30-32. 
exists only one unique equivalent risk-neutral measure $Q^{14}$. This equivalence is called the Second Fundamental Theorem of Finance 15

Conversely, if there are not as many traded securities as there are risk factors, the market is incomplete which renders the valuation of contingent claims ambiguous, since different risk-neutral measures $Q$ will all yield different values for $F_{t_{0}}$. Incompleteness of the market is probably rather the rule than an exception and occurs, if certain risk factors are not traded at all or if only derivatives of these risk factors are traded. Examples of not directly traded sources of risk which lead to market incompleteness are interest rates, electricity, state variables like for example temperature ${ }^{16}$ as well as jump ${ }^{17}$ or volatility ${ }^{18}$ risks in the underlying process.

But although markets are frequently presumed to be incomplete, modern option pricing based on the Black-Scholes model relies on the possibility of replication, which is a sufficient condition for market completeness ${ }^{19}$. As will still be seen, the BlackScholes approach is grounded on the assumption that the option can be perfectly replicated by a portfolio consisting of the underlying and the risk-free account ${ }^{20}$. But however useful this assumption might be to derive an unambiguous option value, it also implies that options are completely redundant and thus cannot contribute to market completeness. Still, the improvement of risk allocation thanks to market completion is often presumed to represent a constituent function of option market ${ }^{21}$. A market completing effect of options on the one hand and their redundancy if priced by replication on the other is an obvious contradiction 22

Risk-neutral valuation is possible in complete and incomplete markets alike, but the role of preferences is different in the two cases. Should the market be complete and perfect replication be possible by just trading the underlying of the claim, then each claim can be valued without the knowledge of preferences. In this case the drift of

See HARRISON/PLISKA (1981).

15 See BJÖRK (2004), p. 33

16 ZIMMERMANN (2003) treats the question, under which conditions claims on non-traded variables like interest rates, electricity or temperature can be priced.

17 See CONT/TANKOV (2004), chapter 9.2.

18 Cf. FOUQUE ET AL. (2000), chapter 2.

19 A complete market is a necessary condition for the fact that each contingent claim can be replicated by a self-financing portfolio involving the underlying, see BJÖRK (2004), p. 111.

20 Cf. HULL (2006), pp. 291-292.

$21 \operatorname{ROSS}(1976)$ for instance holds that options are completing the market and KASERER (1993) also argues in favor of the market completing function of options by providing a simple example of a non-replicable option protection against an exogenously driven downside move of the underlying (p. 85).

22 This contradiction has first been addressed by HAKANSSON (1979) who termed it "The catch 22 of option pricing". 
the process of the underlying can be substituted with the risk-free rate ${ }^{23}$ On the other hand, should the market be incomplete and perfect replication be impossible, then preferences matter and the drift no longer equals the risk-free rate. Instead, risk aversion has to be considered in the specification of the drift of the underlying's process ${ }^{24}$. This can be achieved by estimating the market's risk aversion from the prices of traded derivatives ${ }^{25}$. In this respect derivatives are indeed able to complete a virtually incomplete market ${ }^{26}$.

\subsection{The Role of Risk Aversion as a Link between Physical and Risk-Neutral Probability Density}

The degree of risk aversion can be inferred from the comparison of physical and riskneutral probabilities. While physical probabilities are the statistical probabilities of occurrence, associated with the physical probability measure $P$, risk-neutral probabilities are related to the risk-neutral measure $Q^{27}$.

The risk-neutral probability $q_{t_{0}, T}^{W}$ to reach state $W$ in time $T$ can be expressed as the state price $\pi_{t_{0}, T}^{W}$ compounded at the risk-free rate ${ }^{28}$,

$$
q_{t_{0}, T}^{W}=e^{r\left(T-t_{0}\right)} \pi_{t_{0}, T}^{W}
$$

The state price $\pi_{t_{0}, T}^{W}$ in turn is the price in time $t_{0}$ of a hypothetical security which pays one unit of payment if state $W$ occurs in time $T$ and zero otherwise ${ }^{29}$. Such a hypothetical security is also called an Arrow-Debreu security ${ }^{30}$.

23 See ZIMMERMANN $(1998)$, p. 164.

24 See ZIMMERMANN (1998), p. 158, for the case of a single, but non-traded source of randomness and for example PAN (2002) for not directly traded volatility and jump risks in the process of the underlying.

25 For a derivative $F_{t}$ to be priced in a market where perfect replication by trading in the underlying is impossible, the market price of risk, $\lambda$, can be inferred from a benchmark derivative $G_{t}$ via the relationship $\lambda=\frac{\mu_{G}-r}{\sigma_{C}}$ (see BJÖRK (2004), p. 210).

26 Cf. also for example ZIMMERMANN (1998), pp. 176-177.

27 See ZIMMERMANN (1998), pp. 35-36.

28 See ZIMMERMANN (1998), p. 29.

29 See ZIMMERMANN (1998), pp. 11-16.

30 Securities paying one unit of payment if state $W$ occurs and zero otherwise are also called ArrowDebreu securities, because they represent a fundamental building block of the state preference theory introduced by ARROW (1953) and DEBREU (1959). 
Further, the relationship between physical and risk-neutral probability can be expressed in terms of the state-price-deflator or pricing kernel $\left.\Lambda_{t_{0}, t}^{W}\right]^{31}$

$$
\Lambda_{t_{0}, T}^{W}=e^{-r\left(T-t_{0}\right)} \frac{q_{t_{0}, T}^{W}}{p_{t_{0}, T}^{W}}=\frac{\pi_{t_{0}, T}^{W}}{p_{t_{0}, T}^{W}}
$$

The pricing kernel can be interpreted as an investor's readiness to pay for an ArrowDebreu security after normalization by the statistical probability of occurrence of state $W$ and after correction for the time-value of money. $\operatorname{Be} S_{T}(W)$ and $S_{T}(Z)$ the values of an asset or of the wealth of an investor in dependence of the states $W$ and $Z$ with $S_{T}(W)<S_{T}(Z)$. Then, if the pricing kernel of state $W$ is higher than that of state $Z$ and allowing continuous states 32 , the investor is attributing a higher marginal utility to state $W$ than to state $Z: U^{\prime}\left(S_{T}(W)\right)>U^{\prime}\left(S_{T}(Z)\right)$. Given a concave utility function $U\left(S_{T}\right)$, this also implies a higher risk aversion with regard to state $W$ than to state $Z$. On the other hand, if the pricing kernel is the same in all states irrespective of their level of wealth, physical and risk-neutral probability coincide and investors are indifferent to risk 33 .

For continuous states and in the optimum with regard to an investor's expected utility of current and future consumption, the elasticity of the pricing kernel with respect to the level of his wealth equals the negative Arrow-Pratt measure of relative risk aversion $\eta_{R}\left(S_{T}\right)$ 34,

$$
\frac{d \ln \left(\Lambda_{t_{0}, T}\right)}{d \ln \left(S_{T}\right)}=-\eta_{R}\left(S_{T}\right)
$$

Aggregated across all investors, the elasticity of the pricing kernel with respect to the level of aggregated wealth equals the negative average risk aversion.

In the optimum and with continuous states, the pricing kernel $\Lambda_{t_{0}, T}^{W}$ can also be expressed in terms of the scaled marginal utility, where $w$ represents a constant scaling factor 35 .

$$
\Lambda_{t_{0}, T}=\frac{U^{\prime}\left(S_{T}\right)}{w}
$$

The above remarks show that the comparison of physical and risk-neutral measures conveys important information about the market's risk aversion. This is not only true for the expectation of the risk aversion with regard to all possible states of an asset,

\footnotetext{
See ZimMERMANN (1998), p. 37.

And a differentiable utility function $U(\cdot)$

Regarding the whole paragraph, see ZIMMERMANN (1998), pp. 37-38.

See ZIMMERMANN (1998), pp. 52-60.

See JACKWERTH (2004). In a one-period model, the scaling factor $w$ can be interpreted as the marginal utility of the initial level of wealth: $w=U^{\prime}\left(S_{t_{0}}\right)$ (Cf. ZIMMERMANN (1998), p. 59.)
} 
but also for the risk aversion with respect to certain, specific states of this asset. While the former represents a global risk aversion averaged across possible states, the latter can reveal state specific peculiarities.

The concept of inferring risk aversion from the comparison of physical and riskneutral measure applies to discrete as well as to continuous states. Carried over to the case of continuous states, the counterparts of $p_{t_{0}, T}$ and $q_{t_{0}, T}$ are the terminal physical and risk-neutral probability density functions $f^{P}\left(S_{T}\right)$ and $f^{Q}\left(S_{T}\right)$ which will still be of interest later. 


\section{Option Prices, Volatility Smile, and Risk-Neutral Density}

This chapter introduces the phenomenon of the volatility smile and its relationship with option prices and the risk-neutral density. The chapter also highlights a brief sketch of the prevalent techniques to recover the volatility smile and the risk-neutral density from option prices.

\subsection{The Volatility Smile Phenomenon}

\subsubsection{The Black-Scholes Model}

To ease the understanding of the volatility smile phenomenon, it is necessary to briefly review the Black-Scholes option pricing model.

The Black-Scholes model applies to the valuation of European options on an underlying $S_{t}$ which does not pay dividends. The process for $S_{t}$ is assumed to follow a geometric Brownian motion where $d z_{t}^{P}$ describes a Wiener process under the physical measure $P^{36}$.

$$
d S_{t}=\mu S_{t} d t+\sigma S_{t} d z_{t}^{P}
$$

According to Ito's Lemma, the process of a claim $F_{t}$ contingent on $S_{t}$ can be represented in the following way:

$$
d F_{t}=\left(\frac{\partial F_{t}}{\partial S_{t}} \mu S_{t}+\frac{\partial F_{t}}{\partial t}+\frac{1}{2} \frac{\partial^{2} F_{t}}{\partial S_{t}^{2}} \sigma^{2} S_{t}^{2}\right) d t+\frac{\partial F_{t}}{\partial S_{t}} \sigma S_{t} d z_{t}^{P}
$$

As can be seen, $S_{t}$ and $F_{t}$ both depend on the same source of randomness, $d z_{t}^{P}$, which can be eliminated by constructing a portfolio $\Pi$ consisting of a short position of 1 unit

36 The derivation of the Black-Scholes pricing formulas follows largely HULL (2006), pp. 291-292 and p. 295. 
of $F_{t}$ and a long position of $\frac{\partial F_{t}}{\partial S_{t}}$ units of $S_{t}$. In an infinitesimal small time interval the change in the value of this portfolio is given by the following expression:

$$
d \Pi_{t}=-d F_{t}+\frac{\partial F_{t}}{\partial S_{t}} d S_{t}=\left(-\frac{\partial F_{t}}{\partial t}-\frac{1}{2} \frac{\partial^{2} F_{t}}{\partial S_{t}^{2}} \sigma^{2} S_{t}^{2}\right) d t
$$

Since the change in the portfolio value does not involve the term $d z_{t}^{P}$ any more, it is instantaneously riskless and thus can only earn the risk-free rate $r$ :

$$
d \Pi_{t}=r \Pi d t
$$

Substituting $\Pi_{t}$ by $-F_{t}+\frac{\partial F_{t}}{\partial S_{t}} S_{t}$ and $d \Pi$ from equation 3.3 yields the Black-Scholes partial differential equation ${ }^{37}$.

$$
\frac{\partial F_{t}}{\partial t}+r S_{t} \frac{\partial F_{t}}{\partial S_{t}}+\frac{1}{2} \frac{\partial^{2} F_{t}}{\partial S_{t}^{2}} \sigma^{2} S_{t}^{2}=r F_{t}
$$

This partial differential equation is a general expression and can be used to value diverse derivatives contingent on the terminal state of $S_{t}$ subject to their specific boundary conditions. Be $T$ the time at the option's expiration and $X$ the exercise price, then the boundary condition for a European call is:

$$
F_{T}=\left(S_{T}-X\right)^{+}
$$

And the boundary condition for a European put is:

$$
F_{T}=\left(X-S_{T}\right)^{+}
$$

Based on these boundary conditions, BLACK/SCHOLES (1973) derive the following closed form solutions to the above partial differential equation for the time $t$ value of a European call $c_{t_{0}}^{B S}$ and of a European put $p_{t_{0}}^{B S 38}$,

$$
\begin{gathered}
c_{t_{0}}^{B S}=S_{t} N\left(d_{1}\right)-X e^{-r\left(T-t_{0}\right)} N\left(d_{2}\right) \\
p_{t_{0}}^{B S}=-S_{t} N\left(-d_{1}\right)+X e^{-r\left(T-t_{0}\right)} N\left(-d_{2}\right)
\end{gathered}
$$

\footnotetext{
37 This equation is called Black-Scholes partial differential equation, because it was first used by BLACK/SCHOLES (1973) (see NEFTCI (2000), p. 297.)

38 BLACK/SCHOLES $(1973)$ derive the closed form solutions to the Black-Scholes partial differential equation by recurring to the Fokker-Planck equation.
} 
where

$$
\begin{gathered}
d_{1}=\frac{\ln \left(\frac{S_{t}}{X}\right)+\left(r+\frac{\sigma^{2}}{2}\right)\left(T-t_{0}\right)}{\sigma \sqrt{T-t_{0}}} \\
d_{2}=d_{1}-\sigma \sqrt{T-t_{0}}
\end{gathered}
$$

$N(\cdot)$ denotes the cumulative probability function of the standardized normal distribution.

The portfolio $\Pi_{t}$ consisting of a position in the underlying $S_{t}$ and in the derivative $F_{t}$ has to be refinanced at the risk-free rate. Consequently, the derivative $F_{t}$ can be replicated by a position in the underlying and in the risk-free account. For the replication of the claim to hold at any point in time, the position in the underlying and in the risk-free account has to be rebalanced continuously 39 . As stated in section 2.1.2. the possibility of replicating a contingent claim is a sufficient condition for market completeness, which is a key assumption of the Black-Scholes model.

Another important assumption in the context of the volatility smile phenomenon is the constant volatility $\sigma$ in the process of the underlying $S_{t}$.

\subsubsection{The Shortcomings of the Black-Scholes Model and the Volatility Smile Phenomenon}

All in all the Black-Scholes model imposes very restrictive assumptions on the market microstructure and more importantly on the process of the underlying. Not only does the model solely permit a diffusion with a single source of normally distributed random increments, it also requires the volatility to be constant.

A convenient way to test the Black-Scholes assumption of a constant volatility consists in the calculation of implied volatilities. Be $c_{t_{0}}^{B S}\left(S_{t}, X, T, t_{0}, \sigma, r\right)$ the BlackScholes call option pricing function according to equation 3.8 . Since all parameters except the volatility can easily be observed, the unobservable volatility parameter can be inferred from prices of traded options. This so-called implied volatility $\tilde{\sigma}$ is the volatility which equates the function $c_{t_{0}}^{B S}$ with the price $\hat{c}_{t_{0}}$ of a traded option: $c_{t_{0}}^{B S}\left(S_{t}, X, T, t_{0}, \tilde{\sigma}, r\right)=\hat{c}_{t_{0}}{ }^{40}$. For ease of notation this reasoning has only been shown for call options, although it is naturally also valid for put options.

39 See Musiela/RutKowsKi (2005), p. 93.

40 Cf. FENGLER (2005), p. 19. LATANÉ/RENDLEMAN (1976) were the first to imply the volatility parameter from option prices by inverting the Black-Scholes formula. 
Unfortunately, the closed-form Black-Scholes formula does not apply to the valuation of individual stock options, which are in most of the cases path-depending American options. In this case, the underlying's process of a geometric Brownian motion can be modeled with a binomial tree instead $\sqrt{41}$. For the inference of the volatility implied by an American option, the function $c_{t_{0}}^{B S}$ then simply has to be replaced by the binomial tree.

According to the assumptions of the Black-Scholes model, the implied volatility should be constant throughout time and independent of the option's strike price or its maturity. This however is not the case. Instead, the implied volatility changes through time ${ }^{42}$ and exhibits a certain characteristic shape if plotted against different strikes or maturities of options on the same underlying with otherwise equal attributes.

A plot of volatilities against strike prices is generally called a volatility smile ${ }^{43}$, due to its largely U-shaped pattern. If the smile pattern is rather downward sloping and asymmetric than U-shaped, the smile is also referred to as a skew ${ }^{44}$. Often, implied volatilities are not only plotted against the options' strike, but simultaneously against their maturities. This 3-dimensional representation is labeled a volatility surface 45 , A 2-dimensional visualization of the evolution of the smile in the maturity dimension will be referred to throughout this thesis as the term structure of the smile.

Already soon after the introduction of the Black-Scholes model, practitioners and academics alike observed Black-Scholes pricing biases for the equity option market of diverse and also changing forms 46 , hinting at flaws in the Black-Scholes assumptions. But compared to the dramatic change of the pattern of the equity index smile after the market crash of 1987, these biases of the early days of organized option trading were of rather minor significance. While the equity index smile was more or less fluctuating

41 See HULL (2006), pp. 391-398 for the construction of binomial trees permitting the evaluation of American options.

42 Early evidence of this is provided by MACBETH/MERVILLE (1979).

43 See Hull (2006), p. 375.

44 Cf. Hull (2006), p. 380.

45 See FENGLER (2005), p. 19.

46 Already for the very early days of option trading on the CBOE BLACK (1975) mentions so-called strike price biases. But inconsistent with nowadays smile patterns, he reports that OTM calls are overpriced and ITM calls are underpriced relative to their respective Black-Scholes values.

MERTON (1976) refers to the practitioners' claim that deep ITM and OTM as well as short maturity options seem to be overpriced relative to the Black-Scholes valuation.

One of the first to thoroughly document Black-Scholes pricing biases is RUBINSTEIN (1985), who focuses on individual stock options and finds various pricing biases which change direction between different time periods. 
around a flat line before the crash, it suddenly became a pronounced skew afterwards ${ }^{47}$ and has remained like this since.

A typical nowadays implied volatility surface for the DAX equity index can be viewed in figure 3.1 .

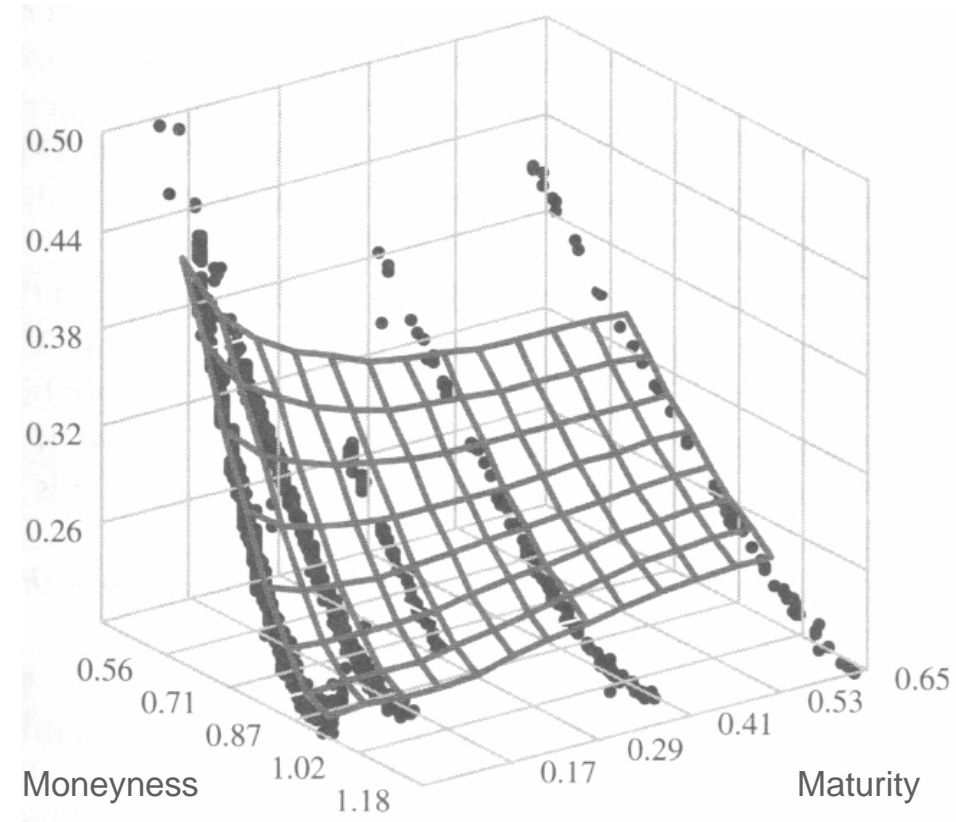

Figure 3.1.: DAX index implied volatility surface ${ }^{a}$

$\bar{a}$ Taken from FENGLER (2005), p. 2. The lower left axis designates moneyness, measured as the ratio of the strike price to the forward price, the lower right axis maturity, measured in years, and the dots signify observations of implied volatility.

The pattern of the index volatility surface visible in figure 3.1 can be summarized by the following 4 stylized facts 48 .

- The smile flattens with increasing maturity. However, it does not seem to completely flatten out which is a mystery, since this would contradict the central limit theorem 49

47 This has first been noted by RUBINSTEIN (1994), who ascribes this crash-related change in the smile pattern to what he calls "crashophobia".

48 See Fengler (2005), pp. 30-32. and cf. ReBOnATO (2004), p. 206.

49 With increasing maturity, the shape of the implied risk-neutral return distribution should more and more resemble a normal distribution due to the central limit theorem. CARR/WU (2003a) however find that even for maturities up to 2 years, the equity index smile does not flatten out. They use this observation as a justification for a model of the index price process which deliberately violates the central limit theorem. 
- The valley of the volatility surface is slightly at the right hand side of the ATM point.

- The smile is asymmetric, which means that OTM puts yield higher implied volatilities than OTM calls.

- The ATM term structure varies throughout time and can be flat, downward or upward sloping.

Literature on the typical smile pattern of individual equity options as compared to index options are extremely rare. Notwithstanding, it can be said that the smile of individual stock options is less steep ${ }^{50}$ and more symmetric and U-shaped ${ }^{51}$.

\subsubsection{The Relationship between Option Prices, Volatility Smile and Risk-Neutral Density}

\subsubsection{The Discounted Risk-Neutral Density as the Second Derivative of a European Call}

Option prices, implied volatility smile and risk-neutral density are intimately related with each other.

To begin with, implied volatilities are equivalent to option prices as has been shown above, given that the pricing model and its input variables are known. In practice, traders find it convenient to quote option prices in terms of their implied volatilities, since the latter do not change as much as the option prices 5 . It is important to note that the pricing model used for the transformation of option prices into implied volatilities is just a transformation vehicle, the use of which does not require that the employed model corresponds to the true model.

In contrast to the relationship between option prices and implied volatilities, the connection between option prices and the risk-neutral density is less obvious. BREEDEN/LITZENBERGER (1978) show that European options can be used to complete the market by the construction of elementary claims, which yield one unit of payment in a certain state of the underlying and zero in all other states.

\footnotetext{
50 See BAKSHI ET AL. (2003).

51 This statement is based on my own personal experience which I gathered when working for Banque Natinale de Paris in 1998 - one of the leading Eurex equity option market makers at that time. My own experience of a U-shaped pattern of the individual stock smile has been confirmed in a fruitful discussion by Boris Leblanc, member of the Global Equity and Derivatives Quantitative R\&D Team at BNP Paribas, Paris.

52 Cf. for example JACKWERTH (2004), p. 5.
} 
$\operatorname{Be} S_{T}(W)$ the value of the underlying depending on state $W$ with $S_{T}(W)=X$. Then such a construction basically consists of a butterfly spread ${ }^{53}$, bracketing $S_{T}(W)=X$ with the combination of two long calls with strike price $X$, one short call with strike $X-\Delta X$ and another short call with strike $X+\Delta X{ }^{54}$. Since the intrinsic value of this butterfly spread in state $S_{T}(W)=X$ amounts to $\Delta X$, the construction has to be divided by $\Delta X$ in order to yield a payoff of 1 in state $S_{T}(W)=X$. Be $c_{t_{0}}(X)$ the price of a European call in time $t_{0}$ maturing in time $T$ and depending on the strike price. Then, if possible states of the underlying are always $\Delta X$ apart from each other, the state price $\pi_{t_{0}, T}^{W}$ of an elementary claim equals the following expression 55 ,

$$
\pi_{t_{0}, T}^{W}=\frac{\left[c_{t_{0}}(X+\Delta X)-c_{t_{0}}(X)\right]-\left[c_{t_{0}}(X)-c_{t_{0}}(X-\Delta X)\right]}{\Delta X}
$$

The expression $\pi_{t_{0}, T}^{W}$ denominates the state price in the case of discrete states of the underlying. If in contrast we allow for continuous states and let $\Delta X$ become infinitesimally small, the state price $\pi_{t_{0}, T}^{W}$ per step size $\Delta X$ in the limit becomes the state price density which in turn equals the second derivative of the call price function $c_{t_{0}}$ with respect to the strike price $X 56$.

$$
\lim _{\Delta x \rightarrow 0} \frac{\pi_{t_{0}, T}^{W}}{\Delta X}=\frac{\left[c_{t_{0}}(X+\Delta X)-c_{t_{0}}(X)\right]-\left[c_{t_{0}}(X)-c_{t_{0}}(X-\Delta X)\right]}{(\Delta X)^{2}}=\frac{\partial^{2} c_{t_{0}}(X)}{\partial X^{2}}
$$

Since the state price density is nothing else than the risk-neutral density $f^{Q}$ of the underlying discounted at the risk-free rate (cf. equation 2.3 for the discrete case), we obtain the central result that the second derivative of the call price with respect to the strike equals the discounted risk-neutral density 57 .

$$
f^{Q}\left(S_{T}=X\right)=e^{r\left(T-t_{0}\right)} \frac{\partial^{2} c_{t_{0}}(X)}{\partial X^{2}}
$$

Equation 3.14 demonstrates that the call price function can be expressed in terms of the risk-neutral density and vice versa. Thanks to the put-call parity this statement is also true for put prices.

\footnotetext{
See ZIMMERMANN (1998), p. 241.

54 See ZIMMERMANN (1998), pp. 241-244.

55 Cf. ZIMMERMANN (1998), pp. 245 or BREEDEN/LITZENBERGER (1978).

56 Cf. ZIMMERMANN (1998), p. 246. or BREEDEN/LITZENBERGER (1978).

57 This result can yet be obtained in another way: The call price function can be represented as $c_{t_{0}}=e^{-r(T-t)} \int_{X}^{\infty}\left(S_{T}-X\right) f^{Q}\left(S_{T}\right) d S_{T}$. Differentiating this expression twice with regard to $X$ yields the desired result $e^{r(T-t)} \frac{\partial^{2} c_{t_{0}}(X)}{\partial X^{2}}=f^{Q}\left(S_{T}=X\right)$ (Cf. ZIMMERMANN (1998), pp. 248-249.).
} 
If the call or put price function is unknown, it has to be estimated based on the set of observed option prices and relying on statistical techniques which will still be discussed.

The equivalence of option prices, implied volatilities and the risk-neutral density is not restricted to European options only. Although strictly speaking, the above reasoning is not valid for American options, it is still applicable using a detour. This detour consists of first implying volatilities out of American option prices using a model which allows for path-dependency. Afterwards, these volatilities can be employed in order to calculate hypothetical European options 58

\subsubsection{The Relationship between the Shape of the Smile and the Form of the Risk-Neutral Density}

The above remarks show that European option prices, implied volatilities and the riskneutral density can be used interchangeably. This insight entails two very important qualitative relationships between the shape of the risk-neutral density and the shape of the corresponding volatility smile. These relationships will still be of great use throughout the remainder of my thesis and shall be explained in terms of the return density:

1. Excess kurtosis of the risk-neutral return density is associated with a U-shaped implied volatility smile 59 Imagine a fat-tailed return density as opposed to the normal return density implied by the Black-Scholes mode ${ }^{60}$. A deep OTM put option with strike $X$ will be more expensive, if evaluated with this density than with the normal density, since the cumulative probability up to a log-return of $\ln \left(X / S_{t_{0}}\right)$ will be higher in the fat-tailed than in the normal case. Consequently, the corresponding Black-Scholes implied volatility will also be higher. Due to the symmetry of both densities, this gives rise to a U-shaped smile.

58 See JACKWERTH (2004), p. 29. Strictly speaking however, this amounts to translating American into European options under the Black-Scholes assumptions and if these assumptions fail to be true, the translation could be biased.

59 HULL (2006) shows that a price density exhibiting more kurtosis than the lognormal density is associated with a U-shaped volatility smile (see p. 377). This can be easily carried over to the case of a return density with excess kurtosis with regard to the normal density.

60 Cf. Hull (2006), p. 283. 
2. Left-skewness of the risk-neutral return density is associated with a downward sloping implied volatility smile This can be shown using the same arguments as in the case of excess kurtosis. Only this time, imagine an asymmetric, leftskewed return density with a fat left tail. Again, a deep OTM put option with strike $X$ will be more expensive, if evaluated with this density than with the normal density. But as opposed to above, this will only give rise to a downward sloping implied volatility smile, since the left-skewed density is asymmetric.

\subsubsection{The Role of Risk Aversion in an Idealized Black-Scholes Economy}

Under the Black-Scholes assumptions, the physical stock price process follows a geometric Brownian motion with drift $\mu$ as specified in equation 3.1. In the complete market framework of the Black-Scholes model, the change from the physical measure $P$ to the risk-neutral measure $Q$ leads to a drift transformation from $\mu$ to the risk free rate $r 6$. Be $d z_{t}^{Q}$ a Wiener process under $Q$, then the risk-neutral stock price process ensues according to the following expression:

$$
d S_{t}=r S_{t} d t+\sigma S_{t} d z_{t}^{Q}
$$

While the physical as well as the risk-neutral stock price densities are lognormal, the corresponding return densities are norma ${ }^{63}$. Graphically, the change of measure from $P$ to $Q$ can be represented as a parallel left-shift of the return density by the amount of the risk premium $\mu-r^{64}$.

As has been laid out in section 2.2, the pricing kernel is essentially determined by the ratio of the risk-neutral to the physical density. In the Black-Scholes world, the left-shift of the return density results in a constant, and more importantly - state independent - elasticity of the pricing kernel $\Lambda^{65}$.

$$
\frac{d \ln \left(\Lambda_{t_{0}, T}\right)}{d \ln \left(S_{T}\right)}=-\frac{\mu-r}{\sigma^{2}}
$$

61 HULL (2006) shows that a price density exhibiting less skewness than the lognormal density is associated with a downward sloping volatility smile (see p. 380). This can be easily carried over to the case of a left-skewed return density.

62 For this and the following statements, see ZIMMERMANN (1998), pp. 250-253.

63 Cf. Hull (2006), p. 283.

64 See ZIMMERMANN (1998), p. 253.

65 See $\overline{\text { ZIMMERMANN }}(\overline{1998})$, pp. 257-259. 
As we have seen above by equation 2.5, in the optimum the elasticity of the pricing kernel is equivalent to the negative coefficient of relative risk aversion $\eta_{R}$. Thus, "using Black-Scholes for pricing options [...] is tantamount to assuming that aggregate relative risk aversion is constant ${ }^{\prime} 66$,

$$
\eta_{R}\left(S_{T}\right)=\frac{\mu-r}{\sigma^{2}}
$$

If this relationship implying a constant and state independent risk aversion held, options would be correctly priced by the Black-Scholes formula and there would be no smile phenomenon ${ }^{67}$.

\subsection{Estimation of the Volatility Smile and the Risk-Neutral Density}

\subsubsection{Introduction}

As has been shown above, option prices, implied volatility smile and risk-neutral density can be used interchangeably and one can be converted into the other two. In practice however, a non-continuous spectrum of option prices poses a challenge to its translation into a continuous smile or density function. As an answer to this challenge, different smoothing and approximation techniques have been developed aiming at the recovery of either a continuous smile or continuous density function 68 .

Following JACKWERTH $(200469$, the abundance of diverse estimation techniques can be differentiated into non-parametric and parametric approaches, meaning in this context that the former do not rely on a prior assumption as to the form of the risk-neutral distribution whereas the latter do.

The non-parametric approach of Bakshi-Madan allows to directly extract the higher moments of the risk-neutral density out of option prices. This method will be treated in some detail, since it will still be of substantial interest for my empirical analysis.

\footnotetext{
66 See BREEDEN/LITZENBERGER (1978) and ZIMMERMANN (1998), p. 258.

67 See FRANKE ET AL. (1999).

68 While it would also be possible to approximate a smooth option price function, this is hardly ever attempted, since option prices differ greatly in magnitude between deep OTM and deep ITM options (See REBONATO (2004), p. 254.).

69 See JACKWERTH (2004), pp. 16-25 and also JACKWERTH (1999).
} 
What is important to note is that the estimation and smoothing techniques presented below do not rely on any assumptions concerning the process of the underlying. Such process based models explaining the smile or the risk-neutral density will be discussed in sections 4 and 5 .

Given the rich body of extant estimation and smoothing techniques, the following passages shall only provide a very brief sketch of the central ideas of the different approaches and literature citations only represent a selection of determinant work in the respective fields.

\subsubsection{Non-Parametric Techniques}

\subsubsection{Polynomial Smoothing of the Volatility Smile}

The polynomial smoothing of the volatility smile has been first proposed by SHIMKO (1993) with the ulterior objective of extracting the risk-neutral density. SHIMKO (1993) estimates the implied volatility function $\hat{\sigma}$ as a function of the strike price $X$ :

$$
\hat{\sigma}(X)=b_{0}+b_{1} \cdot X+b_{2} \cdot X^{2}
$$

The next step consists in replacing the constant volatility $\sigma$ in the Black-Scholes equation 3.8 by the strike dependent volatility function $\hat{\sigma}(X)$. Twice differentiating of the so-modified Black-Scholes formula with respect to the strike $X$ yields the following closed form expression for the cumulative risk-neutral density $F^{Q}$ of the underlying price stock $S_{T}{ }^{70}$, where $n$ designates the standard normal density, $N$ the cumulative standard normal density and $d_{2}$ is defined as in equation 5.5 :

$$
\left.F^{Q}\left(S_{T}\right)\right|_{S_{T}=X}=1+X n\left(d_{2}\right)\left(b_{1}+2 b_{2} X\right)-N\left(d_{2}\right)
$$

A risk-neutral density along the lines sketched above can only be obtained, if $\hat{\sigma}(X)$ is at least twice differentiable $e^{71}$. Thus, the approach of SHIMKO (1993) represents one of the the least complex but still feasible specifications of $\hat{\sigma}(X)$.

Other authors propose polynomials of higher order for $\hat{\sigma}(X)$, more complex specifications like splines or implied volatility functions $\hat{\sigma}(\cdot)$ in the domain of moneyness or

70 According to the Breeden-Litzenberger result: $f^{Q}\left(S_{T}=X\right)=e^{r(T-t)} \frac{\partial^{2} c_{t_{0}}}{\partial X^{2}}$

71 Cf. ANDERSON/WAGENER (2002). 
option delta 2 . However, more complicated specification do not forcibly yield closed form solutions for the risk-neutral density, which then has to be evaluated numerically.

\subsubsection{Kernel Regression}

Kernel regression is a method to find an unknown functional relationship between known inputs and outputs without specifying the form of this function ${ }^{73}$. Let us suppose that the inputs $\mathbf{Z}_{i}$ are some measure of moneyness and / or option maturity and the observable outputs $H_{i}$ are the corresponding implied volatilities. Then the function $H(\mathbf{Z})$ to be looked for is the true implied volatility function. Alternatively, the inputs $\mathbf{Z}_{i}$ can also be the option's observable characteristics like underlying price, strike price, maturity, dividends and risk free rate and the outputs $H_{i}$ the corresponding observable option prices, in which case the unknown function $H(\mathbf{Z})$ would be the option price function.

The relationship between inputs and outputs in terms of the true function $H(\cdot)$ can be represented in the following form, where $u_{i}$ denotes a white noise term:

$$
H_{i}=H\left(\mathbf{Z}_{i}\right)+u_{i}
$$

Imagine a specific set of inputs $\mathbf{Z}_{i_{0}}$ and corresponding outputs $H_{i_{0}}$. If we had repeated observations of $H_{i_{0}}$, then - by the law of large numbers - averaging across those would eliminate the white noise term and yield the desired function $H(\cdot)$. Unfortunately, repeated observations of $H_{i_{0}}$ will rarely be available. The central idea of kernel regression consists in resorting to closely adjacent observations of $\mathbf{Z}_{i_{0}}$ instead and in averaging across the corresponding observations of $H_{i}$. Assuming smoothness of $H(\cdot)$, such an averaging yields an estimate of $H\left(\mathbf{Z}_{i_{0}}\right)$.

72 Like SHIMKO (1993), MALZ (1997a) and MALZ (1997b) also propose a quadratic polynomial, but in delta space, whereas TOMPKINS (2001) resorts to a quadratic polynomial in moneyness space. BRUNNER/HAFNER (2003) on the other hand use a cubic polynomial of the strike and a lower order polynomial of time to maturity to approximate the volatility surface. While HAFNER/WALLMEIER (2001) employ quadratic splines, CAMPA ET AL. (1998), BLISS/PANIGIRTZOGLOU (2002) and GLATZER/SCHEICHER (2003) apply cubic splines and ANDERSON/WAGENER (2002) even fit splines of degree four.

73 Regarding the following passage, cf. FENGLER (2005), pp. 98-102 and AIT-SAHALIA/LO (1998). Kernel regression with the objective of estimating risk-neutral densities or implied volatility functions has first been proposed by AIT-SAHALIA/LO (1998). While AIT-SAHALIA/LO (1998) apply kernel regression to estimate the option price function, AIT-SAHALIA/LO (2000), ROSENBERG (2000) and CONT/DA FONSECA (2002) employ it to approximate the implied volatility function. 
The closer the adjacent $\mathbf{Z}_{i}$ 's are to the reference $\mathbf{Z}_{i_{0}}$, the closer the average of the corresponding $H_{i}$ 's will be to the true function $H\left(\mathbf{Z}_{i_{0}}\right)$. This calls for a weighted average of the $H_{i}$, apportioning the most weight to those $H_{i}$ 's whose corresponding $\mathbf{Z}_{i}$ 's are closest to $\mathbf{Z}_{i_{0}}$.

The weighting scheme is incorporated in a weighting or kernel function $K$ with $\int K(u) d u=$ 1 , which can be the standard normal density. An estimator of the true function $H(\cdot)$ is then given by the Nadaraya-Watson kernel estimator, where the bandwidth $h$ should be a decreasing function of the sample size $n$ :

$$
\hat{H}(\mathbf{Z})=\frac{\sum_{i=1}^{n} K\left[\left(\mathbf{Z}-\mathbf{Z}_{i}\right) / h\right] H_{i}}{\sum_{i=1}^{n} K\left[\left(\mathbf{Z}-\mathbf{Z}_{i}\right) / h\right]}
$$

While kernel regression methods offer a great flexibility, they exhibit the disadvantage of being rather data intensive ${ }^{74}$

\subsubsection{The Estimation of the Moments of the Risk-Neutral Density with the Bakshi-Madan Method}

The approach proposed by BAKSHI/MADAN (2000) and BAKSHI ET AL. (2003) is a non-parametric method to imply risk-neutral return skewness and kurtosis directly out of European option prices. The method is non-parametric in the sense that it dispenses with the assumption of a specific form of the risk-neutral distribution. The Bakshi-Madan method is a very direct approach, because it does not rely on the prior approximation of the risk-neutral density.

The central idea of the Bakshi-Madan method is that any twice differentiable payoff function $H\left(S_{T}\right)$ of a contingent claim on $S_{T}$ can be spanned by a continuum of put and call option contracts on $S_{T}$. More precisely, the risk-neutral expectation of $H\left(S_{T}\right)$ can be expressed as a combination of a position in the risk free account, in the underlying $S_{T}$ and in a continuum of OTM puts and calls.

Further, risk-neutral skewness and kurtosis can be interpreted as functions of riskneutral expectations of particular payoffs and can consequently be represented in terms of a combination of option contracts.

Let $\tau=T-t_{0}$, then $R_{t_{0}, T}=\ln \left[S_{T}\right]-\ln \left[S_{t_{0}}\right]$ is the $\tau$-period return. Be further $E^{Q}[\cdot]$ the expectation under the risk-neutral measure. Define the volatility, the cubic and the quartic payoff functions as $R_{t_{0}, T}^{2}, R_{t_{0}, T}^{3}$ and $R_{t_{0}, T}^{4}$, and let further the risk-

74 Cf. Ait-S Ahalia/Lo (1998). 
lessly discounted risk-neutral expectations of these payoffs be $V_{t_{0}, T} \equiv E_{t_{0}}^{Q}\left[e^{-r \tau} R_{t_{0}, T}^{2}\right]$, $W_{t_{0}, T} \equiv E_{t_{0}}^{Q}\left[e^{-r \tau} R_{t_{0}, T}^{3}\right]$ and $Z_{t_{0}, T} \equiv E_{t_{0}}^{Q}\left[e^{-r \tau} R_{t_{0}, T}^{4}\right]$. Then, risk-neutral skewness $S K E W$ and kurtosis $K U R T$ can be represented in the following form:

$$
\begin{gathered}
S K E W_{t_{0}, T} \equiv \frac{E_{t_{0}}^{Q}\left[\left(R_{t_{0}, T}-E_{t_{0}}^{Q}\left[R_{t_{0}, T}\right]\right)^{3}\right]}{\left(E_{t_{0}}^{Q}\left[\left(R_{t_{0}, T}-E_{t_{0}}^{Q}\left[R_{t_{0}, T}\right]\right)^{2}\right]\right)^{3 / 2}} \\
=\frac{e^{r \tau} W_{t_{0}, T}-3 \mu_{t_{0}, T} e^{r \tau} V_{t_{0}, T}+2 \mu_{t_{0}, T}^{3}}{\left[e^{r \tau} V_{t_{0}, T}-\mu_{t_{0}, T}^{2}\right]^{3 / 2}} \\
K U R T_{t_{0}, T} \equiv \frac{E_{t_{0}}^{Q}\left[\left(R_{t_{0}, T}-E_{t_{0}}^{Q}\left[R_{t_{0}, T}\right]\right)^{4}\right]}{\left(E_{t_{0}}^{Q}\left[\left(R_{t_{0}, T}-E_{t_{0}}^{Q}\left[R_{t_{0}, T}\right]\right)^{2}\right]\right)^{2}} \\
=\frac{e^{r \tau} Z_{t_{0}, T}-4 \mu_{t_{0}, T} e^{r \tau} W_{t_{0}, T}+6 \mu_{t_{0}, T}^{2} e^{r \tau} V_{t_{0}, T}-3 \mu_{t_{0}, T}^{4}}{\left[e^{r \tau} V_{t_{0}, T}-\mu_{t_{0}, T}^{2}\right]^{2}}
\end{gathered}
$$

The risk-neutral expectations $V_{t_{0}, T}, W_{t_{0}, T}$ and $Z_{t_{0}, T}$ are functions of a continuum of European OTM put and call option contracts $p_{t_{0}}$ and $c_{t_{0}}$. With $X$ being the strike price, $V_{t_{0}, T}, W_{t_{0}, T}$ and $Z_{t_{0}, T}$ ensue according to the following expressions:

$$
\begin{gathered}
V_{t_{0}, T}=\int_{S_{t_{0}}}^{\infty} \frac{2\left(1-\ln \left[\frac{X}{S_{t_{0}}}\right]\right)}{X^{2}} c_{t_{0}}(X, T) d X \\
+\int_{0}^{S_{t_{0}}} \frac{2\left(1+\ln \left[\frac{S_{t_{0}}}{X}\right]\right)}{X^{2}} p_{t_{0}}(X, T) d X \\
W_{t_{0}, T}=\int_{S_{t_{0}}}^{\infty} \frac{6 \ln \left[\frac{X}{S_{t_{0}}}\right]-3\left(\ln \left[\frac{X}{S_{t_{0}}}\right]\right)^{2}}{X^{2}} c_{t_{0}}(X, T) d X \\
-\int_{0}^{S_{t_{0}}} \frac{6 \ln \left[\frac{S_{t_{0}}}{X}\right]+3\left(\ln \left[\frac{S_{t_{0}}}{X}\right]\right)^{2}}{X^{2}} p_{t_{0}}(X, T) d X
\end{gathered}
$$




$$
\begin{aligned}
Z_{t_{0}, T} & =\int_{S_{t_{0}}}^{\infty} \frac{12\left(\ln \left[\frac{X}{S_{t_{0}}}\right]\right)^{2}-4\left(\ln \left[\frac{X}{S_{t_{0}}}\right]\right)^{3}}{X^{2}} c_{t_{0}}(X, T) d X \\
& -\int_{0}^{S_{t_{0}}} \frac{12\left(\ln \left[\frac{S_{t_{0}}}{X}\right]\right)^{2}+4\left(\ln \left[\frac{S_{t_{0}}}{X}\right]\right)^{3}}{X^{2}} p_{t_{0}}(X, T) d X
\end{aligned}
$$

$\mu$ is given by:

$$
\mu_{t_{0}, T}=E_{t_{0}}^{Q}\left[\ln \left(\frac{S_{T}}{S_{t_{0}}}\right)\right]=e^{r \tau}-1-\frac{e^{r \tau}}{2} V_{t_{0}, T}-\frac{e^{r \tau}}{6} W_{t_{0}, T}-\frac{e^{r \tau}}{24} Z_{t_{0}, T}
$$

In the absence of continuous call or put price functions, the integrals $V_{t_{0}, T}, W_{t_{0}, T}$ and $Z_{t_{0}, T}$ have to be approximated in discrete steps.

\subsubsection{Parametric Techniques}

\subsubsection{Mixture of Lognormal Distributions}

Based on the Black-Scholes assumption of a lognormal risk-neutral density of the underyling $S_{T}$ and inspired by the suspicion that this assumption is overly simplistic, the risk-neutral density $f^{Q}$ is often approximated with a mixture of several lognormal densities $L N_{i}$ together allowing for non-normal skewness and kurtosis. This approach has first been tested by MELICK/THOMAS (1997) with a mixture of 3 lognormals ${ }^{75}$

With weights $w_{i}$ as well as with drift $\mu_{i}$ and with volatility $\sigma_{i}$ of the underlying Brownian motion of $S_{T}, \hat{f}^{Q}$ as the approximation of the true density $f^{Q}$ can be expressed in the following way ${ }^{76}$,

$$
\hat{f^{Q}}\left(S_{T}\right)=\sum_{i=1}^{n} w_{i} L N_{i}\left(S_{T}\right)
$$

75 Later examples of the application of this approach are CAMPA ET AL. (1998), Clews/Panigirtzoglou (2000), Bliss/Panigirtzoglou (2002), Glatzer/Scheicher (2003), COUTANT ET AL. (2001) and REBONATO/CARDOSO (2004).

76 The following passage follows largely REBONATO (2004), pp. 259-265. 
The estimated call price $\hat{c}_{t_{0}}$ can be represented as a linear combination of $n$ BlackScholes call prices $c_{t_{0}}^{B S}{ }_{i}$ with the same strike $X$ and maturity $T$, but differing drifts $\mu_{i}$ and volatilities $\sigma_{i}$ :

$$
\begin{array}{r}
\hat{c}_{t_{0}}=e^{-r(T-t)} \sum_{i=1}^{n} w_{i} \int_{0}^{\infty}\left(S_{T}-X\right)^{+} L N_{i}\left(S_{T}\right) d S_{T} \\
=\sum_{i=1}^{n} w_{i} c_{t_{0}}^{B S}{ }_{i}\left(X, T, \mu_{i}, \sigma_{i}\right)
\end{array}
$$

With a mixture of $n$ lognormals, $n-1$ weight parameters, $n-1$ drift parameters and $n$ volatility parameters have to be determined. The optimal $3 n-2$ parameters can be found by minimizing the sum of squared differences between actual option prices $c_{t_{0}}$ and model call prices $\hat{c}_{t_{0}}$. This optimization has to be carried out with the constraint that for all $i \in\{1, \ldots, n\}, w_{i}$ is in $[0,1]$ and $\sum_{i=1}^{n} w_{i}=1$ and further that the resulting distribution $\hat{f}^{Q}$ is centered on the forward price of $S_{T}$.

\subsubsection{Expansion Techniques}

If the true risk-neutral density $f^{Q}$ of the underlying $S_{T}$ is unknown, but is suspected to be similar to a known density $g$ and something is known about its moments, then $f^{Q}$ can be developed as an Edgeworth series expansion of $g^{77}$. An Edgeworth series expansion aiming at the approximation of the risk-neutral density has first been applied by JARROW/RUDD (1982), who derive a skewness and kurtosis corrected version of the Black-Scholes formula ${ }^{78}$

77 Cf. JARROW/RUDD $(1982)$.

78 Later examples of a very similar approach involving a Gram-Charlier series expansion of the lognormal distribution in order to estimate the risk-neutral density are CORRADO/SU (1996), ABKEN/MADAN (1996), CORRADO/SU (1997a), NAVATTE/VILLA (2000) and COUTANT ET AL. (2001). 
Be $\kappa_{i}^{f^{Q} / g}$ the $i^{\text {th }}$ cumulant 79 of $f^{Q}$ or $g$, respectively, and be further $g$ four times differentiable. Then, the truncated Edgeworth series expansion of $g$, denominated as $\hat{f}^{Q}$ ensues according to the following expression:

$$
\begin{array}{r}
\hat{f}^{Q}\left(S_{T}\right)=g\left(S_{T}\right)+\frac{\kappa_{2}^{f^{Q}}-\kappa_{2}^{g}}{2 !} \frac{d^{2} g\left(S_{T}\right)}{d S_{T}{ }^{2}} \\
-\frac{\kappa_{3}^{f^{Q}}-\kappa_{3}^{g}}{3 !} \frac{d^{3} g\left(S_{T}\right)}{d S_{T}{ }^{3}} \\
+\frac{\kappa_{4}^{f^{Q}}-\kappa_{4}^{g}}{4 !} \frac{d^{4} g\left(S_{T}\right)}{d S_{T}{ }^{4}}
\end{array}
$$

The truncation of the expansion after the fourth term is justifiable on the grounds that higher order terms become negligible.

By construction the first cumulants or means of the distributions $f^{Q}$ and $g$ are equal, $\kappa_{1}^{f^{Q}}=\kappa_{1}^{g}$, which is not true for the higher cumulants however. The second term in the above expression represents a correction of $g$ for the known difference between the volatilities of $f^{Q}$ and $g$. Likewise the third term stands for a skewness and the fourth for a kurtosis adjustment.

\subsubsection{Other Non-Parametric and Parametric Methods}

This section briefly sketches some less frequently used parametric and non-parametric techniques for the estimation of the smile or the risk-neutral density which have not yet been presented above.

To begin with, BUCHEN/KELLY (1996) introduced the concept of maximum entropy to finance and used it to estimate the risk-neutral density $f^{Q}$ from option prices. The concept of maximum entropy allows to find a density $\hat{f}^{Q}$ which is least prejudiced due to prior information ${ }^{80}$. The entropy $E$ of a distribution $f^{Q}$ is defined as $E \equiv$ $-\int f^{Q}\left(S_{T}\right) \ln \left[f^{Q}\left(S_{T}\right)\right] d S_{T}$. Maximization of $E$ by adequately choosing $\hat{f}^{Q}$ will yield a density with the maximum possible information conten ${ }^{81}$, which means that a minimum of prior information will be involved ${ }^{82}$. The maximization of $E$ has to respect the constraint of correctly replicating the observable option prices.

\footnotetext{
79 The first cumulant $\kappa_{1}$ equals the first moment $\mu_{1}$ or alternatively the mean of the distribution, the second cumulant $\kappa_{2}$ equals the second central moment $\alpha_{2}$, respectively the variance of the distribution, the third cumulant $\kappa_{3}$ equals the third central moment $\alpha_{3}$, and the fourth cumulant is obtained as $\kappa_{4}=\alpha_{4}-3 \alpha_{2}^{2}$ (see JARROW/RUDD (1982)).

80 Cf. BUCHEN/KELLY (1996).

81 Cf. COUTANT ET AL. (2001).

82 Cf. ROCKINGER/JONDEAU (2002).
} 
Another strand in the literature resorts to dimension reduction techniques like principal component analysis to model the dynamics of the implied volatility surface 83 . Since the volatility surface is a high-dimensional object, a low-dimensional representation can facilitate the description of its dynamical behavior ${ }^{84}$. Strictly speaking however, these approaches cannot be called estimation or smoothing techniques in the sense of the other techniques discussed above. The reason is that the modeling of the dynamics requires a prior description of the static shape of the volatility surface, which in turn demands techniques similar to those reviewed above.

While the maximum entropy method as well as the dimension reduction techniques represent non-parametric approaches, the procedure proposed by SHERRICK ET AL. (1992) and SHERRICK ET AL. (1996) to estimate the risk-neutral density with a 3parameter Burr distribution constitutes another example of a parametric approach.

\subsubsection{Summary and Appraisal of the Available Methods}

Despite the diversity of the different estimation and smoothing techniques discussed above, researchers largely find that the methods all yield similar result ${ }^{85}$ and appraise the polynomial smoothing of the volatility smile approach as being the most stable ${ }^{86}$ and easiest to implement 87 A parsimonious specification like a quadratic polynomial smoothing is considered to be sufficient to capture the essential structure of the implied volatility surface ${ }^{88}$

The only disadvantage of the polynomial smoothing consists in the difficulty of obtaining a risk-neutral density spanning the whole domain of the underlying $S$. As opposed to the parametric approaches and also the maximum entropy method, the polynomial smoothing generally precludes the calculation of the higher risk-neutral moments due

83 Examples are AlEXANDER (2001), FENGLER ET AL. (2003), CONT/DA FONSECA (2002), CONT ET AL. (2002) who all use principal component analysis and ANÉ/LABIDI (2001), who employ a related technique called independent component analysis.

84 See FENGLER (2005), p. 125.

85 Comparing a cubic spline smoothing of the volatility smile with the fitting of the risk-neutral density with a mixture of lognormals, CAMPA ET AL. (1998) find that both approaches yield similar results. COUTANT ET AL. (2001) likewise report no differences between the methods they use, which are the maximum entropy method, a mixture of lognormals and an expansion technique.

86 See JACKWERTH (2004), p. BLISS/PANIGIRTZOGLOU (2002) .

24, Clews/Panigirtzoglou (2000) and

87 See JACKWERTH (2004), p. 24.

88 See DUMAS ET AL. (1998). 
to a limited domain of integration 89 , Therefore, the Bakshi-Madan method can be a helpful non-parametric complement to the polynomial smoothing approach as far as the calculation of the higher risk-neutral moments is concerned.

In the face of potentially incomplete markets, the question arises as to the implications for the uniqueness of the extracted risk-neutral density. Theoretically, in the discrete case it is possible that multiple risk-neutral probability functions are compatible with a set of observed option prices, which leads to the question which one to choose in order to price as yet inexistent contracts. It can be argued that the no-arbitrage bounds for new contracts with as yet inexistent strikes - if we want to smooth in the strike domain - grow all the more smaller, the more data points are available in the first place 90 . Carried over to the continuous case, the additional requirement of a smooth density or call price function further restricts the no-arbitrage bounds in a significant way ${ }^{91}$. Therefore, if markets were incomplete, bounds on the obtained risk-neutral density would in general be negligibly small, provided that enough data points are at hand.

89 SHIMKO (1993) circumvents this problem by adding the tails of the lognormal distribution to his distribution inferred from option prices.

90 See JACKWERTH (2004), p. 14.

91 See JACKWERTH (2004), p. 15. 



\section{Explanations of the Equity Volatility Smile Excluding Default Risk}

\subsection{Introduction}

While the previous chapter concentrated on the definition and description of the notion of the implied volatility smile in general, the present section centers on the explanation of this phenomenon, focusing on equity in particular. Thereby, this chapter only embraces those prevalent explanatory approaches which do not explicitly rely on default risk as a cause of the smile phenomenon. These approaches can be coarsely classified into phenomenological and causal explanations. While the first class concentrates on capturing stylized phenomena of stock return behavior without further investigating their origins, the second class searches for fundamental causes of the equity volatility smile. The chapter concludes with a summary and an appraisal of the different explanatory approaches.

In the course of the following passages, alternative models, conditions and setups will be discussed which all deviate from the Black-Scholes assumptions. However, given the vast literature in this field, a detailed and exhaustive treatment would be beyond the scope of my thesis. Therefore, the numerous forms of deviation from the idealized Black-Scholes world will only be outlined with regard to their central ideas, and citations will be limited to substantial and determinant work in the respective domains. 


\subsection{Phenomenological Explanations of the Equity Volatility Smile}

The section starts with a review of the stylized facts of equity returns which deepens the understanding of the equity price process. As will be seen, these stylized facts are another hint besides the existence of the volatility smile that the rather restrictive Black-Scholes assumptions need to be relaxed.

Following this insight, the constant volatility assumption will be abandoned in favor of deterministically and stochastically changing variance. Then the notion of a merely diffusive process will be challenged by the introduction of jumps. And finally, deviating from the Black-Scholes model, also stochastic interest rates will be allowed.

\subsubsection{Stylized Facts of Stock Returns}

Many stylized facts of physical equity returns do not agree with the rather restrictive Black-Scholes assumptions. CONT (2001) provides a substantiated survey of stylized facts of asset returns in general which are also valid for equity returns in particular. The following passage represents a corresponding excerpt of the most relevant facts with regard to the equity volatility smile:

1. Fat tails: The asset return distribution exhibits tails which are heavier than those of the normal distribution.

2. Left-skewness: The physical equity and equity index return distribution is leftskewed.

3. Randomness of volatility: Asset return volatility is considered to be stochastic 92 ,

4. Volatility clustering: Asset return volatility displays a positive autocorrelation over some days, which leads to the clustering of high-volatility events.

5. Asymmetric volatility: The asset return is negatively correlated with its volatility.

6. Aggregational gaussianity: With increasing time horizon the asset return distribution resembles more and more the normal distribution.

92 This is only implicitly mentioned in CONT (2001) and can explicitly be verified in BOLLERSLEV ET AL. (1992) and HULL (2006), chapter 19. 
7. Absence of autocorrelation of returns: Except for very small intraday time scales, asset returns are not autocorrelated.

The first five facts are not at all compatible with the assumption of a geometric Brownian motion of the stock price and raise the question as to the nature of the true stock price process.

However, the above return features only concern the physical return. But as has been elaborated in section 2.2, it has to be distinguished between physical and risk-neutral return behavior. Since we want to investigate the causes of the volatility smile phenomenon, we should be primarily interested in the risk-neutral return. But since already the behavior of the physical return strongly violates the Black-Scholes assumptions, it is highly likely that the same also holds for its risk-neutral counterpart.

\subsubsection{Deterministically Changing Volatility in the Stock Price Process}

The above stylized facts in conjunction with the existence of the smile phenomenon suggest that - if not stochastic - volatility is at least not constant. This insight led to models with deterministically changing variance.

\subsubsection{The CEV Model}

One of the earliest models relaxing the Black-Scholes assumption of a constant diffusion parameter is the constant elasticity of variance (CEV) model introduced by COX/ROSS (1976). According to the CEV model, the instantaneous volatility is a deterministic function of the stock price level $S_{t}{ }^{93}$.

$$
\sigma\left(S_{t}\right)=\sigma S_{t}^{\delta-1}
$$

Substituting the constant volatility $\sigma$ of the geometric Brownian motion in equation 3.1 by $\sigma\left(S_{t}\right)$ and assuming a risk-neutral drift yields the following risk-neutral process specification for the CEV mode ${ }^{94}$.

$$
d S_{t}=r S_{t} d t+\sigma S_{t}^{\delta} d z_{t}^{Q}
$$

93 Cf. Cox/Ross (1976) and HULL (2006), p. 562.

94 Cf. COX/ROSS (1976) and HULL (2006), p. 562.

Incidentally, the CEV model owes its name to the fact that the elasticity of the variance of stock 
For $\delta=1$, the CEV model collapses to the Black-Scholes geometric Brownian motion. If $\delta<1$, the volatility increases as the stock price decreases, and if $\delta>1$, increasing volatility is coupled with decreasing stock price ${ }^{95}$ as can be veryfied by expression 4.1. In the first case the probability density of $S_{t}$ becomes left-skewed, exhibiting a fat left tail: The decreasing stock price level is coupled with increasing volatility, which in turn makes even lower stock price levels possible ${ }^{96}$. Conversely, with $\delta>1$ the probability density becomes right-skewed.

The best fit of the CEV model to the equity volatility smile is obtained with $\delta<$ $11^{97}$. which translates into a downward sloping volatility smile thanks to a left-skewed density (see section 3.1.3). But although the model then performs better than the Black-Scholes model, it still fails to completely explain the smile 98

\subsubsection{The Concept of Local Volatility}

The concept of local volatility has first been introduced by DUPIRE (1994), shortly followed by DERMAN/KANI (1994) and RUBINSTEIN (1994). In this concept the Black-Scholes assumption of a constant volatility $\sigma$ is abandoned in favor of a deterministically varying instantaneous volatility depending not only on the stock price level $S_{t}$, but equally on time $t$. This approach yields the following risk-neutral process specification for $S_{t}$ (cf. equation 3.1 99 .

$$
d S_{t}=r S_{t} d t+\sigma\left(S_{t}, t\right) S_{t} d z_{t}^{Q}
$$

Imagine some future time $T$, some future stock price level $S_{T}=X$ and the corresponding instantaneous volatility $\sigma\left(S_{T}=X, T\right)$. Then the local volatility $\sigma_{S_{t_{0}}, t_{0}}\left(S_{T}=X, T\right)$ is defined as the time $t_{0}$ expectation under the risk-neutral measure $Q$ of the future instantaneous volatility $\sigma\left(S_{T}=X, T\right)$ given the time $t_{0}$ stock price level $\left.S_{t_{0}}\right|^{100}$;

$$
\sigma_{S_{t_{0}}, t_{0}}\left(S_{T}=X, T\right)=E_{t_{0}}^{Q}\left[\sigma\left(S_{T}=X, T\right)\right]
$$

price changes with respect to the stock price is indeed constant (Cf. MACBETH/MERVILLE (1980).):

$$
\frac{d \ln \left[\sigma^{2} S_{t}^{2 \delta}\right]}{d \ln \left[S_{t}\right]}=\frac{d\left(\sigma^{2} S_{t}^{2 \delta}\right)}{d S_{t}} \frac{S_{t}}{\sigma^{2} S_{t}^{2 \delta}}=2 \delta
$$

95 See Hull (2006), p. 562.

96 See HULL (2006), p. 562.

97 See MACBETH/MERVILLE (1980).

98 See EMANUEL/MACBETH (1982).

99 Cf. GATHERAL (2006), p. 9.

100 See. FENGLER (2005), p. 50. 
While the implied volatility treated in section 3.1.2 represents a global average of the squared volatility throughout the life time of an option, the local volatility constitutes a local measure, since it depends on a particular stock price level and time

Following the market expectation of time $t_{0}$, the local volatility evolves deterministically on a two-dimensional path. For some market level $X=S_{t}$ and time $T=t$, the local volatility coincides with the instantaneous volatility ${ }^{102}$.

$$
\sigma_{S_{t}, t}\left(S_{t}, t\right)=\sigma\left(S_{t}, t\right)
$$

The local volatility $\sigma_{S_{t_{0}}, t_{0}}(X, T)$ can be inferred from the time $t_{0}$ price of a European call $c_{t_{0}}$ with strike $X$ maturing in $T$ using the Dupire formula ${ }^{103}$.

$$
\sigma_{S_{t_{0}}, t_{0}}^{2}(X, T)=2 \frac{\frac{\partial c_{t_{0}}(X, T)}{\partial T}+r X \frac{\partial c_{t_{0}}(X, T)}{\partial X}}{X^{2} \frac{\partial^{2} c_{t_{0}}(X, T)}{\partial X^{2}}}
$$

101 See FENGLER (2005), p. 57.

102 This and the sentence before, see FENGLER (2005), p. 50.

103 See FENGLER (2005), p. 53. The Dupire formula can be derived in the following way (cf. DERMAN/KANI (1994)): Be $f^{Q}$ the risk-neutral density of $S_{T}$, then $c_{t_{0}}$ can be represented in the following form:

$$
c_{t_{0}}=e^{-r\left(T-t_{0}\right)} \int_{X}^{\infty}\left(S_{T}-X\right) f^{Q}\left(S_{T}\right) d S_{T}
$$

The density $f^{Q}$ evolves according to the Fokker-Planck equation:

$$
\frac{\partial f^{Q}}{\partial T}=\frac{1}{2} \frac{\partial^{2}}{\partial S_{T}^{2}}\left(\sigma^{2} S_{T}^{2} f^{Q}\right)-\frac{\partial}{\partial S_{T}}\left(r S_{T} f^{Q}\right)
$$

Now imagine the time derivative of the above call price function:

$$
\frac{\partial c_{t_{0}}}{\partial T}=-r c_{t_{0}}+e^{-r\left(T-t_{0}\right)} \int_{X}^{\infty}\left[\frac{\partial f^{Q}}{\partial T}\right]\left(S_{T}-X\right) d S_{T}
$$

Substituting for $\frac{\partial f^{Q}}{\partial T}$ from the Fokker-Planck equation yields:

$$
\frac{\partial c_{t_{0}}}{\partial T}=-r c_{t_{0}}+e^{-r\left(T-t_{0}\right)} \int_{X}^{\infty}\left[\frac{1}{2} \frac{\partial^{2}}{\partial S_{T}^{2}}\left(\sigma^{2} S_{T}^{2} f^{Q}\right)-\frac{\partial}{\partial S_{T}}\left(r S_{T} f^{Q}\right)\right]\left(S_{T}-X\right) d S_{T}
$$

Integrating by parts twice and substituting $f^{Q}$ from equation 3.14 leads to the following expression:

$$
\frac{\partial c_{t_{0}}}{\partial T}=\frac{1}{2} \sigma^{2} X^{2} \frac{\partial^{2} c_{t_{0}}}{\partial X^{2}}-r X \frac{\partial c_{t_{0}}}{\partial X}
$$

Rearranging leads to:

$$
\sigma^{2}=2 \frac{\frac{\partial c_{t_{0}}}{\partial T}+r X \frac{\partial c_{t_{0}}}{\partial X}}{X^{2} \frac{\partial^{2} c_{t_{0}}}{\partial X^{2}}}
$$


Given a set of option prices with a continuum of strikes and maturities, the complete local volatility surface can be extracted from those prices. Since in reality such a continuum is not available, DUPIRE (1994), DERMAN/KANI (1994) and also RUBINSTEIN (1994) propose lattice algorithms - called implied trees - for the recovery of the local volatility surface 104 .

Considering all available European option prices for the construction of the local volatility surface leads to a perfect replication also of the implied volatility surface. More particularly, the inclusion of all available option maturities amounts to the specification of a unique diffusion process according to expression 4.3 which can be used to price other path depending instrument $\$ 105$.

The ability to perfectly match the implied volatility surface is the true value added of the local volatility concept. As we have seen above, the CEV model for example fails to do so due to less flexibility 106 ,

Since the concept of local volatility dispenses with additional risk factors, it is consistent with the assumption of a complete market ${ }^{107}$. While this complete market framework can be interpreted as an advantage, it can at the same time be criticized as being overly simplistic. But even besides the question of market completeness, the concept of local volatility has been challenged due to its unrealistic dynamic properties ${ }^{108}$.

\subsubsection{Stochastically Changing Volatility in the Stock Price Process}

The empirical insight regarding the random nature of volatility in conjunction with the existence of the smile phenomenon led to specifications of a stock price process in which volatility is modeled as a stochastic variable.

\footnotetext{
104 While DERMAN/KANI (1994) and DUPIRE (1994) propose binomial and trinomial trees, respectively, including options of all available maturities, RUBINSTEIN (1994) uses a binomial tree which only considers options of one maturity.

Another way to recover a continuous local volatility surface consists in smoothing the implied volatility surface first and in then generating hypothetical option prices for arbitrary strikes and maturities (cf. FENGLER (2005), pp. 55-56).

105 See DUPIRE (1994).

106 The CEV model can be considered as a special case of the local volatility model with $\sigma_{S_{t_{0}}, t_{0}}(X, T)=\sigma X^{\delta-1}$ (see FENGLER (2005), p. 84).

107 See DUPIRE (1994).

108 Cf. FENGLER (2005), p. 88 and DUMAS ET AL. (1998).
} 


\subsubsection{A General Representation of a Stochastic Volatility Model}

In the class of the stochastic volatility models, the risk-neutral dynamics of the stock price are very similar to those of the models discussed above with the only exception that the volatility or variance now follows its own random process.

One of the first stochastic volatility models has been proposed by HULL/WHITE (1987) 109 . Meanwhile the most popular and most frequently used model in the context of option pricing is that of HESTON (1993a ${ }^{110}$ due to its easy implementable closed-form solution 111 . In contrast to most earlier models, the Heston model allows the innovations of the stock price and the variance to be correlated. Finally, the rather recent SABR ("stochastic alpha beta rho") model of HAGAN ET AL. (2002) should not be left unmentioned. Although providing exact expressions for the implied volatility in the short-term limit, this model is only appropriate for short term expirations ${ }^{112}$.

As an example of the class of stochastic volatility models allowing for correlated innovations, a rather general model will be studied below ${ }^{113}$. This model implies the following risk-neutral dynamics of the underlying and the volatility, where $d z_{t}^{Q}$ and $d w_{t}^{Q}$ are standard normal innovations under a risk-neutral measure $Q$ correlated with the parameter $\rho$ :

$$
\begin{gathered}
d S_{t}=r S_{t} d t+\sigma_{t} S_{t} d z_{t}^{Q} \\
v_{t}=\sigma_{t}^{2} \\
d v_{t}=\alpha_{v}\left(\theta_{v}-v_{t}\right) d t+\varsigma d w_{t}^{Q} \\
\left\langle d z_{t}^{Q}, d w_{t}^{Q}\right\rangle=\rho d t
\end{gathered}
$$

The variance $v_{t}$ follows a mean reverting Ornstein-Uhlenbeck process ${ }^{114}$ whose riskneutral version can be decomposed into a drift term and a term of random innovations. The drift term is mean-reverting with the risk-neutral parameter $\theta_{v}$ denoting the long term mean of the variance. In the long run the process reverts to $\theta_{v}$ at a speed defined by the risk-neutral parameter $\alpha_{v}$. The last term comprises the random innovations $d w_{t}^{Q}$ with the volatility of the variance $\varsigma$. The above risk-neutral specification contains a

109 Hull/White (1987), SCOTT (1987) and WigGINS (1987) in a short time span all proposed rather similar models, but failed to offer a closed form pricing formula which has later been furnished by STEIN/STEIN (1991) Cf. GATHERAL (2006), p. 15.

111 See. GATHERAL (2006), p. 24.

112 This is due to the lacking mean reversion of this model.

113 Regarding this model and its process specification, cf. FOUQUE ET AL. (2000), p. 47.

114 See. FouQUE ET AL. (2000), p. 43. 
risk premium for the additional volatility risk, which is implicitly encapsulated in the risk-neutral parameters $\theta_{v}$ and $\alpha_{v}{ }^{115}$

Although stochastic volatility models are appreciated for being more realistic than deterministic volatility model $\Omega^{116}$, the addition of an unobservable risk factor disables the Black-Scholes type perfect replication principle and thus poses a new challenge to option pricing ${ }^{117}$. However, the complete market framework can easily be restored in a rather simplistic manner by setting $\rho= \pm 1$ which eliminates the additional source of randomness 118 .

An approach similar to the above is not the only way to incorporate random volatility. Exploiting the resemblance of the stochastic volatility specification in option pricing models to GARCH-type time series models, alternative option pricing models building on GARCH models have been proposed 119 .

Another starting point to model stochastic volatility could be the implied or the local volatility surface. Such avenues have indeed been followed in order to model the dynamics of the volatility surface ${ }^{120}$. However, such approaches are of secondary importance for my study, since my primary focus is the relationship between the level of default risk and the statics of the implied volatility smile.

115 BAKSHI/KAPADIA (2003a) find that the volatility risk premium is negative for the stock market as a whole. To a lesser extent they confirm this finding also for individual stocks in BAKSHI/KAPADIA (2003b). A reason for a negative volatility risk premium could be a positive correlation between volatility changes and the economy-wide pricing kernel (see BAKSHI/KAPADIA (2003a)).

LAMOUREUX/LASTRAPES (1993) also claim that in the stock market volatility risk must be priced.

Contrary to these findings, HuLL/WHITE (1987) SCOTT (1987) and WIGGINS (1987) assume that volatility does not incorporate any systematic risk and therefore is unpriced.

116 Cf. BURASCHI/JACKWERTH (2001), e.g..

117 Cf. FOUQUE ET AL. (2000), p. 44.

118 See HESTON/NANDI (1998).

119 Examples are DUAN (1995), KAllsen/TAQQU (1998) or HestON/NANDi (2000).

120 DERMAN/KANI (1998) extends the concept of local volatility to stochastic local volatility, while SCHÖNBUCHER (1999) is one of the first to model a stochastic behavior of the implied volatility smile. Also the approaches of CONT/DA FONSECA (2002) and CONT ET AL. (2002) - already brought up above in the context of the reduced form techniques for the approximation of the implied volatility surface - should not be left unmentioned. In their work, the principal components describing the dynamics of the volatility surface are modeled as random factors. 


\subsubsection{The Ability of Stochastic Volatility Models to Replicate the Equity Volatility Smile}

Models of the type described above manage fairly well to replicate the essential features of the volatility surface, except for very short maturities ${ }^{121}$. The smile and the risk-neutral density generated by this type of model can be controlled with the parameters $\rho$ and $\varsigma$.

A negative $\rho$ leads to a left-skewed return distribution, whereas a positive $\rho$ implies right-skewness $\sqrt{122}$. For equity markets $\rho$ is assumed to be negative ${ }^{123}$ which is tantamount to a left-skewed return distribution and thus a downward sloping smile (see section 3.1.3.2.

The volatility of the variance parameter $\varsigma$ on the other hand is positively linked with the kurtosis of the return density ${ }^{124}$, which translates into a U-shaped smile pattern (see section 3.1.3.2).

Despite the fairly good fit of stochastic volatility models to medium and long term options, the lack of such a fit for shorter maturities can be considered an essential weakness. Further, the capability of replicating the volatility surface of medium and long term options comes at the cost of unrealistic parameter settings ${ }^{125}$.

\subsubsection{Allowing for Jumps in the Stock Price Process}

The above mentioned stylized fact of fat tailed equity return distributions hints at the possibility of relatively likely, extreme events. These are not typical of a continuous diffusion, but should rather be expected in the case of sudden, large jumps in the stock price process 126 . Yet apart from the fat-tailedness of the distribution, already the discontinuous nature of stock prices strongly speaks in favor of the presence of jumps. Corroborating these arguments, several researchers have produced empirical evidence in favor of the existence of jumps ${ }^{127}$. Like in the case of stochastic volatility

121 See Gatheral (2006), p. 50.

122 See HESTON (1993a).

123 See FOUQUE ET AL. (2000), p. 51.

124 See $\overline{\text { HESTON }}$ (1993a).

125 Cf. BATES (1996), BAKSHI ET AL. (1997) and BATES (2000). ERAKER (2004) argues that the unrealistically high volatility of volatility needed to match the volatility surface hints at the necessity to include jumps into the process of the volatility.

126 See CONT/TANKOV (2004), pp. 5-6.

127 As an early example see BALl/TOROUS (1985) and more recently CARR/WU (2003b). CARR/WU (2003b) analyze the prices of options whose maturity converges to zero and find that the options' prices can only be justified in the presence of a jump component. 
above, the introduction of additional jump risks implies a departure from the complete market framework of the Black-Scholes mode 128 .

\subsubsection{Jump-Diffusion Processes}

A jump-diffusion process is the combination of a diffusion and a jump component. While the diffusion part is considered to be due to the steady flow of marginal information, the jump part is assumed to be owed to the arrival of substantial, new information 129 .

The earliest such process has been proposed by MERTON (1976) and consists of a geometric Brownian motion as already studied above and a Poisson process with uncertain jump size. The risk-neutral dynamics of this class of process can be represented in the following way, where $d z_{t}^{Q}$ denotes a standard normal innovation and $d q_{t}^{Q}$ the innovation of a Poisson process, both under a risk-neutral measure $Q^{130}$.

$$
\begin{aligned}
& d S_{t}=\left(r-h \mu_{J}\right) S_{t} d t+\sigma S_{t} d z_{t}^{Q}+J_{t} S_{t} d q_{t}^{Q} \\
& \ln \left(1+J_{t}\right) \sim N\left(\ln \left[1+\mu_{J}\right]-\frac{1}{2} \sigma_{J}^{2}, \sigma_{J}^{2}\right)
\end{aligned}
$$

$h$ is the risk-neutral jump intensity of the Poisson process $d q_{t}^{Q}$. The risk-neutral probability of a jump occurring in the infinitesimal small time interval $d t$ is proportional to this interval, $P\left(d q_{t}^{Q}=1\right)=h d t . J_{t}$ is the lognormally distributed percentage jump size conditional on a jump occurring with mean risk-neutral jump size $\mu_{J} \cdot \ln \left(1+J_{t}\right)$ has the standard deviation $\sigma_{J}$.

The term $-h \mu_{J} d t$ is called the jump compensator which corrects the drift of the process $d S_{t}$ for the average jump effect 131 .

The risk-neutral parameters $h$ and $\mu_{J}$ implicitly reflect a risk premium for jump timing and size uncertainty which makes them differ from their physical counterparts 132 .

Another popular jump-diffusion model is the double-exponential jump model of $\mathrm{KOU}$ (2002). In distinction to the above case of a lognormally distributed jump size this

28 Cf. section 2.1.2 and see CONT/TANKOV (2004), chapter 9.2.

129 See MERTON (1976).

130 Cf. BAKSHI ET AL. (1997) and BATES (1996).

131 Cf. CONT/TANKOV (2004), p. 52.

132 Cf. BATES (1996). 
model proposes the combination of two exponential distributions for $\ln \left(1+J_{t}\right)$. Let $D \operatorname{Exp}(\cdot)$ denote the double-exponential probability distribution, then $n^{133}$.

$$
\ln \left(1+J_{t}\right) \sim \operatorname{DExp}\left(p, \eta_{d}, \eta_{u}\right)
$$

Let $Y$ be a double-exponentially distributed variable with $Y=\ln \left(1+J_{t}\right)$ and let further denote $f_{D E x p}^{Q}(y)$ the density of this variable under some risk-neutral measure $Q$. Then, $f_{D E x p}^{Q}$ is defined as:

$$
f_{D E x p}^{Q}(y)=p \eta_{u} e^{-\eta_{u} y} 1_{(y \geq 0)}+(1-p) \eta_{d} e^{\eta_{d} y} 1_{(y<0)}
$$

The parameters of $f_{D E x p}^{Q}$ are assumed to comply with the risk-neutral measure $Q$. Conditional on the occurrence of a jump, the parameter $p$ denotes the probability that this jump lies on the upside and $(1-p)$ that it lies on the downside. $\eta_{u}$ and $\eta_{d}$ are the respective parameters of the exponential distribution, with:

$$
\eta_{u}>1, \eta_{d}>0
$$

The average jump size is given by:

$$
\mu_{J}=p \frac{\eta_{u}}{\eta_{u}-1}+(1-p) \frac{\eta_{d}}{\eta_{d}+1}-1
$$

In contrast to the Merton jump-diffusion model, the double-exponential jump-diffusion model allows analytic solutions for the valuation of many path-depending instruments thanks to the memoryless property of the exponential distribution ${ }^{134}$.

\subsubsection{Infinite Activity Processes}

In distinction to the jump-diffusion models above, infinite activity processes are characterized by an infinite number of jumps in every time interval ${ }^{135}$. For this reason their dynamics are rich enough to dispense with the diffusion component ${ }^{136}$, earning them also the name pure jump processes. Such specifications are considered by some to be

\footnotetext{
133 For this and the following equations cf. KOU (2002).

134 See KoU (2002) and CONT/TANKOV (2004), p. 111.

135 CONT/TANKOV (2004), pp. 103-104.

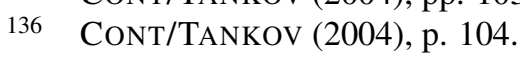


superior to jump-diffusion processes as to their ability to describe the true nature of asset prices 137 .

The most common pure jump model is the Variance-Gamma model which consists of an arithmetic Brownian motion $b(\cdot)$ evaluated at a random time which in turn is given by a gamma process $\gamma(\cdot)^{138}$,

The component of the Brownian motion $b\left(t, \mu_{b}, \sigma\right)$ depends on time $t$, on a drift parameter $\mu_{b}$ and a volatility parameter $\sigma$. With $z_{t}$ being a standard Brownian motion, $b(\cdot)$ can be represented in the following form:

$$
b\left(t, \mu_{b}, \sigma\right)=\mu_{b} t+\sigma z_{t}
$$

The gamma process $\gamma\left(t, \mu_{\gamma}=1, \nu\right)$ in turn has a mean rate $\mu_{\gamma}=1$ and a variance rate $\nu$ and represents the process of independent gamma increments over non-overlapping intervals of time $(t, t+\tau)$, with the density of the increments $\gamma\left(t+\tau, \mu_{\gamma}, \nu\right)-\gamma\left(t, \mu_{\gamma}, \nu\right)$ given by the gamma distribution.

The Variance-Gamma process $V G\left(t, \nu, \mu_{b}, \sigma\right)$ finally as the combination of both is defined as:

$$
V G\left(t, \nu, \mu_{b}, \sigma\right)=b\left(\gamma(t, 1, \nu), \mu_{b}, \sigma\right)
$$

The risk-neutral stock price dynamics according to the Variance-Gamma process can be read from the following expression, where all parameters have to be understood as risk-neutral:

$$
\begin{array}{r}
S_{T}=S_{t_{0}} e^{r\left(T-t_{0}\right)+\left[V G\left(T, \nu, \mu_{b}, \sigma\right)-V G\left(t_{0}, \nu, \mu_{b}, \sigma\right)\right]+\omega\left(T-t_{0}\right)} \\
\omega=\frac{1}{\nu} \ln \left(1-\mu_{b} \nu-\sigma^{2} \nu / 2\right)
\end{array}
$$

In the Variance-Gamma process, the physical calender time is replaced by an economically meaningful time determined by the erratic arrival of economically significant information 139

137 Analyzing time-series and options data CARR ET AL. (2002) conclude that equity processes are pure jump processes and DAAL/MADAN (2005) find that a pure jump model is superior to a jump diffusion model as far as option pricing is concerned.

138 For the following derivation of the Variance-Gamma model see MADAN ET AL. (1998). The Variance-Gamma model has been introduced by MADAN/SENETA (1990) and in a version with an additional parameter also allowing for asymmetric volatility smiles by MADAN ET AL. (1998). Although already COX/ROSS (1976) proposed a pure jump process, their early version of a pure jump process is not directly comparable to the Variance-Gamma model, since it is of finite activity.

139 See REBONATO (2004), pp. 517-518. 
Other examples of pure jump processes are the direct use of the gamma process for the modeling of the stock price dynamics ${ }^{140}$ as well as the normal inverse Gaussian process ${ }^{141}$. However, the conception of the latter originates from time-series analysis and is not motivated by the problem of contingent claim pricing.

\subsubsection{The Ability of Models Incorporating Jumps to Replicate the Equity Volatility Smile}

Jumps are an essential ingredient for the specification of the stock price process, since they yield a great flexibility as to the replication of the implied volatility surface. This flexibility is owed to the levers jump intensity, direction and size.

A general effect of symmetric jump activity on the risk-neutral return density consists in the generation of excess kurtosis 142 , which is associated with a U-shaped implied volatility smile as can be recalled from section 3.1.3.2. Excess kurtosis dies away with increasing time horizon ${ }^{143}$ which can be explained by the central limit theorem 144 . Consequently, excess kurtosis vanishes all the more faster with increasing time horizon, the smaller the jump size ${ }^{145}$.

In jump-diffusion models the jump activity is determined by the interplay of the jump intensity $h$ and the jump size $J_{t}$, whereas in the Variance-Gamma model the jump activity is determined by the parameter $\nu$. Increasing $\nu$ leads to increasing kurtosis ${ }^{146}$,

As opposed to risk-neutral excess kurtosis, risk-neutral skewness can easily be produced by a non zero mean jump size $e^{147}$. While for $\mu_{J}<0$ the distribution grows left-skewed, a $\mu_{J}>0$ leads to right-skewness. In the Variance-Gamma model the same effect can be attained by correspondingly varying the parameter $\mu_{0}^{148}$. As has been elaborated in section 3.1.3.2, risk-neutral left-skewness, as is typical for equity markets, can be carried over to a downward sloping volatility smile.

Stochastic volatility models generally have difficulties fitting the accentuated features of the short term smile, since due to their diffusive character they need a certain time

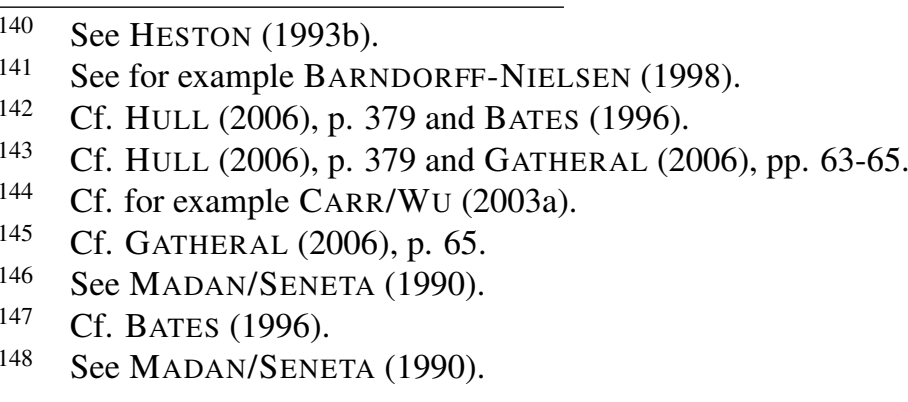


horizon to unfold their effect on the smile. Whereas the effects of jump risks particularly act on the short term smile and fade away with increasing maturity. Therefore, none of the models alone is capable of reproducing the term structure of the smile ${ }^{149}$ but both models together represent an ideal combination 150 . The approach of combining stochastic volatility with jumps in the equity price process constitutes a rather popular practice ${ }^{151}$. Besides an enhanced fit to available option prices, the combination of both types of model also exhibits the beneficial effect of entailing a more realistic volatility of volatility parameter for the stochastic volatility componen 152

In some models, jumps are not only added to the process of the underlying, but also to the process of the volatility ${ }^{153}$. However, if jumps are already present in the process of the underlying, this is not considered to further improve the fitting capabilities of the model 154

\subsubsection{Stochastic Interest Rates}

In the previous sections, the Black-Scholes assumptions have been relaxed in diverse ways in order to explain the equity volatility smile. One assumption which has not yet been challenged above is that of a constant risk free interest rate.

One of the first option pricing models abandoning this assumption in favor of stochastically varying interest rates is that of BAILEY/STULZ (1989). This model does not only feature stochastic interest rates, but also stochastic stock price volatility. This combination is deemed reasonable on grounds of the empirical fact of a negative correlation between interest rates and stock return volatility ${ }^{155}$.

\footnotetext{
149 See DAS/SUNDARAM (1999).

150 See GATHERAL (2006), p. 65.

151 Examples are BATES (1996) and BATES (2000), who apply combined stochastic volatility and jump models, and SCOTT (1997) and BAKSHI ET AL. (1997), who additionally integrate stochastic interest rates.

152 See BATES (2000).

153 See for example ERAKER ET AL. (2003), who claim that a stochastic volatility model with volatility jumps outperforms a simple stochastic volatility model.

154 GATHERAL (2006) shows that a stochastic volatility model with jumps in the underlying and the volatility does not only have more parameters but is also more difficult to fit to market prices than the same model without volatility jumps.

155 See BAiley/STUlZ (1989) and SCOTT (1997).
} 
Although stochastic interest rates and stochastic volatility are frequently modeled together ${ }^{156}$, we will dispense below with the stochastic volatility component for the sake of illustration.

The risk-neutral stock price dynamics assumed by the class of stochastic interest rate option pricing models can be represented in the following, very general form, where $d z_{t}^{Q}$ and $d w_{t}^{Q}$ are standard normal innovations ${ }^{157}$.

$$
\begin{gathered}
d S_{t}=r_{t} S_{t} d t+\sigma S_{t} d z_{t}^{Q} \\
d r_{t}=\alpha_{r}\left(\theta_{r}-r_{t}\right) d t+\sigma_{r} \sqrt{r_{t}} d w_{t}^{Q}
\end{gathered}
$$

The additional risk factor $r_{t}$ follows a mean-reverting Cox-Ingersoll-Ross process with the long term average level of the risk free rate given by $\theta_{r}$ and $\alpha_{r}$ denoting the speed of mean reversion.

Due to the negative correlation between interest rates and equity volatility, it is seemingly natural to allow for stochastic interest rates, if stochastic volatility is already present in a model. However, the effects of the inclusion of stochastic interest rates into an option pricing model with stochastic volatility are marginal and do not improve the performance of the model in a noticeable way 158 . Therefore, it can be concluded that the same, barely noticeable effects arise when adding stochastic interest rates to the Black-Scholes Brownian motion.

\subsection{Causal Explanations of the Equity Volatility Smile}

Above, we concentrated on important, phenomenological explanations of the smile residing in the process of the underlying and representing the mainstream practice of option pricing. In contrast, this section stresses more isolated but also more fundamental explanations.

156 Besides BAILEY/STULZ (1989), see for example AMIN/JARROW (1991), AMIN/NG (1993). Only AMIN/JARROW (1992) propose an option pricing model with stochastic interest rates only.

157 Cf. PAN (2002)

158 BAKSHI ET AL. (1997) test a very generic option pricing model with the components stochastic volatility, stochastic interest rates and jumps and find no noticeable difference between the specification with stochastic volatility and stochastic interest rates on the one hand and the one with only stochastic volatility on the other. 
First, the broad and diverse class of causal explanations related to market microstructure and market efficiency issues will be presented. Secondly, risk aversion as a possible cause of the equity volatility smile will be discussed.

\subsubsection{Market Microstructure and Efficiency Related Explanations}

The market microstructure can be described as the actual setup and the actual processes governing the functioning of the market and determining price discovery, trading volumes and trading behavior 159 . While many financial models assume a frictionless and efficient market, the actual market processes are often subject to cicumstances like non-synchronous trading, transaction costs, illiquidity, asymmetric information, deferred flow of information, trading rules and restrictions, etc 160 . Since the market microstructure can affect the flow and dispersion of information, it can also impact market efficiency.

While all previous explanations of the equity volatility smile have been of rather phenomenological nature, market microstructure related approaches advance more causal, fundamental explanations of the smile.

Most explanations of the smile derived from the microstructure of the market involve market frictions in some form or another. LONGSTAFF (1995) for example holds frictions in the guise of transaction costs and illiquidity responsible for the smile phenomenon. According to LONGSTAFF (1995), market frictions impose bounds on option prices thus invalidating pricing by no-arbitrage considerations as well as the application of the Black-Scholes model. This in turn means that the model implicit, riskneutral lognormal price density of the underlying with the riskless drift as its mean is not valid and other models implying other densities have be considered instead.

Although some authors confirm an effect of frictions like transaction costs and illiquidity on the smile ${ }^{161}$, this is also controverted by others with the argument that transaction cost induced bounds on option prices are too tight to account for the smile phenomenon ${ }^{162}$.

\footnotetext{
159 See MÜNNICH (2003).

160 See MÜNNICH $(2003)$ and CAMPBELL ET AL. (1997), chapter 3.

161 PENA ET AL. (1999) for example confirm a connection between transaction costs and the equity volatility smile for the Spanish market. And the effect of illiquidity on option prices is analyzed by CETIN ET AL. (2006), who model illiquidity as an additional risk factor and hold that a strikedependent illiquidity premium could be responsible for the smile phenomenon.

162 See for example CONSTANTINIDES (1997).
} 
A slightly different stance is taken by BOLLEN/WHALEY (2004) and EDERINGTON/WEI (2002), who claim that the smile is at least partly driven by the demand for OTM puts serving as an insurance against market downturns ${ }^{163}$, This demand cannot be fully satisfied, due to position limits or hedging costs that possible counterparties on the supply side are subject to. This excess demand drives up option prices and implied volatilities in a way not accounted for by the Black-Scholes assumptions.

Such a demand or supply surplus is accompanied by an asymmetric bid-ask spread around the true market value. Consistent with the conjecture of excess demand, BOLLEN/WHALEY (2004) find that transactions are on average executed closer to the ask than to the bid price. For the Swedish market in contrast, NORDEN (2003) claim that for ITM and OTM options true prices are closer to the bid than to the ask quote and conclude that the true smile is flatter than the one calculated by bid-ask midpoints. However, they fail to furnish an explanation for the observed bid-ask asymmetry.

Still another market microstructure related cause of the smile is given by HENTSCHEL (2003), who argues that the finite precision of option prices leads to systematic biases in the measurement of implied volatilities.

Some authors also question market efficiency in an attempt to explain the volatility smile. STERNBERG/YING (2005) hold that market frictions like transaction costs lead to relatively wide no-arbitrage bounds on option prices allowing for a certain extent of serial correlation in the prices of the underlying. This serial correlation in turn leads to a volatility smile. Strictly speaking, STERNBERG/YING (2005) do not really challenge the notion of market efficiency, since they hold that the serial correlation is too small relative to the transaction costs to be exploited by arbitrageurs.

Likewise breaching the assumption of market efficiency, VANDEN (2005) hypothesize the possibility of insider trading as a cause of the smile.

Most of the above approaches offer fundamental causes of the smile which are not forcibly incompatible with the more phenomenological approaches discussed before. However, they remain unsatisfactory, since at best they only furnish partial substantiations of the smile. Further, while some of the above explanations like insider trading for example should loose their impact with increasing time horizon, others like transaction costs possibly do not. Such a persistency however would contradict the fact that the smile eventually flattens out. To this adds that the market microstructure re-

163 BOLLEN/WHALEY (2004) do not only find an excess demand for OTM index puts, but also for OTM calls on individual stocks. 
lated explanations do not share any mainstream beliefs or opinions and are in parts contradictory to each other.

\subsubsection{The Role of Risk Aversion}

When searching for explanations of the smile phenomenon, the role of risk aversion should not be ignored. As has been laid out in section 2.2, risk aversion links the physical with the risk-neutral density.

And as has been demonstrated in section 3.1.4, the idealized Black-Scholes world implies that the coefficient of relative risk aversion must be constant. But the undisputed existence of the smile phenomenon shows that options are apparently not priced correctly by using the Black-Scholes formula. Consequently, if the Black-Scholes model does not hold in reality, relative risk aversion must not forcibly be constant.

Therefore it will first be examined how risk aversion manifests itself in the real world giving rise to the smile phenomenon. But however omnipotent the role of risk aversion might be in the explanation of the smile, it still does not furnish a fundamental cause. The reason is that the existence of a certain pattern of risk aversion might well explain the smile but needs in turn to be explained by fundamental reasoning. This will be addressed in the second part of this section.

\subsubsection{The Manifestation of Risk Aversion in the Real World}

FRANKE ET AL. (1999) demonstrate theoretically that the existence of the smile is synonymous with a non-constant coefficient of relative risk aversion $\eta_{R}$ which declines in the level of wealth or alternatively in the level of the stock price.

On an empirical level several studies confirm the existence of a state dependent function of relative risk aversion. JACKWERTH (2000), AIT-S AHALIA/LO (2000), ROSENBERG/ENGLE (2002) and PÉRIGNON/VILLA (2002) for example all find state dependent risk aversion functions for equity indices by independently estimating and comparing the physical and the option-implied risk-neutral density ${ }^{164}$ All studies employ largely non-parametric techniques for the extraction of the physical and the risk-

164 JACKWERTH (2000) even detects the existence of a so-called prizing kernel puzzle, which consists in a relative risk aversion function implying a marginal utility which is locally increasing in the level of the equity index. RosEnBERG/ENGLE (2002) and PÉRIGNON/VILLA (2002) confirm the existence of the prizing kernel puzzle. 
neutral density ${ }^{165}$ and refrain from imposing any parametric structure on the function of relative risk aversion. However, in exchange for allowing a state independent and time-varying risk aversion function, they are forced to subject the measurement of the physical density to a certain stationarity assumption.

Another, but similar strand in the literature assumes a possibly time-varying but state independent coefficient of relative risk aversion $\sqrt{166}$. The objective of these studies consists either in the estimation of the risk aversion coefficient which balances physical and risk-neutral density ${ }^{167}$, or in the forecast of the physical density with the help of the option-implied risk-neutral density adjusted by the estimated degree of risk aversion ${ }^{168}$ In the light of the above evidence of state dependent risk aversion, the assumption of state independent risk aversion can be considered as a simplistic attempt to make reality comply with the idealized Black-Scholes world.

While the prior approaches focus on the comparison of the physical and the riskneutral density in order to learn more about the market's risk aversion, some researchers instead concentrate on the simultaneous modeling of the physical and the risk-neutral process of the underlying. Such joint specifications of the two processes rely on time series data of option and contemporaneous index prices and involve the estimation of risk premia for volatility and jump risks 169 . As has been discussed above, the accommodation of additional risk factors clearly violates the assumptions of the idealized Black-Scholes world.

What has been said so far demonstrates how risk aversion can explain differences between the physical and the risk-neutral density or differences between the physical and the risk-neutral process, respectively, which are not compatible with the BlackScholes assumptions. Since such deviations from the Black-Scholes world forcibly

165 All four studies except ROSENBERG/ENGLE (2002) use kernel regression techniques to recover the physical distribution. ROSENBERG/ENGLE (2002) use a GARCH based method instead. While kernel regression is also used for the risk-neutral distribution by AIT-SAHALIA/LO (2000) and PÉRIGNON/VILLA (2002), JACKWERTH (2000) and ROSENBERG/ENGLE (2002) on the other hand resort to a smoothing algorithm for the implied volatility smile. See for example BARTUnEK/ChOWdhury (1997), Bliss/PANigirTzOGLOU (2004) and ANAGNOU ET AL. (2005). See BARTUNEK/CHOWDHURY (1997) and Bliss/PANIGIRTZOGLOU (2004).

See ANAGNOU ET AL. (2005) and BLISS/PANIGIRTZOGLOU (2004).

Examples of this approach are PAN (2002), SANTA-CLARA/YAN, LIU ET AL. (2005) and ERAKER (2004).

CHERNOV/GHYSELS (2000) should not be left unmentioned in this context. However, unlike the other studies they do not primarily focus on the extraction of risk premiums. Instead, they use the index time series for the estimation of the amount of conditional kurtosis to be generated by the index process specification. 
result in a smile phenomenon, risk aversion automatically serves as an explanation of the smile.

However, this statement is only true in the absence of a Peso problem ${ }^{170}$. Unfortunately, a gab between the distribution of past returns and the forward looking distribution implied in option prices cannot with absolute certainty be exclusively attributed to risk aversion. The reason is that it could equally be due to market expectations not reflected in the history of past returns. The distinction between the expectation of certain events on the one hand and a risk premium for the possibility of such events on the other hand is a general problem arising any time there is a gap between the distribution of past returns and the forward looking distribution implied in option prices.

\subsubsection{Fundamental Explanations of the Real World Manifestation of Risk Aversion}

As has been shown above, risk aversion in the real world manifests itself in a way incompatible with the Black-Scholes assumptions. This inconsistency is tantamount with the existence of the smile phenomenon. However, nothing has been said so far as to the presumed causes of a form of risk aversion which does not comply with an idealized Black-Scholes economy.

One of the most common explanations of Black-Scholes inconsistent risk aversion is linked to the possibility of rare, but large downward moves like market crashes. The earliest explanations of such sort in connection with the smile phenomenon has been offered by RUBINSTEIN (1994), who interpreted the relative left-skewness of the risk-neutral index density compared to the physical density as a sign of "Crashophobia". However, he did not specify, whether he also suspects the existence of a Peso problem or not. Are the relatively high probabilities of the left tail of the risk-neutral density exclusively caused by risk aversion to large downward moves or also by the expectation that such moves will be more likely in the future?

170 The fact that the market seems to anticipate large moves of a type not included in the history of past returns is called a Peso problem. The Peso problem is associated with a warning by Milton Friedman in the early 1970's that the FX-market anticipated a devaluation of the Mexican Peso. The exchange rate between US-Dollar and Mexican Peso was fixed between 1954 and 1976. During this period there existed an unnatural positive interest rate differential between the Mexican Peso and the US-Dollar. Friedman interpreted this differential as the market's anticipation of a devaluation of the Mexican Peso, which in effect took place in 1976 after the Peso was allowed to freely float against the Dollar. Therefore the seemingly abnormal interest rate differential can be well explained by the market's expectation of a future event which is not part of past experience (see SILL (2000)). 
Other examples of risk aversion to large downward moves are given by AIT-S AHALIA ET AL. (2001) and LIU ET AL. (2005). AIT-S AHALIA ET AL. (2001) find evidence of a Peso problem and claim that the risk-neutral index density can only be explained by the anticipation of and risk aversion to large, yet unheard-of downward moves. LIU ET AL. (2005) on the other hand hold that the uncertainty in connection with rare but large downward moves justifies the existence of a special risk premium for this class of events which is separate from the risk premium for ordinary diffusive moves.

The uncertainty approach of LIU ET AL. (2005) is related to yet another strand in the literature, which postulates a world of incomplete information where investors are exposed to information uncertainty as to the behavior of the underlying ${ }^{171}$. In these models information is revealed in a random fashion which can be considered as the introduction of an additional source of risk. The exposure to this supplementary risk factor causes risk aversion just like the uncertainty with regard to rare events in the model of LIU ET AL. (2005).

Still another strand in the literature holds that the aggregation of possibly BlackScholes-conform, but heterogeneous degrees of risk aversion can cause aggregate, non constant relative risk aversion and thus produce the smile phenomenon ${ }^{172}$

The reason for heterogeneous degrees of risk aversion is believed to consist in different roles played by different market participants. A stylized example of this is the separation of the economy into risk averse asset managers on the one hand and less risk averse hedge fund manager on the other. The relatively risk averse asset managers seek portfolio insurance against downward moves in the form of put options, which are offered by the less risk averse hedge fund managers ${ }^{173}$.

It is worthwhile noting that the idea of portfolio insurance causing the smile can be approached from different directions. While in section 4.3.1 we discussed excess put demand due to market frictions, here different degrees of risk aversion of put buyers and sellers are responsible for the smile phenomenon.

171 Exponents of this strand of the literature are for example DAVID/VERONESI (2000) and GUIDOLIN/TIMMERMANN (2003).

172 Exponents of this strand in the literature are for example GROSSMAN/ZHOU (1996), BENNINGA/MAYSHAR (2000), BATES (2001) and ZIEGLER (2002).

173 While GROSSMAN/ZHOU (1996), BENNINGA/MAYSHAR (2000) and BATES (2001) provide this or a similar explanation for the existence of heterogeneous degrees of risk aversion, ZIEGLER (2002) does not furnish any causes. 


\subsection{Summary and Conclusions}

Above, we discussed the most important phenomenological and causal explanations of the equity volatility smile which are not related to default risk. However, all of the above approaches exhibit certain weaknesses, especially when it comes to the explanation of the individual stock smile.

The phenomenological approaches indeed manage to replicate the smile and its term structure fairly well, especially in the guise of rather complicated process specifications simultaneously involving jumps and stochastic volatility. However, these models arouse the suspicion of being rather arbitrary, because they fail to offer any true causal explanation of the smile.

As opposed to the phenomenological approaches, the causal approaches are able to furnish fundamental reasons for the existence of the smile phenomenon. They can be distinguished into explanations related to the market microstructure on the one hand and into explanations associated with risk aversion on the other.

To begin with, market microstructure related substantiations can often only partly explain the smile. Further, some approaches of this category possibly do not loose their impact with increasing time horizon which contradicts the fact that the smile eventually flattens out. And finally, the literature regarding market microstructure related causes of the smile is fragmented, partly contradictory and does not represent any mainstream opinions or beliefs.

In contrast, risk aversion in a form inconsistent with the Black-Scholes assumptions could be a convincing, causal explanation of the smile. However, risk aversion deviating from the Black-Scholes type needs in turn to be explained itself. Corresponding secondary substantiations all center on aggregate or individual risk aversion as to large downward moves, possibly in conjunction with a Peso problem.

All in all, it seems as if the anticipation of or risk aversion to large downward moves were the only fundamental and at the same time convincing explanation of the smile so far. But since this substantiation has only been proposed in the context of the equity index smile, its significance for the individual stock option smile is not self-evident. Naturally, large downward moves of the index can be interpreted as market crashes. Similarly, a large downward move of the individual stock price could be equated with the default event. However, there exists the slight difference that the crashed market can recover, whereas the defaulted stock cannot. This also means that market crashes can theoretically be part of the history of past market returns, whereas the history of 
past stock returns of the undefaulted company must forcibly be devoid of the default event. It follows that in the case of the individual, defaultable company a Peso problem will always exist and differences between the physical and the risk-neutral density do not only reflect risk aversion as to the default event, but must likewise include its anticipation.

In summary, it can be concluded that almost all of the above approaches attempting to explain the equity volatility smile are inadequate, either because they fail to furnish fundamental causes or because they only offer a partial substantiation. The only convincing explanation amounts to the anticipation of and risk aversion to large downward moves, but only refers to the index smile. This raises the question as to the significance of large downward moves for the individual stock smile and insinuates that these could be associated with financial distress and the default of the individual company. While default risk as an explanation of the smile has deliberately not been addressed so far ${ }^{174}$, it will be dealt with in detail in the next chapter.

174 In none of the above approaches to explain the smile phenomenon, default risk is considered as an explicit factor or variable. The only exception is the jump-to-ruin version of the model of MERTON (1976), which so far has only been presented in its mode of a regular jump-diffusion model. 



\title{
5. Default Risk as an Explanation of the Equity Volatility Smile
}

\begin{abstract}
Although the last chapter initially focused on explanations of the equity volatility smile which are not related to default risk, it still concluded that default risk should be taken into account as a possible cause. This conclusion is heeded by this chapter which centers on the link between smile and default risk. The examination of this link will be grouped into two parts. In the first part, the nature of default risk and its connection with the equity volatility smile will be illustrated by means of traditional credit risk models and extant empirical evidence. In the second part, more recent model enhancements incorporating equity options and establishing a direct link between default risk and the smile will be discussed. These model enhancements build upon the theoretical and empirical insights presented in the first part. The chapter concludes with a critical appraisal of the adequacy of theory and empiricism in explaining the link between default risk and the equity volatility smile.
\end{abstract}

\subsection{The Nature of Default Risk and its Theoretical and Empirical Connection with the Equity Volatility Smile}

The present section starts with a brief sketch of the basic ideas of default risk modeling. It ensues a description of the theoretical link between default risk and the equity volatility smile. Thereafter, this theoretical view will be confronted with established empirical evidence concerning the relationship between default risk and the equity volatility smile. The section concludes with a review of the explanations of the smile of the last chapter in the light of default risk. 


\subsubsection{Default Risk in Theory}

Since credit risk models can be coarsely classified into structural and reduced form model ${ }^{175}$, the theoretical foundation of individual default risk will be briefly sketched using this classification. As will be seen later on, this partition is also useful, since recent enhancements of credit risk models incorporating equity options can also be classified according to this logic. The presentation of the two traditional classes of credit risk models will be accompanied by the important theoretical concept of the leverage effect, which is a cornerstone of most structural credit risk models and represents a mechanism linking default risk and stock price volatility.

\subsubsection{Structural Credit Risk Models}

The firm-value model originally proposed by MERTON (1974) is the prototype of all structural credit risk models 176 . The Merton model assumes that the value of the firm $V$ is composed of the market value of equity $S$ and that of debt $B$ - hence the name firm-value model - and that $V$ follows a geometric Brownian motion. Under a riskneutral measure $Q$, the process of $V$ is given by ${ }^{177}$.

$$
d V_{t}=r V_{t} d t+\sigma_{V} V_{t} d z_{t}^{Q}
$$

The firm's debt is presumed to be a single zero bond maturing in time $T$ with face value $D$. Based on the Black-Scholes option pricing approach, the equity is interpreted as a European call option on the firms assets $V$ with a strike price equal to $D^{178}$.

- If at the option's expiry $T, V_{T}$ is below $D$, the call option expires worthlessly and the equity holders give up the firm and render the firm's control to the bond holders. The bond holders recover the remaining firm value.

- If on the other hand at the option's expiry $T$ the value of the firm $V_{T}$ exceeds the notional value of the debt $D$, the equity holders exercise their call option by paying back the debt $D$.

175 See MCNEIL ET AL. (2005), p. 328

176 See MCNEIL ET AL. (2005), p. 331. Except otherwise indicated, the following exposition of the Merton model follows largely MCNEIL ET AL. (2005), pp. 331-334.

177 See MCNEIL ET AL. (2005), pp. 401-402.

178 Although the firm value model is commonly attributed to MERTON (1974), already BLACK/SCHOLES (1973) reasoned about an application of their option pricing formula to the firm's assets in order to evaluate the firm's debt. 
Given today's value of the firm's assets, $V_{t_{0}}$, the probability that the firm has to default in time $T$, because the assets are worth less than the face value of the debt, is given by the risk-neutral default probability $Q_{d} 179$.

$$
Q_{d}=Q\left(V_{T} \leq D\right)=\Phi\left(\frac{\ln \frac{D}{V_{t_{0}}}-\left(r-\frac{1}{2} \sigma_{V}^{2}\right)\left(T-t_{0}\right)}{\sigma_{V} \sqrt{T-t_{0}}}\right)
$$

The time $t_{0}$ price of $S$ can be represented as the value of a Black-Scholes call option $c_{t_{0}}^{B S}$ :

$$
S_{t_{0}}=c_{t_{0}}^{B S}=V_{t_{0}} N\left(d_{1}\right)-D e^{-r\left(T-t_{0}\right)} N\left(d_{2}\right)
$$

where

$$
\begin{gathered}
d_{1}=\frac{\ln \left(\frac{V_{t_{0}}}{D}\right)+\left(r+\frac{\sigma_{V}^{2}}{2}\right)\left(T-t_{0}\right)}{\sigma_{V} \sqrt{T-t_{0}}} \\
d_{2}=d_{1}-\sigma_{V} \sqrt{T-t_{0}}
\end{gathered}
$$

The time $t_{0}$ price of a European put $p_{t_{0}}^{B S}$ ensues accordingly:

$$
p_{t_{0}}=-V_{t_{0}} N\left(-d_{1}\right)+D e^{-r\left(T-t_{0}\right)} N\left(-d_{2}\right)
$$

Thanks to the put-call-parity, the market value of the defaultable debt $B$ can be expressed as the sum of the discounted notional debt value and a short position in a put contract $p_{t_{0}}$ on the asset's of the firm 180 ,

$$
B_{t_{0}}=D e^{-r\left(T-t_{0}\right)}-p_{t_{0}}
$$

Be $\hat{B}_{t_{0}}=D e^{-r\left(T-t_{0}\right)}$ today's value of $D$, if $D$ were issued by the government and thus were not defaultable. Then the credit spread $s$ as the difference between the continuously compounded rate of return of $B$ and $\hat{B}$ is defined according to the following expression:

$$
s=-\frac{1}{T-t_{0}} \ln \frac{B_{t_{0}}}{\hat{B}_{t_{0}}}
$$

The original, rather simple Merton model has been extended by many authors and in various ways of which only some shall be reported here. While the original model only

179 See MCNEIL ET AL. (2005), pp. 401-402.

180 According to the put-call-parity, the value of the firm $V_{t}$ plus a put contract $p_{t}$ must equal the sum of the exercise price discounted at the risk free rate $D e^{-r(T-t)}$ and a call contract $c_{t}: V_{t}+p_{t}=$ $D e^{-r(T-t)}+c_{t}$. Substituting for $c_{t}$, this can be reexpressed as: $V_{t}=S_{t}+D e^{-r(T-t)}-p_{t}$. Due to the relation $V_{t}=S_{t}+B_{t}$ it follows that $B_{t}=D e^{-r(T-t)}-p_{t}$ (see LANDO (2004), p. 10.) 
allows the default to happen at the end of the option's term, the model of BLACK/COX (1976) also permits default to occur prior to time $T$ as soon as a default barrier has been touched by the path of the firm value $V_{t}$. Besides, the model of BLACK/COX (1976) also features the possibility that the default is endogenously triggered by the equity holders. LONGSTAFF/SCHWARTZ (1995) introduces stochastic interest rates and $\mathrm{ZHOU}$ (2001) adds jumps to the firm value process, thus accommodating credit spreads clearly greater than zero for remaining terms close to zero. Besides also considering taxes and insolvency costs, the endogenous models of LELAND (1994) and LELAND/TOFT (1996) model the default barrier as a zero bond of infinite maturity or a set of finite maturity, but endlessly revolving bonds, respectively. To conclude this short excerpt it is also worthwhile mentioning the model of COLLINDUFRESNE/GOLDSTEIN (2001), who assume that the firm's leverage is constantly adjusted in a way that the firm's leverage ratio remains unchanged.

The main drawback of structural credit risk models consists in the fact that the key input variables - the firm value and its volatility - are not directly observable and have to be inferred from other observable parameters like the stock price, balance sheet data and the stock price volatility 181

\subsubsection{Reduced-Form Credit Risk Models}

In contrast to structural models, reduced-form models do not specify the structural setting of the firm and its capital structure which causes the default event ${ }^{182}$. Instead they model the default time as a random variable, which typically depends on economic covariables 53 somehow linked to default risk like ratings, debt-to-equity ratios, volatility measures, stock prices, yield spreads, industry performance measures and macroeconomic variables 184 Therefore, without recurring to these covariables, reduced-form models are not able to intrinsically explain the level of default risk as do their structural counterparts.

The default time $\xi$ in a reduced-form model is the first jump time of a counting process whose countable events are driven by an intensity or hazard rate process $h^{185}$ Let $h_{t}$ be the process under the risk-neutral measure $Q$, then the risk-neutral probability for

\footnotetext{
181 Cf. for example HULL ET AL. (2004a).

182 See MCNEIL ET AL. (2005), p. 328.

183 See MCNEIL ET AL. (2005), p. 328.

184 Cf. DUFFIE (2005).

185 See DUFFIE
} 
the firm to default between $t_{0}$ and $T$ equals the probability that $\xi$ is less than or equal to $T$, given that the firm has survived until time $t_{0}{ }^{186}$.

$$
Q_{d}=Q\left(\xi \leq T \mid \xi>t_{0}\right)=1-e^{-\int_{t_{0}}^{T} h_{t} d t}
$$

One of the key advantages of intensity models is their closeness to default-free term structure models, which allows to model defaultable securities in a way very similar to the modeling of default free instruments ${ }^{187}$. As in the previous section, let $D$ be the face value of a zero coupon bond maturing in time $T$ and let $r_{t}$ be the process of the risk-free interest rate, then the present value of a default-free bond $\hat{B}_{t_{0}}$ in time $t_{0}$ can be represented in the following form ${ }^{188}$,

$$
\hat{B}_{t_{0}}=D E_{t_{0}}^{Q}\left[e^{-\int_{t_{0}}^{T} r_{t} d t}\right]
$$

The value of the defaultable bond with zero recovery can be obtained by additionally considering the process of the default intensity $h{ }^{189}$.

$$
B_{t_{0}}=D E_{t_{0}}^{Q}\left[e^{-\int_{t_{0}}^{T}\left(r_{t}+h_{t}\right) d t}\right]
$$

Expressions 5.10 and 5.11 show that the intensity $h_{t}$ can be interpreted as a timevarying credit spread. The average credit spread $s$ can be computed as above by means of equation 5.8 .

If the intensity $h_{t}$ driving the random default time $\xi$ is deterministic, the default process is a Poisson process 190 . If on the other hand the intensity $h_{t}$ is itself stochastic, the default process is called a doubly stochastic process 191 .

While the first reduced-form model proposed by JARROW/TURNBULL (1995) assumes a constant default intensity $h$, later models presume a time-varying intensity dependent on exogenous state variables. DUFFIE/SINGLETON (1999) very generally allow $h_{t}$ to be determined by firm-specific or macro-economic variables, whereas MADAN/UNAL (1998) and MADAN/UNAL (2000) let $h_{t}$ to be influenced by the value of the equity. This dependence of the intensity $h_{t}$ on the equity value will still be of interest below when discussing reduced-form models incorporating equity options.

\footnotetext{
186 See. LANDO (2004), p. 84 and p. 113.

187 Cf. LANDO (2004), p. 109.

188 Cf. LANDO (2004), p. 112.

189 Cf. $\overline{\text { LANDO }}(\overline{2004})$, p. 113.

190 See DUFFIE (2005).

191 See DUFFIE (2005).
} 


\subsubsection{The Leverage Effect}

Structural firm value models like the Merton model imply important mechanics concerning the relationship between default risk and the equity volatility. These mechanics are referred to as the leverage effect.

As above let us suppose that the value of the firm $V_{t}$ is composed of the value of the equity $S_{t}$ and the value of the debt $B_{t}, V_{t}=S_{t}+B_{t}$, and that $V_{t}$ follows a geometric Brownian motion under a risk-neutral measure $Q$. Further, if we interpret the equity value $S$ as a derivative of the firm value $V_{t}$, application of Ito's Lemma yields the following expression for changes of $S_{t}{ }^{192}$.

$$
d S_{t}=\left(\frac{\partial S_{t}}{\partial V_{t}} r V_{t}+\frac{\partial S_{t}}{\partial t}+\frac{1}{2} \frac{\partial^{2} S_{t}}{\partial V_{t}^{2}} \sigma_{V}^{2} V_{t}^{2}\right) d t+\frac{\partial S_{t}}{\partial V_{t}} \sigma_{V} V_{t} d z_{t}^{Q}
$$

This means that the standard deviation of absolute stock price changes equals $\frac{\partial S_{t}}{\partial V_{t}} \sigma_{V} V_{t}$. Consequently, the standard deviation of relative stock price changes $\sigma_{S}$ ensues accordingly 193 .

$$
\sigma_{S}=\sigma_{V} \frac{\partial S_{t}}{\partial V_{t}} \frac{V_{t}}{S_{t}}
$$

Since the partial derivative $\frac{\partial S_{t}}{\partial V_{t}}$ is normally unknown, it is often set to $1^{194}$, assuming that all changes in firm value are completely reflected in the change of the equity value and that the market value of the debt remains unaffected 195 .

$$
\sigma_{S}=\sigma_{V} \frac{V_{t}}{S_{t}}=\sigma_{V} \frac{S_{t}+B_{t}}{S_{t}}
$$

Let the leverage ratio $L_{t}$ be the ratio of the market value of debt $B_{t}$ to that of equity $S_{t} 196$.

$$
L_{t}=\frac{B_{t}}{S_{t}}
$$

192 See MERTON (1974).

193 See CHRISTIE (1982) or CHELLEY-STEELEY/STEELEY (2005), e.g.

194 Cf. for example CHRISTIE (1982), CHELlEY-STEELEY/STEELEY (2005) or FIGLEWSKi/WANG (2000).

195 The simplifying assumption, that the partial derivative $\frac{\partial S_{t}}{\partial\left(S_{t}+B_{t}\right)}$ equals 1 can be argued, if the firm is close to default. Then the equity value is close to zero and it is likely that firm value changes also affect the value of debt and not only that of the equity. This would reduce the term $\frac{\partial S_{t}}{\partial\left(S_{t}+B_{t}\right)}$ and consequently dampen the leverage effect.

$196 L_{t}$ can alternatively be defined as the ratio of the notional value of debt $D$ to the market value of equity $S_{t}: L_{t}=\frac{D}{S_{t}}$. While FigLEWSKI/WANG (2000) and CHELLEY-STEELEY/STEELEY (2005) take this stance, CHRISTIE (1982) derives the leverage ratio based on the market value of debt. 
Substituting for $\frac{B_{t}}{S_{t}}$ in equation 5.14 leads to the following expression:

$$
\sigma_{S}=\sigma_{V}\left(1+L_{t}\right)
$$

Equation 5.16 encapsulates the leverage effect, which exhibits two important properties:

- Equation 5.16 illustrates that for a given firm volatility $\sigma_{V}$ a change in leverage leads to an equally directed change in equity volatility $\sigma_{S}$. Recalling the definition of $L_{t}=\frac{B_{t}}{S_{t}}$, it is obvious that a change in leverage can be induced either by a change in the level of debt or a change in the level of equity or both. This entails that for a given level of $B_{t}$, a change of $S_{t}$ leads to an inverse change of $\sigma_{S}{ }^{197}$.

- Further, the magnitude of the leverage effect depends on the leverage ratio $L_{t}$. The same proportional change $d \ln \left(S_{t}\right)$ will have a high effect on $\sigma_{S}$ if $L_{t}$ is high, and a low effect, if $L_{t}$ is low ${ }^{198}$. This can be shown by imagining the extreme case of a leverage $L_{t}$ close to zero, which entails that $B_{t}<<S_{t}$ and $\frac{B_{t}}{S_{t}}<<1$, which signifies that a proportional change $d \ln \left(S_{t}\right)$ has almost no effect on $\sigma_{S}$, since after this change $\frac{B_{t}}{S_{t}}$ will still be very small. On the other hand, if $L_{t}$ is very large, it follows that $B_{t}>>S_{t}$ and $\frac{B_{t}}{S_{t}}>>1$, which in turn implies that the same proportional change $d \ln \left(S_{t}\right)$ will have a much larger effect on $\sigma_{S}$.

\subsubsection{Theoretical Connection between Default Risk and the Implied Volatility Smile}

\subsubsection{Theoretical Connection between Default Risk and the Level of Equity Volatility}

Before addressing the link between default risk and the form of the equity volatility smile let us examine the theoretical relationship between default risk and the level of the equity volatility.

To this end it is helpful to take a closer look at the Merton firm value model which implies a positive relationship between default risk on the one hand and firm volatility and leverage on the other. If we accept the risk-neutral default probability $Q_{d}$ as a

197 See Chelley-Steeley/STEEley (2005).

198 See CHELLEY-STEELEY/STEELEY $(2005)$. 
measure of default risk, the latter can be considered to be essentially a function which is monotonically increasing in the leverage ratio $L_{t}$ and the firm volatility $\sigma_{V} 199$.

$$
Q_{d}=f\left(L_{t}, \sigma_{V}\right), \frac{\partial Q_{d}}{\partial L_{t}} \geq 0, \frac{\partial Q_{d}}{\partial \sigma_{V}} \geq 0
$$

Thanks to the leverage effect, the positive relationship between default risk and firm volatility implied in the Merton model can be translated into a positive relationship between default risk and equity volatility.

More precisely, using equation 5.16 which encapsulates the leverage effect, allows to substitute the firm volatility $\sigma_{V}$ in expression 5.18 in terms of the equity volatility $\sigma_{S}$. Therefore, the default risk $Q_{d}$ can likewise be expressed as a function which is monotonically increasing in the equity volatility $\sigma_{S}$ :

$$
Q_{d}=f\left(L_{t}, \frac{\sigma_{S}}{1+L_{t}}\right), \frac{\partial Q_{d}}{\partial \sigma_{S}} \geq 0
$$

The positive relationship between default risk and equity volatility represents an important finding in its own right. As to the implied volatility smile it predicts that the horizontal level of the smile should be situated all the more higher, the higher the level of default risk. But beyond, nothing much can be learned concerning the exact form of the smile. This question will be addressed in the next section.

199 This can be shown by means of equation 5.2

- As to $\frac{\partial Q_{d}}{\partial L_{t}} \geq 0$ : For reasons of simplicity, let us redefine the initial leverage ratio $L_{t_{0}}$ as the ratio of the fixed notional value of debt $D$ with respect to the initial stock price $S_{t_{0}}$ : $L_{t_{0}}=\frac{D}{S_{t_{0}}}$. An increase of $L_{t_{0}}$ can either be caused by an increase of the level of debt $D$ or by a decrease of the initial stock price $S_{t_{0}}$ or by both at the same time. This in turn implies an increase of the term $\ln \frac{D}{V_{t_{0}}}$ in equation 5.2. Since $\Phi(\cdot)$ is a monotonically increasing function, an increase of the leverage ratio $L_{t_{0}}$ ultimately entails an increase of the default risk $Q_{d}$.

Intuitively speaking, an increasing leverage ratio reduces the relative size of the equity cushion and thus makes default more likely.

- As to $\frac{\partial Q_{d}}{\partial \sigma_{V}} \geq 0$ : The default probability of equation 5.2 can be reexpressed as:

$$
\Phi\left(-\frac{\ln \frac{V_{t_{0}}}{D}+r\left(T-t_{0}\right)}{\sigma_{V} \sqrt{T-t_{0}}}+\frac{1}{2} \sigma_{V} \sqrt{T-t_{0}}\right)
$$

From this representation it is obvious that an increasing firm volatility $\sigma_{V}$ leads to increasing default risk. Intuitively speaking, increasing volatility makes it more likely that the firm value process $V_{t}$ ends up below the nominal value of debt $D$. 


\subsubsection{Theoretical Connection between Default Risk and the Shape of the Smile}

To gain a more thorough understanding of the link between default risk and the form of the smile, let us first follow our intuition. Intuitively, the presence of default risk should lead to the expectation of strong downward moves of the stock price and also to corresponding risk aversion as to these downward moves. Consequently, the anticipation of the default event should be reflected in a left-skewed risk-neutral equity return density, which in turn should manifest itself in a downward sloping implied volatility smile (see section 3.1.3.2).

This intuition can be derived in yet another, more theoretical way by recurring to the mechanism of the leverage effect. To recapitulate, the leverage effect exhibits the following two properties:

1. A rising equity volatility $\sigma_{S}$ is associated with a decreasing stock price level $S_{t}$.

2. The magnitude of effect 1 . depends positively on the leverage ratio $L_{t}$.

It can be shown that these two properties translate into the manifestation of a downward sloping equity volatility smile which is monotonically decreasing and convex in the strike price:

ad 1. The first property of the leverage effect, the negative relationship between $S_{t}$ and $\sigma_{S}$, directly leads to a downward sloping smile which is monotonically decreasing: If $S_{t}$ declines from its current value equaling the ATM strike to a value corresponding to a strike level lower than the ATM strike, $\sigma_{S}$ will increase, giving rise to a downward sloping smile ${ }^{200}$. Since this example applies to any point in the strike dimension, the smile must be monotonically decreasing in the strike price. But if the leverage effect is at work, the firm's equity must be levered with defaultable debt, which means that also default risk is present. Therefore, in theory the mere presence of default risk leads to the manifestation of a monotonically downward sloping equity volatility smile.

ad 2. The second property of the leverage effect has two implications: The first and more obvious is that the steepness of the downward sloping smile depends positively on the leverage ratio and by expression 5.18 also on the level of default risk. The second and less obvious implication is that the downward sloping smile is not only monotonically decreasing, but also convex. This can be shown in the following way: The decline of $S_{t}$ to a level corresponding to a strike price

200 Cf. for example FIGLEWSKI/WANG $(2000)$. 
lower than the ATM strike price, but associated with a higher than the ATM volatility will also raise the leverage ratio $L_{t} 201$. This increase in $L_{t}$ exhibits the secondary effect that a further decline in $S_{t}$ will now lead to an even greater increase of the equity volatility which in turn translates into a convex smile curve.

This theoretical prediction of a default risk induced, monotonically downward sloping equity volatility smile raises the question, how this prediction is compatible with the observation of a generally U-shaped smile curve (see section 3.1.2).

\subsubsection{Existing Empirical Evidence of the Relationship between Default Risk and the Equity Volatility Smile}

While the prior sections elaborated the theoretical link between default risk and the individual stock option smile, this section presents extant empirical evidence of this relationship.

\subsubsection{The Leverage Effect}

As has been shown in section 5.1.1.3, the theory concerning the leverage effect predicts a negative relationship between equity returns and changes in equity volatility. The first to empirically detect such a negative relationship was BLACK (1976). Since then, many academics have explored and confirmed this relationship. While at the beginning, the negative relationship between return and changes in volatility was considered synonymous with the leverage effect, later research furnished alternative explanations. For this reason, BEKAERT/WU (2000) introduced the more general expression asymmetric volatility for the negative relationship between returns and changes in volatility ${ }^{202}$

Besides BLACK (1976), also CHRISTIE (1982) finds evidence of the leverage effect as a cause of asymmetric volatility, but already suspects that it cannot be the only reason. Soon after CHRISTIE (1982), PINDYCK (1984), FRENCH ET AL. (1987) and later CAMPBELL/HENTSCHEL (1992) offer the competing explanation of volatility feedback. Whereas the leverage effect theory holds changes in leverage responsible for changes in volatility, the volatility feedback theory starts the causal chain with

201 Cf. for example JACKWERTH (2004), p. 6.

202 Strictly speaking, BEKAERT/WU (2000) coin the term asymmetric volatility for a negative relationship between unexpected returns and changes in conditional volatility, see BEKAERT/WU (2000). 
changes in volatility. According to this theory, a rise in volatility leads to a higher required return and thus to a decline in prices.

The debate as to the origin of asymmetric volatility is still open. The leverage effect as a possible cause has been advocated by many authors besides BLACK (1976) and CHRISTIE (1982) All find evidence of the existence of the leverage effect, although with varying emphasis. While some suppose that it cannot be the only cause of asymmetric volatility ${ }^{204}$, others see it dominated by volatility feedback effect ${ }^{205}$ FIGLEWSKI/WANG (2000) are the only ones to explicitly rebut the leverage effect as the origin of asymmetric volatility. Instead they suspect systematic causes, which they refer to as a down market effect. DENNIS ET AL. (2006) confirm this finding of systematic driving forces, presuming that these systematic factors could be related to the volatility feedback mechanism. One of the latest studies concerning the asymmetric volatility phenomenon is that of BOLLERSLEV ET AL. (2006), who are the first to use high-frequency data and find strong evidence in favor of the leverage effect theory. Although the asymmetric volatility phenomenon has been treated in abundance in the literature, it is not the goal to reproduce all available studies here. Instead, the above cited studies must be considered as an essential extract in order to sketch the ongoing debate. Table 5.1 summarizes the above cited work with a coarse classification with regard to the suspected causes of the asymmetric volatility phenomenon. As can be seen, not all studies concerned with the leverage effect also use financial data to reconstruct a leverage ratio. Rather, the change in leverage is proxied by the equity return assuming that the value of debt remains constant. Further the volatility is estimated by the gross volatility ${ }^{206}$ by some and by the conditional volatility in the context of an $\mathrm{ARCH}$ or GARCH specification ${ }^{207}$ by others. Only 3 studies use option implied volatilities.

203 Examples are SCHWERT (1990), NELSON (1991), ChEung/NG (1992), Glosten Et AL. (1993), DufFeE (1995), BRAun ET AL. (1995), BEKAERT/Wu (2000), Wu (2001), CHELlEYSTEELEY/STEELEY (2005) and most recently BOLLERSLEV ET AL. (2006). SCHWERT (1990) and BRAUN ET AL. (1995) claim that the leverage effect is too weak as to fully explain the asymmetric volatility phenomenon.

205 BEKAERT/WU (2000) and WU (2001) identify leverage effect and volatility feedback as causes of the asymmetric volatility phenomenon, but claim that the leverage effect is dominated by the volatility feedback effect.

206 Gross volatility refers to simple return changes in contrast to conditional volatility estimated with a GARCH-type model, cf. BEKAERT/WU (2000).

207 There exist essentially two versions of GARCH type models that are used in the context of the analysis of the asymmetric volatility phenomenon: The EGARCH specification developed by NELSON (1991) to accommodate asymmetric conditional volatility as well as the leverage effect (cf. also HAMILTON (1994), p. 668) and the GARCH-in-Mean specification proposed by ENGLE ET AL. (1987) relating the conditional volatility to the expected return and thus incorporating the volatility feedback mechanism (cf. also HAMILTON (1994), p. 667). 


\begin{tabular}{|c|c|c|c|c|c|}
\hline Author & Year & $\begin{array}{l}\text { Suspected cause } \\
\text { of asymmetric } \\
\text { volatility* }\end{array}$ & $\begin{array}{c}\text { Use of } \\
\text { financial } \\
\text { data }\end{array}$ & Volatility estimation ${ }^{* *}$ & $\begin{array}{l}\text { Use of } \\
\text { implied } \\
\text { volatility }\end{array}$ \\
\hline Dennis et al. & 2006 & $\mathrm{~s}$ & & Gross & Yes \\
\hline Bollerslev et al. & 2006 & LE & & Gross & \\
\hline Chelley-Steeley and Steeley & 2005 & LE & Yes & Conditional & \\
\hline Wu & 2001 & $\mathrm{LE} / \mathrm{VF}$ & & Conditional & \\
\hline Bekaert and Wu & 2000 & LE / VF & Yes & Conditional & \\
\hline Figlewski and Wang & 2000 & S & Yes & Gross & Yes \\
\hline Braun et al. & 1995 & LE & & Conditional & \\
\hline Duffee & 1995 & LE & Yes & Gross & \\
\hline Glosten & 1993 & LE & & Conditional & \\
\hline Campbell and Hentschel & 1992 & VF & & Conditional & \\
\hline Cheung and $\mathrm{Ng}$ & 1992 & LE & & Conditional & \\
\hline Nelson & 1991 & LE & & Conditional & \\
\hline Schwert & 1990 & LE & Yes & Conditional, gross & Yes \\
\hline French et al. & 1987 & VF & & Conditional & \\
\hline Pindyck & 1984 & VF & & Gross & \\
\hline Christie & 1982 & LE & Yes & Gross & \\
\hline Black & 1976 & LE & Yes & Gross & \\
\hline
\end{tabular}

Table 5.1.: Extract of the literature analyzing the causes of the asymmetric volatility phenomenon

In summary, it can be said that there exists non negligible evidence of the existence of the leverage effect, although its non-existence has also been advocated. If existent, the leverage effect seems to be dominated by other factors, like systematic forces or the volatility feedback effect.

\subsubsection{Default Risk and the Level of Equity Volatility}

Section 5.1.2.1 elaborated, why default risk is positively coupled with the level of firm value volatility and equity volatility. This positive relationship has been overwhelmingly confirmed by many empirical studies 208 , of which the most important are summarized in table 5.2. As can be seen in table 5.2, preferences for the default risk measure are about equally split between bond and CDS spreads and for the equity volatility measure between implied and historical volatility. Deviating from these choices, COLLIN-DUFRESNE ET AL. (2001) use a a volatility index as a substitute for individual volatility.

208 Examples are CAO ET AL. (2006), AVRAMOV ET AL. (2006), CREMERS ET AL. (2005b), ZHANG ET AL. (2005), HULL ET AL. (2004a), BENKERT (2004), ERICSSON ET AL., CAMPBELlTAKSLER (2003), CONSIGLi (2004) and COLLIN-DUfRESNE ET AL. (2001). 


\begin{tabular}{l|c|c|c|c}
\multicolumn{1}{c|}{ Author } & Year & $\begin{array}{c}\text { Relationship } \\
\text { between default } \\
\text { risk and equity } \\
\text { volatility }\end{array}$ & Default risk measure & $\begin{array}{c}\text { Equity volatility } \\
\text { measure }\end{array}$ \\
\hline Cao et al. & 2006 & + & CDS spread & Implied \\
Avramov & 2006 & + & Bond spread & Historical \\
Cremers et al. & 2005 & + & Bond spread & Implied \\
Zhang et al. & 2005 & + & CDS spread & Historical \\
Hull et al. & 2004 & + & CDS spread & Implied \\
Benkert & 2004 & + & CDS spread & Implied \\
Ericsson et al. & 2004 & + & CDS spread & Implied \\
Consigli & 2004 & + & Bond spread & Historical \\
Campbell and Taksler & 2003 & + & Bond spread & Volatility index \\
Collin-Dufresne et al. & 2001 & + &
\end{tabular}

Table 5.2.: Extract of the literature analyzing the relationship between default risk and equity volatility

Most studies regress their default risk measure on certain firm-specific and systematic variables, one of which is the equity volatility measure. All studies unanimously find a positive relationship between the equity volatility and the default risk measure. Equity volatility alone or in union with other variables is found to explain up to $56 \%$ of the default risk measure 209 Generally, the studies using implied volatility are able to detect a stronger degreee of dependence between default risk and the level of equity volatility than the studies based on historical volatility measures.

The three studies of ERICSSON ET AL., of AVRAMOV ET AL. (2006) and of COLLINDUFRESNE ET AL. (2001) rely on regressions in differences, but only AVRAMOV ET AL. (2006) do so accosting the question of possible non-stationarity of the respective series. COLLIN-DUFRESNE ET AL. (2001) claim to focus on differences for economic reasons.

\subsubsection{Default Risk and the Shape of the Equity Volatility Smile}

As discussed in section 5.1.2.2, the theory predicts that the slope of the equity volatility smile should be all the more steeper the higher the level of default risk. However, empirical evidence concerning this relationship is very rare. Altogether only five studies analyze the link between default risk and the slope of the smile. This scarcity of empirical evidence is marginally remedied by the fact that two additional studies analyze the relationship between default risk and the higher moments of the equity return distribution.

209 Among the reviewed studies, CAO ET AL. (2006) report the highest explanatory power in terms of $R^{2}$, namely $56 \%$. 
Table 5.3 summarizes the extant work investigating the dependence between default risk on the one hand and the slope of the equity volatility smile and the higher moments of the equity return distribution on the other and shows the identified directions of dependence.

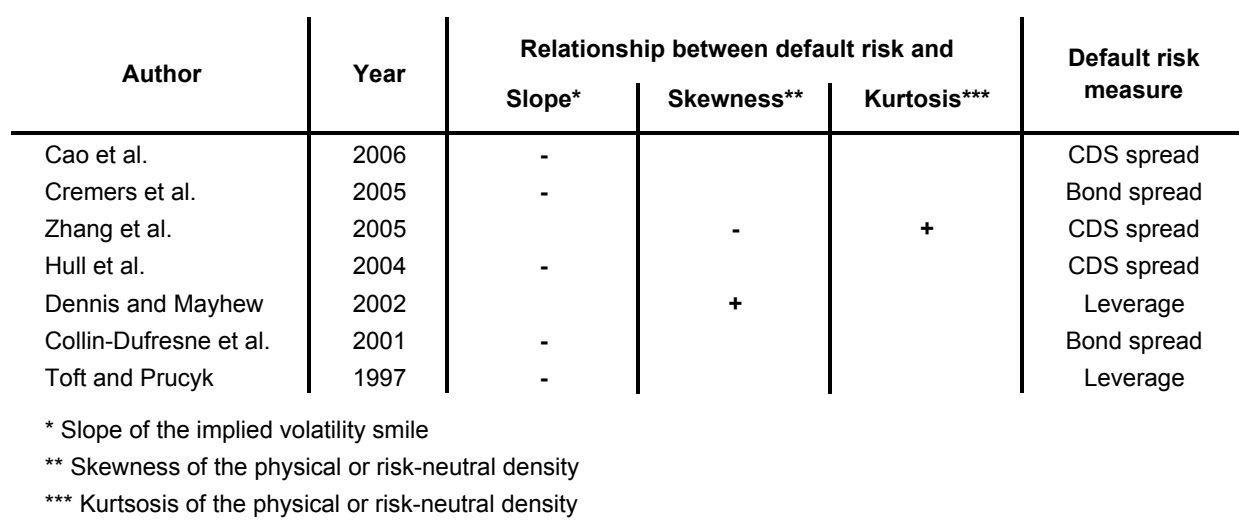

Table 5.3.: Compilation of the literature analyzing the relationship between default risk and the slope of the equity volatility smile

In most cases default risk is proxied by bond or CDS spreads, only TOFT/PRUCYK (1997) and DENNIS/MAYHEW (2002) use financial leverage instead.

The five studies concentrating on the slope of the implied volatility smile all use similar slope measures based on the difference between the implied volatility of an option with a strike-to-spot-ratio of less than one and the ATM implied volatility ${ }^{210}$. Only TOFT/PRUCYK (1997) deviate from this approach by constructing a slope measure of options that are symmetrically spaced around the ATM point. But since their slope measure construction also involves a division by the level of the ATM volatility, their measure becomes arguable ${ }^{211}$. Of the researchers focusing on the slope of the smile, COLLIN-DUFRESNE ET AL. (2001) are the only ones who derive their slope measure from the smile of a stock index instead of from the smile of individual stocks, arguing that for the purposes of their analysis, the stock index smile is representative of

210 CAO ET AL. (2006) and CREMERS ET AL. (2005b) use the difference between the implied volatility of the option nearest to a strike-to-spot-ratio of 0,92 and that of the option nearest to the ATM point. COLLIN-DUFRESNE ET AL. (2001) carry out a polynomial regression of the implied volatility on the strike-to-spot-ratio and calculate the difference between the corresponding approximated implied volatility with a strike-to-spot-ratio of 0.9 and that with a strike-to-spot-ratio of 1. HULL ET AL. (2004a) use the difference between the implied volatility of a 0,25-delta and a 0,5 -delta put option.

211 Rightly, DENNIS/MAYHEW (2002) point out that the result of TOFT/PRUCYK (1997) is questionable, since their slope measure implies division by the implied ATM volatility, which raises the question whether a covariation of their default risk and their slope measures has to be attributed to a change of the slope of the smile or rather to a change of the level of implied ATM volatility. 
the individual smile. In the context of our research question however, neglecting the individual slope seems questionable.

All slope measures except that of TOFT/PRUCYK (1997) would imply a positive sign for a downward sloping smile. Since this contradicts the convention of notation adopted in my thesis, the direction of dependence indicated in table 5.3 is equivalent with the one which would have been reported, if the first derivative of the implied volatility with respect to the strike-to-spot-ratio had been used.

All studies testing the link between default risk on the one hand and the slope of the smile on the other rely on simple regression analysis and agree upon a negative relationship between default risk and slope measure. Thus, the level of default risk seems indeed to be negatively coupled with the slope of the smile. However, due to the construction of the slope measure, this relationship only refers to strike levels at or slightly below the ATM point.

DENNIS/MAYHEW (2002) and ZHANG ET AL. (2005) also analyze the relationship between default risk and the higher moments of the equity return distribution. While DENNIS/MAYHEW (2002) use the Bakshi-Madan approach (see section 3.2.2.3) to imply the risk-neutral return skewness out of option prices, ZHANG ET AL. (2005) resort to daily equity returns to calculate historical skewness and kurtosis measures. DENNIS/MAYHEW (2002) find weak evidence of a positive relationship between the level of leverage and their skewness measure. ZHANG ET AL. (2005) on the other hand identify a negative relationship between their skewness measure and the CDS spread level and a positive relationship between their kurtosis measure and the CDS spread level.

Besides their firm-level study CREMERS ET AL. (2005b) mentioned in table 5.3, the same authors also analyze the link between option-implied jump risk measures on the aggregate CDS spread level in CREMERS ET AL. (2005a). They find that jump risk measures implied out of index options can contribute substantially to the explanation of the market wide CDS spread level, which corroborates the role of systematic influences.

All in all, the five studies analyzing the link between default risk and the slope of the smile seem to corroborate the theoretical prediction that the slope of the smile is negatively coupled with the level of default risk. However, with only five studies this evidence remains scarce. Further, the distinct studies are not directly comparable due to different slope and default risk measures and two studies rely on questionable slope variables. And what is more, beyond the ATM point no insights at all seem 
to exist. Further, there exist only two studies with moreover contradicting results targeting the relationship between default risk and the higher moments of the equity return distribution. All this underlines the necessity of further and more thorough empirical research.

\subsubsection{Review of the Smile Explanations which are not Related to Default Risk}

\subsubsection{Introduction}

The explanations of the equity volatility smile in chapter 4 were limited to approaches not related to default risk. In the following, this lack will be complemented by their review in the light of default risk. In doing so, it will be concentrated on the phenomenological explanations of section 4.2, since these are unbiased in the sense that they do not presume any fundamental cause of the smile. Therefore, their smile fitting ability can easily be reassessed assuming that default risk is present.

In the following, it will be of interest in how far the phenomenological approaches of the last chapter are able to reproduce certain features and phenomena of default risk. But however successful they might be in mimicking the manifestations of default risk, it has to be stressed that they will always fail to integrate default risk as an explicit variable ${ }^{212}$ However, they can serve as an example for credit risk models as to a realistic modeling of the smile.

Above it has been shown that the presence of default risk should be accompanied by the leverage effect and should manifest itself in a downward sloping volatility smile. Both should be associated with a left-skewed equity return distribution. Therefore, the phenomenological approaches of the last chapter will principally be gaged by their ability to reproduce a left-skewed equity return distribution. In doing so it will be distinguished between continuous and discontinuous models of the stock price process.

\subsubsection{Continuous Process Models in the Light of Default Risk}

The continuous models of the stock price process worthwhile discussing at this point are the Black-Scholes model, the CEV model, the concept of local volatility and the class of the stochastic volatility models.

212 With the only exception of Merton's jump-to-ruin model, whose direct link to default risk will be discussed below. 
- Black-Scholes model: The Black-Scholes model represents a trivial case, since by definition it is not able to produce a volatility smile. Consequently, it is neither able to generate a left-skewed return distribution nor to accommodate the symptoms of the leverage effect. Furthermore, the Black-Scholes model implicit lognormal law of the stock price distribution precludes that the stock price will ever attain a value of zerd 213 . This however would be a prerequisite for the event of default.

- CEV model: In the non trivial case of $\delta<1$, the CEV model induces a leftskewed return distribution which is accompanied by a negative correlation between the stock price level $S_{t}$ and the volatility $\sigma\left(S_{t}\right)=\sigma S_{t}^{\delta-1}$. This negative relationship is mimicking the leverage effect and therefore leads to a convex, downward sloping smile. In contrast to the Black-Scholes model, in the CEV model the stock price can reach zero as an absorbing barrier, if $\frac{1}{2} \leq \delta<11^{214}$.

- Local volatility: The local volatility framework allowing the equity volatility to vary freely in function of the stock price level and time can theoretically reproduce any terminal stock price distribution. If fitted to actual equity option prices, the local volatility surface exhibits a volatility which is increasing with a decreasing level of the underlying. This is schematically represented in figure 5.1, which illustrates a typical implied binomial tree obtained by fitting a local volatility model to stock index options. As can be seen, the implied tree seems to be distorted compared to a regular binomial tree and the vertical distance between the terminal nodes increases with decreasing level of the underlying. This increasing vertical distance is equivalent to an increasing level of volatility.

213 See for example ATLAN/LEBLANC (2005).

214 See for example $\overline{\overline{A T L A N} / L E B L A N C}(\overline{2005})$. 


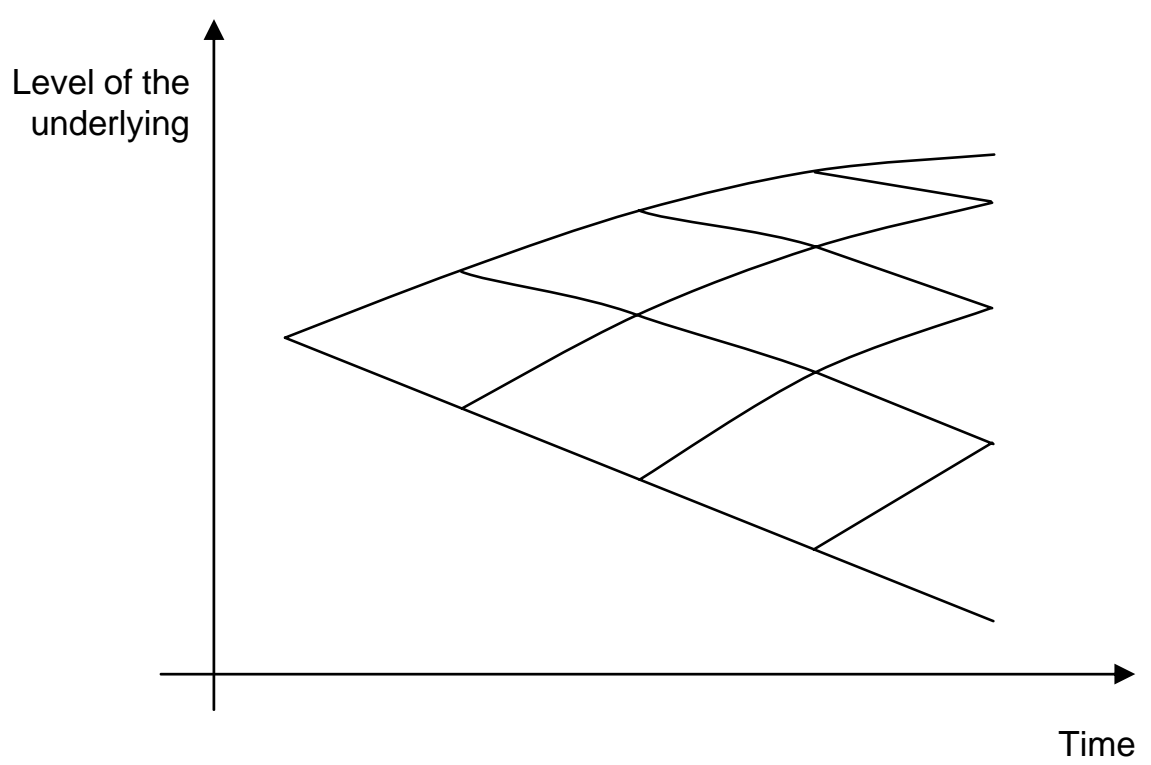

Figure 5.1.: Schematic representation of a fitted local volatility surface ${ }^{a}$

a Schematic representation of a typical implied binomial tree obtained by fitting a local volatility model to stock index option prices (figure follows DERMAN ET AL. (1996)).

Therefore, the distortion of the binomial tree in figure 5.1 can be interpreted as a manifestation of the leverage effect. Since the concept of local volatility offers even greater flexibility than the CEV model, it must consequently be possible to parameterize the model in a way as to accommodate the possibility that $S_{t}$ reaches zero.

- Stochastic volatility model: By letting the correlation between the innovations of the stock price and that of the volatility process be negative, the stochastic volatility model is able to reproduce a left-skewed return distribution (see section 4.2.3.2, which can be viewed as a symptom of the leverage effect. As to the attainability of a stock price of zero, the same can be said as for the local volatility model.

\subsubsection{Discontinuous Process Models in the Light of Default Risk}

Thanks to the possibility to vary the size, direction and intensity of jumps, discontinuous models of the stock price process offer a great flexibility as to the reproduction of a certain price process or terminal distribution. Letting the mean jump size $\mu_{J}$ of a jump-diffusion model or the drift parameter $\mu_{b}$ in an infinite activity model to be neg- 
ative produces a left-skewed return distribution which is symptomatic of the presence of default risk and leads to a downward sloping volatility smile.

Left-skewness can equally be caused by many small jumps or also by just a single large jump into the state of default. The latter is the case in the jump-to-ruin mode of Merton's jump-diffusion mode ${ }^{215}$ which represents a special case of the general jumpdiffusion model discussed in section 4.2.4.1. In the jump-to-ruin mode of Merton's model there exists only one constant jump size, $J=-1$, which brings the equity value to zero in the case of a jump. This setting dramatically simplifies the evaluation of stock options with this model, since it is reduced to the Black-Scholes model with a risk-free rate $r$ augmented by the jump intensity $h 216$. The evaluation of a European call, $c_{t_{0}}$, or a European put, $p_{t_{0}}$, under jump-diffusion with jump-to-ruin reduces to 217 .

$$
\begin{aligned}
& c_{t_{0}}(J=-1)=c_{t_{0}}^{B S}\left(S_{t_{0}}, X, t_{0}, T, \sigma, r+h\right) \\
& p_{t_{0}}(J=-1)=p_{t_{0}}^{B S}\left(S_{t_{0}}, X, t_{0}, T, \sigma, r+h\right)
\end{aligned}
$$

The Merton's jump-diffusion model in its jump-to-ruin mode is the only smile explanation of chapter 4, which explicitly contains a variable which can be interpreted as being related to the level of default risk. This variable is the jump intensity $h$, which can be viewed as a credit spread 218 .

\subsection{Enhanced Credit Risk Models Incorporating the Implied Volatility Smile}

\subsubsection{Introduction}

While the smile explanations of chapter 4 do not explicitly consider default risk and while on the other hand the traditional credit risk models discussed in section 5.1.1 do

215 See MERTON (1976).

216 See $\overline{\text { MERTON }}(1976)$.

217 In this case, Black-Scholes type perfect replication necessitates hedging the option position with default risky instead of default free bonds. The bonds have to be issued by the same firm which has issued the stocks. Then, in the case of a sudden jump to ruin stock and bond position will simultaneously become worthless (assuming no recovery on the bond). The call option will likewise become worthless, which ensures a perfect hedge. The put option however, will not become worthless, unless it has been written by the firm which has also issued the stocks and the bonds. Therefore, perfect replication of the put additionally necessitates that the put has been written by the default risky firm. See GATHERAL (2006), pp. 75-78.

See GATHERAL (2006), p. 75 and cf. section 5.1.1.2. 
not explicitly incorporate the smile, the type of models presented in this section bridge the gab between smile and default risk.

The advent of products like CDS and more recently EDS contracts, but also the wish, not to neglect the default risk component in the pricing of convertible bonds, have spurred the development of hybrid models offering a unifying framework which embraces debt, equity and equity options 219

These hybrid models are extensions of the traditional credit risk models and are inspired by the theoretical insights regarding the relationship between default risk and the smile which have been presented above. They can be distinguished from the traditional credit risk models in that their calibration involves at least two implied volatilities of differing strikes, which accommodates the existence of a volatility smile.

Following the classification of the traditional credit risk models, the models discussed in this section can likewise be coarsely grouped into structural and reduced form models.

\subsubsection{Structural Credit Risk Models Incorporating the Implied Volatility Smile}

Bridging the gap between default risk and the smile within a structural setting centers on the idea of considering equity options as compound options. This concept can be approached from two directions:

The first is based on a fully specified model of the firm, the firm value process and its density. Unknown variables can be inferred by means of two implied equity volatilities related to different strike levels. This direction can further be decomposed in approaches considering equity as a European call and those regarding it as a downand-out barrier call.

The second direction exploits the full spectrum of available option prices in order to infer the risk-neutral equity or firm value density. Given this density and a corresponding default barrier, the default probability can be deduced.

219 Although the models presented below encompass equity options and default risk, they have to be delimited clearly against models for the pricing of OTC options subject to counterparty risk. Options exposed to the risk that the writer of the option defaults are called vulnerable options. Their valuation is treated in JOHNSON/STULZ (1987), for example. 


\subsubsection{Options on a European Call on the Firm's Assets}

The notion that equity options can be considered as compound options since equity itself can be viewed as an option on the firm's assets was first introduced by GESKE (1979). Following Merton, GESKE (1979) models the equity $S_{t}$ as a European call on the firm's assets $V_{t}$ with final maturity $T$ and the strike price equaling the nominal value of debt $D^{220}$. The risk-neutral process of $V_{t}$ is given by expression 5.1. The equity option in turn - for example the call $c_{t}$ - is a European option on $S_{t}$ with strike price $X$ and maturing in $t^{*}$ with $t^{*} \leq T$.

The dynamics of both $S_{t}$ and $c_{t}$ can be expressed in terms of functions of $V_{t}$ :

$$
\begin{aligned}
& \frac{\partial c_{t}}{\partial t}=r c_{t}-r V_{t} \frac{\partial c_{t}}{\partial V}-\frac{1}{2} \sigma_{V}^{2} V^{2} \frac{\partial^{2} c_{t}}{\partial V^{2}} \\
& \frac{\partial S_{t}}{\partial t}=r S_{t}-r V_{t} \frac{\partial S_{t}}{\partial V}-\frac{1}{2} \sigma_{V}^{2} V^{2} \frac{\partial^{2} S_{t}}{\partial V^{2}}
\end{aligned}
$$

The corresponding boundary conditions for the solution of the above stochastic differential equations are:

$$
\begin{aligned}
& c_{t^{*}}=\left(S_{t^{*}}-X\right)^{+} \\
& S_{T}=\left(S_{T}-D\right)^{+}
\end{aligned}
$$

The stochastic differential equation 5.22 cannot be solved independently of the stochastic differential equation 5.23, since its boundary condition 5.24 hinges on the solution of 5.23 . To solve both equations 5.22 and 5.23 simultaneously, the critical stock price $\tilde{S}_{t^{*}}=X$ separating exercise from worthless expiry has to be translated into the corresponding firm value $\tilde{V}$ so that according to equation 5.3 . $\tilde{S}_{t^{*}}(\tilde{V})=X$.

Be $N(\cdot)$ the univariate and $N_{2}(\cdot)$ the bivariate, cumulative standard normal density function and be further $\tau_{1}=t^{*}-t_{0}$ and $\tau_{2}=T-t_{0}$, then a solution to the above problem is given by the following expression for the compound call option $c_{t_{0}}$ :

$$
\begin{aligned}
& c_{t_{0}}=V_{t} N_{2}\left(m+\sigma_{V} \sqrt{\tau_{1}}, l+\sigma_{V} \sqrt{\tau_{2}}, \sqrt{\tau_{1} / \tau_{2}}\right) \\
& -D e^{-r \tau_{2}} N_{2}\left(m, l, \sqrt{\tau_{1} / \tau_{2}}\right)-X e^{-r \tau_{1}} N(m)
\end{aligned}
$$

220 For the following presentation of Geske's compound option model, see GESKE (1979). 


$$
\begin{array}{r}
m=\frac{\ln \left(V_{t} / \tilde{V}\right)+\left(r-\frac{1}{2} \sigma_{V}^{2}\right) \tau_{1}}{\sigma_{V} \sqrt{\tau_{1}}} \\
l=\frac{\ln \left(V_{t} / D\right)+\left(r-\frac{1}{2} \sigma_{V}^{2}\right) \tau_{2}}{\sigma_{V} \sqrt{\tau_{2}}}
\end{array}
$$

In two cases the compound option model collapses to the Merton firm value model or to a simple option model, respectively. Either if the equity becomes unlevered, for example due to $T=\infty$ or $D=0$, or if the maturity of the debt $T$ coincides with the maturity of the equity option $t^{*}$. In the latter the compound option model becomes a simple option model with the strike price equaling $D+X$.

Since the compound option model is derived from the Merton firm value model it inherits the leverage effect and therefore accommodates a downward sloping implied volatility smile 221 .

Therefore it can be applied in a way to exploit the information which is contained in the smile. This is demonstrated by HuLL ET AL. (2004a) who compare the implementation of the standard Merton firm value model with that of Geske's compound option model. While the determination of default probability and credit spread in the context of the traditional implementation of the Merton model necessitates the prior estimation of the face value of the debt, $D$, the compound option model allows to also infer the latter by the use of two implied volatilities with differing strikes 222 .

\subsubsection{Options on a Down-And-Out Barrier Call on the Firm's Assets}

As discussed in section 5.1.1.1, in many extensions of the original Merton firm value model default can happen any time and is triggered as soon as the path of the firm

\footnotetext{
221 Although this is not specified further, GESKE (1979) holds that his model is likewise consistent with the observation of Black-Scholes pricing biases in the time to maturity dimension.

222 While in the traditional Merton model the equity as a call option on the firm's assets is a function of 6 variables, $S_{t_{0}}=c_{t_{0}}^{B S}=f\left(V_{t_{0}}, D, r, t_{0}, T, \sigma_{V}\right)$ (see equation 5.3 , the compound option in Geske's model is a function of 8 variables, $c_{t_{0}}=g\left(V_{t_{0}}, D, X, r, t_{0}, t^{*}, T, \sigma_{V}\right)$ (see equation 5.26.

Usually, the traditional implementation of the Merton model involves estimating the face value of the debt $D$ by means of financial statement data. Provided with an estimate of $D$ and of the equity volatility $\sigma_{S}$, the two unobservable unknowns $V_{t_{0}}$ and $\sigma_{V}$ can be determined using the leverage effect relationship (equation 5.14) and the stock price function $S_{t_{0}}=c_{t_{0}}^{B S}=f(\cdot)$. This allows to ultimately solve for the default probability (equation 5.2) and the credit spread (equation 5.8).

As an alternative, HULL ET AL. (2004a) propose to simultaneously solve the compound option formula $c_{t_{0}}=g(\cdot)$ for $V_{t_{0}}, \sigma_{V}$ and also $D$ by using the prices of two options with different strikes, $\tilde{c}_{1}$ and $\tilde{c}_{2}$. This amounts to solving for the three unknowns $V_{t_{0}}, \sigma_{V}$ and $D$ with the system of the three equations $\tilde{c}_{1}=g\left(X_{1}, \ldots\right), \tilde{c}_{2}=g\left(X_{2}, \ldots\right)$ and $S_{t_{0}}=c_{t_{0}}^{B S}=f(\cdot)$.
} 
value $V_{t}$ touches a default barrier $V_{B}$ from above. In this type of model the equity $S_{t}$ no longer is a European but instead a down-and-out barrier call[223 which ceases to exist as soon as the barrier has been reached 224 . The exact specification of the call price function $S_{t}=f\left(V_{t}, V_{B} \ldots\right)$ naturally depends on the nature of the barrier $V_{B}$.

Prior to default, the structural credit risk models with a default barrier behave similar to the original Merton model in that the leverage effect is present 225 .

The compound option approach can likewise be applied to the case in which equity is modeled as a barrier option. Be $t_{d}$ the first time the firm value $V_{t}$ hits the default barrier $V_{B}, t_{d}=\inf \left\{t \geq t_{0}: V_{t} \leq V_{B}\right\}$, then the compound option - for example the call $c_{t_{0}}$ - is a European option on a barrier option and can be expressed in the following very general manner 226 .

$$
c_{t_{0}}=e^{-r\left(T-t_{0}\right)} E_{t_{0}}^{Q}\left[\left(S_{T}-X\right)^{+} 1_{t_{d} \geq T}\right]
$$

In the following, three different specifications of the stock price function $S_{t}=f\left(V_{t}, V_{B} \ldots\right)$ and the barrier $V_{B}$ will be sketched briefly. All three approaches have in common that they are embedded in a compound option framework allowing a connection between default risk and the smile.

5.2.2.2. Toft/Prucyk Model TOFT/PRUCYK (1997) base their compound option model on the structural firm value model of LELAND (1994). The stock price function $S_{t}=f(\cdot)$ of the model of LELAND (1994) offers a great flexibility as to the firm's capital structure and the default trigger, which can be determined endogenously at the discretion of the equity holders or alternatively, exogenously due to a debt covenant.

TOFT/PRUCYK (1997) derive an expression for the compound option $c_{t_{0}}$, which reduces to the Black-Scholes formula for a European call on the firm's assets if the firm is unlevered.

TOFT/PRUCYK (1997) calculate values of $c_{t_{0}}$ and corresponding Black-Scholes implied volatilities for various combinations of input variables. The tabulated implied volatilities reveal the existence of a downward sloping volatility smile which is all the more steeper, the higher the leverage ratio $L$. This can be explained with the presence

223 See for example FINGER/STAMICAR (2005).

224 A down-and-out call is a knock-out option which ceases to exist as soon as the underlying reaches a certain barrier level (see HULL (2006), p. 533).

225 This is shown by FINGER/STAMICAR (2005) for the case of the CreditGrades model.

226 See TOFT/PRUCYK (1997). 
of the leverage effect. Further, the tabulated figures disclose that the downward sloping smile is steeper in the cases where the debt is protected by debt covenants than in the cases where it is not. And lastly, the smile mostly flattens with increasing option maturity, except in the cases of high leverage ratios in conjunction with protected debt. In the latter cases the smile even steepens substantially with increasing option maturity.

While the Toft/Prucyk compound option model features constant, infinite maturity debt with regularly paying coupons, HANKE (2002) proposes a similar model with exponentially increasing, finite maturity debt. He numerically shows that the downward sloping smile is all the more steeper, the shorter the maturity and the higher the growth rate of the debt.

5.2.2.2. EBIT-Based Model GENSER (2004) develops a compound option model which builds on an EBIT-based structural credit risk model. In EBIT-based models the primary variable of interest is not the firm value $V_{t}$, but the EBIT ${ }^{227} \eta_{t}$. Be $\lambda$ a risk premium, then the risk-neutral dynamics of the non-traded variable $\eta_{t}$ are given by the following expression 228 ,

$$
d \eta_{t}=\left(\mu_{\eta}-\lambda \sigma_{\eta}\right) d t+\sigma_{\eta} d z_{t}^{Q}
$$

Default is triggered if $\eta_{t}$ reaches a constant bankruptcy boundary $\eta_{B}$ and the equity $S_{t}$ is modeled as a residual claim to EBIT after all other claimants have been satisfied.

GENSER (2004) derives an expression for a compound option $c_{t_{0}}$ on the equity $S_{t}$ which has to be evaluated numerically. As a by-product of this numerical evaluation GENSER (2004) also determines the standard deviation, skewness and kurtosis of the terminal risk-neutral equity return density for three different levels of leverage. As dictated by the leverage effect, the standard deviation increases with increasing leverage ratio. Further, for low and medium leverage ratios he finds that negative risk-neutral return skewness is prevailing, whereas highly leveraged firms seem to exhibit positive risk-neutral return skewness. Lastly, he detects risk-neutral excess kurtosis in all three leverage cases which increases with increasing leverage ratio.

GENSER (2004) also calculates Black-Scholes implied volatilities and demonstrates the corresponding smile effects of the interplay of the risk-neutral moments. Basically,

227 EBIT stands for earnings before interest and taxes.

228 Strictly speaking GENSER (2004) develops two sub-models, one in which $\eta_{t}$ follows an arithmetic Brownian motion as depicted here and one in which it follows a geometric Brownian motion. However, as to the implications for the implied volatility smile, this distinction is irrelevant. 
he observes a convex, monotonically decreasing smile. But in contrast to the common theoretical prediction that increasing leverage should lead to a steepening of the smile, his model astonishingly produces the inverse effect, namely a flattening.

5.2.2.3. Extension of the CreditGrades Model The CreditGrades model is a commercial, structural credit risk model with a random default threshold 229 . The stock price function $S_{t}=f\left(V_{t}, V_{B t} \ldots\right)$ prior to default is simply given by

$$
S_{t}=V_{t}-V_{B t}
$$

As an extension to the original CreditGrades model FINGER/STAMICAR (2005) derive an expression for a European compound option $c_{t_{0}}$ on $S_{t}$, which has to be evaluated numerically. Since originally based on the Merton firm value model, the CreditGrades extension accommodates a leverage effect ${ }^{231}$ and thus a downward sloping volatility smile.

For an unlevered firm the compound option expression, $c_{t_{0}}$, collapses to the BlackScholes formula like in the other enhanced structural models above. FINGER/STAMICAR (2005) show that - all things being equal - rising leverage leads to increasing values of $c_{t_{0}}$. That this also leads to a steepening of the smile is not demonstrated explicitly, although it should be expected due to the existence of the leverage effect (see section 5.1 .2 .2 .

Similar to HULL ET AL. (2004a) (see section 5.2.2.1), FINGER/STAMICAR (2005) apply the CreditGrades extension in order to exploit the information content of a downward sloping smile. To this end and assuming a deterministic default barrier they use implied equity volatilities of two options with different strikes to simultaneously infer the default barrier $V_{B t}$ and the asset volatility $\sigma_{V}$ which in turn allows to calculate the default probability and the credit spread.

229 See FINGER ET AL. (2002). The random default threshold enables short term credit spreads to be significantly different from zero.

230 See FINGER/STAMICAR (2005).

231 Prior to default the model implicit leverage effect ensues according to $\sigma_{S}=\sigma_{V}\left(1+\frac{V_{B_{t}}}{S_{t}}\right)$. 


\subsubsection{Option-Implied Density Approach}

The option-implied density approach tries to infer the risk-neutral firm value density and ultimately the default probability from a set of equity option prices. This approach has been proposed by DELZIO (2006b)

As in the models above, DELZIO (2006b) assumes that prior to default the firm's assets follow a geometric Brownian motion and models the stock price function $S_{t}=$ $f\left(V_{t}, V_{B} \ldots\right)$ as a down-and-out barrier call on $V_{t}$. The default barrier $V_{B}$ is assumed to be constant and equal to the recovery portion of the face value of the company's debt. Be $f_{V}^{Q}\left(V_{t^{*}}\right)$ the risk-neutral probability density of the firm value in time $t^{*}$ with $t^{*}>t_{0}$ and be further $t_{d}$ the first hitting time of $V_{B}$ with $t_{d}=\inf \left\{t \geq t_{0}: V_{t} \leq V_{B}\right\}$, then the risk-neutral probability of default $Q_{d}$ equals the probability of having reached the barrier $V_{B}$ up to time $t^{*}$ :

$$
Q_{d}=Q\left(t_{d} \leq t^{*}\right)=\int_{0}^{V_{B}} f_{V}^{Q}\left(V_{t^{*}}\right) d V_{t^{*}}
$$

The risk-neutral firm value density $f_{V}^{Q}$ in expression 5.32 can be inferred from the risk-neutral stock price density $f_{S}^{Q}$ by means of the following relation:

$$
f_{V}^{Q}\left(V_{t^{*}}\right)=f_{S}^{Q}\left(S_{t^{*}}\right) \frac{d S_{t^{*}}}{d V_{t^{*}}}
$$

DELZIO (2006b) infers the stock price density $f_{S}^{Q}\left(S_{t^{*}}\right)$ in turn from the prices of equity options with maturity $t^{*}$ by using a smile smoothing procedure (see section 3.2.2.1.

The second term on the right hand side of equation 5.33 can be determined by means of the stock price function $S_{t}=f(\cdot)$ or the leverage effect relationship (see equation 5.14). Both $S_{t}=f(\cdot)$ and the leverage effect relationship involve the two unknowns $V_{t}$ and $\sigma_{V}$ which can be solved for by means of these two equations 233 .

While the approach of DELZIO (2006b) seems to represent an elegant way of exploiting all available equity option prices to infer default risk given the default barrier $V_{B}$ is known, one critical remark has to be made:

In the model the asset price density $f_{V}^{Q}$ is deduced by means of the stock price function $S_{t}=f(\cdot)$ which in turn relies on certain assumptions regarding the processes fol-

\footnotetext{
232 While the underlying theory of this approach is described in DELZIO (2006b), a practical application is furnished in DELZIO (2006a).

$233 \sigma_{S}$ is assumed to be given exogenously.
} 
lowed by $V_{t}$ and $S_{t}$ as well as the form of their respective terminal densities $f_{V}^{Q}\left(V_{t^{*}}\right)$ and $f_{S}^{Q}\left(S_{t^{*}}\right)$. But since the actually inferred asset price density $\tilde{f}_{V}^{Q}$ is derived from the actual, market-implicit stock price density $\tilde{f}_{S}^{Q}$, it is well possible that neither $\tilde{f}_{V}^{Q}$ nor $\tilde{f}_{S}^{Q}$ comply with the original assumptions regarding $f_{V}^{Q}$ and $f_{V}^{Q}$.

Another version of the option-implied density approach is followed by MIZRACH (2006) who focuses on the equity instead of the asset price density. He exogenously fixes a "bankruptcy strike" and calculates the default probability as the probability with which this barrier strike level is reached from above by the equity price process 234

\subsubsection{Reduced Form Credit Risk Models Incorporating the Implied Volatility Smile}

\subsubsection{Introduction and Overview}

In section 5.1 .4 we reviewed the phenomenological smile explanations of chapter 4 in the light of default risk. Regarding the continuous process models we observed two weaknesses as to their ability to reflect the existence of default risk: Firstly, all of them are lacking an explicit variable or parameter related to default risk. And secondly, some of them are not able to reach an equity value of zero. However, we also saw that these two defects are remedied by the hazard rate driven jump-to-ruin in Merton's jump-diffusion model.

Like the Merton jump-diffusion model the models discussed in the following allow a continuous equity price process to be killed by a hazard rate process. The inclusion of a hazard rate process stresses that these models spring from the class of the reduced form credit risk models discussed in section 5.1.1.2.

The combination of a continuous equity price process with a default intensity process establishes a direct link between credit and equity market. Thanks to their ability to exploit liquid equity and equity options market data, such approaches have recently become very popular for the pricing of hybrid products like convertible bonds 235

234 Applying this procedure to the case of the Enron bankruptcy, MIZRACH (2006) finds that the equity option market underestimated Enron's default risk.

235 In this particular respect they have supplanted the use of structural models which depend on the unobservable firm value. See AYACHE ET AL. (2003), who furnish a brief account of the history of the pricing of convertible bonds. 
Besides exhibiting the feature that they combine a continuous diffusion with a hazard rate process, the models discussed below all have in common that they consist of the following three general building blocks which can assume different forms in the individual models:

- A hazard rate process $h_{t}$

- A process of the risk free rate $r_{t}$

- A stock price diffusion volatility process $\sigma_{t}$

Most of the models presented below try to combine the three building blocks in a way as to mimic some or all of the following three stylized features related to the leverage effect 236 .

1. A negative relationship between the stock price level and the level of equity volatility 237

2. A positive relationship between the equity volatility and the default intensity 238

3. A negative relationship between the stock price level and the default intensity

Table 5.4 furnishes an overview of the models presented below and provides details concerning the three building blocks and the model-implicit shape of the smile.

236 See for example DAS/SUNDARAM (2006), CARR/LINETSKY (2006) or CARR/WU (2006).

237 This relationship is due to the leverage effect (cf. section 5.1.1.3 for the theoretical and section 5.1.3.1 for the empirical foundation of the leverage effect).

238 This relationship can be explained by means of the Merton model in conjunction with the leverage effect (cf. section 5.1.2 for the theoretical and section 5.1.3.2 for the empirical foundation of the relationship between equity return and volatility).

239 This follows from 1 . and 2. 


\begin{tabular}{|c|c|c|c|c|c|}
\hline Author & Year & Hazard rate $h$ & Interest rate $r$ & $\begin{array}{l}\text { Stock price diffusion } \\
\text { volatility } \sigma^{*}\end{array}$ & Smile shape ${ }^{* *}$ \\
\hline Linetsky & 2006 & Det., $h=f(S)$ & Det., $r=$ constant & Det., $\sigma=$ constant & Decreasing \\
\hline Das and Sundaram & 2006 & Det., $h=f(S, r)$ & Stoch. & Det., $\sigma=f(S)$ (CEV) & $?$ \\
\hline Carr and Linetsky & 2006 & Det., $h=f(S, t)$ & Det., $r=f(t)$ & Det., $\sigma=f(S, t)(C E V)$ & Decreasing \\
\hline Carr and Wu & 2006 & Stoch. & Det., $r=f(t)$ & Stoch. & U-shaped \\
\hline Campi et al. & 2005 & Det., $\mathrm{h}=$ constant & Det., $r=$ constant & Det., $\sigma=f(S)$ (CEV) & Decreasing \\
\hline Bloch & 2005 & Det., $h=f(S)$ & Det., $r=f(t)$ & Stoch. & U-shaped \\
\hline Atlan and Leblanc & 2005 & Det., $h=f(S)$ & Det., $r=$ constant & Stoch. (CEV) & U-shaped \\
\hline Andersen and Buffum & 2003 & Det., $h=f(S)$ & Det., $r=f(t)$ & Det., $\sigma=f(S, t)$ & Decreasing \\
\hline Ayache et al. & 2003 & Det., $h=f(S, t)$ & Det., $r=f(t)$ & Det., $\sigma=$ constant & Decreasing \\
\hline Takahashi et al. & 2001 & Det., $h=f(S)$ & Det., $r=f(t)$ & Det., $\sigma=$ constant & Decreasing \\
\hline Davis and Lischka & 1999 & Det., $h=f(S, t)$ & Stoch. & Det., $\sigma=$ constant & $?$ \\
\hline Merton & 1976 & Det., $\mathrm{h}=$ constant & Det., $r=$ constant & Det., $\sigma=$ constant & Decreasing \\
\hline
\end{tabular}

Det. $=$ deterministic, Stock. $=$ stochastic

* In the case that the diffusion volatility comprises a CEV specification, this is indicated with CEV.

** Shape of the smile in the strike dimension; decreasing means monotonically decreasing; ? denotes that the

smile shape remains unmentioned in the respective paper and that it cannot be deduced on account of the

model characteristics, if it is monotonically decreasing or U-shaped.

Table 5.4.: Overview of enhanced reduced form credit risk models incorporating the implied volatility smile

While most of the literature cited in table 5.4 directly alludes to the pricing of equity options or the shape of the implied volatility smile or both, some work in table 5.4 exclusively focuses on the pricing of convertible bonds. Nevertheless such papers have also been included, as long as the models therein exhibit very similar features as the models explicitly addressing the issue of equity options pricing.

\subsubsection{Model Mechanics}

Very generally, the risk-neutral, pre-default stock price process $S_{t}$ can be represented as a composition of a regular diffusion dependent on $r_{t}$ and $\sigma_{t}$ and a counting process $N_{t} . N_{t}$ in turn is driven by the hazard rate process $h_{t}$ and is compensated by $h_{t} d t$ to ensure the Martingale property of $S_{t} 240$.

$$
d S_{t}=\left(r_{t}+h_{t}\right) S_{t} d t+\sigma_{t} S_{t} d z_{t}^{Q}-S_{t} d N_{t}
$$

The jump-to-default occurs at the first jump time $\xi$ of the counting process $N_{t}$ :

$$
\xi=\inf \left\{t>t_{0}: N_{t}=1\right\}
$$

$240 \mathrm{Cf}$ AtLAN/LEBlANC 2005) and CAMPI ET AL. (2005). 
Depending on the specification of $\sigma_{t}$, the process $S_{t}$ might not only reach $S_{t}=0$ by a jump-to-default, but also by mere diffusion. Be $t_{d}$ the first time $S_{t}$ reaches zero by diffusion only or by a jump-to-default, whichever happens first ${ }^{241}$.

$$
t_{d}=\inf \left\{t>t_{0}: S_{t}=0\right\}
$$

Then the price of a European call option $c_{t_{0}}$ on the stock $S_{t}$ can be represented very generally in the following form 242 .

$$
c_{t_{0}}=e^{-\int_{t_{0}}^{T} r_{u} d u} E_{t}^{Q}\left[\left(S_{T}-X\right)^{+} 1_{t_{d}>T}\right]
$$

Thanks to the existence of default risk, all models in table 5.4 are able to produce a monotonically decreasing smile shape. Some however, are also able to generate a more realistic U-shaped smile pattern due to the integration of stochastic diffusion volatility. Therefore, with regard to their implications for the shape of the smile the models will be coarsely classified depending on whether they exhibit a deterministic or a stochastic stock price diffusion volatility.

5.2.3.1. Models with a Deterministic Stock Price Diffusion Volatility The jump-diffusion model of MERTON (1976) with its special case of a jump-to-ruin can be considered as a precursor of the reduced form credit risk models incorporating the smile. Although the three building blocks $h_{t}, r_{t}$ and $\sigma_{t}$ are only constants in this model, it is still able to produce a downward sloping smile thanks to the model implicit left-skewness of the risk-neutral equity return density as can be recalled from section 5.1.4.3.

The first "modern" model linking a continuous equity price and a hazard rate process is that of DAVIS/LISCHKA (1999), who model $h_{t}$ as depending on time and the stock price process $S_{t}$. A similar specification been adopted in the models of LINETSKY (2006), AYACHE ET AL. (2003), TAKAHASHI ET AL. (2001), DAS/SUNDARAM (2006), ANDERSEN/BUFFUM (2003) and CARR/LINETSKY (2006). In most of these approaches the functional relationship between $h_{t}$ and $S_{t}$ can be represented in the following, very general form:

$$
h_{t}\left(t, S_{t}\right)=a(t)+d(t) S_{t}^{-\omega}
$$

241 Cf. CARR/LINETSKY $(2006)$.

242 Again, the representation of the call option price is given exemplarily for call and put options alike. For the expression of $c_{t_{0}}$, cf. CARR/LINETSKY (2006). 
For $w>0$ and $d>0$ the above expression guarantees a negative relationship between the hazard rate and the stock price level and thus between default risk and the equity return. As we have seen in section 5.1.2.2, the mere presence of default risk manifests itself in a left-skewed equity return density which in turn produces a downward sloping volatility smile. Just like a decreasing stock price $S_{t}$ leads to an increasing leverage ratio $L_{t}$ in the leverage effect relationship and thus further accentuates the leverage effect and entails a convex smile curve (see section 5.1.2.2), the negative link between $h_{t}$ and $S_{t}$ likewise generates convexity.

As to the effects on the term structure of the smile, the above specification seems to lead to a smile which flattens with increasing option maturity 243 .

The only other model besides the one of MERTON (1976) which does not assume $h_{t}$ to be a function of $S_{t}$ is the model of CAMPI ET AL. (2005). In exchange, their model features a CEV-type specification of the diffusion volatility $\sigma_{t}$ which can be generalized in the following form:

$$
\sigma_{t}\left(t, S_{t}\right)=v(t) S_{t}^{\delta-1}
$$

For $\delta<1$ this specification assures a negative relationship between the equity return and its volatility and thus the existence of the leverage effect as well as a convex, monotonically downward sloping smile (cf. sections 5.1.2.2 and 5.1.4.2). A CEV specification of the diffusion volatility has been likewise adopted by DAS/SUNDARAM (2006) and CARR/LINETSKY (2006).

As can be easily verified, the combination of a hazard rate process according to expression 5.38 and of a diffusion volatility process consistent with equation 5.39 also ensures a negative relationship between the hazard rate and the level of the diffusion volatility, given that $w>0, d>0$ and $\delta<1$.

While most models feature a deterministic interest rate process $r_{t}$, which is either constant or time-dependent, the models of DAS/SUNDARAM (2006) and DAVIS/LISCHKA (1999) assume a stochastic specification of $r_{t}$. Theoretically this could result in a different than a merely downward sloping smile. But as can be recalled from section 4.2.5, the contribution of stochastic interest rates to the explanation of the smile is marginal. Therefore it is unlikely, that the inclusion of stochastic interest rates in the type of model discussed here will create a smile shape any different from a monotonically downward sloping curve. Nevertheless, in table 4.2.5 the smile shape of the two models with stochastic interest rates has been marked indeterminate.

243 Cf. LINETSKY 2006) 


\subsubsection{Models with a Stochastic Stock Price Diffusion Volatility As}

has been shown in section 4.2.3, allowing for stochastic volatility in the stock price process introduces new degrees of freedom in reproducing the smile. Similar as above, this extra flexibility can also be exploited in the framework of a reduced form model in order to produce a more realistic, U-shaped smile pattern. This avenue has been followed by CARR/WU (2006), BLOCH (2005) and ATLAN/LEBLANC (2005).

The most sophisticated and flexible of the three models is that of CARR/WU (2006), which also accommodates a stochastic hazard rate. The stochastic hazard rate $h_{t}$ covaries with the diffusion volatility $\sigma_{t}$ while not being perfectly correlated with the latter, thus reflecting a realistic dependency pattern between default risk and the level of the equity volatility. The risk-neutral dynamics of $\sigma_{t}$ and $h_{t}$ are assumed to be of the following form:

$$
\begin{gathered}
\sigma_{t}=\sqrt{v_{t}} \\
d v_{t}=\alpha_{v}\left(\theta_{v}-v_{t}\right)+\sigma_{v} \sqrt{v_{t}} d w_{v t}^{Q} \\
d x_{t}=\alpha_{x}\left(\theta_{x}-x_{t}\right)+\sigma_{x} \sqrt{x_{t}} d w_{x t}^{Q} \\
h_{t}=\zeta v_{t}+x_{t} \\
\left\langle d w_{v t}^{Q}, d w_{x t}^{Q}\right\rangle=0 \\
\left\langle d w_{x t}^{Q}, d z_{t}^{Q}\right\rangle=0 \\
\left\langle d w_{v t}^{Q}, d z_{t}^{Q}\right\rangle=\rho d t
\end{gathered}
$$

$v_{t}$ and $x_{t}$ each follow a mean reverting Cox-Ingersoll-Ross process (CIR) ${ }^{244}$ with long term mean reversion level $\theta$ and mean reversion speed $\alpha$. While the standard normal increments of $v_{t}$ and $x_{t}$ as well as of $x_{t}$ and $S_{t}$ are uncorrelated, the innovations of the stock price $S_{t}$ and its variance $v_{t}$ are dependent on each other. Despite the independence between $d w_{v t}^{Q}$ and $d w_{x t}^{Q}$, the equity volatility $\sigma_{t}$ and the hazard rate $h_{t}$ are positively correlated thanks to the functional relationship 5.43 .

The above model of the joint, stochastic dynamics of $h_{t}$ and $\sigma_{t}$ offers a great degree of flexibility as to the reproduction of real-world implied volatility smiles of individual stock 245

In contrast to the rather flexible hazard rate specification of CARR/WU (2006), BLOCH (2005) and ATLAN/LEBLANC (2005) follow the approach of a functional relationship

\footnotetext{
244 Cf. FOUQUE ET AL. $(2000)$, p. 42.

245 See CARR/WU (2006).
} 
between $h_{t}$ and $S_{t}$ along the lines of equation 5.38 and with the corresponding effects on the smile and its term structure.

But likewise as CARR/WU (2006), BLOCH (2005) and ATLAN/LEBLANC (2005) also allow for stochastic diffusion volatility enabling a U-shaped smile pattern. ATLAN/LEBLANC (2005) even combine the stochastic diffusion volatility with a CEV specification, thus automatically ensuring a built-in leverage effect.

All three models with a stochastic diffusion volatility adhere to a deterministic specification of $r_{t}$.

\subsection{Summary and Conclusions}

In chapter 4 we had come to the conclusion that default risk should be taken into account as a possible determinant of the smile.

Consequently, in this chapter we focused on the link between default risk and the smile. To this end, we studied in a first step the theoretical and empirical connections between default risk and the smile and then in a second step concentrated on more recent model enhancements bridging the gap between default risk and equity options.

The beginning of the first step centered on the main features of traditional credit risk models as well as the mechanics of the leverage effect. But although an implicit, theoretical connection between default risk and the smile could be derived, it became evident that the traditional credit risk models fail to explicitly incorporate equity options and the smile phenomenon.

From the theoretical contemplation of the link between default risk and the smile further emerged that the existence of default risk should lead to a left-skewed riskneutral stock return density which in turn manifests itself in a convex, monotonically downward sloping volatility smile, whose steepness increases with increasing level of default risk. However, it also became evident that this prediction conflicts with the observation of a generally U-shaped individual stock option smile.

The subsequent survey of the extant empirical research as to the connection between the level of default risk and the smile disclosed an abundance of findings on some fields and a substantial lack on others. While the positive relationship between the level of default risk and the level of equity volatility seem to be well documented, findings regarding the link between the level of default risk on the one hand and the shape of the smile and the risk-neutral equity return density on the other are very scarce, or 
lacking completely. The few studies addressing the slope of the smile all produce weak evidence corroborating the theoretical prediction of a default risk induced steepening of the smile. However, these studies are without exception restricted to the slope of the smile at or around the ATM strike level. Further, only one study at all examines the connection between the level of default risk and the shape of the risk-neutral return density 246 Further, despite the multitude of work treating the asymmetric volatility phenomenon, it is still not concludingly answered whether the leverage effect really exists or not.

Thanks to the insights gained from the analysis of the traditional credit risk models as well as of the extant empirical research, we were able to review the phenomenological smile explanations of the last chapter in the light of default risk. Although these fail to incorporate default risk explicitly, their reassessment revealed that all of them can at least accommodate the manifestations of the leverage effect.

The second step of the analysis was dedicated to the investigation of credit risk models which are enhanced in a way as to explicitly incorporate equity options and the volatility smile phenomenon. Similar to traditional credit risk models, this class of enhanced models can be partitioned into exponents based on the structural firm value setting on the one hand and into the more recent strand of models derived from the reduced form approach on the other. It was shown that all these enhanced models build on the leverage effect as well as its implication of a monotonically downward sloping implied volatility smile. However, beyond this it was found that they do not offer any further relevant insights regarding the link between the level of default risk and the smile. Moreover, it was demonstrated that due to the built-in leverage effect most enhanced models merely generate a monotonically downward sloping smile and fail to produce a more realistic U-shaped curve. And those few models forming an exception all rely on a stochastic diffusion volatility which in turn implies unrealistic parameters, as we have seen earlier. In addition, the enhanced models are in parts mutually contradictory as to the consequences of increasing default risk: While the majority of the models predicts a steepening of the smile with increasing default risk, one model forecasts the contrary 247 . Further, the smile term structure implications of the enhanced credit risk models are not always evident. While the smile generally

\footnotetext{
246 Namely the study of DENNIS/MAYHEW (2002), who analyze the relationship between the level of default risk and the level of risk-neutral skewness, but are only able to produce weak evidence of such a relationship (see section 5.1.3.3).

247 Namely the EBIT-based model of GENSER (2004) (see section 5.2.2.2.2.
} 
seems to flatten with increasing maturity and consistently with empirical observations, there is at least one model for which this does not always seem to be true 248 .

Despite their relatively limited theoretical contributions, it has to be noted that the enhanced credit risk models exhibit the advantage of exploiting the richness of market data to a broad extent by allowing inferences from the market of the company's equity and equity options to that of its debt and vice versa.

In summary, the literature-based study of the link between default risk and the smile revealed theoretical and partly also empirical evidence of the following:

1. The asymmetric volatility phenomenon is caused by the leverage effect.

2. There exists a positive relationship between the level of default risk and the level of equity volatility.

3. The existence of default risk leads to a left-skewed risk-neutral equity return density which in turn manifests itself in a convex, monotonically downward sloping volatility smile. This smile is all the more steeper the higher the level of default risk.

However, empirical findings regarding 1. are contradictory and evidence of 3 . is extremely scarce and - if existent - is limited to the contemplation of the smile slope around the ATM strike level. Beyond this point of the smile or concerning the behavior of the risk-neutral density, almost no insights are available. And more importantly, 3. contradicts the observation of a generally U-shaped individual stock option smile.

Although most enhanced models incorporate the leverage effect as well as a default risk induced, monotonically downward sloping smile, they have to be treated with care with regard to their implications for the implied volatility smile:

a. As to the strike structure of the implied volatility surface, they largely ignore the fact of a U-shaped individual stock smile.

b. The effects of increasing default risk on the smile are not the same in all models.

c. The term structure of the implied volatility surface does not flatten out in all models, although this should be expected.

Altogether, this chapter emphasized the necessity of further empirical research regarding the link between default risk on the one hand and the smile and the risk-neutral density on the other. This necessity follows from the scarcity and incompleteness of

248 In the case of a high leverage ratio in conjunction with protected debt, the model of TOFT/PRUCYK (1997) predicts a steepening of the smile with increasing maturity (see section 5.2.2.2.1. 
extant empirical findings, further from the contradiction between the theoretical prediction of a default risk induced monotonically downward sloping smile on the one hand and its evident static feature of a generally U-shaped pattern on the other and finally from the insufficiencies and contradictions of the enhanced credit risk models. 


\section{Empirical Analysis of the Relationship between the Equity Volatility Smile and Default Risk}

\subsection{Introduction}

While chapter 4 revealed that a fundamental and at the same time convincing explanation of the individual stock smile is still missing, it also suggested that such an explanation could be related to default risk. Chapter 5 consequently analyzed the theoretical link between default risk and the individual stock smile and also presented the extant empirical evidence as to this link which was found to be partly contradictory or scarce, if not missing at all.

This deficiency on the empirical side is accompanied by an obvious contradiction on the theoretical side. While consistently with the leverage effect theory enhanced credit risk models produce a monotonically downward sloping smile, the smile pattern in reality resembles a U-shaped curve.

The obscurities on the empirical as well as on the model side both stress the necessity of a thorough empirical analysis of the relationship between default risk and the individual stock smile, which is the topic of this chapter.

Virtually, the smile is a surface spanning the dimension of strike prices and maturities and a thorough analysis should cover both dimensions. However, the following examination is restricted to the strike dimension for two reasons: First, the strike dimension of the volatility surface is the most important, because unlike the maturity dimension it can be carried over to a terminal risk-neutral density and also because it is the mostly referred to in the literature. And secondly, data points in the maturity dimension are scarce, since in the case of individual stocks they are restricted to at most a handful 
of liquid option maturities 249 . This poses a difficult challenge to the approximation of the smile in the maturity dimension.

The structure of the analysis is as follows: Grounded on the literature-based part of this thesis, section 6.2 starts with a derivation of the hypotheses to be tested. Then section 6.3 outlines the statistical methods employed in the analysis. Section 6.4 continues with the description of the raw data sources and the processing of the raw data into the variables which will be used in the subsequent regression analysis. The regression analysis itself as well as its results are presented in section 6.5. Finally, section 6.6 summarizes the key contributions of the empirical study.

\subsection{Hypotheses}

As has been mentioned above in section 6.1. contradictory, scarce or lacking empirical findings as well as weaknesses of existing models call for a thorough analysis of the relationship between default risk and the equity volatility smile. This insight leads to the central, but general empirical research question: What exactly is the nature of the link between default risk and the individual stock option smile? Based on the theoretical and empirical insights gained in chapter 5, four testable hypotheses will be derived:

- Section 5.1.1.3 underlines the fundamental theoretical importance of the leverage effect for the relationship between default risk and the equity volatility smile. This importance is further stressed by the fact that the leverage effect relationship represents an intrinsic mechanism of all firm value based models and is also mimicked by all enhanced reduced form models. But the overall importance of the leverage effect relationship is contrasted by the fact that its existence is still subject of much debate. As discussed in section 5.1.3.1, the literature agrees upon the existence of the asymmetric volatility phenomenon, but it disagrees on its causes. Some studies suggest that asymmetric volatility is indeed induced by the leverage effect, whereas others ascribe it to volatility feedback or even expressly reject the existence of the leverage effect. This antagonism between theoretical significance and empirical doubts leads to the first hypothesis:

Hypothesis 1: The asymmetric volatility phenomenon is caused by the leverage effect.

249 FENGLER (2005) describes the available data pattern as maturity-wise "strings" (see p. 24). 
- Section 5.1.2.1 showed that rising equity volatility is accompanied by increasing default risk. Due to the leverage effect relationship, rising equity volatility can in turn be caused by increasing firm volatility or increasing leverage ratio. As demonstrated in section 5.1.3.2 the positive link between default risk and the level of equity volatility has already been confirmed in many studies. Yet, for reasons of completeness and consistency within the analysis, this relationship is also to be tested on the available data sample. This leads us to the second hypothesis:

Hypothesis 2: The level of equity volatility depends positively on the level of default risk.

- Section 5.1.2.2 elaborated how the leverage effect should lead to a convex, monotonically downward sloping equity volatility smile, which is all the more steeper the higher the level of default risk. However, as depicted in section 5.1.3.3. empirical evidence this concerning is very scarce and the corresponding studies partly rely on questionable measures of the slope of the smile. And what is more, all studies are limited to an analysis of the slope of the smile around the ATM point. Still, the available evidence seems to corroborate the theoretically predicted negative sign of dependence between default risk and the slope of the smile. However, the theoretical prediction of a default risk induced monotonically downward sloping smile seems to contradict the observation that the individual stock option smile generally exhibits a U-shaped pattern. This contradiction makes the theoretical prediction altogether vulnerable and raises the question whether the hypothesized relationship between the level of default risk and the smile slope is really this simple. Therefore, the scarcity of existing empirical evidence as well as the questionable simplicity of the theoretical prediction suggests the third hypothesis:

Hypothesis 3: The slope of the equity volatility smile depends negatively on the level of default risk.

- As has been illustrated in section 5.1.2.2, the theory associates the existence of default risk with a left-skewed equity return density, which should be all the more left-skewed, the higher the level of default risk. But corresponding empirical evidence is even more scarce than in the case of the link between default risk and the slope of the smile and consists of only two studies with conflicting results 250 . The link between default risk and the level of risk-neutral skewness is

250 Strictly speaking, the available evidence only consists of the study of DENNIS/MAYHEW (2002), since the work of ZHANG ET AL. (2005) treats the physical density. 
worthwhile to be tested also for another reason: While the smile itself is difficult to interpret, the risk-neutral distribution speaks a much clearer language. As to the possible specification of the underlying risk-neutral return process, more can be learned from the concrete moments of the risk-neutral density than from the abstract form of the smile. This entails the fourth hypothesis:

Hypothesis 4: The skewness of the risk-neutral equity return density depends negatively on the level of default risk.

\subsection{Statistical Methods Used}

\subsubsection{Introduction}

The present section focuses on statistical methods that have been used in the analysis and that deviate from basic standard practice and are therefore worthwhile mentioning. Section 6.3.2 starts out with a brief sketch of the basic concept of the ordinary least squares (OLS) regression 251 . Then the very restrictive assumptions of the basic OLS regression are successively relaxed to accommodate the more realistic conditions which are met throughout the present analysis. Especially autocorrelated residuals and to some extent also non-stationarity are encountered in the context of the subsequent analysis. Autocorrelation will be treated in section 6.3 .3 and non-stationarity is subject of section 6.3.4

\subsubsection{Ordinary Least Squares Regression}

\subsubsection{General Assumptions and Properties of the Ordinary Least Squares Regression}

Very generally, and without specifying whether the regression analysis is to be conducted in the cross-section or in the time-series dimension or in both domains simultaneously, the basic principle of the ordinary least squares regression can be summarized in the following way ${ }^{252}$.

\footnotetext{
251 In the following, the term ordinary least squares and its abbreviation OLS will be used interchangeably.

252 For the concept of ordinary least squares regression, cf. HAMILTON (1994), p. 200-201.
} 
Be $y_{i}$ a dependent variable to be explained by a vector $\mathbf{x}_{i}$ of $k$ explanatory variables. Further, the relationship between observations of $y_{i}$ and $\mathbf{x}_{i}$ is assumed as a linear model of the form:

$$
y_{i}=\mathbf{x}_{i}{ }^{\prime} \boldsymbol{\beta}+u_{i}
$$

Then, given a sample of $n$ observations $\left(y_{1}, y_{2}, \ldots, y_{n}\right)$, the ordinary least squares estimate $\mathbf{b}$ of the true coefficient vector $\boldsymbol{\beta}$ is the value for $\boldsymbol{\beta}$, that minimizes the residual sum of squares:

$$
\sum_{i=1}^{n}\left(y_{i}-\mathbf{x}_{i}^{\prime} \boldsymbol{\beta}\right)^{2} \longrightarrow \min !
$$

The ordinary least squares estimator is given by:

$$
\mathbf{b}=\left[\sum_{i=1}^{n}\left(\mathbf{x}_{i} \mathbf{x}_{i}^{\prime}\right)\right]^{-1}\left[\sum_{i=1}^{n}\left(\mathbf{x}_{i} y_{i}\right)\right]
$$

Which in matrix notation can be written as:

$$
\mathbf{b}=\left(\mathbf{X}^{\prime} \mathbf{X}\right)^{-1} \mathbf{X}^{\prime} \mathbf{y}
$$

This estimator $\mathbf{b}$ is unbiased and the most efficient among all linear, unbiased estimators if the following conditions hold 253 .

1. The explanatory variables $\mathbf{x}_{i}$ are non-stochastic.

2. The error term $u_{i}$ is stationary, homoscedastic and not autocorrelated. More formally this means that $u_{i}$ is i.i.d. with mean 0 and constant variance $\sigma^{2}$.

\subsubsection{Application of the Ordinary Least Squares Regression in the Time-Series Domain}

Unfortunately, the rather restrictive assumptions of the previous section regarding the use of the ordinary least squares method cannot be expected to apply everywhere throughout the subsequent analysis. The main reason is that a substantial part of the subsequent analysis is conducted in the time-series domain. The property of time-series data of being ordered data in contrast to cross-sectional date 254 , makes regression analysis more involved. In addition, time-series data is often governed by a stochastic process.

253 See Hamilton (1994), p. 202-203 and AlEXANDER (2004), p. 417.

254 Cf. WOOLDRIDGE(2003), p. 324. 
Carried over to the time-series domain, the above mentioned conditions for unbiasedness and efficiency of the estimator $\mathbf{b}$ have to be reformulated more realistically, where the general index $i$ is replaced with the time index $t$ :

1. The linear model of equation 6.1 is assumed to hold, but in contrast to the above requirement of non stochasticity, the explanatory variables $\mathbf{x}_{t}$ are allowed to follow a stationary, weakly dependent stochastic process. Non technically speaking, weak dependence means that autocorrelation between $x_{t}$ and $x_{x+h}$ vanishes "sufficiently quickly" as $h \rightarrow \propto 255$.

2. The explanatory variables $\mathbf{x}_{t}$ are contemporaneously exogenous, which means that the error term $u_{t}$ has zero expectation given the contemporaneous explanatory variables $\mathbf{x}_{t}\left(E\left(u_{t} \mid \mathbf{x}_{t}\right)=0\right)$.

3. There exists no perfect collinearity between the explanatory variables.

4. The error term $u_{t}$ is contemporaneously homoscedastic: $\operatorname{Var}\left(u_{t} \mid \mathbf{x}_{t}\right)=\sigma^{2}$.

5. The error term $u_{t}$ is not autocorrelated: $\forall t \neq s, E\left(u_{t} u_{s} \mid \mathbf{x}_{t}, \mathbf{x}_{s}\right)=0$.

If conditions 1 to 5 apply in the time-series domain, the estimator $\mathbf{b}$ is not forcibly unbiased any more. However, by the law of large numbers, the estimator is consistent and asymptotically normally distributed which means that OLS standard errors and $t$ and $F$ statistics are still asymptotically valid 256 .

\subsubsection{Autocorrelation and Heteroscedasticity in the Residuals and their Treatment}

\subsubsection{Causes and Effects of Autocorrelation and Heteroscedasticity}

Contrary to the requirements 4 . and 5. of the previous section, error terms in time series analysis often exhibit serial correlation $\left(E\left(u_{t} u_{s}\right) \neq 0\right)^{257}$, and heteroscedasticity in the residuals ( $\operatorname{Var}\left(u_{t}\right)$ not constant). Possible causes of autocorrelation are structural breaks or regime switches 258 , omission of key explanatory variables in

255 See WOOLDRIDGE (2003), p. 362.

256 Cf. WOOLDRIDGE (2003), p. 365-369.

257 See MONTGOMERY/PECK (1992), p. 366.

258 See ALEXANDER (2004), p. 430-431. 
the regression $\sqrt{259}$ or non-stationarity $\sqrt{260}$ A possible origin of heteroscedasticity on the other hand consists likewise the omission of key explanatory variables ${ }^{261}$.

In the presence of serial correlation and/or heteroscedasticity the estimator $\mathbf{b}$ is still consistent, but standard errors as well as $t$ and $F$ statistics become - even asymptotically - invalid ${ }^{262}$. For this reason, autocorrelation and heteroscedasticity have to be treated when encountered in the context of a regression analysis.

\subsubsection{Detection of Autocorrelation and Heteroscedasticity}

Autocorrelation in the residuals can be detected with the Durbin-Watson test statistic, where $n$ is the number of periods and $\hat{u}_{t}$ are the OLS residuals ${ }^{263}$;

$$
D W=\frac{\sum_{t=2}^{n}\left(\hat{u}_{t}-\hat{u}_{t-1}\right)^{2}}{\sum_{t=1}^{n}\left(\hat{u}_{t}\right)^{2}}
$$

The Durbin-Watson statistic $D W$ and the estimated first order autocorrelation coefficient $\hat{\rho}$ are linked in the following way:

$$
D W \approx 2 \cdot(1-\hat{\rho})
$$

Therefore, $D W$ approximately covers the range [0,4] and approximately equals 2 in the absence of autocorrelation. In the case of positive autocorrelation, $D W$ will be smaller then 2 and conversely greater than 2 in the case of negative autocorrelation. The Durbin-Watson test statistic does not detect autocorrelation of higher than first order ${ }^{264}$. Testing for heteroscedasticity in the presence of serial correlation can lead to erroneous results. Therefore it is best to first test for serial correlation, then remedy the serial correlation and finally test for heteroscedasticity ${ }^{265}$. One common test for heteroscedasticity is the White test which regresses the OLS residuals on all squares and cross products of the explanatory variables in the search of omitted factors ${ }^{266}$.

259 See Neter ET AL. (1989), p. 484; AleXANDER (2004), p. 430 or Montgomery/PeCK (1992), p. 366.

260 See ALEXANDER (2004), p. 431.

261 See ALEXANDER (2004), p. 432.

262 See WOOLDRIDGE (2003), p. 392 and p. 414.

263 See WOOLDRIDGE (2003), p. 398.

264 See MONTGOMERY/PECK (1992), p. 371

265 See WOOLDRIDGE (2003), p. 414.

266 See ALEXANDER (2004), p. 432. 


\subsubsection{Treatment of Autocorrelation and Heteroscedasticity}

If autocorrelation or heteroscedasticity are due to omitted explanatory variables, the most natural way to overcome these effects is a respecification of the regression model ${ }^{267}$. Another way to remedy the effects of autocorrelation and/or heteroscedasticity consists in specifying the exact form of the covariance matrix $\mathbf{V}$ of the residuals, which has no longer the form $\mathbf{V}(\mathbf{u})=\sigma^{2} \mathbf{I}$ as in the base case (see section 6.3.2.2). If the exact form of $\mathbf{V}$ is known and is used in a least squares estimation instead of $\sigma^{2} \mathbf{I}$, this procedure is called generalized least squares (GLS). In this case, deviating from equation 6.4 , the estimator $\mathbf{b}$ is given by 268 ,

$$
\mathbf{b}=\left(\mathbf{X}^{\prime} \mathbf{V}^{-1} \mathbf{X}\right)^{-1} \mathbf{X}^{\prime} \mathbf{V}^{-1} \mathbf{y}
$$

This estimator is unbiased and efficient, but GLS estimation poses a challenge in that the unknown covariance matrix $\mathbf{V}$ has to be guessed first.

But instead of estimating the covariance matrix $\mathbf{V}$ of the residuals in order to be able to perform a GLS regression, it is also possible to conduct an OLS regression first and then to correct the covariance matrix $\Sigma$ of the coefficient estimator. This procedure also ensures a consistent estimator $\mathbf{b}$ in connection with correct statistical inferences ${ }^{269}$. One such heteroscedasticity- and autocorrelation-consistent method to estimate the coefficient covariance matrix $\Sigma$ is that of Newey-West, where $n$ equals the number of periods and $k$ the number of coefficients 270 .

$$
\hat{\mathbf{\Sigma}}_{N W}=\frac{n}{n-k}\left(\mathbf{X}^{\prime} \mathbf{X}\right)^{-1} \hat{\mathbf{\Omega}}\left(\mathbf{X}^{\prime} \mathbf{X}\right)^{-1}
$$

The matrix $\hat{\Omega}$ ensues according to the following expression, where $q$ represents a truncation parameter ${ }^{271}$.

$$
\hat{\boldsymbol{\Omega}}=\frac{n}{n-k}\left\{\sum_{t=1}^{n} u_{t}^{2} x_{t} x_{t}^{\prime}+\sum_{i=1}^{q}\left[\left(1-\frac{i}{q+1}\right) \sum_{t=i+1}^{n}\left(x_{t} u_{t} u_{t-i} x_{t-i}^{\prime}+x_{t-i} u_{t-i} u_{t} x_{t}^{\prime}\right)\right]\right\}
$$

\footnotetext{
267 Cf. ALEXANDER (2004), p. 432.

268 Cf. MONTGOMERY/PECK (1992), p. 378-380.

269 Cf. WOOLDRIDGE (2003), p. 410.

270 See Evi (2005), p. 471 and cf. HAMILTON (1994), p. 281.

271 In the statistical package which is used in the subsequent analysis the truncation is set to $q=$

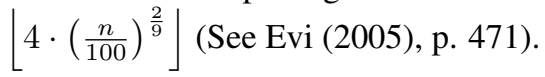


While the Newey-West method is robust to autocorrelation and heteroscedasticity, the alternative White method is only robust to heteroscedasticity. The White method will also be sketched briefly, because in the subsequent analysis it is used for statistical inferences in connection with panel regressions ${ }^{272}$.

$$
\hat{\mathbf{\Sigma}}_{W}=\frac{n}{n-k}\left(\mathbf{X}^{\prime} \mathbf{X}\right)^{-1}\left(\sum_{t=1}^{n} u_{t}^{2} x_{t} x_{t}^{\prime}\right)\left(\mathbf{X}^{\prime} \mathbf{X}\right)^{-1}
$$

\subsubsection{Non-Stationarity}

\subsubsection{Non-Stationarity and its Causes}

Time series data can be non-stationary, which means that it exhibits either a time trend or a stochastic trend or both. A non-stationary behavior of time-series data is inconsistent with the OLS assumptions laid out above and can lead to erroneous regression results. The reason is that a regression analysis of two completely independent series trending in the same direction can suggest a strong dependence, although in reality there is none at all. This phenomenon is called spurious regression 273 ,

A time trend is deterministic and can be described by the following mode ${ }^{274}$, where $c$ denotes a constant and $u_{t}$ is i.i.d and normally distributed, $u_{t} \sim N\left(0, \sigma^{2}\right)$ :

$$
y_{t}=c+\rho t+u_{t}
$$

A stochastic trend on the other hand is not predictable, which is typical of a random walk for example. Under the efficient market hypothesis, a random walk is the process which is generally assumed for asset price movements 275 .

$$
y_{t}=c+y_{t-1}+u_{t}
$$

A stochastic trend of the form of expression 6.12 is also called an integrated process of order 1 - denoted with $I(1)$ - or a difference-stationary process, since in contrast to

272 For the White method, see Evi (2005), p. 470.

273 Spurious regression has originally been termed by GRANGER/NEWBOLD (1974) who performed regression analysis on simulated independent and stochastically trending series. They found that standard regression analysis suggests a strong dependence and very high explanatory power although there is absolutely no dependence between the series. The term spurious regression is not only used in connection with stochastic trends, but also when referring to time trends (cf. e.g. Wooldridge2003, p. 347).

Cf. AlexANDER (2004), p. 322

275

Cf. ALEXANDER (2004), p. 320. 
the series itself the series of its differences is stationary and is therefore denoted with $I(0)$ 276,

$$
\Delta y_{t}=c+u_{t}
$$

A random walk can also be represented as an autoregressive process of order $1(A R(1))$ with a coefficient $\rho=1$ :

$$
y_{t}=c+\rho y_{t-1}+u_{t}
$$

An $A R(1)$ process with a coefficient $\rho$ equaling 1 is said to be a unit root proces 277 . Since $\rho=1$, a unit root process exhibits high persistence in contrast to the above required weak dependence for OLS regressions (cf. section 6.3.2.2).

\subsubsection{Detection and Treatment of Non-Stationarity}

6.3.4.2.1. Detection and Treatment of a Time Trend The detection and treatment of a time-trend is not as critical as that of a stochastic trend. If a time-trend seems likely due to economic reasoning or visual inspection of the series, the variable time can easily be integrated into the regression model according to equation 6.11, which ensures that the assumptions of section 6.3.2.2 will be respected, the estimator $\mathbf{b}$ will be consistent and statistical inference is possible in the usual way ${ }^{278}$. If the assumption of a time trend is justified, the respective coefficient for the time variable will be significantly different from 0 . The only complication arising is that of possibly inflated explanatory power $R^{2}$, if the time variable contributes substantially to the explanation of the dependent variable. If such an increase in $R^{2}$ is not wished, the time trend has to be eliminated before the regression analysis 279 .

6.3.4.2. Detection and Treatment of a Stochastic Trend In the case of a stochastic trend, things are more complicated. A stochastic trend can be detected with a so-called unit root test. There exists a variety of unit root tests. The most popular and easiest to implement single series unit root test is the augmented Dickey-Fuller (ADF) test ${ }^{280}$ For this reason the following passage concentrates on this test. The Dickey-

\footnotetext{
276 See ALEXANDER (2004), p. 322.

277 See WOOLDRIDGE (2003), p. 374.

278 See WOOLDRIDGE (2003), p. 346.

279 See WOOLDRIDGE (2003), p. 351-352.

280 See WOOLDRIDGE $(\overline{2003})$, p. 638.
} 
Fuller test in its basic version is grounded on the $A R(1)$ model. After subtraction of $y_{t-1}$ from both sides of equation 6.14, one arrives at the following model:

$$
\Delta y_{t}=c+(\rho-1) y_{t-1}+u_{t}
$$

The ADF test tests the null hypothesis $H_{0}: \rho=1$ against the alternative hypothesis $H_{1}: \rho<1^{281}$ In its augmented version, the Dickey-Fuller test assumes an $A R(p)$ process instead of an $A R(1)$ process by adding $p$ lagged differences of $y$, thus allowing for the case of auto-correlated residuals 282

Unfortunately, single series unit root tests are not considered to be very powerful, especially for small sample sizes 283 . MADDALA/KIM (2000) even suggest that due to a poor testing power the null hypothesis of a unit root can be rejected on a significance level as high as $25 \%$ and that usual significance levels of $1 \%$ or $5 \%$ are not advisable ${ }^{284}$. However, the poor testing power can be increased by the use of a panel unit root test in the context of a panel regression 285. Panel unit root tests simultaneously test for the presence of unit roots in the individual cross-sections $i$ of the panel and are based on the following regression approach 286 , where $\mathbf{x}$ represents a vector of exogeneous variables:

$$
y_{i, t}=\rho_{i} y_{t, i-1}+\mathbf{x}_{t, i}{ }^{\prime} \boldsymbol{\beta}_{i}+u_{i, t}
$$

As in the single series case, the tests distinguish between $\rho_{i}=1$ for the presence of a unit root and $\rho_{i}<1$ for the absence of a unit root. The tests can be coarsely classified into those testing for a common unit root, which means that $\rho$ is the same for all cross-sections, and those testing each cross-section individually and combining the individual results afterwards to form a common conclusion concerning the whole panel. 287

\footnotetext{
See Evi (2005), p. 520.

282 See Evi $(2005)$, p. 521.

283 See MADDALA/KIM (2000), p. 134 and LEVIN ET AL. (2002).

284 Cf. MADDALA/KIM (2000), p. 146.

285 See MADDALA/KIM (2000), p. 133-134 and LEVIN ET AL. (2002).

286 For the approach of panel unit root tests, cf. Evi (2005), p. 532-539.

287 The statistical package of Eviews, which has been used for the present analysis, offers the following panel unit root tests:

1. The test of Levin, Lin and Chu and that of Breitung with the null hypothesis of a common unit root.

2. The test of Hadri with the null hypothesis of no common unit root.

3. The test of of Im, Pesaran and Shin as well as two derivatives of the test of Fisher with the null hypothesis of individual unit roots.
} 
If a unit root is present, the concerning series can be differenced to eliminate the stochastic trend 288 . While it might still seem natural to transform a $I(1)$ price series by first differencing into a $I(0)$ return series, in other cases such a procedure can lead to an unacceptable loss of information. This is true for the subsequent analysis, where for some series - like the CDS spread for example - the level of the series is relevant and not its first differences. But even if unit roots are unquestionably present, it is still possible to generate valid regression results in 2 special cases:

- If two series $x_{t}$ and $y_{t}$ are $I(1)$, but $y_{t}$ can be expressed as a linear function of $x_{t}$, such that the series $y_{t}-\beta x_{t}$ is $I(0)$. Then the series $x_{t}$ and $y_{t}$ are said to be cointegrated ${ }^{289}$ and the expression $y_{t}-\beta x_{t}$ is called a cointegration equation. Cointegrated series move in lock step in the long term and the cointegration equation reflects their long run relationship 290 . The easiest way to test for cointegration of two $I(1)$ series is the Engle-Granger methodology which consists in performing a standard OLS regression from one series on the other and then in testing the residuals for stationarity with an ADF test 291 .

In the case that the ADF residual test according to the Engle-Granger method is applied in the course of the subsequent analysis, it is resorted to especially tabulated critical values which are not furnished by the statistical program used 292

- In the case of only one possibly $I(1)$ series involved in a regression model, the regression results will still be valid. The reason is that spurious regression is probable between two independent $I(1)$ series, but not between one $I(1)$ and one $I(0)$ series: A spurious result in the sense that the two series trend by chance into directions which make them seem correlated is not possible, since the $I(0)$ series does not trend by definition.

\footnotetext{
See WOOLDRIDGE (2003), p. 615.

See WOOLDRIDGE $(2003)$, p. 615.

Cf. ALEXANDER (2004), p. 350-351.

291 See ALEXANDER (2004), p. 354-355.

292 The critical values for the ADF test supplied by Evi (2005) are only valid for the test of a raw data series, but not for a series of residuals, which are only estimates of the true disturbance term (see Evi (2005), p. 519 and cf. MADDALA/KIM (2000), p. 200). For the case of the residual ADF test in the context of the Engle-Granger method, adequate critical values are tabulated in PHILliPS/OULIARIS (1990).
} 


\subsection{Data Processing and Conditioning}

\subsubsection{Introduction}

This section describes the process from the raw data sources to the ready-to-use variables that have finally entered the regression analysis. Due to the huge amount of data 293 to be handled, this process poses technical and computational challenges. These challenges have been met by first partitioning the raw data into separate Access databases ${ }^{294}$. All data handling across these separate databases has then been managed by a $\mathrm{C}++$ program which has been embedded into one of the databases as a so-called dynamic link library. The program has been implemented in $\mathrm{C}++$, since this language is superior to most other languages as far as computation speed is concerned. The data processing controlled by the $\mathrm{C}++$ program includes the following steps, which will be further elucidated in the subsequent sections:

- Raw data treatment including the computation of a risk free interest rate for each option trade as well as the identification of the respective most recent underlying price

- Computation of the historical moments of the equity return time series

- Computation of implied volatilities with analytical and numerical methods

- Approximation of the volatility smile for each trading day, each underlying and each available option maturity with a polynomial regression

- Calculation of the moments of the risk-neutral density for each trading day, each underlying and each available option maturity

- For hypothetical, constant maturities of 36, 60, 180 and 240 days, computation of the corresponding smile and the corresponding risk-neutral moments for each underlying and each trading day

Where necessary, extracts of the $\mathrm{C}++$ code will be furnished in appendix $\mathrm{A}$

\subsubsection{Data Sources}

The analysis is based on daily and intraday data and covers the 3 year period from January, $2^{\text {nd }} 2002$ through to December, $28^{\text {th }}$ 2004. Raw data has been collected for

293 As will be seen later on, more than 5,3 million option and more than 32,1 million stock trades have been processed.

294 This has been done in order to overcome the Access file size restriction of 2 GB. 
individual DAX companies as well as for the DAX index. The firm's default risk has been proxied by CDS spreads which are preferred to credit ratings or bond spreads as default risk indicators. The reason that CDS spreads have been preferred to credit ratings is twofold:

- First, the analysis has been conducted on a daily basis, but ratings are not available at this frequency.

- Secondly, credit ratings are slower than CDS spreads in their reaction to changes in credit quality 295

Further, the reason that CDS spreads have been preferred to bond spreads is also twofold:

- First, in contrast to corporate bonds, CDS are standardized contracts with standardized maturities. They do not depend on the properties and features of a specific bond issue. Whereas bond data would have to be laboriously processed to produce credit spreads that are representative of the respective firm, this problem does not exist with CDS data.

- Secondly, empirical evidence suggests that CDS spreads are a much better indicator of default risk than are bond spreads. On the one hand and in contrast to CDS spreads, bond spreads seem to contain a significant premium for illiquidity and are also affected by tax issues 296 . On the other hand the CDS and the equity market seem to lead the bond market ${ }^{297}$. This means that CDS and equity markets are more aligned than bond and equity markets. Also for this reason CDS spreads are preferable for the following analysis.

Only those 23 companies of the altogether 30 DAX members have been considered, for whom CDS spread data was available during at least a part of the three year period (see table 6.1 298 . The stocks in table 6.1 are ordered decreasingly by the number of actually used option quotes. This can be understood as a ranking of importance for the subsequent analysis, since the quality of the results depends strongly on the amount of available data. The amount of data available for the analysis of smile and default risk can also be assessed by the rightmost column in table 6.1. This column reports the number of days in the 3 year period for that a CDS spread has been available and

\footnotetext{
295 There exists an abundance of empirical evidence that credit ratings are slower in assessing the credit quality than CDS spreads. HULL ET AL. (2004b), e.g., find evidence that credit rating changes are anticipated by CDS spread changes.

296 See LONGSTAFF ET AL. (2003), LONGSTAFF ET AL. (2005) and CHEN ET AL. (2007).

297 see LONGSTAFF ET AL. (2003) and LONGSTAFF ET AL. (2005)

298 Due to the importance of bank loans compared to bond issues as a means of refinancing for German corporates, there were after all 7 companies with no CDS spreads available.
} 
at the same time at least one smile could be estimated. The raw data stems from 4

\begin{tabular}{|c|c|c|c|c|c|}
\hline (1) & (2) & $\begin{array}{l}\text { ISIN } \\
\text { (3) } \\
\end{array}$ & $\begin{array}{l}\text { Number of } \\
\text { used option } \\
\text { quotes } \\
\text { (4) }\end{array}$ & $\begin{array}{l}\text { Average } \\
\text { CDS } \\
\text { spread } \\
\text { [bp] } \\
\text { (5) }\end{array}$ & $\begin{array}{c}\text { Days with } \\
\text { smile and } \\
\text { spread } \\
\text { (6) }\end{array}$ \\
\hline Dax Performance Index & DAX & DE0008469008 & 2.447 .306 & - & 763 \\
\hline Allianz - Holding & ALL & DE0008404005 & 372.052 & 41 & 729 \\
\hline Siemens & _SIE & DE0007236101 & 365.777 & 40 & 757 \\
\hline Deutsche Telekom & _DTE & DE0005557508 & 309.174 & 134 & 757 \\
\hline DaimlerChrysler & _DAIM & DE0007100000 & 296.702 & 114 & 755 \\
\hline Deutsche Bank & _DBANK & DE0005140008 & 283.419 & 26 & 760 \\
\hline Münchener Rückversicherung & _MRE & DE0008430026 & 189.798 & 37 & 668 \\
\hline Bayer & BAY & DE0005752000 & 127.009 & 58 & 740 \\
\hline VW & _VW & DE0007664005 & 105.847 & 59 & 755 \\
\hline BASF & BASF & DE0005151005 & 103.362 & 20 & 752 \\
\hline E.ON & EON & DE0007614406 & 95.933 & 37 & 755 \\
\hline Commerzbank & _COB & DE0008032004 & 82.112 & 58 & 725 \\
\hline HypoVereinsbank & _HYPV & DE0008022005 & 64.007 & 56 & 747 \\
\hline RWE & _RWE & DE0007037129 & 63.667 & 47 & 671 \\
\hline BMW & _BMW & DE0005190003 & 63.463 & 33 & 756 \\
\hline Lufthansa & _LH & DE0008232125 & 42.130 & 107 & 699 \\
\hline Thyssen Krupp & _TYSS & DE0007500001 & 33.715 & 145 & 646 \\
\hline Metro & _METRO & DE0007257503 & 23.577 & 74 & 610 \\
\hline TUI AG & _TUI & DE0006952005 & 14.431 & 246 & 243 \\
\hline Linde & _LIN & DE0006483001 & 13.995 & 43 & 478 \\
\hline Henkel & _HEN & DE0006048432 & 9.828 & 27 & 383 \\
\hline Continental & _CONT & DE0005439004 & 8.072 & 73 & 277 \\
\hline Deutsche Post & _DP & DE0005552004 & 1.897 & 20 & 58 \\
\hline Degussa & _DEG & DE0005421903 & 266 & 51 & 3 \\
\hline
\end{tabular}

Table 6.1.: Companies considered in the analysis

sources:

- Option prices: Option prices are essential for the calculation of implied volatilities. Option prices consist of intraday trade data and originate from the Eurex 299 electronic trading platform. Eurex intraday data consists of actual trade data and is therefore generally preferable to mere quote data, which would pose the challenge of estimating the fair market value. Besides, Eurex intraday data is also preferable to end-of-day or settlement data: First, using intraday instead of endof-day data multiplies the amount of available data. Secondly, Eurex intraday data represents actual trade data, whereas end-of-day data consists of settlement prices. These settlement prices are based on the last trade of the day and if this is older than 15 minutes, Eurex reserves the right to arbitrarily determine the settlement price ${ }^{300}$. For less liquid OTM options this practice is very frequent

299 Derivative exchange belonging to the Deutsche Börse AG

300 See Eurex product specifications for equity options (www.eurexchange). 
and sometimes leads to questionable results 301 . Initially the raw data sample consisted of 2,51 million regular ${ }^{302}$ stock and 2,81 million regular index option trades for options of all maturities. Of these only 2,45 million stock and 2,67 million index trades respectively have been used in the analysis. The remainder had to be eliminated in the process of data cleaning.

- Underlying prices: Underlying prices are stock and index prices. They are needed to calculate historical moments like standard deviation, skewness and kurtosis and also to compute implied volatilities. As far as implied volatilities are concerned, it is absolutely necessary to dispose of contemporaneous observations of the prices of the option and its underlying. For this reason intraday underlying price data is indispensable. The stock data for the 23 titles of table 6.1 comprehends 32,1 million intraday trades and stems from Xetra 303 . As to the preference of actual trade data over mere quote data, the same can be said as in the previous paragraph. The underlying of the index options is the DAX performance index which is calculated by Deutsche Börse and made available every 15 seconds between $9 \mathrm{~h} 00$ and $17 \mathrm{~h} 45$.

- Dividends: Dividends are indispensable for the calculation of implied volatilities. All dividends that have been paid out in the period from January, $2^{\text {nd }} 2001$ through to the mid of June 2006 have been considered in the analysis. Dividends have been extracted from Bloomberg.

- CDS spreads: CDS spreads are a vital component of the analysis. They have also been extracted from Bloomberg, are based on mid market quotes for a maturity of 5 years and represent an average across market makers as determined by Bloomberg 304 .

- Risk free discount factor curve: The risk free rate is a necessary input for the calculation of implied volatilities. The risk free discount factor curve has been obtained from the trading system of the Siemens AG treasury department 305 .

301 Working for Banque Natinale de Paris in 1998 - one of the leading Eurex equity options market makers at that time - I experienced myself, that OTM option settlement prices are often only based on an arbitrary quote of the leading market maker.

302 Non regular in contrast to regular trades are cancellations, e.g..

303 Xetra is the electronic spot trading platform of Deutsche Börse AG.

304 The so-called Bloomberg generic price.

305 The Siemens AG treasury system during the time of the analysis was Wall Street Systems and was fed with Euribor and interbank swap rates from Reuters. 


\subsubsection{Computation of Historical Moments}

\subsubsection{Observation Period and Frequency}

The historical moments calculated in the analysis are the second, third and fourth central moment of the equity return. These moments are primarily needed for the comparison of the statistical with the respective option implied or risk-neutral moments. This requires that the historical moments are estimated for each underlying and each day.

Both sets of moments are comparable, if both are representative of the momentary market perception. It can be argued that this is the case, since risk-neutral moments reflect the market's momentary anticipation of the future with this anticipation being in turn based on the momentary market behavior. This momentary market behavior in turn can be expressed in terms of the momentary statistical moments of the equity return. The shorter the observation period and the higher the observation frequency, the closer the historical moment comes to a momentary moment. The requirement of a short observation period and high observation frequency for the calculation of the historical moments calls for the use of intraday high frequency data. This settled, the question remains, what observation period and what frequency exactly to pick. Answering this question, at least 4 issues have to addressed:

- Regarding the observation period, it can be argued that the market's anticipation of the 15 day volatility for example - as expressed in prices of options with that term - relies on an equally long history of return observations. For this reason the ideal period of observation for the calculation of historical moments represents a trade-off between timeliness and the proper matching of the options' maturities.

- The longer the period of observation, the more computation time is required 306

- Given a certain frequency of observation, the shorter the period of observation, the smaller will also be the number of observations, at the limit leading to a high variance of the estimator.

- High frequency data exhibits autocorrelation in returns $s^{307}$ This could induce moment estimates which are not valid for longer horizons. However the autocorrelation of returns dies away quickly with declining frequency of observation

306 The more days the observation period consists of, the more queries on the tables containing the stock trades have to be launched. Since these tables are very large (containing 32.1 million entries altogether), a query represents a very time-consuming operation.

307

See ALEXANDER (2004), pp. 391-392. 
and at a frequency of 5 minutes most of the autocorrelation can be supposed to have vanished 308 .

The simultaneous consideration of these 4 issues heuristically led to a frequency of 5 minutes and a period of observation of 12 trading days, which ideally results in 2.448 observations for the estimate of volatility, skewness and kurtosis of a particular trading day.

\subsubsection{Generation of the Return Series}

The historical moments have been estimated for each stock and each trading day at 13h00. This requires that a time series of 5 minute log returns is established, which stretches 12 trading days or 102 trading hours into the past starting from 13h00:

Be $t_{i}$ the sequence of 5 minute interval points in time with $i \in\{0,1, \ldots, n=2447\}$, with $t_{0}=13 h 00$ and with $t_{i-1}-t_{i}=5$ minutes $\forall i$. Be further $p_{\tau_{j}}$ the sequence of $m$ actual, irregularly spaced price observations. If $\tau_{i}=\max \left\{\tau_{j \in\{1,2, \ldots, m\}} \mid \tau_{j} \leq t_{i}\right\} \forall i \in$ $\{0,1, \ldots, n=2.447\}$, then $p_{\tau_{i}}$ is the sequence of regularly spaced price observations used to calculate the series of 5 minute $\log$ returns $R_{\tau_{i}}=\frac{\ln \left(p_{\tau_{i}}\right)}{\ln \left(p_{\tau_{i-1}}\right)} . R_{\tau_{i}}$ finally is the return series, which the estimation of the second, third and fourth central moment is based upon.

\subsubsection{Computation of Moments}

The historical volatility $H V$ has been estimated by the population standard deviation $\hat{s}$, with $\bar{R}$ being the sample mean:

$$
H V=\hat{s}=\sqrt{\frac{1}{n-1} \cdot \sum_{i=1}^{n}\left(R_{\tau_{i}}-\bar{R}\right)^{2}}
$$

The historical skewness $H S K E W$ has been calculated according to the following expression 309 .

$$
H S K E W=\frac{1}{n} \cdot \sum_{i=1}^{n}\left(\frac{R_{\tau_{i}}-\bar{R}}{\hat{s}}\right)^{3}
$$

\footnotetext{
308 GOODHART/FIGLIUOLI (1991) analyzes foreign exchange markets and finds that autocorrelation is substantially reduced at a frequency of 5 minutes and has almost completely vanished at a frequency of 10 minutes.

309 See RINNE (1995), p. 64-65.
} 
The calculation of the historical kurtosis $H K U R T$ ensues accordingly

$$
H K U R T=\frac{1}{n} \cdot \sum_{i=1}^{n}\left(\frac{R_{\tau_{i}}-\bar{R}}{\hat{s}}\right)^{4}
$$

\subsubsection{Computation of Implied Volatilities}

\subsubsection{Introduction}

As has been laid out in section 3.1.2, implied volatilities are calculated by inverting the option valuation function and by solving it for the volatility parameter. The solution for the volatility represents a root-finding problem and will be dealt with in section 6.4 .4 .5 .

Assuming a geometric Brownian motion with constant volatility for the process of the underlying, the option valuation function can either be the Black-Scholes closed form formula (see equations 3.8 and 3.9 or some numerical method 311 . In the case of American options, which are path depending due to the possibility of early exercise, the Black-Scholes formula cannot be used and numerical methods have to be employed. European options on the other hand, with no possibility of exercise before the final maturity, can be evaluated with both approaches.

In the context of the present analysis the underlying can either be the index level or the stock price. Options on the DAX performance index are European options and could be evaluated analytically and also numerically. For reasons of simplicity they have been evaluated analytically with the Black-Scholes formula. Eurex stock options on the other hand are American options and therefore need to be evaluated numerically. The numerical algorithm which is chosen is the Cox-Ross-Rubinstein binomial tree which will be described in more detail in section 6.4.4.4.

\subsubsection{Input Variables for the Option Evaluation Function}

The inverted option valuation function requires the following input variables:

- The underlying's price, which is either the stock price or the index level. Similarly to the proceedings for the generation of the return series (see section 6.4.3.2) the price of the most recent trade or index level with regard to the time

310 See RINNE (1995), p. 66.

311 Theoretically, in the case of this study 3 different numerical methods come into question: lattice methods, Monte-Carlo simulation or finite difference methods; cf. HULL (2006), chapter 17. 
of the option trade is taken as underlying price. For the liquid high volume stocks this method ensures almost perfect concurrence of option and underlying price observation.

- The risk free interest rate. According to banking practice, discount factors $D F_{t_{i}}$ have been interpolated linearly for a given day of maturity $d_{i}$. The continuously compounded risk free interest rate $R_{t_{i}}$ has been calculated according to the following expression:

$$
R_{t_{i}}=-\frac{\ln \left(D F_{t_{i}}\right)}{y f} \quad \text { with } \quad y f=\frac{t_{i}-t_{0}}{365}
$$

- The exercise price.

- The option price.

- The term to maturity.

- In the case of stock options, also dividends have to be considered. In the case of the DAX index however, dividends are not needed, since the DAX index is a performance index which already includes dividends.

\subsubsection{Data Quality Requirements}

Incorrect option prices have been identified by checking whether the option price is in- or outside certain admissible bounds. The so identified have been discarded. Be $S_{t_{0}}$ the current value of the underlying and $X$ the option's exercise price. Be further $c_{t_{0}}^{E}$ the value of a European call on the DAX performance index and $p_{t_{0}}^{E}$ the value of the corresponding put. Be finally $c_{t_{0}}^{A}$ the value of an American call on an individual stock and $p_{t_{0}}^{A}$ the value of the corresponding put. Using arbitrage arguments and for simplicity ignoring the role of dividends, the following bounds can be derived 312 .

- Upper bound for $c_{t_{0}}^{E}$ and $c_{t_{0}}^{A}$ :

$$
c_{t_{0}}^{E}, c_{t_{0}}^{A} \leq S_{t_{0}}
$$

- Upper bound for $p_{t_{0}}^{E}$ and $p_{t_{0}}^{A}$ :

$$
p_{t_{0}}^{E}, p_{t_{0}}^{A} \leq X
$$

312 See Hull (2006), p. 209-219. 
- Lower bound for $c_{t_{0}}^{E}$ and $c_{t_{0}}^{A}$ :

$$
c_{t_{0}}^{E} \geq \max \left(S-X e^{-r\left(T-t_{0}\right)}, 0\right)
$$

- Lower bound for $p_{t_{0}}^{E}$ :

$$
p_{t_{0}}^{E} \geq \max \left(X e^{-r\left(T-t_{0}\right)}-S_{t_{0}}, 0\right)
$$

- Lower bound for $p_{t_{0}}^{A}$ :

$$
p_{t_{0}}^{A} \geq \max \left(X-S_{t_{0}}, 0\right)
$$

\subsubsection{Option Price Evaluation with the Cox-Ross-Rubinstein Binomial Tree}

Stock options have been evaluated using an extension of the Cox-Ross-Rubinstein binomial tree allowing for early exercise and multiple dividend payments. In its original version this tree only permits single dividends paying a constant dividend yield ${ }^{313}$ whereas the extension of the tree can also cope with multiple EUR dividends 314 . The implementation of the tree in C++ code follows closely HULL (2006) and can be seen in appendix A.1. For the purposes of the present analysis, stock options have been evaluated by discretizing the term to maturity into 150 time steps.

\subsubsection{Root-finding Algorithm}

For ease of notation the root-finding algorithm will only be presented by means of the call price evaluation function $c=f(\sigma, \ldots)$, although it could equally be shown using the put function $p . c$ depends among other parameters on the equity return volatility $\sigma$ and represents either the analytical or the numerical evaluation method as detailed above. The goal of the root-finding algorithm consists in finding the implied volatility $\tilde{\sigma}$ which is the the value of $\sigma$ that makes the evaluation function $c$ equal the actual option price $\hat{c}, c(\tilde{\sigma})=\hat{c}$.

$c$ is a strictly monotone function of $\sigma$ and so is the difference $d$ between $c(\sigma)$ and the option's price $\hat{c}$, since $\hat{c}$ is a constant term. Further $c(\sigma=0)$ equals the option's intrinsic value. Now assuming that $\hat{c}$ is always greater than or equal to the intrinsic

313 For the original version of the Cox-Ross-Rubinstein binomial tree see COX ET AL. (1979).

314 The extension of the Cox-Ross-Rubinstein tree allowing for multiple EUR dividends is detailed in HULL (2006), pp. 401-405. 
value, the difference function $d$ must have a root in the intervall $\left[\sigma_{1}=0, \sigma_{2}\right]$, if $\sigma_{2}$ is a positive constant with $\sigma_{2} \geq \sigma_{1}$.

As a root-finding algorithm the bisection method has been chosen ${ }^{315}$. The method can be summarized as follows: The initial interval $\left[\sigma_{1}, \sigma_{2}\right]$ is halved. The half not containing the root is discarded, the other half is halved again. This procedure is repeated on and on until a subinterval is identified, that contains the root and at the same time does not exceed a pre-specified constant $\epsilon$.

The parameter for the bisection method are set to $\sigma_{1}=0, \sigma_{2}=20$ and $\epsilon=10^{-4}$. Thus, $\tilde{\sigma}$ is limited to 20 which is reasonable, since it is highly improbable that values above still represent an economic meaning. As can easily be checked with the help of equation 6.26, the parameter setting leads to at least $n=18$ iteration steps.

$$
\frac{\sigma_{2}-\sigma_{1}}{2^{n}}<\epsilon \Leftrightarrow n>\log _{2} \frac{\sigma_{2}-\sigma_{1}}{\epsilon}
$$

\subsubsection{The Smoothing of the Implied Volatility Smile}

\subsubsection{Assessment of the Available Implied Volatilities}

Before smoothing the smile, it is worthwhile assessing the implied volatilities that are generated with the methods described in the prior section. This assessment will lead to a better understanding regarding the choice of the adequate method to estimate the smile.

Figure 6.1 displays implied volatilities of options for the 6 most important stocks as prioritized in table 6.1. For each of the 6 stocks, figure 6.1 shows the volatilities for a day with a particularly high and for a day with a particularly low CDS spread, since the focus of the present analysis is a possible connection between smile and default risk. Further, figure 6.1 distinguishes between call and put contracts to find out whether they have to be analyzed separately. All volatilities of a specific stock and a specific day have been implied out of options with the same maturity. All options used to generate figure 6.1 exhibit a remaining term between 61 and 90 days. Contemplating figure 6.1, 6 observations can be made:

1. At least on high CDS spread days, implied volatilities do not seem to lie on a monotonically downward sloping curve as theoretically predicted in section

315 The implementation of the bisection method in $\mathrm{C}++$ code follows exactly PRESS ET AL. (2002), p. 358. 
5.1.2.2. Instead, they seem to describe a U-shaped course as is consistent with the stylized facts regarding the implied volatility surface (see section 3.1.2).

2. Implied volatilities of high CDS spread days are positioned on a higher volatility level than those of a low CDS spread day. This seems reasonable, since theory as well as empiricism suggest a positive relationship between volatility and credit spread (see chapter 5).

3. Volatility observations of high CDS spread days seem to cover a much larger extension in the strike price dimension than those of low CDS spread days. This is due to the fact that in periods of high volatility the strike prices of new options are set at accordingly larger intervals.

4. On high CDS spread days the covered strike-to-spot-price range seems to be more asymmetric than on low CDS spread days.

5. On low CDS spread days there is no difference between call and put contracts discernible. On high CDS spread days and for high strike-to-spot-price ratios however, put implied volatilities tend to be higher than call implied volatilities.

6. On high CDS spread days and for high strike-to-spot-price ratios implied volatilities seem to fluctuate substantially. This is due to bid-ask-bounces that are the more accentuated the greater the distance from the ATM point 316

The following conclusion can be drawn from these observations: Since time-series and cross-sectional comparisons are to be made in the course of the present analysis, the strike-to-spot price ranges of different days and cross-sections should be as comparable as possible. If the range is rather balanced on low CDS spread days, it should be alike on high CDS spread days and the asymmetry of high CDS spread days should be corrected. Further, available data should be exploited to maximum extent, calling for the simultaneous use of call and put implied volatilities. Call and put implied volatilities can be considered being equivalent as long as options with high strike-to-spot price ratios are exempted from the analysis. This would also eliminate the problem of bid-ask-bounces for high strike prices. For these three reasons, volatilities implied out of options with strike-to-spot-price ratios above $150 \%$ have been excluded from the analysis.

316 FENGLER (2005) describes this phenomenon for DAX index options (see p. 22-24). 

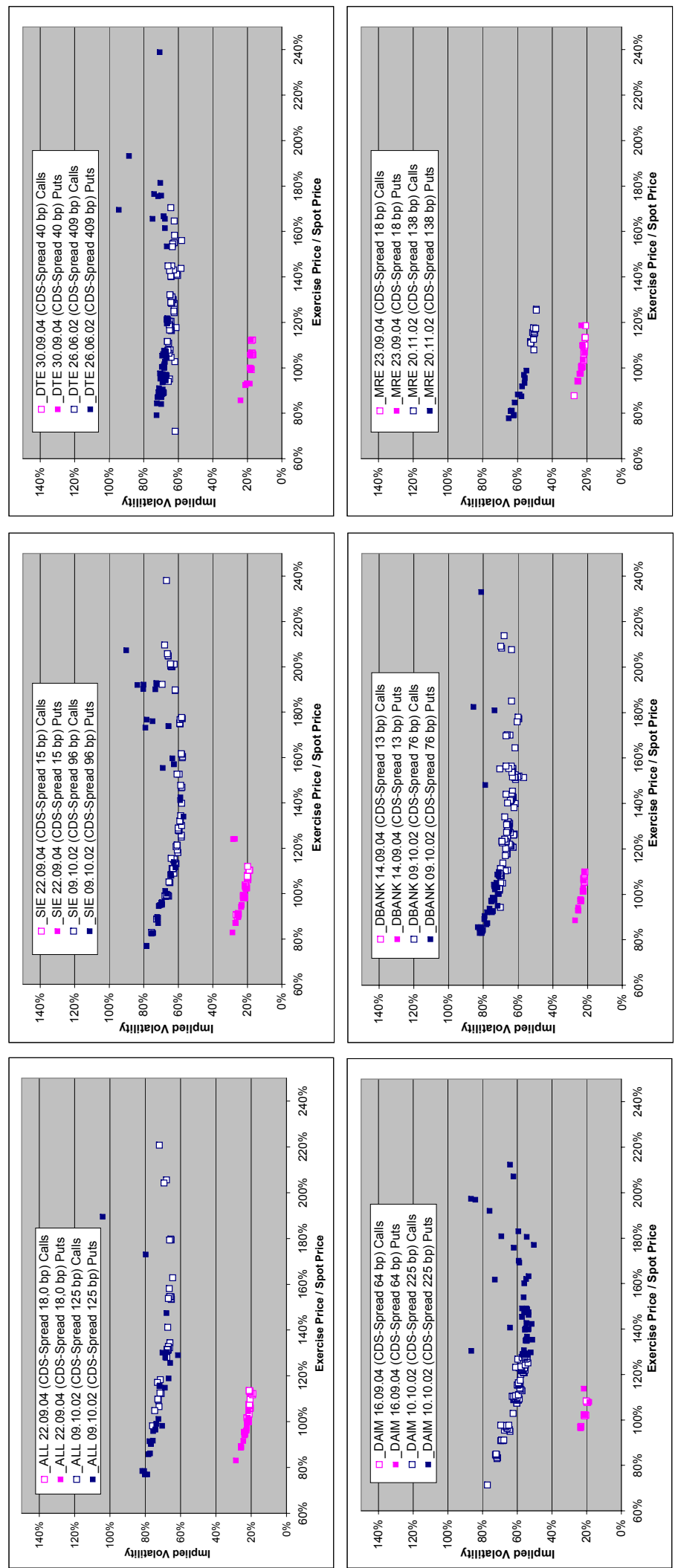

Figure 6.1.: Implied volatilities for a high CDS spread and a low CDS spread day for the 6 most important stocks 


\subsubsection{Quadratic Polynomial as the Method of Choice for the Smoothing of the Implied Volatility Smile}

For each trading day, each underlying and each available maturity, implied volatilities have been smoothed and a volatility smile function has been estimated. The underlying is either one of the 23 stocks or the DAX index. Altogether, this leads to roughly 72.000 smiles to be approximated. In section 3.2, different methods for the smile approximation have been discussed and in section 3.2 .5 it was concluded that the polynomial smile smoothing is the most stable and easiest to implement procedure and that a parsimonious specification like a quadratic polynomial is sufficient to capture the essential features of the smile. Therefore, the method of choice for the smile approximation is indeed the quadratic polynomial procedure originally proposed by SHIMKO (1993). In the light of the specificities of the present analysis this choice can be further substantiated:

- Computation time: The quadratic polynomial approach is a simple method allowing to compute more than 72.000 smiles in a reasonable time. More complex techniques, like for example kernel regression, would be too time consuming.

- Available data: The simpler the method, the less data is required to reach robust estimates of the smile. With only very few data points available for some stock options, complex and data intensive approaches like kernel regression are not feasible. But even polynomials of higher than second order would induce the risk of overfitting in the face of scarce data which for some combinations of trading day, underlying and maturity amounts to just a handful of observations of implied volatilities.

- Accurate reproduction of the smile's essential features: As illustrated in figure 6.2 the quadratic polynomial manages to capture the essential feature of a U-shaped volatility smile as is typical for individual stock options. Obviously a linear approximation would be too simplistic and could even fail in certain cases. And higher than second order approaches either do not lead to a substantial improvement - or even worse - result in overfitting due to outliers. 

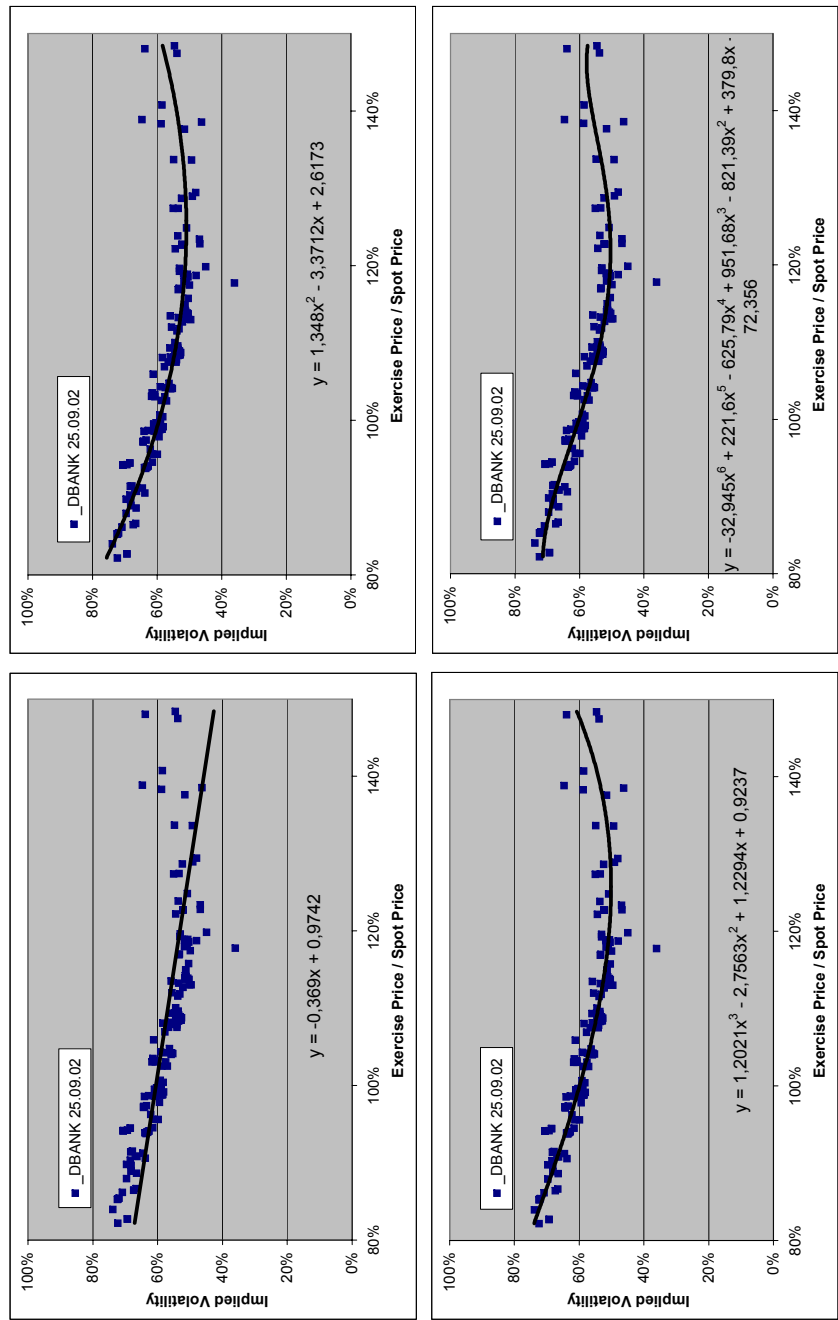

Figure 6.2.: Implied volatility smile approximation with polynomials of order 1, 2, 3 and 6 for a typical short term maturity of 26 days 


\subsubsection{Regression Model}

Be $X$ the option's exercise price and $S$ the spot price of the underlying, then the ratio $\frac{X}{S}$ denotes the moneyness measure. The implied volatility $\sigma$ has been regressed on $\frac{X}{S}$ using the standard OLS procedure for all trading days, all underlyings and all available maturities with the following regression model:

$$
\sigma=\beta_{0}+\beta_{1} \cdot \frac{X}{S}+\beta_{2} \cdot\left(\frac{X}{S}\right)^{2}+u
$$

Be $B 0, B 1$ and $B 2$ the estimates of the true coefficients $\beta_{0}, \beta_{1}$ and $\beta_{2}$, then the estimation of the above model leads to the implied volatility function $\hat{\sigma}$ :

$$
\hat{\sigma}=B 0+B 1 \cdot \frac{X}{S}+B 2 \cdot\left(\frac{X}{S}\right)^{2}
$$

The coefficient estimation as well as the calculation of the regression statistics has been implemented in $\mathrm{C}++\operatorname{code}^{317}$ and is displayed in appendix A.2. The estimation has been carried out with the following restrictions:

- All implied volatilities which are smaller than 5\% have been eliminated, since they do not represent any economically meaningful information 318 .

- All implied volatilities that are greater than $100 \%$ for the DAX index and $300 \%$ for individual stock options have been discarded. There are two reasons for this rule: First, in the case of very short maturities the root-finding algorithm does not terminate with an economically meaningful value and yields the prespecified limit of $2.000 \%$ instead 319 . Secondly, the bounds of $100 \%$ for the index and $300 \%$ for the individual stock options avoid outliers that would unduly bias the overall result.

- With the same argument of avoiding outliers and also to tie in with what was said in section 6.4.5.1, the admissible range of $\frac{X}{S}$ has been restricted to the interval $[0,5 ; 1,5]$.

317 The implementation follows RINNE (1995), part A, chapter 4.4.

318 This case can occur when the option's intrinsic value is close to zero.

319 Especially in the case of DAX index options where the remaining term can be zero days with the option's last trading and maturity day coinciding, the implied volatility can theoretically be infinite. 
- The minimum number of implied volatility observations for a combination of underlying, trading day and maturity that a regression model has been estimated upon was 4.

- The minimum maturity a smile approximation has been carried out for, was 7 days. The reason is that smiles of shorter maturities tend to be rather V-than U-shaped which renders the quadratic polynomial approach less suitable.

\subsubsection{Regression Results}

6.4.5.1. Regression Results and Regression Statistics Generally, a polynomial model specification as that of equation 6.27 introduces the risk of multicollinearity. The effects of multicollinearity can be twofold: First, the dependency among the independent variables leads to redundancy of information and ultimately to an increase of the standard errors of the coefficients of the variables, thus making the individual coefficient estimates less reliable $e^{320}$. Secondly, the regression coefficients become unstable, if only a small number of data points is available; only few additional data points can dramatically change the original regression estimates ${ }^{321}$.

However, our primary goal is not to fundamentally explain the smile. Instead, we want to smooth the implied volatilities and - based on a given functional approach find the function's parameters which best describe the shape of the smile. Therefore, as long as the explanatory power and the overall significance of our approach are high, possible insignificance of individual coefficients is only of minor importance and only matters, if the coefficients of the smile function $B 0, B 1$ and $B 2$ are used individually in a secondary analysis. It is true, that this will the case, though in a subordinate part of my analysis. Therefore, in order to be on the safe side, we will not only judge the quality of the implied volatility smoothing by its explanatory power and its overall significance, but also by the individual coefficient's significance.

On a cross-sectional basis table 6.2 displays the median coefficient estimates and regression statistics for the smoothing of the short term smiles which have ultimately been used for the construction of the 36 days constant maturity variables. The results for the longer maturities can be viewed in appendix B.1. The cross-sections are ordered by decreasing importance as measured by the number of used option trades (cf. table 6.1).

\footnotetext{
320 Cf. for example DraPer/Smith (1998), p. 369, ChanG (1996) or GRAPENTINE (1997).

321 See CHANG (1996).
} 
As can be seen in table 6.2, the explanatory power in terms of $R^{2}$ and the overall significance in terms of the $F$-statistic and its $p$ value are satisfactory for all crosssections. Further, the median number $n$ of data points involved in the smile smoothing decreases with decreasing importance of the cross-sections. At least for the 5 most important stocks, the median number of data points is around 100 and thus can be considered to be sufficient.

Taking a closer look at the results of the individual smile function's coefficients reveals that increasing importance of the cross-sections, as measured in option liquidity, leads to increasing significance of $B 0, B 1$ and $B 2$. For roughly $70 \%$ of all cross-sections, the coefficients are significant at the $5 \%$ level. Further, the smile function's intercept, $B 0$, as well as its coefficient for the quadratic term, $B 2$, are positive, and the coefficient of its linear term, $B 1$, is negative for all cross-sections. This very clear picture stresses the overall quality of the results.

Therefore, considering the high explanatory power, the relatively high number of data points as well as the high overall and individual significance, multicollinearity need not worry us, at least not for the shorter maturities.

As can be inspected in the appendix, the results for the longer maturities yield a similar picture. However, it has to be conceded that the results on the basis of the individual coefficients are slightly degrading in quality with increasing maturity. For the longest maturity the fraction of cross-sections with median significant coefficients declines to roughly $30 \%$. This maturity-dependent worsening of the results is due to the decreasing importance of the quadratic term with increasing, maturity-related flattening of the smile. 


\begin{tabular}{|c|c|c|c|c|c|c|c|c|c|c|c|c|c|}
\hline ID & \multicolumn{3}{|c|}{ B0 } & \multicolumn{3}{|c|}{ B1 } & \multicolumn{3}{|c|}{ B2 } & $\mathbf{R}^{2}$ & \multicolumn{2}{|c|}{$\mathbf{F}$} & $\mathbf{n}$ \\
\hline DAX & 2,27 & $103,39]$ & $(0,00)$ & $-3,60$ & {$[-74,42]$} & $(0,00)$ & 1,58 & {$[63,39]$} & $(0,00)$ & 0,97 & 18.363 & $(0,00)$ & 1.004 \\
\hline ALL & 2,36 & {$[22,64]$} & $(0,00)$ & $-3,66$ & {$[-16,24]$} & $(0,00)$ & 1,67 & {$[14,90]$} & $(0,00)$ & 0,87 & 507 & $(0,00)$ & 130 \\
\hline SIE & 2,30 & {$[26,67]$} & $(0,00)$ & $-3,54$ & {$[-20,01]$} & $(0,00)$ & 1,60 & {$[17,77]$} & $(0,00)$ & 0,92 & 820 & $(0,00)$ & 120 \\
\hline DTE & 2,75 & {$[17,91]$} & $(0,00)$ & $-4,46$ & {$[-13,89]$} & $(0,00)$ & 2,12 & {$[13,38]$} & $(0,00)$ & 0,80 & 238 & $(0,00)$ & 98 \\
\hline DAIM & 2,48 & {$[18,01]$} & $(0,00)$ & $-3,92$ & {$[-14,10]$} & $(0,00)$ & 1,78 & {$[12,85]$} & $(0,00)$ & 0,88 & 392 & $(0,00)$ & 94 \\
\hline DBANK & 2,48 & {$[22,17]$} & $(0,00)$ & $-3,84$ & {$[-17,41]$} & $(0,00)$ & 1,74 & {$[15,90]$} & $(0,00)$ & 0,93 & 669 & $(0,00)$ & 92 \\
\hline MRE & 2,44 & {$[12,56]$} & $(0,00)$ & $-3,80$ & {$[-9,43]$} & $(0,00)$ & 1,76 & {$[8,74]$} & $(0,00)$ & 0,84 & 181 & $(0,00)$ & 56 \\
\hline BAY & 2,78 & {$[10,19]$} & $(0,00)$ & $-4,55$ & {$[-7,97]$} & $(0,00)$ & 2,14 & {$[7,38]$} & $(0,00)$ & 0,85 & 123 & $(0,00)$ & 39 \\
\hline _VW & 2,46 & {$[9,11]$} & $(0,00)$ & $-3,89$ & {$[-7,01]$} & $(0,00)$ & 1,75 & {$[6,40]$} & $(0,00)$ & 0,89 & 162 & $(0,00)$ & 33 \\
\hline BASF & 3,06 & {$[8,51]$} & $(0,00)$ & $-5,19$ & {$[-6,97]$} & $(0,00)$ & 2,39 & {$[6,40]$} & $(0,00)$ & 0,90 & 181 & $(0,00)$ & 32 \\
\hline EON & 2,98 & {$[7,10]$} & $(0,00)$ & $-5,16$ & {$[-5,74]$} & $(0,00)$ & 2,41 & {$[5,34]$} & $(0,01)$ & 0,88 & 129 & $(0,00)$ & 28 \\
\hline COB & 2,98 & {$[6,65]$} & $(0,00)$ & $-4,83$ & {$[-5,49]$} & $(0,01)$ & 2,30 & {$[5,21]$} & $(0,01)$ & 0,76 & 52 & $(0,00)$ & 26 \\
\hline _HYPV & 2,44 & {$[5,19]$} & $(0,01)$ & $-3,74$ & {$[-3,95]$} & $(0,03)$ & 1,74 & {$[3,68]$} & $(0,03)$ & 0,79 & 48 & $(0,00)$ & 20 \\
\hline RWE & 3,33 & {$[5,90]$} & $(0,01)$ & $-5,87$ & {$[-5,14]$} & $(0,01)$ & 2,81 & {$[4,90]$} & $(0,01)$ & 0,86 & 84 & $(0,00)$ & 20 \\
\hline BMW & 2,54 & {$[5,97]$} & $(0,01)$ & $-4,12$ & {$[-4,67]$} & $(0,02)$ & 1,90 & {$[4,18]$} & $(0,03)$ & 0,88 & 95 & $(0,00)$ & 21 \\
\hline _LH & 3,19 & {$[4,16]$} & $(0,03)$ & $-5,38$ & {$[-3,56]$} & $(0,04)$ & 2,59 & {$[3,41]$} & $(0,05)$ & 0,80 & 41 & $(0,00)$ & 14 \\
\hline _TYSS & 3,61 & {$[3,66]$} & $(0,04)$ & $-6,24$ & {$[-3,19]$} & $(0,06)$ & 2,93 & {$[3,07]$} & $(0,06)$ & 0,82 & 37 & $(0,00)$ & 12 \\
\hline METRO & 2,66 & {$[3,02]$} & $(0,06)$ & $-4,41$ & {$[-2,34]$} & $(0,09)$ & 2,08 & {$[2,21]$} & $(0,10)$ & 0,87 & 45 & $(0,00)$ & 10 \\
\hline _TUI & 2,85 & {$[3,08]$} & $(0,04)$ & $-4,61$ & {$[-2,57]$} & $(0,07)$ & 2,19 & {$[2,43]$} & $(0,07)$ & 0,82 & 34 & $(0,01)$ & 12 \\
\hline _LIN & 2,90 & {$[2,55]$} & $(0,07)$ & $-4,85$ & {$[-2,30]$} & $(0,09)$ & 2,24 & {$[2,10]$} & $(0,10)$ & 0,94 & 68 & $(0,00)$ & 7 \\
\hline _HEN & 2,69 & {$[2,05]$} & $(0,08)$ & $-4,54$ & {$[-1,68]$} & $(0,09)$ & 2,19 & {$[1,56]$} & $(0,11)$ & 0,94 & 59 & $(0,01)$ & 7 \\
\hline _CONT & 2,63 & {$[1,98]$} & $(0,06)$ & $-4,39$ & {$[-1,62]$} & $(0,08)$ & 1,97 & {$[1,52]$} & $(0,08)$ & 0,93 & 63 & $(0,00)$ & 8 \\
\hline DP & 3,38 & {$[2,73]$} & $(0,06)$ & $-5,94$ & {$[-2,43]$} & $(0,08)$ & 2,82 & {$[2,33]$} & $(0,08)$ & 0,85 & 32 & $(0,01)$ & 10 \\
\hline _DEG & 3,02 & {$[1,25]$} & $(0,10)$ & $-4,97$ & {$[-1,02]$} & $(0,13)$ & 2,19 & {$[0,89]$} & $(0,14)$ & 0,91 & 40 & $(0,01)$ & 8 \\
\hline
\end{tabular}

The table reports the median regression results and statistics for the daily smoothing of all those smiles which have ultimately been used for the construction of the variables with a constant maturity of 36 days.

t-stats in [ ] and corresponding p-values in ( )

Table 6.2.: Median regression results and statistics for the smoothing of the smiles which have been used for the construction of the 36 days constant maturity variables 
6.4.5.4.2. Interpretation of the Regression Results Figure 6.3 visualizes the regression results for the 6 most important stocks as prioritized in table 6.1. The smiles displayed are all calculated on the basis of options maturing in the next 61 to 90 days. Figure 6.3 juxtaposes for each stock the smile of a day with a particularly high CDS spread and that of a day with a particularly low CDS spread. Contemplating figure 6.3 , it becomes obvious that there exists a qualitative difference between the smile of a day with a high CDS spread and that of a day with a low CDS spread:

- First, the smile seems to be flatter on high CDS spread days compared to low CDS spread days. This visual impression is also reflected in the difference of the regression coefficients which are also displayed in figure 6.3. In all six cases the coefficient for the quadratic term is lower and that for the linear term is higher on high CDS spread days.

- Secondly, on high CDS spread days the smile appears to be translated to the right in the direction of high strike prices as compared to its position on low CDS spread days.

This observation calls for further systematic and quantitative analyses of the relationship between the smile pattern and the CDS spread level as will be undertaken in section 6.5 . 

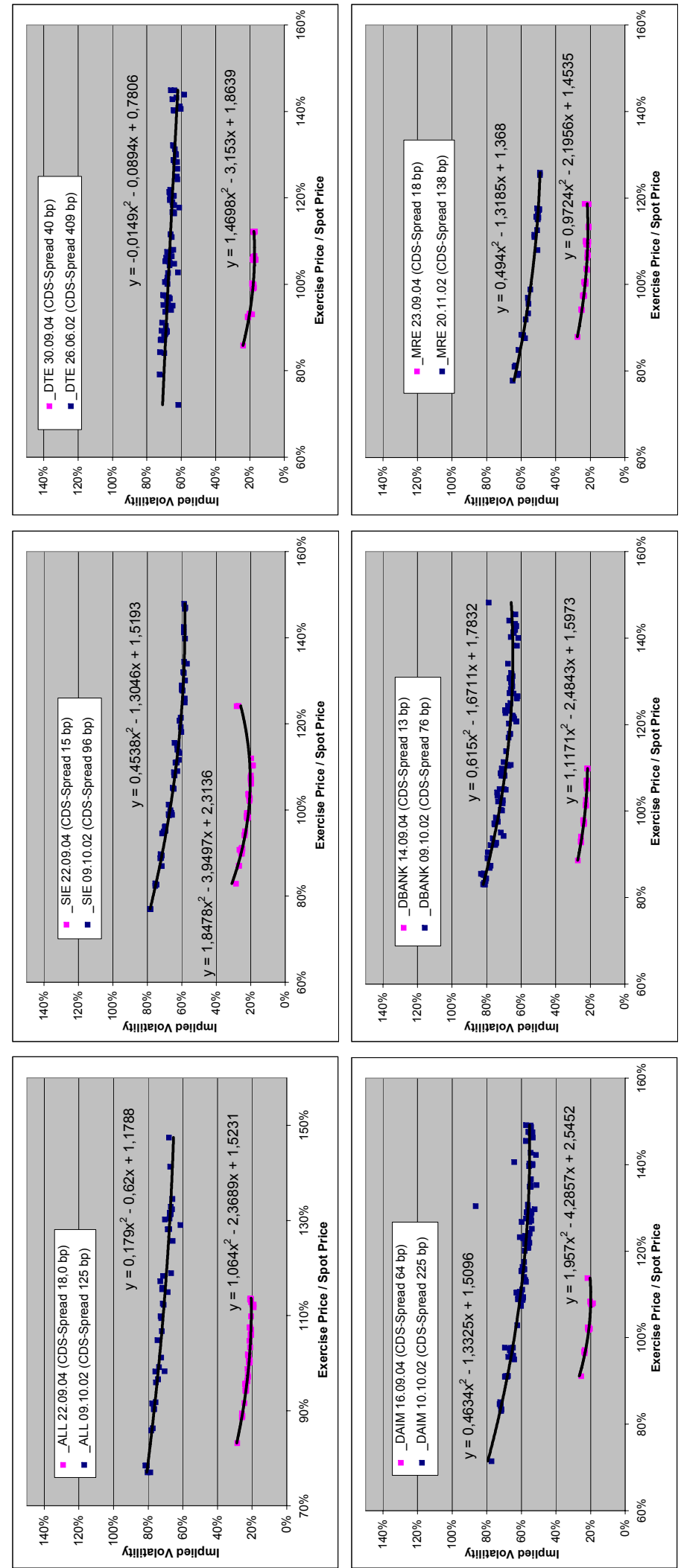

Figure 6.3.: Implied volatility smiles approximated with quadratic polynomials for a high CDS spread and a low CDS spread day for the 6 most important stocks 


\subsubsection{Computation of Risk-Neutral Skewness and Kurtosis}

\subsubsection{Qualitative Assessment of the Risk-Neutral Return Density}

The smile itself is a rather abstract concept as opposed to the risk-neutral density, which is intuitively more revealing. Following Shimko (see section 3.2.2.1), the smiles of high and low CDS spread days of the 6 most important stocks as displayed in figure 6.3 have been translated into corresponding risk-neutral densities.

However, the different volatility levels associated with high and low CDS spread days would make a visual and qualitative comparison rather difficult. Therefore deviating from SHIMKO (1993), the risk-neutral densities have been corrected for differing means and volatilities. $\mathrm{Be} \phi^{Q}(R)$ the density function of the risk-neutral log return $R=\ln \left(\frac{X}{S}\right)$ and $\tau$ the time to maturity, then for the visual comparison between high and low volatility days, the density should be evaluated at $\hat{R}$ instead of $R$ :

$$
\phi^{Q}(\hat{R})=\phi^{Q}\left(\frac{R-\mu \cdot \tau}{\sigma \cdot \sqrt{\tau}}\right)
$$

Since mean $\mu$ and volatility $\sigma$ are unknown, they have to be guessed. Given the density across the complete strike price or return spectrum, this could be done numerically. However, in our case a complete density stretching across the whole return spectrum is only available if the underlying smile is extrapolated beyond the range of observable option prices. But it is arguable, if such an extrapolation is admissible. Besides, this method would lead to numerical problems due to extreme values at the fringes of the extrapolated strike price range 322 Therefore another method has been chosen: The return volatility $\sigma$ has been proxied by the ATM implied volatility. And the mean $\mu$ of the risk-neutral return has been estimated as $\mu=r-\frac{\sigma^{2}}{2}$, assuming that the equity price is approximately lognormally distributed.

Figure 6.4 displays the standardized risk-neutral log return densities that have been derived from the smiles of figure 6.3 . Like the smile in figure 6.3 , the risk-neutral density also seems to exhibit a qualitative difference between high and low CDS spread days. It seems as if the risk-neutral density were more left-skewed and in 5 out of 6 cases also less high peaked on high CDS spread days. However, apart from a first hint at what to look for in the subsequent quantitative analysis, it is very difficult to reach any sound conclusions at this point. What is needed instead, are measures of the risk-neutral moments that can be systematically employed in a regression analysis.

322 SHIMKO (1993) solves this challenge by replacing the tails of the risk-neutral density with those of the normal distribution. 

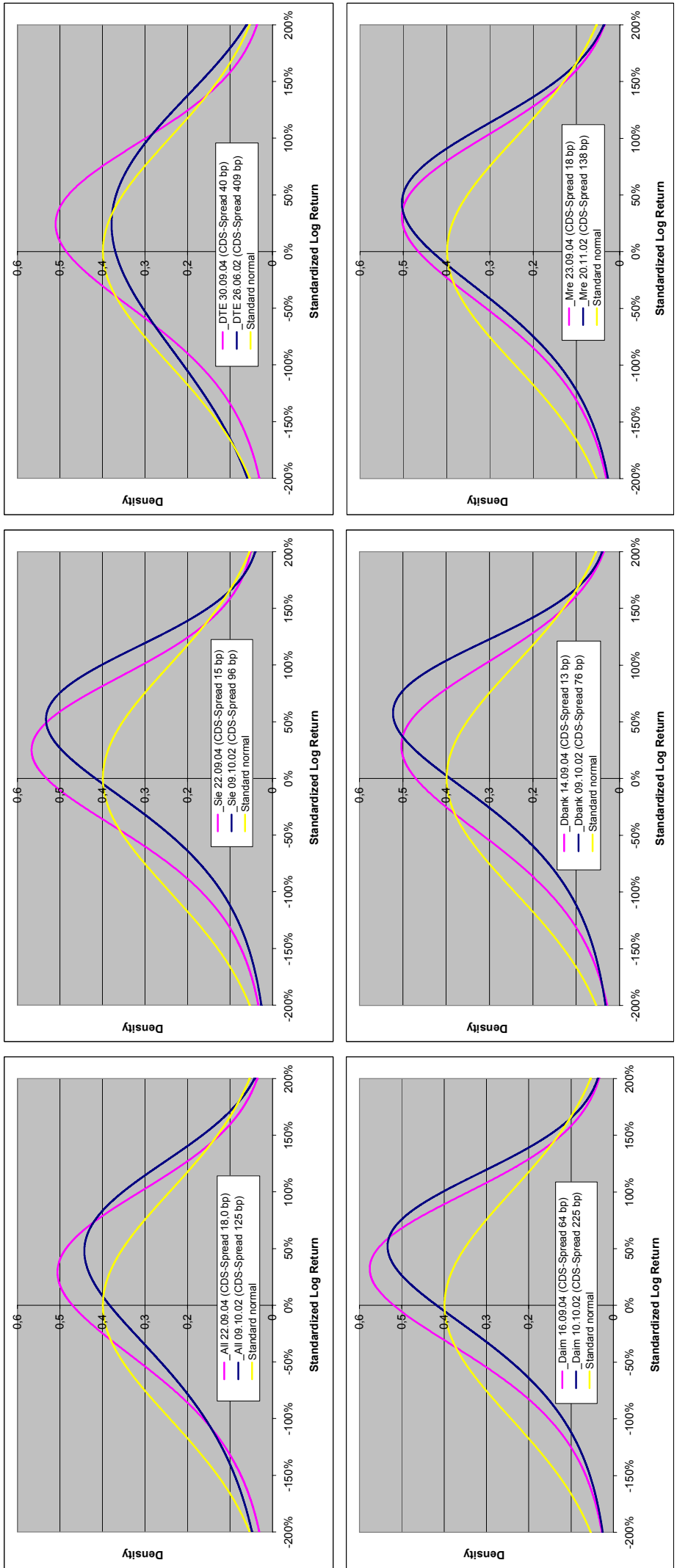

Figure 6.4.: Standardized risk-neutral log return densities for a high CDS spread and a low CDS spread day for the 6 most important stocks 


\subsubsection{Computation of the Moments of the Risk-Neutral Return Density}

\subsection{Practical Issues and Problems Concerning the Implementation} of the Bakshi-Madan Method Like in the case of the smile approximation, riskneutral moments have been computed for each available underlying, trading day and maturity combination. The implementation follows the method originally proposed by BAKSHI/MADAN (2000) and described in section 3.2.2.3. This approach represents a nonparametric technique to imply risk-neutral return skewness and kurtosis out of European option prices. The Bakshi-Madan method relies in theory on a continuum of European call and put option prices. But as has been seen in section 6.4.5.1 and can be verified in figure 6.1, in practice and especially for individual stock options, no such continuum exists. Besides, individual stock options are American and not European. In detail, the following 5 problems will be encountered when applying the Bakshi-Madan method to individual stock option prices:

1. First, strike prices are not continuous. Instead they are distributed across the strike price domain at discrete intervals ${ }^{323}$.

2. Secondly, option prices are irregularly spaced across the spectrum of strike prices. As can be seen in figure 6.1, the density of observations is highest around the ATM point and decreases in both directions.

3. Thirdly, as is obvious from figure 6.1, the range of strike prices is not symmetric around the ATM point 324 .

4. Fourthly, strike prices do not cover the whole domain of possible underlying prices. Therefore the resulting moments can never be identical with those of the complete distribution.

5. Implying risk-neutral moments out of American options following the BakshiMadan method, which has originally been designed for European options, can lead to distorted results, since American options contain a premium for early exercise.

How these problems have been addressed by a modification of the original BakshiMadan method is described in the next section.

323 DENNIS/MAYHEW (2002) apply the Bakshi-Madan method to extract risk-neutral skewness out of individual stock option prices and find a negative bias of the skewness measure on account of discretely spaced strike prices.

324 DENNIS/MAYHEW (2002) apply the Bakshi-Madan method to extract risk-neutral skewness out of individual stock option prices and find a negative bias of the skewness measure on account of an asymmetric domain of strike prices. 
6.4.6.2.2. Modification of the Bakshi-Madan Method In order to address the problems discussed above, the Bakshi-Madan method has been enhanced in the following 3 ways:

\section{Replacement of actual option prices with $\hat{\sigma}$-based continuum of approxi-} mated prices: To remedy the bias of discretely and irregularly spaced strike prices, actual option prices have been replaced with a virtual continuum of theoretical option prices. In 1.000 steps of $\frac{X}{S}$, theoretical Black-Scholes prices for calls and puts have been calculated on the basis of the estimated implied volatility function $\hat{\sigma}$. Besides the remedy of non-continuous strike prices, this procedure features yet 3 other advantages:

- Problem 5. of the previous section concerning the use of American instead of European option prices is completely remedied by the computation of hypothetical Black-Scholes prices, which are by definition European prices $\$ 25$.

- Instead of only using put contracts with strikes up to the current spot price $S$ and only calls with strikes above $S$, like prescribed by the Bakshi-Madan method, all available put and call prices have been used implicitly, since they have all contributed to the estimation of the implied volatility function $\hat{\sigma}$.

- The use of the implied volatility function $\hat{\sigma}$ has a smoothing effect which eliminates the noise present in actual option prices.

2. Symmetric standardized return interval: To remedy the effects of fluctuating strike price domains, the strike price range used for the computation of moments has been regularized. However, it has not been made symmetric as to fixed, limiting strike-to-spot-price ratios, since this would have resulted in a varying spectrum of standardized log returns $\tilde{R}$ depending on the prevailing level of volatility. Instead, $\tilde{R}$ has been symmetrically bounded in order to enable comparisons between differing levels of volatility. Two issues have to be considered when fixing the bounds for the standardized return $\tilde{R}$ :

325 As pointed out in section 3.1.3.1 it is admissible to infer the risk-neutral density out of American option prices, if these have been transformed into European prices before. This transformation is based on first implying volatilities out of American option prices using a method which allows for path dependency and on then computing corresponding European prices with these volatilities. Exactly this avenue has been taken here: As the reader might remember, the implied volatility function $\hat{\sigma}$ has been estimated using volatilities which have been implied by means of a CoxRoss-Rubinstein binomial tree, thus properly allowing for early exercise. 
- The objective of the computation of risk-neutral moments consists in capturing the differences in the risk-neutral densities between high and low CDS spread days. An interval that is too small might not be able to distinguish between different levels of skewness and kurtosis as they have been detected above (see section 6.4.6.1).

- The return interval should be representative of the actual spectrum of price observations. An interval that is too wide might extend the strike price range too far beyond actually observed option prices.

Weighting these two issues, the bounds for the standardized return $\tilde{R}$ have been set to $\pm 100 \%$. Reorganizating equation 6.29 leads to corresponding, volatility dependent lower $(\mathrm{d})$ and upper $(\mathrm{u})$ bound for the strike-to-spot-price ratio $\frac{X}{S}$ :

$$
\left(\frac{X}{S}\right)_{d / u}=e^{\mp 1 \cdot \sigma^{2} \cdot \tau+\left(r-\frac{\sigma^{2}}{2}\right) \cdot \tau}
$$

Contemplating figure 6.4, an interval of $\pm 100 \%$ for the standardized return $\tilde{R}$ seems to be adequate to capture the relevant features of the risk-neutral density. On the other hand, an interval of this size also appears to be well supported by actually observable option prices as indicated by table 6.3 . Table 6.3 shows the average minimum and maximum strike-to-spot-price ratios $\frac{X}{S}$ as well as the average corresponding minimum and maximum standardized returns $\tilde{R}$ that would have resulted, if the domain of actual option prices had not been limited to a standardized return of $\pm 100 \%$. At least for the most important stocks, the fixed interval of $\pm 100 \%$ lies well inside the average actual interval delimitations.

3. Bias control with the computed moments of the normal distribution: The bias not yet addressed is that of the strike prices not covering the whole domain of possible underlying prices. This bias has been alleviated by parallel computation of the Bakshi-Madan skewness and kurtosis measures for a hypothetical underlying with normally distributed returns. This hypothetical underlying has been created by calculating Black-Scholes put and call prices not with the strike dependent implied volatility function $\hat{\sigma}\left(\frac{X}{S}\right)$, but instead with the constant ATM implied volatility $\hat{\sigma}_{A T M}$. Since skewness and kurtosis of the normal distribution are known to be 0 and 3, respectively, the bias resulting from limiting the domain of $\frac{X}{S}$ can be determined. Be $S K E W_{r}$ and $K U R T_{r}$ the actually calculated moments based on the approximated volatility smile, be further $S K E W_{n}$ and 


\begin{tabular}{|c|c|c|c|c|}
\hline ID* & Average $\mathrm{X} / \mathrm{S}_{\text {min }}$ & Average $X / S_{\text {max }}$ & Average $\hat{R}_{\min }$ & Average $\hat{R}_{\max }$ \\
\hline DAX & $66 \%$ & $143 \%$ & $-394 \%$ & $254 \%$ \\
\hline _ALL & $85 \%$ & $138 \%$ & $-119 \%$ & $144 \%$ \\
\hline _SIE & $82 \%$ & $133 \%$ & $-148 \%$ & $140 \%$ \\
\hline DTE & $87 \%$ & $136 \%$ & $-117 \%$ & $144 \%$ \\
\hline _DAIM & $86 \%$ & $128 \%$ & $-122 \%$ & $125 \%$ \\
\hline _DBANK & $85 \%$ & $129 \%$ & $-150 \%$ & $135 \%$ \\
\hline _MRE & $87 \%$ & $131 \%$ & $-89 \%$ & $138 \%$ \\
\hline _BAY & $89 \%$ & $123 \%$ & $-87 \%$ & $107 \%$ \\
\hline -VW & $90 \%$ & $120 \%$ & $-91 \%$ & $112 \%$ \\
\hline _BASF & $90 \%$ & $112 \%$ & $-119 \%$ & $84 \%$ \\
\hline EON & $92 \%$ & $113 \%$ & $-86 \%$ & $86 \%$ \\
\hline _COB & $91 \%$ & $126 \%$ & $-69 \%$ & $108 \%$ \\
\hline _HYPV & $94 \%$ & $124 \%$ & $-41 \%$ & $101 \%$ \\
\hline _RWE & $92 \%$ & $112 \%$ & $-89 \%$ & $82 \%$ \\
\hline _BMW & $92 \%$ & $112 \%$ & $-71 \%$ & $87 \%$ \\
\hline _LH & $95 \%$ & $116 \%$ & $-36 \%$ & $89 \%$ \\
\hline _TYSS & $94 \%$ & $113 \%$ & $-54 \%$ & $75 \%$ \\
\hline _METRO & $94 \%$ & $109 \%$ & $-70 \%$ & $57 \%$ \\
\hline _TUI & $92 \%$ & $109 \%$ & $-54 \%$ & $66 \%$ \\
\hline _LIN & $96 \%$ & $105 \%$ & $-44 \%$ & $41 \%$ \\
\hline _HEN & $96 \%$ & $104 \%$ & $-110 \%$ & $28 \%$ \\
\hline _CONT & $96 \%$ & $103 \%$ & $-54 \%$ & $24 \%$ \\
\hline _DP & $98 \%$ & $106 \%$ & $-48 \%$ & $68 \%$ \\
\hline _DEG & $98 \%$ & $99 \%$ & $-5 \%$ & $10 \%$ \\
\hline
\end{tabular}

*Avererages are taken across all approximated smiles of the respective cross section.

Table 6.3.: Average minimum and maximum $\frac{X}{S}$ and corresponding $\tilde{R}$ resulting from actual option prices

$K U R T_{n}$ the corresponding moments calculated assuming that the underlying follows a lognormal distribution, then the bias corrected moments $S K E W$ and $K U R T$ ensue according to the following equations:

$$
\begin{gathered}
S K E W=S K E W_{r}-S K E W_{n} \\
K U R T=K U R T_{r}-\left(K U R T_{n}-3\right)
\end{gathered}
$$

The implementation of the modified Bakshi-Madan method can be verified in appendix A.3. 


\subsubsection{The Variables in the Regression Analysis}

\subsubsection{Generation of Constant Maturity Smile, Skewness and Kurtosis}

To facilitate time-series comparisons, constant maturity variables have been calculated. The variables concerned are the smile coefficients $B 0, B 1$ and $B 2$, the ATM implied volatility $I M P V$ and the risk-neutral skewness and kurtosis, $S K E W$ and $K U R T$, respectively.

The constant maturity variables have been generated for the standard maturities of 36 , 60, 180 and 240 days by interpolating linearly between the next lower and the next higher maturity. 36 days is the shortest constant maturity possible, since the shortest at the Eurex exchange traded option contract is the $1 \mathrm{M}$ contract with an initial term of 30 days ${ }^{326}$ and since the lowest maturity for which a smile has been approximated is 7 days (see section 6.4.5.3 327 As highest constant maturity, the term of 240 days has been chosen, since for higher terms the smile flattens substantially and thus ceases to be fertile for the purposes of the subsequent analysis.

\subsubsection{Synopsis of the Variables}

The variables generated for the subsequent regression analysis are summarized and explained in table 6.4. They can be coarsely classified into smile dependent and smile independent variables. The former are ideally available for all 4 constant maturities. Further, depending on the context throughout the analysis all variables possibly carry an ID subscript which can be either the stock or the index ID. They possibly also carry a time subscript, since ideally they are not only available for almost all IDs, but also for all trading days during the 3 year period from January, $2^{\text {nd }} 2002$ through to December, $28^{\text {th }}$ 2004. In practice however, for some stocks the variables are not disposable for all trading days. This is either due to low option trading activity or lacking CDS quotes. The number of days for which at least one set of smile based variables as well as a CDS spread is disposable can be read from table 6.1. It follows a brief description of the variables that have not yet been explained in detail:

326 During the period covered by the analysis, the $1 \mathrm{M}$ contract was the one with the shortest initial term. Nowadays, shorter maturities are available.

327 The constant maturity variables for the lowest possible constant maturity of 36 days are first interpolated between the variables with a maturity of 7 days and those with a maturity of 37 days. The next day they are interpolated between the variables with a maturity of 8 days and those with a maturity of 38 days, and so on. 
- Smile slope variables: The slope variables SLP70, SLP90, SLP100, SLP110 and $S L P 130$ represent the slope of the smile function $\hat{\sigma}$ at different levels of moneyness $\frac{X}{S}$ :

$$
S L P X=\left.\frac{\partial \hat{\sigma}}{\partial\left(\frac{X}{S}\right)}\right|_{\left(\frac{X}{S}\right)=X \%}=2 \cdot B 2 \cdot X \%+B 1
$$

- PRC: The underlying price $P R C$ of a certain day equals the price of the first trade after $13 \mathrm{~h} 00$ of that day.

- XSMIN: The position of the smile's valley in terms of $\frac{X}{S}$ ensues according to the following expression:

$$
\begin{gathered}
\frac{\partial \hat{\sigma}}{\partial\left(\frac{X}{S}\right)} \stackrel{!}{=} 0 \\
\Rightarrow \frac{X}{S}=-\frac{B 1}{2 \cdot B 2}
\end{gathered}
$$

- IMPV: To obtain the ATM implied volatility, the implied volatility function $\hat{\sigma}$ has to be evaluated at a moneyness of $\frac{X}{S}=100 \%$ :

$$
I M P V=\hat{\sigma}\left(\frac{X}{S}=100 \%\right)=B 0+B 1+B 2
$$

- CDS: The CDS spread in basis points. 


\begin{tabular}{c|c|c|c} 
Name & Explanation & $\begin{array}{c}\text { Available for } \\
\text { each constant } \\
\text { maturity* }\end{array}$ & $\begin{array}{c}\text { Available for } \\
\text { the Dax } \\
\text { Index }\end{array}$ \\
\hline Smile dependant variables
\end{tabular}

\section{Smile dependant variables}

\begin{tabular}{l|l|l} 
B0 & Coefficient of the smile function & Yes
\end{tabular}

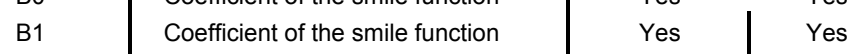

\begin{tabular}{l|l|l} 
B2 & Coefficient of the smile function & Yes
\end{tabular}

\begin{tabular}{l|l|l} 
SLP70 & Smile slope at $X / S=70 \%$ & Yes
\end{tabular}

\begin{tabular}{l|l|l} 
SLP90 & Smile slope at $X / S=90 \%$ & Yes
\end{tabular}

\begin{tabular}{l|l|l} 
SLP100 Smile slope at $X / S=100 \%$ & Yes & Yes
\end{tabular}

\begin{tabular}{l|l|l} 
SLP110 Smile slope at $X / S=110 \%$ & Yes & Yes
\end{tabular}

\begin{tabular}{l|l|l|l} 
SLP130 & smile slope at $X / S=130 \%$ & Yes & Yes
\end{tabular}

\begin{tabular}{l|l|l} 
XSMIN & $\mathrm{X} / \mathrm{S}$ where the smile has its bottom & Yes
\end{tabular}

\begin{tabular}{l|l|l} 
IMPV & ATM implied volatility & Yes
\end{tabular}

\begin{tabular}{l|l|l} 
SKEW & Risk-neutral skewness measure & Yes
\end{tabular}

\begin{tabular}{c|l|l|l} 
KURT & Risk-neutral kurtosis measure & Yes & Yes \\
\hline
\end{tabular}

Smile independant variables

\begin{tabular}{l|l|c|c} 
PRC & Price & - & Yes \\
HV & Historical volatility & - & - \\
HSKEW & Historical skewness measure & - & - \\
HKURT & Historical kurtosis measure & - & - \\
CDS & CDS spread or CDS spread index & - & -
\end{tabular}

CDS

CDS spread or CDS spread index

${ }^{*}$ Available for a constant maturity of $36,60,180$ and 240 days

Table 6.4.: Variables in the regression analysis 


\subsubsection{Summary Statistics of the Variables}

6.4.7.3.1. Summary Statistics of All Variables The overall summary statistics of the variables can be read from table 6.5, which subsumes all stock crosssections and all constant maturities. The DAX index is the only cross-sections which is reported separately. As can be seen, table 6.5 is segmented into smile dependent and smile independent variables with the smile dependent variable section not only merging all stock cross-sections, but also all 4 constant maturities. The summary statistics of the smile dependent variables on an individual constant maturity basis, but pooling all stock cross-sections can be viewed in appendix B.2. Whereas the summary statistics of all variables on an individual constant maturity as well as on an individual cross-section basis can be consulted in appendix B.3.

\begin{tabular}{|c|c|c|c|c|c|c|}
\hline & Mean & Median & Max & Min & StdDev & Obs \\
\hline \multicolumn{7}{|c|}{ Smile dependent variables (all constant maturities) } \\
\hline $\mathrm{BO}_{\text {Stocks }}$ & 7,91 & 1,39 & $45.714,21$ & $-24.556,99$ & 609,45 & 54.896 \\
\hline B1 $1_{\text {Stocks }}$ & $-12,68$ & $-1,77$ & $92.971,62$ & $-95.651,84$ & $1.361,33$ & 54.896 \\
\hline B2 Stocks $_{1}$ & 3,95 & 0,75 & $50.035,39$ & $-87.993,11$ & 883,73 & 54.896 \\
\hline $\mathrm{BO}_{\mathrm{Dax}}$ & 1,03 & 1,09 & 46,50 & $-944,23$ & 18,08 & 54.896 \\
\hline B1 $1_{\text {Dax }}$ & $-1,14$ & $-1,18$ & $1.985,48$ & $-90,22$ & 38,00 & 54.896 \\
\hline B2 & 0,39 & 0,43 & 44,04 & $-1.043,45$ & 19,97 & 54.896 \\
\hline SLP70 $_{\text {Stocks }}$ & $-7,15$ & $-0,72$ & $15.379,99$ & $-30.218,73$ & 410,86 & 54.896 \\
\hline SLP90 ${ }_{\text {Stocks }}$ & $-5,57$ & $-0,41$ & $6.627,78$ & $-65.415,98$ & 499,85 & 54.896 \\
\hline SLP100 ${ }_{\text {Stocks }}$ & $-4,78$ & $-0,24$ & $4.418,95$ & $-83.014,60$ & 619,75 & 54.896 \\
\hline SLP110 ${ }_{\text {Stocks }}$ & $-3,99$ & $-0,10$ & $14.426,03$ & $-100.613,20$ & 762,11 & 54.896 \\
\hline SLP130 ${ }_{\text {Stocks }}$ & $-2,41$ & 0,18 & $34.440,18$ & $-135.810,50$ & $1.077,81$ & 54.896 \\
\hline SLP70 & $-0,60$ & $-0,58$ & 524,65 & $-28,56$ & 10,05 & 54.896 \\
\hline $\mathrm{SLP}_{\text {Dax }}$ & $-0,45$ & $-0,40$ & 107,27 & $-18,26$ & 2,10 & 54.896 \\
\hline SLP100 Dax & $-0,37$ & $-0,31$ & 4,19 & $-101,42$ & 1,99 & 54.896 \\
\hline SLP110 Dax & $-0,29$ & $-0,20$ & 6,91 & $-310,11$ & 5,95 & 54.896 \\
\hline SLP130 & $-0,14$ & $-0,05$ & 24,29 & $-727,49$ & 13,92 & 54.896 \\
\hline XSMIN $_{\text {Stocks }}$ & 0,43 & 1,12 & 447,81 & $-41.090,33$ & 175,48 & 54.896 \\
\hline IMPV $_{\text {Stocks }}$ & $-0,82$ & 0,30 & 469,02 & $-19.578,48$ & 145,57 & 54.896 \\
\hline IMPV $_{\text {Dax }}$ & 0,27 & 0,24 & 0,60 & $-4,29$ & 0,14 & 54.896 \\
\hline SKEW $_{\text {Stocks }}$ & $-0,16$ & $-0,17$ & 5,05 & $-20,10$ & 0,41 & 54.896 \\
\hline $\mathrm{KURT}_{\text {Stocks }}$ & 3,02 & 3,02 & 19,06 & 0,02 & 0,37 & 54.896 \\
\hline SKEW $_{\text {Dax }}$ & $-0,24$ & $-0,24$ & 0,13 & $-0,38$ & 0,05 & 54.896 \\
\hline KURT $_{\text {Dax }}$ & 3,02 & 3,03 & 3,12 & $-27,12$ & 0,65 & 54.896 \\
\hline \multicolumn{7}{|c|}{ Smile independent variables } \\
\hline PRC $_{\text {Stocks }}$ & 41,15 & 35,94 & 291,80 & 5,13 & 33,46 & 13.724 \\
\hline PRC $_{\text {Dax }}$ & $3.744,54$ & $3.815,37$ & $5.449,80$ & $2.247,49$ & 664,22 & 13.724 \\
\hline $\mathrm{HV}_{\text {Stocks }}$ & 0,38 & 0,33 & 2,35 & 0,09 & 0,19 & 13.724 \\
\hline HSKEW $_{\text {Stocks }}$ & $-0,06$ & $-0,03$ & 12,16 & $-12,56$ & 1,52 & 13.724 \\
\hline HKURT $_{\text {Stocks }}$ & 7,37 & 3,27 & 178,15 & $-0,71$ & 13,28 & 13.724 \\
\hline CDS $_{\text {Stocks }}$ & 64,28 & 44,50 & 408,50 & 10,83 & 55,64 & 13.724 \\
\hline $\mathrm{CDS}_{\mathrm{Dax}}$ & 80,44 & 65,18 & 203,12 & 34,05 & 39,37 & 13.724 \\
\hline
\end{tabular}

Table 6.5.: Summary statistics of all cross-sections and all constant maturities pooled together 


\subsection{Summary Statistics of Specific Variables Deserving a Closer}

Examination The summary statistics of some variables deserve a closer look, since they will still play an important role in our later analysis.

To begin with, table 6.6 reports the median smile coefficients $B 0, B 1$ and $B 2$. Table 6.6 is divided into an upper portion for all stock cross-sections together and a lower portion for the DAX index only. Naturally, the intercept of the smile polynomial $B 0$ is positive for all constant maturities. The linear term $B 1$ is always negative and the quadratic term $B 2$ is positive in all cases, as should be expected for a U-shaped smile curve. What can also be seen is that the absolute values of all coefficients are declining with increasing constant maturity, which is symptomatic of the flattening of the smile. Comparing the individual stock cross-sections with the DAX index, it stands out that the coefficients are smaller in absolute value for the DAX index than for the individual stock cross-sections. We will now examine what this means in terms of the smile slope and the risk-neutral moments. The median slope variables

\begin{tabular}{c|l|l|l}
$\begin{array}{c}\text { Constant } \\
\text { maturity }\end{array}$ & B0 & B1 & B2 \\
\hline $\begin{array}{r}\text { Stocks } \\
36\end{array}$ & 2,68 & $-4,38$ & 2,03 \\
60 & 1,67 & $-2,37$ & 1,05 \\
180 & 0,92 & $-0,94$ & 0,36 \\
240 & 0,83 & $-0,78$ & 0,30 \\
\hline Dax & & & \\
36 & 2,29 & $-3,67$ & 1,62 \\
60 & 1,46 & $-2,05$ & 0,84 \\
180 & 0,83 & $-0,84$ & 0,29 \\
240 & 0,73 & $-0,68$ & 0,22
\end{tabular}

Table 6.6.: Median smile coefficients of all stock cross-sections and of the DAX index

SLP70, SlP90, SLP100, SLP110 and SLP130 as well as the horizontal coordinate $X S M I N$ for the individual stock cross-sections as well as the DAX index are shown in table 6.7. Let us first consider the case of the individual stock cross-sections:

The slope of the left wing of the smile, represented by SLP70 and SLP90, is negative in all 4 constant maturity cases, which should be expected in the case of a U-shaped smile pattern. The slope at the ATM point, $S L P 100$ is also always negative. Further, the slope of the right wing of the smile, represented by SLP110 and SLP130 is positive for a constant maturity of 36 days, as is also consistent with a U-shaped smile pattern. However, with increasing maturity the sign of the slope is changing, first for $S L P 110$ and finally at a constant maturity of 240 days also for the rightmost section of the smile, represented by $S L P 130$. This change of signs is characteristic of the 
transformation of the U-shaped smile into a downward sloping skew with increasing maturity.

As to the DAX index, the picture is similar, but with two important exceptions: First, the middle, ATM portion of the smile seems to be steeper in the case of the index as compared to the case of the individual stocks. This finding is consistent with other studies ${ }^{328}$. However, at the far left wing the index smile seems to be flatter than the individual smile, at least in the constant maturity cases of 36 and 60 days. Secondly, the feature of an upwards sloping right wing of the smile seems to be less accentuated in the case of the index as compared to the individual smile. Finally, the summary

\begin{tabular}{|c|c|c|c|c|c|c|}
\hline $\begin{array}{l}\text { Constant } \\
\text { maturity }\end{array}$ & SLP70 & SLP90 & SLP100 & SLP110 & SLP130 & XSMIN \\
\hline \multicolumn{7}{|l|}{ Stocks } \\
\hline 36 & $-1,54$ & $-0,73$ & $-0,31$ & 0,11 & 0,92 & 1,06 \\
\hline 60 & $-0,91$ & $-0,50$ & $-0,28$ & $-0,07$ & 0,35 & 1,10 \\
\hline 180 & $-0,43$ & $-0,28$ & $-0,21$ & $-0,13$ & 0,02 & 1,20 \\
\hline 240 & $-0,37$ & $-0,25$ & $-0,19$ & $-0,13$ & $-0,01$ & 1,20 \\
\hline \multicolumn{7}{|l|}{ Dax } \\
\hline 36 & $-1,40$ & $-0,75$ & $-0,41$ & $-0,10$ & 0,55 & - \\
\hline 60 & $-0,87$ & $-0,54$ & $-0,37$ & $-0,21$ & 0,12 & - \\
\hline 180 & $-0,44$ & $-0,32$ & $-0,27$ & $-0,21$ & $-0,10$ & - \\
\hline 240 & $-0,37$ & $-0,28$ & $-0,24$ & $-0,20$ & $-0,11$ & - \\
\hline
\end{tabular}

Table 6.7.: Median smile slopes of all stock cross-sections and the DAX index

statistics of the risk-neutral moments are worth being discussed briefly. As can be viewed in table 6.8, the risk-neutral density is always left-skewed and leptokurtic for individual stocks as well as for the index. This finding is consistent with other studies ${ }^{329}$. Further, it can be gathered from table 6.8 that left-skewness and excess kurtosis are more accentuated in the case of the index than in the case of individual stocks. The finding of a more accentuated left-skewness in the case of the index is in line with the corresponding literature ${ }^{330}$, whereas that of its more pronounced excess kurtosis conflicts with other finding 331 . Moreover, the physical left-skewness of individual stocks seems to be less pronounced than the risk-neutral left-skewness, but excess kurtosis

328 BAKSHI ET AL. (2003) for instance, focusing on the ATM point detect a more negative slope of the S\&P index smile as compared to the smile of its constituents. BRANGER/SCHLAG (2004) confirm this finding of a generally steeper index smile and try to explain the slope difference with the dependence structure among the index constituent stocks.

329 DENNIS/MAYHEW (2002) and CORRADO/SU (1997b) report risk-neutral left-skewness for individual stocks, in doing so CORRADO/SU/(1997b) also give account of risk-neutral excess kurtosis for individual stocks. As far as the index is concerned, JACKWERTH/RUBINSTEIN (1996), AITSAHALIA/LO (1998), CORRADO/SU (1997a) and NAVATTE/VILLA (2000) find corroborating evidence of risk-neutral left-skewness and excess kurtosis.

330 See BAKSHI ET AL. (2003), e.g..

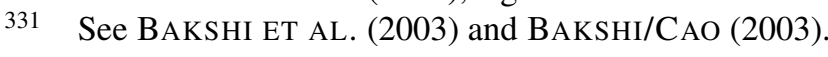


appears to be more distinct in the physical than in the risk-neutral case. Literature on the comparison of risk-neutral and physical moments is lacking in the case of individual stocks 332 , but the research conducted on stock indexes suggests a similar pattern as I have found for individual stocks 333 .

Interestingly, the left-skewness seems to increase with increasing option maturity, both for individual stocks and the index. Whereas increasing option maturity appears to lead to slightly decreasing excess kurtosis for individual stocks and to increasing excess kurtosis for the index 334 .

\begin{tabular}{c|c|c|c|c}
$\begin{array}{c}\text { Constant } \\
\text { maturity }\end{array}$ & SKEW & KURT & HSKEW & HKURT \\
\hline Stocks & & & \\
36 & $-0,141$ & 3,023 & & \\
60 & $-0,163$ & 3,022 & $-0,028$ & 3,266 \\
180 & $-0,193$ & 3,022 & & \\
240 & $-0,195$ & 3,021 & & \\
\hline Dax & & & & \\
36 & $-0,185$ & 3,027 & & \\
60 & $-0,219$ & 3,033 & & \\
180 & $-0,264$ & 3,052 & \\
240 & $-0,274$ & 3,060 &
\end{tabular}

Table 6.8.: Median smile risk-neutral moments of all stock cross-sections and the DAX index

$332 \quad$ MACHADO-SANTOS/FERNANDES $(2005)$ is one rare study in this context, focusing only on individual physical skewness. Contrary to my findings, MACHADO-SANTOS/FERNANDES (2005) report largely positive physical skewness of the leading Portuguese stocks.

333 See JACKWERTH (2004), e.g..

334 For the index, increasing left-skewness and increasing excess kurtosis due to increasing option maturity has also been observed by AIT-SAHALIA/LO (1998). 


\subsubsection{Assessment of the Stationarity of the Variables}

As has been pointed out in section 6.3.4, stationarity of time-series is a prerequisite of a regression analysis. Therefore the degree of stationarity of the potential regression variables has been assessed with single series ADF tests, allowing for a time trend in the test equation 335 . A time trend has been included into the test specification, since visual inspection of all series suggests the possibility of a slight time trend for most of them. Table 6.9 shows the one-sided MACKINNON (1996) probabilities of error ${ }^{\sqrt{36}}$ rounded to the next $\frac{1}{10}$ for the rejection of the null hypothesis of a unit root. Table 6.9 only displays the 19 most important underlyings, since for the remainder the data is insufficient to carry out meaningful unit root tests. Following MADDALA/KIM (2000) in that the poor power of single series unit root tests should be countered with significance levels as high as $25 \% 33$, the presence of a unit root can be rejected in most cases. Only for the variables $P R C$ and $C D S$ and partly also for $I M P V$, the null hypothesis of a unit root cannot be rejected. This does not mean however, that a unit root must in any case be present. It only signifies that there exists a high probability that a rejection of a unit root is a wrong decision.

For $P R C$ a result suggesting non-stationarity must be expected, since asset prices are assumed to follow a random walk under the efficient market hypothesis (Cf. section 6.3.4.

Compared to $P R C$, the evidence for non-stationarity of $C D S$ is somewhat weaker. This also makes sense, considering the hybrid nature of interest rates and also credit spreads as a mixture of prices and returns: On the one hand, it is assumed that bond prices follow a random walk, which implies a stochastic trend. As a consequence, yields or interest rates have to be interpreted as returns and as a differenced price series and therefore should be stationary. On the other hand it is likewise practice to suppose that interest rates follow a random walk and are therefore non-stationary 338 .

Finally, compared to $P R C$ and $C D S$, evidence for non-stationarity of $I M P V$ is the weakest, but surprisingly stronger than for $H V$. Regarding the possible non-

335 The number of lagged terms in the test equation has been set automatically such that all serial correlation in the residuals has been removed, using a Schwarz information criterion, cf. Evi (2005), pp. 521 and 527. This setting resulted in the number of lags varying between 0 and 9.

336 The coefficients of the lagged variables in the ADF test do not follow the conventional tdistribution. Therefore, Evi (2005) calculates p-values following the approach of MACKINNON (1996) (see Evi (2005) pp. 520-522).

337 See MADDALA/KIM (2000), p. 146 and cf. section 6.3.4.2.2

338 According to HULL (2006) it is inconsistent that the Black option pricing model, assuming a geometric Brownian motion for the underlying, is likewise employed for the pricing of interest rate caps and bond options (see p. 630-631). 
stationarity of $I M P V$, it can equally be argued in both directions: On the one hand equity volatility is often assumed stochastic and is also modeled as such (Cf. section 4.2.3); further financial market volatility is often considered to be persistent 339 . Both assumptions speak in favor of a stochastic trend. On the other hand volatility also exhibits mean reversion, which in turn should lead to a certain degree of stationarity.

For the sake of the subsequent analysis, it will be supposed that all series except $P R C$, but including $C D S$ and $I M P V$ are stationary. In the light of rather weak evidence for a possible non-stationarity of $C D S$ and $I M P V$ and due to the fact that in both cases it can be argued in either direction, stationarity will be explicitly assumed for those two series. Where deemed necessary to be on the safe side, this view will be challenged throughout the analysis by the application of cointegration and panel unit root tests.

339 Cf. ANDERSEN ET AL. (2001) or BOLLERSLEV/MiKKELSEN (1996), e.g.. 


\begin{tabular}{|c|c|c|c|c|c|c|c|c|c|c|c|c|c|c|c|c|c|c|c|}
\hline & $\underset{1}{x}$ & $\vec{\psi}_{1}$ & $\frac{w^{\prime}}{\omega_{1}}$ & ${\stackrel{w}{\sigma_{1}}}$ & $\underset{⿱ 亠 幺}{\Sigma_{1}}$ & $\begin{array}{l}\frac{y}{z} \\
\frac{1}{\alpha} \\
\text { â, }\end{array}$ & 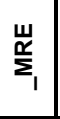 & 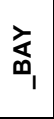 & $\xi_{1}$ & $\begin{array}{l}\frac{u}{0} \\
\frac{1}{\Phi_{1}}\end{array}$ & zo $_{\text {u }}$ & ö & $\sum_{x_{1}}^{a}$ & $\sum_{\mathbb{x}_{1}}^{w_{1}}$ & $\sum_{\infty_{1}}^{3}$ & $I_{1}$ & $\stackrel{\mathscr{D}}{\mathscr{L}}_{1}$ & 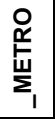 & $\bar{F}_{1}$ \\
\hline \multicolumn{20}{|c|}{ Smile independent variables } \\
\hline PRC & 0,8 & 0,6 & 0,4 & 0,1 & 0,5 & 0,5 & 0,5 & 0,8 & 0,3 & 0,6 & 0,8 & 0,5 & 0,9 & 0,6 & 0,4 & 0,5 & 0,7 & 0,1 & 0,7 \\
\hline HV & & 0,0 & 0,0 & 0,0 & 0,0 & 0,0 & 0,0 & 0,0 & 0,0 & 0,0 & 0,1 & 0,0 & 0,3 & 0,0 & 0,0 & 0,0 & 0,0 & 0,0 & 0,0 \\
\hline HSKEW & & 0,0 & 0,0 & 0,0 & 0,0 & 0,0 & 0,0 & 0,0 & 0,0 & 0,0 & 0,0 & 0,0 & 0,0 & 0,0 & 0,0 & 0,0 & 0,0 & 0,0 & 0,0 \\
\hline HKURT & & 0,0 & 0,0 & 0,0 & 0,0 & 0,0 & 0,0 & 0,0 & 0,0 & 0,0 & 0,0 & 0,0 & 0,0 & 0,0 & 0,0 & 0,0 & 0,0 & 0,0 & 0,0 \\
\hline CDS & 0,4 & 0,3 & 0,1 & 0,1 & 0,0 & 0,3 & 0,0 & 0,3 & 0,3 & 0,4 & 0,6 & 0,5 & 0,7 & 0,3 & 0,1 & 0,7 & 0,9 & 0,6 & 0,9 \\
\hline \multicolumn{20}{|c|}{ Smile dependent variables - Constant maturity of 36 days } \\
\hline B0 & 0,0 & 0,0 & 0,0 & 0,0 & 0,0 & 0,0 & 0,0 & 0,0 & 0,0 & 0,0 & 0,0 & 0,0 & 0,0 & 0,0 & 0,0 & 0,0 & 0,0 & 0,0 & 0,0 \\
\hline B1 & 0,0 & 0,0 & 0,0 & 0,0 & 0,0 & 0,0 & 0,0 & 0,0 & 0,0 & 0,0 & 0,0 & 0,0 & 0,0 & 0,0 & 0,0 & 0,0 & 0,0 & 0,0 & 0,0 \\
\hline B2 & 0,0 & 0,0 & 0,0 & 0,0 & 0,0 & 0,0 & 0,0 & 0,0 & 0,0 & 0,0 & 0,0 & 0,0 & 0,0 & 0,0 & 0,0 & 0,0 & 0,0 & 0,0 & 0,0 \\
\hline SLP70 & 0,0 & 0,0 & 0,0 & 0,0 & 0,0 & 0,0 & 0,0 & 0,0 & 0,0 & 0,0 & 0,0 & 0,0 & 0,0 & 0,0 & 0,0 & 0,0 & 0,0 & 0,0 & 0,0 \\
\hline SLP90 & 0,0 & 0,0 & 0,0 & 0,0 & 0,0 & 0,0 & 0,0 & 0,0 & 0,0 & 0,0 & 0,0 & 0,0 & 0,0 & 0,0 & 0,0 & 0,0 & 0,0 & 0,0 & 0,0 \\
\hline SLP100 & 0,1 & 0,0 & 0,1 & 0,1 & 0,3 & 0,0 & 0,1 & 0,0 & 0,0 & 0,0 & 0,0 & 0,0 & 0,0 & 0,0 & 0,0 & 0,0 & 0,0 & 0,9 & 0,0 \\
\hline SLP110 & 0,0 & 0,0 & 0,0 & 0,0 & 0,0 & 0,0 & 0,0 & 0,0 & 0,0 & 0,0 & 0,0 & 0,0 & 0,0 & 0,0 & 0,0 & 0,0 & 0,0 & 0,9 & 0,0 \\
\hline SLP130 & 0,0 & 0,0 & 0,0 & 0,0 & 0,0 & 0,0 & 0,0 & 0,0 & 0,0 & 0,0 & 0,0 & 0,0 & 0,0 & 0,0 & 0,0 & 0,0 & 0,0 & 1,0 & 0,0 \\
\hline XSMIN & & 0,0 & 0,0 & 0,0 & 0,0 & 0,0 & 0,0 & 0,0 & 0,0 & 0,0 & 0,0 & 0,0 & 0,0 & 0,0 & 0,0 & 0,0 & 0,0 & 0,0 & 0,0 \\
\hline IMPV & 0,2 & 0,4 & 0,1 & 0,3 & 0,2 & 0,1 & 0,0 & 0,2 & 0,2 & 0,3 & 0,2 & 0,2 & 0,0 & 0,0 & 0,0 & 0,0 & 0,0 & 1,0 & 0,0 \\
\hline SKEW & 0,3 & 0,0 & 0,1 & 0,1 & 0,1 & 0,0 & 0,1 & 0,0 & 0,0 & 0,0 & 0,0 & 0,0 & 0,0 & 0,0 & 0,0 & 0,0 & 0,0 & 0,0 & 0,0 \\
\hline KURT & 0,3 & 0,1 & 0,0 & 0,0 & 0,0 & 0,0 & 0,0 & 0,0 & 0,0 & 0,0 & 0,0 & 0,0 & 0,0 & 0,0 & 0,0 & 0,0 & 0,0 & 0,0 & 0,0 \\
\hline
\end{tabular}

Smile dependent variables - Constant maturity of 60 days

\begin{tabular}{l|l|l|l|l|l|l|l|l|l|l|l|l|l|l|l|l|l|l|l|l}
\hline 0 & 0,0 & 0,0 & 0,0 & 0,0 & 0,0 & 0,0 & 0,0 & 0,0 & 0,0 & 0,0 & 0,0 & 0,0 & 0,0 & 0,0 & 0,0 & 0,0 & 0,0 & 0,0 & 0,0
\end{tabular} \begin{tabular}{l|l|l|l|l|l|l|l|l|l|l|l|l|l|l|l|l|l|l|l|l|l|l|l|l|l} 
B1 & 0,0 & 0,0 & 0,0 & 0,0 & 0,0 & 0,0 & 0,0 & 0,0 & 0,0 & 0,0 & 0,0 & 0,0 & 0,0 & 0,0 & 0,0 & 0,0 & 0,0 & 0,0 & 0,0
\end{tabular}

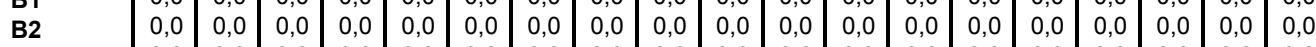
$\begin{array}{lllllllllllllllllllllll}\text { SLP70 } & 0,0 & 0,0 & 0,0 & 0,0 & 0,0 & 0,0 & 0,0 & 0,0 & 0,0 & 0,0 & 0,0 & 0,0 & 0,0 & 0,0 & 0,0 & 0,0 & 0,0 & 0,0 & 0,0\end{array}$

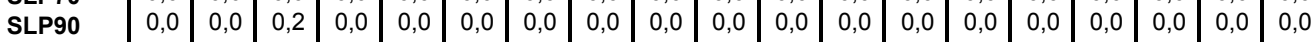

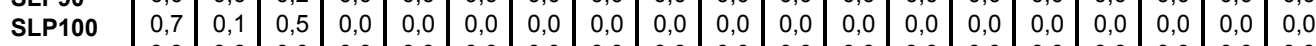
$\begin{array}{lllllllllllllllllllll}\text { SLP110 } & 0,3 & 0,0 & 0,0 & 0,0 & 0,0 & 0,0 & 0,0 & 0,0 & 0,0 & 0,0 & 0,0 & 0,0 & 0,0 & 0,0 & 0,0 & 0,0 & 0,0 & 0,0 & 0,0\end{array}$ \begin{tabular}{llllllllllllllll|l|l|l|l|l|l} 
SLP130 & 0,1 & 0,0 & 0,0 & 0,0 & 0,0 & 0,0 & 0,0 & 0,0 & 0,0 & 0,0 & 0,0 & 0,0 & 0,0 & 0,0 & 0,0 & 0,0 & 0,0 & 0,0 & 0,0
\end{tabular} \begin{tabular}{lc|c|c|c|c|c|c|c|c|c|c|c|c|c|c|c|c|c|c|c|c|c|c|} 
XSMIN & - & 0,0 & 0,0 & 0,0 & 0,0 & 0,0 & 0,0 & 0,0 & 0,0 & 0,0 & 0,0 & 0,0 & 0,0 & 0,0 & 0,0 & 0,0 & 0,0 & 0,0 & 0,0
\end{tabular} $\begin{array}{lllllllllllllllllllll}\text { IMPV } & 0,4 & 0,6 & 0,2 & 0,3 & 0,4 & 0,2 & 0,0 & 0,4 & 0,3 & 0,1 & 0,1 & 0,0 & 0,0 & 0,0 & 0,0 & 0,0 & 0,0 & 0,0 & 0,0\end{array}$ \begin{tabular}{lllllllllllllllllllllll} 
SKEW & 0,7 & 0,1 & 0,4 & 0,0 & 0,4 & 0,0 & 0,0 & 0,0 & 0,0 & 0,0 & 0,0 & 0,0 & 0,0 & 0,0 & 0,0 & 0,0 & 0,0 & 0,0 & 0,0 \\
KURT & 0,4 & 0,5 & 0,1 & 0,0 & 0,0 & 0,0 & 0,0 & 0,0 & 0,0 & 0,0 & 0,0 & 0,0 & 0,0 & 0,0 & 0,0 & 0,0 & 0,0 & 0,0 & 0,0 \\
\hline
\end{tabular}

Smile dependent variables - Constant maturity of 180 days

\begin{tabular}{l|l|l|l|l|l|l|l|l|l|l|l|l|l|l|l|l|l|l|l|l|l} 
B0 & 0,0 & 0,0 & 0,0 & 0,0 & 0,0 & 0,0 & 0,0 & 0,0 & 0,0 & 0,0 & 0,0 & 0,0 & 0,0 & 0,0 & 0,0 & 0,0 & 0,0 & 0,0 & 0,0 \\
B1 & 0,0 & 0,0 & 0,0 & 0,0 & 0,0 & 0,0 & 0,0 & 0,0 & 0,0 & 0,0 & 0,0 & 0,0 & 0,0 & 0,0 & 0,0 & 0,0 & 0,0 & 0,0 & 0,0
\end{tabular} \begin{tabular}{l|l|l|l|l|l|l|l|l|l|l|l|l|l|l|l|l|l|l|l|l|l|l|l} 
B1 & 0,0 & 0,0 & 0,0 & 0,0 & 0,0 & 0,0 & 0,0 & 0,0 & 0,0 & 0,0 & 0,0 & 0,0 & 0,0 & 0,0 & 0,0 & 0,0 & 0,0 & 0,0 & 0,0 \\
\hline & 0,0 & 0,0 & 0,0 & 0,0 & 0,0 & 0,0 & 0,0 & 0,0 & 0,0 & 0,0 & 0,0 & 0,0 & 0,0 & 0,0 & 0,0 & 0,0 & 0,0 & 0,0 & 0,0
\end{tabular} $\begin{array}{llllllllllllllllllllllllllll}\text { B2 } & 0,0 & 0,0 & 0,0 & 0,0 & 0,0 & 0,0 & 0,0 & 0,0 & 0,0 & 0,0 & 0,0 & 0,0 & 0,0 & 0,0 & 0,0 & 0,0 & 0,0 & 0,0 & 0,0\end{array}$

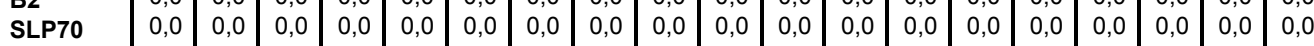
$\begin{array}{llllllllllllllllllllll}\text { SLP90 } & 0,0 & 0,0 & 0,0 & 0,0 & 0,0 & 0,0 & 0,0 & 0,0 & 0,0 & 0,0 & 0,0 & 0,0 & 0,0 & 0,0 & 0,0 & 0,0 & 0,0 & 0,0 & 0,0\end{array}$ $\begin{array}{lllllllllllllllllllllll}\text { SLP100 } & 0,0 & 0,0 & 0,0 & 0,0 & 0,0 & 0,0 & 0,0 & 0,0 & 0,0 & 0,0 & 0,0 & 0,0 & 0,0 & 0,0 & 0,0 & 0,0 & 0,0 & 0,0 & 0,0\end{array}$ \begin{tabular}{lllllllllllllll|l|l|l|l|l|l|l|l|l|l|l|l|l} 
SLP110 & 0,0 & 0,0 & 0,0 & 0,0 & 0,0 & 0,0 & 0,0 & 0,0 & 0,0 & 0,0 & 0,0 & 0,0 & 0,0 & 0,0 & 0,0 & 0,0 & 0,0 & 0,0 & 0,0
\end{tabular} $\begin{array}{llllllllllllllllllllll}\text { SLP130 } & 0,0 & 0,0 & 0,0 & 0,0 & 0,0 & 0,0 & 0,0 & 0,0 & 0,0 & 0,0 & 0,0 & 0,0 & 0,0 & 0,0 & 0,0 & 0,0 & 0,0 & 0,0 & 0,0\end{array}$ $\begin{array}{llllllllllllllllllllll}\text { XSMIN } & - & 0,0 & 0,0 & 0,0 & 0,0 & 0,0 & 0,0 & 0,0 & 0,0 & 0,0 & 0,0 & 0,0 & 0,0 & 0,0 & 0,0 & 0,0 & 0,0 & 0,0 & 0,0\end{array}$

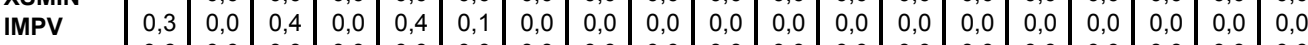

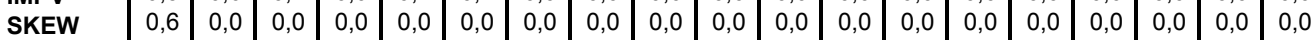
\begin{tabular}{llllllllllllllllllll} 
KURT & 0,7 & 0,5 & 0,0 & 0,0 & 0,0 & 0,0 & 0,0 & 0,0 & 0,0 & 0,0 & 0,0 & 0,0 & 0,0 & 0,0 & 0,0 & 0,0 & 0,0 & 0,0 & 0,0 \\
\hline
\end{tabular} Smile dependent variables - Constant maturity of 240 days

\begin{tabular}{l|c|c|c|c|c|c|c|c|c|c|c|c|c|c|c|c|c|c|c} 
B0 & 0,0 & 0,0 & 0,0 & 0,0 & 0,0 & 0,0 & 0,0 & 0,0 & 0,0 & 0,0 & 0,0 & 0,0 & 0,0 & 0,0 & 0,0 & 0,0 & 0,0 & 0,0 & 0,0 \\
B1 & 0,0 & 0,0 & 0,0 & 0,0 & 0,0 & 0,0 & 0,0 & 0,0 & 0,0 & 0,0 & 0,0 & 0,0 & 0,0 & 0,0 & 0,0 & 0,0 & 0,0 & 0,0 & 0,0 \\
B2 & 0,0 & 0,0 & 0,0 & 0,0 & 0,0 & 0,0 & 0,0 & 0,0 & 0,0 & 0,0 & 0,0 & 0,0 & 0,0 & 0,0 & 0,0 & 0,0 & 0,0 & 0,0 & 0,0 \\
SLP70 & 0,0 & 0,0 & 0,0 & 0,0 & 0,0 & 0,0 & 0,0 & 0,0 & 0,0 & 0,0 & 0,0 & 0,0 & 0,0 & 0,0 & 0,0 & 0,0 & 0,0 & 0,0 & 0,0 \\
SLP90 & 0,0 & 0,0 & 0,0 & 0,0 & 0,0 & 0,0 & 0,0 & 0,0 & 0,0 & 0,0 & 0,0 & 0,0 & 0,0 & 0,0 & 0,0 & 0,0 & 0,0 & 0,0 & 0,0 \\
SLP100 & 0,0 & 0,0 & 0,0 & 0,0 & 0,0 & 0,0 & 0,0 & 0,0 & 0,0 & 0,0 & 0,0 & 0,0 & 0,0 & 0,0 & 0,0 & 0,0 & 0,0 & 0,0 & 0,0 \\
SLP110 & 0,0 & 0,0 & 0,0 & 0,0 & 0,0 & 0,0 & 0,0 & 0,0 & 0,0 & 0,0 & 0,0 & 0,0 & 0,0 & 0,0 & 0,0 & 0,0 & 0,0 & 0,0 & 0,0 \\
SLP130 & 0,0 & 0,0 & 0,0 & 0,0 & 0,0 & 0,0 & 0,0 & 0,0 & 0,0 & 0,0 & 0,0 & 0,0 & 0,0 & 0,0 & 0,0 & 0,0 & 0,0 & 0,0 & 0,0 \\
XSMIN & - & 0,0 & 0,0 & 0,0 & 0,0 & 0,0 & 0,0 & 0,0 & 0,0 & 0,0 & 0,0 & 0,0 & 0,0 & 0,0 & 0,0 & 0,0 & 0,0 & 0,0 & 0,0 \\
IMPV & 0,0 & 0,0 & 0,0 & 0,0 & 0,0 & 0,0 & 0,0 & 0,0 & 0,0 & 0,0 & 0,0 & 0,0 & 0,0 & 0,0 & 0,0 & 0,0 & 0,0 & 0,0 & 0,0 \\
SKEW & 0,5 & 0,0 & 0,0 & 0,0 & 0,0 & 0,0 & 0,0 & 0,0 & 0,0 & 0,0 & 0,0 & 0,0 & 0,0 & 0,0 & 0,0 & 0,0 & 0,0 & 0,0 & 0,0 \\
KURT & 0,0 & 0,0 & 0,0 & 0,0 & 0,0 & 0,0 & 0,0 & 0,0 & 0,0 & 0,0 & 0,0 & 0,0 & 0,0 & 0,0 & 0,0 & 0,0 & 0,0 & 0,0 & 0,0
\end{tabular}

p-values are calculated according to MacKinnon(1996)

Only the 19 most important cross sections are reported, since for the remainder the available data is insufficien to carry out a meaningful unit root test.

Table 6.9.: Rounded ADF probabilities of error for the rejection of the null hypothesis of a unit root 


\subsection{Analysis and Results}

\subsubsection{Introduction}

\subsubsection{Generalities}

The models that have been tested in the course of the following analysis have all been estimated with the OLS method. True coefficients are always denominated with $\beta$, whereas the respective estimates are labeled $b$. Regressions have been carried out in the time-series dimension for all stocks and in the framework of a pooled series regression for the five most important stocks as prioritized in table 6.1. Pure cross-sectional comparisons have been avoided, due to an insufficient number of cross-sections altogether and due to a relatively poor data quality for the cross-sections with low option trading volume.

For regressions in the time-series dimension, the coefficient covariance matrix has been estimated according to the Newey-West method. For regressions in the pooled series framework, the White method has been applied (see section 6.3.3.3).

The pooled series model has always been estimated with a cross-section specific fixed effect in order to allow for the possibility of firm specific particularities. To keep the documentation as parsimonious as possible, the cross-section specific intercept will never be reported in detail on an individual cross-sectional basis but rather as an aggregate figure.

Further, a time trend has always been included into the specification of the regression models, since the visual inspection of the series suggests the existence of a slight deterministic trend for most of them. Only in the case of the test of the leverage effect hypothesis, a time trend is redundant, since the analysis has been carried in differences.

If not stated otherwise, in the course of the subsequent analysis regression coefficients are considered to be significant, if the error probability for the rejection of the null hypothesis of the coefficient equaling zero is less than or equal to $5 \%$.

Finally, the cross-sections displayed in the results tables are always ordered decreasingly according to their importance as measured by the number of used option trades (cf. table 6.1). 


\subsubsection{Robustness Checks}

For all regressions robustness checks have been carried out addressing the issue of possible non-stationarity as well as the robustness as to the inclusion of further independent variables into the regression model.

6.5.1.2.1. The Issue of Possible Non-Stationarity The possibility of nonstationarity must be considered and possibly be treated, since the preliminary analysis of the variables in section 6.4.7.3 suggests that unit roots cannot be completely ruled out for at least three series, namely $P R C, C D S$ and $I M P V$. This issue has been addressed in the following way:

- In the case of the test of Hypothesis 1, the leverage effect hypothesis, the regression has been carried out in differences, thus circumventing the problem of possible non-stationarity.

- The test of Hypothesis 2, which concerns to the relationship between default risk and the level of equity volatility, simultaneously involved the 2 possibly non-stationary variables $C D S$ and $I M P V$. In this case, tests for conintegration and panel unit root tests have been carried out.

- The remainder of the analysis only involved one possibly trending series at a time, namely $C D S$. In this case, spurious regression need not concern us, as has been laid out in section 6.3.4.2.2. While the regression models of the remainder of the analysis indeed did not contain more than one possibly trending variable, this is not true for the corresponding robustness checks. Strictly speaking, the issue of possible non-stationarity should also have been addressed there. But for reasons of simplicity and readability of this document, this has been dispensed with.

\subsection{Robustness in the Presence of Additional Explanatory Vari-}

ables The robustness of the regression model in the presence of additional explanatory variables is crucial for the following reason: First, imagine the case that a significant regression relationship between two variables is owed to a common, underlying factor driving both these variables. Then the addition of this underlying factor to the regression model will probably lead to a loss of significance of the original relationship. Or secondly, imagine the case that the two variables of a regression relationship both depend on two common, underlying factors, but that these factors taken individually influence the regression relationship in opposite directions. If this regression 
relationship is significant, then this means that one of the opposing factors dominates the other. If this dominating factor is added as a control variable to the regression model, its inclusion will probably lead to a sign inversion of the original regression relationship. With regard to the possibility of such constellations, two effects have been tested:

- The effect of systematic influences: The role of systematic influences on the regression model has been analyzed in the context of the examination of the leverage effect (Hypothesis 1) and the study of the relationship between default risk and the level of implied volatility (Hypothesis 2). For this purpose the variables $I M P V_{D A X}$ and $P R C_{D A X}$ have been included as additional variables proxying for systematic influences.

- The effect of individual volatility: The influence of individual volatility on the relationship between default risk on the one hand and the shape of the smile and the risk-neutral moments on the other (Hypotheses 3 and 4) could be important: As suggested by our preliminary analysis of section 6.4.5, a high CDS spread level seems to be associated with a flattening and right-translation of the volatility smile. But as can also be seen in figure 6.3 above and as is to be expected on account of (Hypothesis 2), a high CDS spread level in turn is also connected with a high level of equity volatility. This raises the question, whether a possible relationship between the CDS spread level and the smile is indeed a relationship between default risk and the smile, or is in reality rather a relationship between the level of volatility and the smile. To distinguish between these two effects on the smile, the terms default-risk-only effect and volatility-only effect will be used throughout the remainder of this document. To shed light onto the interaction of these two effects, two approaches have been followed:

- Volatility has been included as a supplementary variable into the regression models. To this end, both volatility measures - IMPV and $H V$ - have been used. It is to be expected that $I M P V$ exerts a more detrimental effect on the relationship between the CDS spread level and the smile than $H V$, since it is known that implied volatility is a better predictor of the CDS spread than is historical volatility (see section 5.1.3.2).

- The default-risk-only and the volatility-only effect have further been dissociated in the following way: First, to neutralize the role of the volatilityonly effect in the relationship between the CDS spread level and the smile and to expose the default-risk-only effect, the regression analysis has been conducted on a sample limited to datasets with the same or about the same 
implied volatility $I M P V$. Secondly, to eliminate the default-risk-only effect and to emphasize the role of the volatility-only effect, $C D S$ as the dependent variable in the regression models has been replaced by $I M P V$ and $H V$, respectively. Then, in order to isolate and emphasize the role of the volatility-only effect while eliminating that of the default-risk-only effect, the analysis has been conducted on a sample limited to datasets with the same or about the same CDS spread. In order to keep this document as lean and readable as possible, this exercise has only been performed in a pooled series context. Since the limitation of the bandwidth of IMPV and of $C D S$, respectively, substantially reduces the number of available datasets, the pooled series framework has been expanded to the 10 most important stock cross-sections.

\subsubsection{Structure of the Empirical Analysis}

The documentation of the empirical analysis is structured as follows: The leverage effect hypothesis Hypothesis 1 will be treated in section 6.5.2. Hypothesis 2 concerning the relationship between default risk and the level of equity volatility will be probed in section 6.5.3. Afterwards, Hypothesis 3 regarding the connection between default risk and the pattern of the volatility smile will examined in section 6.5.4. Section 6.5.5 finally concludes with the test of Hypothesis 4, which assumes a dependency between default risk and the risk-neutral moments.

In most cases, regressions have been carried out several times, once for each constant maturity. In all cases, the constant maturity of 36 days yielded the most meaningful results. For this reason and to avoid an overcharging of the subsequent documentation, most of the time only the results of the constant maturity case of 36 days will be reported and discussed in detail. The results of the other maturity cases, will only be reported and discussed as aggregated figures. Notwithstanding, the totality of detailed results will always be available in the appendix.

\subsubsection{The Leverage Effect}

This section is dedicated to Hypothesis 1: The asymmetric volatility phenomenon is caused by the leverage effect. 


\subsubsection{Derivation of the Regression Model}

As has been laid out in section 5.1.1.3, the leverage effect implies that changes in the company's leverage lead to subsequent, equally directed changes in the company's equity volatility. As illustrated by equation 5.14 , the change in leverage can be caused by a change in the debt or a change in the equity value. For the purposes of this study, the change in leverage will be proxied by the change in equity value, which is consistent with many other studies (cf. section 5.1.3.1). Assuming that the level of debt remains constant, this approach ensures that the change in leverage will be completely tracked by the change in the equity value.

To reflect the chain of causation, the observations of the change in the equity value and the change in the volatility should not be contemporaneous. Instead, the change in the level of volatility should be lagged with regard to the stock price change. The fact that implied volatility is a good proxy for subsequently realized volatility ${ }^{340}$, makes it an ideal variable in this context.

A natural approach to track the relationship between changes in leverage expressed as proportional changes in equity value $d \ln (S)$ and subsequent changes in equity volatility $d \sigma_{S}$ would be the following regression model:

$$
d \sigma_{S t}=\beta_{0}+\beta_{1} \cdot d \ln \left(S_{t}\right)+u_{t}
$$

Due to the leverage effect, we would expect a negative value for the coefficient $\beta_{1}$ in the above model 6.37. However, a negative value is only a necessary, but not a sufficient condition for the existence of the leverage effect. It can be argued that a negative $\beta_{1}$ could equally well have been caused by volatility feedback or systematic factors.

It is true that volatility feedback as a cause of a negative $\beta_{1}$ should be unlikely, since implied volatility as a proxy for subsequently realized volatility has been used: If volatility feedback were at work, there should be a negative relationship between past volatility changes and current return and not between current return and future volatility changes.

But additionally to the use of implied volatility as a proxy of future volatility, something can be done to more adequately track the leverage effect: As has been elaborated

340 This is among others confirmed by Christensen/PRABHALA (1996) and by MAYHEW/STIVERS (2003). However, an earlier study claims that this is not the case (See CANINA/FIGLEWSKI (1993)). MAYHEW/STIVERS (2003) find that at least for firms with high option trading volume, implied volatility provides substantial information regarding future volatility. 
in section 5.1.1.3, the level of the leverage ratio $L=\frac{B}{S}$ is decisive for the magnitude of the leverage effect. The distinction between low and high levels of $L$ should lead to respectively different responses of $\sigma_{S}$ to changes in $L$. Assuming a constant level of debt value $B$, the level of $L$ can be proxied by $S^{-1}$. Be $\tilde{S}$ the median of $S_{t}$ and be $I$ an indicator variable, then the model of equation 6.37 can be altered in the following way:

$$
d \sigma_{S t}=\beta_{0}+\beta_{1} \cdot d \ln \left(S_{t}\right) \cdot I_{S_{t} \leq \tilde{S}}+\beta_{2} \cdot d \ln \left(S_{t}\right) \cdot I_{S_{t}>\tilde{S}}+u_{t}
$$

If the leverage effect is present, we would expect, that the coefficient $\beta_{1}$ is more negative than the coefficient $\beta_{2}$. In terms of the variables of section 6.4.7 and with $I_{S_{t} \leq \tilde{S}}$ replaced by $D_{L}$ and $I_{S_{t}>\tilde{S}}$ substituted by $D_{H}$, the model of equation 6.38 can be reformulated:

$$
d I M P V_{t}=\beta_{0}+\beta_{1} \cdot d \ln \left(P R C_{t}\right) \cdot D_{L}+\beta_{2} \cdot d \ln \left(P R C_{t}\right) \cdot D_{H}+u_{t}
$$

While this model only covers the time-series dimension, the cross-sectional dimension has also been examined in the framework of a pooled series regression with crosssection individual constants $\beta_{0 i}$ :

$$
d I M P V_{i, t}=\beta_{0 i}+\beta_{1} \cdot d \ln \left(P R C_{i, t}\right) \cdot D_{L}+\beta_{2} \cdot d \ln \left(P R C_{i, t}\right) \cdot D_{H}+u_{i, t}
$$

Both models, 6.39 and 6.40, have been tested and the corresponding results will be discussed in the following section.

\subsubsection{Regression Results}

6.5.2.2.1. Examination in the Time Series Dimension The model of equation 6.39 has been estimated with the OLS method for all individual stocks. Only ${ }_{-} D E G$ has been excluded due to an insufficient number of observations. Thanks to the regression in differences, possible non-stationarity of the series $P R C$ need not concern us here. For the variable $I M P V$, only the constant maturities of 36 and 60 have been used. Regressions with longer terms did not yield any significant results, probably because with rising maturity the horizon mismatch between daily returns and long dated implied volatilities becomes non negligible at some point.

The results of the regression according to the model of equation 6.39 can be viewed in table 6.10 for a constant maturity of 36 days and in table 6.11 for a constant maturity of 60 days. Both tables only report those cross-sections for whom the regression has yielded coefficient estimates $b_{1}$ and $b_{2}$ that are significant at a level of 5\%. All 
cross-sections, also those with insignificant coefficients, can be viewed in appendix C.1. As can be seen from tables 6.10 and 6.11. both coefficients $b_{1}$ and $b_{2}$ are

\begin{tabular}{|c|c|c|c|c|c|c|c|}
\hline \multicolumn{8}{|c|}{$d I M P V_{t}=\beta_{0}+\beta_{1} \cdot d \ln \left(P R C_{t}\right) \cdot D_{L}+\beta_{2} \cdot d \ln \left(P R C_{t}\right) \cdot D_{H}+u_{t}$} \\
\hline ID * & $b_{0}$ & $b_{1}$ & $b_{2}$ & $b_{2}-b_{1}$ & R2 cor. & $F$ & DW \\
\hline ALL & $\begin{array}{r}0,00 \\
{[-1,06]} \\
(0,29)\end{array}$ & $\begin{array}{r}-0,35 \\
{[-15,76]} \\
(0,00)\end{array}$ & $\begin{array}{r}-0,44 \\
{[-20,67]} \\
(0,00)\end{array}$ & $-0,08$ & 0,48 & 337,46 & 1,77 \\
\hline _SIE & $\begin{array}{r}0,00 \\
{[-1,18]} \\
(0,24)\end{array}$ & $\begin{array}{r}-0,49 \\
{[-28,10]} \\
(0,00)\end{array}$ & $\begin{array}{r}-0,40 \\
{[-14,30]} \\
(0,00)\end{array}$ & 0,09 & 0,57 & 497,25 & 1,95 \\
\hline _DTE & $\begin{array}{r}0,00 \\
{[-1,16]} \\
(0,25)\end{array}$ & $\begin{array}{r}-0,51 \\
{[-26,01]} \\
(0,00)\end{array}$ & $\begin{array}{r}-0,33 \\
{[-8,60]} \\
(0,00)\end{array}$ & 0,17 & 0,50 & 375,37 & 1,86 \\
\hline _DAIM & $\begin{array}{r}0,00 \\
{[-0,87]} \\
(0,38)\end{array}$ & $\begin{array}{r}-0,40 \\
{[-15,93]} \\
(0,00)\end{array}$ & $\begin{array}{r}-0,52 \\
{[-21,03]} \\
(0,00)\end{array}$ & $-0,12$ & 0,48 & 348,58 & 1,87 \\
\hline _DBANK & $\begin{array}{r}0,00 \\
{[-0,76]} \\
(0,45)\end{array}$ & $\begin{array}{r}-0,53 \\
{[-23,47]} \\
(0,00)\end{array}$ & $\begin{array}{r}-0,51 \\
{[-13,75]} \\
(0,00)\end{array}$ & 0,02 & 0,49 & 370,03 & 1,83 \\
\hline _MRE & $\begin{array}{r}0,00 \\
{[-1,40]} \\
(0,16)\end{array}$ & $\begin{array}{r}-0,42 \\
{[-16,36]} \\
(0,00)\end{array}$ & $\begin{array}{r}-0,52 \\
{[-25,47]} \\
(0,00)\end{array}$ & $-0,10$ & 0,58 & 457,77 & 1,84 \\
\hline BAY & $\begin{array}{r}0,00 \\
{[-0,02]} \\
(0,99)\end{array}$ & $\begin{array}{r}-0,31 \\
{[-10,43]} \\
(0,00)\end{array}$ & $\begin{array}{r}-0,43 \\
{[-10,12]} \\
(0,00)\end{array}$ & $-0,12$ & 0,22 & 105,67 & 2,29 \\
\hline _VW & $\begin{array}{r}0,00 \\
{[-0,93]} \\
(0,35)\end{array}$ & $\begin{array}{r}-0,40 \\
{[-14,28]} \\
(0,00)\end{array}$ & $\begin{array}{r}-0,50 \\
{[-17,86]} \\
(0,00)\end{array}$ & $-0,10$ & 0,41 & 261,75 & 2,42 \\
\hline _BASF & $\begin{array}{r}0,00 \\
{[-0,35]} \\
(0,73)\end{array}$ & $\begin{array}{r}-0,44 \\
{[-14,50]} \\
(0,00)\end{array}$ & $\begin{array}{r}-0,41 \\
{[-7,31]} \\
(0,00)\end{array}$ & 0,02 & 0,26 & 132,11 & 2,49 \\
\hline EON & $\begin{array}{r}0,00 \\
{[-0,08]} \\
(0,94)\end{array}$ & $\begin{array}{r}-0,38 \\
{[-5,94]} \\
(0,00)\end{array}$ & $\begin{array}{r}-0,34 \\
{[-3,09]} \\
(0,00)\end{array}$ & 0,04 & 0,05 & 22,41 & 2,84 \\
\hline$-\mathrm{COB}$ & $\begin{array}{r}0,00 \\
{[-0,31]} \\
(0,76)\end{array}$ & $\begin{array}{r}-0,47 \\
{[-8,44]} \\
(0,00)\end{array}$ & $\begin{array}{r}-0,29 \\
{[-2,30]} \\
(0,02)\end{array}$ & 0,18 & 0,09 & 38,23 & 2,80 \\
\hline
\end{tabular}

Table 6.10.: Time-series results for the leverage effect hypothesis for a constant maturity of 36 days

significantly negative, which is consistent with the asymmetric volatility phenomenon. The expectation of a more negative $b_{1}$ with regard to $b_{2}$ has been met by 6 out of 11 cross-sections for both constant maturities cases. This can be interpreted as a weak evidence for the existence of the leverage effect. Remarkably, the cross-sections that yielded the expected result, are the same in both constant maturity cases. Further, at least for the most important cross-sections, the explanatory power $R^{2}$ as well as the overall significance as measured by the $F$ statistic are relatively high and the DurbinWatson statistic is around 2 , ruling out autocorrelated residuals.

In conclusion, it has to be put on record that the existence of the asymmetric volatility phenomenon can be confirmed and that there exists non negligible evidence for the leverage effect hypothesis as well. The robustness of these results will be discussed 


\begin{tabular}{|c|c|c|c|c|c|c|c|}
\hline \multicolumn{8}{|c|}{$d I M P V_{t}=\beta_{0}+\beta_{1} \cdot d \ln \left(P R C_{t}\right) \cdot D_{L}+\beta_{2} \cdot d \ln \left(P R C_{t}\right) \cdot D_{H}+u_{t}$} \\
\hline ID * & $b_{0}$ & $b_{1}$ & $b_{2}$ & $b_{2}-b_{1}$ & R2 cor. & $\mathbf{F}$ & DW \\
\hline ALL & $\begin{array}{r}0,00 \\
{[-1,18]} \\
(0,24)\end{array}$ & $\begin{array}{r}-0,29 \\
{[-16,49]} \\
(0,00)\end{array}$ & $\begin{array}{r}-0,38 \\
{[-22,86]} \\
(0,00)\end{array}$ & $-0,09$ & 0,52 & 396,92 & 1,82 \\
\hline SIE & $\begin{array}{r}0,00 \\
{[-1,53]} \\
(0,13) \\
\end{array}$ & $\begin{array}{r}-0,42 \\
{[-33,13]} \\
(0,00)\end{array}$ & $\begin{array}{r}-0,33 \\
{[-16,13]} \\
(0,00)\end{array}$ & 0,09 & 0,64 & 679,11 & 1,99 \\
\hline _DTE & $\begin{array}{r}0,00 \\
{[-0,82]} \\
(0,41)\end{array}$ & $\begin{array}{r}-0,43 \\
{[-17,15]} \\
(0,00)\end{array}$ & $\begin{array}{r}-0,24 \\
{[-4,74]} \\
(0,00)\end{array}$ & 0,19 & 0,30 & 158,30 & 2,57 \\
\hline _DAIM & $\begin{array}{r}0,00 \\
{[-1,13]} \\
(0,26)\end{array}$ & $\begin{array}{r}-0,36 \\
{[-18,03]} \\
(0,00)\end{array}$ & $\begin{array}{r}-0,44 \\
{[-22,38]} \\
(0,00)\end{array}$ & $-0,08$ & 0,52 & 413,56 & 1,87 \\
\hline _DBANK & $\begin{array}{r}0,00 \\
{[-0,80]} \\
(0,42)\end{array}$ & $\begin{array}{r}-0,46 \\
{[-23,42]} \\
(0,00)\end{array}$ & $\begin{array}{r}-0,45 \\
{[-13,94]} \\
(0,00)\end{array}$ & 0,01 & 0,49 & 371,54 & 1,97 \\
\hline _MRE & $\begin{array}{r}0,00 \\
{[-0,33]} \\
(0,74)\end{array}$ & $\begin{array}{r}-0,25 \\
{[-2,64]} \\
(0,01)\end{array}$ & $\begin{array}{r}-0,45 \\
{[-6,07]} \\
(0,00)\end{array}$ & $-0,21$ & 0,06 & 21,88 & 3,03 \\
\hline _BAY & $\begin{array}{r}0,00 \\
{[-0,29]} \\
(0,77)\end{array}$ & $\begin{array}{r}-0,33 \\
{[-11,35]} \\
(0,00)\end{array}$ & $\begin{array}{r}-0,35 \\
{[-8,54]} \\
(0,00)\end{array}$ & $-0,02$ & 0,22 & 100,91 & 2,59 \\
\hline _VW & $\begin{array}{r}0,00 \\
{[-0,50]} \\
(0,61)\end{array}$ & $\begin{array}{r}-0,34 \\
{[-7,12]} \\
(0,00)\end{array}$ & $\begin{array}{r}-0,39 \\
{[-8,08]} \\
(0,00)\end{array}$ & $-0,05$ & 0,13 & 58,08 & 2,97 \\
\hline _BASF & $\begin{array}{r}0,00 \\
{[-0,26]} \\
(0,80)\end{array}$ & $\begin{array}{r}-0,28 \\
{[-2,54]} \\
(0,01)\end{array}$ & $\begin{array}{r}0,12 \\
{[0,57]} \\
(0,57)\end{array}$ & 0,40 & 0,01 & 3,38 & 2,98 \\
\hline EON & $\begin{array}{r}0,00 \\
{[-0,08]} \\
(0,94)\end{array}$ & $\begin{array}{r}-0,57 \\
{[-4,32]} \\
(0,00)\end{array}$ & $\begin{array}{r}-0,25 \\
{[-1,11]} \\
(0,27)\end{array}$ & 0,32 & 0,02 & 9,93 & 2,96 \\
\hline _COB & $\begin{array}{r}0,00 \\
{[-0,01]} \\
(0,99)\end{array}$ & $\begin{array}{r}-0,68 \\
{[-0,34]} \\
(0,74)\end{array}$ & $\begin{array}{r}-0,59 \\
{[-0,13]} \\
(0,90)\end{array}$ & 0,09 & 0,00 & 0,07 & 3,03 \\
\hline
\end{tabular}

t-stats in [ ] and corresponding two-sided p-values in ( )

* Cross sections are only reported, if $p$-values for $b_{1}$ and $b_{2}$ are below 0,05

Table 6.11.: Time-series regression results for the leverage effect hypothesis for a constant maturity of 60 days

in section 6.5.2.3.1. A comparison with the results of other studies follows in section 6.5.2.4 


\subsection{Examination in the Framework of a Pooled Series Regression}

The model of equation 6.40 has been estimated with the OLS method as a pooled series regression for the 5 most important stocks as prioritized in table 6.1. As mentioned above, possible non-stationarity is not an issue thanks to the regression in differences. Likewise as above, the regression has only been carried out for the two constant maturities of 36 and 60 days. The regression results can be viewed in table 6.12. As

\begin{tabular}{|c|c|c|c|c|c|c|}
\hline \multicolumn{7}{|c|}{$d I M P V_{i, t}=\beta_{0_{i}}+\beta_{1} \cdot d \ln \left(P R C_{i, t}\right) \cdot D_{L_{i}}+\beta_{2} \cdot d \ln \left(P R C_{i, t}\right) \cdot D_{H_{i}}+u_{i, t}$} \\
\hline$b_{0}$ & $b_{1}$ & $b_{2}$ & $b_{2}-b_{1}$ & R2 cor. & $\mathrm{F}$ & DW \\
\hline \multicolumn{7}{|c|}{ Constant maturity of 36 days } \\
\hline 0,00 & $-0,46$ & $-0,46$ & 0,002 & 0,50 & 585,41 & 1,85 \\
\hline$[-18,77]$ & {$[-14,77]$} & {$[-18,01]$} & & & & \\
\hline$(0,00)$ & $(0,00)$ & $(0,00)$ & & & & \\
\hline \multicolumn{7}{|c|}{ Constant maturity of 60 days } \\
\hline 0,00 & $-0,40$ & $-0,39$ & 0,007 & 0,45 & 489,97 & 2,20 \\
\hline$[-18,31]$ & {$[-13,68]$} & {$[-17,23]$} & & & & \\
\hline$(0,00)$ & $(0,00)$ & $(0,00)$ & & & & \\
\hline
\end{tabular}

Table 6.12.: Pooled series regression results for the leverage effect hypothesis for constant maturities of 36 and 60 days

depicted in table 6.12, both coefficients $b_{1}$ and $b_{2}$ are significantly negative which is a clear sign of the existence of the asymmetric volatility phenomenon. Further, the difference between $b_{2}$ and $b_{2}$ is slightly positive in both constant maturity cases, which suggests that the leverage effect is at work. As to the explanatory power, the overall significance and the Durbin-Watson statistic the same can be said as in the time-series analysis. The discussion of the findings will be continued in section 6.5.2.3.1 as far as their robustness is concerned and in section 6.5.2.4 with respect to the results of other analyses. 


\subsubsection{Robustness of the Regression Results}

As discussed in section 5.1.3.1, the leverage effect is not the only hypothesized cause of the asymmetric volatility phenomenon. Competing explanations all rely on systematic influences: FIGLEWSKI/WANG (2000) and DENNIS ET AL. (2006) claim the existence of strong market-wide factors and the volatility feedback theory also requires systematic determinants, since it supposes a time-varying risk premium ${ }^{341}$. This raises the question, whether the results of the previous section are really related to the leverage of the individual firm. Instead, it could be that in reality they have been predominantly or even exclusively caused by latent systematic factors in the guise of individual stock returns. If this were the case, the inclusion of these latent systematic variables into the regression model could induce one or more of the following:

- The estimates of the firm specific coefficients $b_{1}$ and $b_{2}$ could possibly loose their significance, because the systematic variables are more apt in explaining changes in individual volatility.

- The estimates of the firm specific coefficients $b_{1}$ and $b_{2}$ could possibly be lower in absolute value than before, because the systematic variables and the systematically caused portion of the individual stock return now both contribute to the explanation of $d I M P V$.

- Due to dependencies between the latent systematic variables and the individual stock return, the positive difference between $b_{2}$ and $b_{1}$ - until now interpreted as evidence of the existence of the leverage effect - could become negative.

Candidate variables for latent systematic influences are $d \ln \left(P R C_{D A X}\right)$ and $d I M P V_{D A X}$. Their inclusion has been tested in the times series dimension as well as in the framework of a pooled series regression. The results of the robustness checks will be discussed in the two following sections.

6.5.2.3.1. Robustness Check of the Time Series Regression Results When comparing the coefficients of $d \ln \left(P R C_{i}\right)$ and $d \ln \left(P R C_{D A X}\right)$, the lower index volatility with regard to that of its components has to be taken into account. If this lower volatility were not corrected for, the index could yield higher coefficients, only because the amplitudes of the index return are not as high as those of the individual stock return. Therefore, the time series of index returns $d \ln \left(P R C_{D A X}\right)$ has been

341 Cf. FRENCH ET AL. (1987), e.g.. 
divided by its volatility and multiplied by the volatility of the respective series of the individual stock returns:

$$
\hat{R}_{D A X} \equiv d \ln \left(P R C_{D A X}\right) \cdot \frac{\sigma\left[d \ln \left(P R C_{i}\right)\right]}{\sigma\left[d \ln \left(P R C_{D A X}\right)\right]}
$$

Definition 6.41 ensures that the square root of the average squared amplitude of index and individual stock return are equalized, assuming that the mean return of both is zero. The inclusion of the systematic variables $d \ln \left(P R C_{D A X}\right)$ and $d I M P V_{D A X}$, where the former is replaced by $\hat{R}_{D A X}$, leads to the following alternative model as a robustness check of the model of equation 6.39 .

$$
\begin{array}{r}
d I M P V_{t}=\beta_{0}+\beta_{1} \cdot d \ln \left(P R C_{t}\right) \cdot D_{L}+\beta_{2} \cdot d \ln \left(P R C_{t}\right) \cdot D_{H} \\
+\beta_{3} \cdot \hat{R}_{D A X} \cdot D_{L}+\beta_{4} \cdot \hat{R}_{D A X} \cdot D_{H}+\beta_{5} \cdot d I M P V_{D A X}+u_{t}
\end{array}
$$

The results of the regression according to the model of equation 6.42 are displayed in

\begin{tabular}{|c|c|c|c|c|c|c|c|c|c|c|c|}
\hline \multicolumn{12}{|c|}{$d I M P_{t}=\beta_{0}+\beta_{1} \cdot d \ln \left(P R C_{t}\right) \cdot D_{L}+\beta_{2} \cdot d \ln \left(P R C_{t}\right) \cdot D_{H}+\beta_{3} \cdot \hat{R}_{D A X, t} \cdot D_{L}+\beta_{4} \cdot \hat{R}_{D A X, t} \cdot D_{H}+\beta_{5} \cdot d I M P V_{D a x, t}+u_{t}$} \\
\hline ID* & $b_{0}$ & $b_{1}$ & $b_{2}$ & $b_{3}$ & $b_{4}$ & $b_{5}$ & $b_{2}-b_{1}^{* *}$ & $b_{4}-b_{3}{ }^{* *}$ & R2 cor. & $F$ & DW \\
\hline ALL & $\begin{array}{r}0,00 \\
{[-0,46]} \\
(0,64)\end{array}$ & $\begin{array}{r}-0,09 \\
{[-2,88]} \\
(0,00)\end{array}$ & $\begin{array}{r}-0,25 \\
{[-8,18]} \\
(0,00)\end{array}$ & $\begin{array}{r}-0,07 \\
{[-2,10]} \\
(0,04)\end{array}$ & $\begin{array}{r}0,09 \\
{[2,68]} \\
(0,01)\end{array}$ & $\begin{array}{r}0,89 \\
{[22,19]} \\
(0,00)\end{array}$ & $-0,17$ & 0,16 & 0,73 & 399,02 & 1,95 \\
\hline _SIE & $\begin{array}{r}0,00 \\
{[-0,74]} \\
(0,46) \\
\end{array}$ & $\begin{array}{r}-0,32 \\
{[-10,22]} \\
(0,00) \\
\end{array}$ & $\begin{array}{r}-0,29 \\
{[-6,99]} \\
(0,00)\end{array}$ & $\begin{array}{r}0,14 \\
{[4,46]} \\
(0,00)\end{array}$ & $\begin{array}{r}0,17 \\
{[3,41]} \\
(0,00) \\
\end{array}$ & $\begin{array}{r}0,79 \\
{[26,92]} \\
(0,00)\end{array}$ & 0,03 & 0,03 & 0,79 & 557,14 & 2,03 \\
\hline _DTE & $\begin{array}{r}0,00 \\
{[-0,98]} \\
(0,33)\end{array}$ & $\begin{array}{r}-0,29 \\
{[-11,48]} \\
(0,00)\end{array}$ & $\begin{array}{r}-0,27 \\
{[-6,23]} \\
(0,00)\end{array}$ & $\begin{array}{r}0,08 \\
{[2,67]} \\
(0,01)\end{array}$ & $\begin{array}{r}0,22 \\
{[4,43]} \\
(0,00)\end{array}$ & $\begin{array}{r}0,83 \\
{[21,53]} \\
(0,00)\end{array}$ & 0,02 & 0,15 & 0,71 & 370,76 & 1,94 \\
\hline _DAIM & $\begin{array}{r}0,00 \\
{[-0,51]} \\
(0,61) \\
\end{array}$ & $\begin{array}{r}-0,18 \\
{[-5,10]} \\
(0,00) \\
\end{array}$ & $\begin{array}{r}-0,19 \\
{[-5,66]} \\
(0,00) \\
\end{array}$ & $\begin{array}{r}0,05 \\
{[1,34]} \\
(0,18) \\
\end{array}$ & $\begin{array}{r}-0,02 \\
{[-0,44]} \\
(0,66) \\
\end{array}$ & $\begin{array}{r}0,82 \\
{[24,13]} \\
(0,00)\end{array}$ & $-0,01$ & & 0,75 & 440,37 & 2,07 \\
\hline _DBANK & $\begin{array}{r}0,00 \\
{[-0,44]} \\
(0,66) \\
\end{array}$ & $\begin{array}{r}-0,33 \\
{[-8,47]} \\
(0,00)\end{array}$ & $\begin{array}{r}-0,15 \\
{[-2,69]} \\
(0,01)\end{array}$ & $\begin{array}{r}0,10 \\
{[2,26]} \\
(0,02) \\
\end{array}$ & $\begin{array}{r}0,00 \\
{[-0,03]} \\
(0,97) \\
\end{array}$ & $\begin{array}{r}0,85 \\
{[20,66]} \\
(0,00)\end{array}$ & 0,19 & & 0,70 & 356,60 & 1,87 \\
\hline _MRE & $\begin{array}{r}0,00 \\
{[-1,17]} \\
(0,24) \\
\end{array}$ & $\begin{array}{r}-0,41 \\
{[-11,53]} \\
(0,00)\end{array}$ & $\begin{array}{r}-0,32 \\
{[-8,88]} \\
(0,00) \\
\end{array}$ & $\begin{array}{r}0,19 \\
{[4,68]} \\
(0,00) \\
\end{array}$ & $\begin{array}{r}0,06 \\
{[1,69]} \\
(0,09) \\
\end{array}$ & $\begin{array}{r}0,83 \\
{[17,22]} \\
(0,00)\end{array}$ & 0,09 & & 0,72 & 343,70 & 1,96 \\
\hline _VW & $\begin{array}{r}0,00 \\
{[-0,63]} \\
(0,53) \\
\end{array}$ & $\begin{array}{r}-0,31 \\
{[-7,13]} \\
(0,00)\end{array}$ & $\begin{array}{r}-0,29 \\
{[-6,21]} \\
(0,00) \\
\end{array}$ & $\begin{array}{r}0,11 \\
{[2,09]} \\
(0,04) \\
\end{array}$ & $\begin{array}{r}0,04 \\
{[0,80]} \\
(0,42) \\
\end{array}$ & $\begin{array}{r}0,61 \\
{[12,69]} \\
(0,00)\end{array}$ & 0,02 & & 0,53 & 171,36 & 2,68 \\
\hline BASF & $\begin{array}{r}0,00 \\
{[-0,23]} \\
(0,82)\end{array}$ & $\begin{array}{r}-0,18 \\
{[-3,69]} \\
(0,00)\end{array}$ & $\begin{array}{r}-0,24 \\
{[-3,48]} \\
(0,00)\end{array}$ & $\begin{array}{r}-0,02 \\
{[-0,44]} \\
(0,66)\end{array}$ & $\begin{array}{r}0,07 \\
{[0,86]} \\
(0,39)\end{array}$ & $\begin{array}{r}0,58 \\
{[11,07]} \\
(0,00)\end{array}$ & $-0,06$ & & 0,41 & 104,01 & 2,73 \\
\hline
\end{tabular}
table 6.13 for a constant maturity of 36 days and in table 6.14 for a constant maturity of 60 days, respectively. Similar to the prior section, both tables only report those

Table 6.13.: Robustness check of the time-series regression results for the leverage effect hypothesis for a constant maturity of 36 days 


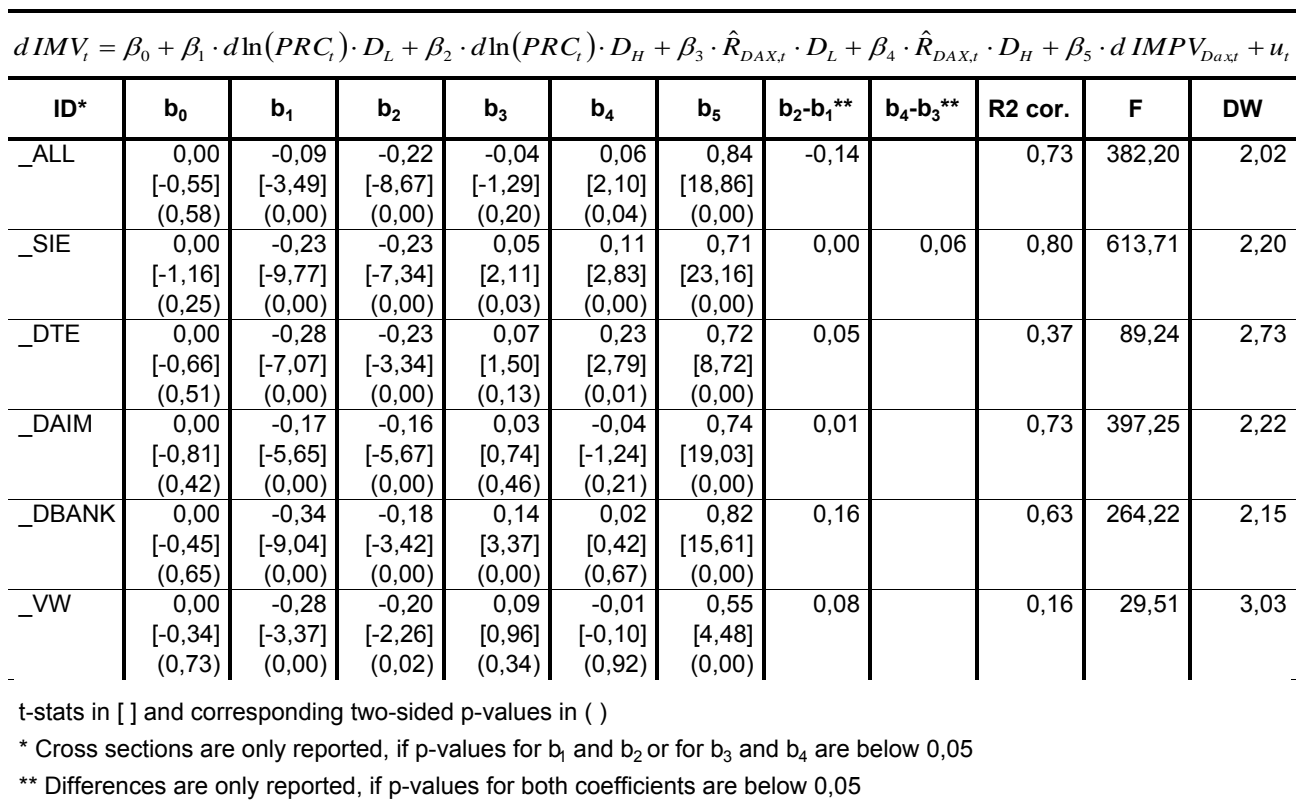

Table 6.14.: Robustness check of the time-series regression results for the leverage effect hypothesis for a constant maturity of 60 days

cross-sections for whom the regression has yielded coefficient estimates $b_{1}$ and $b_{2}$ or $b_{3}$ and $b_{4}$ that are significant at a level of $5 \%$. All cross-sections, also those with insignificant coefficients, can be viewed in appendix C.1. Comparing tables 6.10 and 6.11 with tables 6.13 and 6.14 , it can be seen that overall the explanatory power as well as the significance of the regression have increased. This is mainly due to the highly significant coefficient $b_{5}$ for $d I M P V_{D A X, t}$, which confirms the suspicion, that systematic influences do matter. However, the coefficients $b_{3}$ and $b_{4}$ for the corrected index return fall short of being significant in most cases. In addition, they are smaller in absolute terms than those of the individual return and exhibit the wrong sign in most cases. But more importantly, the sign of the coefficients $b_{1}$ and $b_{2}$ of the individual return has not changed in any case. It is true that the absolute value as well as the $t$-statistics of $b_{1}$ and $b_{2}$ have almost halved, but the coefficients are still highly significant. The fraction of cross-sections for whom the difference between $b_{2}$ and $b_{1}$ yields the expected positive sign has even increased thanks to the integration of systematic control variables.

In summary, it can be concluded, that systematic influences are important, but do not dominate the individual return. The stock price level dependent sensitivity of $d I M P V$ with respect to the individual stock return becomes even more pronounced in 
the presence of systematic factors. Thus, the robustness check confirms the existence of the leverage effect. 
6.5.2.3.2. Robustness of the Pooled Series Regression Results As discussed in the previous section, the lower index volatility with regard to that of individual stocks has to be taken into account. In the framework of the pooled series regression this is achieved by an analogue approach as above, with the distinction that the return volatility of all 5 pooled cross-sections has been considered:

$$
\hat{R}_{D A X}=d \ln \left(P R C_{D A X}\right) \cdot \frac{\frac{1}{5} \sum_{i=1}^{5} \sigma\left[d \ln \left(P R C_{i}\right)\right]}{\sigma\left[d \ln \left(P R C_{D A X}\right)\right]}
$$

The robustness check for the pooled series regression has been conducted according to the following model:

$$
\begin{array}{r}
d I M P V_{i, t}=\beta_{0 i}+\beta_{1} \cdot d \ln \left(P R C_{i, t}\right) \cdot D_{L}+\beta_{2} \cdot d \ln \left(P R C_{i, t}\right) \cdot D_{H} \\
+\beta_{3} \cdot \hat{R}_{D A X, t} \cdot D_{L}+\beta_{4} \cdot \hat{R}_{D A X, t} \cdot D_{H}+\beta_{5} \cdot d I M P V_{D A X, t}+u_{i, t}
\end{array}
$$

\begin{tabular}{|c|c|c|c|c|c|c|c|c|c|c|}
\hline$b_{0}$ & $b_{1}$ & $b_{2}$ & $b_{3}$ & $\mathbf{b}_{4}$ & $b_{5}$ & $b_{2}-b_{1}$ & $b_{4}-b_{3}$ & R2 cor. & $\mathbf{F}$ & DW \\
\hline \multicolumn{11}{|c|}{ Constant maturity of 36 days } \\
\hline 0,00 & $-0,24$ & $-0,23$ & 0,06 & 0,08 & 0,84 & 0,005 & 0,019 & 0,73 & 1099,8 & 1,95 \\
\hline$[-13,03]$ & {$[-5,51]$} & {$[-10,28]$} & {$[1,76]$} & {$[1,90]$} & {$[58,59]$} & & & & & \\
\hline$(0,00)$ & $(0,00)$ & $(0,00)$ & $(0,08)$ & $(0,06)$ & $(0,00)$ & & & & & \\
\hline \multicolumn{11}{|c|}{ Constant maturity of 60 days } \\
\hline 0,00 & $-0,22$ & $-0,21$ & 0,05 & 0,05 & 0,77 & 0,013 & 0,004 & 0,60 & 618,65 & 2,43 \\
\hline$[-12,66]$ & {$[-4,97]$} & {$[-11,04]$} & {$[1,77]$} & {$[1,44]$} & {$[32,00]$} & & & & & \\
\hline$(0,00)$ & $(0,00)$ & $(0,00)$ & $(0,08)$ & $(0,15)$ & $(0,00)$ & & & & & \\
\hline
\end{tabular}

The regression results of the model of equation 6.44 can be viewed in table 6.15. Comparing tables 6.12 and 6.15 , the overall picture is similar to that of the robust-

Table 6.15.: Robustness check of the pooled series regression results for the leverage effect hypothesis for constant maturities of 36 and 60 days

ness check in the time series case: Explanatory power and significance of the regression have increased, mainly due to the highly significant coefficient of $d I M P V_{D A X, t}$. However, the coefficients $b_{3}$ and $b_{4}$ of the corrected index return fail to be significant at the $5 \%$ level. Moreover $b_{3}$ and $b_{4}$ show the wrong sign and are lower in absolute value compared to $b_{1}$ and $b_{2}$. Further, $t$-statistics and absolute values of $b_{1}$ and $b_{2}$ have decreased, but have kept the expected negative sign and the difference between $b_{2}$ and $b_{1}$ is still positive.

In conclusion, it can be said, that the robustness check of the pooled series regression also confirms the original evidence in favor of the leverage effect hypothesis. 


\subsubsection{Summary and Discussion of the Results in the Light of Hitherto Existing Findings}

6.5.2.4.1. Summary of the Results The above analysis clearly confirms the existence of the asymmetric volatility phenomenon. Further, clear evidence has been produced of the existence of the leverage effect as well. This has been achieved in two ways: First, the leverage effect specific chain of causation has been respected, using implied volatility as a proxy for future volatility. Secondly, the sensitivity of volatility with regard to leverage changes has been detected to be more pronounced when the level of leverage is high than when it is low.

In order to examine the influence of systematic variables on asymmetric volatility and the presumed leverage effect relationship, the DAX return and its volatility have been included into the regression models. Both additional effects seem to matter for the explanation of individual volatility changes. But while the DAX volatility seems to play an important role, the DAX return is far from unfolding the same effects as the individual return. Anyhow, the leverage effect relationship - though less accentuated seems to remain largely intact after the inclusion of systematic variables. Altogether, this confirms Hypothesis 1 that the asymmetric volatility phenomenon is caused by the leverage effect. However, it cannot be concluded that the leverage effect is the exclusive cause of asymmetric volatility. Instead, the non negligible role of systematic influences leaves room for additional explanations.

\subsection{Discussion of the Results in the Light of Hitherto Existing Find-} ings While the above results use a volatility measure based on gross implied volatility and a leverage measure derived from the stock price level, previous studies partly use other measures as has been mentioned in section 5.1.3.1 $\left.\right|^{42}$. Therefore, the above results can only be selectively compared with the existing literature.

Deviating from the above model of equation 6.39 some authors use relative changes in stock return volatility like $\frac{d \sigma_{S t}}{\sigma_{S t}}{ }^{343}$ or $d \ln \left(\sigma_{S t}\right)^{\sqrt{344}}$ as dependent variable instead of

342 While some studies likewise imply changes in leverage out of changes in the stock price level, others use debt ratios of financial statements. And as to the volatility measure, some work is based on gross volatility, whereas other papers employ conditional volatility estimated with GARCHtype models. Only three studies also use implied volatility.

343 See for instance BLACK (1976).

344 See for instance CHRISTIE (1982) and FIGLEWSKI/WANG (2000). 
absolute changes like $d \sigma_{t}$. All the same they arrive at similar ranges for the coefficient of the equity return. In the case of a regression according to the following model

$$
d \ln \left(\sigma_{S t}\right)=\beta_{0}+\beta_{1} \cdot d \ln \left(S_{t}\right)+u_{t}
$$

the coefficient $\beta_{1}$ can be interpreted as a measure of the elasticity $\epsilon$ of the stock return volatility with respect to the stock price ${ }^{345}$ and should lie in the interval $[-1,0]^{346}$ Contrary to such an approach, I have preferred absolute changes in return volatility over relative ones in my study. The reason is that relative changes in return volatility are difficult to interpret economically, since return volatility is already a relative figure. Further, the purpose of the above analysis consisted in showing that the magnitude of the dependency of stock return volatility changes on the stock return hinges upon the level of the leverage ratio. If the leverage effect hypothesis holds, a high leverage ratio also entails a high level of equity return volatility and high absolute volatility changes in the wake of changes in the leverage ratio. But since the volatility level is already high, this does not forcibly also entail high relative changes in volatility as presumed by some of the other studies. It could well be that absolute changes in volatility are higher at high leverage ratios than at low leverage ratios, but that the converse is true for relative changes, since relative volatility changes imply a division by the level of the volatility.

CHRISTIE (1982) examines the dependency of the elasticity $\epsilon$ on the leverage ratio by partitioning his sample in quartiles of ascending leverage, where the latter has been measured with the help of accounting data. Consistent with the above results, he finds $\epsilon$ decreasing with increasing leverage. This is remarkable in the light of what has just been reasoned concerning the use of relative and absolute changes in volatility. But it could have to do with the way CHRISTIE (1982) measures leverage. While my study completely relies on the stock price level only, CHRISTIE (1982) uses the stock price level and the level of nominal debt, which in the long run yields more exact estimates of the true leverage ratio. This gain in exactitude could possibly compensate the weakness of a measure of volatility changes that is blurred by the level of volatility in the denominator.

\footnotetext{
345 To see this, consider the elasticity $\epsilon$ of the stock return volatility with respect to the stock price (with subscripts for $t$ suppressed for ease of notation): $\epsilon=\frac{d \sigma_{S}}{\sigma_{S}} \cdot \frac{S}{d S}=\frac{d \ln \left(\sigma_{S}\right)}{d \ln (S)}$.

346 Recalling from section 5.1.1.3 that $\sigma_{S}=\sigma(1+L)=\sigma\left(1+\frac{B}{S}\right)$, the elasticity $\epsilon=\frac{d \sigma_{S}}{d S} \cdot \frac{S}{\sigma_{S}}$ can be reexpressed as $\epsilon=-\frac{\sigma B}{\sigma_{S} S}$. Substituting for $\sigma$ yields $\epsilon=-\frac{B}{S+B}$. It can easily be seen that this term must lie in the interval $[-1,0]$. Cf. FIGLEWSKI/WANG (2000).
} 
The above results are also in line with FIGLEWSKI/WANG (2000) and DENNIS ET AL. (2006) or the literature on volatility feedback as far as the important role of systematic influences in the context of asymmetric volatility is concerned. In the above results this importance is reflected by the fact that the coefficients $b_{1}$ and $b_{2}$ - testifying the existence of asymmetric volatility by their negativity - grow less important in the presence of changes in systematic volatility.

In summary, it can be said that my findings are consistent with the comparable literature in producing non negligible evidence of the existence of the leverage effect.

\subsubsection{The Credit Spread Level and the Implied Volatility Level}

The following section is devoted to the test of Hypothesis 2: The implied volatility depends positively on the level of default risk.

\subsubsection{Derivation of the Regression Models}

The expectation of a positive relationship between the level of equity volatility and the level of default risk as suggested by Hypothesis $\mathbf{2}$ has been tested in the time-series dimension and also in the context of a pooled series approach with the following regression models:

Time-series dimension:

$$
C D S_{t}=\beta_{0}+\beta_{1} \cdot I M P V_{t}+\beta_{2} \cdot t+u_{t}
$$

Pooled series regression:

$$
C D S_{i, t}=\beta_{0 i}+\beta_{1} \cdot I M P V_{i, t}+\beta_{2} \cdot t+u_{i, t}
$$

Equity volatility has been proxied by implied volatility, as the superordinate focus of my analysis is the implied volatility smile.

\subsubsection{Regression Results}

6.5.3.2.1. Examination in the Time-Series Dimension The test of the relationship between CDS spread and implied volatility according to model 6.46 has been 
carried out for all stocks except $\_D E G$ due to an insufficient number of observations. Table 6.16 shows the regression results for the constant maturity case of 36 days in detail, whereas table 6.17 exhibits a summary of all constant maturity cases. This summary is grouped according to the constant maturity cases and contains averages, once across all cross-sections and once only across the 5 most important cross-sections. The results of an ADF residual test are also reported in the rightmost column of the two tables, but will be discussed later on in section 6.5.3.3. Table 6.16 only reports those cross-sections for whom the coefficient $b_{1}$ is significant at the $5 \%$ level and for whom the ADF residual test has yielded error probabilities below or equal to 5\%. The totality of detailed results for all cross-sections and all 4 constant maturity cases can be seen in appendix C.2. As can be viewed in table 6.16, all cross-sections which are significant at the 5\% level and which yield ADF error probabilties below or equal to $5 \%$ also show positive coefficients $b_{1}$. Further, the coefficient $b_{1}$ always remains positive even if also averaged across possibly non-significant cross-sections as can be gathered from table 6.17. In the case of the lower 2 constant maturities and for the top 5 cross-sections in table 6.17, $b_{1}$ is even significant on average. Altogehter, this clearly confirms Hypothesis 2.

In most of the cases in table 6.16 the coefficient $b_{2}$ for the time trend is also significant, which justifies the initial assumption of the existence of a time trend. Further, looking at table 6.17, it is obvious that increasing constant maturity leads to weaker results: The explanatory power $R^{2}$, the overall significance $F$, the individual significance of $b_{1}$ and the significance of the ADF test are all declining with increasing maturity.

The explanatory power is relatively high, reaching $87 \%$ on average for the 5 most important cross-sections as can be verified in table 6.16. However, the low DurbinWatson statistic DW indicates a high positive serial correlation between the residuals, which could be caused by non-stationarity. This issue will be further addressed in section 6.5.3.3.

In summary, it can be put on record that Hypothesis $\mathbf{2}$ seems to be confirmed, subject to further robustness checks. 


\begin{tabular}{|c|c|c|c|c|c|c|c|}
\hline \multicolumn{8}{|c|}{$C D S_{t}=\beta_{0}+\beta_{1} \cdot I M P V_{t}+\beta_{2} \cdot t+u_{t}$} \\
\hline ID* & $b_{0}$ & $b_{1}$ & $b_{2}$ & R2 cor. & $\mathbf{F}$ & DW & $\mathrm{ADF}^{* *}$ \\
\hline ALL & $\begin{array}{r}-17,21 \\
{[-5,64]} \\
(0,00) \\
\end{array}$ & $\begin{array}{r}143,69 \\
{[20,82]} \\
(0,00) \\
\end{array}$ & $\begin{array}{r}0,01 \\
{[2,34]} \\
(0,02) \\
\end{array}$ & 0,85 & $2.029,15$ & 0,10 & $\begin{array}{r}{[-4,15]} \\
(<=0,05)\end{array}$ \\
\hline _SIE & $\begin{array}{r}5,75 \\
{[1,39]} \\
(0,16)\end{array}$ & $\begin{array}{r}110,74 \\
{[15,62]} \\
(0,00)\end{array}$ & $\begin{array}{r}-0,01 \\
{[-3,22]} \\
(0,00)\end{array}$ & 0,89 & $3.013,43$ & 0,12 & $\begin{array}{r}{[-4,89]} \\
(<=0,01)\end{array}$ \\
\hline _DTE & $\begin{array}{r}-24,85 \\
{[-1,38]} \\
(0,17) \\
\end{array}$ & $\begin{array}{r}483,67 \\
{[18,46]} \\
(0,00) \\
\end{array}$ & $\begin{array}{r}-0,04 \\
{[-1,79]} \\
(0,07) \\
\end{array}$ & 0,93 & $4.879,13$ & 0,12 & $\begin{array}{r}{[-5,24]} \\
(<=0,01)\end{array}$ \\
\hline _DAIM & $\begin{array}{r}109,79 \\
{[11,50]} \\
(0,00) \\
\end{array}$ & $\begin{array}{r}104,34 \\
{[5,81]} \\
(0,00) \\
\end{array}$ & $\begin{array}{r}-0,08 \\
{[-8,77]} \\
(0,00) \\
\end{array}$ & 0,80 & $1.528,79$ & 0,08 & $\begin{array}{r}{[-4,50]} \\
(<=0,01)\end{array}$ \\
\hline DBANK & $\begin{array}{r}-2,04 \\
{[-1,01]} \\
(0,31)\end{array}$ & $\begin{array}{r}82,12 \\
{[17,24]} \\
(0,00)\end{array}$ & $\begin{array}{r}0,00 \\
{[-0,24]} \\
(0,81)\end{array}$ & 0,86 & $2.248,48$ & 0,15 & $\begin{array}{r}{[-5,50]} \\
(<=0,01)\end{array}$ \\
\hline _MRE & $\begin{array}{r}-8,11 \\
{[-2,17]} \\
(0,03)\end{array}$ & $\begin{array}{r}103,03 \\
{[19,93]} \\
(0,00) \\
\end{array}$ & $\begin{array}{r}0,01 \\
{[2,74]} \\
(0,01) \\
\end{array}$ & 0,78 & $1.172,68$ & 0,53 & $\begin{array}{l}{[-10,18]} \\
(<=0,01)\end{array}$ \\
\hline -VW & $\begin{array}{r}2,90 \\
{[1,13]} \\
(0,26)\end{array}$ & $\begin{array}{r}107,35 \\
{[17,15]} \\
(0,00) \\
\end{array}$ & $\begin{array}{r}0,05 \\
{[24,69]} \\
(0,00) \\
\end{array}$ & 0,68 & 795,00 & 0,11 & $\begin{array}{r}{[-4,44]} \\
(<=0,01)\end{array}$ \\
\hline BASF & $\begin{array}{r}18,23 \\
{[19,45]} \\
(0,00) \\
\end{array}$ & $\begin{array}{r}35,85 \\
{[12,09]} \\
(0,00) \\
\end{array}$ & $\begin{array}{r}-0,02 \\
{[-21,89]} \\
(0,00)\end{array}$ & 0,88 & $2.711,94$ & 0,11 & $\begin{array}{r}{[-4,11]} \\
(<=0,05)\end{array}$ \\
\hline EON & $\begin{array}{r}-5,46 \\
{[-1,33]} \\
(0,18)\end{array}$ & $\begin{array}{r}171,10 \\
{[14,69]} \\
(0,00)\end{array}$ & $\begin{array}{r}0,00 \\
{[-0,57]} \\
(0,57)\end{array}$ & 0,83 & $1.804,88$ & 0,42 & $\begin{array}{r}{[-5,55]} \\
(<=0,01)\end{array}$ \\
\hline _COB & $\begin{array}{r}-40,16 \\
{[-6,27]} \\
(0,00)\end{array}$ & $\begin{array}{r}255,27 \\
{[18,01]} \\
(0,00)\end{array}$ & $\begin{array}{r}0,00 \\
{[-0,50]} \\
(0,62)\end{array}$ & 0,80 & $1.462,33$ & 0,33 & $\begin{array}{r}{[-4,99]} \\
(<=0,01)\end{array}$ \\
\hline _HYPV & $\begin{array}{l}19,89 \\
{[0,83]} \\
(0,41) \\
\end{array}$ & $\begin{array}{r}113,72 \\
{[2,62]} \\
(0,01) \\
\end{array}$ & $\begin{array}{r}-0,03 \\
{[-1,84]} \\
(0,07) \\
\end{array}$ & 0,48 & 338,73 & 0,70 & $\begin{array}{r}{[-4,72]} \\
(<=0,01)\end{array}$ \\
\hline _LH & $\begin{array}{r}100,18 \\
{[7,25]} \\
(0,00) \\
\end{array}$ & $\begin{array}{r}119,73 \\
{[4,04]} \\
(0,00) \\
\end{array}$ & $\begin{array}{r}-0,09 \\
{[-8,62]} \\
(0,00)\end{array}$ & 0,57 & 466,93 & 0,45 & $\begin{array}{r}{[-2,92]} \\
(<=0,05)\end{array}$ \\
\hline _TUI & $\begin{array}{r}295,52 \\
{[7,44]} \\
(0,00) \\
\end{array}$ & $\begin{array}{l}52,65 \\
{[1,97]} \\
(0,05) \\
\end{array}$ & $\begin{array}{r}-0,10 \\
{[-1,54]} \\
(0,12) \\
\end{array}$ & 0,11 & 15,90 & 0,17 & $\begin{array}{r}{[-1,98]} \\
(<=0,05)\end{array}$ \\
\hline _HEN & $\begin{array}{r}20,99 \\
{[26,31]} \\
(0,00)\end{array}$ & $\begin{array}{r}-0,03 \\
{[-3,96]} \\
(0,00)\end{array}$ & $\begin{array}{r}0,01 \\
{[5,18]} \\
(0,00)\end{array}$ & 0,19 & 45,15 & 0,04 & $\begin{array}{r}{[-5,55]} \\
(<=0,01)\end{array}$ \\
\hline
\end{tabular}

t-stats in [ ] and corresponding p-values in ( )

*Only those cross sections are reported, for whom $b_{1}$ is significant at the $5 \%$ level and the ADF residual test yields an error probability of less than $5 \%$ for the rejection of the null hypothesis of a unit root

**Phillips and Ouliaris (1990) p-values

Table 6.16.: Time-series regression results for the test of the relationship between default risk and implied volatility for a constant maturity of 36 days 


\begin{tabular}{|c|c|c|c|c|c|c|c|c|c|c|c|c|c|c|c|}
\hline \multicolumn{16}{|c|}{$C D S_{t}=\beta_{0}+\beta_{1} \cdot I M P V_{t}+\beta_{2} \cdot t+u_{t}$} \\
\hline $\mathbf{C S}^{*}$ & Variable & & $b_{0}$ & & & $b_{1}$ & & & $b_{3}$ & & $\begin{array}{l}\text { R2 } \\
\text { cor. }\end{array}$ & $\mathbf{F}$ & DW & & $A D F^{* *}$ \\
\hline \multicolumn{16}{|c|}{ Constant maturity of 36 days } \\
\hline Top 5 & IMPV & 14,3 & {$[1,0]$} & $(0,13)$ & $\mid 184,9$ & {$[15,6]$} & ] $(0,00)$ & $\mid-0,03$ & {$[-2,3]$} & $(0,18)$ & 0,86 & 2.740 & 0,11 & {$[-4,9]$} & $<=(0,02)$ \\
\hline All & IMPV & 53,8 & {$[7,1]$} & $(0,07)$ & 98,6 & {$[8,2]$} & $(0,12)$ & $-0,04$ & {$[-2,6]$} & $(0,10)$ & 0,60 & 1.110 & 0,19 & {$[-4,2]$} & $<=(0,29)$ \\
\hline \multicolumn{16}{|c|}{ Constant maturity of 60 days } \\
\hline Top 5 & IMPV & 8,7 & {$[-0,1]$} & $(0,19)$ & $\mid 196,8$ & {$[16,0]$} & $(0,00)$ & $\mid-0,02$ & {$[-2,0]$} & $(0,22)$ & 0,87 & 2.747 & 0,11 & {$[-4,5]$} & $<=(0,02)$ \\
\hline All & IMPV & 66,1 & {$[8,9]$} & $(0,13)$ & 75,5 & {$[5,9]$} & $(0,14)$ & $\mid-0,05$ & {$[-3,3]$} & $(0,09)$ & 0,54 & 949 & 0,15 & {$[-3,7]$} & $<=(0,25)$ \\
\hline \multicolumn{16}{|c|}{ Constant maturity of 180 days } \\
\hline Top 5 & IMPV & 77,3 & {$[5,1]$} & $(0,09)$ & 83,8 & {$[5,3]$} & $(0,11)$ & $\mid-0,09$ & {$[-5,3]$} & $(0,04)$ & 0,64 & 1.176 & 0,26 & {$[-3,8]$} & $<=(0,03)$ \\
\hline All & IMPV & 92,0 & {$[13,7]$} & $(0,05)$ & 26,8 & {$[2,1]$} & $(0,30)$ & $-0,07$ & {$[-5,5]$} & $(0,03)$ & 0,41 & 420 & 0,13 & {$[-2,8]$} & $<=(0,31)$ \\
\hline \multicolumn{16}{|c|}{ Constant maturity of 240 days } \\
\hline Top 5 & IMPV & 111,7 & {$[16,2]$} & $(0,00)$ & 10,3 & {$[2,0]$} & $(0,28)$ & $\mid-0,11$ & {$[-9,8]$} & $(0,00)$ & 0,49 & 483 & 0,05 & {$[-3,1]$} & $<=(0,05)$ \\
\hline All & IMPV & 102,9 & {$[16,8]$} & $(0,00)$ & 2,5 & {$[0,9]$} & $(0,31)$ & $\mid-0,08$ & {$[-6,6]$} & $(0,00)$ & 0,36 & 251 & 0,05 & {$[-2,5]$} & $<=(0,36)$ \\
\hline \multicolumn{16}{|c|}{ t-stats in [ ] and corresponding p-values in ( ) } \\
\hline \multirow{2}{*}{\multicolumn{16}{|c|}{ *Cross sections }} \\
\hline Phillips & ind Oul & & & & & & & & & & & & & & \\
\hline
\end{tabular}

Table 6.17.: Cross-sectional averages of time-series regression results for the test of the relationship between default risk and implied volatility 


\subsection{Examination in the Framework of a Pooled Series Regression}

The examination of the relationship between CDS spread and implied volatility according to model 6.47 has been carried out in the context of a pooled series regression for the five most important stocks. All constant maturity cases have been analyzed, but only the results for 36,60 and 180 days are reported in table 6.18 , since the examination for 240 days has not yielded any meaningful results. This deterioration of results with increasing constant maturity is consistent with the observations made before in the context of the time-series examination and the analysis of the leverage effect. As

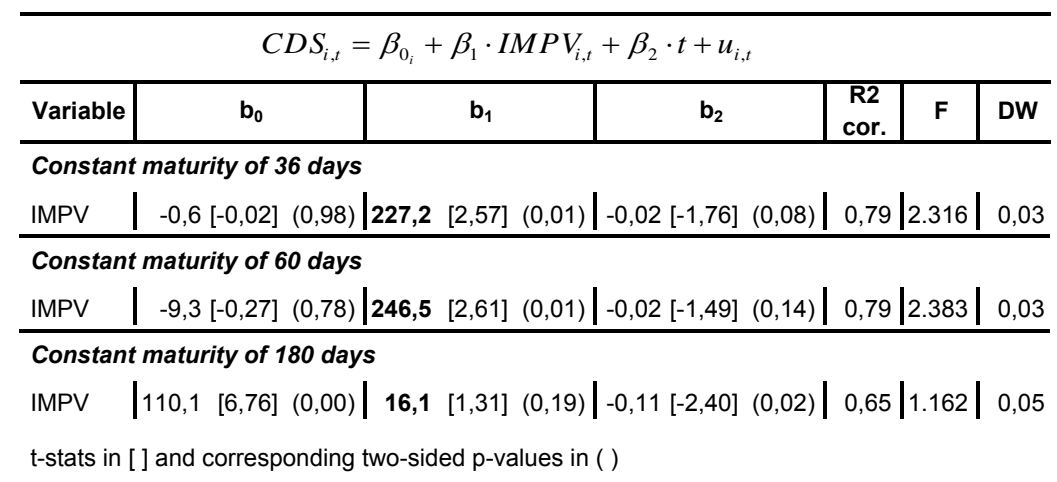

Table 6.18.: Pooled series regression results for the test of the relationship between default risk and implied volatility for constant maturities of 36, 60 and 180 days

can be seen in table 6.18, the coefficient $b_{1}$ is positive in all 3 constant maturity cases. However, it is only significant for 36 and 60 days. Further, a common time trend does not seem to be present in the constant maturity cases of 36 and 60 days as can be inferred from the high error probabilities for the coefficient $b_{2}$. Also the cross-section specific constant $b_{0}$ is highly insignificant for those 2 maturities. High significance of $b_{1}$ seems to be mirrored by low significance for the other coefficients $b_{0}$ and $b_{2}$ and vice versa.

Like above, the explanatory power $R^{2}$ is relatively high and the Durbin-Watson statistic DW is extremely low. The former could be partially induced by the cross-section specific, but insignificant intercepts and the latter could be associated with non-stationarity, which will be further explored in section 6.5.3.3.

Likewise as the cross-sectional analysis, the pooled series regression results confirm Hypothesis 2 subject to further robustness checks. 


\subsubsection{Robustness of the Regression Results}

6.5.3.3.1. Examination in the Time-Series Dimension The single series ADF tests in section 6.4.7.4nourished the suspicion that the series $C D S$ and $I M P$ could be non-stationary. Let us assume that this were indeed the case, then the above regression results would still be valid, if the two series were cointegrated. Economically it is well imaginable that this is the case, which means that $C D S$ and $I M P V$ show a common stochastic trend. To test for a possible cointegrating relationship, the Engle-Granger approach has been applied and the residuals of the time-series regressions above have been tested for the presence of a unit root with an ADF tes ${ }^{347}$. The results of the residual unit root tests are displayed in the rightmost column of tables 6.16 and 6.17 and can also be consulted in appendix C.2 for the totality of cross-sections. As can be read from table 6.17, the ADF residual test yields average error probabilities of less than or equal to $5 \%$ for the top 5 cross-sections. Therefore it can be concluded that the residuals are stationary and that $C D S$ and $I M P V$ are cointegrated, which validates the above regression results.

The robustness of the above results as to the inclusion of supplementary variables has been tested by independently adding $H V$ and $I M P V_{D A X}$ to model 6.46 .

$$
\begin{gathered}
C D S_{t}=\beta_{0}+\beta_{1} \cdot I M P V_{t}+\beta_{2} \cdot H V_{t}+\beta_{3} \cdot t+u_{t} \\
C D S_{t}=\beta_{0}+\beta_{1} \cdot I M P V_{t}+\beta_{2} \cdot I M P V_{D A X, t}+\beta_{3} \cdot t+u_{t}
\end{gathered}
$$

The inclusion of $H V$ does not only help to assess the significance of $I M P V$ in the presence of an alternative volatility measure. It also allows a comparison between the two measures as to their capacity to explain $C D S$. The results of this comparison are not only interesting in their own right, but will also serve another purpose later on. The inclusion of $I M P V_{D A X}$ on the other hand accounts for possible systematic influences on the relationship between default risk and the level of individual implied volatility. Table 6.19 juxtaposes the estimates $b_{1}$, the respective $p$-values and the respective ADF error probabilities for the estimation of the original model 6.46, the model including

347 The number of lagged terms in the test equation has been automatically set such that all serial correlation in the residuals has been removed, using a Schwarz information criterion, cf. Evi (2005), p. 521 and 527. This setting resulted in the number of lags varying between 1 and 15 . The test equation included an intercept and a time trend. The reason is that above, we assumed the possibility that both series $C D S$ and $I M P V$ exhibit a time trend. Consequently it is straightforward to likewise assume this for a linear combination of the two. $p$-values for the ADF $t$-statistics have been taken from PHILLIPS/OULIARIS (1990), since they are not provided by Evi (2005) for the case of an ADF residual test (see section 6.3.4.2.2. 
$H V 6.48$ and the model including $I M P V_{D A X} 6.49$ for a constant maturity of 36 days. Only those cross-sections are reported, which yielded significant coefficients $b_{1}$ and ADF error probabilities of less than or equal to $5 \%$ when estimating the original model. As can be seen in table 6.19, in all cases the sign of the originally positive

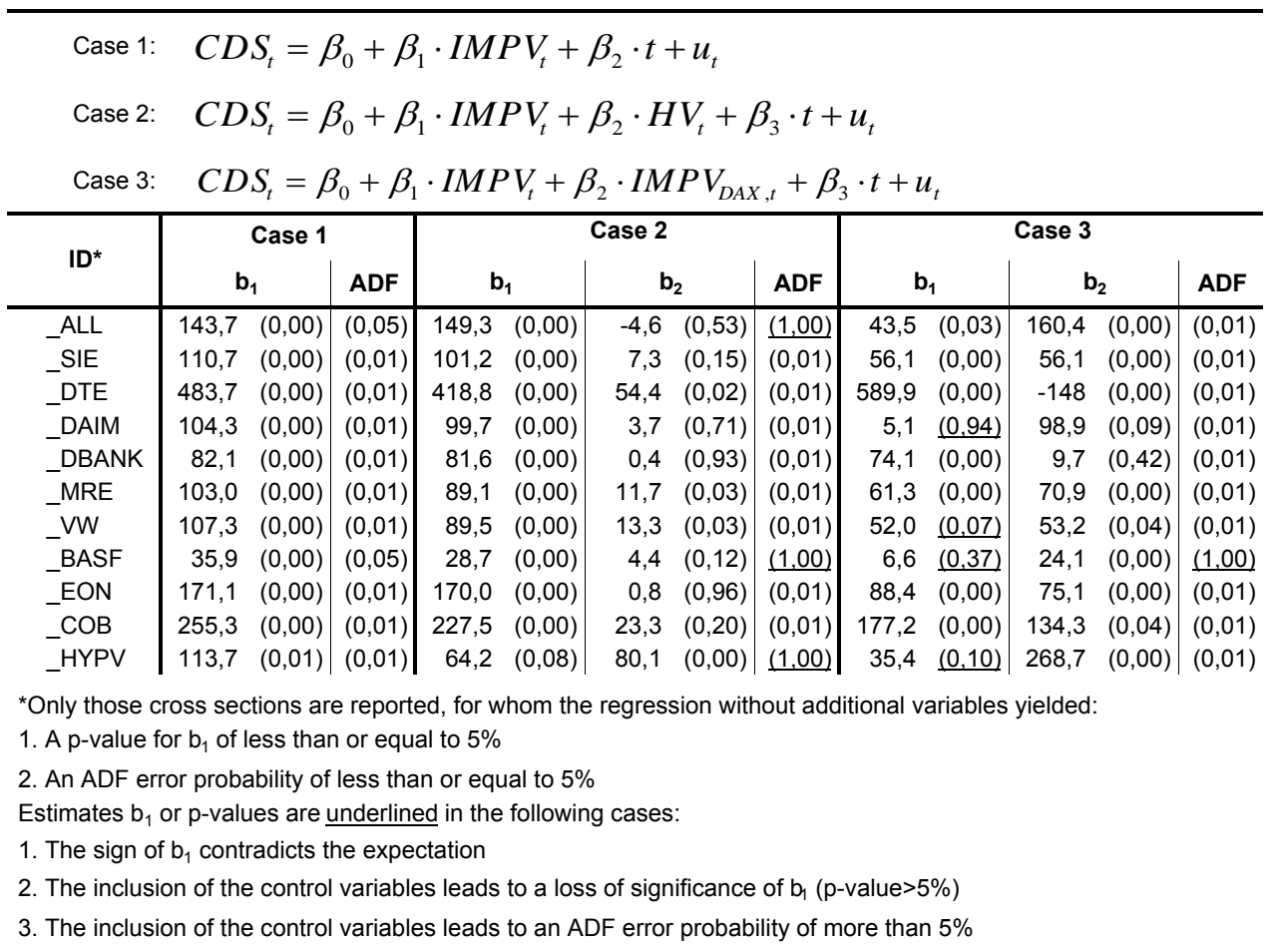

Table 6.19.: Robustness check of the time-series regression results for the test of the relationship between default risk and implied volatility for a constant maturity of 36 days

coefficient $b_{1}$ remains unchanged. However, in 3 cases the inclusion of $H V$ leads to an inadmissible increase of the ADF error probabilities. The inclusion of $I M P V_{D A X}$ in contrast only leads to one insignificant ADF test result, but additionally produces a loss of significance of $b_{1}$ in 4 cases. Comparing $H V$ and $I M P V_{D A X}$, it can be seen that the coefficient $b_{2}$ is significant in almost all cases for $I M P V_{D A X}$, while the same is not true for $H V$.

Altogether, it can be concluded that systematic implied volatility does matter for the explanation of the individual CDS spread, but does not dominate the influence of individual implied volatility. Further, $I M P V$ seems to be a better measure of volatility than $H V$ as far as the explanation of the CDS spread level is concerned. 


\subsection{Examination in the Framework of a Pooled Series Regression}

In the pooled series framework, the stability as to the inclusion of further variables and the degree of stationarity have been assessed successively, on top of each other and in inverse order as compared to the time-series case. First and analogue to the analysis in the time-series dimension, $H V$ and $I M P V_{D A X}$ have been independently included as additional variables into the original model of 6.47

$$
\begin{gathered}
C D S_{i, t}=\beta_{0 i}+\beta_{1} \cdot I M P V_{i, t}+\beta_{2} \cdot H V_{i, t}+\beta_{3} \cdot t+u_{i, t} \\
C D S_{i, t}=\beta_{0 i}+\beta_{1} \cdot I M P V_{i, t}+\beta_{2} \cdot I M P V_{D A X, t}+\beta_{3} \cdot t+u_{i, t}
\end{gathered}
$$

Models 6.50 and 6.51 have been tested for the most meaningful constant maturity cases of 36, 60 and 180 days. The results are displayed in table 6.20. Comparing

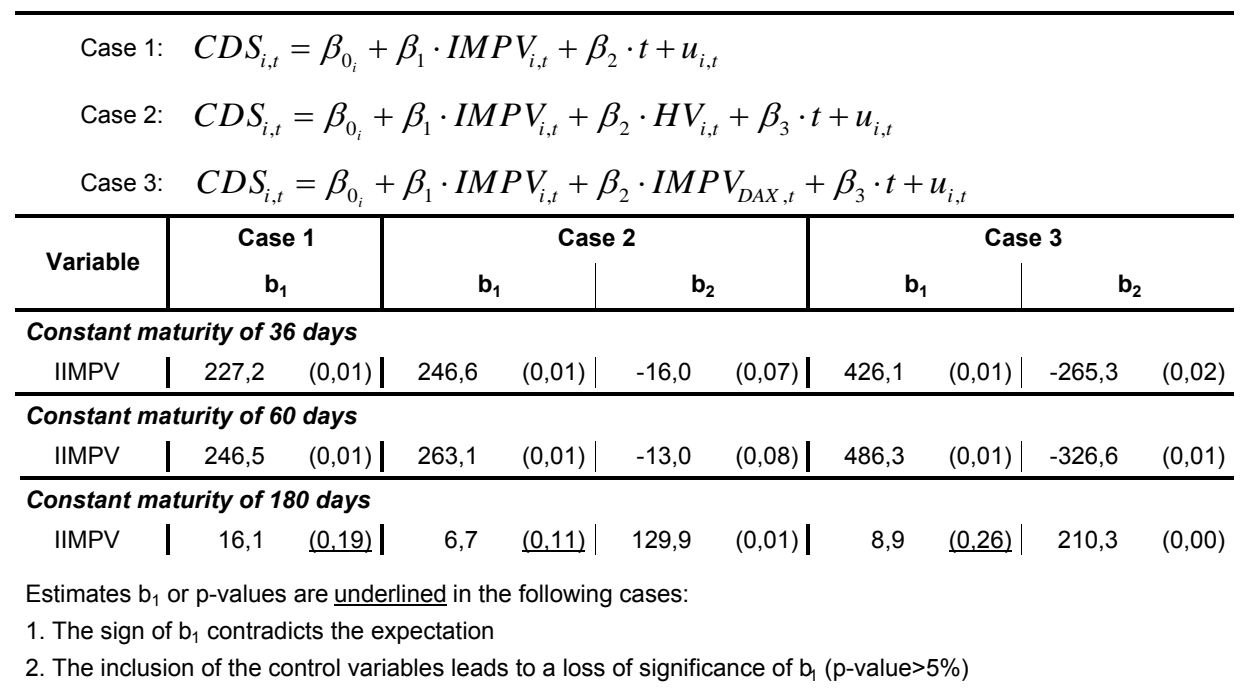

Table 6.20.: Robustness check of the pooled series regression results for the test of the relationship between default risk and implied volatility

tables 6.18 and 6.20 , it can be seen that the sign and the significance of $b_{1}$ remain unchanged after the inclusion of $H V$ and $I M P V_{D A X}$, respectively. This is true for the originally significant constant maturities of 36 and 60 days as well as for the originally insignificant case of 180 days. For 36 and 60 days, the absolute value of $b_{1}$ has even increased and the significance of $b_{1}$ is higher than that of $b_{2}$ in both cases. Noteworthily, $b_{2}$ is negative for both $H V$ and $I M P V_{D A X}$ in both constant maturity cases, which hints at the strong explanatory power of the idiosyncratic portion of $I M P V$. Further, it stands out that $b_{2}$ is smaller in absolute value and less significant in the case of $H V$ as compared to the case of $I M P V_{D A X}$. 
Altogether, and likewise as for the time-series robustness check, it can be concluded that $H V$ is not as powerful in explaining $C D S$ as is $I M P V$, and moreover that systematic influences in the guise of $I M P V_{D A X}$ seem to matter but are far from dominating the individual implied volatility $I M P V$.

As to the issue of possible non-stationarity, the test for a conintegrating relationship as carried out in the case of the time-series examination does not make sense economically in the case of the pooled series approach. Admittedly, it is well possible that a cointegrating relationship exists between the series $C D S$ and $I M P V$ on a single firm basis. However, such a relationship is economically less likely between all involved series, if the 5 most important firms are pooled together, since it would mean, that all 5 firms follow a common stochastic trend. Therefore, within the pooled series context, the issue of non-stationarity has not been approached by a test for cointegration as carried out in the time-series setting. Instead, the possible non-stationarity of $C D S$ and $I M P V$ has been assessed by resorting to a panel unit root test. The panel setting allows to exploit the superior unit root testing power of the panel unit root test as compared to the single series test. Panel unit root tests have been carried out for all series involved in the case of the original pooled series regression and in the case of the robustness check including $H V$ and in that including $I M P V_{D A X}$, respectively. The results are displayed in table 6.21, which is partitioned accordingly into the 3 cases. For all 3 constant maturities of 36, 60 and 180 days and in all three test cases the null hypothesis of the Hadri-test of the absence of a unit root must be rejected. However, for the other 5 tests featuring the null hypothesis of the presence of a unit root, a unit root can be rejected at a vote of at least 4 to 1 . Altogether, these results can be interpreted as a majority vote in favor of stationarity, thus validating the above findings.

In summary, it can be put on record that the robustness check of the pooled series regression results confirms the positive relationship between CDS spread level and the level of implied volatility. 


\begin{tabular}{|c|c|c|c|c|c|c|}
\hline & \multicolumn{2}{|c|}{ Case $1^{*}$} & \multicolumn{2}{|c|}{ Case $2^{*}$} & \multicolumn{2}{|c|}{ Case $3^{*}$} \\
\hline & Stat & Prob $^{* *}$ & Stat & Prob $^{* *}$ & Stat & Prob $^{* *}$ \\
\hline \multicolumn{7}{|l|}{ Constant maturity of 36 days } \\
\hline \multicolumn{7}{|c|}{ Null: Unit root (assumes common unit root process) } \\
\hline Levin, Lin \& Chu t* & $-4,5$ & 0,00 & $-5,7$ & 0,00 & $-5,4$ & 0,00 \\
\hline Breitung t-stat & $-2,4$ & 0,01 & $-3,0$ & 0,00 & $-2,7$ & 0,00 \\
\hline \multicolumn{7}{|c|}{ Null: Unit root (assumes individual unit root process) } \\
\hline Im, Pesaran and Shin W-stat & $-3,0$ & 0,00 & $-5,3$ & 0,00 & $-4,9$ & 0,00 \\
\hline ADF - Fisher Chi-square & 39,7 & 0,01 & 82,5 & 0,00 & 78,4 & 0,00 \\
\hline PP - Fisher Chi-square & 29,9 & $\underline{0,07}$ & 326,7 & 0,00 & 324,6 & 0,00 \\
\hline \multicolumn{7}{|c|}{ Null: No unit root (assumes common unit root process) } \\
\hline Hadri Z-stat & 17,7 & $\underline{0,00} \mid$ & 21,6 & $\underline{0,00} \mid$ & 21,6 & $\underline{0,00}$ \\
\hline \multicolumn{7}{|l|}{ Constant maturity of 60 days } \\
\hline \multicolumn{7}{|c|}{ Null: Unit root (assumes common unit root process) } \\
\hline Levin, Lin \& Chu t* & $-4,3$ & 0,00 & $-4,8$ & 0,00 & $-4,4$ & 0,00 \\
\hline Breitung t-stat & $-2,1$ & 0,02 & $-2,6$ & 0,00 & $-2,3$ & 0,01 \\
\hline \multicolumn{7}{|c|}{ Null: Unit root (assumes individual unit root process) } \\
\hline Im, Pesaran and Shin W-stat & $-2,5$ & 0,01 & $-3,1$ & 0,00 & $-2,5$ & 0,01 \\
\hline ADF - Fisher Chi-square & 35,7 & 0,02 & 42,7 & 0,01 & 37,8 & 0,02 \\
\hline PP - Fisher Chi-square & 27,8 & $\underline{0,11}$ & 31,4 & $\underline{0,09}$ & 29,1 & $\underline{0,14}$ \\
\hline \multicolumn{7}{|c|}{ Null: No unit root (assumes common unit root process) } \\
\hline Hadri Z-stat & 17,7 & $\underline{0,00}$ & 18,5 & $\underline{0,00}$ & 18,5 & $\underline{0,00}$ \\
\hline \multicolumn{7}{|l|}{ Constant maturity of 180 days } \\
\hline \multicolumn{7}{|c|}{ Null: Unit root (assumes common unit root process) } \\
\hline Levin, Lin \& Chu t* & $-1,8$ & 0,03 & $-2,3$ & 0,01 & $-1,9$ & 0,03 \\
\hline Breitung t-stat & $-3,1$ & 0,00 & $-4,0$ & 0,00 & $-3,2$ & 0,00 \\
\hline \multicolumn{7}{|c|}{ Null: Unit root (assumes individual unit root process) } \\
\hline Im, Pesaran and Shin W-stat & $-11,7$ & 0,01 & $-11,3$ & 0,00 & $-11,1$ & 0,00 \\
\hline ADF - Fisher Chi-square & 239,1 & 0,02 & 265,1 & 0,01 & 240,4 & 0,00 \\
\hline PP - Fisher Chi-square & 491,4 & $\underline{0,11}$ & 649,3 & $\underline{0,09}$ & 538,9 & 0,00 \\
\hline \multicolumn{7}{|l|}{ Null: No unit root (assumes comm } \\
\hline Hadri Z-stat & 47,7 & $\underline{0,00} \mid$ & 18,5 & $\underline{0,00} \mid$ & 50,0 & $\underline{0,00}$ \\
\hline
\end{tabular}

${ }^{*}$ Panel unit root test for all cross section / series combinations of the cross sections

ALL, SIE, DDTE, DAIM and_DBANK and the series:

Case 1: CDS, IMPV

Case 2: CDS, IMPV and HV

Case 3: CDS, IMPV IMPV DAX

**Probabilities for Fisher tests are computed using an asympotic Chi-square distribution. All other tests assume asymptotic normality.

Probabilities are underlined, if either the presence of a unit root cannot be rejected with an error probability of less than or equal to $5 \%$, or if the absence of a unit root can be rejected with an error probability of less than or equal to $5 \%$.

Table 6.21.: Results of the panel unit root tests for the robustness check of the pooled series regression results for the test of the relationship between default risk and implied volatility 


\subsubsection{Summary and Discussion of the Results in the Light of Hitherto Existing Findings}

6.5.3.4.1. Summary of the Results The above analysis confirms Hypothesis 2 of a positive relationship between default risk and the level of implied volatility. This relationship has been demonstrated to exist in the time-series as well as in the cross-sectional dimension, the latter at least for the 5 most important stocks. The above results have further been shown to be stable also in the presence of important additional variables and to be valid, even if possible non-stationarity is taken into account: It can either be argued that a cointegrating relationship exists as in the timeseries setting, or it can be claimed that non-stationarity is not present at all as suggested by the panel unit root tests 348 .

\subsection{Discussion of the Results in the Light of Hitherto Existing Find-} ings The results are well in line with the hitherto existing findings which have been discussed in section 5.1.3.2. The most powerful results in the literature for the linkage between equity volatility and default risk have been established by proxying the former with the level of implied volatility and the latter by the CDS spread level as has been done in my analysis above. In turn, among these studies the most powerful relation has been detected by CAO ET AL. (2006), who reports a cross-sectional average $R^{2}$ of $56 \%$ for the regression of the CDS spread level on the level of implied volatility. This compares with my finding of a cross-sectional average of $R^{2}$ of $86 \%$ for the top 5 and of $60 \%$ for all 22 stocks in the constant maturity case of 36 days. The superiority of my finding could have to do with my relatively high data quality 349 or also with the implied volatility measure in CAO ET AL. (2006) ${ }^{350}$. Further, as the inclusion of the historical volatility measure in the context of the robustness check revealed, implied volatility seems indeed to be the better of the two variables when it comes to explaining the CDS spread level. This finding is consistent with the fact

348 This does not imply a contradiction, since it is well possible that the series as well as the residuals are both stationary. Then a cointegrating relationship exists and a panel unit root can be rejected.

349 CAO ET AL. (2006) construct averages accross 220 firms in the United States. Forcibly, the average option trading volume and ensuing data quality cannot be as high as in my case focusing on just 22 firms which rank among those with the highest option trading volume.

350 CAO ET AL. (2006) also use all available option prices to calculate their implied volatility measure, but do not use the ATM implied volatility like I do. Instead, they compute an average across all available strike-to-spot ratios. 
that the most powerful linkage between default risk and equity volatility is reported by studies using implied volatility instead of historical volatility ${ }^{351}$

In summary, it can be retained that my finding of a positive relationship between the CDS spread level and the level of implied volatility is consistent with the comparable literature. In addition, it represents a value added to the existing literature in that the issue of non-stationarity has been addressed without differencing and further in that very high values of $R^{2}$ have been achieved.

351 CAO ET AL. (2006) for example compute an average cross-sectional $R^{2}$ of only $36 \%$ for the regression of the CDS spread level on the level of historical volatility as compared to $56 \%$ for the regression on the level of implied volatility. 


\subsubsection{The Credit Spread Level and the Shape of the Equity Volatility Smile}

This section engages in the test of Hypothesis 3: The slope of the equity volatility smile depends negatively on the level of default risk. However, in the light of our preliminary findings in connection with the smile approximation (see section 6.4.5), Hypothesis 3 has to be refined as we will see.

\subsubsection{Derivation of the Regression Models}

Hypothesis 3 has mainly been derived from the theoretical predictions regarding the relationship between default risk and the shape of the smile (see section 5.1.2.2) and therefore implies the existence of a default risk induced downward sloping smile which is monotonically decreasing and convex in the strike price. Further, according to Hypothesis 3, this smile should be all the more steeper, the higher the level of default risk. However, as suggested by the preliminary analysis of section 6.4.5 and as confirmed by the summary statistics of the variables in section 6.4.7.3.2, the smile seems instead to be U-shaped and not downward sloping. And what is more, the preliminary analysis of section 6.4 .5 showed that default risk seems to lead to a flattening and right-translation instead of a steepening of the smile.

This obvious contradiction can only be resolved by further thorough examination. To this end, the relationship between credit spread level and equity volatility smile has first been examined by regressing $C D S$ on the smile coefficients $B 0, B 1$ and $B 2$, since each coefficient influences the smile pattern in a specific way. Each coefficient's influence on $C D S$ has been tested separately for 2 reasons: First, the influence of the totality of the three smile coefficients has already been tested by the models 6.46 and 6.47, since the variable $I M P V$ is nothing else than the sum of the three coefficients $B 0, B 1$ and $B 2$. And secondly, a simultaneous regression on all three coefficients could possibly blur the results due to probable construction related interdependencies between the coefficients. The relationship between $C D S$ and each of the smile coefficients $B 0, B 1$ and $B 2$ has been examined in the time-series dimension as well as in the pooled series context:

Time-series dimension:

$$
C D S_{t}=\beta_{0}+\beta_{1} \cdot B j_{t}+\beta_{2} \cdot t+u_{t}, \quad j \in(0,1,2)
$$




\section{Pooled series regression:}

$$
C D S_{i, t}=\beta_{0 i}+\beta_{1} \cdot B j_{i, t}+\beta_{2} \cdot t+u_{i, t}, \quad j \in(0,1,2)
$$

Following Hypothesis 3, we should expect a negative coefficient for the linear term $B 1$. Regarding the coefficient $B 2$, we should expect that this coefficient is zero or slightly positive owed to the assumed convexity of the smile. But as opposed to Hypothesis 3, our preliminary finding of a default risk induced flattening and righttranslation of the smile suggests slightly other expectations: Increasing CDS spread levels should be associated with a decreasing influence of the quadratic term, an increasing influence of the linear term and a decreasing influence of the constant term. Consequently, if we believe our preliminary findings, we should expect negative signs for the coefficients of $B 0$ and $B 2$ and a positive sign for the coefficient of $B 1$.

To further test the relationship between smile pattern and default risk, also the slope of the smile has been examined by directly regressing $C D S$ on the slope variables $S L P j$. In addition, the smile's horizontal behavior has been analyzed by regressing $C D S$ on $X S M I N$. Since a joint regression on the collectivity of the variables $S L P 70$, SLP90, SLP100, SLP110, SLP130 and XSMIN together would probably yield misleading results or would even be impossible due to likely high multicollinearity, the influence of each variable has been tested separately in the time-series dimension as well as in the pooled series setting:

\section{Time-series dimension:}

$$
\begin{gathered}
C D S_{t}=\beta_{0}+\beta_{1} \cdot S L P j_{t}+\beta_{2} \cdot t+u_{t}, \quad j \in(70,90,100,110,130) \\
C D S_{t}=\beta_{0}+\beta_{1} \cdot X S M I N_{t}+\beta_{2} \cdot t+u_{t}
\end{gathered}
$$

\section{Pooled series regression:}

$$
\begin{gathered}
C D S_{i, t}=\beta_{0 i}+\beta_{1} \cdot S L P j_{i, t}+\beta_{2} \cdot t+u_{i, t}, \quad j \in(70,90,100,110,130) \\
C D S_{i, t}=\beta_{0 i}+\beta_{1} \cdot X S M I N_{i, t}+\beta_{2} \cdot t+u_{i, t}
\end{gathered}
$$

As to our expectations regarding the signs of the slope coefficients, we again have to distinguish between what is implied by Hypothesis $\mathbf{3}$ on the one hand and what should be expected due to our preliminary findings on the other: According to Hypothesis 3 we should expect negative sings for all slope coefficients. Due to the convex, downward sloping nature of the smile implied by Hypothesis 3, the coefficients should be 
highest in absolute value on the left wing of the slope and decrease in absolute value with increasing strike-to-spot-price ratio. However, these expectations are largely incompatible with our preliminary observation of a default risk associated flattening and right-translation of the smile. Relying on these preliminary findings, we should instead expect an increase of the slope of the left wing of the smile, which would result in a positive coefficient for $S L P 70$ and $S L P 90$. Further, the right-translation should manifest itself in a positive coefficient of the horizontal coordinate XSMIN. Moreover, due to the right-translation, the largely positive slope of the right wing of the smile should decrease with increasing default risk, which should entail a negative sign for the coefficients of $S L P 110$ and $S L P 130$. As to the combined effect of flattening and right-translation on $S L P 100$ we should be indifferent, because this effect is difficult to predict.

Throughout the following analysis, expectations as to the sign of $b_{1}$ will always be formulated based on what is suggested by our preliminary findings of section 6.4.5 and not on what insinuates Hypothesis 3.

\subsubsection{Examination of the Relationship between the CDS Spread Level and the Smile Coefficients}

This section is dedicated to the test of the relationship between $C D S$ and the smile coefficients $B 0, B 1$ and $B 2$ by estimating and validating the regression models 6.52 and 6.53 in the time-series dimension as well as in the pooled series context.

\subsection{Regression Results}

6.5.4.2.1. Examination in the Time-Series Dimension The test of the relationship between $C D S$ and the smile coefficients $B 0, B 1$ and $B 2$ according to model 6.52 has been carried out for all stocks except $D E G$ due to an insufficient number of observations. Tables 6.22, 6.23 and 6.24 show the detailed regression results for the constant maturity case of 36 days, whereas table 6.17 exhibits a constant-maturitywise cross-sectional summary of all results, once averaged across all cross-sections and once only across the 5 most important stocks. Only the constant maturity case of 240 days has been excluded, since it has not yielded any meaningful results. In order to keep this documentation as lean and readable as possible, tables 6.22, 6.23, 6.24 only report those cross-sections for whom the coefficient $b_{1}$ is significant at the $1 \%$ 
level. The totality of detailed results for all cross-sections and all constant maturities except 240 days can be viewed in appendix C.3.1.

\begin{tabular}{|c|c|c|c|c|c|c|}
\hline \multicolumn{7}{|c|}{$C D S_{t}=\beta_{0}+\beta_{1} \cdot B 0_{t}+\beta_{2} \cdot t+u_{t}$} \\
\hline ID $^{*}$ & $b_{0}$ & $b_{1}$ & $b_{2}$ & R2 cor. & $F$ & DW \\
\hline _ALL & $\begin{array}{r}77,34 \\
{[12,85]} \\
(0,00)\end{array}$ & $\begin{array}{r}-9,09 \\
{[-4,41]} \\
(0,00)\end{array}$ & $\begin{array}{r}-0,03 \\
{[-3,02]} \\
(0,00)\end{array}$ & 0,27 & 137,16 & 0,08 \\
\hline _DBANK & $\begin{array}{r}39,73 \\
{[14,33]} \\
(0,00)\end{array}$ & $\begin{array}{r}-1,89 \\
{[-2,59]} \\
(0,01)\end{array}$ & $\begin{array}{r}-0,02 \\
{[-5,76]} \\
(0,00)\end{array}$ & 0,30 & 162,90 & 0,03 \\
\hline _MRE & $\begin{array}{r}60,91 \\
{[14,82]} \\
(0,00)\end{array}$ & $\begin{array}{r}-2,10 \\
{[-3,34]} \\
(0,00)\end{array}$ & $\begin{array}{r}-0,04 \\
{[-5,36]} \\
(0,00)\end{array}$ & 0,34 & 176,10 & 0,21 \\
\hline BASF & $\begin{array}{r}30,90 \\
{[39,61]} \\
(0,00)\end{array}$ & $\begin{array}{r}-0,02 \\
{[-5,58]} \\
(0,00)\end{array}$ & $\begin{array}{r}-0,03 \\
{[-20,48]} \\
(0,00)\end{array}$ & 0,75 & $1.124,78$ & 0,04 \\
\hline _LH & $\begin{array}{r}155,86 \\
{[24,22]} \\
(0,00)\end{array}$ & $\begin{array}{r}0,00 \\
{[-3,71]} \\
(0,00)\end{array}$ & $\begin{array}{r}-0,12 \\
{[-11,21]} \\
(0,00)\end{array}$ & 0,43 & 268,08 & 0,01 \\
\hline _TYSS & $\begin{array}{r}205,46 \\
{[17,36]} \\
(0,00)\end{array}$ & $\begin{array}{r}0,01 \\
{[4,14]} \\
(0,00)\end{array}$ & $\begin{array}{r}-0,14 \\
{[-7,23]} \\
(0,00)\end{array}$ & 0,28 & 129,06 & 0,03 \\
\hline
\end{tabular}

Table 6.22.: Time-series regression results for the test of the relationship between default risk and the smile coefficient $B 0$ for a constant maturity of 36 days

As can be read from tables 6.22, 6.23 and 6.24 displaying the highly significant crosssections, the coefficient of $B 0$ and $B 2$ is largely negative, whereas the coefficient of $B 1$ is largely positive. This picture is confirmed by table 6.25 for $B 1$ and $B 2$. For $B 0$ however, it is only confirmed in the case of a constant maturity of 36 days; in the cases of the higher maturities in contrast, the coefficient of $B 0$ changes the sign and becomes positive. If we concentrate on the cases in table 6.25 which yield on average a significant coefficient $b_{1}$, that is the case of a constant maturity of 36 days and the summary of the top 5 stocks, then the result is almost congruent with the detailed representation of the highly significant cross sections in tables 6.22, 6.23 and 6.24. Further, if we stretch the notion of significance a little bit and interpret the error probability of $7 \%$ for the coefficient of $B 0$ as still being admissible, we end up with even perfect congruence between the detailed and the summary representation. This result of a negative coefficient for $B 0$ and $B 2$ and a positive one for $B 1$ rather confirms the findings of our preliminary analysis above than the predictions implied by Hypothesis 3.

What is further noteworthy is that the assumption of an intercept and a time-trend seems to be justified considering the significance of the coefficients $b_{0}$ and $b_{2}$. Moreover, it is worthwhile mentioning the extremely low Durbin-Watson statistic, which 


\begin{tabular}{|c|c|c|c|c|c|c|}
\hline \multicolumn{7}{|c|}{$C D S_{t}=\beta_{0}+\beta_{1} \cdot B 1_{t}+\beta_{2} \cdot t+u_{t}$} \\
\hline ID* & $b_{0}$ & $b_{1}$ & $b_{2}$ & R2 cor. & $\mathbf{F}$ & DW \\
\hline ALL & $\begin{array}{r}74,22 \\
{[14,58]} \\
(0,00) \\
\end{array}$ & $\begin{array}{r}6,33 \\
{[5,95]} \\
(0,00) \\
\end{array}$ & $\begin{array}{r}-0,02 \\
{[-1,98]} \\
(0,05) \\
\end{array}$ & 0,37 & 211,07 & 0,17 \\
\hline _SIE & $\begin{array}{r}68,08 \\
{[23,21]} \\
(0,00) \\
\end{array}$ & $\begin{array}{r}2,13 \\
{[3,67]} \\
(0,00)\end{array}$ & $\begin{array}{r}-0,05 \\
{[-7,99]} \\
(0,00) \\
\end{array}$ & 0,62 & 609,36 & 0,07 \\
\hline DAIM & $\begin{array}{r}162,24 \\
{[42,19]} \\
(0,00)\end{array}$ & $\begin{array}{r}1,33 \\
{[2,64]} \\
(0,01)\end{array}$ & $\begin{array}{r}-0,11 \\
{[-14,74]} \\
(0,00)\end{array}$ & 0,72 & 966,66 & 0,09 \\
\hline DBANK & $\begin{array}{r}40,98 \\
{[15,33]} \\
(0,00) \\
\end{array}$ & $\begin{array}{r}1,95 \\
{[4,12]} \\
(0,00) \\
\end{array}$ & $\begin{array}{r}-0,02 \\
{[-4,75]} \\
(0,00) \\
\end{array}$ & 0,35 & 204,01 & 0,10 \\
\hline _MRE & $\begin{array}{r}59,67 \\
{[15,01]} \\
(0,00)\end{array}$ & $\begin{array}{r}1,52 \\
{[4,21]} \\
(0,00)\end{array}$ & $\begin{array}{r}-0,04 \\
{[-4,86]} \\
(0,00)\end{array}$ & 0,37 & 196,37 & 0,26 \\
\hline BASF & $\begin{array}{r}30,89 \\
{[39,61]} \\
(0,00) \\
\end{array}$ & $\begin{array}{r}0,01 \\
{[5,01]} \\
(0,00)\end{array}$ & $\begin{array}{r}-0,03 \\
{[-20,48]} \\
(0,00)\end{array}$ & 0,75 & $1.126,17$ & 0,04 \\
\hline LLH & $\begin{array}{r}155,86 \\
{[24,22]} \\
(0,00) \\
\end{array}$ & $\begin{array}{r}0,00 \\
{[3,72]} \\
(0,00)\end{array}$ & $\begin{array}{r}-0,12 \\
{[-11,20]} \\
(0,00)\end{array}$ & 0,43 & 268,09 & 0,01 \\
\hline _TYSS & $\begin{array}{r}205,46 \\
{[17,36]} \\
(0,00)\end{array}$ & $\begin{array}{r}0,00 \\
{[-4,09]} \\
(0,00)\end{array}$ & $\begin{array}{r}-0,14 \\
{[-7,23]} \\
(0,00)\end{array}$ & 0,28 & 129,05 & 0,03 \\
\hline & and corr & onding & alues in & & & \\
\hline
\end{tabular}

Table 6.23.: Time-series regression results for the test of the relationship between default risk and the smile coefficient $B 1$ for a constant maturity of 36 days

hints at auto-correlated residuals which could be caused by non-stationarity. But since only one candidate series for non-stationarity is present in the regression, namely $C D S$, spurious regression need not concern us here.

In summary, the regression has confirmed our preliminary finding that the constant and the quadratic term of the smile polynomial are negatively associated with default risk, whereas the linear term is positively connected with the CDS spread level. Hypothesis 3 in contrast does not seem to be supported by the above findings. 


\begin{tabular}{|c|c|c|c|c|c|c|}
\hline \multicolumn{7}{|c|}{$C D S_{t}=\beta_{0}+\beta_{1} \cdot B 2_{t}+\beta_{2} \cdot t+u_{t}$} \\
\hline ID* & $b_{0}$ & $b_{1}$ & $b_{2}$ & R2 cor. & $F$ & DW \\
\hline \multirow[t]{3}{*}{-ALL } & 72,38 & $-13,25$ & $-0,02$ & \multirow[t]{3}{*}{0,38} & \multirow[t]{3}{*}{227,31} & \multirow[t]{3}{*}{0,19} \\
\hline & {$[14,99]$} & {$[-6,20]$} & {$[-1,75]$} & & & \\
\hline & $(0,00)$ & $(0,00)$ & $(0,08)$ & & & \\
\hline \multirow[t]{3}{*}{ _SIE } & 67,83 & $-4,99$ & $-0,05$ & \multirow[t]{3}{*}{0,63} & \multirow[t]{3}{*}{632,31} & \multirow[t]{3}{*}{0,10} \\
\hline & {$[23,74]$} & {$[-4,08]$} & {$[-7,69]$} & & & \\
\hline & $(0,00)$ & $(0,00)$ & $(0,00)$ & & & \\
\hline \multirow[t]{3}{*}{ _DAIM } & 161,90 & $-2,86$ & $-0,11$ & \multirow[t]{3}{*}{0,72} & \multirow[t]{3}{*}{972,12} & \multirow[t]{3}{*}{0,10} \\
\hline & {$[42,79]$} & {$[-2,74]$} & {$[-14,53]$} & & & \\
\hline & $(0,00)$ & $(0,01)$ & $(0,00)$ & & & \\
\hline \multirow[t]{3}{*}{ _DBANK } & 40,47 & $-4,28$ & $-0,02$ & \multirow[t]{3}{*}{0,36} & \multirow[t]{3}{*}{218,17} & \multirow[t]{3}{*}{0,12} \\
\hline & {$[16,09]$} & {$[-4,44]$} & {$[-4,42]$} & & & \\
\hline & $(0,00)$ & $(0,00)$ & $(0,00)$ & & & \\
\hline \multirow[t]{3}{*}{-MRE } & 59,13 & $-3,26$ & $-0,04$ & \multirow[t]{3}{*}{0,38} & \multirow[t]{3}{*}{201,17} & \multirow[t]{3}{*}{0,27} \\
\hline & {$[15,01]$} & {$[-4,38]$} & {$[-4,73]$} & & & \\
\hline & $(0,00)$ & $(0,00)$ & $(0,00)$ & & & \\
\hline \multirow[t]{3}{*}{ BASF } & 30,88 & $-0,03$ & $-0,03$ & \multirow[t]{3}{*}{0,75} & \multirow[t]{3}{*}{$1.126,54$} & \multirow[t]{3}{*}{0,04} \\
\hline & {$[39,60]$} & {$[-4,80]$} & {$[-20,48]$} & & & \\
\hline & $(0,00)$ & $(0,00)$ & $(0,00)$ & & & \\
\hline \multirow[t]{3}{*}{ _LH } & 155,86 & 0,00 & $-0,12$ & \multirow[t]{3}{*}{0,43} & \multirow[t]{3}{*}{268,09} & \multirow[t]{3}{*}{0,01} \\
\hline & {$[24,22]$} & {$[-3,72]$} & {$[-11,20]$} & & & \\
\hline & $(0,00)$ & $(0,00)$ & $(0,00)$ & & & \\
\hline \multirow[t]{3}{*}{ _TYSS } & 205,45 & 0,01 & $-0,14$ & 0,28 & 129,05 & 0,03 \\
\hline & {$[17,36]$} & {$[4,04]$} & {$[-7,23]$} & & & \\
\hline & $(0,00)$ & $(0,00)$ & $(0,00)$ & & & \\
\hline _HEN & 20,99 & 0,00 & 0,01 & 0,19 & 45,16 & 0,04 \\
\hline & {$[26,25]$} & {$[-2,61]$} & {$[5,18]$} & & & \\
\hline & $(0,00)$ & $(0,01)$ & $(0,00)$ & & & \\
\hline & & ding & alus in & & & \\
\hline & & & & & & \\
\hline
\end{tabular}

Table 6.24.: Time-series regression results for the test of the relationship between default risk and the smile coefficient $B 2$ for a constant maturity of 36 days 


\begin{tabular}{|c|c|c|c|c|c|c|c|c|c|c|}
\hline \multicolumn{11}{|c|}{$C D S_{t}=\beta_{0}+\beta_{1} \cdot B j_{t}+\beta_{2} \cdot t+u_{t}, \quad j \in\{0,1,2\}$} \\
\hline $\mathbf{C S}^{*}$ & Variable & & $b_{0}$ & & $b_{1}$ & & $\mathbf{b}_{3}$ & $\begin{array}{c}\mathbf{R} 2 \\
\text { cor. }\end{array}$ & $\mathbf{F}$ & DW \\
\hline \multicolumn{11}{|c|}{ Constant maturity of 36 days } \\
\hline Top 5 & B0 & 122,2 & {$[21,2]$} & $(0,00)$ & $\mid-3,06[-2,39]$ & $(0,07)$ & $-0,11[-9,00](0,00)$ & 0,50 & 484,6 & 0,05 \\
\hline Top 5 & B1 & 122,0 & {$[22,4]$} & $(0,00)$ & $2,53[3,58]$ & $(0,03)$ & $-0,10[-8,05](0,01)$ & 0,53 & 523,1 & 0,09 \\
\hline Top 5 & B2 & 121,4 & {$[22,9]$} & $(0,00)$ & $-5,48[-3,79]$ & $(0,03)$ & $-0,10[-7,82](0,02)$ & 0,54 & 535,2 & 0,11 \\
\hline All & B0 & 105,6 & {$[18,0]$} & $(0,00)$ & $-0,83[-1,29]$ & $(0,23)$ & $-0,08[-6,41](0,00)$ & 0,36 & 251,6 & 0,05 \\
\hline All & B1 & 105,6 & {$[18,3]$} & $(0,00)$ & $0,67[1,65]$ & $(0,23)$ & $-0,08[-6,17](0,01)$ & 0,37 & 261,4 & 0,06 \\
\hline All & B2 & 105,4 & {$[18,4]$} & $(0,00)$ & $-1,44[-1,71]$ & $(0,23)$ & $|-0,08[-6,11](0,01)|$ & 0,37 & 264,4 & 0,06 \\
\hline \multicolumn{11}{|c|}{ Constant maturity of 60 days } \\
\hline Top 5 & B0 & 115,6 & {$[20,9]$} & $(0,00)$ & $1,09[2,56]$ & $(0,31)$ & $\mid-0,12 \cdot 10,20](0,00) \mid$ & 0,48 & $\mid 471,7$ & 0,03 \\
\hline Top 5 & B1 & 118,9 & {$[21,8]$} & $(0,00)$ & $2,15[0,45]$ & $(0,29)$ & $-0,11[-9,31](0,00)$ & 0,50 & 482,8 & 0,06 \\
\hline Top 5 & B2 & 118,5 & {$[21,9]$} & $(0,00)$ & $-5,17[-0,83]$ & $(0,22)$ & $-0,11[-9,14](0,01)$ & 0,50 & 490,4 & 0,07 \\
\hline All & B0 & 104,0 & {$[17,9]$} & $(0,00)$ & $\mathbf{0 , 2 0}[0,27]$ & $(0,30)$ & $-0,08[-6,72](0,00)$ & 0,36 & 247,8 & 0,04 \\
\hline All & B1 & 104,7 & {$[18,1]$} & $(0,00)$ & $\mathbf{0 , 5 3}[0,44]$ & $(0,31)$ & $-0,08[-6,51](0,00)$ & 0,36 & 250,6 & 0,05 \\
\hline All & B2 & 104,6 & {$[18,1]$} & $(0,00)$ & $-1,26[-0,53]$ & $(0,30)$ & $|-0,08[-6,47](0,01)|$ & 0,36 & 252,3 & 0,05 \\
\hline \multicolumn{11}{|c|}{ Constant maturity of 180 days } \\
\hline Top 5 & B0 & 116,8 & {$[20,9]$} & $(0,00)$ & $\mathbf{0 , 0 3}[0,82]$ & $(0,39)$ & $\mid-0,12 \cdot 10,43](0,00) \mid$ & 0,48 & $\mid 469,2$ & 0,02 \\
\hline Top 5 & B1 & 116,9 & {$[21,0]$} & $(0,00)$ & $\mathbf{0 , 0 3}[-0,26]$ & $(0,24)$ & $-0,12 \cdot 10,44](0,00)$ & 0,48 & 469,2 & 0,02 \\
\hline Top 5 & B2 & 116,9 & {$[21,0]$} & $(0,00)$ & $-0,07[-0,01]$ & $(0,21)$ & $-0,12 \cdot 10,44](0,00)$ & 0,48 & 469,3 & 0,02 \\
\hline All & B0 & 104,2 & {$[17,9]$} & $(0,00)$ & $\mathbf{0 , 0 0}[-0,06]$ & $(0,35)$ & $-0,08[-6,79](0,00)$ & 0,36 & 247,2 & 0,04 \\
\hline All & B1 & 104,2 & {$[17,9]$} & $(0,00)$ & $\mathbf{0 , 0 1}[0,29]$ & $(0,28)$ & $-0,08[-6,78](0,00)$ & 0,36 & 247,2 & 0,04 \\
\hline All & B2 & 104,2 & {$[17,9]$} & $(0,00)$ & $-0,02[-0,39]$ & $(0,27)$ & $|-0,08[-6,78](0,00)|$ & 0,36 & 247,2 & 0,04 \\
\hline
\end{tabular}

${ }^{*}$ Cross sections

t-stats in [ ] and corresponding two-sided p-values in ( )

Table 6.25.: Cross-sectional averages of the time-series regression results for the test of the relationship between default risk and the smile coefficients 


\subsection{Examination in the Framework of a Pooled Series Regres-}

sion The relationship between $C D S$ and the smile coefficients $B 0, B 1$ and $B 2$ has also been tested in a pooled series regression according to model 6.53 . The case of a constant maturity of 240 days is not reported here, because it did not yield any meaningful results. The outcome for the first 3 constant maturities can be viewed in table 6.26. As depicted in table 6.26, the coefficient $b_{1}$ of the smile coefficients yields a neg-

\begin{tabular}{|c|c|c|c|c|c|c|c|c|c|c|}
\hline \multicolumn{6}{|c|}{$C D S_{i, t}=\beta_{0_{i}}+\beta_{1} \cdot B j_{i, t}+\beta_{2} \cdot t+u_{i, t}}$, & \multicolumn{5}{|c|}{$j \in\{0,1,2\}$} \\
\hline Variable & & $b_{0}$ & $b_{1}$ & & & $b_{2}$ & & $\begin{array}{l}\text { R2 } \\
\text { cor. }\end{array}$ & $\mathbf{F}$ & DW \\
\hline \multicolumn{11}{|c|}{ Constant maturity of 36 days } \\
\hline B0 & $\mid 125,0$ & {$[7,03](0,00)$} & | -4,24[-6,83] & $(0,00)$ & $-0,11[$ & {$[-2,17]$} & $(0,03)$ & 0,65 & 1.183 & 0,07 \\
\hline B1 & 123,0 & {$[6,98](0,00)$} & $2,65[6,16]$ & $(0,00)$ & $-0,10[$ & {$[-2,05]$} & $(0,04)$ & 0,66 & 1.220 & 0,10 \\
\hline B2 & 122,1 & {$[6,90](0,00)$} & $-5,52[-6,17]$ & $(0,00)$ & $-0,10$ & {$[-2,02]$} & $(0,04)$ & 0,66 & 1.228 & 0,11 \\
\hline \multicolumn{11}{|c|}{ Constant maturity of 60 days } \\
\hline B0 & 117,8 & {$[5,95](0,00)$} & $\mid-0,06[-0,84]$ & $(0,40)$ & $-0,12[$ & {$[-2,35]$} & $(0,02)$ & 0,64 & 1.123 & 0,01 \\
\hline B1 & 117,8 & {$[5,96](0,00)$} & $0,05[1,04]$ & $(0,30)$ & $-0,12[$ & {$[-2,36]$} & $(0,02)$ & 0,64 & 1.123 & 0,01 \\
\hline B2 & 117,8 & {$[5,96](0,00)$} & $-0,11[-1,06]$ & $(0,29)$ & $-0,12[$ & {$[-2,36]$} & $(0,02)$ & 0,64 & 1.124 & 0,01 \\
\hline \multicolumn{11}{|c|}{ Constant maturity of 180 days } \\
\hline BO & 117,8 & {$[5,96](0,00)$} & $\mid-0,01[-3,81]$ & $(0,00)$ & $\mid-0,12[$ & {$[-2,35]$} & $(0,02)$ & 0,64 & 1.123 & 0,01 \\
\hline B1 & 117,7 & {$[5,96](0,00)$} & $\mathbf{0 , 0 1}[4,09]$ & $(0,00)$ & $-0,12$ & {$[-2,35]$} & $(0,02)$ & 0,64 & 1.124 & 0,01 \\
\hline B2 & 117,7 & {$[5,96](0,00)$} & $-0,01[-4,28]$ & $(0,00)$ & $-0,12 \mid$ & {$[-2,35]$} & $(0,02)$ & 0,64 & 1.124 & 0,01 \\
\hline
\end{tabular}

Table 6.26.: Pooled series regression results for the test of the relationship between default risk and the smile coefficients

ative sign for $B 0$ and $B 2$ and a positive sign for $B 1$ in all 3 constant maturity cases. The absolute values of $b_{1}$ are decreasing with increasing constant maturity, which has to do with the flattening of the smile with increasing maturity. Astonishingly, $b_{1}$ is significant for constant maturities of 36 and 180 days, but not for a constant maturity of 60 days. The finding of negative coefficients for $B 0$ and $B 2$ and a positive coefficient for $B 1$ are consistent with the results of the examination in the time-series dimension. Again, the results confirm our preliminary observation of a default risk induced flattening of the smile. However, they seem to bely Hypothesis 3 of a steepening of the smile. Regarding the intercept $b_{0}$, the coefficient of the time trend $b_{2}$ as well as the regression statistics, the same can be said as in the discussion of the times-series results above. 
6.5.4.2.2. Robustness of the Regression Results The robustness check of the above results is restricted to the inclusion of the additional variables $I M P V$ and $H V$. The motivation for the inclusion of $I M P V$ consists in testing, whether the relationship between the CDS spread level and the smile coefficients is not possibly dominated by the level of volatility. Non-stationarity need not concern us, since only one possibly stochastically trending series is involved in the regression, namely $C D S$.

6.5.4.2.1. Examination in the Time-Series Dimension The robustness of the time-series regression results has been tested by including $H V$ and $I M P V$ in the regression model 6.52 .

$$
\begin{array}{r}
C D S_{t}=\beta_{0}+\beta_{1} \cdot B j_{t}+\beta_{2} \cdot H V_{t}+\beta_{3} \cdot t+u_{t}, \quad j \in(0,1,2) \\
C D S_{t}=\beta_{0}+\beta_{1} \cdot B j_{t}+\beta_{2} \cdot I M P V_{t}+\beta_{3} \cdot t+u_{t}, \quad j \in(0,1,2)
\end{array}
$$

Table 6.27 juxtaposes the estimates $b_{1}$ and their respective $p$-values for the estimation of the original model 6.52, the model including $H V 6.58$ and the model including $I M P V 6.59$ for a constant maturity of 36 days. Only those cross-sections are reported, which yielded significant coefficients $b_{1}$ in the estimation of the original model. As can be seen in table 6.27 and as expected, volatility in general seems to play an important role in explaining the CDS spread level in the face of the smile coefficients $B 0, B 1$ and $B 2$, since the coefficient $b_{2}$ of $H V$ as well as of $I M P V$ is positive and significant in almost all cases. Further, $I M P V$ seems to unfold a more detrimental effect on the sign and significance of $b_{1}$ than does $H V$. While after the inclusion of $I M P V$, not a single cross-section exhibits a significant coefficient $b_{1}$ with its original sign any more, the inclusion of $H V$ generally leads to losses of significance, but not to sign inversions. If in the case of the inclusion of $H V$ a less restrictive significance requirement of for example $15 \%$ were adopted, it could be argued that the original pattern of dependence remains unchanged. The only effect would consist in absolutely shrinking coefficients $b_{1}$.

Altogether, the robustness check can be interpreted in the following way: Volatility in the guise of $H V$ and $I M P V$ seems to contribute to the explanation of $C D S$ in a non-negligible way, considering the largely significant coefficient $b_{2}$. However, in the presence of $H V$, the original pattern of dependence between the CDS spread level and the smile coefficients remains largely unchanged. This is probably due to the weaker explanatory power of $H V$ as to the CDS spread level as compared to that of $I M P V$. Altogether, the robustness check casts light doubts on our preliminary finding of a 
default risk induced flattening and right-translation of the smile. However, a default risk induced flattening of the smile is still more likely than its steepening as predicted by Hypothesis 3. 


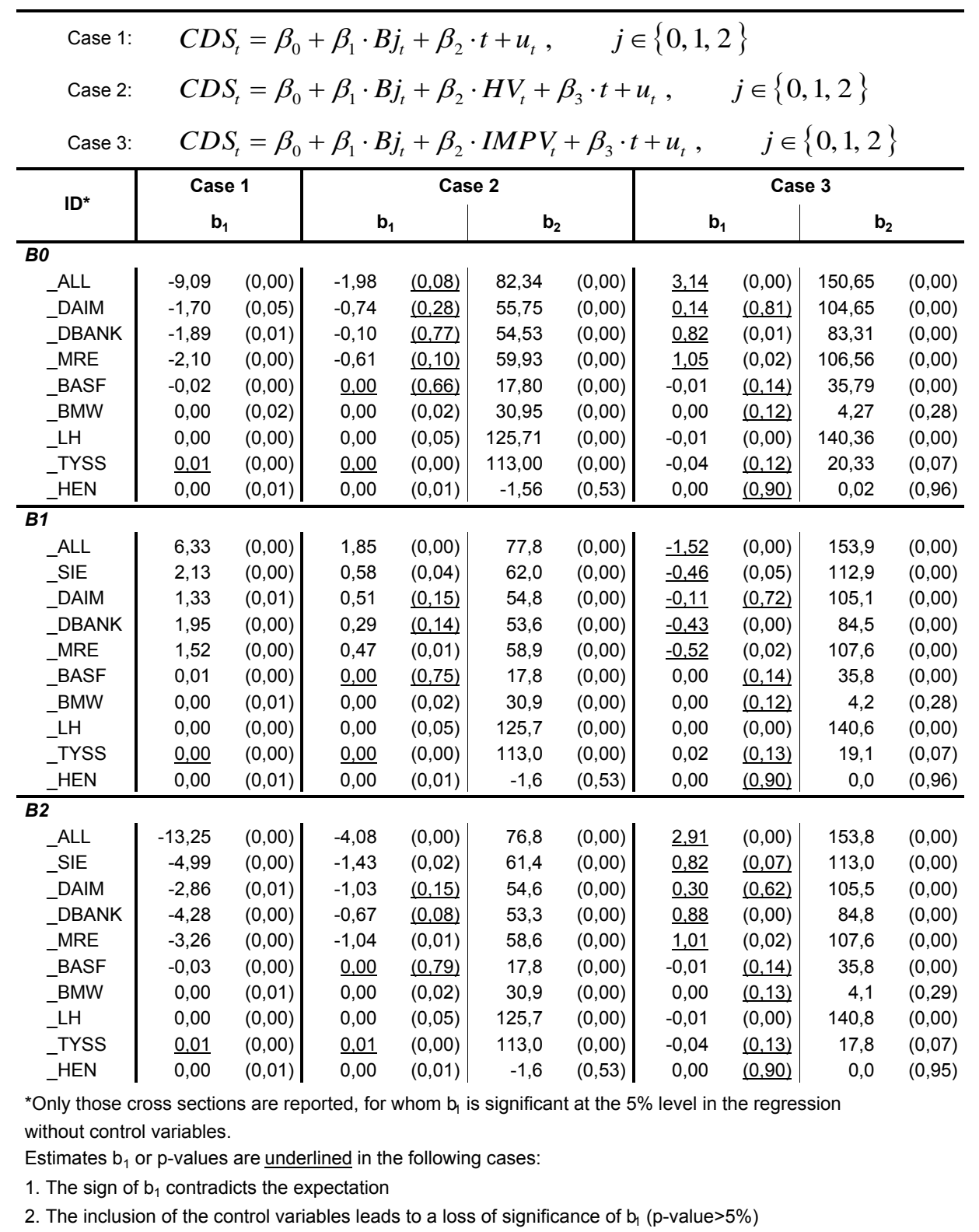

Table 6.27.: Robustness check of the time-series regression results for the test of the relationship between default risk and the smile coefficients for a constant maturity of 36 days 


\subsection{Examination in the Framework of a Pooled Series Regression}

Like the robustness check in the time-series dimension, the pooled series regression results have been challenged by including the variables $H V$ and $I M P V$ into the original regression model 6.53 .

$$
\begin{gathered}
C D S_{i, t}=\beta_{0 i}+\beta_{1} \cdot B j_{i, t}+\beta_{2} \cdot H V_{i, t}+\beta_{3} \cdot t+u_{i, t}, \quad j \in(0,1,2) \\
C D S_{i, t}=\beta_{0 i}+\beta_{1} \cdot B j_{i, t}+\beta_{2} \cdot I M P V_{i, t}+\beta_{3} \cdot t+u_{i, t}, \quad j \in(0,1,2)
\end{gathered}
$$

Table 6.28 juxtaposes the estimates $b_{1}$ and their respective $p$-values for the estimation of the original model 6.53 , the model including $H V 6.60$ and the model including $I M P V 6.61$ for constant maturities of 36,60 and 180 days.

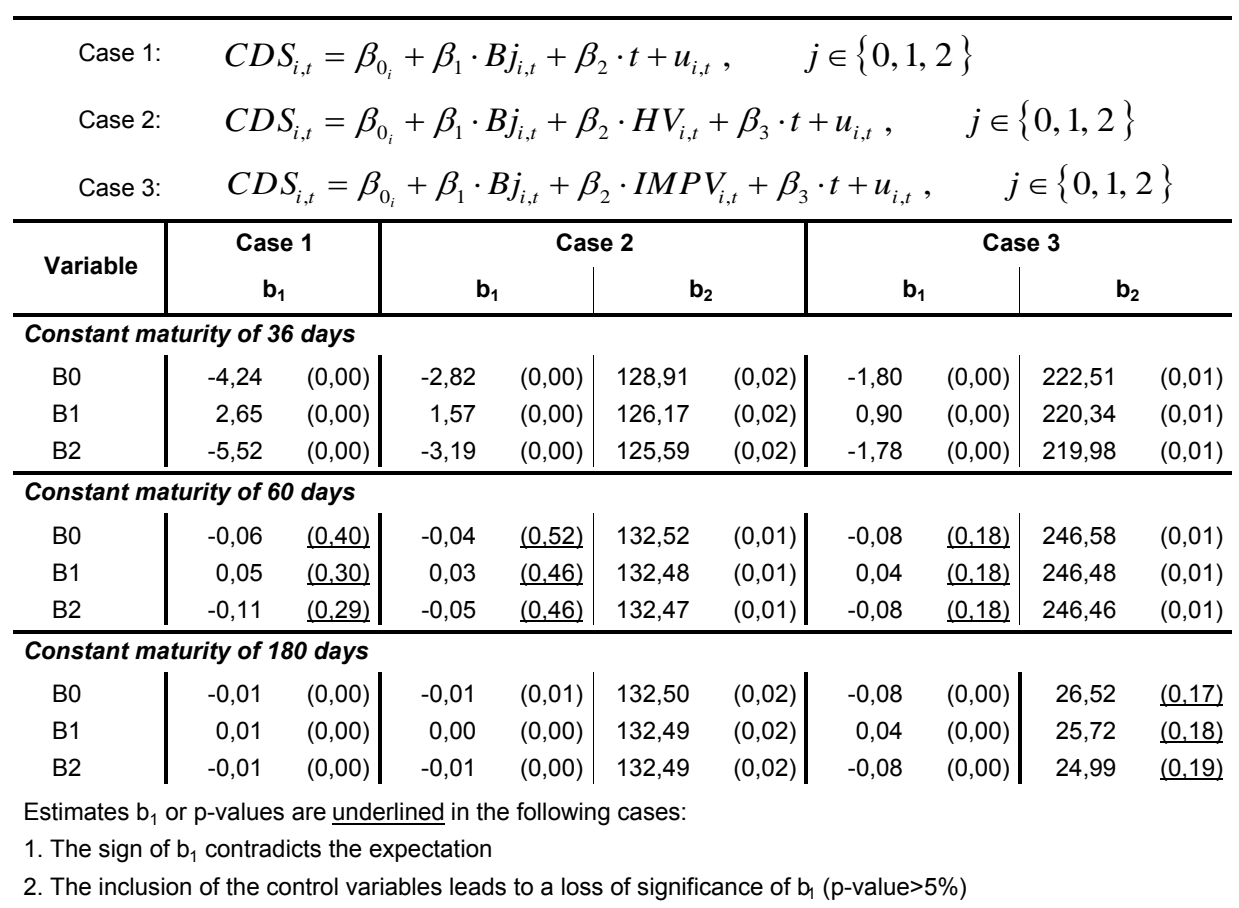

Table 6.28.: Robustness check of the pooled series regression results for the test of the relationship between default risk and the smile coefficients

As in the robustness check of the time-series results, $H V$ and $I M P V$ yield positive and significant coefficients in almost all cases which documents the important role of volatility in general in explaining the CDS spread level. It is worth noting however that $I M P V$ does not yield significant coefficients in the constant maturity case of 180 days. This weakening dependence between $C D S$ and $I M P V$ with growing maturity has already been observed in section 6.5.3. Further, the original pattern of a negative coefficient $b_{1}$ for $B 0$ and $B 2$ and a positive one for $B 1$ remains unchanged in all cases. 
And what is more, for the constant maturity case of 36 and 180 days, $b_{1}$ remains significant even after the inclusion of $H V$ and $I M P V$. Unfortunately, this is not true for the maturity case of 60 days, but in this case $b_{1}$ has been insignificant from the beginning. Altogether, the robustness check can be viewed as a confirmation of the above regression results which suggested a default risk induced flattening and righttranslation of the smile. 


\subsubsection{Examination of the Relationship between the CDS Spread Level and the Slope of the Smile}

This section is dedicated to the test of the relationship between $C D S$ and the slope coefficients SLP70, SLP90, SLP100, SLP110 and SLP130 as well as the horizontal coordinate $X S M I N$. This relationship has been tested by estimating and validating the regression models 6.54, 6.56, 6.55 and 6.57 in the time-series dimension as well as in the pooled series context.

\subsection{Regression Results}

6.5.4.3.1.1. Examination in the Time-Series Dimension The test of the relationship between $C D S$ and the slope variables SLP70, SLP90, SLP100, SLP110 and $S L P 130$ as well as the horizontal coordinate $X S M I N$ according to the models 6.54 and 6.55 has been carried out for all stocks. Only _DEG has been excluded due to an insufficient number of observations. Tables 6.29, 6.30, 6.31, 6.32, 6.33 and 6.34 show the detailed regression results for the constant maturity case of 36 days, whereas table 6.35 exhibits a constant-maturity-wise cross-sectional summary of all results, once averaged across all cross-sections and once only across the 5 most important stocks. Only the constant maturity case of 240 days has been excluded, since it has not yielded any meaningful results. In order to keep this documentation as lean and readable as possible, Tables 6.29, 6.30, 6.31, 6.32, 6.33 and 6.34 only report those cross-sections for whom the coefficient $b_{1}$ is significant at the $1 \%$ level. The totality of the detailed results for all cross-sections and all constant maturities except 240 days can be viewed in appendix C.3.2.

As can be gathered from tables 6.29, 6.30, 6.31, 6.32, 6.33 and 6.34, which display the highly significant cross-sections, the coefficient $b_{1}$ of the slope variables is largely positive for the left wing of the smile and negative for the right wing. Further, the coefficient of the horizontal coordinate is largely positive. A similar pattern can be discerned in the summary representation of table 6.35. The summary representation reveals that $b_{1}$ is on average positive for the slope variables of the left wing of the smile and negative for those of the right wing of the smile. For a constant maturity of 36 days, $b 1$ is positive for the whole left wing consisting of SLP70 and SLP90, whereas for higher maturities it is only positive for the utmost left part of the smile, namely $S L P 70$. In contrast, the coefficient for the right wing is negative on average for all maturities. Further the coefficient of the horizontal coordinate $X S M I N$ is likewise 


\begin{tabular}{|c|c|c|c|c|c|c|}
\hline \multicolumn{7}{|c|}{$C D S_{t}=\beta_{0}+\beta_{1} \cdot \operatorname{SLP} 70_{t}+\beta_{2} \cdot t+u_{t}$} \\
\hline ID* & $b_{0}$ & $b_{1}$ & $b_{2}$ & R2 cor. & $\mathbf{F}$ & DW \\
\hline \multirow[t]{3}{*}{ _ALL } & 77,28 & 18,29 & $-0,02$ & \multirow{3}{*}{0,33} & \multirow{3}{*}{176,72} & \multirow{3}{*}{0,12} \\
\hline & {$[13,69]$} & {$[5,30]$} & {$[-2,50]$} & & & \\
\hline & $(0,00)$ & $(0,00)$ & $(0,01)$ & & & \\
\hline \multirow[t]{3}{*}{ _DBANK } & 41,04 & 4,59 & $-0,02$ & \multirow[t]{3}{*}{0,32} & \multirow[t]{3}{*}{176,73} & \multirow[t]{3}{*}{0,05} \\
\hline & {$[13,91]$} & {$[3,23]$} & {$[-5,52]$} & & & \\
\hline & $(0,00)$ & $(0,00)$ & $(0,00)$ & & & \\
\hline \multirow[t]{3}{*}{ _MRE } & 60,58 & 4,20 & $-0,04$ & \multirow[t]{3}{*}{0,36} & \multirow[t]{3}{*}{186,52} & \multirow[t]{3}{*}{0,23} \\
\hline & {$[14,94]$} & {$[3,78]$} & {$[-5,15]$} & & & \\
\hline & $(0,00)$ & $(0,00)$ & $(0,00)$ & & & \\
\hline \multirow[t]{3}{*}{ BASF } & 30,90 & 0,04 & $-0,03$ & \multirow[t]{3}{*}{0,75} & \multirow[t]{3}{*}{$1.125,37$} & \multirow[t]{3}{*}{0,04} \\
\hline & {$[39,63]$} & {$[5,52]$} & {$[-20,48]$} & & & \\
\hline & $(0,00)$ & $(0,00)$ & $(0,00)$ & & & \\
\hline \multirow[t]{3}{*}{ _LH } & 155,86 & 0,00 & $-0,12$ & \multirow[t]{3}{*}{0,43} & \multirow[t]{3}{*}{268,08} & \multirow[t]{3}{*}{0,01} \\
\hline & {$[24,22]$} & {$[3,72]$} & {$[-11,21]$} & & & \\
\hline & $(0,00)$ & $(0,00)$ & $(0,00)$ & & & \\
\hline \multirow[t]{3}{*}{ _TYSS } & 205,46 & $-0,01$ & $-0,14$ & \multirow[t]{3}{*}{0,28} & \multirow[t]{3}{*}{129,06} & \multirow[t]{3}{*}{0,03} \\
\hline & {$[17,36]$} & {$[-4,14]$} & {$[-7,23]$} & & & \\
\hline & $(0,00)$ & $(0,00)$ & $(0,00)$ & & & \\
\hline \multicolumn{7}{|c|}{$\begin{array}{l}\text { *Only those cross sections are reported, for whom } b_{1} \text { is significant } \\
\text { at the } 1 \% \text { level }\end{array}$} \\
\hline & & & & & & \\
\hline
\end{tabular}

Table 6.29.: Time-series regression results for the test of the relationship between default risk and the slope variable $S L P 70$ for a constant maturity of 36 days

positive for all 3 constant maturities. This pattern is observable for the averages across all stocks as well as for the averages across the top 5 stocks only. For the top 5 crosssections and a constant maturity of 36 days, this pattern is on average even significant for the coefficients of SLP70, SLP100, SLP110 and SLP130.

As in the case of the smile coefficients above, the overall quality of the regression results, as measured in the explanatory power $R^{2}$, the overall significance $F$ and the significance of the coefficient $b_{1}$ degrades with increasing maturity. This is due to the maturity-related worsening of the significance of the smile coefficients $B 0, B 1$ and $B 2$ in connection with the flattening of the smile. Further, the assumption of an intercept and a time-trend are proved to be justified in the light of the ubiquitously significant coefficients $b_{0}$ and $b_{2}$. Moreover, the average explanatory power $R^{2}$ is nonnegligible, ranging between $20 \%$ and roughly $60 \%$ and the Durbin-Watson statistic is again very small, hinting at autocorrelated residuals, which could be due to possible non-stationarity of the series $C D S$. But since $C D S$ is the only possible non-stationary series involved, non-stationarity need not concern us here.

The detected signs of the coefficient $b_{1}$ can be interpreted in the following way:

- A positive relationship between the CDS spread level on the one hand and the negative slope of the left wing of the smile on the other is consistent with our preliminary suspicion of a default risk induced flattening of the smile. It contra- 


\begin{tabular}{|c|c|c|c|c|c|c|}
\hline \multicolumn{7}{|c|}{$C D S_{t}=\beta_{0}+\beta_{1} \cdot S L P 90_{t}+\beta_{2} \cdot t+u_{t}$} \\
\hline ID* & $b_{0}$ & $b_{1}$ & $b_{2}$ & R2 cor. & $F$ & DW \\
\hline A ALL & $\begin{array}{r}75,91 \\
{[11,55]} \\
(0,00)\end{array}$ & $\begin{array}{l}26,87 \\
{[3,05]} \\
(0,00)\end{array}$ & $\begin{array}{r}-0,04 \\
{[-3,76]} \\
(0,00)\end{array}$ & 0,24 & 113,68 & 0,04 \\
\hline BASF & $\begin{array}{r}30,91 \\
{[39,64]} \\
(0,00)\end{array}$ & $\begin{array}{r}0,09 \\
{[5,58]} \\
(0,00)\end{array}$ & $\begin{array}{r}-0,03 \\
{[-20,48]} \\
(0,00)\end{array}$ & 0,75 & $1.123,44$ & 0,04 \\
\hline LLH & $\begin{array}{r}155,86 \\
{[24,21]} \\
(0,00)\end{array}$ & $\begin{array}{r}0,00 \\
{[3,68]} \\
(0,00)\end{array}$ & $\begin{array}{r}-0,12 \\
{[-11,21]} \\
(0,00)\end{array}$ & 0,43 & 268,08 & 0,01 \\
\hline -TYSS & $\begin{array}{r}205,47 \\
{[17,36]} \\
(0,00)\end{array}$ & $\begin{array}{r}-0,02 \\
{[-4,07]} \\
(0,00)\end{array}$ & $\begin{array}{r}-0,14 \\
{[-7,22]} \\
(0,00)\end{array}$ & 0,28 & 129,07 & 0,03 \\
\hline & ross se & ns are & orted, $f$ & whom $b$ & signific & \\
\hline
\end{tabular}

Table 6.30.: Time-series regression results for the test of the relationship between default risk and the slope variable $S L P 90$ for a constant maturity of 36 days

dicts Hypothesis 3 however, which would require the slope of the left wing to decrease with increasing CDS spread level.

- A negative relationship between the CDS spread level on the one hand and the positive slope of the right wing of the smile on the other is likewise consistent with a default risk induced flattening of the smile, but is also in line with Hypothesis 3 .

- Finally, a positive relationship between the CDS spread level and the horizontal coordinate $X S M I N$ clearly is symptomatic of our initial finding of a default risk induced right-translation of the smile.

Altogether it can be concluded that our preliminary findings have been confirmed. So far, Hypothesis 3 must be rejected, since it is only compatible with the findings concerning the right wing of the smile, but not with those regarding the left wing. 


\begin{tabular}{|c|c|c|c|c|c|c|}
\hline \multicolumn{7}{|c|}{$C D S_{t}=\beta_{0}+\beta_{1} \cdot S L P 100_{t}+\beta_{2} \cdot t+u_{t}$} \\
\hline ID* & $b_{0}$ & $b_{1}$ & $b_{2}$ & R2 cor. & $\mathbf{F}$ & DW \\
\hline ALL & $\begin{array}{l}12,71 \\
{[1,57]} \\
(0,12) \\
\end{array}$ & $\begin{array}{r}-132,48 \\
{[-7,50]} \\
(0,00)\end{array}$ & $\begin{array}{r}-0,03 \\
{[-3,67]} \\
(0,00) \\
\end{array}$ & 0,39 & 237,62 & 0,15 \\
\hline _SIE & $\begin{array}{l}27,24 \\
{[6,65]} \\
(0,00)\end{array}$ & $\begin{array}{r}-96,29 \\
{[-10,18]} \\
(0,00)\end{array}$ & $\begin{array}{r}-0,05 \\
{[-13,29]} \\
(0,00)\end{array}$ & 0,78 & $1.347,49$ & 0,31 \\
\hline _DTE & $\begin{array}{r}177,58 \\
{[7,92]} \\
(0,00) \\
\end{array}$ & $\begin{array}{r}-264,53 \\
{[-4,97]} \\
(0,00) \\
\end{array}$ & $\begin{array}{r}-0,29 \\
{[-11,12]} \\
(0,00) \\
\end{array}$ & 0,71 & 934,86 & 0,30 \\
\hline DBANK & $\begin{array}{l}14,35 \\
{[5,08]} \\
(0,00)\end{array}$ & $\begin{array}{r}-45,64 \\
{[-7,48]} \\
(0,00) \\
\end{array}$ & $\begin{array}{r}-0,01 \\
{[-4,51]} \\
(0,00)\end{array}$ & 0,48 & 350,50 & 0,15 \\
\hline _MRE & $\begin{array}{l}44,30 \\
{[7,78]} \\
(0,00) \\
\end{array}$ & $\begin{array}{r}-33,35 \\
{[-4,02]} \\
(0,00) \\
\end{array}$ & $\begin{array}{r}-0,04 \\
{[-5,26]} \\
(0,00) \\
\end{array}$ & 0,37 & 195,87 & 0,24 \\
\hline _VW & $\begin{array}{r}45,25 \\
{[15,72]} \\
(0,00)\end{array}$ & $\begin{array}{r}-9,34 \\
{[-2,65]} \\
(0,01)\end{array}$ & $\begin{array}{r}0,03 \\
{[6,45]} \\
(0,00)\end{array}$ & 0,24 & 119,43 & 0,10 \\
\hline _TYSS & $\begin{array}{r}205,50 \\
{[17,33]} \\
(0,00) \\
\end{array}$ & $\begin{array}{r}-0,05 \\
{[-3,42]} \\
(0,00)\end{array}$ & $\begin{array}{r}-0,14 \\
{[-7,21]} \\
(0,00) \\
\end{array}$ & 0,28 & 129,05 & 0,03 \\
\hline _HEN & $\begin{array}{r}20,99 \\
{[26,27]} \\
(0,00)\end{array}$ & $\begin{array}{r}0,00 \\
{[-4,11]} \\
(0,00)\end{array}$ & $\begin{array}{r}0,01 \\
{[5,18]} \\
(0,00)\end{array}$ & 0,19 & 45,15 & 0,04 \\
\hline $\begin{array}{l}{ }^{*} \text { Only tho } \\
\text { at the } 1 \%\end{array}$ & $\begin{array}{l}\text { cross se } \\
\text { el }\end{array}$ & ons are & ported, $\mathrm{fc}$ & r whom $b$ & is signific & \\
\hline
\end{tabular}

Table 6.31.: Time-series regression results for the test of the relationship between default risk and the slope variable $S L P 100$ for a constant maturity of 36 days 


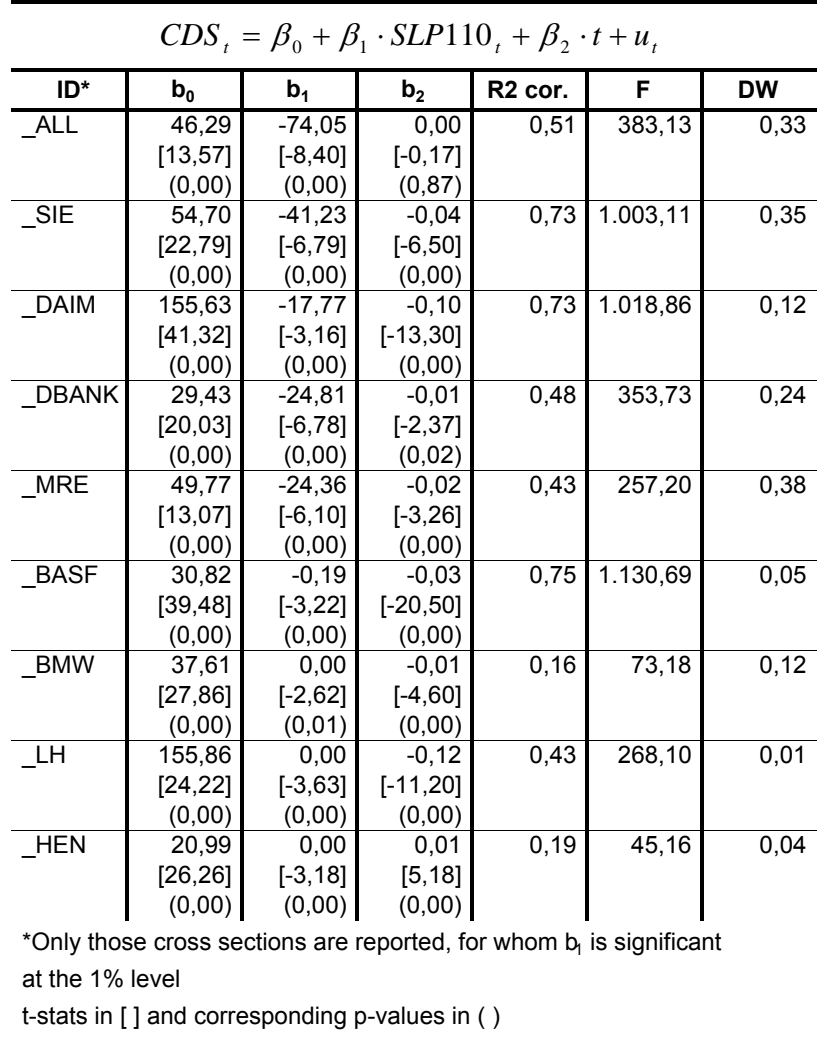

Table 6.32.: Time-series regression results for the test of the relationship between default risk and the slope variable $S L P 110$ for a constant maturity of 36 days 


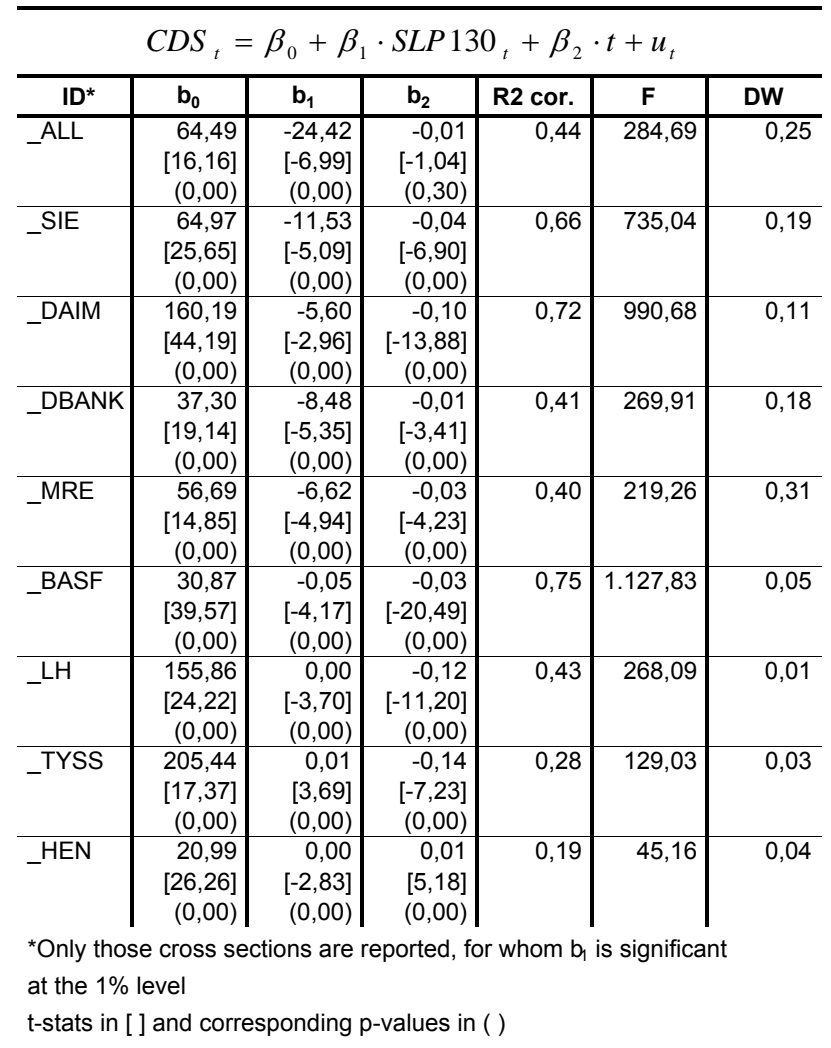

Table 6.33.: Time-series regression results for the test of the relationship between default risk and the slope variable $S L P 130$ for a constant maturity of 36 days

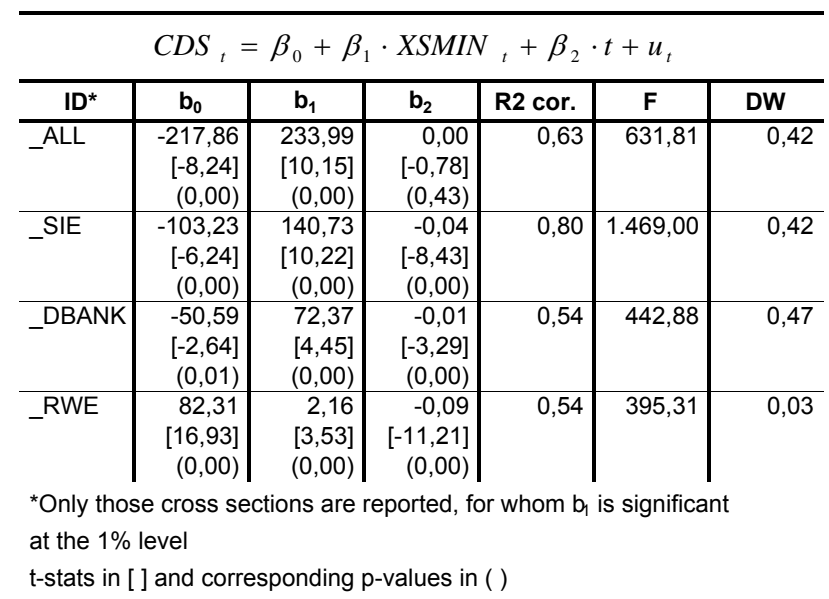

Table 6.34.: Time-series regression results for the test of the relationship between default risk and the horizontal coordinate $X S M I N$ for a constant maturity of 36 days 


\begin{tabular}{|c|c|c|c|c|c|c|c|c|c|c|c|c|c|}
\hline \multicolumn{14}{|c|}{$\begin{array}{l}C D S_{t}=\beta_{0}+\beta_{1} \cdot S L P j_{t}+\beta_{2} \cdot t+u_{t}, j \in\{70,90,100,110,130\} \\
C D S_{t}=\beta_{0}+\beta_{1} \cdot X S M I N_{t}+\beta_{2} \cdot t+u_{t}\end{array}$} \\
\hline $\mathrm{CS}^{*}$ & Variable & & $b_{0}$ & & & $b_{1}$ & & & $b_{3}$ & & $\begin{array}{l}\text { R2 } \\
\text { cor. }\end{array}$ & $\mathbf{F}$ & DW \\
\hline \multicolumn{14}{|c|}{ Constant maturity of 36 days } \\
\hline Top 5 & SLP70 & 122,7 & {$[21,4]$} & ] $(0,00)$ & 6,6 & {$[2,94]$} & $(0,04)$ & $\mid-0,11$ & {$[-8,6]$} & $(0,00)$ & 0,52 & 500 & 0,06 \\
\hline Top 5 & SLP90 & 118,8 & {$[17,9]$} & $(0,00)$ & 3,9 & {$[0,36]$} & $(0,22)$ & $-0,12$ & {$[-9,9]$} & $(0,00)$ & 0,49 & 478 & 0,03 \\
\hline Top 5 & SLP100 & 75,9 & {$[8,9]$} & $(0,02)$ & $-113,8[$ & {$[-6,49]$} & $(0,00)$ & $-0,10$ & {$[-9,8]$} & $(0,00)$ & 0,62 & 772 & 0,20 \\
\hline Top 5 & SLP110 & 109,0 & {$[22,9]$} & ] $(0,00)$ & $-35,4[$ & {$[-5,35]$} & ] $(0,02)$ & $-0,09$ & {$[-6,4]$} & $(0,18)$ & 0,62 & 683 & 0,23 \\
\hline Top 5 & SLP130 & 118,0 & {$[24,4]$} & ] $(0,00)$ & $-10,9[$ & {$[-4,38]$} & $(0,03)$ & $-0,10$ & {$[-7,1]$} & $(0,06)$ & 0,57 & 583 & 0,16 \\
\hline Top 5 & XSMIN & $-3,8$ & {$[-0,4]$} & ] $(0,00)$ & 101,5 & {$[5,61]$} & ] $(0,10)$ & $-0,10$ & {$[-7,7]$} & $(0,09)$ & 0,66 & 839 & 0,30 \\
\hline All & SLP70 & 105,8 & {$[18,1]$} & ] $(0,00)$ & 1,8 & {$[1,47]$} & $(0,23)$ & $-0,08$ & {$[-6,3]$} & $(0,00)$ & 0,37 & 256 & 0,05 \\
\hline All & SLP90 & 104,9 & {$[17,2]$} & $(0,00)$ & 1,4 & {$[0,67]$} & $(0,21)$ & $-0,08$ & {$[-6,6]$} & $(0,00)$ & 0,36 & 250 & 0,04 \\
\hline All & SLP100 & 93,9 & {$[14,6]$} & $(0,01)$ & $-28,2$ & {$[-2,28]$} & $(0,21)$ & $-0,07$ & {$[-6,5]$} & $(0,00)$ & 0,39 & 319 & 0,09 \\
\hline All & SLP110 & 102,0 & {$[18,3]$} & $(0,00)$ & $-9,5[$ & {$[-2,17]$} & $(0,20)$ & $-0,07$ & {$[-5,7]$} & $(0,04)$ & 0,39 & 301 & 0,10 \\
\hline All & SLP130 & 104,5 & {$[18,8]$} & $(0,00)$ & $-2,9[$ & {$[-1,87]$} & ] $(0,23)$ & $-0,07$ & {$[-5,9]$} & $(0,02)$ & 0,38 & 276 & 0,08 \\
\hline All & XSMIN & 74,3 & {$[12,0]$} & $(0,00)$ & 25,1 & {$[2,02]$} & ] $(0,24)$ & $\mid-0,07$ & {$[-6,1]$} & $(0,02)$ & 0,40 & 332 & 0,11 \\
\hline \multicolumn{14}{|c|}{ Constant maturity of 60 days } \\
\hline Top 5 & SLP70 & 118,7 & {$[21,6]$} & $(0,00)$ & $3,6[$ & {$[-1,08]$} & $(0,35)$ & $\mid-0,11$ & {$[-9,7]$} & $(0,00)$ & 0,49 & 473 & 0,03 \\
\hline Top 5 & SLP90 & 112,8 & {$[18,6]$} & $(0,00)$ & $-10,4[$ & {$[-2,51]$} & ] $(0,27)$ & $-0,12$ & {$[-10,8]$} & $(0,00)$ & 0,49 & 497 & 0,05 \\
\hline Top 5 & SLP100 & 96,4 & {$[13,9]$} & $(0,02)$ & $-65,2[$ & {$[-4,38]$} & $(0,06)$ & $-0,11$ & {$[-10,9]$} & $(0,00)$ & 0,58 & 731 & 0,15 \\
\hline Top 5 & SLP110 & 106,8 & {$[17,7]$} & ] $(0,00)$ & $-40,0[$ & {$[-2,41]$} & ] $(0,15)$ & $-0,10$ & {$[-8,4]$} & $(0,11)$ & 0,57 & 627 & 0,22 \\
\hline Top 5 & SLP130 & 115,4 & {$[22,0]$} & ] $(0,00)$ & $-12,2$ & {$[-1,53]$} & $(0,14)$ & $-0,11$ & {$[-8,7]$} & $(0,05)$ & 0,53 & 529 & 0,13 \\
\hline Top 5 & XSMIN & 106,9 & {$[14,4]$} & ] $(0,00)$ & 7,4 & {$[1,15]$} & $(0,29)$ & $-0,11$ & {$[-10,3]$} & $(0,00)$ & 0,49 & 484 & 0,06 \\
\hline All & SLP70 & 104,7 & {$[18,0]$} & ] $(0,00)$ & 0,9 & {$[0,08]$} & $(0,33)$ & $-0,08$ & {$[-6,6]$} & $(0,00)$ & 0,36 & 248 & 0,04 \\
\hline All & SLP90 & 103,4 & {$[17,3]$} & $(0,00)$ & $-2,1$ & {$[-0,29]$} & ] $(0,27)$ & $\mid-0,08$ & {$[-6,9]$} & $(0,00)$ & 0,36 & 253 & 0,05 \\
\hline All & SLP100 & 99,5 & {$[16,2]$} & ] $(0,01)$ & $-15,0$ & {$[-1,54]$} & ] $(0,34)$ & $-0,08$ & {$[-6,9]$} & $(0,00)$ & 0,38 & 307 & 0,07 \\
\hline All & SLP110 & 101,8 & {$[17,1]$} & ] $(0,00)$ & $-9,6[$ & {$[-1,09]$} & ] $(0,27)$ & $-0,08$ & {$[-6,3]$} & $(0,03)$ & 0,38 & 284 & 0,09 \\
\hline All & SLP130 & 103,9 & {$[18,1]$} & ] $(0,00)$ & $-2,9$ & {$[-0,76]$} & $(0,26)$ & $-0,08$ & {$[-6,4]$} & $(0,02)$ & 0,37 & 261 & 0,07 \\
\hline All & XSMIN & 100,8 & {$[16,2]$} & $(0,00)$ & 2,6 & {$[1,20]$} & ] $(0,28)$ & $\mid$\begin{tabular}{|l}
$\mid$ \\
$\mid$
\end{tabular} & {$[-6,7]$} & $(0,00)$ & 0,36 & 252 & 0,05 \\
\hline \multicolumn{14}{|c|}{ Constant maturity of 180 days } \\
\hline Top 5 & SLP70 & 116,9 & & $(0,00)$ & $0,04[$ & {$[-0,61]$} & ] $(0,44)$ & $\mid-0,12$ & & $(0,00)$ & 0,48 & 469 & 0,02 \\
\hline Top 5 & SLP90 & 116,8 & {$[20,9]$} & $(0,00)$ & $-0,08$ & {$[-0,67]$} & $(0,39)$ & $-0,12$ & {$[-10,4]$} & $(0,00)$ & 0,48 & 469 & 0,02 \\
\hline Top 5 & SLP100 & 116,5 & {$[20,3]$} & $(0,00)$ & $-1,45$ & {$[-0,09]$} & $(0,32)$ & $-0,12$ & {$[-10,5]$} & $(0,00)$ & 0,48 & 471 & 0,03 \\
\hline Top 5 & SLP110 & 116,6 & {$[20,9]$} & ] $(0,00)$ & $-1,79$ & {$[0,02]$} & ] $(0,28)$ & $-0,12$ & {$[-10,4]$} & $(0,00)$ & 0,48 & 472 & 0,03 \\
\hline Top 5 & SLP130 & 116,8 & {$[21,0]$} & $(0,00)$ & $-0,23$ & {$[0,11]$} & ] $(0,25)$ & $-0,12$ & {$[-10,4]$} & $(0,00)$ & 0,48 & 470 & 0,02 \\
\hline Top 5 & XSMIN & 116 & {$[19,5]$} & $(0,00)$ & 0,57 & {$[0,60]$} & $(0,25)$ & $-0,12$ & {$[-10,4]$} & $(0,00)$ & 0,49 & 477 & 0,04 \\
\hline All & SLP70 & 104,2 & {$[17,9]$} & ] $(0,00)$ & 0,02 & {$[0,14]$} & $(0,36)$ & $\mid-0,08$ & {$[-6,8]$} & $(0,00)$ & 0,36 & 247 & 0,04 \\
\hline All & SLP90 & 104,2 & {$[17,9]$} & $(0,00)$ & $-0,02$ & {$[0,09]$} & $(0,39)$ & $-0,08$ & {$[-6,8]$} & $(0,00)$ & 0,36 & 247 & 0,04 \\
\hline All & SLP100 & 104,1 & {$[17,7]$} & ] $(0,00)$ & $-0,38[$ & {$[-0,36]$} & $(0,35)$ & $-0,08$ & {$[-6,8]$} & $(0,00)$ & 0,36 & 248 & 0,04 \\
\hline All & SLP110 & 104,1 & {$[17,8]$} & $(0,00)$ & $-0,46[$ & {$[-0,62]$} & $(0,22)$ & $-0,08$ & {$[-6,8]$} & $(0,00)$ & 0,36 & 248 & 0,04 \\
\hline All & SLP130 & 104,2 & {$[17,9]$} & ] $(0,00)$ & $-0,06[$ & {$[-0,39]$} & ] $(0,23)$ & $-0,08$ & {$[-6,8]$} & $(0,00)$ & 0,36 & 247 & 0,04 \\
\hline All & XSMIN & 104 & {$[17,4]$} & ] $(0,00)$ & 0,32 & {$[0,92]$} & ] $(0,34)$ & $\mid-0,08$ & {$[-6,8]$} & $(0,00)$ & 0,36 & 249 & 0,05 \\
\hline
\end{tabular}

${ }^{*}$ Cross sections

t-stats in [ ] and corresponding two-sided p-values in ( )

Table 6.35.: Cross-sectional averages of time-series regression results for the test of the relationship between default risk and the slope of the smile 


\subsection{Examination in the Framework of a Pooled Series Regression}

The relationship between $C D S$ and the slope variables SLP70, SLP90, SLP100, $S L P 110$ and $S L P 130$ as well as the horizontal coordinate $X S M I N$ has also been tested in a pooled series regression by estimating the models 6.56 and 6.57 . The case of a constant maturity of 240 days is not reported here, because it did not yield any meaningful results. The outcome for the first 3 constant maturities can be viewed in table 6.36 .

\begin{tabular}{|c|c|c|c|c|c|c|}
\hline \multicolumn{7}{|c|}{$\begin{array}{l}C D S_{i, t}=\beta_{0_{i}}+\beta_{1} \cdot S L P j_{i, t}+\beta_{2} \cdot t+u_{i, t} \\
\operatorname{CDS}_{i, t}=\beta_{0_{i}}+\beta_{1} \cdot X S M I N_{i, t}+\beta_{2} \cdot t+u_{i, t}\end{array}$} \\
\hline Variable & $b_{0}$ & $b_{1}$ & $b_{2}$ & $\begin{array}{l}\text { R2 } \\
\text { cor. }\end{array}$ & $\mathbf{F}$ & DW \\
\hline \multicolumn{7}{|c|}{ Constant maturity of 36 days } \\
\hline SLP70 & $|124,6[7,12](0,00)|$ & $7,9[6,28](0,00)$ & $-0,10[-2,11](0,04) \mid$ & 0,66 & 1.203 & 0,09 \\
\hline SLP90 & $126,6[7,01](0,00)$ & $16,1[7,17](0,00)$ & $-0,11[-2,26](0,02)$ & 0,65 & 1.163 & 0,05 \\
\hline SLP100 & $80,1[6,83](0,00)$ & $-96,9[-2,10](0,04)$ & $-0,10[-2,25](0,02)$ & 0,66 & 1.241 & 0,04 \\
\hline SLP110 & $109,5[5,85](0,00)$ & $-35,9[-8,39] \quad(0,00)$ & $-0,09[-1,82](0,07)$ & 0,68 & 1.312 & 0,17 \\
\hline SLP130 & $118,7[6,56](0,00)$ & $-10,3[-6,48] \quad(0,00)$ & $-0,10[-1,94](0,05)$ & 0,67 & 1.256 & 0,13 \\
\hline XSMIN & $97,1[3,04](0,00)$ & $17,6[1,65](0,10)$ & $-0,12[-2,20] \quad(0,03)$ & 0,64 & 1.137 & 0,03 \\
\hline \multicolumn{7}{|c|}{ Constant maturity of 60 days } \\
\hline SLP70 & $|117,8[5,96](0,00)|$ & $\mathbf{0 , 1}[0,98](0,33)$ & $|-0,12[-2,36](0,02)|$ & 0,64 & 1.123 & 0,01 \\
\hline SLP90 & $117,9[5,95](0,00)$ & $0,2[0,77](0,44)$ & $-0,12[-2,35] \quad(0,02)$ & 0,64 & 1.123 & 0,01 \\
\hline SLP100 & $114,0[5,69](0,00)$ & $-12,1[-2,71](0,01)$ & $-0,12[-2,32](0,02)$ & 0,64 & 1.132 & 0,02 \\
\hline SLP110 & $117,5[5,98](0,00)$ & $-1,0[-1,23](0,22)$ & $-0,12[-2,35] \quad(0,02)$ & 0,64 & 1.125 & 0,01 \\
\hline SLP130 & $117,7[5,97](0,00)$ & $-0,2[-1,12] \quad(0,26)$ & $-0,12[-2,35] \quad(0,02)$ & 0,64 & 1.124 & 0,01 \\
\hline XSMIN & $111,2[6,87](0,00)$ & $4,9[1,56](0,12)$ & $-0,12[-2,36](0,02)$ & 0,64 & 1.131 & 0,02 \\
\hline \multicolumn{7}{|c|}{ Constant maturity of 180 days } \\
\hline SLP70 & $|117,8[5,96](0,00)|$ & $\mathbf{0 , 0 2}[3,68](0,00)$ & $-0,12[-2,35](0,02)$ & 0,64 & 1.123 & 0,01 \\
\hline SLP90 & $117,8[5,96](0,00)$ & $\mathbf{0 , 0 3}[2,83](0,00)$ & $-0,12[-2,35](0,02)$ & 0,64 & 1.123 & 0,01 \\
\hline SLP100 & $117,8[5,96](0,00)$ & $\mathbf{0 , 0 4}[1,03](0,30)$ & $-0,12[-2,35](0,02)$ & 0,64 & 1.123 & 0,01 \\
\hline SLP110 & $117,7[5,97](0,00)$ & $-0,14[-9,64](0,00)$ & $-0,12[-2,35](0,02)$ & 0,64 & 1.124 & 0,01 \\
\hline SLP130 & $117,7[5,96](0,00)$ & $-0,03[-4,93](0,00)$ & $-0,12[-2,35](0,02)$ & 0,64 & 1.124 & 0,01 \\
\hline XSMIN & $|117,8[5,93](0,00)|$ & $-0,02[-0,26] \quad(0,79)$ & $-0,12[-2,35] \quad(0,02)$ & 0,64 & 1.123 & 0,01 \\
\hline
\end{tabular}

Table 6.36.: Pooled series regression results for the test of the relationship between default risk and slope variables

As reported in table 6.36, the coefficient $b_{1}$ is positive for the slope variables of the left wing of the smile and negative for the slope variables of the right wing of the smile, which is consistent with a default risk induced flattening of the smile. The coefficient of the slope at the ATM point, $S L P 100$, is negative and significant for the first 2 constant maturities and then turns positive and insignificant for a constant maturity of 180 days. The coefficient of $X S M I N$ is positive and slightly insignificant for the first 2 constant maturities and then turns negative and highly insignificant for 180 days. The initially positive - though slightly insignificant - coefficient $b_{1}$ can be 
interpreted as weak evidence of a default risk associated right-translation of the smile. Generally, the coefficient $b_{1}$ is declining in absolute value with increasing maturity which hints at the maturity dependent flattening of the smile. Consistently with what has been observed for the smile coefficients $B 0, B 1$ and $B 2$ in section 6.5.4.2.1.2. the coefficient $b_{1}$ of the slope variables is largely significant for the constant maturity cases of 36 and 180 days, but fails to be so for 60 days.

Altogether, the pooled series regression results confirm the findings of the time-series regression and assert our preliminary finding of a default risk induced flattening and right-translation of the smile. The positive and largely significant coefficients of SLP70 and SLP90 are not compatible with Hypothesis 3 and should lead to its rejection. 
6.5.4.3.2. Robustness of the Regression Results The robustness check of the above results consists of two approaches: First, the additional variables $I M P V$ and $H V$ have been included into the regression models in order to test, whether the smile behavior is really related to the level of default risk and not primarily to the level of volatility. The inclusion of the additional variables is first studied in the timeseries dimension (section 6.5.4.3.2.1) and then in the pooled series framework (section 6.5.4.3.2.2. Secondly, the volatility-only and the default-risk-only effect have been eliminated each at a time in order to test the respective remaining effect on the shape of the smile (section 6.5.4.3.2.3).

As to the issue of possible non-stationarity, this question need not concern us here, since only one possibly stochastically trending series has been involved in the regression, namely $C D S$.

6.5.4.3.2.1. Examination in the Time-Series Dimension The robustness of the time-series regression results has been tested by including $H V$ and $I M P V$ in the regression models 6.54 and 6.55 .

$$
\begin{gathered}
C D S_{t}=\beta_{0}+\beta_{1} \cdot S L P j_{t}+\beta_{2} \cdot H V_{t}+\beta_{3} \cdot t+u_{t}, \quad j \in(70,90,100,110,130) \\
C D S_{t}=\beta_{0}+\beta_{1} \cdot X S M I N_{t}+\beta_{2} \cdot H V_{t}+\beta_{3} \cdot t+u_{t} \\
C D S_{t}=\beta_{0}+\beta_{1} \cdot S L P j_{t}+\beta_{2} \cdot I M P V_{t}+\beta_{3} \cdot t+u_{t}, \quad j \in(70,90,100,110,130) \\
C D S_{t}=\beta_{0}+\beta_{1} \cdot X S M I N_{t}+\beta_{2} \cdot I M P V_{t}+\beta_{3} \cdot t+u_{t}
\end{gathered}
$$

Tables 6.37 and 6.38 juxtapose the estimates $b_{1}$ and their respective $p$-values for the estimation of the original models 6.54 and 6.55 , the models including $H V, 6.62$ and 6.63 , and lastly the models including $I M P V, 6.64$ and 6.65, all for a constant maturity of 36 days. Only those cross-sections are reported, which yielded significant coefficients $b_{1}$ in the estimation of the original models.

As can be gathered from tables 6.64 and 6.65, $H V$ and $I M P V$ largely yield positive and significant coefficients $b 2$ which underlines their importance in explaining $C D S$. Further, both additional variables seem to affect the relationship between $C D S$ and the slope variables in a detrimental way. Altogether, the inclusion of $I M P V$ seems to lead to more sign inversions and losses of significance of $b_{1}$ than the addition of $H V$ which hints at a closer relationship between IMPV and $C D S$ than between $H V$ and $C D S$. The most robust relationship appears to be that between the CDS spread and the slope at the ATM point, SLP100. 


\begin{abstract}
Although $H V$ leads to losses of significance in numerous cases, the original pattern in terms of the signs of the coefficient $b_{1}$ remains largely unchanged, which could be interpreted as a confirmation of the above regression results. However, the dominating effect of $I M P V$, visible in sign inversions coupled with losses of significance, calls into question the robustness of the above results. Especially the sign inversions suggest that the explicit presence of volatility in the regression model exposes the defaultrisk-only effect. To further analyze the interplay between the volatility-only and the default-risk-only effect, let us examine the results of the robustness check in the pooled series context and afterwards the dissociation of both effects by alternately eliminating one of them.
\end{abstract}




\begin{tabular}{|c|c|c|c|c|c|c|c|c|c|c|}
\hline $\begin{array}{l}\text { Case 1: } \\
\text { Case 2: }\end{array}$ & \multicolumn{10}{|c|}{$C D S_{t}=\beta_{0}+\beta_{1} \cdot X S M I N_{t}+\beta_{2} \cdot H V_{t}+\beta_{3} \cdot t+u_{t}$} \\
\hline \multirow{3}{*}{$\begin{array}{c}\text { Case 3: } C \\
\mathbf{I D}^{*}\end{array}$} & \multicolumn{10}{|c|}{$\begin{array}{l}D D S_{t}=\beta_{0}+\beta_{1} \cdot S L P j_{t}+\beta_{2} \cdot I M P V_{t}+\beta_{3} \cdot t+u_{t}, \quad j \in\{70,90,100,110,130\} \\
D D S_{t}=\beta_{0}+\beta_{1} \cdot X S M I N_{t}+\beta_{2} \cdot I M P V_{t}+\beta_{3} \cdot t+u_{t}\end{array}$} \\
\hline & Cas & & & Cas & & & & Cas & & \\
\hline & $b_{1}$ & & $b_{1}$ & & $b_{2}$ & & $b_{1}$ & & $b_{2}$ & \\
\hline \multicolumn{11}{|l|}{$\overline{S L P 70}$} \\
\hline ALL & 18,29 & $(0,00)$ & 4,69 & $(0,02) \mid$ & 80,03 & $(0,00)$ & $-5,40$ & $(0,00)$ & 153,55 & $(0,00)$ \\
\hline SIE & 4,06 & $(0,02)$ & 0,92 & $(0,31)$ & 62,91 & $(0,00)$ & $\frac{-1,83}{-1,83}$ & $(0,02)$ & 112,57 & $(0,00)$ \\
\hline DAIM & 3,58 & $(0,02)$ & 1,61 & $(0,18)$ & 55,28 & $(0,00)$ & $\underline{-0,08}$ & $(0,94)$ & 104,47 & $(0,00)$ \\
\hline DDBANK & 4,59 & $(0,00)$ & 0,59 & $(0,35)$ & 54,19 & $(0,00)$ & $-1,28$ & $(0,01)$ & 83,68 & $(0,00)$ \\
\hline _MRE & 4,20 & $(0,00)$ & 1,23 & $(0,05)$ & 59,43 & $(0,00)$ & $-1,78$ & $(0,02)$ & 107,54 & $(0,00)$ \\
\hline BASF & 0,04 & $(0,00)$ & 0,00 & $(0,68)$ & 17,80 & $(0,00)$ & 0,01 & $(0,14)$ & 35,79 & $(0,00)$ \\
\hline BMW & 0,00 & $(0,02)$ & 0,00 & $(0,02)$ & 30,95 & $(0,00)$ & 0,01 & $(0,11)$ & 4,42 & $(0,27)$ \\
\hline LLH & 0,00 & $(0,00)$ & 0,00 & $(0,05)$ & 125,71 & $(0,00)$ & 0,01 & $(0,00)$ & 140,10 & $(0,00)$ \\
\hline TYSS & $-0,01$ & $(0,00)$ & $\underline{-0,01}$ & $(0,00)$ & 113,00 & $(0,00)$ & 0,07 & $(0,12)$ & 21,93 & $(0,07)$ \\
\hline HEN & $\overline{0,00}$ & $(0,02)$ & $\overline{0,00}$ & $(0,02)$ & $-1,56$ & $(0,53) \mid$ & 0,00 & $(0,90)$ & 0,02 & $(0,96)$ \\
\hline \multicolumn{11}{|l|}{ SLP90 } \\
\hline ALL & 26,87 & $(0,00)$ & 3,29 & $(0,53)$ & 83,9 & $(0,00)$ & $\underline{-16,00}$ & $(0,00)$ & 150,7 & $(0,00)$ \\
\hline _SIE & $-10,56$ & $(0,03)$ & $-4,10$ & $(0,14)$ & 62,8 & $(0,00)$ & $-\underline{-6,81}$ & $(0,00)$ & 110,1 & $(0,00)$ \\
\hline MRE & $\frac{6,35}{6,35}$ & $(0,02)$ & 1,36 & $(0,46)$ & 60,4 & $(0,00)$ & $\frac{-5,15}{-5,15}$ & $(0,02)$ & 106,7 & $(0,00)$ \\
\hline VW & $-0,75$ & $(0,02)$ & $-0,49$ & $(0,03)$ & 60,1 & & $-0,09$ & $\begin{array}{l}(0,75) \\
(0,0)\end{array}$ & 107,3 & $(0,00)$ \\
\hline BASF & 0,09 & $(0,00)$ & $\frac{-0,02}{-0,2}$ & $(0,48)$ & 17,8 & $(0,00)$ & $\frac{0,03}{0,03}$ & $(0,20)$ & 35,8 & $(0,00)$ \\
\hline BMW & 0,00 & $(0,03)$ & 0,01 & $(0,02)$ & 31,0 & $(0,00)$ & 0,04 & $(0,07)$ & 5,5 & $(0,21)$ \\
\hline _LH & 0,00 & $(0,00)$ & 0,00 & $(0,05$ & 125,7 & $(0,00)$ & 0,04 & $(0,00)$ & 138,9 & $(0,00)$ \\
\hline TYSS & $\underline{-0,02}$ & $(0,00)$ & $-0,02$ & $(0,00)$ & 113,0 & $(0,00)$ & 0,25 & $(0,10)$ & 29,5 & $(0,06)$ \\
\hline \multicolumn{11}{|l|}{ SLP100 } \\
\hline ALL & $-132,48$ & $(0,00)$ & $-55,76$ & $(0,00)$ & 74,9 & $(0,00)$ & $-32,78$ & $(0,00)$ & 135,2 & $(0,00)$ \\
\hline SIE & $-96,29$ & $(0,00)$ & $-48,78$ & $(0,00)$ & 45,0 & & $-24,26$ & $(0,00)$ & & $(0,00)$ \\
\hline DTE & $-264,53$ & $(0,00)$ & $-111,36$ & $(0,00)$ & 283,0 & $(0,00)$ & $-19,38$ & $(0,18)$ & 473,5 & $(0,00)$ \\
\hline _DAIM & $-29,91$ & $(0,02)$ & $-0,20$ & $(0,98)$ & 56,3 & $(0,00)$ & 13,16 & $(0,11)$ & 112,7 & $(0,00)$ \\
\hline DBANK & $-45,64$ & $(0,00)$ & $-10,51$ & $(0,00)$ & 50,1 & $(0,00)$ & 2,03 & $(0,53)$ & & $(0,00)$ \\
\hline MRE & $-33,35$ & $(0,00)$ & $-14,35$ & $(0,02)$ & 58,7 & $(0,00)$ & $-13,88$ & $(0,01)$ & 100,4 & $(0,00)$ \\
\hline _vW & $-9,34$ & $(0,01)$ & $-2,75$ & $(0,01)$ & 59,0 & $(0,00)$ & 0,56 & $(0,57)$ & 107,9 & $(0,00)$ \\
\hline TYSS & $-0,05$ & $(0,00)$ & $-0,04$ & $(0,00)$ & 112,9 & $(0,00)$ & $\underline{0,94}$ & $\frac{\left(\frac{1}{0}, 011\right)}{(0,06)}$ & 37,4 & $(0,05)$ \\
\hline HEN & 0,00 & $(0,00)$ & 0,00 & $(0,00)$ & $-1,6$ & $(0,53)$ & 0,00 & $(0,99)$ & 0,0 & $(0,95)$ \\
\hline _DP & 0,03 & $(0,04)$ & 0,03 & $(0,02)$ & $-27,3$ & $(0,00)$ & $\underline{0,03}$ & $(0,00)$ & 1,0 & $(0,10)$ \\
\hline
\end{tabular}

*Only those cross sections are reported, for whom $b_{1}$ is significant at the $5 \%$ level in the regression without control variables.

Estimates $b_{1}$ or $p$-values are underlined in the following cases:

1. The sign of $b_{1}$ contradicts the expectation

2. The inclusion of the control variables leads to a loss of significance of $b_{1}(p-v a l u e>5 \%)$

Table 6.37.: Robustness check of the time-series regression results for the test of the relationship between default risk and the slope of the smile for a constant maturity of 36 days - Part I 


$$
\begin{array}{ll}
\hline \text { Case 1: } & C D S_{t}=\beta_{0}+\beta_{1} \cdot S L P j_{t}+\beta_{2} \cdot t+u_{t}, j \in\{70,90,100,110,130\} \\
& C D S_{t}=\beta_{0}+\beta_{1} \cdot X S M I N_{t}+\beta_{2} \cdot t+u_{t} \\
\text { Case 2: } & C D S_{t}=\beta_{0}+\beta_{1} \cdot S L P j_{t}+\beta_{2} \cdot H V_{t}+\beta_{3} \cdot t+u_{t}, j \in\{70,90,100,110,130\} \\
& C D S_{t}=\beta_{0}+\beta_{1} \cdot X S M I N_{t}+\beta_{2} \cdot H V_{t}+\beta_{3} \cdot t+u_{t} \\
\text { Case 3: } & C D S_{t}=\beta_{0}+\beta_{1} \cdot S L P j_{t}+\beta_{2} \cdot I M P V_{t}+\beta_{3} \cdot t+u_{t}, j \in\{70,90,100,110,130\} \\
& C D S_{t}=\beta_{0}+\beta_{1} \cdot X S M I N_{t}+\beta_{2} \cdot I M P V_{t}+\beta_{3} \cdot t+u_{t}
\end{array}
$$

\begin{tabular}{|c|c|c|c|c|c|c|c|c|c|c|}
\hline \multirow{2}{*}{ ID* } & \multirow{2}{*}{\multicolumn{2}{|c|}{$\begin{array}{c}\text { Case } 1 \\
b_{1}\end{array}$}} & \multicolumn{4}{|c|}{ Case 2} & \multicolumn{4}{|c|}{ Case 3} \\
\hline & & & b & & b & & b & & b & \\
\hline \multicolumn{11}{|l|}{ SLP110 } \\
\hline ALL & $-74,05$ & $(0,00)$ & $-31,46$ & $(0,00)$ & 67,1 & $(0,00)$ & $\underline{3,24}$ & $(0,38)$ & 146,8 & $(0,00)$ \\
\hline SIE & $-41,23$ & $(0,00)$ & $-16,91$ & $(0,00)$ & 52,6 & $(0,00)$ & $-1,70$ & $(0,54)$ & 108,7 & $(0,00)$ \\
\hline DAIM & $-17,77$ & $(0,00)$ & $-4,41$ & $(0,18)$ & 53,2 & $(0,00)$ & 4,72 & $(0,14)$ & 110,9 & $(0,00)$ \\
\hline DBANK & $-24,81$ & $(0,00)$ & $-5,41$ & $(0,00)$ & 50,2 & $(0,00)$ & $\underline{3,91}$ & $(0,00)$ & 87,0 & $(0,00)$ \\
\hline MRE & $-24,36$ & $(0,00)$ & $-9,41$ & $(0,00)$ & 55,6 & $(0,00)$ & $\overline{1,91}$ & $(0,24)$ & 105,1 & $(0,00)$ \\
\hline _VW & $-2,87$ & $(0,02)$ & $-0,18$ & $(0,68)$ & 60,1 & $(0,00)$ & $\overline{0,46}$ & $(0,27)$ & 107,9 & $(0,00)$ \\
\hline BASF & $-0,19$ & $(0,00)$ & $-0,01$ & $(0,87)$ & 17,8 & $(0,00)$ & $-\overline{0,06}$ & $(0,13)$ & 35,7 & $(0,00)$ \\
\hline BMW & 0,00 & $(0,01)$ & 0,00 & $(0,02)$ & 30,9 & $(0,00)$ & $-0,01$ & $(0,19)$ & 3,4 & $(0,32)$ \\
\hline L LH & 0,00 & $(0,00)$ & 0,00 & $(0,06)$ & 125,7 & $(0,00)$ & $-0,05$ & $(0,00)$ & 143,0 & $(0,00)$ \\
\hline _TYSS & $\underline{0,04}$ & $(0,03)$ & $\underline{0,04}$ & $(0,01)$ & 113,1 & $(0,00)$ & $-0,09$ & $(0,26)$ & 8,0 & $(0,12)$ \\
\hline -HEN & $\overline{0,00}$ & $(0,00)$ & $\overline{0,00}$ & $(0,00)$ & $-1,6$ & $(0,53)$ & 0,00 & $(0,90)$ & 0,0 & $(0,94)$ \\
\hline $\mathrm{DP}$ & $\underline{0,00}$ & $(0,02)$ & $\underline{0,00}$ & $(0,09)$ & $-26,5$ & $(0,00)$ & $\underline{0,01}$ & $(0,00)$ & $-2,2$ & $(0,05)$ \\
\hline \multicolumn{11}{|l|}{$S L P 130$} \\
\hline ALL & $-24,42$ & $(0,00)$ & $-8,66$ & $(0,00)$ & 73,2 & $(0,00)$ & $\underline{3,81}$ & $(0,00)$ & 152,6 & $(0,00)$ \\
\hline SIE & $-11,53$ & $(0,00)$ & $-3,79$ & $(0,00)$ & 58,9 & $(0,00)$ & $\underline{\overline{0,76}}$ & $(0,34)$ & 112,5 & $(0,00)$ \\
\hline DAIM & $-5,60$ & $(0,00)$ & $-1,73$ & $(0,13)$ & 53,9 & $(0,00)$ & $\overline{0,94}$ & $(0,36)$ & 107,1 & $(0,00)$ \\
\hline DBANK & $-8,48$ & $(0,00)$ & $-1,54$ & $(0,02)$ & 52,2 & $(0,00)$ & $\overline{1,54}$ & $(0,00)$ & 85,9 & $(0,00)$ \\
\hline MRE & $-6,62$ & $(0,00)$ & $-2,28$ & $(0,00)$ & 57,7 & $(0,00)$ & $\overline{1,46}$ & $(0,03)$ & 107,3 & $(0,00)$ \\
\hline BASF & $-0,05$ & $(0,00)$ & $\underline{0,00}$ & $(0,91)$ & 17,8 & $(0,00)$ & $-\overline{0,02}$ & $(0,14)$ & 35,8 & $(0,00)$ \\
\hline BMW & 0,00 & $(0,01)$ & $\overline{0,00}$ & $(0,02)$ & 30,9 & $(0,00)$ & $-0,01$ & $(0,15)$ & 3,8 & $(0,30)$ \\
\hline LH & 0,00 & $(0,00)$ & 0,00 & $(0,06)$ & 125,7 & $(0,00)$ & $-0,01$ & $\overline{(0,00)}$ & 141,5 & $(0,00)$ \\
\hline -TYSS & $\underline{0,01}$ & $(0,00)$ & $\underline{0,01}$ & $(0,00)$ & 113,0 & $(0,00)$ & $-0,05$ & $(0,16)$ & 14,0 & $(0,08)$ \\
\hline _HEN & $\overline{0,00}$ & $(0,00)$ & $\overline{0,00}$ & $(0,00)$ & $-1,6$ & $(0,53)$ & 0,00 & $(0,90)$ & 0,0 & $(0,95)$ \\
\hline \multicolumn{11}{|l|}{ XSMIN } \\
\hline ALL & 233,99 & $(0,00)$ & 124,18 & $(0,00)$ & 53,4 & $(0,00)$ & 19,69 & $(0,28)$ & 135,9 & $(0,00)$ \\
\hline SIE & 140,73 & $(0,00)$ & 71,97 & $(0,00)$ & 42,4 & $(0,00)$ & 14,19 & $(0,24)$ & 103,4 & $(0,00)$ \\
\hline DAIM & 50,28 & $(0,01)$ & 17,02 & $(0,20)$ & 51,9 & $(0,00)$ & $\underline{-2,29}$ & $(0,83)$ & 105,5 & $(0,00)$ \\
\hline DBANK & 72,37 & $(0,00)$ & 21,14 & $(0,03)$ & 47,3 & $(0,00)$ & $\overline{0,29}$ & $(0,94)$ & 82,0 & $(0,00)$ \\
\hline HYPV & 4,54 & $(0,01)$ & 1,97 & $(0,04)$ & 114,5 & $(0,00)$ & 2,39 & $(0,03)$ & 113,3 & $(0,01)$ \\
\hline RWE & 2,16 & $(0,00)$ & 1,68 & $(0,00)$ & 85,5 & $(0,00)$ & 2,15 & $(0,00)$ & 5,3 & $(0,21)$ \\
\hline _TUI & 7,93 & $(0,03)$ & 8,14 & $(0,02)$ & 85,8 & $(0,00)$ & 7,51 & $(0,03)$ & 52,2 & $(0,05)$ \\
\hline LIN & 0,58 & $(0,03)$ & $\underline{-0,22}$ & $(0,20)$ & 64,3 & $(0,00)$ & 0,58 & $(0,03)$ & 0,0 & $(0,54)$ \\
\hline
\end{tabular}

*Only those cross sections are reported, for whom $b_{1}$ is significant at the $5 \%$ level in the regression

without control variables.

Estimates $b_{1}$ or $p$-values are underlined in the following cases:

1. The sign of $b_{1}$ contradicts the expectation

2. The inclusion of the control variables leads to a loss of significance of $b_{1}$ ( $p$-value $>5 \%$ )

Table 6.38.: Robustness check of the time-series regression results for the test of the relationship between default risk and the slope of the smile for a constant maturity of 36 days - Part II 


\subsection{Examination in the Framework of a Pooled Series Regression}

The robustness of the pooled series regression results has been tested by including $H V$ and $I M P V$ in the regression models 6.56 and 6.57 .

$$
\begin{array}{r}
C D S_{i, t}=\beta_{0 i}+\beta_{1} \cdot S L P j_{i, t}+\beta_{2} \cdot H V_{i, t}+\beta_{3} \cdot t+u_{i, t}, \quad j \in(70,90,100,110,130) \\
C D S_{i, t}=\beta_{0 i}+\beta_{1} \cdot X S M I N_{i, t}+\beta_{2} \cdot H V_{i, t}+\beta_{3} \cdot t+u_{i, t} \\
C D S_{i, t}=\beta_{0 i}+\beta_{1} \cdot S L P j_{i, t}+\beta_{2} \cdot I M P V_{i, t}+\beta_{3} \cdot t+u_{i, t}, \quad j \in(70,90,100,110,130) \\
C D S_{i, t}=\beta_{0 i}+\beta_{1} \cdot X S M I N_{i, t}+\beta_{2} \cdot I M P V_{i, t}+\beta_{3} \cdot t+u_{i, t}
\end{array}
$$

Table 6.39 juxtaposes the estimates $b_{1}$ and their respective $p$-values for the estimation of the original models 6.56 and 6.57, the models including $H V$, 6.66 and 6.67, and the models including $I M P V$, 6.68 and 6.69, for constant maturities of 36, 60 and 180 days. The picture which can be gathered from table 6.39 differs from the results of the robustness check in the time-series dimension in 3 respects: First, $I M P V$ fails to yield significant coefficients everywhere, namely in the constant maturity case of 180 days. This is due to the maturity related decreasing power of $I M P V$ to explain $C D S$ which has already been noted in section 6.5.3. Secondly, while in the time-series dimension SLP100 produces the most robust results of all slope variables, it ranks besides $X S M I N$ among those generating the least stable results in the pooled series context. And thirdly, IMPV does not appear to have a much more detrimental effect on the existing relationship between the CDS spread level and the slope variables than $H V$. Further and not surprisingly, the constant maturity case of 60 days - exhibiting insignificant results from the beginning - does not seem to pass the robustness check in the least. Overall, in the constant maturity cases of 36 and 180 days, sign inversions of $b_{1}$ and losses of significance are less frequent and the latter are less severe than in the time-series robustness check.

Altogether, the robustness check in the pooled series setting seems to produce more validating evidence of the initial results than the robustness check in the time-series dimension. 


$$
\begin{array}{ll}
\text { Case 1: } & C D S_{i, t}=\beta_{0_{i}}+\beta_{1} \cdot S L P j_{i, t}+\beta_{2} \cdot t+u_{i, t}, \quad j \in\{70,90,100,110,130\} \\
& C D S_{i, t}=\beta_{0_{i}}+\beta_{1} \cdot X S M I N_{i, t}+\beta_{2} \cdot t+u_{i, t} \\
\text { Case 2: } \quad & C D S_{i, t}=\beta_{0_{i}}+\beta_{1} \cdot S L P j_{i, t}+\beta_{2} \cdot H V_{i, t}+\beta_{3} \cdot t+u_{i, t}, j \in\{70,90,100,110,130\} \\
& C D S_{i, t}=\beta_{0_{i}}+\beta_{1} \cdot X S M I N_{i, t}+\beta_{2} \cdot H V_{i, t}+\beta_{3} \cdot t+u_{i, t} \\
\text { Case 3: } & C D S_{i, t}=\beta_{0_{i}}+\beta_{1} \cdot S L P j_{i, t}+\beta_{2} \cdot I M P V_{i, t}+\beta_{3} \cdot t+u_{i, t}, \quad j \in\{70,90,100,110,130\} \\
& C D S_{i, t}=\beta_{0_{i}}+\beta_{1} \cdot X S M I N_{i, t}+\beta_{2} \cdot I M P V_{i, t}+\beta_{3} \cdot t+u_{i, t}
\end{array}
$$

\begin{tabular}{|c|c|c|c|c|c|c|c|c|c|c|}
\hline \multirow{2}{*}{ Variable } & \multirow{2}{*}{\multicolumn{2}{|c|}{$\begin{array}{c}\text { Case } 1 \\
b_{1}\end{array}$}} & \multicolumn{4}{|c|}{ Case 2} & \multicolumn{4}{|c|}{ Case 3} \\
\hline & & & \multicolumn{2}{|c|}{$b_{1}$} & \multicolumn{2}{|c|}{$b_{2}$} & \multicolumn{2}{|c|}{$b_{1}$} & \multicolumn{2}{|c|}{$b_{2}$} \\
\hline \multicolumn{11}{|c|}{ Constant maturity of 36 days } \\
\hline SLP70 & 7,94 & $(0,00)$ & 4,97 & $(0,00)$ & 127,47 & $(0,02)$ & 3,02 & $(0,00)$ & 221,22 & $(0,01)$ \\
\hline SLP90 & 16,12 & $(0,00)$ & 12,56 & $(0,00)$ & 130,66 & $(0,02)$ & 8,97 & $(0,00)$ & 223,99 & $(0,01)$ \\
\hline SLP100 & $-96,86$ & $(0,04)$ & $-9,86$ & $\underline{(0,72)}$ & 129,72 & $(0,02)$ & 30,03 & $\underline{(0,25)}$ & 240,81 & $(0,01)$ \\
\hline SLP110 & $-35,88$ & $(0,00)$ & $-17,76$ & $(0,00)$ & 119,75 & $(0,03)$ & $-7,32$ & $(0,01)$ & 217,92 & $(0,01)$ \\
\hline SLP130 & $-10,34$ & $(0,00)$ & $-5,61$ & $(0,00)$ & 123,62 & $(0,02)$ & $-2,85$ & $(0,00)$ & 218,97 & $(0,01)$ \\
\hline XSMIN & 17,59 & $(0,10)$ & $\underline{-6,33}$ & $(0,08)$ & 134,39 & $(0,02)$ & $-11,47$ & $(0,00)$ & 231,94 & $(0,01)$ \\
\hline \multicolumn{11}{|c|}{ Constant maturity of 60 days } \\
\hline SLP70 & 0,14 & $(0,33)$ & 0,08 & $(0,46)$ & 132,49 & $(0,01)$ & 0,14 & $(0,18)$ & 246,52 & $(0,01)$ \\
\hline SLP90 & 0,23 & $(0,44)$ & 0,21 & $(0,48)$ & 132,53 & $(0,01)$ & 0,45 & $(0,18)$ & 246,65 & $(0,01)$ \\
\hline SLP100 & $-12,06$ & $(0,01)$ & $-0,80$ & $(0,75)$ & 132,37 & $(0,02)$ & 7,95 & $(0,08)$ & 250,07 & $(0,01)$ \\
\hline SLP110 & $-0,96$ & $(0,22)$ & $-0,36$ & $(0,43)$ & 132,38 & $(0,01)$ & $-0,32$ & $(0,19)$ & 246,33 & $(0,01)$ \\
\hline SLP130 & $-0,22$ & $\underline{(0,26)}$ & $-0,10$ & $(0,45)$ & 132,44 & $(0,01)$ & $-0,13$ & $\underline{(0,18)}$ & 246,41 & $(0,01)$ \\
\hline XSMIN & 4,88 & $(0,12)$ & 1,26 & $(0,31)$ & 132,09 & $(0,01)$ & $-1,03$ & $(0,04)$ & 247,26 & $(0,01)$ \\
\hline \multicolumn{11}{|c|}{ Constant maturity of 180 days } \\
\hline SLP70 & 0,02 & $(0,00)$ & 0,01 & $(0,02)$ & 132,49 & $(0,02)$ & 0,13 & $(0,00)$ & 27,35 & $(0,16)$ \\
\hline SLP90 & 0,03 & $(0,00)$ & 0,02 & $(0,09)$ & 132,50 & $(0,02)$ & 0,39 & $(0,00)$ & 31,35 & $(0,11)$ \\
\hline SLP100 & 0,04 & $(0,30)$ & 0,02 & $(0,76)$ & 132,53 & $(0,01)$ & 2,22 & $(0,00)$ & 54,97 & $(0,00)$ \\
\hline SLP110 & $-0,14$ & $(0,00)$ & $-0,13$ & $(0,00)$ & 132,48 & $(0,01)$ & $-0,27$ & $(0,26)$ & 17,35 & $(0,27)$ \\
\hline SLP130 & $-0,03$ & $(0,00)$ & $-0,02$ & $(0,00)$ & 132,49 & $(0,02)$ & $-0,13$ & $(0,00)$ & 22,41 & $(0,23)$ \\
\hline XSMIN & $\underline{-0,02}$ & $(0,79)$ & 0,01 & $(0,89)$ & 132,54 & $(0,01)$ & $\underline{-0,02}$ & $(0,79)$ & 16,14 & $(0,19)$ \\
\hline
\end{tabular}

Estimates $b_{1}$ or $p$-values are underlined in the following cases:

1. The sign of $b_{1}$ contradicts the expectation

2. The inclusion of the control variables leads to a loss of significance of $b_{1}$ ( $p$-value $>5 \%$ )

Table 6.39.: Robustness check of the pooled series regression results for the test of the relationship between default risk and slope variables 


\subsection{Disentangling the Volatility-Only from the Default-Risk-Only-}

Effect In order to dissociate the volatility-only from the default-risk-only-effect, both have been eliminated each at a time in order to study the respective remaining effect on the shape of the smile. This exercise has been carried out in a pooled series framework consisting of the 10 most important stock cross-sections and for a constant maturity of 36 days.

The elimination of the volatility-only-effect has been studied by testing the regression models 6.56 and 6.57 on a data sample in which the admissible range of IMPV has been limited to the interval $[23,26 \% ; 25,51 \%]$. This interval has been determined under the restrictions that the resulting sample must contain 800 datasets and that the maximum difference of $I M P V$ within this sample is minimal. The regression results after the elimination of the volatility-only effect are displayed in table 6.40 .

\begin{tabular}{|c|c|c|c|c|c|c|c|c|c|c|c|c|}
\hline \multicolumn{7}{|c|}{$\begin{array}{l}\operatorname{CDS}_{i, t}=\beta_{0_{i}}+\beta_{1} \cdot S L P j_{i, t}+\beta_{2} \cdot t+u_{i, t} \\
\operatorname{CDS}_{i, t}=\beta_{0_{i}}+\beta_{1} \cdot X \operatorname{SMIN}_{i, t}+\beta_{2} \cdot t+u_{i, t}\end{array}$} & \multicolumn{6}{|c|}{$j \in\{70,90,100,110,130\}$} \\
\hline Variable & \multicolumn{3}{|c|}{$b_{0}$} & \multicolumn{3}{|c|}{$b_{1}$} & \multicolumn{3}{|c|}{$b_{2}$} & \multirow{2}{*}{$\begin{array}{l}\mathbf{R 2} \\
\text { cor. }\end{array}$} & \multirow{2}{*}{$\begin{array}{l}\mathbf{F} \\
941\end{array}$} & \multirow{2}{*}{$\begin{array}{c}\text { DW } \\
0,04\end{array}$} \\
\hline SLP70 & 39,4 & {$[12,3]$} & $(0,00)$ & $-0,34$ & {$[-2,2]$} & $(0,03)$ & 0,00 & {$[0,11]$} & $(0,91)$ & & & \\
\hline SLP90 & 39,2 & {$[12,0]$} & $(0,00)$ & $-1,06$ & {$[-2,2]$} & $(0,03)$ & 0,00 & {$[0,11]$} & $(0,91)$ & 0,93 & 943 & 0,04 \\
\hline SLP100 & 38,1 & {$[10,8]$} & $(0,00)$ & $-6,62$ & {$[-3,1]$} & $(0,00)$ & 0,00 & {$[0,13]$} & $(0,90)$ & 0,93 & 948 & 0,05 \\
\hline SLP110 & 39,9 & {$[13,1]$} & $(0,00)$ & 0,66 & {$[1,8]$} & $(0,07)$ & 0,00 & {$[0,13]$} & $(0,90)$ & 0,93 & 938 & 0,03 \\
\hline SLP130 & 39,7 & {$[12,7]$} & $(0,00)$ & 0,29 & {$[2,1]$} & $(0,04)$ & 0,00 & {$[0,12]$} & $(0,91)$ & 0,93 & 940 & 0,04 \\
\hline XSMIN & 40,1 & {$[13,0]$} & $(0,00)$ & $-0,11$ & {$[-0,6]$} & $(0,56)$ & 0,00 & {$[0,14]$} & $(0,89)$ & 0,93 & 936 & 0,03 \\
\hline \multicolumn{13}{|c|}{ t-stats in [ ] and corresponding two-sided p-values in ( ) } \\
\hline \multicolumn{13}{|c|}{ All values are calculated for a constant maturity of 36 days. } \\
\hline \multicolumn{13}{|c|}{$\begin{array}{l}\text { IMPV is restricted to the interval }[23,26 \% ; 25,51 \%] \text {. In order to obtain a sufficient number } \\
\text { of datasets, the sample includes the top } 10 \text { cross-sections, yielding } 800 \text { datasets } \\
\text { altogether. }\end{array}$} \\
\hline
\end{tabular}

Table 6.40.: Dissecting the default-risk-only effect by eliminating the volatility-only effect in the test of the relationship between default risk and slope variables

Comparing the results of table 6.40 with the unrestricted pooled series regression results of table 6.36 and focusing on the case of 36 days there, the following striking observations can be made: The coefficient $b_{1}$ seems to have changed signs in all cases except for $S L P 100$. Although the significance of $b_{1}$ is not sufficient in all cases - especially not for $X S M I N$ - the comparison hints at the following: If the volatility-only effect is eliminated, increasing default risk seems indeed to lead to a steepening of the left wing of the smile as predicted by Hypothesis $\mathbf{3}$ and not to a flattening and right translation as suggested by our preliminary findings. However, the positive coefficient of the slope variables SLP110 and SLP130 also seems to indicate a default risk induced steepening of the right wing of the smile, which has been neither predicted by 
Hypothesis 3 nor by our preliminary finding of a flattening and right-translation of the smile. But this finding has to be interpreted with care, since the coefficients of $S L P 110$ and $S L P 130$ are relatively small compared to those of the slope variables of the left wing of the smile and since the coefficient of $S L P 110$ is further slightly insignificant. Finally, it is worthwhile to note the high explanatory power of the results in table 6.40, which could be due to the highly insignificant time trend.

In a second step, the default-risk-only effect has been eliminated. To this end the dependent variable $C D S$ in the regression models 6.56 and 6.57 has been substituted by the volatility variables $I M P V$ and $H V$, respectively, and the admissible range of $C D S$ in the data sample has been restricted to the interval $[21,44 b p ; 27,63 b p]$. Similar as above, the interval has been determined under the conditions that the resulting sample must contain 1.200 datasets ${ }^{352}$ and that the maximum difference of $I M P V$ within this sample is minimal. The regression results after the elimination of the default-risk-only effect are displayed in table 6.41. While the results with $I M P V$ as dependent variable are shown in the upper panel, the lower panel contains the results with $H V$ as dependent variable.

Comparing the upper portion of table 6.41 with table 6.40, it seems as if the volatilityonly effect in the guise of $I M P V$ acted in the same direction on the slope variables of the smile as does the default-risk-only effect, since the pattern of dependence is almost the same in both cases. Considering the lower portion of table 6.41 however, it seems as if the volatility-only effect in the guise of $H V$ behaved opposite to the defaultrisk-only effect, since all coefficients except that of SLP100 show inverse signs as compared to table 6.40. But it is obvious that $H V$ exerts a much weaker effect on the slope of the smile than $I M P V$, since in the case of $H V$ the coefficient $b_{1}$ is insignificant for all slope variables. It remains unclear whether this difference in significance between $I M P V$ and $H V$ is due to true, economic reasons or is rather owed to the construction related proximity of $I M P V$ and the smile variables 353 . Assuming the latter, the results can be interpreted in the following way: While the results of $I M P V$ in the upper panel of table 6.41 are mainly due to the construction related proximity of $I M P V$ and the slope variables, the results of $H V$ in the lower panel are less significant, but reflect the true impact of the volatility-only effect which is a flattening and right-translation of the smile.

\footnotetext{
352 The number of 1.200 datasets as compared to 800 in the case of the elimination of $I M P V$ can be justified by the relatively greater standard deviation of $C D S$ as compared to that of $I M P V$.

353 Since $I M P V$ equals the sum of the three smile coefficients $B 0, B 1$ and $B 3$ (cf. section 6.4.7.2), it is highly correlated with all smile dependent variables.
} 


\begin{tabular}{|c|c|c|c|c|c|c|c|c|c|c|c|c|}
\hline \multirow{2}{*}{$\begin{array}{l}\text { Case 1: } \\
\text { Case 2: }\end{array}$} & \multicolumn{7}{|c|}{$\begin{array}{l}I M P V_{i, t}=\beta_{0_{i}}+\beta_{1} \cdot S L P j_{i, t}+\beta_{2} \cdot t+u_{i, t} \\
I M P V_{i, t}=\beta_{0}+\beta_{1} \cdot X S M I N_{i, t}+\beta_{2} \cdot t+u_{i, t}\end{array}$} & \multicolumn{5}{|c|}{$j \in\{70,90,100,110,130\}$} \\
\hline & \multicolumn{7}{|c|}{$\begin{array}{l}H V_{i, t}=\beta_{0_{i}}+\beta_{1} \cdot S L P j_{i, t}+\beta_{2} \cdot t+u_{i, t} \\
H V_{i, t}=\beta_{0_{i}}+\beta_{1} \cdot X S M I N_{i, t}+\beta_{2} \cdot t+u_{i, t}\end{array}$} & \multicolumn{5}{|c|}{$j \in\{70,90,100,110,130\}$} \\
\hline Variable & & $b_{0}$ & & & $b_{1}$ & & & $b_{2}$ & & $\begin{array}{l}\text { R2 } \\
\text { cor. }\end{array}$ & $F$ & DW \\
\hline \multicolumn{13}{|l|}{ Case1: } \\
\hline SLP70 & 0,29 & {$[12,8]$} & $(0,00)$ & $-0,002$ & {$[-2,42]$} & $(0,02)$ & 0,00 & {$[-1,23]$} & $(0,22)$ & 0,34 & 77,3 & 0,07 \\
\hline SLP90 & 0,29 & {$[12,6]$} & $(0,00)$ & $-0,007$ & {$[-1,42]$} & $(0,16)$ & 0,00 & {$[-1,23]$} & $(0,22)$ & 0,33 & 75,1 & 0,09 \\
\hline SLP100 & 0,30 & {$[12,9]$} & $(0,00)$ & 0,018 & {$[8,50]$} & $(0,00)$ & 0,00 & {$[-1,21]$} & $(0,23)$ & 0,35 & 82,8 & 0,05 \\
\hline SLP110 & 0,29 & {$[12,9]$} & $(0,00)$ & 0,004 & {$[4,05]$} & $(0,00)$ & 0,00 & {$[-1,22]$} & $(0,22)$ & 0,34 & 79,4 & 0,06 \\
\hline SLP130 & 0,29 & {$[12,8]$} & $(0,00)$ & 0,002 & {$[3,31]$} & $(0,00)$ & 0,00 & {$[-1,23]$} & $(0,22)$ & 0,34 & 78,6 & 0,07 \\
\hline XSMIN & 0,23 & {$[6,7]$} & $(0,00)$ & 0,054 & {$[1,71]$} & $(0,09)$ & 0,00 & {$[-1,18]$} & $(0,24)$ & 0,33 & 74,1 & 0,20 \\
\hline \multicolumn{13}{|l|}{ Case 2: } \\
\hline SLP70 & 0,32 & {$[9,76]$} & $(0,00)$ & 0,001 & {$[1,23]$} & $(0,22)$ & 0,00 & {$[-1,05]$} & $(0,29)$ & 0,23 & 45,8 & 0,63 \\
\hline SLP90 & 0,32 & {$[9,87]$} & $(0,00)$ & 0,005 & {$[1,26]$} & $(0,21)$ & 0,00 & {$[-1,04]$} & $(0,30)$ & 0,23 & 45,9 & 0,63 \\
\hline SLP100 & 0,32 & {$[9,42]$} & $(0,00)$ & $-0,005$ & {$[-1,30]$} & $(0,20)$ & 0,00 & {$[-1,07]$} & $(0,29)$ & 0,23 & 45,5 & 0,63 \\
\hline SLP110 & 0,32 & {$[9,63]$} & $(0,00)$ & $-0,002$ & {$[-1,23]$} & $(0,22)$ & 0,00 & {$[-1,06]$} & $(0,29)$ & 0,23 & 45,7 & 0,63 \\
\hline SLP130 & 0,32 & {$[9,69]$} & $(0,00)$ & $-0,001$ & {$[-1,23]$} & $(0,22)$ & 0,00 & {$[-1,05]$} & $(0,29)$ & 0,23 & 45,7 & 0,63 \\
\hline XSMIN & 0,24 & {$[5,31]$} & $(0,00)$ & 0,071 & {$[2,05]$} & $(0,04)$ & 0,00 & {$[-1,08]$} & $(0,28)$ & 0,23 & 46,9 & 0,64 \\
\hline $\begin{array}{l}\text { t-stats in }[ \\
\text { All values } \\
\text { CDS is re } \\
\text { of datase }\end{array}$ & $\begin{array}{l}\text { and cor } \\
\text { are calc }\end{array}$ & $\begin{array}{l}\text { responc } \\
\text { llated fo }\end{array}$ & $\begin{array}{l}\text { ing two- } \\
\text { a const }\end{array}$ & $\begin{array}{l}\text { ided } p-v \\
\text { ant matu } \\
4 \text { bp; } 2 \\
\text { top } 10\end{array}$ & $\begin{array}{l}\text { values in } \\
\text { urity of } 36 \\
7,63 \mathrm{bp}]\end{array}$ & $\begin{array}{l}\text { () } \\
\text { days. }\end{array}$ & & . & sets & & & \\
\hline
\end{tabular}

Table 6.41.: Dissecting the volatility-only effect by eliminating the default-risk-only effect in the test of the relationship between equity volatility and slope variables

At least as to the implication of a right-translation, this interpretation is not arguable, since in both the upper and the lower panel of table 6.41 the coefficient of XSMIN is positive and significant. This contrasts with the negative, though insignificant coefficient of $X S M I N$ in the case of the default-risk-only effect as depicted in table 6.40 .

Altogether, it can be concluded that the default-risk-only effect seems to steepen both wings of the smile, whereas the volatility-only effect seems to contribute to the flattening and right-translation of the smile. Especially the first finding contrasts somewhat with the originally detected default risk induced flattening of the smile. This contradiction now explains why the inclusion of $H V$ and $I M P V$ in the original regression models leads to sign inversions and losses of significance. 


\subsubsection{Summary and Discussion of the Results in the Light of Hitherto Existing Findings}

6.5.4.4.1. Summary of the Results Our expectations regarding the results of the above analysis have been governed by Hypothesis 3, and more importantly by our preliminary findings in the wake of the smile approximation. While Hypothesis 3 simply claims a negative relationship between the level of default risk and the slope of the smile, our preliminary findings instead suggest a default risk induced flattening and right-translation of the smile. As far as the left wing of the smile is concerned, the two predictions are incompatible with each other: While Hypothesis 3 requires a steepening of the smile with increasing default risk, our preliminary findings in contrast suggest a flattening.

The above analysis has first focused on the relationship between the CDS spread level and the coefficients of the smile polynomial. It has then centered on the examination of the link between the CDS spread level on the one hand and the slope of the smile for different strike-to-spot ratios as well as the horizontal coordinate of the valley of the smile on the other. The first part of the analysis clearly indicates a default risk induced flattening of the smile, since the intercept and the quadratic term of the smile polynomial correlate negatively with the CDS spread level, whereas the linear term correlates positively with the latter. The second part of the analysis confirmed this picture: The slope of the downward sloping left wing of the smile depends positively and that of the upward sloping right wing negatively on the CDS spread level. Further, the horizontal coordinate of the valley of the smile is correlated positively with the level of default risk. Both these findings also clearly hint at a default risk induced flattening and right-translation of the smile.

The robustness check of these results revealed that in the time-series dimension the relationship between the CDS spread level and the pattern of the smile is dominated by $I M P V$. However, in the pooled series context the original pattern of dependence remains largely unchanged after the inclusion of $I M P V$. As opposed to IMPV, $H V$ unfolds a much less detrimental effect, leaving the original pattern largely unaltered in the time-series dimension as well as in the pooled series context. Altogether, the inclusion of equity volatility into the regression models casts light doubts on our initial observation of a default risk induced flattening and right-translation of the smile.

But to shed more light on the interaction of equity volatility and CDS spread, the volatility-only effect has been disentangled from the default-risk-only effect by eliminating both, but each at a time in order to study the respective remaining effect. This 
dissociation of effects shows that the default-risk-only effect indeed entails a steepening of the left wing of the smile as predicted by Hypothesis 3. But it also implies a slight steepening of the originally upwards sloping right wing of the smile, which contradicts Hypothesis 3 and seems in the first instance inexplicable. The dissociation of effects further demonstrates that in turn the volatility-only effect is responsible for a flattening and right-translation of the smile. Relating these findings to our initial observation of a default risk induced flattening and right-translation now explains why the inclusion of $I M P V$ and $H V$ in the original regression models leads to sign inversions and losses of significance.

Considering the combined impact of the default-risk-only and the volatility-only effect resulting in a default risk induced flattening and right-translation of the smile, Hypothesis 3 does not seem to be confirmed, since it predicts a steepening.

\subsection{Discussion of the Results in the Light of Hitherto Existing Find-}

ings As discussed in section 5.1.3.3, hitherto existing studies aiming at the link between default risk and the slope of the smile are very scarce. Further, out of 5 existing studies altogether only only 3 analyze the link between the credit spread level and the slope of the individual volatility smile 354 . Interestingly, of these 3 studies in turn, only CREMERS ET AL. (2005b) manage to obtain a significant coefficient for the slope estimate in a joint regression of the credit spread on the the slope measure and the level of implied volatility 355 . This is consistent with my finding that the inclusion of implied volatility into the regression model unfolds a certain detrimental effect on the relationship between the CDS spread level and the slope variables.

More importantly, all existing studies limit their attention to the slope at the ATM point. And consistently with my findings, all existing studies observe a negative relationship between default risk and the slope at the ATM point. They interpret this

354 The 2 studies deviating from this approach are COLLIN-DUfRESNE ET AL. (2001) and TOFT/PRUCYK (1997): While COLLIN-DUfRESNE ET AL. (2001) use the index slope instead of the individual slope, TOFT/PRUCYK (1997) regress their slope measure on financial leverage and not on credit spreads (cf. table 5.3.

355 However, it has to be noted that the t-statistic of CREMERS ET AL. (2005b) for their implied volatility variable is surprisingly low compared to that of the slope variable. This suggests that their finding of a significant coefficient for the slope variable is only owed to a relatively poor volatility estimate. Performing a similar exercise by regressing the credit spread simultaneously on historical and implied volatility, financial leverage, stock return and slope measure, CAO ET AL. (2006) fail to obtain a significant result for the coefficient of the latter. Finally.HULL ET AL. (2004a) only manage to obtain a significant coefficient for the slope estimate in a joint regression of the credit spread level on the slope measure and on the level of implied volatility after orthogonalizing the volatility measure with regard to the slope variable. 
as a default risk induced general steepening of the whole smile. As opposed to this conclusion however, my findings reveal something else: In reality, increasing default risk is accompanied by a combined flattening and right-translation of the smile, which is incompatible with an ubiquitous steepening. So why do existing studies as well as mine observe a negative dependence between default risk and the slope at the ATM point? The reason is the default risk associated right-translation which moves the relatively steep left wing in direction of the ATM point, thus creating the impression of a generally steepening smile, if the viewpoint is restricted to a strike-to-spot ratio around one.

\subsubsection{The Credit Spread Level and the Moments of the Risk-Neutral Equity Return Density}

The following section is devoted to the test of Hypothesis 4: The skewness of the risk-neutral equity return density depends negatively on the level of default risk.

\subsubsection{Derivation of the Regression Models}

Consistent with the theoretical link between the shape of the smile and that of the risk-neutral return density (see section 5.1.2.2), decreasing skewness in turn should be coupled with an increasing steepening of the smile, which however does not seem to be the case in reality. Instead, as we have just seen above, increasing default risk is rather associated with a flattening and right-translation of the smile. But despite the seeming contradiction between the notion of a steepening smile and our finding of a flattening and right-translation instead, it is well imaginable that the combination of flattening and right-translation still accommodates the possibility of decreasing return skewness.

Independent of the issue of skewness, our observation of a flattening of the smile could also be related to decreasing risk-neutral kurtosis, as in theory the existence of a U-shaped smile is often associated with excess kurtosis (cf. section 3.1.3.2).

Therefore, based on Hypothesis $\mathbf{4}$ as well as on our preliminary findings above, it is reasonable to expect a negative relationship between the CDS spread level on the one hand and the level of risk-neutral skewness and kurtosis on the other. To challenge this expectation, the CDS spread level has simply been regressed on the third and forth moment of the risk-neutral density in the time-series dimension as well as in a pooled series setting: 
Time-series dimension:

$$
\begin{aligned}
& C D S_{t}=\beta_{0}+\beta_{1} \cdot S K E W_{t}+\beta_{2} \cdot t+u_{t} \\
& C D S_{t}=\beta_{0}+\beta_{1} \cdot K U R T_{t}+\beta_{2} \cdot t+u_{t}
\end{aligned}
$$

Pooled series regression:

$$
\begin{aligned}
& C D S_{i, t}=\beta_{0 i}+\beta_{1} \cdot S K E W_{i, t}+\beta_{2} \cdot t+u_{i, t} \\
& C D S_{i, t}=\beta_{0 i}+\beta_{1} \cdot K U R T_{i, t}+\beta_{2} \cdot t+u_{i, t}
\end{aligned}
$$

The influence of $S K E W$ and $K U R T$ has been tested separately, because our goal is not to explain the CDS spread level as completely as possible, but rather to detect patterns of dependency. These patterns could be blurred by a joint regression, since both moments are correlated by construction. 


\subsubsection{Regression Results}

6.5.5.2.1. Examination in the Time-Series Dimension The test of the relationship between $C D S$ and the risk-neutral moments $S K E W$ and $K U R T$ according to models 6.70 and 6.71 has been carried out for all stocks except _DEG due to an insufficient number of observations. Tables 6.42 and 6.43 show the detailed regression results for the constant maturity case of 36 days, whereas table 6.44 exhibits a constant-maturity-wise cross-sectional summary of all results, once averaged across all cross-sections and once only across the 5 most important stocks. Tables 6.42 and 6.43 only report those cross-sections for whom the coefficient $b_{1}$ is significant at the $1 \%$ level, in order to keep this documentation as lean and readable as possible. The totality of detailed results for all cross-sections and constant maturities can be viewed in appendix C.4.

\begin{tabular}{|c|c|c|c|c|c|c|}
\hline \multicolumn{7}{|c|}{$C D S_{t}=\beta_{0}+\beta_{1} \cdot S K E W_{t}+\beta_{2} \cdot t+u_{t}$} \\
\hline ID* & $b_{0}$ & $b_{1}$ & $b_{2}$ & R2 cor. & $\mathbf{F}$ & DW \\
\hline _ALL & \begin{tabular}{l|}
13,47 \\
{$[1,56]$} \\
$(0,12)$
\end{tabular} & $\begin{array}{r}-292,33 \\
{[-6,86]} \\
(0,00)\end{array}$ & $\begin{array}{r}-0,03 \\
{[-3,82]} \\
(0,00)\end{array}$ & 0,37 & 211,52 & 0,12 \\
\hline SIIE & $\begin{array}{l}24,39 \\
{[5,64]} \\
(0,00)\end{array}$ & $\begin{array}{r}-233,88 \\
{[-10,23]} \\
(0,00)\end{array}$ & $\begin{array}{r}-0,05 \\
{[-13,49]} \\
(0,00)\end{array}$ & 0,78 & $1.369,90$ & 0,31 \\
\hline DTTE & $\begin{array}{r}157,03 \\
{[8,02]} \\
(0,00)\end{array}$ & $\begin{array}{r}-722,56 \\
{[-7,83]} \\
(0,00)\end{array}$ & $\begin{array}{r}-0,28 \\
{[-11,26]} \\
(0,00)\end{array}$ & 0,72 & 987,01 & 0,29 \\
\hline -DBANK & $\begin{array}{l}13,83 \\
{[4,68]} \\
(0,00)\end{array}$ & $\begin{array}{r}-105,46 \\
{[-7,41]} \\
(0,00)\end{array}$ & $\begin{array}{r}-0,01 \\
{[-4,61]} \\
(0,00)\end{array}$ & 0,46 & 330,57 & 0,13 \\
\hline MRE & $\begin{array}{l}45,85 \\
{[7,89]} \\
(0,00)\end{array}$ & $\begin{array}{r}-67,85 \\
{[-3,72]} \\
(0,00)\end{array}$ & $\begin{array}{r}-0,04 \\
{[-5,42]} \\
(0,00)\end{array}$ & 0,36 & 187,24 & 0,22 \\
\hline BASF & $\begin{array}{r}28,14 \\
{[27,58]} \\
(0,00)\end{array}$ & $\begin{array}{r}-13,39 \\
{[-3,23]} \\
(0,00)\end{array}$ & $\begin{array}{r}-0,03 \\
{[-21,58]} \\
(0,00)\end{array}$ & 0,76 & $1.212,71$ & 0,12 \\
\hline EON & $\begin{array}{r}46,90 \\
{[11,52]} \\
(0,00) \\
\end{array}$ & $\begin{array}{r}-36,33 \\
{[-3,38]} \\
(0,00)\end{array}$ & $\begin{array}{r}-0,04 \\
{[-6,17]} \\
(0,00) \\
\end{array}$ & 0,30 & 159,54 & 0,08 \\
\hline COB & $\begin{array}{l}84,33 \\
{[7,67]} \\
(0,00)\end{array}$ & $\begin{array}{r}-95,35 \\
{[-3,15]} \\
(0,00)\end{array}$ & $\begin{array}{r}-0,09 \\
{[-5,47]} \\
(0,00)\end{array}$ & 0,29 & 150,12 & 0,17 \\
\hline -HYPV & $\begin{array}{l}72,49 \\
{[8,31]} \\
(0,00)\end{array}$ & $\begin{array}{r}-44,78 \\
{[-3,36]} \\
(0,00)\end{array}$ & $\begin{array}{r}-0,05 \\
{[-3,64]} \\
(0,00)\end{array}$ & 0,15 & 67,78 & 0,07 \\
\hline LLH & $\begin{array}{r}154,34 \\
{[24,00]} \\
(0,00)\end{array}$ & $\begin{array}{r}-9,69 \\
{[-3,12]} \\
(0,00)\end{array}$ & $\begin{array}{r}-0,12 \\
{[-11,19]} \\
(0,00)\end{array}$ & 0,44 & 272,03 & 0,03 \\
\hline tats in [ & and corre & onding $p$ & alues in & & & \\
\hline
\end{tabular}

Table 6.42.: Time-series regression results for the test of the relationship between default risk and risk-neutral skewness for a constant maturity of 36 days

As can be read from tables 6.42 and 6.43, the results for the highly significant crosssections yield without exception a negative coefficient $b_{1}$ for both risk-neutral mo- 


\begin{tabular}{|c|c|c|c|c|c|c|}
\hline \multicolumn{7}{|c|}{$C D S_{t}=\beta_{0}+\beta_{1} \cdot K U R T_{t}+\beta_{2} \cdot t+u_{t}$} \\
\hline ID & $b_{0}$ & $b_{1}$ & $b_{2}$ & R2 cor. & $F$ & DW \\
\hline _ALL & $\begin{array}{r}4.451,47 \\
{[15,96]} \\
(0,00)\end{array}$ & $\begin{array}{r}-1.460,5 \\
{[-15,83]} \\
(0,00)\end{array}$ & $\begin{array}{r}-0,01 \\
{[-3,45]} \\
(0,00)\end{array}$ & 0,71 & 895,25 & 0,39 \\
\hline _SIE & $\begin{array}{r}3.052,63 \\
{[11,16]} \\
(0,00)\end{array}$ & $\begin{array}{r}-994,56 \\
{[-10,92]} \\
(0,00)\end{array}$ & $\begin{array}{r}-0,03 \\
{[-6,95]} \\
(0,00)\end{array}$ & 0,80 & $1.500,64$ & 0,40 \\
\hline DAIM & $\begin{array}{r}2.426,08 \\
{[5,15]} \\
(0,00)\end{array}$ & $\begin{array}{r}-753,16 \\
{[-4,81]} \\
(0,00)\end{array}$ & $\begin{array}{r}-0,10 \\
{[-12,71]} \\
(0,00)\end{array}$ & 0,77 & $1.275,74$ & 0,27 \\
\hline DBANK & $\begin{array}{r}2.025,82 \\
{[10,81]} \\
(0,00)\end{array}$ & $\begin{array}{r}-660,73 \\
{[-10,64]} \\
(0,00)\end{array}$ & $\begin{array}{r}-0,01 \\
{[-6,87]} \\
(0,00)\end{array}$ & 0,69 & 832,91 & 0,42 \\
\hline -MRE & $\begin{array}{r}1.682,00 \\
{[6,88]} \\
(0,00)\end{array}$ & $\begin{array}{r}-542,02 \\
{[-6,63]} \\
(0,00)\end{array}$ & $\begin{array}{r}-0,02 \\
{[-3,41]} \\
(0,00)\end{array}$ & 0,52 & 368,50 & 0,53 \\
\hline _HYPV & $\begin{array}{r}382,48 \\
{[3,63]} \\
(0,00)\end{array}$ & $\begin{array}{r}-100,79 \\
{[-2,88]} \\
(0,00)\end{array}$ & $\begin{array}{r}-0,06 \\
{[-4,13]} \\
(0,00)\end{array}$ & 0,16 & 71,24 & 0,09 \\
\hline -RWE & $\begin{array}{r}125,02 \\
{[7,67]} \\
(0,00)\end{array}$ & $\begin{array}{r}-13,37 \\
{[-2,58]} \\
(0,01)\end{array}$ & $\begin{array}{r}-0,09 \\
{[-11,20]} \\
(0,00)\end{array}$ & 0,54 & 396,20 & 0,03 \\
\hline t- & and corre & onding & values in & & & \\
\hline
\end{tabular}

Table 6.43.: Time-series regression results for the test of the relationship between default risk and risk-neutral kurtosis for a constant maturity of 36 days

ments. This picture is confirmed by the summary representation of table 6.44 which shows on average negative coefficients $b_{1}$ for risk-neutral skewness and kurtosis in all constant maturity cases. In the constant maturity cases of 36 and 60 days and for the top 5 stocks the result for $b_{1}$ is on average even significant. Interestingly, the explanatory power $R^{2}$ is astonishingly high, reaching on average $73 \%$ for $S K E W$ and $61 \%$ for $K U R T$ for the top 5 cross-sections. However, as already experienced before, the quality of the results is degrading with increasing constant maturity, which can be concluded from the declining values for the explanatory power $R^{2}$, for the overall significance $F$ and for the significance of $b_{1}$. Moreover, the Durbin-Watson statistic is noticeably small, hinting at auto-correlated residuals, possibly due to omitted key variables or non-stationarity. But since there is only one possibly highly persistent series involved in the regression, namely $C D S$, non-stationarity need not concern us here. Further, the significant coefficients $b_{0}$ and $b_{2}$ corroborate the assumption of an intercept and a time trend.

Altogether, the time-series results indicate as expected that an increasing CDS spread level is associated with decreasing skewness and kurtosis of the risk-neutral return distribution. 


\begin{tabular}{|c|c|c|c|c|c|c|c|c|c|c|}
\hline \multicolumn{11}{|c|}{$\begin{array}{l}C D S_{t}=\beta_{0}+\beta_{1} \cdot S K E W_{t}+\beta_{2} \cdot t+u_{t} \\
C D S_{t}=\beta_{0}+\beta_{1} \cdot K U R T_{t}+\beta_{2} \cdot t+u_{t}\end{array}$} \\
\hline $\mathbf{C S}^{*}$ & Variable & & $\mathbf{b}_{0}$ & $b_{1}$ & & & $b_{2}$ & $\begin{array}{l}\text { R2 } \\
\text { cor. }\end{array}$ & $\mathbf{F}$ & DW \\
\hline \multicolumn{11}{|c|}{ Constant maturity of 36 days } \\
\hline Top 5 & SKEW & 3.364 & {$[9,1](0,00)$} & $-1.080[-8,89]$ & $(0,00)$ & $|-0,08|$ & {$[-7,4](0,00)$} & 0,73 & 1.069 & 0,36 \\
\hline Top 5 & KURT & & {$[8,3](0,02)$} & $-285[-6,90]$ & $(0,01)$ & $-0,10$ & {$[-9,9](0,00)$} & 0,61 & 777 & 0,19 \\
\hline All & SKEW & & {$[13,4](0,01)$} & $-80[-2,88]$ & $(0,16)$ & $-0,07$ & {$[-6,6](0,00)$} & 0,40 & 327 & 0,10 \\
\hline All & KURT & 1.038 & {$[7,6](0,00)$} & $-310[-3,20]$ & $(0,19)$ & $-0,07$ & {$[-6,0](0,00)$} & 0,43 & 399 & 0,15 \\
\hline \multicolumn{11}{|c|}{ Constant maturity of 60 days } \\
\hline Top 5 & SKEW & 1.606 & {$[7,7](0,03)$} & $-496[-7,24]$ & $(0,05)$ & $|-0,10|$ & {$[-8,3] \quad(0,00)$} & 0,66 & 938 & 0,27 \\
\hline Top 5 & KURT & & {$[8,4](0,01)$} & $-195[-5,65]$ & $(0,00)$ & $-0,11[-$ & $-10,9] \quad(0,00)$ & 0,59 & 748 & 0,17 \\
\hline All & SKEW & & {$[14,1](0,00)$} & $-53[-2,33]$ & $(0,19)$ & $-0,08$ & {$[-6,8](0,00)$} & 0,39 & 315 & 0,08 \\
\hline All & KURT & 491 & {$[7,8](0,02)$} & $-129[-2,52]$ & $(0,23)$ & $-0,07$ & {$[-6,3](0,00)$} & 0,40 & 358 & 0,11 \\
\hline \multicolumn{11}{|c|}{ Constant maturity of 180 days } \\
\hline Top 5 & SKEW & & {$[6,2](0,00)$} & $-252[-4,32]$ & $(0,09)$ & $|-0,10|$ & {$[-9,4](0,00)$} & 0,62 & 724 & 0,26 \\
\hline Top 5 & KURT & 109 & {$[14,4](0,00)$} & $-44[-1,86]$ & $(0,07)$ & $-0,12[-$ & $-10,9] \quad(0,00)$ & 0,50 & 508 & 0,09 \\
\hline All & SKEW & 102 & {$[16,1](0,00)$} & $-10[-0,37]$ & $(0,34)$ & $-0,08 \mid$ & {$[-6,9] \quad(0,00)$} & 0,36 & 257 & 0,06 \\
\hline All & KURT & 294 & {$[9,3](0,00)$} & $-64[-1,91]$ & $(0,21)$ & $-0,07$ & {$[-6,6](0,00)$} & 0,39 & 309 & 0,11 \\
\hline \multicolumn{11}{|c|}{ Constant maturity of 240 days } \\
\hline Top 5 & SKEW & 409 & {$[4,9](0,00)$} & $-98[-2,37]$ & $(0,08)$ & $\mid-0,11[-$ & $-10,0](0,00)$ & 0,55 & 557 & 0,21 \\
\hline Top 5 & KURT & 115 & {$[18,3] \quad(0,00)$} & $-8[-1,17]$ & $(0,41)$ & $-0,12[-$ & $-10,5] \quad(0,00)$ & 0,49 & 479 & 0,04 \\
\hline All & SKEW & 104 & {$[17,2] \quad(0,00)$} & $-2[-0,06]$ & $(0,47)$ & $-0,08$ & {$[-6,8] \quad(0,00)$} & 0,36 & 250 & 0,04 \\
\hline All & KURT & 179 & {$[10,2](0,00)$} & $-25[-1,34]$ & $(0,23)$ & $|-0,08|$ & {$[-6,7](0,00)$} & 0,37 & 269 & 0,09 \\
\hline
\end{tabular}

Table 6.44.: Cross-sectional averages of time-series regression results for the test of the relationship between default risk and risk-neutral moments 


\subsection{Examination in the Framework of a Pooled Series Regression}

The relationship between $C D S$ and the risk-neutral moments $S K E W$ and $K U R T$ has also been tested in a pooled series regression according to models 6.72 and 6.73 . The case of a constant maturity of 240 days is not reported here, because it did not yield any meaningful results. The outcome for the first 3 constant maturities can be viewed in table 6.45. The picture which can be gathered from table 6.45 corroborates

\begin{tabular}{|c|c|c|c|c|c|c|c|c|}
\hline \multicolumn{9}{|c|}{$\begin{array}{l}\operatorname{CDS}_{i, t}=\beta_{0_{i}}+\beta_{1} \cdot \operatorname{SKEW_{i,t}}+\beta_{2} \cdot t+u_{i, t} \\
\operatorname{CDS}_{i, t}=\beta_{0_{i}}+\beta_{1} \cdot \operatorname{KURT}_{i, t}+\beta_{2} \cdot t+u_{i, t}\end{array}$} \\
\hline Variable & & $\mathbf{b}_{0}$ & $b_{1}$ & $b_{2}$ & & $\begin{array}{c}\mathbf{R 2} \\
\text { cor. }\end{array}$ & $\mathbf{F}$ & DW \\
\hline \multicolumn{9}{|c|}{ Constant maturity of 36 days } \\
\hline SKEW & 75 & {$[6,36](0,00)$} & $|-245[-1,93](0,05)|$ & $-0,10[-2,31]$ & $(0,02)$ & 0,67 & 1.250 & 0,04 \\
\hline KURT & 4.409 & {$[5,82](0,00)$} & $|-1.427[-5,79](0,00)|$ & $-0,08[-1,90]$ & $(0,06)$ & 0,71 & 1.537 & 0,19 \\
\hline \multicolumn{9}{|c|}{ Constant maturity of 60 days } \\
\hline SKEW & & {$[6,54](0,00)$} & $-191[-2,67](0,01)$ & $-0,11[-2,28]$ & $(0,02)$ & 0,66 & $\mid 1.224$ & 0,06 \\
\hline KURT & & {$[4,48](0,00)$} & $\mathbf{- 2 2 8}[-3,71](0,00)$ & $-0,11[-2,15]$ & $(0,03)$ & 0,65 & 1.186 & 0,07 \\
\hline \multicolumn{9}{|c|}{ Constant maturity of 180 days } \\
\hline SKEW & 115 & {$[5,86](0,00)$} & $-16[-1,61](0,11)$ & $-0,12[-2,35]$ & $(0,02)$ & 0,64 & 1.125 & 0,01 \\
\hline KURT & 175 & {$[2,41](0,02)$} & $-19[-1,00](0,32)$ & $-0,12[-2,37]$ & $(0,02)$ & 0,64 & $\mid 1.135$ & 0,02 \\
\hline
\end{tabular}

Table 6.45.: Pooled series regression results for the test of the relationship between default risk and risk-neutral moments

the findings in the time-series dimension, since $S K E W$ and $K U R T$ yield negative coefficients in all 3 constant maturity cases, which are also significant in the cases of 36 and 60 days. Further, in all 3 constant maturity cases the explanatory power is rather high, with $R^{2}$ ranging between $64 \%$ and $71 \%$. Finally, as to the Durbin-Watson statistic, the intercept and the time trend, the same can be said as in the time-series case.

In summary, the pooled series regression results confirm our expectations of default risk associated decreasing skewness and kurtosis of the risk-neutral return density. 


\subsubsection{Robustness of the Regression Results}

As in the case of the analysis of the slope of the smile, the robustness check consists of two approaches: First, the additional variables $I M P V$ and $H V$ have been included into the regression models. The inclusion of these additional variables is first studied in the time-series dimension (section 6.5.5.3.1) and then in the pooled series framework (section 6.5.5.3.2). Secondly, the volatility-only and the default-risk-only effect have been eliminated each at a time in order to test the respective remaining effect on the moments of the risk-neutral density (section 6.5.5.3.3).

As to the issue of possible non-stationarity, this question need not concern us here, since only one possibly stochastically trending series has been involved in the regression, namely $C D S$.

6.5.5.3.1. Examination in the Time-Series Dimension The robustness of the time-series regression results has been challenged by including $H V$ and $I M P V$ in the regression models 6.70 and 6.71 .

$$
\begin{gathered}
C D S_{t}=\beta_{0}+\beta_{1} \cdot S K E W_{t}+\beta_{2} \cdot H V_{t}+\beta_{3} \cdot t+u_{t} \\
C D S_{t}=\beta_{0}+\beta_{1} \cdot S K E W_{t}+\beta_{2} \cdot I M P V_{t}+\beta_{3} \cdot t+u_{t} \\
C D S_{t}=\beta_{0}+\beta_{1} \cdot K U R T_{t}+\beta_{2} \cdot H V_{t}+\beta_{3} \cdot t+u_{t} \\
C D S_{t}=\beta_{0}+\beta_{1} \cdot K U R T_{t}+\beta_{2} \cdot I M P V_{t}+\beta_{3} \cdot t+u_{t}
\end{gathered}
$$

Table 6.46 juxtaposes the estimates $b_{1}$ and their respective $p$-values for the estimation of the original models 6.70 and 6.71, the models including $H V, 6.74$ and 6.76, and lastly the models including $I M P V, 6.75$ and 6.77 , all for a constant maturity of 36 days. Only those cross-sections are reported, which yielded significant coefficients $b_{1}$ in the estimation of the original models.

Similar to the analysis of the relationship between the CDS spread level and the shape of the smile, the robustness check reveals the important role of the volatility variables $H V$ and $I M P V$ in explaining the CDS spread level, even in the face of third variables like $S K E W$ and $K U R T$. This is stressed by the positivity and significance of the coefficient $b_{2}$ in almost all cases. While the inclusion of $H V$ leaves the sign as well as the significance of the coefficient $b_{1}$ of the risk-neutral moments rather unaffected in most cases, $I M P V$ exerts a more detrimental influence on the coefficients of the risk-neutral moments, especially so in the case of $K U R T$. 
Distinguishing between the robustness of the coefficient of $S K E W$ on the on hand and that of $K U R T$ on the other, it can be concluded that the robustness check in the time-series dimension seems to corroborate the initial observation of a negative link between default risk and the level of risk-neutral skewness, but casts doubts on the existence of a similar link between default risk and the level of risk-neutral kurtosis. 


\begin{tabular}{|c|c|c|c|c|c|c|c|c|c|c|}
\hline \multirow[t]{6}{*}{ 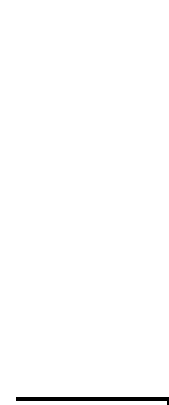 } & \multirow[t]{2}{*}{ Case 1: } & \multicolumn{9}{|c|}{$C D S_{t}=\beta_{0}+\beta_{1} \cdot S K E W_{t}+\beta_{2} \cdot t+u_{t}$} \\
\hline & & \multicolumn{9}{|c|}{$C D S_{t}=\beta_{0}+\beta_{1} \cdot K U R T_{t}+\beta_{2} \cdot t+u_{t}$} \\
\hline & Case 2: & \multicolumn{9}{|c|}{$C D S_{t}=\beta_{0}+\beta_{1} \cdot S K E W_{t}+\beta_{2} \cdot H V_{t}+\beta_{3} \cdot t+u_{t}$} \\
\hline & & \multicolumn{9}{|c|}{$C D S_{t}=\beta_{0}+\beta_{1} \cdot K U R T_{t}+\beta_{2} \cdot H V_{t}+\beta_{3} \cdot t+u_{t}$} \\
\hline & Case 3: & \multirow{2}{*}{\multicolumn{9}{|c|}{$\begin{array}{l}C D S_{t}=\beta_{0}+\beta_{1} \cdot S K E W_{t}+\beta_{2} \cdot I M P V_{t}+\beta_{3} \cdot t+u_{t} \\
C D S_{t}=\beta_{0}+\beta_{1} \cdot K U R T_{t}+\beta_{2} \cdot I M P V_{t}+\beta_{3} \cdot t+u_{t}\end{array}$}} \\
\hline & & & & & & & & & & \\
\hline \multirow{2}{*}{$I^{*}$} & \multirow{2}{*}{\multicolumn{2}{|c|}{$\begin{array}{c}\text { Case } 1 \\
b_{1}\end{array}$}} & \multicolumn{4}{|c|}{ Case 2} & \multicolumn{4}{|c|}{ Case 3} \\
\hline & & & $b_{1}$ & & $b_{2}$ & & $b_{1}$ & & $b_{2}$ & \\
\hline \multicolumn{11}{|l|}{ SKEW } \\
\hline _ALL & $-292,3$ & $(0,00)$ & $-122,6$ & $(0,00)$ & 76,5 & $(0,00)$ & $-78,9$ & $(0,00)$ & 136,1 & $(0,00)$ \\
\hline _SIE & $-233,9$ & $(0,00)$ & $-122,5$ & $(0,00)$ & 44,6 & $(0,00)$ & $-63,4$ & $(0,00)$ & 94,2 & $(0,00)$ \\
\hline _DTE & $-722,6$ & $(0,00)$ & $-309,8$ & $(0,00)$ & 278,6 & $(0,00)$ & $-42,5$ & $(0,34)$ & 474,5 & $(0,00)$ \\
\hline DAIM & $-69,7$ & $(0,03)$ & 1,7 & $(0,94)$ & 56,6 & $(0,00)$ & $\underline{35,2}$ & $(0,09)$ & 113,5 & $(0,00)$ \\
\hline DBANK & $-105,5$ & $(0,00)$ & $-2 \overline{6,0}$ & $(0,00)$ & 50,2 & $(0,00)$ & $\overline{\underline{2,0}}$ & $(0,78)$ & 82,6 & $(0,00)$ \\
\hline -MRE & $-67,8$ & $(0,00)$ & $-31,3$ & $(0,02)$ & 59,1 & $(0,00)$ & $-3 \overline{3,4}$ & $(0,01)$ & 101,0 & $(0,00)$ \\
\hline BAY & $-43,0$ & $(0,02)$ & 10,6 & $(0,42)$ & 94,2 & $(0,00)$ & 19,8 & $(0,41)$ & 233,4 & $(0,00)$ \\
\hline BASF & $-13,4$ & $(0,00)$ & $-4,8$ & $\overline{(0,04)}$ & 17,2 & $(0,00)$ & $\overline{-1,3}$ & $(0,61)$ & 35,5 & $(0,00)$ \\
\hline EON & $-36,3$ & $(0,00)$ & $-4,9$ & $(0,26)$ & 85,2 & $(0,00)$ & $-10,4$ & $(0,16)$ & 169,1 & $(0,00)$ \\
\hline COB & $-95,3$ & $(0,00)$ & $-30,0$ & $(0,01)$ & 152,9 & $(0,00)$ & $-17,7$ & $(0,05)$ & 251,5 & $(0,00)$ \\
\hline _HYPV & $-44,8$ & $(0,00)$ & $-23,7$ & $(0,03)$ & 112,8 & $(0,00)$ & $-30,7$ & $(0,01)$ & 111,6 & $(0,01)$ \\
\hline LH & $-9,7$ & $(0,00)$ & $-8,4$ & $(0,00)$ & 125,4 & $(0,00)$ & $-31,8$ & $(0,00)$ & 140,4 & $(0,00)$ \\
\hline DP & $-0,7$ & $(0,05)$ & $-0,7$ & $(0,03)$ & $-27,0$ & $(0,00)$ & $-0,7$ & $\underline{(0,06)}$ & 0,2 & $(0,84)$ \\
\hline \multicolumn{11}{|l|}{ KURT } \\
\hline _ALL & -1.461 & $(0,00)$ & $-951,1$ & $(0,00)$ & 41,3 & $(0,00)$ & 221,5 & $(0,08)$ & 161,3 & $(0,00)$ \\
\hline _SIE & $-994,6$ & $(0,00)$ & $-496,7$ & $(0,00)$ & 41,6 & $(0,00)$ & $\underline{93,3}$ & $(0,28)$ & 118,1 & $(0,00)$ \\
\hline _DTE & -1.532 & $(0,02)$ & $-412,4$ & $(0,05)$ & 296,3 & $(0,00)$ & $\underline{113,5}$ & $(0,16)$ & 492,3 & $(0,00)$ \\
\hline _DAIM & $-753,2$ & $(0,00)$ & $-454,2$ & $(0,00)$ & 36,4 & $(0,00)$ & $-141,6$ & $(0,16)$ & 92,4 & $(0,00)$ \\
\hline DBANK & $-660,7$ & $(0,00)$ & $-293,1$ & $(0,00)$ & 36,9 & $(0,00)$ & $\underline{176,6}$ & $(0,00)$ & 98,4 & $(0,00)$ \\
\hline _MRE & $-542,0$ & $(0,00)$ & $-220,6$ & $(0,00)$ & 50,4 & $(0,00)$ & 126,9 & $(0,02)$ & 114,8 & $(0,00)$ \\
\hline BAY & $-559,2$ & $(0,01)$ & $-292,2$ & $(0,04)$ & 82,7 & $(0,00)$ & 12,7 & $(0,63)$ & 232,1 & $(0,00)$ \\
\hline BASF & $-22,2$ & $(0,05)$ & $\underline{3,2}$ & $(0,45)$ & 17,9 & $(0,00)$ & $\overline{14,0}$ & $(0,00)$ & 37,2 & $(0,00)$ \\
\hline EON & $-130,5$ & $(0,03)$ & $-5 \overline{0,1}$ & $(0,01)$ & 155,3 & $(0,00)$ & $-\overline{46,5}$ & $(0,21)$ & 252,7 & $(0,00)$ \\
\hline COB & $-100,8$ & $(0,00)$ & $-44,3$ & $(0,02)$ & 112,3 & $(0,00)$ & $-89,4$ & $(0,00)$ & 112,5 & $(0,01)$ \\
\hline _HYPV & $-13,4$ & $(0,01)$ & $-9,4$ & $(0,01)$ & 85,4 & $(0,00)$ & $-13,8$ & $(0,01)$ & 5,4 & $(0,21)$ \\
\hline \multirow{2}{*}{\multicolumn{11}{|c|}{$\begin{array}{l}\text { *Only those cross sections are reported, for whom } b_{1} \text { is significant at the } 5 \% \text { level in the regression } \\
\text { without control variables. } \\
\text { Estimates } b_{1} \text { or } p \text {-values are underlined in the following cases: }\end{array}$}} \\
\hline & & & & & & & & & & \\
\hline \multicolumn{11}{|c|}{ 1. The sign of $b_{1}$ contradicts the expectation } \\
\hline 2. The inclus & ion of the $\mathrm{c}$ & 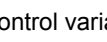 & 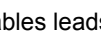 & 1 & & & & & & \\
\hline
\end{tabular}

Table 6.46.: Robustness check of the time-series regression results for the test of the relationship between default risk and the risk-neutral moments for a constant maturity of 36 days 


\subsection{Examination in the Framework of a Pooled Series Regression}

The robustness of the pooled series regression results has been tested by including $H V$ and $I M P V$ in the regression models models 6.72 and 6.73

$$
\begin{gathered}
C D S_{i, t}=\beta_{0}+\beta_{1} \cdot S K E W_{i, t}+\beta_{2} \cdot H V_{i, t}+\beta_{3} \cdot t+u_{i, t} \\
C D S_{i, t}=\beta_{0}+\beta_{1} \cdot S K E W_{i, t}+\beta_{2} \cdot I M P V_{i, t}+\beta_{3} \cdot t+u_{i, t} \\
C D S_{i, t}=\beta_{0}+\beta_{1} \cdot K U R T_{i, t}+\beta_{2} \cdot H V_{i, t}+\beta_{3} \cdot t+u_{i, t} \\
C D S_{i, t}=\beta_{0}+\beta_{1} \cdot K U R T_{i, t}+\beta_{2} \cdot I M P V_{i, t}+\beta_{3} \cdot t+u_{i, t}
\end{gathered}
$$

Table 6.47 juxtaposes the estimates $b_{1}$ and their respective $p$-values for the estimation of the original models 6.72 and 6.73 , the models including $H V, 6.78$ and 6.80 , and lastly the models including $I M P V, 6.79$ and 6.81 , for the constant maturities of 36 , 60 and 180 days.

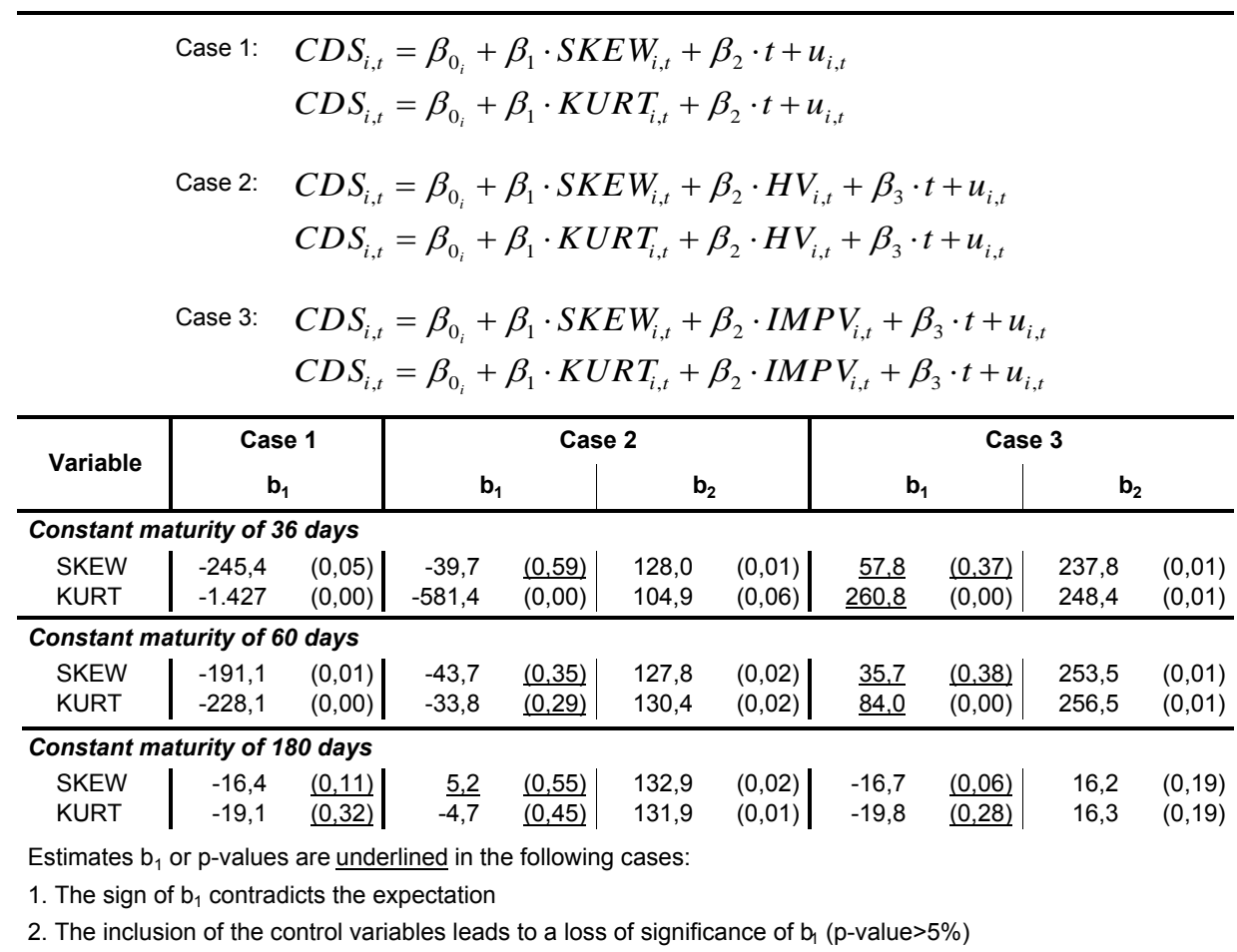

Table 6.47.: Robustness check of the pooled series regression results for the test of the relationship between default risk and risk-neutral moments

As can be seen in table 6.47, both the inclusion of $H V$ and $I M P V$ seem to invalidate most of the above obtained regression results. Even if the case of a constant maturity of 180 days is excluded from the examination on account of insignificant results from 
the beginning, only in 1 out of 8 cases the originally negative sign of $b_{1}$ remains unaltered without a loss of significance, namely for $K U R T$ after the inclusion of $H V$ in the case of a constant maturity of 36 days. Again, IMPV seems to exert a more detrimental influence on the original regression results than does $H V$, considering that the effect of $H V$ is restricted to one loss of significance, whereas IMPV leads without exception to inversions of the originally negative sign of $b_{1}$. A difference as to the effects of equity volatility on the coefficients of $S K E W$ on the one hand and $K U R T$ on the other like in the time-series dimension are not observable in the pooled series context.

Altogether, the examination in the pooled series setting raises doubts as to the robustness of the relationship between the CDS spread level and the risk-neutral moments in the presence of volatility. Before drawing final conclusions let us first dissociate the volatility-only from the default-risk-only effect as will be done in the next section. 


\subsection{Disentangling the Volatility-Only from the Default-Risk-Only Ef-}

fect Similarly to the approach of section 6.5.4.3.2.3, the volatility-only and the default-risk-only effect have both been eliminated each at a time in order to study the respective remaining effect on the moments of the risk-neutral equity return density. As above, this exercise has been carried out in a pooled series framework consisting of the 10 most important stock cross-sections and for a constant maturity of 36 days.

The elimination of the volatility-only effect has been studied by testing the regression models 6.72 and 6.73 on a data sample in which the admissible range of IMPV has been limited as above. The regression results after the elimination of the volatility effect are displayed in table 6.48 .

\begin{tabular}{|c|c|c|c|c|c|c|c|c|c|c|c|}
\hline \multicolumn{12}{|c|}{$\begin{array}{l}\operatorname{CDS}_{i, t}=\beta_{0_{i}}+\beta_{1} \cdot \operatorname{SKEW}_{i, t}+\beta_{2} \cdot t+u_{i, t} \\
\operatorname{CDS}_{i, t}=\beta_{0_{i}}+\beta_{1} \cdot \operatorname{KURT}_{i, t}+\beta_{2} \cdot t+u_{i, t}\end{array}$} \\
\hline Variable & & $\mathbf{b}_{0}$ & & & $b_{1}$ & & $\mathbf{b}_{2}$ & & $\begin{array}{c}\text { R2 } \\
\text { cor. }\end{array}$ & $\mathbf{F}$ & DW \\
\hline SKEW & 37,8 & {$[10,7]$} & $(0,00)$ & $-16,95$ & {$[-3,3] \quad(0,00)$} & 0,00 & {$[0,13] \quad($} & $(0,89)$ & 0,93 & 948 & 0,05 \\
\hline KURT & $-2,8$ & {$[-0,1]$} & $(0,93)$ & 14,10 & {$[1,6] \quad(0,12)$} & 0,00 & {$[0,16]$} & $(0,87)$ & 0,93 & 937 & 0,03 \\
\hline \multicolumn{12}{|c|}{ t-stats in [ ] and corresponding two-sided p-values in ( ) } \\
\hline \multicolumn{12}{|c|}{ All values are calculated for a constant maturity of 36 days. } \\
\hline \multicolumn{12}{|c|}{$\begin{array}{l}\text { IMPV is restricted to the interval }[23,26 \% ; 25,51 \%] \text {. In order to obtain a sufficient number } \\
\text { of datasets, the smple includes the } 10 \text { cross-sections vielding } 800 \text { datasets }\end{array}$} \\
\hline
\end{tabular}

Table 6.48.: Dissecting the default-risk-only effect by eliminating the volatility-only effect in the test of the relationship between default risk and the risk-neutral moments

The negative and highly significant coefficient of the variable $S K E W$ in table 6.48 clearly shows that the default-risk-only effect implies a negative relationship between the CDS spread level the skewness of the risk-neutral return distribution. Comparing this result with the corresponding, barely significant result of the unrestricted regression depicted in the upper panel of table 6.45, it is obvious that in the absence of the volatility-only effect the negative relationship between the CDS spread level and the risk-neutral return skewness is much more significant than in its presence. In contrast to the likewise negative relationship between $C D S$ and $K U R T$ in the unrestricted regression case, this relationship seems to change signs in the absence of the volatilityonly effect as can be read from the positive - though slightly insignificant - coefficient of $K U R T$ in table 6.48. Again, the explanatory power $R^{2}$ of the results in table 6.48 is relatively high, which could be due to the highly insignificant time trend.

After the elimination of the volatility-only effect, the default-risk-only effect has been suppressed. Likewise as above in section 6.5.4.3.2.3, the dependent variable $C D S$ in 
the regression models 6.72 and 6.73 has been substituted by the volatility variables $I M P V$ and $H V$, respectively, and the admissible range of $C D S$ in the data sample has been restricted to a very small bandwidth. The regression results after the elimination of the default-risk-only effect are displayed in table 6.49. Again, the results with $I M P V$ as dependent variable are shown in the upper panel, while the lower panel contains those with $H V$ as response variable.

\begin{tabular}{|c|c|c|c|c|c|c|c|c|c|c|c|c|}
\hline \multicolumn{13}{|c|}{$\begin{array}{ll}\text { Case 1: } & I M P V_{i, t}=\beta_{0_{i}}+\beta_{1} \cdot S K E W_{i, t}+\beta_{2} \cdot t+u_{i, t} \\
& I M P V_{i, t}=\beta_{0_{i}}+\beta_{1} \cdot K U R T_{i, t}+\beta_{2} \cdot t+u_{i, t} \\
\text { Case 2: } & H V_{i, t}=\beta_{0_{i}}+\beta_{1} \cdot S K E W_{i, t}+\beta_{2} \cdot t+u_{i, t} \\
& H V_{i, t}=\beta_{0_{i}}+\beta_{1} \cdot K U R T_{i, t}+\beta_{2} \cdot t+u_{i, t}\end{array}$} \\
\hline Variable & \multicolumn{3}{|c|}{$b_{0}$} & & $b_{1}$ & & \multicolumn{3}{|c|}{$b_{2}$} & $\begin{array}{l}\text { R2 } \\
\text { cor. }\end{array}$ & $F$ & DW \\
\hline \multicolumn{13}{|l|}{ Case1: } \\
\hline SKEW & 0,30 & {$[9,6]$} & $(0,00)$ & 0,037 & {$[0,4]$} & $(0,66)$ & 0,00 & {$[-1,17]$} & $(0,24)$ & 0,32 & 71,5 & 0,16 \\
\hline KURT & 3,21 & {$[2,7]$} & $(0,01)$ & $-0,963$ & {$[-2,5]$} & $(0,01)$ & 0,00 & {$[-1,31]$} & $(0,19)$ & 0,39 & 96,7 & 0,33 \\
\hline \multicolumn{13}{|l|}{ Case 2: } \\
\hline SKEW & 0,30 & {$[7,40]$} & $(0,00)$ & $-0,140$ & {$[-1,6]$} & $(0,11)$ & 0,00 & {$[-1,12]$} & $(0,26)$ & 0,23 & 46,9 & 0,64 \\
\hline KURT & 3,27 & {$[2,38]$} & $(0,02)$ & $-0,975$ & {$[-2,1]$} & $(0,03)$ & 0,00 & {$[-1,16]$} & $(0,25)$ & 0,26 & 53,2 & 0,71 \\
\hline \multicolumn{13}{|c|}{$\begin{array}{l}\text { CDS is restricted to the interval }[21,44 \mathrm{bp} ; 27,63 \mathrm{bp}] \text {. In order to obtain a sufficient number } \\
\text { of datasets, the sample includes the top } 10 \text { cross-sections, yielding } 1.200 \text { datasets } \\
\text { altogether. }\end{array}$} \\
\hline
\end{tabular}

Table 6.49.: Dissecting the volatility-only effect by eliminating the default-risk-only effect in the test of the relationship between equity volatility and the risk-neutral moments

Both the upper as well as the lower panel of table 6.49 show a significant negative relationship between the level of equity volatility and the level of risk-neutral kurtosis in the absence of the default-risk-only effect. As to the link between the level of equity volatility and that of risk-neutral skewness on the other hand, not much can be said due to an insignificant coefficient which additionally exhibits opposite signs in the two panels.

While the default-risk-only effect primarily implies a negative relationship between the CDS spread level and the level of risk-neutral skewness, the volatility-only effect primarily acts on the level of risk-neutral kurtosis entailing a negative relationship between the latter and the level of equity volatility. The combination of the two effects on the other hand simultaneously acts upon both risk-neutral moments in the same direction as indicated by our original regression results. The weak evidence of the fact that the default-risk-only effect also leads to increasing kurtosis explains by the way, 
why the inclusion of equity volatility in the original regression models provokes sign inversions and losses of significance for the coefficient of $K U R T$. 


\subsubsection{Summary and Discussion of the Results in the Light of Hitherto Existing Findings}

6.5.5.4.1. Summary of the Results Our expectations regarding the relationship between the CDS spread level and the risk-neutral moments have been originally determined by Hypothesis 4, predicting a risk-neutral return density which is all the more left-skewed, the higher the level of default risk. Generally, this prediction is also compatible with our expectations emanating from our preliminary finding of a default risk induced flattening and simultaneous right-translation of the smile. Moreover, the flattening suggested the additional expectation that increasing credit risk should also be accompanied by decreasing risk-neutral kurtosis.

The time-series analysis as well as the examination in the pooled series context largely yielded negative and significant coefficients for both risk-neutral moments, thus producing clear evidence of a negative relationship between the CDS spread level on the one hand and the level of risk-neutral skewness and kurtosis, respectively, on the other.

But as we have seen earlier, high CDS spread levels are associated with high levels of equity volatility. Therefore it is important to find out, whether the detected relationship between the CDS spread level and that of the risk-neutral moments is indeed primarily owed to the effect of default risk or rather to that of equity volatility. In a first step, this has been tested in a robustness check by including the additional variables $H V$ and $I M P V$ into the original regression models. The robustness check disclosed that equity volatility partly dominates the relationship between the CDS spread level and that of the risk-neutral moments, leading to losses of significance and sign inversions of the coefficients of the latter.

To shed more light onto the role of volatility, the volatility-only effect has been disentangled from the default-risk-only effect by eliminating both, but each at a time and by analyzing the respective remaining effect. This exercise revealed that the defaultrisk-only effect primarily acts upon the level of risk-neutral skewness, whereas the volatility-only effect primarily affects the level of risk-neutral kurtosis. The former entails a negative relationship between the level of default risk and the level of riskneutral skewness and secondarily also a positive relationship between the level of default risk and the level of risk-neutral kurtosis. Whereas the latter implies a negative relationship between the level of volatility and the level of risk-neutral kurtosis. Both effects in union lead to a negative relationship between the CDS spread level and the level of risk-neutral skewness and kurtosis, respectively. This finding confirms Hypothesis 4 as well as our expectations emanating from our preliminary observations. 
The slightly insignificant evidence of the fact that the default-risk-only effect also implies a positive relationship between the level of default risk and the level of riskneutral kurtosis is by the way consistent with the observation above that it also involves a steepening of both wings of the smile.

\subsection{Discussion of the Results in the Light of Hitherto Existing Find-}

ings The hitherto existing literature concerning the relationship between default risk and the higher moments of the equity return distribution is even more scarce than that regarding the slope of the smile. Altogether there only exist 2 other studies which can be cited in this context (cf. section 5.1.3.3): Like myself, DENNIS/MAYHEW (2002) also use the Bakshi-Madan method to extract individual risk-neutral return skewness out of option prices. They find evidence of a positive relationship between the level of risk-neutral skewness and the level of financial leverage. Interpreting financial leverage as a measure of default risk, this result contradicts my finding of a negative relationship. Further they also detect a positive relationship between the level of risk-neutral skewness and that of implied volatility, which I cannot confirm either 356 ,

A possible reason for the conflicting results of the study of DENNIS ET AL. (2006) on the one hand and mine on the other could reside in our different approaches to measuring risk-neutral skewness and default risk. It is true that DENNIS/MAYHEW (2002) also use the Bakshi-Madan method, but they use it in its raw version and base it on always the same strike-to-spot price range. Whereas I rely on smoothed option prices, apply a bias correction and more importantly, vary the strike-to-spot price range in dependence of the current volatility level which ensures a constant standardized return interval.

The other interesting study besides the paper of DENNIS ET AL. (2006) is that of ZHANG ET AL. (2005), who use historically based measures of return skewness and return kurtosis, respectively, and agree with my finding of a negative relationship between the CDS spread level and the level of skewness. However, in contrast to my results they observe a positive relationship between the CDS spread level and their kurtosis measure. But strictly speaking, their results are not directly comparable with mine above, since it is well possible that the statistical and the risk-neutral distribution differ with regard to their relationship with the CDS spread level.

356 Considering the findings of the above empirical analysis, namely the high, positive correlation between the CDS spread level and the level of implied volatility as well as the substantial, negative correlation between the CDS spread level and the level of risk-neutral skewness, the latter should also be negatively correlated with the level of implied volatility. 
Regarding the relationship between the smile and the risk-neutral moments, the results of BAKSHI ET AL. (2003) cannot be left unmentioned at this point. Among other things, BAKSHI ET AL. (2003) analyze the relationship between the slope of the individual volatility smile on the one hand and the level of risk-neutral skewness and kurtosis on the other. To this end, they linearly regress the logarithm of implied volatility on the logarithm of moneyness, to obtain an estimate of the implied volatility slope. Carried over to my second order polynomial approximation of the smile, their slope measure is soonest comparable to the slope at the ATM point, SLP100. Consistently with the findings above, they report a largely negative slope at the ATM point (cf. table 6.7). Having computed the risk-neutral moments according to the original Bakshi-Madan method, BAKSHI ET AL. (2003) then simultaneously regress the slope on both risk-neutral moments and find a positive relationship in both cases. This induces them to conclude that decreasing skewness and kurtosis lead to a steepening of the smile, which is inconsistent with our findings above. There to the contrary, it has been shown that increasing default risk leads to a flattening and right translation of the smile as well as to decreasing skewness and kurtosis. Therefore, there must exist a negative relationship between the slope of the smile and the level of risk-neutral skewness and kurtosis, respectively.

The finding of BAKSHI ET AL. (2003) is due to their restriction of the smile analysis to the ATM point, which has also been adopted by all other authors analyzing the link between default risk and the behavior of the smile. However, such a restriction of the viewpoint is misleading, since the combination of a global flattening and right-translation of the smile leads to the local impression of a steepening. This local impression is owed to the fact that the right-translation of the smile makes its steeper left wing pass through the ATM point, thus falsely suggesting a steepening, although the smile globally flattens. The claim of BAKSHI ET AL. (2003) of a positive relationship between the smile slope and the level of risk-neutral kurtosis is by the way counterintuitive, since it would mean that increasing kurtosis is coupled with a flattening of the smile, whereas we have seen above that excess kurtosis of the risk-neutral density is considered to be associated with an accentuated U-shape of the smile (see section 3.1.3.2.

So far, the possible economical meaning of the above findings has not yet been discussed. Strictly speaking, the interpretation of the risk-neutral moments should span the two aspects anticipation and risk aversion. While default risk associated increasing left-skewness clearly indicates the anticipation of large negative returns, decreasing kurtosis could just be the consequence of increasing left-skewness in that kurtosis is 
exchanged for left-skewness. This would mean that probability mass from the right tail and the center of the distribution is shifted to its left tail. Regarding the aspect of risk aversion, it is likely that increasing left-skewness also has to do with increasing risk aversion as to the expectation of large downward moves. However, without the additional knowledge of the behavior of the physical density, not much more can be said in this context.

\subsection{Summary and Conclusions}

As stated in the introduction of this chapter, a fundamental and at the same time convincing explanation of the equity volatility smile could be the presence of default risk. However, corresponding empirical insights regarding the link between the level of default risk and the individual stock smile are extremely scarce, partly contradictory or even missing at all. This paucity of extant empirical findings is aggravated by the evident contradiction between the theoretical prediction of a default risk induced, monotonically downward sloping smile on the one hand and the observation of a generally U-shaped individual stock smile on the other. This situation was judged unsatisfactory and therefore called for a thorough empirical analysis of the relationship between the level of default risk and the equity volatility smile. Such an examination was the subject of this chapter.

Based on theoretical predictions and extant empirical evidence, the following 4 hypotheses were derived in order to examine the relationship between default risk and the equity volatility smile:

- Hypothesis 1: The asymmetric volatility phenomenon is caused by the leverage effect.

- Hypothesis 2: The level of implied volatility depends positively on the level of default risk.

- Hypothesis 3: The slope of the equity volatility smile depends negatively on the level of default risk.

- Hypothesis 4: The skewness of the risk-neutral equity return density depends negatively on the level of default risk.

These hypotheses were tested by means of a regression analysis involving a sample comprising altogether 23 DAX companies and stretching across the 3 year period from the beginning of 2002 until the end of 2004. While the CDS spread level served as 
a default risk measure, variables describing the equity volatility smile were derived by smoothing the smile with a quadratic polynomial of the strike-to-spot-price ratio. Further, the Bakshi-Madan method was modified in several respects in order to allow an unbiased estimation of the levels of risk-neutral return skewness and kurtosis. The results of the analysis can be summarized as follows:

1. To begin with, the analysis of the summary statistics of the regression variables disclosed the following findings which partly represent new insights:

- For short maturities, the individual stock smile and the index smile are first downward and then upward sloping which leads to an asymmetric, U-shaped smile pattern.

- For individual stocks, the smile is less steep and more symmetric and Ushaped than for the index.

- The index as well as the individual stock smile flatten with increasing option maturity.

- The risk-neutral return density of individual stocks and of the index is leftskewed and exhibits excess kurtosis.

- The left-skewness and excess kurtosis of the risk-neutral return density are more pronounced in the case of the index than in the case of individual stocks.

- The left-skewness of the risk-neutral return density is increasing with increasing option maturity in the case of the index as well as in the case of individual stocks. Whereas the risk-neutral excess kurtosis is increasing for the index, but decreasing for individual stocks.

- The physical return density of individual stocks is likewise left-skewed and also exhibits excess kurtosis.

- For individual stocks, the left-skewness of the risk-neutral return density is more accentuated than that of the physical return density. The converse is true for the level of excess kurtosis.

2. In accordance with most other studies in this field, it was shown that the asymmetric volatility phenomenon as well as the leverage effect are both existent. This result was obtained in the time-series dimension as well as in a pooled series setting and allows the conclusion that the asymmetric volatility phenomenon is at least partly caused by the leverage effect, which confirms Hypothesis 1. Notwithstanding, this result does not preclude the coexistence of other causes 
of the asymmetric volatility phenomenon, like the volatility feedback mechanism for instance.

3. In agreement with the mainstream of the relevant empirical literature, a strong positive relationship between the level of default risk and that of implied volatility could be confirmed. Again, this was achieved in the time-series dimension as well as in the context of a pooled series analysis and clearly verifies Hypothesis 2.

In the time series dimension the explanatory power of implied volatility as to the CDS spread level as a proxy for default risk has been found to be as high as over 90\% for some cross-sections. The relationship between the CDS spread level and that of historical volatility proved to be weaker. Since the time-series of CDS spreads and implied volatilities have shown to be potentially persistent, the suspicion of possible non-stationarity has been addressed with adequate tests, but has proven to be unsubstantiated.

4. The analysis of the relationship between the CDS spread level and the pattern of the smile disclosed that rising default risk unfolds two competing effects on the smile: a default-risk-only effect on the one hand and a volatility-only-effect on the other. While the default-risk-only-effect entails a steepening of both wings of the U-shaped smile, the volatility-only-effect implies its flattening and right-translation. Both effects in union however lead to a default risk induced flattening and right-translation of the smile which shows that the default-riskonly-effect is dominated by the volatility-only-effect.

While the default-risk-only effect of a steepening of the downward sloping left wing of the smile seems to corroborate Hypothesis 3, the latter is rather belied by the effect of a simultaneous steepening of the upward sloping right wing. Further, considering the combined impact of the default-risk-only and the volatility-only effect which results in a default risk induced flattening and right-translation of the smile, Hypothesis 3 has been refuted.

Further, the impact of a default risk induced flattening and right-translation is consistent with the scarce literature in this field and also with Hypothesis 3 in that it confirms a steepening of the slope of the smile at the ATM point in the event of increasing default risk. It is inconsistent however, with the conclusions commonly drawn from this observation. While existing studies and models as well as Hypothesis 3 wrongly assume a globally steepening smile in conjunction with increasing default risk, the above results uncovered something else: 
While the smile horizontally shifts to the right, its steeper descending left wing passes through the ATM point, thus creating the local impression of a steepening of the smile, whereas in reality it globally flattens, thanks to the volatility-onlyeffect.

5. Similarly as above, the examination of the connection between the CDS spread level and that of the risk-neutral moments revealed that rising default risk embraces two partly competing effects: a default-risk-only effect on the one hand and a volatility-only-effect on the other. The default-risk-only-effect primarily acts upon the level of risk-neutral skewness and secondarily on the level of riskneutral kurtosis and involves a negative relationship between the CDS spread level and the level of risk-neutral skewness and a weaker, positive relationship between the CDS spread level and the level of risk-neutral kurtosis. Whereas the volatility-only-effect solely affects the level of risk-neutral kurtosis and implies a negative relationship between the CDS spread level and the level of riskneutral kurtosis. Both effects together lead to a negative relationship between the CDS spread level on the one hand and the level of risk-neutral skewness and kurtosis on the other. This shows that the volatility-only effect dominates the default-risk-only effect as to the impact on the level of risk-neutral kurtosis.

As to the negative link between the level of default risk and the level of riskneutral skewness, Hypothesis 4 was confirmed. But while the default-risk-onlyeffect of decreasing risk-neutral skewness has been predicted by the literature, its impact of increasing kurtosis seems to be largely unknown 357 . Also the existence of the volatility-only-effect seems to be ignored in the literature, let alone the interaction of the default-risk-only and the volatility-only effect and their combined impact on the shape of the smile and the risk-neutral moments.

What remains is the interpretation of the observed effects. While the defaultrisk-only effect of increasing left-skewness could be explained by the anticipation of and risk aversion to large downward moves, increasing kurtosis could have to do with the augmented rate of information arrival in times of financial distress 358 However, the economic meaning of decreasing kurtosis in the wake of the volatility-only effect is more difficult to guess. It could have to do with

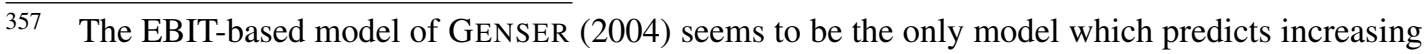
levels of excess kurtosis with increasing levels of default risk or the leverage ratio, respectively (see section 5.2.2.2.2).

358 Excess kurtosis is often attributed to the presence of jumps (cf. for example HULL (2006), p. 379) which are in turn considered to be associated with the arrival of information as has been indicated in section 4.2.4.1. 
the already skewed return distribution. While in a Black-Scholes world with a normally distributed equity return the level of kurtosis is independent of the level of volatility, this could be different in a world with an already left-skewed return distribution. In this world, decreasing kurtosis could simply be the result of a volatility induced exchange of kurtosis for left-skewness. As the volatility increases, large downward moves become even more likely and small moves less likely as compared to the normal distribution, entailing a shift of probability mass from the center of the distribution to the left tail. 



\section{Conclusions and Implications}

\subsection{Conclusions}

The question as to the link between default risk and the equity volatility smile has been first approached from a theoretical, literature-based stance and subsequently by means of a thorough empirical analysis.

\subsubsection{Summary and Conclusions of the Theoretical Part}

In chapter 2, the literature-based part of this thesis first focused on the ubiquitous principle of risk-neutral valuation as well as on the notion of risk aversion and its role as a key element linking the physical with the option-implied, risk-neutral equity return density.

In a second step, chapter 3 introduced and described the phenomenon of the implied volatility smile which was shown to be asymmetrically U-shaped in the case of individual stock options. Further, chapter 3 demonstrated that option prices, the implied volatility smile and the risk-neutral density can all be used interchangeably. Moreover, chapter 3 also provided a survey of the available techniques for the smoothing of the implied volatility smile and the extraction of the option-implied, risk-neutral density. This survey concluded with the appraisal of the quadratic polynomial smoothing due its simplicity paired with its capability to capture the essential features of the smile. In conjunction with the polynomial smoothing of the smile, the Bakshi-Madan method was identified as the method of choice for the estimation of the moments of the riskneutral density.

Chapter 4 was dedicated to the study of those explanations of the equity volatility smile phenomenon which are not explicitly related to default risk. This study embraced phenomenological as well as fundamental approaches. While the phenomenological approaches manage fairly well to replicate the smile and its term structure by recurring to rather intricate process specifications of the underlying stock price 
or index level, they fail to offer a true, fundamental explanation of the smile. Most fundamental approaches on the other hand are in parts mutually contradictory, do not represent any mainstream beliefs or opinions and more importantly, only provide a partial substantiation of the smile. The only fundamental and at the same time convincing explanation of the equity volatility smile was identified to be the anticipation of and risk aversion to large downward moves. Carried over to the case of the individual stock smile, large downward moves can be interpreted as being related to the default event. This conclusion emphasized the necessity to analyze the relationship between default risk and the individual stock smile, which - at a literature-based level - was the subject of chapter 5 .

Chapter 5 first demonstrated how default risk in the guise of the leverage effect theoretically leads to a left-skewed risk-neutral stock return density which in turn should manifest itself in a convex, monotonically downward sloping equity volatility smile, which is all the more steeper the higher the level of default risk. However, it emerged that the theoretical prediction of a monotonically downward sloping smile conflicts with the observation of a generally U-shaped individual stock option smile as it had been discussed in chapter 3. Further, a survey of the empirical literature revealed that evidence concerning the link between the level of default risk on the one hand and the smile and the risk-neutral density on the other is extremely scarce, if not missing at all. It is true that the few extant studies all confirm a negative relationship between the level of default risk and the slope of the smile, thus seemingly corroborating the theoretical prediction of a default risk induced steepening of the smile. However, they are all limited to the contemplation of the smile at or around the ATM strike level. Examinations beyond this point or studies addressing the default risk dependent behavior of the risk-neutral density are lacking almost entirely. Moreover, it was demonstrated that despite the fundamental importance of the leverage effect for the theoretical connection between the level of default risk and the smile, it is still not concludingly substantiated that the leverage effect relationship really exists.

The second part of chapter 5 was devoted to the presentation of credit risk models which are enhanced in a way that they are able to incorporate equity options as well as the volatility smile phenomenon. Similar to traditional credit risk models, this class of enhanced models can be partitioned into exponents based on the structural firm value setting on the one hand and into the more recent strand of models derived from the reduced form approach on the other. It was shown that all these enhanced models build on the leverage effect as well as its implication of a monotonically downward sloping implied volatility smile. However, beyond this it was found that they do not 
offer any further relevant insights regarding the link between the level of default risk and the smile. Moreover, it was demonstrated that due to the built-in leverage effect most enhanced models merely generate a monotonically downward sloping smile and fail to produce a more realistic U-shaped curve. And lastly, it emerged that not all enhanced models imply the same smile behavior in the wake of increasing default risk.

Altogether, chapter 5 emphasized the necessity of further empirical research regarding the link between default risk on the one hand and the smile and the risk-neutral density on the other. This necessity followed from the scarcity and lack of corresponding extant empirical evidence, further from the contradiction between the theoretical prediction of a default risk induced monotonically downward sloping smile on the one hand and its evident static feature of a generally U-shaped pattern on the other, moreover from the very limited theoretical contribution of even the most recent enhanced models and finally from the in parts mutually contradictory smile behavior of the enhanced models in the wake of increasing default risk.

\subsubsection{Summary and Conclusions of the Empirical Part}

The objective of the empirical part, namely chapter 6 , consisted in elucidating the relationship between default risk and the equity volatility smile. To this end, 4 hypotheses were deduced from the insights of the theoretical part of this thesis. These hypotheses involved the leverage effect relationship, further the link between the level of default risk and that of implied volatility, moreover the relationship between the level of default risk and the smile and finally the connection between the level of default risk and the shape of the risk-neutral density.

The 4 hypotheses were tested in time-series and pooled series regression analyses on a data sample which stretched across a 3 year period from the beginning of 2002 to the end of 2004 and comprised those 23 DAX companies for whom sufficient data was available during this 3 year period. While the CDS spread level served as a default risk measure, the smile was described in terms of variables derived by smoothing the implied volatilities with a quadratic polynomial as had been recommended in chapter 3 of the theoretical part. The risk-neutral equity return density in turn was proxied by the levels of risk-neutral skewness and kurtosis estimated by means of the BakshiMadan method. Thanks to the following 3 modifications, the Bakshi-Madan method produced stable, comparable and unbiased estimates of return skewness and kurtosis: 
- Stable estimates were mainly achieved by replacing actual option prices with smoothed option prices based on the polynomial smile approximation.

- Estimates comparable across differing levels of equity volatility were obtained by varying the relevant strike-to-spot-price range in a way as to ensure a constant interval with regard to the standardized log-return.

- Unbiased estimates were basically enabled by subtracting the bias generated for the skewness and kurtosis of the normal distribution.

The results of the empirical analysis can be summarized as follows:

1. To begin with, the analysis of the summary statistics of the regression variables disclosed the following findings which partly represent new insights:

- For short maturities, the individual stock smile and the index smile are first downward and then upward sloping which leads to an asymmetric, U-shaped smile pattern.

- For individual stocks, the smile is less steep and more symmetric and Ushaped than for the index.

- The index as well as the individual stock smile flatten with increasing option maturity.

- The risk-neutral return density of individual stocks and of the index is leftskewed and exhibits excess kurtosis.

- The left-skewness and excess kurtosis of the risk-neutral return density are more pronounced in the case of the index than in the case of individual stocks.

- The left-skewness of the risk-neutral return density is increasing with increasing option maturity in the case of the index as well as in the case of individual stocks. Whereas the risk-neutral excess kurtosis is increasing for the index, but decreasing for individual stocks.

- The physical return density of individual stocks is likewise left-skewed and also exhibits excess kurtosis.

- For individual stocks, the left-skewness of the risk-neutral return density is more accentuated than that of the physical return density. The converse is true for the level of excess kurtosis. 
2. In accordance with many other studies in this field, it was shown that the leverage effect is indeed existent. Notwithstanding, the coexistence of other causes of the symptoms of the leverage effect cannot be precluded 359

3. In agreement with the mainstream of the relevant empirical literature, a strong positive relationship between the level of default risk and that of implied volatility could be confirmed. In the time series dimension the explanatory power of implied volatility as to the CDS spread level as a proxy for default risk was found to be as high as over $90 \%$ for some cross-sections. As expected, the relationship between the CDS spread level and that of historical volatility turned out to be weaker. Since the time-series of CDS spreads and implied volatilities showed to be potentially persistent, the suspicion of possible non-stationarity was addressed with adequate tests, but proved to be unsubstantiated.

4. The analysis of the relationship between the CDS spread level and the shape of the smile disclosed that the impact of rising default risk on the risk-neutral return density embraces two competing effects: a default-risk-only effect on the one hand and a volatility-only-effect on the other. The default-risk-only effect on the risk-neutral density is that of a rising level of default risk, as measured by the CDS spread level, if the level of equity volatility is held constant. Whereas the volatility-only effect on the risk-neutral density is that of a rising level of equity volatility, if the level of default risk proxied by the CDS spread level is kept unchanged. While the default-risk-only effect leads to increasing left-skewness and increasing kurtosis of the risk-neutral equity return density, the volatilityonly effect entails decreasing risk-neutral kurtosis. Both effects together imply a negative relationship between the level of default risk on the one hand and the level of risk-neutral return skewness and kurtosis on the other. This shows that with regard to the level of risk-neutral kurtosis, the default-risk-only effect seems to be dominated by the volatility-only effect.

Carried over to the smile behavior, the default-risk-only effect implies a steepening of both wings of the U-shaped smile, whereas the volatility-only effect involves a flattening and right-translation of the smile. Again, the volatilityonly effect dominates the default-risk-only effect in that both effects in union lead to a default risk induced flattening and right-translation of the smile.

Indeed, the default-risk-only effect of increasing left-skewness of the risk-neutral return density and of a steepening of the left wing of the smile has been pre-

359 One other possibly coexisting cause could be the mechanism of volatility feedback (see section 5.1.3.1. 
dicted by the literature. However, its effect of increasing risk-neutral kurtosis and of a corresponding steepening of the right wing of the smile is largely ignored in the literature. Also the volatility-only effect and its interplay with the default-risk-only effect seem to be completely unknown.

Extant studies comparable to the above analysis are extremely scarce and are all limited to the link between the level of default risk and the slope of the smile at or around the at-the-money strike level. Although they all find weak evidence of a default risk induced, local steepening of the smile and from this observation deduce a global steepening, the empirical analysis of this thesis shows something else: While the smile horizontally shifts to the right, its steeper descending left wing passes through the at-the-money point, thus creating the local impression of a steepening of the smile, whereas in reality it globally flattens, thanks to the dominating volatility-only-effect.

What remains is the interpretation of the observed effects. While the defaultrisk-only effect of increasing left-skewness could be explained by the anticipation of and risk aversion to large downward moves, increasing kurtosis could have to do with the augmented rate of information arrival in times of financial distress 360 . However, the economic meaning of decreasing kurtosis in the wake of the volatility-only effect is more difficult to guess. It could have to do with the already skewed return distribution. While in a Black-Scholes world with a normally distributed equity return the level of kurtosis is independent of the level of volatility, this could be different in a world with an already left-skewed return distribution. In this world, decreasing kurtosis could simply be the result of a volatility induced exchange of kurtosis for left-skewness. As the volatility increases, large downward moves become even more likely and small moves less likely as compared to the normal distribution, entailing a shift of probability mass from the center of the distribution to the left tail.

360 Excess kurtosis is often attributed to the presence of jumps (cf. for example HULL (2006), p. 379) which are in turn considered to be associated with the arrival of information as has been indicated in section 4.2.4.1. 


\subsection{Implications}

\subsubsection{Implications for Future Research}

One issue not addressed in detail in the previous analysis, but raising some interesting questions, is the term structure of the individual stock option smile and its behavior in dependence of the level of default risk 361 . In section 3.1 .2 is was stated that the equity smile generally flattens with increasing maturity, but that inconsistent with the central limit theorem, it does not seem to flatten out completely. Could - in the case of the individual stock smile - this persistency be due to the presence of default risk? And, should not the presence of default risk prevent the smile from flattening at all, assuming that an increasing term also leads to an increasing cumulative probability of default $\sqrt{362}$ ? An answer to these questions could be that the default-risk-only effect indeed causes a persistency and even a maturity related increasing steepness of the smile, but is more than offset by the volatility-only effect. Like the default-risk-only effect, the volatility-only effect grows stronger with increasing time horizon ${ }^{363}$, but possibly it grows faster so than the default-risk-only effect. Due to this prevalence of the volatility-only effect, both effects together lead to a maturity dependent flattening of the smile. This hypothesis could be tested by analyzing the default-risk-only effect on the smile in dependence of a changing time horizon 364

Another question related to the term structure of the smile is raised by the observation in section 6.5.3 that the dependence between the CDS spread level and the level of implied volatility weakens with increasing time horizon. This weakening relationship

361 The detailed examination of the term structure of the smile was explicitly excluded from the analysis, since it is rarely referred to in the literature as compared to the strike structure and also due to the fact that even for the most liquid stock cross-sections data points in the maturity dimension are often only restricted to a handful of observations coinciding with the series of option maturities (see section 6.1).

362 This assumption is based on the observation that default rates are monotonically increasing with time, as documented in VAZZA ET AL. (2007).

363 Given a constant annualized equity return volatility, it seems reasonable to assume that the absolute equity return volatility increases with a growing time horizon, similar to the absolute standard deviation of the normal distribution which is proportional to the square root of time.

364 To this end, the data sample would have to be restricted to datasets with the same CDS spread level and the same time horizon adjusted level of absolute return volatility $\sigma^{*}$, but with differing time horizons $\tau_{i}$. As an approximation, it could be assumed that the time horizon adjusted level of absolute return volatility $\sigma^{*}$ equals the product of the annualized return volatility $\sigma$ and the square root of time: $\sigma^{*}=\sigma \sqrt{\tau}$. 
could be due to the anticipated mean reversion of the level of volatility ${ }^{365}$ and should likewise be examined in more detail.

Still another interesting issue is that of the dual impact of the default-risk-only effect. While its consequence of increasing left-skewness of the risk-neutral equity return density is consistent with theoretical predictions, its effect of increasing kurtosis is unheard-of so far. Since the economic meaning of increasing kurtosis can only be guessed, it should be the subject of further research.

But also the causes of the volatility-only effect deserve a closer examination. As we have seen, in a Black-Scholes world with normal return densities the volatilityonly effect must not exist at all. Obviously its existence in the real world depends on the non-normality of the risk-neutral equity return density. Therefore, it would be interesting to see, if the extent of the volatility-only effect is related to the extent of risk-neutral skewness and excess kurtosis caused by the default-risk-only effect.

Further, partly in accordance with and also partly in excess of the existing literature, the empirical analysis revealed a difference between the risk-neutral density of the index and that of the individual stock (see section 6.4.7.3.2). It would be worthwhile to scrutinize the causes of this difference and to analyze, whether it could be related to the singularity of the default event which is characteristic of the individual company, but is naturally not shared by the stock index.

Finally, it could be interesting to repeat the present analysis with a more recent data sample. Thanks to the impressive growth rates of both the organized equity options market as well as of the CDS market (see section 1.1), it is to be expected that the amount of available data has substantially increased meanwhile, now also allowing more extensive cross-sectional studies. But also the rather data intensive dissociation of the default-risk-only from the volatility-only effect should benefit from a more recent and thus larger data sample.

365 In an extra examination not reported in this document, I found that in times of high levels of implied volatility, the ATM term structure of the smile exhibits the tendency to decrease, and conversely in times of low levels of volatility it shows a tendency to increase. This phenomenon could have to do with a market-implied anticipation of a mean reverting volatility and has the following effect: While the level of the short term implied volatility fluctuates substantially and mostly in lockstep with the CDS spread level, the level of the long term implied volatility remains more or less the same, due to the mean reverting term structure of the smile. Therefore, a growing time horizon leads to a weakening dependence between the level of implied volatility and that of the CDS spread. 


\subsubsection{Implications for Practice}

A very challenging, but all the more promising objective could be the conception of a holistic model which is able to replicate the implied volatility smile and its term structure as well as the term structure of credit spreads and at the same time accommodates the default-risk-only and the volatility-only effect on the smile detected in the empirical part of this thesis. Such a model could be employed for risk management and hedging as well as for pricing and arbitrage purposes, both in connection with equity options, credit derivatives and hybrid products and will be called a holistic model in the following.

As we have seen in section 5.2, most existing enhanced credit risk models are only able to generate a monotonically downward sloping smile, except for example the model of CARR/WU (2006) which thanks to a stochastic volatility specification is also capable of producing a more realistic U-shaped smile pattern. This model could be the starting point for the development of our holistic model. As to the default-riskonly effect of increasing left-skewness, this effect is already reflected in the model. What remains to be integrated are the default-risk-only effect of increasing kurtosis and the antagonistic volatility-only effect of decreasing kurtosis. These effects could be considered by making the volatility of volatility parameter in this model positively dependent on the level of default risk and negatively dependent on the level of volatility. Besides capturing the default-risk-only effect of increasing kurtosis as well as the volatility-only effect of decreasing kurtosis 366 , this approach would render the model more parsimonious by saving the volatility of volatility parameter.

First of all, such a holistic model could be used to improve the quality of inferences from the equity options to the CDS or bond market for the purposes of pricing and arbitrage. As already mentioned in chapter 1, such inferences are potentially very rewarding thanks to the rich data source of the equity options market and the lead-lag relationship between the latter on the one hand and the CDS and the bond market on the other ${ }^{367}$. The more parsimonious such a model is specified, the less data input is needed in order to generate meaningful inferences. Therefore, the saving of the volatility of volatility parameter due to the integration of the volatility-only effect

366 As was shown in section 4.2.3.2 an increasing volatility of volatility parameter in a stochastic volatility specification increases the kurtosis of the risk-neutral equity return density. Therefore, positively linking the volatility of volatility parameter $\sigma_{v}$ in the model of CARR/WU/2006) (see section 5.2.3.2.2) to the level of default risk should create a positive relationship between the level of default risk and that of return kurtosis as implied by the default-risk-only effect. The reasoning for the volatility-only effect ensues conversely.

As to the lead-lag relationship, cf. section 1.1 . 
would possibly even allow to make inferences from the equity options market to the debt market, if the latter is not existent at al 368

Finally, also hedging and risk management of equity options in a developed and liquid equity options market could be improved by means of our holistic model which incorporates the default-risk-only and the volatility-only effect on the smile. It is true that option pricing in liquid markets relies on intricate models capable of statically fitting all given option prices - which is tantamount to the replication of the volatility smile and its term structure - and therefore can dispense with fundamental smile explanations like default risk (cf. section 4.2). Therefore, for mere valuation purposes, also the dissection of default risk into a default-risk-only and a volatility-only effect on the smile would only be of limited value added. However, things change dramatically in the case of hedging or risk management whose objective consists in forecasting changes of option prices in dependence of changes of underlying risk factors like for example the level of volatility or that of default risk. Therefore, in the context of hedging or risk management, the knowledge about the default-risk-only and the volatility-only effect on the smile and ultimately also on option prices could matter and could be exploited to generate valuable price forecasts.

368 Especially in Germany, many sizable, publicly listed corporations rely on bank loans as primary source of debt. For these firms neither a bond nor a CDS market is available, but exchange traded equity options are existing. Prominent examples of such German companies with no existing CDS market, but with equity options traded on EUREX during the time covered by the empirical study of this thesis would be Addidas, Epcos, Infineon, Beiersdorf, Fresenius, Deutsche Börse, MAN, KarstadtQuelle, MLP, Mobilcom, Porsche, Puma, Schering and Altana, just to name a few. 


\section{A. C++ Code Extracts}

\section{A.1. Cox-Ross-Rubinstein Binomial Tree Allowing Multiple Dividends}

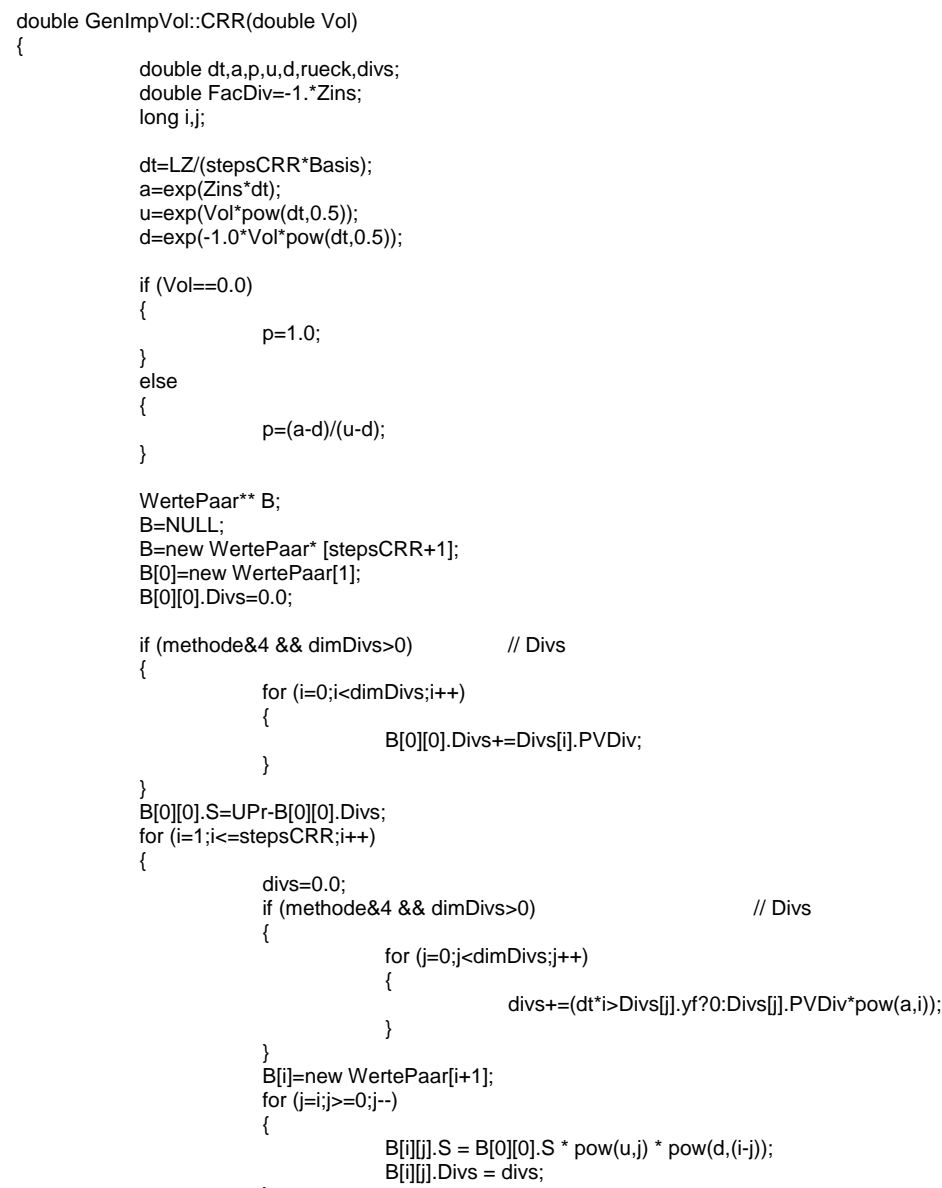




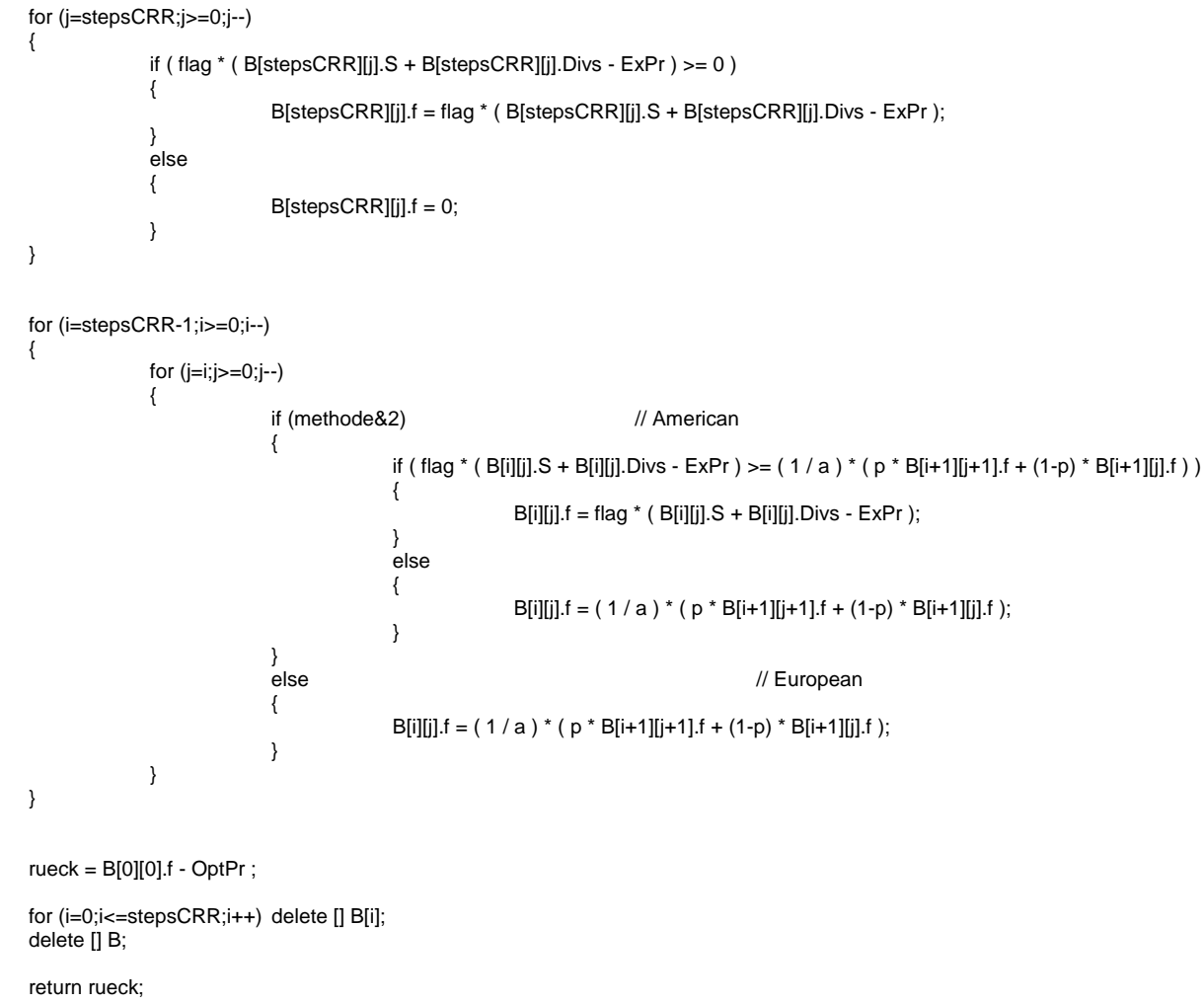




\section{A.2. Quadratic Polynomial Smoothing of the Implied Volatility Smile}

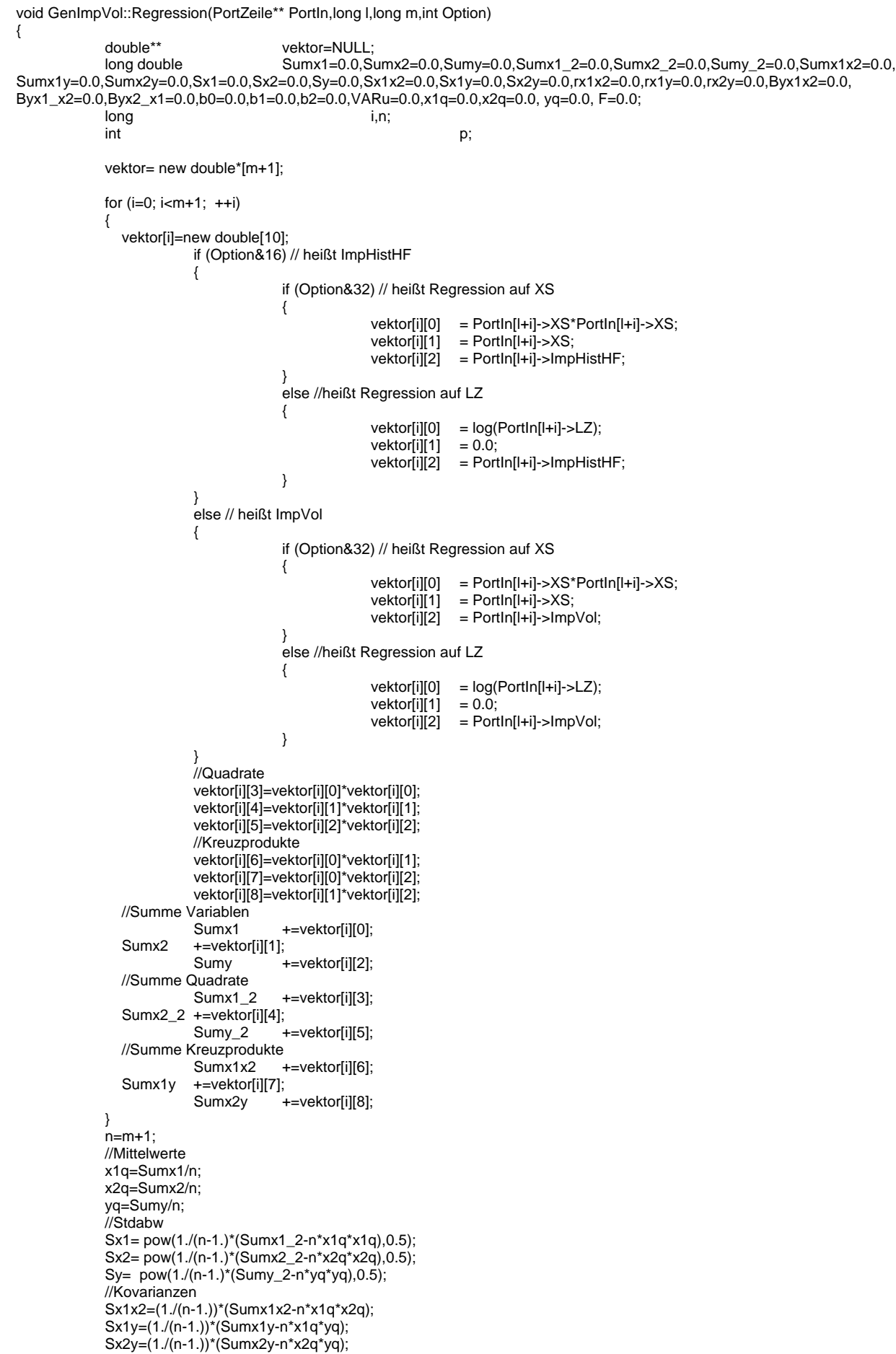




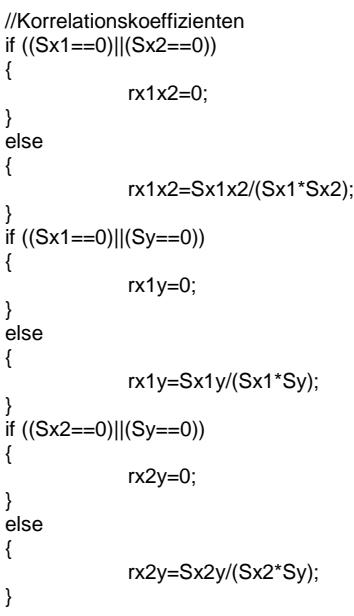

//Regressionskoeffizienten if $(\mathrm{S} \times 1==0)$

b1=0;

else

\{

if $(\mathrm{S} \times 2==0)$

$b 1=S y / S x 1^{*}\left(r \times 1 y-r \times 2 y^{*} r \times 1 \times 2\right) /(1-r \times 1 \times 2 * r x 1 \times 2) ;$

else

$b 0=y q-b 1 * x 1 q-b 2 * x 2 q$

//Multiples Bestimmtheitsmaß:=Byx1x2

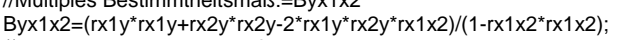

/Partielle Bestimmtheitsmaße, Byx1_x2:"ohne $\times 2$ ", Byx2_x1:"ohne $\times 1$ "

Byx1_x2 $=\left(r \times 1 y-r \times 2 y^{\star} r \times 1 \times 2\right)^{\star}\left(r \times 1 y-r \times 2 y^{\star} r x 1 \times 2\right) /\left(\left(1-r \times 2 y^{\star} r \times 2 y\right)^{*}\left(1-r \times 1 \times 2^{*} r \times 1 \times 2\right)\right)$

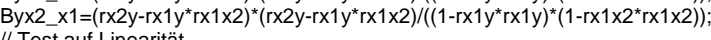

TARu=0.0;

VARu $=0.0 ;$

for $(\mathrm{i}=0 ; \mathrm{i}<\mathrm{n} ;++\mathrm{i})$

vektor[i][9]=vektor[i][2]-b0-b1*vektor[i][0]-b2*vektor[i][1]; VARu+=vektor[i][9] ${ }^{\star}$ vektor[i][9];

VARu/=n-2;

$\mathrm{p}=2$;

$\mathrm{F}=\operatorname{Byx} 1 \times 2 /(1-\operatorname{Byx} 1 \times 2) *(\mathrm{n}-\mathrm{p}-1) / \mathrm{p}$

II in alle Datensätze für einen bestimmten Tag und eine bestimmte ISIN Regressionsergebnisse reinschreiben, da noch nicht feststeht, welcher Datensatz zurückgeliefert wird (min(ImpVol) oder ?)

for $(i=0 ; i<m+1 ;++i)$

Portln[l+i]->b0=b0;

Portln[l+i]->b1=b2; //Achtung Vertauschung der Koeffizienten für die Ausgabe

Portln[l+i] $>>b 2=b 1$;

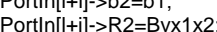

Portln[l+i]->minXS=-1.*b2/(2.*b1);

Portln[l+i]->y minXS = b0 + b1 * Portln[l+i] ->minXS * Portln[l+i]->minXS + b2 * Portln[l+i]->minXS:

Portln[l+i]->y_minXS $=$ b0 + b1 * Portln[l+i]->minXS * Portln[l+i] ->minXS + b2 * Portln[l+i]->minXS;
Portln[l+i]->n=n;

Portln[l+i]->F=F;

for $(\mathrm{i}=0 ; \mathrm{i}<\mathrm{n} ;++\mathrm{i})$

delete vektor[i];

delete [] vektor; 


\section{A.3. Implementation of the Modified Bakshi-Madan Method}

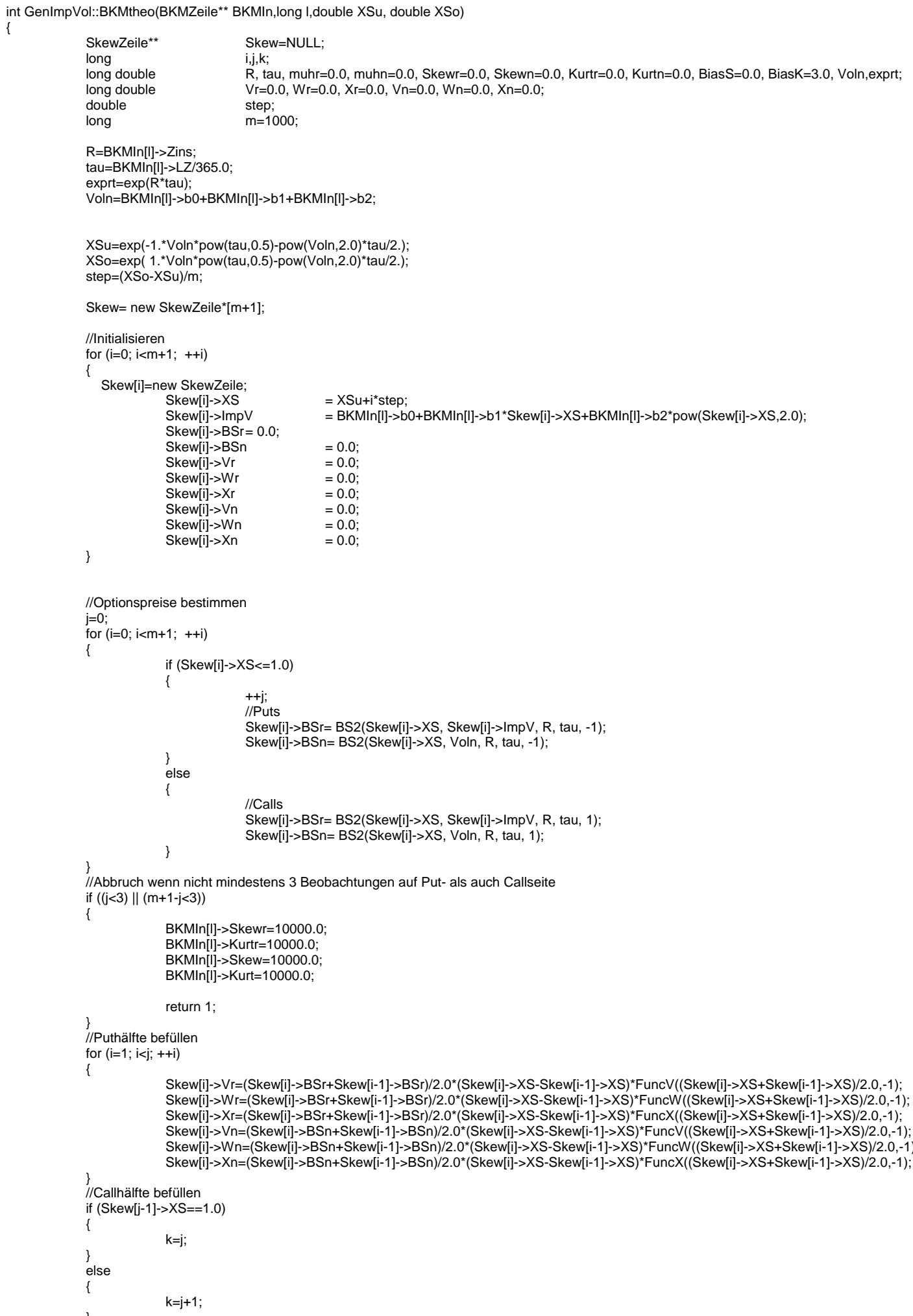

Skew[i]->Vr=(Skew[i]->BSr+Skew[i-1]->BSr)/2.0*(Skew[i]->XS-Skew[i-1]->XS)*FuncV $((\operatorname{Skew}[i]->X S+S k e w[i-1]->X S) / 2.0,-1)$; Skew[i] $>$ Wr $=($ Skew[i] $>$ BSr+Skew[i-1] $>>$ BSr $) / 2.0^{*}(\text { Skew }[i]->X S-S k e w[i-1]->X S)^{*}$ FuncW $(($ Skew $[i]->X S+S k e w[i-1]->X S) / 2.0,-1)$

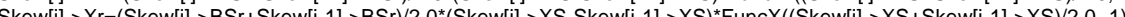
Sken $]>X r=($ Skw

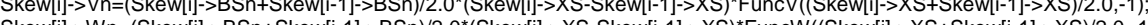

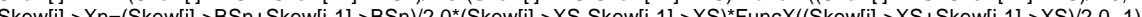
//Callhälfte befüllen 


\section{Appendices}

for $(\mathrm{i}=\mathrm{k} ; \mathrm{i}<\mathrm{m}+1 ;++\mathrm{i})$

Skew[i]->Vr=(Skew[i]->BSr+Skew[i-1]->BSr)/2.0*(Skew[i]->XS-Skew[i-1]->XS)*FuncV $(($ Skew $[i]->X S+$ Skew $[i-1]->X S) / 2.0,1)$; Skew[i]->Wr=(Skew[i]->BSr+Skew[i-1]->BSr)/2.0*(Skew[i]->XS-Skew[i-1]->XS)*FuncW $(($ Skew[i]->XS+Skew[i-1]->XS $) / 2.0,1)$; Skew[i]->Xr=(Skew[i]->BSr+Skew[i-1]->BSr)/2.0*(Skew[i]->XS-Skew[i-1]->XS)*FuncX((Skew[i]->XS+Skew[i-1]->XS)/2.0,1); Skew[i]->Vn=(Skew[i]->BSn+Skew[i-1]->BSn)/2.0*(Skew[i]->XS-Skew[i-1]->XS)*FuncV $(($ Skew[i]->XS+Skew[i-1]->XS)/2.0,1); Skew[i]->Wn=(Skew[i]->BSn+Skew[i-1]->BSn)/2.0*(Skew[i]->XS-Skew[i-1]->XS)*FuncW $(($ Skew[i]->XS+Skew[i-1] ->XS $) / 2.0,1)$ Skew[i]->Xn=(Skew[i]->BSn+Skew[i-1]->BSn)/2.0*(Skew[i]->XS-Skew[i-1]->XS)*FuncX $(($ Skew[i]->XS+Skew[i-1]->XS)/2.0,1);

IN $, \mathrm{W}, \mathrm{X}$ aufaddieren

for $(\mathrm{i}=0 ; \mathrm{i}<\mathrm{m}+1 ;++\mathrm{i})$

$\mathrm{Vr}+=$ Skew[i] $>\mathrm{Vr}$;

$\mathrm{Wr}+=$ Skew[i] $->\mathrm{Wr}$

$\mathrm{Xr}+=$ Skew $[i]->\mathrm{Xr}$;

$\mathrm{Vn}+=$ Skew[i] $\rightarrow \mathrm{Vn}$;

$W n+=S k e w[i]->W n$

$X n+=S k e w[i]->X n$;

//muh, Vol, Skew und Kurt berechnen

muhr=exprt-1.0-exprt* ${ }^{\star} / r / 2.0$-exprt* $W r / 6.0$-exprt ${ }^{\star} \times r / 24.0$

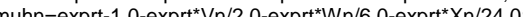

Skewr=(exprt*Wr-3.0*muhr*exprt*Vr+2.0*pow $(m u h r, 3.0)) / \operatorname{pow}\left(\left(\operatorname{exprt}{ }^{\star} V r-p o w(m u h r, 2.0)\right), 3 . / 2.\right)$;

Skewn $=\left(\operatorname{exprt} t^{*} W n-3.0^{*} m u h n * e x p r t^{\star} V n+2.0^{*} \operatorname{pow}(m u h n, 3.0)\right) / \operatorname{pow}\left(\left(\operatorname{exprt}{ }^{\star} V n-p o w(m u h n, 2.0)\right), 3 . / 2.\right) ;$

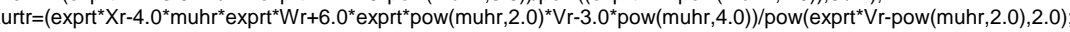

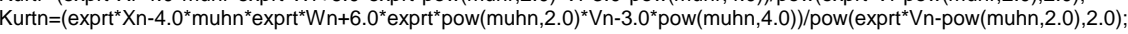

BKMIn[l]->Skewr=Skewr;

BKMIn[l]->Kurtr=Kurtr;

BKMIn[1]->Skew=Skewr-Skewn+BiasS:

BKMIn[l]->Kurt=Kurtr-Kurtn+BiasK

for $(\mathrm{i}=0 ; \mathrm{i}<\mathrm{m}+1 ;++\mathrm{i})$

delete Skew[i];

delete $\square$ Skew;

return 1;

double GenImpVol::FuncV(double KS, int pc)

long double rueck;

if $(\mathrm{pc}==-1)$

\{ rueck=2.0* $(1.0+\log (1.0 / \mathrm{KS})) / \operatorname{pow}(\mathrm{KS}, 2.0)$;

\}

rueck $=2.0^{*}(1.0-\log (K S)) / \operatorname{pow}(\mathrm{KS}, 2.0)$;

return rueck;

double GenImpVol::FuncW(double KS, int pc)

long double rueck;

if $(\mathrm{pc}==-1)$

rueck $=-1.0^{*}\left(6.0 * \log (1.0 / \mathrm{KS})+3.0^{*} \operatorname{pow}(\log (1.0 / \mathrm{KS}), 2.0)\right) / \operatorname{pow}(\mathrm{KS}, 2.0)$

else

rueck=(6.0*log(KS)-3.0*pow(log(KS),2.0))/pow(KS,2.0);

return rueck

double GenImpVol::FuncX(double KS, int pc)

long double rueck;

if $(\mathrm{pc}==-1)$

rueck=(12.0* $\left.\operatorname{pow}(\log (1.0 / \mathrm{KS}), 2.0)+4.0^{\star} \operatorname{pow}(\log (1.0 / \mathrm{KS}), 3.0)\right) / \operatorname{pow}(\mathrm{KS}, 2.0)$;

\{

return rueck

rueck=(12.0* $\left.\operatorname{pow}(\log (\mathrm{KS}), 2.0)-4.0^{\star} \operatorname{pow}(\log (\mathrm{KS}), 3.0)\right) / \operatorname{pow}(\mathrm{KS}, 2.0)$ 


\section{B. Summary Statistics}

\section{B.1. Quadratic Polynomial Smoothing of the Implied Volatility Smile}

\begin{tabular}{|c|c|c|c|c|c|c|c|c|c|c|c|c|c|}
\hline ID & \multicolumn{3}{|c|}{ B0 } & \multicolumn{3}{|c|}{ B1 } & \multicolumn{3}{|c|}{ B2 } & $\mathbf{R}^{2}$ & \multicolumn{2}{|c|}{$F$} & $\mathbf{n}$ \\
\hline DAX & 1,45 & {$[77,25]$} & $(0,00)$ & $-2,03$ & {$[-49,99]$} & $(0,00)$ & 0,83 & {$[39,58]$} & $(0,00)$ & 0,99 & 13.708 & $(0,00)$ & 398 \\
\hline ALL & 1,47 & {$[16,64]$} & $(0,00)$ & $-1,86$ & {$[-10,56]$} & $(0,00)$ & 0,79 & {$[9,19]$} & $(0,00)$ & 0,92 & 443 & $(0,00)$ & 73 \\
\hline _SIE & 1,47 & {$[21,07]$} & $(0,00)$ & $-1,81$ & {$[-13,34]$} & $(0,00)$ & 0,75 & {$[11,58]$} & $(0,00)$ & 0,95 & 737 & $(0,00)$ & 72 \\
\hline DTE & 1,62 & {$[13,30]$} & $(0,00)$ & $-2,07$ & {$[-8,46]$} & $(0,00)$ & 0,91 & {$[7,69]$} & $(0,00)$ & 0,83 & 180 & $(0,00)$ & 55 \\
\hline DAIM & 1,57 & {$[13,25]$} & $(0,00)$ & $-2,08$ & {$[-9,17]$} & $(0,00)$ & 0,89 & {$[8,16]$} & $(0,00)$ & 0,92 & 354 & $(0,00)$ & 59 \\
\hline DBANK & 1,59 & {$[16,99]$} & $(0,00)$ & $-2,04$ & {$[-11,32]$} & $(0,00)$ & 0,86 & {$[9,47]$} & $(0,00)$ & 0,95 & 617 & $(0,00)$ & 54 \\
\hline MRE & 1,57 & {$[10,06]$} & $(0,00)$ & $-2,08$ & {$[-6,47]$} & $(0,00)$ & 0,92 & {$[5,73]$} & $(0,00)$ & 0,90 & 178 & $(0,00)$ & 34 \\
\hline BAY & 1,70 & {$[7,89]$} & $(0,00)$ & $-2,42$ & {$[-5,63]$} & $(0,01)$ & 1,06 & {$[5,07]$} & $(0,01)$ & 0,88 & 113 & $(0,00)$ & 27 \\
\hline _VW & 1,52 & {$[6,89]$} & $(0,00)$ & $-2,06$ & {$[-4,72]$} & $(0,02)$ & 0,88 & {$[4,05]$} & $(0,03)$ & 0,93 & 145 & $(0,00)$ & 23 \\
\hline BASF & 1,86 & {$[6,36]$} & $(0,00)$ & $-2,75$ & {$[-4,82]$} & $(0,01)$ & 1,20 & {$[4,24]$} & $(0,02)$ & 0,93 & 190 & $(0,00)$ & 23 \\
\hline EON & 1,71 & {$[5,53]$} & $(0,01)$ & $-2,58$ & {$[-4,13]$} & $(0,02)$ & 1,15 & {$[3,62]$} & $(0,04)$ & 0,91 & 135 & $(0,00)$ & 20 \\
\hline COB & 1,86 & {$[5,42]$} & $(0,01)$ & $-2,64$ & {$[-3,83]$} & $(0,03)$ & 1,18 & {$[3,43]$} & $(0,04)$ & 0,80 & 44 & $(0,00)$ & 18 \\
\hline _HYPV & 1,63 & {$[4,84]$} & $(0,02)$ & $-2,17$ & {$[-3,21]$} & $(0,05)$ & 0,97 & {$[2,92]$} & $(0,06)$ & 0,84 & 45 & $(0,00)$ & 15 \\
\hline _RWE & 1,99 & {$[4,96]$} & $(0,02)$ & $-3,23$ & {$[-3,98]$} & $(0,04)$ & 1,48 & {$[3,69]$} & $(0,05)$ & 0,87 & 73 & $(0,00)$ & 15 \\
\hline _BMW & 1,57 & {$[4,68]$} & $(0,02)$ & $-2,18$ & {$[-3,21]$} & $(0,06)$ & 0,95 & {$[2,83]$} & $(0,09)$ & 0,92 & 101 & $(0,00)$ & 15 \\
\hline _LH & 2,19 & {$[3,72]$} & $(0,05)$ & $-3,33$ & {$[-2,92]$} & $(0,07)$ & 1,54 & {$[2,72]$} & $(0,08)$ & 0,84 & 35 & $(0,01)$ & 12 \\
\hline _TYSS & 2,50 & {$[3,67]$} & $(0,05)$ & $-4,09$ & {$[-2,93]$} & $(0,07)$ & 1,90 & {$[2,73]$} & $(0,08)$ & 0,84 & 34 & $(0,01)$ & 11 \\
\hline _METRO & 1,91 & {$[2,91]$} & $(0,06)$ & $-2,92$ & {$[-2,07]$} & $(0,10)$ & 1,31 & {$[1,86]$} & $(0,11)$ & 0,90 & 45 & $(0,01)$ & 9 \\
\hline _TUI & 1,85 & {$[2,80]$} & $(0,07)$ & $-2,58$ & {$[-1,96]$} & $(0,11)$ & 1,20 & {$[1,81]$} & $(0,11)$ & 0,83 & 32 & $(0,01)$ & 10 \\
\hline _LIN & 2,31 & {$[2,20]$} & $(0,08)$ & $-3,66$ & {$[-1,84]$} & $(0,11)$ & 1,66 & {$[1,79]$} & $(0,11)$ & 0,95 & 74 & $(0,01)$ & 7 \\
\hline _HEN & 2,61 & {$[1,90]$} & $(0,09)$ & $-4,44$ & {$[-1,56]$} & $(0,11)$ & 2,01 & {$[1,44]$} & $(0,11)$ & 0,95 & 58 & $(0,01)$ & 6 \\
\hline _CONT & 1,97 & {$[1,76]$} & $(0,10)$ & $-3,15$ & {$[-1,44]$} & $(0,12)$ & 1,49 & {$[1,24]$} & $(0,11)$ & 0,94 & 57 & $(0,00)$ & 7 \\
\hline DP & 2,42 & {$[2,18]$} & $(0,08)$ & $-3,84$ & {$[-1,86]$} & $(0,10)$ & 1,81 & {$[1,80]$} & $(0,11)$ & 0,87 & 32 & $(0,02)$ & 9 \\
\hline _DEG & 2,04 & {$[1,05]$} & $(0,13)$ & $-3,09$ & {$[-0,81]$} & $(0,15)$ & 1,05 & {$[0,70]$} & $(0,16)$ & 0,89 & 29 & $(0,01)$ & 8 \\
\hline
\end{tabular}

The table reports the median regression results and statistics for the daily smoothing of all those smiles which have ultimately been used for the construction of the variables with a constant maturity of 60 days. t-stats in [ ] and corresponding p-values in ( )

Table B.1.: Median regression results and statistics for the smoothing of the smiles which have been used for the construction of the 60 days constant maturity variables 


\begin{tabular}{|c|c|c|c|c|c|c|c|c|c|c|c|c|c|}
\hline ID & \multicolumn{3}{|c|}{ B0 } & \multicolumn{3}{|c|}{ B1 } & \multicolumn{3}{|c|}{ B2 } & $\mathbf{R}^{2}$ & \multicolumn{2}{|l|}{$F$} & $\mathbf{n}$ \\
\hline DAX & 0,83 & {$[80,49]$} & $(0,00)$ & $-0,84$ & {$[-39,65]$} & $(0,00)$ & 0,29 & {$[25,86]$} & $(0,00)$ & 0,99 & 10.282 & $(0,00)$ & 129 \\
\hline ALL & 0,88 & {$[22,77]$} & $(0,00)$ & $-0,75$ & {$[-10,04]$} & $(0,00)$ & 0,27 & {$[7,47]$} & $(0,00)$ & 0,97 & 606 & $(0,00)$ & 36 \\
\hline _SIE & 0,85 & {$[24,73]$} & $(0,00)$ & $-0,74$ & {$[-11,61]$} & $(0,00)$ & 0,26 & {$[8,67]$} & $(0,00)$ & 0,98 & 799 & $(0,00)$ & 34 \\
\hline _DTE & 0,89 & {$[13,34]$} & $(0,00)$ & $-0,79$ & {$[-6,24]$} & $(0,00)$ & 0,30 & {$[5,00]$} & $(0,01)$ & 0,91 & 179 & $(0,00)$ & 30 \\
\hline DAIM & 0,87 & {$[14,30]$} & $(0,00)$ & $-0,84$ & {$[-7,25]$} & $(0,00)$ & 0,30 & {$[5,70]$} & $(0,01)$ & 0,96 & 390 & $(0,00)$ & 30 \\
\hline DBANK & 0,90 & {$[20,37]$} & $(0,00)$ & $-0,86$ & {$[-10,03]$} & $(0,00)$ & 0,31 & {$[7,44]$} & $(0,00)$ & 0,98 & 766 & $(0,00)$ & 26 \\
\hline MRE & 0,87 & {$[12,85]$} & $(0,00)$ & $-0,82$ & {$[-5,99]$} & $(0,01)$ & 0,31 & {$[4,53]$} & $(0,02)$ & 0,95 & 274 & $(0,00)$ & 20 \\
\hline BAY & 0,90 & {$[7,65]$} & $(0,00)$ & $-0,94$ & {$[-4,09]$} & $(0,03)$ & 0,37 & {$[3,29]$} & $(0,05)$ & 0,94 & 140 & $(0,00)$ & 15 \\
\hline _VW & 0,86 & {$[8,16]$} & $(0,00)$ & $-0,84$ & {$[-4,06]$} & $(0,04)$ & 0,32 & {$[3,21]$} & $(0,07)$ & 0,97 & 245 & $(0,00)$ & 12 \\
\hline BASF & 0,92 & {$[6,52]$} & $(0,00)$ & $-1,06$ & {$[-3,93]$} & $(0,03)$ & 0,42 & {$[2,99]$} & $(0,05)$ & 0,97 & 260 & $(0,00)$ & 13 \\
\hline EON & 0,87 & {$[6,80]$} & $(0,01)$ & $-1,01$ & {$[-3,95]$} & $(0,04)$ & 0,41 & {$[3,22]$} & $(0,06)$ & 0,96 & 187 & $(0,00)$ & 12 \\
\hline $\mathrm{COB}$ & 0,97 & {$[5,97]$} & $(0,01)$ & $-1,00$ & {$[-3,13]$} & $(0,05)$ & 0,41 & {$[2,63]$} & $(0,08)$ & 0,90 & 62 & $(0,00)$ & 12 \\
\hline _HYPV & 1,02 & {$[4,57]$} & $(0,02)$ & $-1,02$ & {$[-2,27]$} & $(0,07)$ & 0,41 & {$[2,03]$} & $(0,09)$ & 0,90 & 45 & $(0,00)$ & 10 \\
\hline RWE & 0,89 & {$[4,50]$} & $(0,03)$ & $-1,06$ & {$[-2,73]$} & $(0,08)$ & 0,43 & {$[2,39]$} & $(0,10)$ & 0,94 & 110 & $(0,00)$ & 10 \\
\hline BMW & 0,96 & {$[5,05]$} & $(0,02)$ & $-1,00$ & {$[-2,76]$} & $(0,07)$ & 0,40 & {$[2,18]$} & $(0,10)$ & 0,96 & 134 & $(0,00)$ & 10 \\
\hline LLH & 1,18 & {$[3,22]$} & $(0,05)$ & $-1,36$ & {$[-1,98]$} & $(0,09)$ & 0,57 & {$[1,76]$} & $(0,10)$ & 0,88 & 38 & $(0,01)$ & 9 \\
\hline _TYSS & 1,11 & {$[3,62]$} & $(0,05)$ & $-1,40$ & {$[-2,21]$} & $(0,09)$ & 0,59 & {$[1,97]$} & $(0,11)$ & 0,90 & 41 & $(0,00)$ & 9 \\
\hline _METRO & 1,17 & {$[2,67]$} & $(0,07)$ & $-1,55$ & {$[-1,67]$} & $(0,11)$ & 0,68 & {$[1,41]$} & $(0,12)$ & 0,94 & 45 & $(0,00)$ & 7 \\
\hline _TUI & 1,18 & {$[2,69]$} & $(0,06)$ & $-1,37$ & {$[-1,52]$} & $(0,13)$ & 0,59 & {$[1,37]$} & $(0,14)$ & 0,90 & 37 & $(0,01)$ & 8 \\
\hline LLIN & 1,62 & {$[1,98]$} & $(0,08)$ & $-2,50$ & {$[-1,50]$} & $(0,12)$ & 1,15 & {$[1,40]$} & $(0,12)$ & 0,97 & 76 & $(0,00)$ & 6 \\
\hline HEN & 1,76 & {$[1,91]$} & $(0,08)$ & $-2,96$ & {$[-1,37]$} & $(0,10)$ & 1,31 & {$[1,28]$} & $(0,11)$ & 0,96 & 77 & $(0,00)$ & 6 \\
\hline _CONT & 1,56 & {$[1,76]$} & $(0,08)$ & $-2,32$ & {$[-1,39]$} & $(0,10)$ & 1,07 & {$[1,25]$} & $(0,09)$ & 0,95 & 65 & $(0,00)$ & 7 \\
\hline DP & 1,43 & {$[1,97]$} & $(0,07)$ & $-2,07$ & {$[-1,42]$} & $(0,10)$ & 0,96 & {$[1,36]$} & $(0,11)$ & 0,90 & 29 & $(0,01)$ & 8 \\
\hline DEG & 1,19 & {$[1,12]$} & $(0,13)$ & $-1,58$ & {$[-0,84]$} & $(0,18)$ & 0,71 & {$[0,69]$} & $(0,18)$ & 0,90 & 29 & $(0,01)$ & 8 \\
\hline
\end{tabular}

The table reports the median regression results and statistics for the daily smoothing of all those smiles which have ultimately been used for the construction of the variables with a constant maturity of 180 days.

t-stats in [ ] and corresponding p-values in ( )

Table B.2.: Median regression results and statistics for the smoothing of the smiles which have been used for the construction of the 180 days constant maturity variables 


\begin{tabular}{|c|c|c|c|c|c|c|c|c|c|c|c|c|c|}
\hline ID & \multicolumn{3}{|c|}{ B0 } & \multicolumn{3}{|c|}{ B1 } & \multicolumn{3}{|c|}{ B2 } & $\mathbf{R}^{2}$ & \multicolumn{2}{|c|}{$\mathbf{F}$} & $\mathbf{n}$ \\
\hline DAX & 0,73 & {$[70,08]$} & $(0,00)$ & $-0,67$ & {$[-31,08]$} & $(0,00)$ & 0,22 & {$[19,21]$} & $(0,00)$ & 1,00 & 8.072 & $(0,00)$ & 69 \\
\hline ALL & 0,77 & {$[17,01]$} & $(0,00)$ & $-0,59$ & {$[-6,68]$} & $(0,00)$ & 0,20 & {$[4,72]$} & $(0,02)$ & 0,97 & 440 & $(0,00)$ & 21 \\
\hline _SIE & 0,75 & {$[21,20]$} & $(0,00)$ & $-0,60$ & {$[-9,00]$} & $(0,00)$ & 0,20 & {$[6,49]$} & $(0,00)$ & 0,98 & 728 & $(0,00)$ & 20 \\
\hline DTE & 0,79 & {$[10,77]$} & $(0,00)$ & $-0,65$ & {$[-4,45]$} & $(0,02)$ & 0,23 & {$[3,46]$} & $(0,05)$ & 0,92 & 151 & $(0,00)$ & 19 \\
\hline DAIM & 0,76 & {$[11,51]$} & $(0,00)$ & $-0,67$ & {$[-5,23]$} & $(0,01)$ & 0,24 & {$[4,00]$} & $(0,03)$ & 0,96 & 277 & $(0,00)$ & 19 \\
\hline DBANK & 0,81 & {$[17,57]$} & $(0,00)$ & $-0,67$ & {$[-7,76]$} & $(0,00)$ & 0,23 & {$[5,37]$} & $(0,01)$ & 0,98 & 659 & $(0,00)$ & 17 \\
\hline _MRE & 0,78 & {$[10,94]$} & $(0,00)$ & $-0,67$ & {$[-4,73]$} & $(0,02)$ & 0,24 & {$[3,61]$} & $(0,04)$ & 0,96 & 261 & $(0,00)$ & 14 \\
\hline BAY & 0,81 & {$[6,52]$} & $(0,01)$ & $-0,76$ & {$[-3,21]$} & $(0,05)$ & 0,29 & {$[2,49]$} & $(0,07)$ & 0,95 & 127 & $(0,00)$ & 12 \\
\hline _VW & 0,80 & {$[7,55]$} & $(0,00)$ & $-0,73$ & {$[-3,54]$} & $(0,05)$ & 0,27 & {$[2,76]$} & $(0,08)$ & 0,98 & 256 & $(0,00)$ & 9 \\
\hline BASF & 0,83 & {$[6,03]$} & $(0,01)$ & $-0,91$ & {$[-3,45]$} & $(0,05)$ & 0,35 & {$[2,72]$} & $(0,07)$ & 0,97 & 251 & $(0,00)$ & 11 \\
\hline EON & 0,78 & {$[6,12]$} & $(0,01)$ & $-0,87$ & {$[-3,44]$} & $(0,05)$ & 0,34 & {$[2,84]$} & $(0,06)$ & 0,97 & 191 & $(0,00)$ & 10 \\
\hline COB & 0,89 & {$[5,42]$} & $(0,01)$ & $-0,82$ & {$[-2,76]$} & $(0,07)$ & 0,32 & {$[2,21]$} & $(0,10)$ & 0,92 & 63 & $(0,00)$ & 10 \\
\hline _HYPV & 1,00 & {$[4,09]$} & $(0,02)$ & $-0,96$ & {$[-2,08]$} & $(0,08)$ & 0,38 & {$[1,72]$} & $(0,10)$ & 0,91 & 50 & $(0,00)$ & 10 \\
\hline _RWE & 0,80 & {$[3,87]$} & $(0,04)$ & $-0,88$ & {$[-2,18]$} & $(0,09)$ & 0,35 & {$[1,77]$} & $(0,12)$ & 0,94 & 88 & $(0,00)$ & 9 \\
\hline BMW & 0,90 & {$[4,73]$} & $(0,02)$ & $-0,95$ & {$[-2,37]$} & $(0,08)$ & 0,36 & {$[1,89]$} & $(0,11)$ & 0,97 & 127 & $(0,00)$ & 9 \\
\hline _LH & 1,19 & {$[2,87]$} & $(0,05)$ & $-1,33$ & {$[-1,70]$} & $(0,09)$ & 0,55 & {$[1,46]$} & $(0,11)$ & 0,90 & 37 & $(0,01)$ & 9 \\
\hline _TYSS & 1,10 & {$[3,33]$} & $(0,04)$ & $-1,37$ & {$[-2,07]$} & $(0,08)$ & 0,58 & {$[1,82]$} & $(0,11)$ & 0,92 & 40 & $(0,00)$ & 8 \\
\hline _METRO & 1,17 & {$[2,56]$} & $(0,06)$ & $-1,53$ & {$[-1,60]$} & $(0,10)$ & 0,65 & {$[1,32]$} & $(0,11)$ & 0,95 & 45 & $(0,00)$ & 7 \\
\hline _TUI & 1,13 & {$[2,57]$} & $(0,06)$ & $-1,23$ & {$[-1,41]$} & $(0,12)$ & 0,52 & {$[1,30]$} & $(0,14)$ & 0,91 & 37 & $(0,01)$ & 7 \\
\hline LLIN & 1,64 & {$[2,02]$} & $(0,07)$ & $-2,52$ & {$[-1,59]$} & $(0,10)$ & 1,19 & {$[1,43]$} & $(0,11)$ & 0,97 & 79 & $(0,00)$ & 6 \\
\hline HEN & 1,71 & {$[1,91]$} & $(0,07)$ & $-2,78$ & {$[-1,42]$} & $(0,10)$ & 1,27 & {$[1,27]$} & $(0,11)$ & 0,97 & 77 & $(0,00)$ & 6 \\
\hline _CONT & 1,55 & {$[1,71]$} & $(0,06)$ & $-2,32$ & {$[-1,38]$} & $(0,08)$ & 1,06 & {$[1,25]$} & $(0,08)$ & 0,95 & 64 & $(0,00)$ & 7 \\
\hline DP & 1,27 & {$[1,86]$} & $(0,07)$ & $-1,80$ & {$[-1,40]$} & $(0,10)$ & 0,83 & {$[1,30]$} & $(0,10)$ & 0,90 & 28 & $(0,01)$ & 7 \\
\hline DEG & 1,12 & {$[1,08]$} & $(0,13)$ & $-1,49$ & {$[-0,70]$} & $(0,18)$ & 0,67 & {$[0,59]$} & $(0,17)$ & 0,91 & 27 & $(0,01)$ & 8 \\
\hline
\end{tabular}

The table reports the median regression results and statistics for the daily smoothing of all those smiles which have ultimately been used for the construction of the variables with a constant maturity of 240 days.

t-stats in [ ] and corresponding p-values in ( )

Table B.3.: Median regression results and statistics for the smoothing of the smiles which have been used for the construction of the 240 days constant maturity variables 
B.2. Smile Dependent Regression Variables on an Individual Constant Maturity Basis - All Cross-Sections Pooled Together 


\begin{tabular}{|c|c|c|c|c|c|c|}
\hline & Mean & Median & Max & Min & StdDev & Obs \\
\hline \multicolumn{7}{|c|}{ Constant maturity of 36 days } \\
\hline $\mathrm{B}_{\text {Stocks }}$ & 12,97 & 2,68 & $45.714,21$ & $-5.739,73$ & 564,18 & 13.724 \\
\hline B1 $1_{\text {Stocks }}$ & $-25,02$ & $-4,38$ & $16.579,96$ & $-95.651,84$ & $1.156,01$ & 13.724 \\
\hline B2 $2_{\text {Stocks }}$ & 12,12 & 2,03 & $50.035,39$ & $-15.701,01$ & 600,88 & 13.724 \\
\hline $\mathrm{B}_{\text {Dax }}$ & 2,37 & 2,29 & 4,63 & 1,32 & 0,61 & 13.724 \\
\hline B1 $1_{\text {Dax }}$ & $-3,77$ & $-3,67$ & $-1,18$ & $-8,59$ & 1,44 & 13.724 \\
\hline B2 Dax & 1,68 & 1,62 & 4,12 & 0,39 & 0,74 & 13.724 \\
\hline SLP70 ${ }_{\text {Stocks }}$ & $-8,05$ & $-1,54$ & $4.060,24$ & $-25.602,29$ & 331,81 & 13.724 \\
\hline $\mathrm{SLP}_{\text {Stocks }}$ & $-3,20$ & $-0,73$ & $2.640,21$ & $-11.681,85$ & 140,35 & 13.724 \\
\hline SLP100 ${ }_{\text {Stocks }}$ & $-0,78$ & $-0,31$ & $4.418,95$ & $-14.822,05$ & 133,51 & 13.724 \\
\hline SLP110 ${ }_{\text {Stocks }}$ & 1,65 & 0,11 & $14.426,03$ & $-17.962,26$ & 211,74 & 13.724 \\
\hline SLP130 ${ }_{\text {Stocks }}$ & 6,50 & 0,92 & $34.440,18$ & $-24.242,66$ & 430,71 & 13.724 \\
\hline SLP70 $_{\text {Dax }}$ & $-1,42$ & $-1,40$ & $-0,63$ & $-2,82$ & 0,42 & 13.724 \\
\hline SLP90 & $-0,75$ & $-0,75$ & $-0,40$ & $-1,20$ & 0,13 & 13.724 \\
\hline SLP100 & $-0,41$ & $-0,41$ & $-0,20$ & $-0,59$ & 0,07 & 13.724 \\
\hline SLP110 Dax & $-0,08$ & $-0,10$ & 0,55 & $-0,44$ & 0,19 & 13.724 \\
\hline SLP130 Dax & 0,60 & 0,55 & 2,12 & $-0,22$ & 0,48 & 13.724 \\
\hline XSMIN $_{\text {Stocks }}$ & 1,13 & 1,06 & 344,43 & $-50,73$ & 3,71 & 13.724 \\
\hline IMPV $_{\text {Stocks }}$ & 0,07 & 0,29 & 97,76 & $-3.495,85$ & 29,94 & 13.724 \\
\hline IMPV $_{\text {Dax }}$ & 0,28 & 0,24 & 0,60 & 0,12 & 0,12 & 13.724 \\
\hline SKEW $_{\text {Stocks }}$ & $-0,14$ & $-0,14$ & 5,05 & $-15,72$ & 0,32 & 13.724 \\
\hline KURT $_{\text {Stocks }}$ & 3,02 & 3,02 & 18,95 & 0,02 & 0,27 & 13.724 \\
\hline SKEW $_{\text {Dax }}$ & $-0,19$ & $-0,19$ & $-0,09$ & $-0,26$ & 0,03 & 13.724 \\
\hline KURT $_{\text {Dax }}$ & 3,02 & 3,03 & 3,04 & 2,99 & 0,01 & 13.724 \\
\hline \multicolumn{7}{|c|}{ Constant maturity of 60 days } \\
\hline $\mathrm{B}_{\text {Stocks }}$ & 7,64 & 1,67 & $45.714,21$ & $-24.556,99$ & 613,56 & 13.724 \\
\hline B1 $1_{\text {Stocks }}$ & $-11,59$ & $-2,37$ & $92.971,62$ & $-95.651,84$ & $1.418,53$ & 13.724 \\
\hline B2 ${ }_{\text {Stocks }}$ & 2,85 & 1,05 & $50.035,39$ & $-87.993,11$ & 961,65 & 13.724 \\
\hline$B 0_{\text {Dax }}$ & 1,51 & 1,46 & 2,46 & 1,05 & 0,25 & 13.724 \\
\hline B1 $1_{\text {Dax }}$ & $-2,09$ & $-2,05$ & $-0,82$ & $-4,41$ & 0,69 & 13.724 \\
\hline B2 Dax & 0,86 & 0,84 & 2,09 & 0,21 & 0,35 & 13.724 \\
\hline SLP70 ${ }_{\text {Stocks }}$ & $-7,60$ & $-0,91$ & $13.942,34$ & $-30.218,73$ & 423,93 & 13.724 \\
\hline SLP90 ${ }_{\text {Stocks }}$ & $-6,46$ & $-0,50$ & $5.311,42$ & $-65.415,98$ & 567,44 & 13.724 \\
\hline SLP $100_{\text {Stocks }}$ & $-5,89$ & $-0,28$ & $4.418,95$ & $-83.014,60$ & 709,99 & 13.724 \\
\hline SLP110 ${ }_{\text {Stocks }}$ & $-5,32$ & $-0,07$ & $14.426,03$ & $-100.613,20$ & 871,88 & 13.724 \\
\hline SLP130 ${ }_{\text {Stocks }}$ & $-4,19$ & 0,35 & $34.440,18$ & $-135.810,50$ & $1.222,41$ & 13.724 \\
\hline SLP70 $_{\text {Dax }}$ & $-0,89$ & $-0,87$ & $-0,47$ & $-1,48$ & 0,20 & 13.724 \\
\hline SLP90 & $-0,54$ & $-0,54$ & $-0,34$ & $-0,70$ & 0,07 & 13.724 \\
\hline SLP100 Dax & $-0,37$ & $-0,37$ & $-0,20$ & $-0,52$ & 0,06 & 13.724 \\
\hline SLP110 & $-0,20$ & $-0,21$ & 0,19 & $-0,41$ & 0,11 & 13.724 \\
\hline SLP130 Dax & 0,15 & 0,12 & 1,03 & $-0,29$ & 0,24 & 13.724 \\
\hline XSMIN $_{\text {Stocks }}$ & 1,17 & 1,10 & 447,81 & $-557,02$ & 7,71 & 13.724 \\
\hline IMPV $_{\text {Stocks }}$ & $-1,10$ & 0,30 & 97,76 & $-19.578,48$ & 167,13 & 13.724 \\
\hline IMPV $_{\text {Dax }}$ & 0,28 & 0,24 & 0,57 & 0,13 & 0,10 & 13.724 \\
\hline SKEW $_{\text {Stocks }}$ & $-0,16$ & $-0,16$ & 5,05 & $-15,72$ & 0,33 & 13.724 \\
\hline $\mathrm{KURT}_{\text {Stocks }}$ & 3,02 & 3,02 & 18,95 & 0,02 & 0,31 & 13.724 \\
\hline SKEW $_{\text {Dax }}$ & $-0,22$ & $-0,22$ & $-0,12$ & $-0,30$ & 0,03 & 13.724 \\
\hline KURT $_{\text {Dax }}$ & 3,03 & 3,03 & 3,06 & 2,99 & 0,02 & 13.724 \\
\hline
\end{tabular}

Table B.4.: Summary statistics for smile dependent variables with constant maturities of 36 and 60 days (all cross sections pooled together 


\begin{tabular}{|c|c|c|c|c|c|c|}
\hline & Mean & Median & Max & Min & StdDev & Obs \\
\hline \multicolumn{7}{|c|}{ Constant maturity of 180 days } \\
\hline $\mathrm{BO}_{\text {Stocks }}$ & 5,66 & 0,92 & $42.448,98$ & $-24.556,99$ & 606,57 & 13.724 \\
\hline B1 $1_{\text {Stocks }}$ & $-7,68$ & $-0,94$ & $92.971,62$ & $-88.819,67$ & $1.395,83$ & 13.724 \\
\hline B2 Stocks & 0,90 & 0,36 & $46.461,49$ & $-87.993,11$ & 950,50 & 13.724 \\
\hline $\mathrm{BO}_{\text {Dax }}$ & 0,83 & 0,83 & 1,27 & $-1,02$ & 0,10 & 13.724 \\
\hline$B 1_{\text {Dax }}$ & $-0,85$ & $-0,84$ & 5,59 & $-1,77$ & 0,31 & 13.724 \\
\hline B2 & 0,29 & 0,29 & 0,72 & $-4,96$ & 0,22 & 13.724 \\
\hline SLP70 ${ }_{\text {Stocks }}$ & $-6,42$ & $-0,43$ & $13.942,34$ & $-30.218,73$ & 424,03 & 13.724 \\
\hline SLP90 ${ }_{\text {Stocks }}$ & $-6,06$ & $-0,28$ & $5.311,42$ & $-65.415,98$ & 570,07 & 13.724 \\
\hline SLP100 ${ }_{\text {Stocks }}$ & $-5,88$ & $-0,21$ & $4.103,31$ & $-83.014,60$ & 711,33 & 13.724 \\
\hline SLP110 ${ }_{\text {Stocks }}$ & $-5,70$ & $-0,13$ & $13.395,60$ & $-100.613,20$ & 871,36 & 13.724 \\
\hline SLP130 ${ }_{\text {Stocks }}$ & $-5,33$ & 0,02 & $31.980,20$ & $-135.810,50$ & $1.217,64$ & 13.724 \\
\hline SLP70 $_{\text {Dax }}$ & $-0,45$ & $-0,44$ & $-0,26$ & $-1,35$ & 0,08 & 13.724 \\
\hline SLP90 $_{\text {Dax }}$ & $-0,33$ & $-0,32$ & $-0,21$ & $-3,34$ & 0,12 & 13.724 \\
\hline SLP100 & $-0,28$ & $-0,27$ & $-0,19$ & $-4,33$ & 0,15 & 13.724 \\
\hline SLP110 Dax & $-0,22$ & $-0,21$ & $-0,12$ & $-5,32$ & 0,19 & 13.724 \\
\hline SLP130 & $-0,10$ & $-0,10$ & 0,11 & $-7,31$ & 0,28 & 13.724 \\
\hline XSMIN $_{\text {Stocks }}$ & 1,21 & 1,20 & 344,43 & $-424,65$ & 6,65 & 13.724 \\
\hline IMPV $_{\text {Stocks }}$ & $-1,12$ & 0,31 & 90,80 & $-19.578,48$ & 167,17 & 13.724 \\
\hline IMPV $_{\text {Dax }}$ & 0,27 & 0,24 & 0,48 & $-0,38$ & 0,08 & 13.724 \\
\hline SKEW $_{\text {Stocks }}$ & $-0,18$ & $-0,19$ & 5,05 & $-20,10$ & 0,45 & 13.724 \\
\hline $\mathrm{KURT}_{\text {Stocks }}$ & 3,02 & 3,02 & 18,95 & 0,02 & 0,41 & 13.724 \\
\hline SKEW $_{\text {Dax }}$ & $-0,27$ & $-0,26$ & $-0,19$ & $-0,36$ & 0,03 & 13.724 \\
\hline KURT $_{\text {Dax }}$ & 3,05 & 3,05 & 3,12 & 2,98 & 0,03 & 13.724 \\
\hline \multicolumn{7}{|c|}{ Constant maturity of 240 days } \\
\hline $\mathrm{B} 0_{\text {Stocks }}$ & 5,39 & 0,83 & $27.378,69$ & $-24.556,99$ & 650,45 & 13.724 \\
\hline B1 $1_{\text {Stocks }}$ & $-6,44$ & $-0,78$ & $92.971,62$ & $-57.286,60$ & $1.454,72$ & 13.724 \\
\hline B2 ${ }_{\text {Stocks }}$ & $-0,06$ & 0,30 & $29.966,55$ & $-87.993,11$ & 966,82 & 13.724 \\
\hline $\mathrm{B0}_{\text {Dax }}$ & $-0,60$ & 0,73 & 46,50 & $-944,23$ & 36,10 & 13.724 \\
\hline$B 1_{\text {Dax }}$ & 2,14 & $-0,68$ & $1.985,48$ & $-90,22$ & 75,86 & 13.724 \\
\hline B2 ${ }_{\text {Dax }}$ & $-1,28$ & 0,22 & 44,04 & $-1.043,45$ & 39,87 & 13.724 \\
\hline SLP70 ${ }_{\text {Stocks }}$ & $-6,53$ & $-0,37$ & $15.379,99$ & $-30.218,73$ & 453,46 & 13.724 \\
\hline SLP90 ${ }_{\text {Stocks }}$ & $-6,55$ & $-0,25$ & $6.627,78$ & $-65.415,98$ & 576,86 & 13.724 \\
\hline SLP100 ${ }_{\text {Stocks }}$ & $-6,57$ & $-0,19$ & $2.825,48$ & $-83.014,60$ & 713,10 & 13.724 \\
\hline SLP110 ${ }_{\text {Stocks }}$ & $-6,58$ & $-0,13$ & $8.639,80$ & $-100.613,20$ & 871,22 & 13.724 \\
\hline SLP130 ${ }_{\text {Stocks }}$ & $-6,60$ & $-0,01$ & $20.626,42$ & $-135.810,50$ & $1.218,36$ & 13.724 \\
\hline SLP70 $_{\text {Dax }}$ & 0,35 & $-0,37$ & 524,65 & $-28,56$ & 20,06 & 13.724 \\
\hline SLP90 Dax & $-0,17$ & $-0,28$ & 107,27 & $-18,26$ & 4,18 & 13.724 \\
\hline SLP100 Dax & $-0,43$ & $-0,24$ & 4,19 & $-101,42$ & 3,97 & 13.724 \\
\hline SLP110 Dax & $-0,68$ & $-0,20$ & 6,91 & $-310,11$ & 11,88 & 13.724 \\
\hline SLP130 & $-1,20$ & $-0,11$ & 24,29 & $-727,49$ & 27,81 & 13.724 \\
\hline XSMIN $_{\text {Stocks }}$ & $-1,77$ & 1,20 & 344,43 & $-41.090,33$ & 350,80 & 13.724 \\
\hline IMPV $_{\text {Stocks }}$ & $-1,11$ & 0,31 & 469,02 & $-19.578,48$ & 167,31 & 13.724 \\
\hline IMPV $_{\text {Dax }}$ & 0,26 & 0,24 & 0,60 & $-4,29$ & 0,21 & 13.724 \\
\hline SKEW $_{\text {Stocks }}$ & $-0,18$ & $-0,19$ & 5,05 & $-19,66$ & 0,51 & 13.724 \\
\hline $\mathrm{KURT}_{\text {Stocks }}$ & 3,02 & 3,02 & 19,06 & 0,02 & 0,47 & 13.724 \\
\hline SKEW $_{\text {Dax }}$ & $-0,28$ & $-0,27$ & 0,13 & $-0,38$ & 0,04 & 13.653 \\
\hline KURT $_{\text {Dax }}$ & 2,98 & 3,06 & 3,12 & $-27,12$ & 1,31 & 13.724 \\
\hline
\end{tabular}

Table B.5.: Summary statistics for smile dependent variables with constant maturities of 180 and 240 days (all cross sections pooled together 


\section{B.3. All Regression Variables on an Individual Cross-Sectional and an Individual Constant Maturity Basis}

\section{B.3.1. DAX}

\begin{tabular}{|c|c|c|c|c|c|c|}
\hline DAX & Mean & Median & Max & Min & StdDev & Obs \\
\hline \multicolumn{7}{|c|}{ Constant maturity of 36 days } \\
\hline Bo & 2,35 & 2,27 & 4,63 & 1,32 & 0,60 & 763 \\
\hline B1 & $-3,72$ & $-3,60$ & $-1,18$ & $-8,59$ & 1,42 & 763 \\
\hline B2 & 1,66 & 1,58 & 4,12 & 0,39 & 0,72 & 763 \\
\hline SLP70 & $-1,41$ & $-1,38$ & $-0,63$ & $-2,82$ & 0,41 & 763 \\
\hline SLP90 & $-0,74$ & $-0,74$ & $-0,40$ & $-1,20$ & 0,13 & 763 \\
\hline SLP100 & $-0,41$ & $-0,41$ & $-0,20$ & $-0,59$ & 0,07 & 763 \\
\hline SLP110 & $-0,08$ & $-0,10$ & 0,55 & $-0,44$ & 0,19 & 763 \\
\hline SLP130 & 0,58 & 0,53 & 2,12 & $-0,22$ & 0,47 & 763 \\
\hline IMPV & 0,28 & 0,25 & 0,60 & 0,12 & 0,12 & 763 \\
\hline SKEW & $-0,19$ & $-0,19$ & $-0,09$ & $-0,26$ & 0,03 & 763 \\
\hline KURT & 3,02 & 3,03 & 3,04 & 2,99 & 0,01 & 763 \\
\hline \multicolumn{7}{|c|}{ Constant maturity of 60 days } \\
\hline B0 & 1,50 & 1,46 & 2,46 & 1,05 & 0,24 & 763 \\
\hline B1 & $-2,07$ & $-2,04$ & $-0,82$ & $-4,41$ & 0,67 & 763 \\
\hline B2 & 0,85 & 0,83 & 2,09 & 0,21 & 0,35 & 763 \\
\hline SLP70 & $-0,88$ & $-0,86$ & $-0,47$ & $-1,48$ & 0,19 & 763 \\
\hline SLP90 & $-0,54$ & $-0,54$ & $-0,34$ & $-0,70$ & 0,07 & 763 \\
\hline SLP100 & $-0,37$ & $-0,37$ & $-0,20$ & $-0,52$ & 0,06 & 763 \\
\hline SLP110 & $-0,20$ & $-0,20$ & 0,19 & $-0,41$ & 0,11 & 763 \\
\hline SLP130 & 0,14 & 0,12 & 1,03 & $-0,29$ & 0,24 & 763 \\
\hline IMPV & 0,28 & 0,25 & 0,57 & 0,13 & 0,10 & 763 \\
\hline SKEW & $-0,22$ & $-0,22$ & $-0,12$ & $-0,30$ & 0,03 & 763 \\
\hline KURT & 3,03 & 3,03 & 3,06 & 2,99 & 0,02 & 763 \\
\hline \multicolumn{7}{|c|}{ Constant maturity of 180 days } \\
\hline B0 & 0,83 & 0,83 & 1,27 & $-1,02$ & 0,10 & 763 \\
\hline B1 & $-0,85$ & $-0,84$ & 5,59 & $-1,77$ & 0,31 & 763 \\
\hline B2 & 0,29 & 0,29 & 0,72 & $-4,96$ & 0,21 & 763 \\
\hline SLP70 & $-0,45$ & $-0,43$ & $-0,26$ & $-1,35$ & 0,08 & 763 \\
\hline SLP90 & $-0,33$ & $-0,32$ & $-0,21$ & $-3,34$ & 0,12 & 763 \\
\hline SLP100 & $-0,27$ & $-0,27$ & $-0,19$ & $-4,33$ & 0,15 & 763 \\
\hline SLP110 & $-0,22$ & $-0,21$ & $-0,12$ & $-5,32$ & 0,19 & 763 \\
\hline SLP130 & $-0,10$ & $-0,10$ & 0,11 & $-7,31$ & 0,27 & 763 \\
\hline IMPV & 0,27 & 0,25 & 0,48 & $-0,38$ & 0,08 & 763 \\
\hline SKEW & $-0,27$ & $-0,26$ & $-0,19$ & $-0,36$ & 0,03 & 763 \\
\hline KURT & 3,05 & 3,05 & 3,12 & 2,98 & 0,03 & 763 \\
\hline \multicolumn{7}{|c|}{ Constant maturity of 240 days } \\
\hline B0 & $-0,47$ & 0,73 & 46,50 & $-944,23$ & 34,33 & 763 \\
\hline B1 & 1,87 & $-0,67$ & $1.985,48$ & $-90,22$ & 72,14 & 763 \\
\hline B2 & $-1,14$ & 0,22 & 44,04 & $-1.043,45$ & 37,91 & 763 \\
\hline SLP70 & 0,28 & $-0,37$ & 524,65 & $-28,56$ & 19,08 & 763 \\
\hline SLP90 & $-0,18$ & $-0,28$ & 107,27 & $-18,26$ & 3,98 & 763 \\
\hline SLP100 & $-0,41$ & $-0,24$ & 4,19 & $-101,42$ & 3,78 & 763 \\
\hline SLP110 & $-0,63$ & $-0,20$ & 6,91 & $-310,11$ & 11,30 & 763 \\
\hline SLP130 & $-1,09$ & $-0,11$ & 24,29 & $-727,49$ & 26,45 & 763 \\
\hline IMPV & 0,26 & 0,25 & 0,60 & $-4,29$ & 0,20 & 763 \\
\hline SKEW & $-0,28$ & $-0,27$ & 0,13 & $-0,38$ & 0,04 & 763 \\
\hline KURT & 2,99 & 3,06 & 3,12 & $-27,12$ & 1,27 & 763 \\
\hline \multicolumn{7}{|c|}{ Smile independent variables } \\
\hline PRC & $3.781,99$ & $3.820,15$ & $\begin{array}{r}5.449,80 \\
\end{array}$ & $\begin{array}{r}2.247,49 \\
\end{array}$ & $\begin{array}{r}712,08 \\
3926\end{array}$ & 763 \\
\hline CDS & 81,89 & 69,00 & 203,12 & 34,05 & 39,26 & 763 \\
\hline
\end{tabular}

Table B.6.: Summary statistics for cross section DAX 


\section{B.3.2. ALL}

\begin{tabular}{|c|c|c|c|c|c|c|}
\hline _ALL & Mean & Median & Max & Min & StdDev & Obs \\
\hline \multicolumn{7}{|c|}{ Constant maturity of 36 days } \\
\hline B0 & 2,58 & 2,37 & 6,91 & 0,90 & 0,84 & 729 \\
\hline B1 & $-4,07$ & $-3,68$ & $-1,13$ & $-12,96$ & 1,92 & 729 \\
\hline B2 & 1,88 & 1,70 & 6,29 & 0,38 & 0,97 & 729 \\
\hline SLP70 & $-1,44$ & $-1,32$ & $-0,46$ & $-4,16$ & 0,56 & 729 \\
\hline SLP90 & $-0,69$ & $-0,65$ & $-0,27$ & $-1,64$ & 0,19 & 729 \\
\hline SLP100 & $-0,31$ & $-0,30$ & $-0,02$ & $-0,55$ & 0,09 & 729 \\
\hline SLP110 & 0,06 & 0,06 & 1,05 & $-0,38$ & 0,23 & 729 \\
\hline SLP130 & 0,82 & 0,72 & 3,39 & $-0,16$ & 0,61 & 729 \\
\hline XSMIN & 1,11 & 1,08 & 1,51 & 1,00 & 0,08 & 729 \\
\hline IMPV & 0,39 & 0,33 & 0,81 & 0,17 & 0,16 & 729 \\
\hline SKEW & $-0,14$ & $-0,14$ & $-0,02$ & $-0,24$ & 0,04 & 729 \\
\hline KURT & 3,02 & 3,02 & 3,06 & 2,98 & 0,01 & 729 \\
\hline \multicolumn{7}{|c|}{ Constant maturity of 60 days } \\
\hline B0 & 1,55 & 1,47 & 5,94 & 0,06 & 0,43 & 729 \\
\hline B1 & $-2,05$ & $-1,88$ & 0,57 & $-10,72$ & 0,99 & 729 \\
\hline B2 & 0,88 & 0,80 & 5,32 & $-0,39$ & 0,50 & 729 \\
\hline SLP70 & $-0,81$ & $-0,76$ & 0,03 & $-3,70$ & 0,30 & 729 \\
\hline SLP90 & $-0,46$ & $-0,44$ & $-0,13$ & $-1,69$ & 0,11 & 729 \\
\hline SLP100 & $-0,28$ & $-0,27$ & 0,26 & $-0,69$ & 0,07 & 729 \\
\hline SLP110 & $-0,10$ & $-0,10$ & 1,32 & $-0,40$ & 0,13 & 729 \\
\hline SLP130 & 0,25 & 0,21 & 3,45 & $-0,52$ & 0,31 & 729 \\
\hline XSMIN & 1,23 & 1,16 & 8,29 & 0,43 & 0,35 & 729 \\
\hline IMPV & 0,39 & 0,33 & 0,77 & 0,19 & 0,15 & 729 \\
\hline SKEW & $-0,16$ & $-0,16$ & 0,04 & $-0,38$ & 0,03 & 729 \\
\hline KURT & 3,01 & 3,02 & 3,07 & 2,94 & 0,02 & 729 \\
\hline \multicolumn{7}{|c|}{ Constant maturity of 180 days } \\
\hline B0 & 3,19 & 0,88 & $1.555,58$ & $-357,93$ & 61,90 & 729 \\
\hline B1 & $-4,92$ & $-0,75$ & 717,68 & $-2.758,09$ & 111,64 & 729 \\
\hline B2 & 2,13 & 0,28 & $1.222,74$ & $-359,23$ & 50,51 & 729 \\
\hline SLP70 & $-1,94$ & $-0,37$ & 214,76 & $-1.046,26$ & 41,10 & 729 \\
\hline SLP90 & $-1,08$ & $-0,26$ & 71,06 & $-557,17$ & 21,18 & 729 \\
\hline SLP100 & $-0,66$ & $-0,20$ & 0,67 & $-312,62$ & 11,58 & 729 \\
\hline SLP110 & $-0,23$ & $-0,14$ & 86,06 & $-72,63$ & 4,88 & 729 \\
\hline SLP130 & 0,62 & $-0,03$ & 421,02 & $-216,32$ & 20,39 & 729 \\
\hline XSMIN & 1,32 & 1,34 & 43,85 & $-136,50$ & 5,81 & 729 \\
\hline IMPV & 0,41 & 0,33 & 20,22 & 0,21 & 0,75 & 729 \\
\hline SKEW & $-0,20$ & $-0,19$ & 0,54 & $-0,40$ & 0,04 & 729 \\
\hline KURT & 3,01 & 3,02 & 3,20 & 2,86 & 0,03 & 729 \\
\hline \multicolumn{7}{|c|}{ Constant maturity of 240 days } \\
\hline B0 & 13,10 & 0,77 & $6.527,59$ & $-465,67$ & 257,01 & 729 \\
\hline B1 & $-25,55$ & $-0,60$ & 933,30 & $-13.729,07$ & 532,98 & 729 \\
\hline B2 & 12,88 & 0,21 & $7.219,30$ & $-467,11$ & 277,30 & 729 \\
\hline SLP70 & $-7,52$ & $-0,31$ & 279,34 & $-3.622,05$ & 145,85 & 729 \\
\hline SLP90 & $-2,37$ & $-0,22$ & 92,50 & $-734,33$ & 39,41 & 729 \\
\hline SLP100 & 0,21 & $-0,18$ & 709,54 & $-406,33$ & 30,34 & 729 \\
\hline SLP110 & 2,78 & $-0,13$ & $2.153,40$ & $-94,35$ & 80,24 & 729 \\
\hline XSMIN & 7,93 & $-0,05$ & $5.041,12$ & $-281,19$ & 189,55 & 729 \\
\hline SLP130 & 1,19 & 1,36 & 25,72 & $-260,08$ & 9,91 & 729 \\
\hline IMPV & 0,43 & 0,33 & 26,21 & $-1,60$ & 1,17 & 729 \\
\hline SKEW & $-0,19$ & $-0,20$ & 1,52 & $-1,20$ & 0,11 & 729 \\
\hline KURT & 3,01 & 3,01 & 3,76 & 2,67 & 0,05 & 729 \\
\hline \multicolumn{7}{|c|}{ Smile independent variables } \\
\hline PRC & 111,61 & & & & 58,16 & 729 \\
\hline HV & 0,41 & 0,34 & 1,43 & 0,11 & 0,23 & 729 \\
\hline HSKEW & $-0,04$ & $-0,07$ & 11,31 & $-9,61$ & 2,01 & 729 \\
\hline HKURT & 10,66 & 3,87 & 154,16 & $-0,08$ & 17,79 & 729 \\
\hline CDS & 41,03 & 28,75 & 125,00 & 18,03 & 24,38 & 729 \\
\hline
\end{tabular}

Table B.7.: Summary statistics for cross section ALL 
B.3.3. SIE

\begin{tabular}{|c|c|c|c|c|c|c|}
\hline _SIE & Mean & Median & Max & Min & StdDev & Obs \\
\hline \multicolumn{7}{|c|}{ Constant maturity of 36 days } \\
\hline B0 & 2,49 & 2,30 & 7,41 & 1,20 & 0,77 & 757 \\
\hline B1 & $-3,93$ & $-3,54$ & $-1,29$ & $-13,89$ & 1,72 & 757 \\
\hline B2 & 1,80 & 1,60 & 6,72 & 0,46 & 0,87 & 757 \\
\hline SLP70 & $-1,41$ & $-1,29$ & $-0,64$ & $-4,49$ & 0,51 & 757 \\
\hline SLP90 & $-0,69$ & $-0,67$ & $-0,39$ & $-1,80$ & 0,17 & 757 \\
\hline SLP100 & $-0,33$ & $-0,32$ & $-0,14$ & $-0,62$ & 0,09 & 757 \\
\hline SLP110 & 0,03 & 0,02 & 0,89 & $-0,42$ & 0,22 & 757 \\
\hline SLP130 & 0,75 & 0,64 & 3,58 & $-0,17$ & 0,55 & 757 \\
\hline XSMIN & 1,12 & 1,09 & 1,43 & 1,02 & 0,07 & 757 \\
\hline IMPV & 0,36 & 0,31 & 0,73 & 0,14 & 0,13 & 757 \\
\hline SKEW & $-0,15$ & $-0,14$ & $-0,07$ & $-0,26$ & 0,04 & 757 \\
\hline KURT & 3,02 & 3,02 & 3,05 & 2,98 & 0,01 & 757 \\
\hline \multicolumn{7}{|c|}{ Constant maturity of 60 days } \\
\hline B0 & 1,55 & 1,47 & 5,99 & 0,43 & 0,41 & 757 \\
\hline B1 & $-2,08$ & $-1,81$ & 0,02 & $-11,31$ & 0,95 & 757 \\
\hline B2 & 0,89 & 0,75 & 5,54 & $-0,14$ & 0,48 & 757 \\
\hline SLP70 & $-0,83$ & $-0,77$ & $-0,18$ & $-3,55$ & 0,28 & 757 \\
\hline SLP90 & $-0,47$ & $-0,45$ & $-0,23$ & $-1,34$ & 0,10 & 757 \\
\hline SLP100 & $-0,29$ & $-0,28$ & $-0,15$ & $-0,51$ & 0,07 & 757 \\
\hline SLP110 & $-0,11$ & $-0,11$ & 0,88 & $-0,39$ & 0,13 & 757 \\
\hline SLP130 & 0,24 & 0,18 & 3,09 & $-0,37$ & 0,31 & 757 \\
\hline XSMIN & 1,22 & 1,18 & 14,60 & $-0,05$ & 0,51 & 757 \\
\hline IMPV & 0,36 & 0,32 & 0,70 & 0,16 & 0,12 & 757 \\
\hline SKEW & $-0,17$ & $-0,17$ & $-0,09$ & $-0,28$ & 0,03 & 757 \\
\hline KURT & 3,02 & 3,02 & 3,07 & 2,97 & 0,02 & 757 \\
\hline \multicolumn{7}{|c|}{ Constant maturity of 180 days } \\
\hline B0 & 1,09 & 0,85 & 148,84 & $-9,90$ & 5,43 & 757 \\
\hline B1 & $-1,24$ & $-0,74$ & 19,10 & $-284,98$ & 10,43 & 757 \\
\hline B2 & 0,51 & 0,26 & 136,63 & $-9,05$ & 5,00 & 757 \\
\hline SLP70 & $-0,53$ & $-0,38$ & 6,43 & $-93,70$ & 3,43 & 757 \\
\hline SLP90 & $-0,32$ & $-0,26$ & 2,81 & $-39,05$ & 1,42 & 757 \\
\hline SLP100 & $-0,22$ & $-0,21$ & 1,00 & $-11,72$ & 0,43 & 757 \\
\hline SLP110 & $-0,12$ & $-0,14$ & 15,61 & $-0,81$ & 0,58 & 757 \\
\hline SLP130 & 0,08 & $-0,04$ & 70,26 & $-4,43$ & 2,58 & 757 \\
\hline XSMIN & 1,43 & 1,37 & 7,09 & $-6,92$ & 0,58 & 757 \\
\hline IMPV & 0,36 & 0,34 & 0,60 & 0,15 & 0,10 & 757 \\
\hline SKEW & $-0,20$ & $-0,20$ & 0,87 & $-0,66$ & 0,06 & 757 \\
\hline KURT & 3,01 & 3,02 & 3,57 & 2,59 & 0,04 & 757 \\
\hline \multicolumn{7}{|c|}{ Constant maturity of 240 days } \\
\hline B0 & 20,48 & 0,75 & $14.346,85$ & $-1.626,52$ & 528,09 & 757 \\
\hline B1 & $-40,92$ & $-0,61$ & $2.981,06$ & $-29.060,94$ & $1.067,61$ & 757 \\
\hline B2 & 20,80 & 0,20 & $14.716,75$ & $-1.365,55$ & 539,96 & 757 \\
\hline SLP70 & $-11,79$ & $-0,31$ & $1.069,30$ & $-8.457,49$ & 312,08 & 757 \\
\hline SLP90 & $-3,47$ & $-0,23$ & 523,08 & $-2.570,79$ & 97,37 & 757 \\
\hline SLP100 & 0,69 & $-0,19$ & 372,56 & $-325,18$ & 22,61 & 757 \\
\hline SLP110 & 4,85 & $-0,13$ & $3.315,91$ & $-128,82$ & 121,93 & 757 \\
\hline XSMIN & 13,17 & $-0,05$ & $9.202,61$ & $-569,36$ & 336,97 & 757 \\
\hline SLP130 & 1,54 & 1,41 & 72,43 & $-6,09$ & 2,67 & 757 \\
\hline IMPV & 0,36 & 0,33 & 27,25 & $-25,11$ & 1,52 & 757 \\
\hline SKEW & $-0,20$ & $-0,21$ & 1,38 & $-0,59$ & 0,09 & 757 \\
\hline KURT & 3,01 & 3,02 & 3,83 & 2,16 & 0,07 & 757 \\
\hline \multicolumn{7}{|c|}{ Smile independent variables } \\
\hline PRC & 55,11 & & & 31,27 & 10,59 & 757 \\
\hline HV & 0,36 & 0,31 & 1,29 & 0,12 & 0,17 & 757 \\
\hline HSKEW & $-0,02$ & $-0,04$ & 7,78 & $-9,92$ & 1,93 & 757 \\
\hline HKURT & 9,83 & 3,33 & 122,57 & $-0,63$ & 16,51 & 757 \\
\hline CDS & 39,66 & 36,33 & 96,10 & 14,00 & 18,17 & 757 \\
\hline
\end{tabular}

Table B.8.: Summary statistics for cross section_SIE 


\section{B.3.4. DTE}

\begin{tabular}{|c|c|c|c|c|c|c|}
\hline _DTE & Mean & Median & Max & Min & StdDev & Obs \\
\hline \multicolumn{7}{|c|}{ Constant maturity of 36 days } \\
\hline B0 & 3,44 & 2,74 & 75,61 & $-1,72$ & 3,55 & 757 \\
\hline B1 & $-5,89$ & $-4,46$ & 4,10 & $-149,55$ & 7,19 & 757 \\
\hline B2 & 2,81 & 2,11 & 74,11 & $-2,18$ & 3,58 & 757 \\
\hline SLP70 & $-1,95$ & $-1,53$ & 1,04 & $-45,79$ & 2,18 & 757 \\
\hline SLP90 & $-0,83$ & $-0,72$ & 0,17 & $-16,15$ & 0,75 & 757 \\
\hline SLP100 & $-0,27$ & $-0,26$ & 0,03 & $-1,33$ & 0,11 & 757 \\
\hline SLP110 & 0,29 & 0,17 & 13,49 & $-0,70$ & 0,70 & 757 \\
\hline SLP130 & 1,42 & 1,02 & 43,14 & $-1,57$ & 2,13 & 757 \\
\hline XSMIN & 1,07 & 1,05 & 5,50 & $-9,28$ & 0,42 & 757 \\
\hline IMPV & 0,36 & 0,30 & 0,83 & 0,13 & 0,17 & 757 \\
\hline SKEW & $-0,12$ & $-0,12$ & 0,02 & $-0,25$ & 0,04 & 757 \\
\hline KURT & 3,02 & 3,02 & 3,07 & 2,74 & 0,02 & 757 \\
\hline \multicolumn{7}{|c|}{ Constant maturity of 60 days } \\
\hline B0 & 2,09 & 1,62 & 200,32 & $-146,39$ & 11,48 & 757 \\
\hline B1 & $-3,19$ & $-2,06$ & 292,20 & $-397,07$ & 22,73 & 757 \\
\hline B2 & 1,47 & 0,90 & 196,94 & $-145,66$ & 11,24 & 757 \\
\hline SLP70 & $-1,13$ & $-0,82$ & 88,28 & $-121,35$ & 7,00 & 757 \\
\hline SLP90 & $-0,54$ & $-0,46$ & 30,02 & $-42,58$ & 2,51 & 757 \\
\hline SLP100 & $-0,25$ & $-0,24$ & 3,86 & $-6,04$ & 0,37 & 757 \\
\hline SLP110 & 0,05 & $-0,05$ & 36,20 & $-28,24$ & 2,02 & 757 \\
\hline SLP130 & 0,64 & 0,30 & 114,98 & $-86,51$ & 6,50 & 757 \\
\hline XSMIN & 1,17 & 1,12 & 11,29 & $-3,51$ & 0,50 & 757 \\
\hline IMPV & 0,37 & 0,31 & 0,77 & 0,14 & 0,17 & 757 \\
\hline SKEW & $-0,14$ & $-0,15$ & 0,22 & $-0,95$ & 0,06 & 757 \\
\hline KURT & 3,01 & 3,02 & 3,12 & 2,21 & 0,06 & 757 \\
\hline \multicolumn{7}{|c|}{ Constant maturity of 180 days } \\
\hline B0 & 7,28 & 0,89 & $4.493,74$ & $-11,79$ & 163,65 & 757 \\
\hline B1 & $-13,14$ & $-0,80$ & 33,58 & $-8.637,88$ & 314,60 & 757 \\
\hline B2 & 6,23 & 0,30 & $4.151,12$ & $-25,30$ & 151,19 & 757 \\
\hline SLP70 & $-4,41$ & $-0,38$ & 7,76 & $-2.826,31$ & 102,93 & 757 \\
\hline SLP90 & $-1,92$ & $-0,26$ & 11,40 & $-1.165,87$ & 42,46 & 757 \\
\hline SLP100 & $-0,67$ & $-0,18$ & 14,88 & $-335,64$ & 12,25 & 757 \\
\hline SLP110 & 0,58 & $-0,11$ & 494,58 & $-22,07$ & 18,05 & 757 \\
\hline SLP130 & 3,07 & 0,00 & $2.155,03$ & $-32,19$ & 78,51 & 757 \\
\hline XSMIN & 1,17 & 1,25 & 26,64 & $-47,54$ & 3,37 & 757 \\
\hline IMPV & 0,38 & 0,34 & 6,98 & $-2,17$ & 0,30 & 757 \\
\hline SKEW & $-0,17$ & $-0,18$ & 0,56 & $-2,01$ & 0,10 & 757 \\
\hline KURT & 3,01 & 3,01 & 3,45 & 2,58 & 0,05 & 757 \\
\hline \multicolumn{7}{|c|}{ Constant maturity of 240 days } \\
\hline B0 & 6,12 & 0,79 & $2.445,81$ & $-264,40$ & 99,10 & 757 \\
\hline B1 & $-11,01$ & $-0,65$ & 509,98 & $-4.700,96$ & 190,86 & 757 \\
\hline B2 & 5,25 & 0,23 & $2.259,04$ & $-245,49$ & 91,92 & 757 \\
\hline SLP70 & $-3,65$ & $-0,32$ & 166,29 & $-1.538,30$ & 62,22 & 757 \\
\hline SLP90 & $-1,55$ & $-0,22$ & 68,10 & $-634,68$ & 25,55 & 757 \\
\hline SLP100 & $-0,50$ & $-0,17$ & 38,98 & $-182,88$ & 7,58 & 757 \\
\hline SLP110 & 0,55 & $-0,11$ & 268,93 & $-50,00$ & 11,75 & 757 \\
\hline XSMIN & 2,65 & $-0,03$ & $1.172,55$ & $-128,29$ & 48,24 & 757 \\
\hline SLP130 & 1,45 & 1,26 & 95,69 & $-40,22$ & 3,92 & 757 \\
\hline IMPV & 0,37 & 0,34 & 3,89 & $-5,70$ & 0,32 & 757 \\
\hline SKEW & $-0,17$ & $-0,18$ & 1,28 & $-2,61$ & 0,15 & 757 \\
\hline KURT & 3,00 & 3,01 & 4,59 & 2,02 & 0,10 & 757 \\
\hline \multicolumn{7}{|c|}{ Smile independent variables } \\
\hline PRC & 13,53 & 13,49 & & 8,30 & 2,11 & 757 \\
\hline HV & 0,37 & 0,29 & 1,32 & 0,11 & 0,21 & 757 \\
\hline HSKEW & $-0,01$ & 0,02 & 9,13 & $-11,13$ & 1,52 & 757 \\
\hline HKURT & 6,83 & 2,58 & 151,58 & $-0,54$ & 13,84 & 757 \\
\hline CDS & 134,95 & 93,93 & 408,50 & 33,63 & 94,69 & 757 \\
\hline
\end{tabular}

Table B.9.: Summary statistics for cross section DTE 
B.3.5. DAIM

\begin{tabular}{|c|c|c|c|c|c|c|}
\hline _DAIM & Mean & Median & Max & Min & StdDev & Obs \\
\hline \multicolumn{7}{|c|}{ Constant maturity of 36 days } \\
\hline B0 & 2,72 & 2,48 & 11,99 & $-0,46$ & 1,10 & 755 \\
\hline B1 & $-4,43$ & $-3,92$ & 1,53 & $-22,60$ & 2,32 & 755 \\
\hline B2 & 2,05 & 1,78 & 10,94 & $-0,92$ & 1,17 & 755 \\
\hline SLP70 & $-1,56$ & $-1,43$ & 0,35 & $-7,29$ & 0,69 & 755 \\
\hline SLP90 & $-0,74$ & $-0,71$ & 0,02 & $-2,91$ & 0,24 & 755 \\
\hline SLP100 & $-0,33$ & $-0,31$ & 0,02 & $-0,73$ & 0,12 & 755 \\
\hline SLP110 & 0,08 & 0,05 & 1,91 & $-0,54$ & 0,28 & 755 \\
\hline SLP130 & 0,90 & 0,76 & 5,84 & $-0,85$ & 0,73 & 755 \\
\hline XSMIN & 1,11 & 1,08 & 1,84 & 0,29 & 0,10 & 755 \\
\hline IMPV & 0,34 & 0,31 & 0,71 & 0,14 & 0,12 & 755 \\
\hline SKEW & $-0,15$ & $-0,14$ & 0,00 & $-0,32$ & 0,05 & 755 \\
\hline KURT & 3,02 & 3,02 & 3,07 & 2,98 & 0,01 & 755 \\
\hline \multicolumn{7}{|c|}{ Constant maturity of 60 days } \\
\hline B0 & 1,12 & 1,57 & 16,43 & $-427,77$ & 15,67 & 755 \\
\hline B1 & $-1,26$ & $-2,08$ & 847,12 & $-31,61$ & 31,00 & 755 \\
\hline B2 & 0,48 & 0,89 & 15,44 & $-419,12$ & 15,33 & 755 \\
\hline SLP70 & $-0,58$ & $-0,85$ & 260,35 & $-9,99$ & 9,53 & 755 \\
\hline SLP90 & $-0,39$ & $-0,49$ & 92,70 & $-3,82$ & 3,40 & 755 \\
\hline SLP100 & $-0,29$ & $-0,29$ & 8,87 & $-1,16$ & 0,35 & 755 \\
\hline SLP110 & $-0,20$ & $-0,10$ & 2,36 & $-74,95$ & 2,73 & 755 \\
\hline SLP130 & $-0,01$ & 0,25 & 8,54 & $-242,60$ & 8,86 & 755 \\
\hline XSMIN & 1,19 & 1,15 & 4,04 & $-12,13$ & 0,55 & 755 \\
\hline IMPV & 0,34 & 0,31 & 0,68 & 0,17 & 0,11 & 755 \\
\hline SKEW & $-0,18$ & $-0,17$ & $-0,01$ & $-0,39$ & 0,05 & 755 \\
\hline KURT & 3,02 & 3,02 & 3,08 & 2,88 & 0,02 & 755 \\
\hline \multicolumn{7}{|c|}{ Constant maturity of 180 days } \\
\hline B0 & 0,89 & 0,87 & 16,03 & $-23,98$ & 1,35 & 755 \\
\hline B1 & $-0,91$ & $-0,84$ & 46,70 & $-29,10$ & 2,57 & 755 \\
\hline B2 & 0,35 & 0,30 & 13,41 & $-22,53$ & 1,23 & 755 \\
\hline SLP70 & $-0,42$ & $-0,40$ & 15,15 & $-10,32$ & 0,85 & 755 \\
\hline SLP90 & $-0,28$ & $-0,27$ & 6,14 & $-4,96$ & 0,37 & 755 \\
\hline SLP100 & $-0,21$ & $-0,21$ & 1,63 & $-2,28$ & 0,15 & 755 \\
\hline SLP110 & $-0,14$ & $-0,14$ & 1,98 & $-2,87$ & 0,17 & 755 \\
\hline SLP130 & $-0,01$ & $-0,02$ & 6,04 & $-11,89$ & 0,64 & 755 \\
\hline XSMIN & 1,38 & 1,32 & 16,51 & $-25,21$ & 1,51 & 755 \\
\hline IMPV & 0,33 & 0,31 & 0,58 & 0,16 & 0,08 & 755 \\
\hline SKEW & $-0,20$ & $-0,20$ & 1,12 & $-0,62$ & 0,08 & 755 \\
\hline KURT & 3,02 & 3,02 & 3,08 & 1,47 & 0,07 & 755 \\
\hline \multicolumn{7}{|c|}{ Constant maturity of 240 days } \\
\hline B0 & 0,13 & 0,76 & 63,99 & $-243,20$ & 12,52 & 755 \\
\hline B1 & 0,51 & $-0,68$ & 423,33 & $-118,09$ & 23,07 & 755 \\
\hline B2 & $-0,32$ & 0,25 & 58,67 & $-183,90$ & 10,74 & 755 \\
\hline SLP70 & 0,07 & $-0,33$ & 165,86 & $-41,63$ & 8,15 & 755 \\
\hline SLP90 & $-0,06$ & $-0,24$ & 92,30 & $-19,85$ & 4,04 & 755 \\
\hline SLP100 & $-0,12$ & $-0,18$ & 55,52 & $-9,81$ & 2,27 & 755 \\
\hline SLP110 & $-0,19$ & $-0,13$ & 18,74 & $-39,08$ & 1,77 & 755 \\
\hline XSMIN & $-0,32$ & $-0,04$ & 34,45 & $-97,61$ & 5,18 & 755 \\
\hline SLP130 & 1,19 & 1,33 & 18,73 & $-104,30$ & 4,09 & 755 \\
\hline IMPV & 0,32 & 0,31 & 1,52 & $-3,77$ & 0,18 & 755 \\
\hline SKEW & $-0,20$ & $-0,20$ & 1,83 & $-0,62$ & 0,13 & 755 \\
\hline KURT & 3,01 & 3,03 & 3,49 & 0,62 & 0,11 & 755 \\
\hline \multicolumn{7}{|c|}{ Smile independent variables } \\
\hline PRC & 36,57 & & & 24,08 & 7,14 & 755 \\
\hline HV & 0,37 & 0,32 & 1,08 & 0,11 & 0,16 & 755 \\
\hline HSKEW & $-0,05$ & $-0,04$ & 12,16 & $-10,41$ & 1,50 & 755 \\
\hline HKURT & 6,78 & 2,76 & 160,27 & $-0,41$ & 13,68 & 755 \\
\hline CDS & 114,41 & 114,39 & 225,00 & 64,00 & 30,57 & 755 \\
\hline
\end{tabular}

Table B.10.: Summary statistics for cross section DAIM 


\section{B.3.6. DBANK}

\begin{tabular}{|c|c|c|c|c|c|c|}
\hline _DBANK & Mean & Median & Max & Min & StdDev & Obs \\
\hline \multicolumn{7}{|c|}{ Constant maturity of 36 days } \\
\hline B0 & 2,67 & 2,48 & 7,32 & 0,97 & 0,79 & 760 \\
\hline B1 & $-4,31$ & $-3,84$ & $-0,72$ & $-13,94$ & 1,76 & 760 \\
\hline B2 & 1,98 & 1,74 & 6,84 & 0,16 & 0,91 & 760 \\
\hline SLP70 & $-1,54$ & $-1,44$ & $-0,48$ & $-4,36$ & 0,50 & 760 \\
\hline SLP90 & $-0,75$ & $-0,73$ & $-0,33$ & $-1,63$ & 0,17 & 760 \\
\hline SLP100 & $-0,36$ & $-0,34$ & 0,10 & $-0,97$ & 0,13 & 760 \\
\hline SLP110 & 0,04 & 0,01 & 1,11 & $-0,49$ & 0,27 & 760 \\
\hline SLP130 & 0,83 & 0,70 & 3,85 & $-0,30$ & 0,62 & 760 \\
\hline XSMIN & 1,12 & 1,10 & 2,22 & 0,98 & 0,09 & 760 \\
\hline IMPV & 0,34 & 0,30 & 0,84 & 0,16 & 0,13 & 760 \\
\hline SKEW & $-0,16$ & $-0,15$ & 0,03 & $-0,40$ & 0,05 & 760 \\
\hline KURT & 3,02 & 3,02 & 3,05 & 2,97 & 0,01 & 760 \\
\hline \multicolumn{7}{|c|}{ Constant maturity of 60 days } \\
\hline B0 & 1,56 & 1,59 & 11,05 & $-41,08$ & 1,75 & 760 \\
\hline B1 & $-2,12$ & $-2,04$ & 79,71 & $-21,02$ & 3,40 & 760 \\
\hline B2 & 0,90 & 0,86 & 10,17 & $-38,37$ & 1,65 & 760 \\
\hline SLP70 & $-0,86$ & $-0,87$ & 26,00 & $-6,78$ & 1,10 & 760 \\
\hline SLP90 & $-0,50$ & $-0,51$ & 10,65 & $-2,71$ & 0,45 & 760 \\
\hline SLP100 & $-0,32$ & $-0,31$ & 2,97 & $-0,86$ & 0,16 & 760 \\
\hline SLP110 & $-0,14$ & $-0,13$ & 1,36 & $-4,70$ & 0,25 & 760 \\
\hline SLP130 & 0,22 & 0,19 & 5,42 & $-20,05$ & 0,89 & 760 \\
\hline XSMIN & 1,22 & 1,18 & 18,32 & $-10,36$ & 0,80 & 760 \\
\hline IMPV & 0,34 & 0,30 & 0,81 & 0,18 & 0,12 & 760 \\
\hline SKEW & $-0,19$ & $-0,18$ & 0,21 & $-0,41$ & 0,06 & 760 \\
\hline KURT & 3,02 & 3,02 & 3,07 & 2,14 & 0,04 & 760 \\
\hline \multicolumn{7}{|c|}{ Constant maturity of 180 days } \\
\hline B0 & 0,16 & 0,90 & 59,33 & $-471,97$ & 18,08 & 760 \\
\hline B1 & 0,68 & $-0,86$ & 970,12 & $-100,90$ & 37,26 & 760 \\
\hline B2 & $-0,50$ & 0,31 & 43,06 & $-498,18$ & 19,23 & 760 \\
\hline SLP70 & $-0,02$ & $-0,43$ & 272,66 & $-40,62$ & 10,37 & 760 \\
\hline SLP90 & $-0,22$ & $-0,29$ & 73,39 & $-23,40$ & 2,82 & 760 \\
\hline SLP100 & $-0,32$ & $-0,22$ & 2,74 & $-31,98$ & 1,59 & 760 \\
\hline SLP110 & $-0,42$ & $-0,16$ & 2,40 & $-125,88$ & 5,16 & 760 \\
\hline SLP130 & $-0,62$ & $-0,04$ & 11,04 & $-325,15$ & 12,79 & 760 \\
\hline XSMIN & 1,18 & 1,36 & 179,55 & $-424,65$ & 16,82 & 760 \\
\hline IMPV & 0,34 & 0,31 & 1,49 & $-0,83$ & 0,11 & 760 \\
\hline SKEW & $-0,21$ & $-0,22$ & 3,71 & $-0,77$ & 0,16 & 760 \\
\hline KURT & 3,03 & 3,03 & 12,94 & 2,12 & 0,36 & 760 \\
\hline \multicolumn{7}{|c|}{ Constant maturity of 240 days } \\
\hline B0 & $-3,38$ & 0,81 & 64,95 & $-1.825,17$ & 74,69 & 760 \\
\hline B1 & 10,37 & $-0,67$ & $5.586,25$ & $-110,51$ & 214,86 & 760 \\
\hline B2 & $-7,32$ & 0,23 & 47,16 & $-4.264,59$ & 159,05 & 760 \\
\hline SLP70 & 0,12 & $-0,36$ & 506,81 & $-384,18$ & 24,17 & 760 \\
\hline SLP90 & $-2,81$ & $-0,26$ & 136,58 & $-2.090,02$ & 76,00 & 760 \\
\hline SLP100 & $-4,28$ & $-0,20$ & 14,65 & $-2.942,93$ & 106,80 & 760 \\
\hline SLP110 & $-5,74$ & $-0,15$ & 22,08 & $-3.795,85$ & 138,07 & 760 \\
\hline XSMIN & $-8,67$ & $-0,06$ & 37,98 & $-5.501,69$ & 201,11 & 760 \\
\hline SLP130 & 1,30 & 1,40 & 89,07 & $-233,42$ & 9,19 & 760 \\
\hline IMPV & $-0,33$ & 0,31 & 1,66 & $-503,51$ & 18,28 & 760 \\
\hline SKEW & $-0,21$ & $-0,22$ & 4,09 & $-3,01$ & 0,21 & 760 \\
\hline KURT & 3,03 & 3,03 & 13,90 & 1,72 & 0,40 & 760 \\
\hline \multicolumn{7}{|c|}{ Smile independent variables } \\
\hline PRC & 59,26 & & & & 10,39 & 760 \\
\hline HV & 0,34 & 0,29 & 1,11 & 0,09 & 0,16 & 760 \\
\hline HSKEW & $-0,08$ & 0,00 & 6,71 & $-8,38$ & 1,62 & 760 \\
\hline HKURT & 8,06 & 3,21 & 110,63 & $-0,37$ & 13,13 & 760 \\
\hline CDS & 25,52 & 20,58 & 75,50 & 13,08 & 11,26 & 760 \\
\hline
\end{tabular}

Table B.11.: Summary statistics for cross section DBANK 


\section{B.3.7. MRE}

\begin{tabular}{|c|c|c|c|c|c|c|}
\hline _MRE & Mean & Median & Max & Min & StdDev & Obs \\
\hline \multicolumn{7}{|c|}{ Constant maturity of 36 days } \\
\hline B0 & 2,67 & 2,45 & 16,36 & $-4,13$ & 1,32 & 668 \\
\hline B1 & $-4,27$ & $-3,80$ & 8,60 & $-30,93$ & 2,81 & 668 \\
\hline B2 & 1,99 & 1,76 & 14,88 & $-4,29$ & 1,40 & 668 \\
\hline SLP70 & $-1,49$ & $-1,36$ & 2,59 & $-10,10$ & 0,85 & 668 \\
\hline SLP90 & $-0,70$ & $-0,67$ & 0,87 & $-4,15$ & 0,30 & 668 \\
\hline SLP100 & $-0,30$ & $-0,29$ & 0,01 & $-1,17$ & 0,12 & 668 \\
\hline SLP110 & 0,10 & 0,07 & 1,81 & $-1,07$ & 0,31 & 668 \\
\hline SLP130 & 0,89 & 0,73 & 7,76 & $-2,58$ & 0,85 & 668 \\
\hline XSMIN & 1,13 & 1,08 & 5,91 & $-2,12$ & 0,33 & 668 \\
\hline IMPV & 0,39 & 0,37 & 0,85 & 0,15 & 0,16 & 668 \\
\hline SKEW & $-0,14$ & $-0,13$ & 0,08 & $-0,44$ & 0,05 & 668 \\
\hline KURT & 3,02 & 3,02 & 3,07 & 2,89 & 0,02 & 668 \\
\hline \multicolumn{7}{|c|}{ Constant maturity of 60 days } \\
\hline B0 & 1,49 & 1,58 & 7,86 & $-58,60$ & 2,81 & 668 \\
\hline B1 & $-1,94$ & $-2,08$ & 114,43 & $-14,46$ & 5,34 & 668 \\
\hline B2 & 0,84 & 0,92 & 6,90 & $-55,28$ & 2,53 & 668 \\
\hline SLP70 & $-0,76$ & $-0,81$ & 37,03 & $-4,80$ & 1,81 & 668 \\
\hline SLP90 & $-0,43$ & $-0,46$ & 14,92 & $-2,03$ & 0,83 & 668 \\
\hline SLP100 & $-0,26$ & $-0,27$ & 8,60 & $-1,09$ & 0,39 & 668 \\
\hline SLP110 & $-0,09$ & $-0,08$ & 4,28 & $-7,20$ & 0,37 & 668 \\
\hline SLP130 & 0,25 & 0,28 & 3,58 & $-29,31$ & 1,28 & 668 \\
\hline XSMIN & 1,31 & 1,13 & 74,80 & $-1,85$ & 2,91 & 668 \\
\hline IMPV & 0,39 & 0,36 & 0,80 & $-0,31$ & 0,15 & 668 \\
\hline SKEW & $-0,16$ & $-0,16$ & 0,59 & $-0,41$ & 0,06 & 668 \\
\hline KURT & 3,02 & 3,02 & 4,51 & 2,76 & 0,06 & 668 \\
\hline \multicolumn{7}{|c|}{ Constant maturity of 180 days } \\
\hline B0 & 0,77 & 0,88 & 310,35 & $-566,89$ & 26,05 & 668 \\
\hline B1 & $-1,11$ & $-0,82$ & 793,17 & $-715,15$ & 43,10 & 668 \\
\hline B2 & 0,67 & 0,31 & 412,70 & $-277,26$ & 19,95 & 668 \\
\hline SLP70 & $-0,17$ & $-0,39$ & 405,01 & $-137,37$ & 17,28 & 668 \\
\hline SLP90 & 0,09 & $-0,26$ & 294,10 & $-44,55$ & 11,81 & 668 \\
\hline SLP100 & 0,23 & $-0,20$ & 238,65 & $-36,62$ & 10,38 & 668 \\
\hline SLP110 & 0,36 & $-0,14$ & 192,79 & $-28,68$ & 10,39 & 668 \\
\hline SLP130 & 0,63 & $-0,02$ & 357,87 & $-23,65$ & 14,27 & 668 \\
\hline XSMIN & 1,18 & 1,27 & 8,80 & $-76,04$ & 3,23 & 668 \\
\hline IMPV & 0,33 & 0,35 & 8,82 & $-50,98$ & 2,04 & 668 \\
\hline SKEW & $-0,19$ & $-0,19$ & 1,65 & $-3,49$ & 0,17 & 668 \\
\hline KURT & 3,00 & 3,01 & 6,52 & 1,45 & 0,17 & 668 \\
\hline \multicolumn{7}{|c|}{ Constant maturity of 240 days } \\
\hline B0 & $-0,09$ & 0,78 & 647,83 & $-1.431,43$ & 70,33 & 668 \\
\hline B1 & 0,55 & $-0,67$ & $2.683,07$ & $-1.480,53$ & 129,27 & 668 \\
\hline B2 & $-0,06$ & 0,24 & 854,58 & $-1.256,92$ & 61,92 & 668 \\
\hline SLP70 & 0,46 & $-0,32$ & 923,38 & $-461,67$ & 44,98 & 668 \\
\hline SLP90 & 0,43 & $-0,23$ & 420,62 & $-340,35$ & 24,23 & 668 \\
\hline SLP100 & 0,42 & $-0,18$ & 230,98 & $-279,69$ & 18,15 & 668 \\
\hline SLP110 & 0,41 & $-0,13$ & 399,54 & $-219,02$ & 19,45 & 668 \\
\hline XSMIN & 0,38 & $-0,04$ & 741,38 & $-584,92$ & 37,37 & 668 \\
\hline SLP130 & 1,27 & 1,31 & 6,91 & $-15,55$ & 1,17 & 668 \\
\hline IMPV & 0,40 & 0,35 & 64,84 & $-49,33$ & 3,27 & 668 \\
\hline SKEW & $-0,20$ & $-0,20$ & 1,65 & $-3,42$ & 0,24 & 668 \\
\hline KURT & 3,00 & 3,01 & 6,52 & 1,12 & 0,21 & 668 \\
\hline \multicolumn{7}{|c|}{ Smile independent variables } \\
\hline PRC & 111,60 & & & & 48,51 & 668 \\
\hline HV & 0,42 & 0,36 & 1,40 & 0,15 & 0,22 & 668 \\
\hline HSKEW & $-0,04$ & $-0,06$ & 8,78 & $-9,79$ & 1,95 & 668 \\
\hline HKURT & 10,29 & 4,03 & 119,45 & $-0,29$ & 16,85 & 668 \\
\hline CDS & 36,95 & 28,89 & 137,67 & 18,33 & 16,97 & 668 \\
\hline
\end{tabular}

Table B.12.: Summary statistics for cross section MRE 


\section{B.3.8. BAY}

\begin{tabular}{|c|c|c|c|c|c|c|}
\hline _BAY & Mean & Median & Max & Min & StdDev & Obs \\
\hline \multicolumn{7}{|c|}{ Constant maturity of 36 days } \\
\hline B0 & 2,99 & 2,77 & 68,54 & $-49,96$ & 3,86 & 740 \\
\hline B1 & $-5,00$ & $-4,54$ & 101,38 & $-144,07$ & 7,96 & 740 \\
\hline B2 & 2,36 & 2,14 & 75,87 & $-51,09$ & 4,08 & 740 \\
\hline SLP70 & $-1,70$ & $-1,58$ & 29,86 & $-37,84$ & 2,26 & 740 \\
\hline SLP90 & $-0,76$ & $-0,74$ & 9,42 & $-7,49$ & 0,67 & 740 \\
\hline SLP100 & $-0,28$ & $-0,29$ & 7,68 & $-1,14$ & 0,35 & 740 \\
\hline SLP110 & 0,19 & 0,13 & 22,86 & $-11,01$ & 1,07 & 740 \\
\hline SLP130 & 1,13 & 0,98 & 53,21 & $-31,45$ & 2,68 & 740 \\
\hline XSMIN & 1,06 & 1,07 & 4,52 & $-7,18$ & 0,47 & 740 \\
\hline IMPV & 0,34 & 0,31 & 0,82 & 0,15 & 0,13 & 740 \\
\hline SKEW & $-0,14$ & $-0,14$ & 1,13 & $-0,40$ & 0,08 & 740 \\
\hline KURT & 3,02 & 3,02 & 3,17 & 2,79 & 0,02 & 740 \\
\hline \multicolumn{7}{|c|}{ Constant maturity of 60 days } \\
\hline B0 & 1,76 & 1,70 & 35,28 & $-27,51$ & 2,70 & 740 \\
\hline B1 & $-2,59$ & $-2,41$ & 51,54 & $-73,73$ & 5,40 & 740 \\
\hline B2 & 1,17 & 1,06 & 38,72 & $-23,79$ & 2,69 & 740 \\
\hline SLP70 & $-0,95$ & $-0,92$ & 18,24 & $-19,52$ & 1,64 & 740 \\
\hline SLP90 & $-0,49$ & $-0,50$ & 8,72 & $-4,03$ & 0,61 & 740 \\
\hline SLP100 & $-0,25$ & $-0,27$ & 3,96 & $-2,03$ & 0,29 & 740 \\
\hline SLP110 & $-0,02$ & $-0,07$ & 11,45 & $-2,74$ & 0,61 & 740 \\
\hline SLP130 & 0,45 & 0,35 & 26,94 & $-11,40$ & 1,64 & 740 \\
\hline XSMIN & 1,30 & 1,12 & 38,51 & $-16,92$ & 2,52 & 740 \\
\hline IMPV & 0,34 & 0,31 & 0,78 & 0,16 & 0,12 & 740 \\
\hline SKEW & $-0,16$ & $-0,16$ & 2,05 & $-0,62$ & 0,11 & 740 \\
\hline KURT & 3,02 & 3,02 & 3,15 & 1,80 & 0,06 & 740 \\
\hline \multicolumn{7}{|c|}{ Constant maturity of 180 days } \\
\hline B0 & 0,78 & 0,90 & 38,41 & $-113,93$ & 6,70 & 740 \\
\hline B1 & $-0,73$ & $-0,94$ & 231,20 & $-72,85$ & 12,96 & 740 \\
\hline B2 & 0,28 & 0,37 & 39,57 & $-116,93$ & 6,32 & 740 \\
\hline SLP70 & $-0,33$ & $-0,43$ & 67,50 & $-24,26$ & 4,17 & 740 \\
\hline SLP90 & $-0,22$ & $-0,28$ & 30,63 & $-10,38$ & 1,79 & 740 \\
\hline SLP100 & $-0,16$ & $-0,20$ & 20,62 & $-4,59$ & 0,95 & 740 \\
\hline SLP110 & $-0,11$ & $-0,13$ & 15,94 & $-26,04$ & 1,34 & 740 \\
\hline SLP130 & 0,01 & 0,02 & 31,77 & $-72,82$ & 3,63 & 740 \\
\hline XSMIN & 1,48 & 1,23 & 121,53 & $-13,55$ & 5,22 & 740 \\
\hline IMPV & 0,33 & 0,31 & 0,74 & $-1,82$ & 0,13 & 740 \\
\hline SKEW & $-0,20$ & $-0,20$ & 0,49 & $-2,05$ & 0,12 & 740 \\
\hline KURT & 3,03 & 3,02 & 14,71 & 2,04 & 0,44 & 740 \\
\hline \multicolumn{7}{|c|}{ Constant maturity of 240 days } \\
\hline Bo & $-0,30$ & 0,82 & 222,20 & $-578,53$ & 28,47 & 740 \\
\hline B1 & 1,19 & $-0,77$ & 945,87 & $-360,35$ & 50,79 & 740 \\
\hline B2 & $-0,57$ & 0,30 & 146,34 & $-422,39$ & 23,31 & 740 \\
\hline SLP70 & 0,40 & $-0,37$ & 404,90 & $-155,48$ & 18,85 & 740 \\
\hline SLP90 & 0,17 & $-0,25$ & 250,34 & $-96,95$ & 10,54 & 740 \\
\hline SLP100 & 0,06 & $-0,19$ & 173,06 & $-67,68$ & 7,35 & 740 \\
\hline SLP110 & $-0,06$ & $-0,13$ & 95,78 & $-93,38$ & 6,37 & 740 \\
\hline XSMIN & $-54,13$ & 1,22 & 59,44 & $-41.090,33$ & $1.510,57$ & 740 \\
\hline SLP130 & $-0,28$ & $-0,01$ & 114,50 & $-262,33$ & 12,00 & 740 \\
\hline IMPV & 0,32 & 0,31 & 8,18 & $-19,06$ & 0,84 & 740 \\
\hline SKEW & $-0,20$ & $-0,20$ & 4,97 & $-1,90$ & 0,25 & 740 \\
\hline KURT & 3,02 & 3,02 & 13,62 & 1,77 & 0,40 & 740 \\
\hline \multicolumn{7}{|c|}{ Smile independent variables } \\
\hline PRC & 23,23 & 21,96 & & 10,09 & 6,28 & 740 \\
\hline HV & 0,41 & 0,34 & 2,35 & 0,13 & 0,22 & 740 \\
\hline HSKEW & 0,02 & $-0,04$ & 11,64 & $-9,09$ & 1,54 & 740 \\
\hline HKURT & 6,99 & 2,81 & 159,71 & $-0,67$ & 13,99 & 740 \\
\hline CDS & 58,12 & 45,88 & 260,50 & 23,05 & 33,46 & 740 \\
\hline
\end{tabular}

Table B.13.: Summary statistics for cross section BAY 
B.3.9. _VW

\begin{tabular}{|c|c|c|c|c|c|c|}
\hline _VW & Mean & Median & Max & Min & StdDev & Obs \\
\hline \multicolumn{7}{|c|}{ Constant maturity of 36 days } \\
\hline B0 & 2,69 & 2,46 & 45,97 & $-27,28$ & 3,26 & 755 \\
\hline B1 & $-4,34$ & $-3,87$ & 52,26 & $-86,21$ & 6,39 & 755 \\
\hline B2 & 1,99 & 1,74 & 40,71 & $-24,84$ & 3,13 & 755 \\
\hline SLP70 & $-1,56$ & $-1,43$ & 17,49 & $-29,21$ & 2,02 & 755 \\
\hline SLP90 & $-0,76$ & $-0,71$ & 7,55 & $-12,92$ & 0,79 & 755 \\
\hline SLP100 & $-0,36$ & $-0,34$ & 2,58 & $-4,78$ & 0,26 & 755 \\
\hline SLP110 & 0,04 & 0,00 & 5,42 & $-5,18$ & 0,54 & 755 \\
\hline SLP130 & 0,83 & 0,68 & 21,63 & $-13,45$ & 1,76 & 755 \\
\hline XSMIN & 1,52 & 1,09 & 241,38 & $-0,28$ & 8,97 & 755 \\
\hline IMPV & 0,34 & 0,31 & 0,65 & 0,14 & 0,10 & 755 \\
\hline SKEW & $-0,16$ & $-0,15$ & 2,04 & $-0,59$ & 0,10 & 755 \\
\hline KURT & 3,02 & 3,02 & 3,12 & 1,61 & 0,06 & 755 \\
\hline \multicolumn{7}{|c|}{ Constant maturity of 60 days } \\
\hline B0 & 1,33 & 1,53 & 41,92 & $-164,84$ & 6,75 & 755 \\
\hline B1 & $-1,69$ & $-2,06$ & 324,00 & $-78,69$ & 13,18 & 755 \\
\hline B2 & 0,69 & 0,89 & 37,22 & $-158,97$ & 6,44 & 755 \\
\hline SLP70 & $-0,71$ & $-0,83$ & 101,44 & $-26,58$ & 4,17 & 755 \\
\hline SLP90 & $-0,44$ & $-0,48$ & 37,85 & $-11,69$ & 1,60 & 755 \\
\hline SLP100 & $-0,30$ & $-0,29$ & 6,05 & $-4,25$ & 0,40 & 755 \\
\hline SLP110 & $-0,16$ & $-0,12$ & 4,63 & $-25,74$ & 1,03 & 755 \\
\hline SLP130 & 0,12 & 0,22 & 18,08 & $-89,33$ & 3,58 & 755 \\
\hline XSMIN & 1,18 & 1,15 & 5,63 & $-6,04$ & 0,48 & 755 \\
\hline IMPV & 0,34 & 0,31 & 0,63 & 0,00 & 0,09 & 755 \\
\hline SKEW & $-0,18$ & $-0,17$ & 0,91 & $-0,61$ & 0,08 & 755 \\
\hline KURT & 3,01 & 3,02 & 3,18 & 2,31 & 0,05 & 755 \\
\hline \multicolumn{7}{|c|}{ Constant maturity of 180 days } \\
\hline B0 & 2,52 & 0,86 & $1.438,71$ & $-800,12$ & 69,00 & 755 \\
\hline B1 & $-3,06$ & $-0,84$ & $1.391,67$ & $-2.409,10$ & 123,25 & 755 \\
\hline B2 & 0,84 & 0,31 & $1.008,66$ & $-936,23$ & 58,56 & 755 \\
\hline SLP70 & $-1,88$ & $-0,41$ & 554,63 & $-996,97$ & 45,85 & 755 \\
\hline SLP90 & $-1,55$ & $-0,28$ & 329,85 & $-593,51$ & 28,84 & 755 \\
\hline SLP100 & $-1,38$ & $-0,22$ & 217,46 & $-480,80$ & 24,65 & 755 \\
\hline SLP110 & $-1,21$ & $-0,15$ & 105,08 & $-668,04$ & 25,65 & 755 \\
\hline SLP130 & $-0,88$ & $-0,03$ & 213,43 & $-1.042,53$ & 39,77 & 755 \\
\hline XSMIN & 1,16 & 1,29 & 19,04 & $-132,03$ & 5,02 & 755 \\
\hline IMPV & 0,30 & 0,31 & 38,28 & $-61,18$ & 2,80 & 755 \\
\hline SKEW & $-0,20$ & $-0,20$ & 0,70 & $-0,99$ & 0,11 & 755 \\
\hline KURT & 3,01 & 3,02 & 4,54 & 1,83 & 0,11 & 755 \\
\hline \multicolumn{7}{|c|}{ Constant maturity of 240 days } \\
\hline B0 & 0,26 & 0,80 & $2.276,51$ & $-3.326,03$ & 158,92 & 755 \\
\hline B1 & 1,72 & $-0,72$ & $5.573,29$ & $-3.812,28$ & 281,20 & 755 \\
\hline B2 & $-1,82$ & 0,26 & $1.596,19$ & $-2.334,51$ & 131,98 & 755 \\
\hline SLP70 & $-0,83$ & $-0,37$ & $2.304,98$ & $-1.577,62$ & 106,28 & 755 \\
\hline SLP90 & $-1,56$ & $-0,26$ & $1.371,18$ & $-939,14$ & 66,88 & 755 \\
\hline SLP100 & $-1,92$ & $-0,20$ & 904,27 & $-1.046,11$ & 56,14 & 755 \\
\hline SLP110 & $-2,29$ & $-0,15$ & 437,37 & $-1.453,74$ & 56,78 & 755 \\
\hline XSMIN & 1,10 & 1,29 & 28,53 & $-132,03$ & 5,93 & 755 \\
\hline SLP130 & $-3,02$ & $-0,04$ & 337,82 & $-2.268,99$ & 86,88 & 755 \\
\hline IMPV & 0,15 & 0,31 & 60,42 & $-133,70$ & 6,31 & 755 \\
\hline SKEW & $-0,20$ & $-0,21$ & 1,36 & $-1,86$ & 0,18 & 755 \\
\hline KURT & 3,01 & 3,02 & 7,22 & 1,71 & 0,20 & 755 \\
\hline \multicolumn{7}{|c|}{ Smile independent variables } \\
\hline PRC & 40,29 & & & 28,65 & 7,76 & 755 \\
\hline HV & 0,36 & 0,32 & 1,07 & 0,13 & 0,15 & 755 \\
\hline HSKEW & $-0,08$ & $-0,02$ & 9,54 & $-6,84$ & 1,38 & 755 \\
\hline HKURT & 6,83 & 3,39 & 123,25 & $-0,70$ & 10,82 & 755 \\
\hline CDS & 59,34 & 63,50 & 96,88 & 29,67 & 12,97 & 755 \\
\hline
\end{tabular}

Table B.14.: Summary statistics for cross section _VW 


\section{B.3.10. BASF}

\begin{tabular}{|c|c|c|c|c|c|c|}
\hline _BASF & Mean & Median & Max & Min & StdDev & Obs \\
\hline \multicolumn{7}{|c|}{ Constant maturity of 36 days } \\
\hline B0 & 2,96 & 3,07 & 35,16 & $-229,06$ & 9,60 & 752 \\
\hline B1 & $-5,01$ & $-5,19$ & 452,09 & $-69,33$ & 18,99 & 752 \\
\hline B2 & 2,31 & 2,41 & 34,35 & $-222,63$ & 9,39 & 752 \\
\hline SLP70 & $-1,77$ & $-1,83$ & 140,40 & $-21,24$ & 5,86 & 752 \\
\hline SLP90 & $-0,85$ & $-0,88$ & 51,35 & $-7,50$ & 2,12 & 752 \\
\hline SLP100 & $-0,38$ & $-0,38$ & 6,82 & $-7,48$ & 0,43 & 752 \\
\hline SLP110 & 0,08 & 0,10 & 6,24 & $-37,70$ & 1,71 & 752 \\
\hline SLP130 & 1,01 & 1,08 & 19,98 & $-126,76$ & 5,44 & 752 \\
\hline XSMIN & 1,11 & 1,08 & 7,21 & $-0,02$ & 0,33 & 752 \\
\hline IMPV & 0,26 & 0,24 & 0,56 & 0,05 & 0,09 & 752 \\
\hline SKEW & $-0,18$ & $-0,17$ & 0,77 & $-0,50$ & 0,07 & 752 \\
\hline KURT & 3,03 & 3,03 & 3,10 & 2,78 & 0,02 & 752 \\
\hline \multicolumn{7}{|c|}{ Constant maturity of 60 days } \\
\hline B0 & 4,09 & 1,87 & $1.123,06$ & $-101,85$ & 44,52 & 752 \\
\hline B1 & $-7,20$ & $-2,76$ & 217,32 & $-2.226,14$ & 87,93 & 752 \\
\hline B2 & 3,38 & 1,21 & $1.103,39$ & $-115,56$ & 43,43 & 752 \\
\hline SLP70 & $-2,47$ & $-1,08$ & 55,54 & $-681,40$ & 27,15 & 752 \\
\hline SLP90 & $-1,12$ & $-0,58$ & 9,32 & $-240,04$ & 9,83 & 752 \\
\hline SLP100 & $-0,44$ & $-0,33$ & 1,28 & $-36,76$ & 1,61 & 752 \\
\hline SLP110 & 0,23 & $-0,10$ & 201,32 & $-36,91$ & 7,72 & 752 \\
\hline SLP130 & 1,58 & 0,38 & 642,67 & $-83,13$ & 25,02 & 752 \\
\hline XSMIN & 1,18 & 1,12 & 22,95 & $-2,38$ & 0,90 & 752 \\
\hline IMPV & 0,27 & 0,25 & 1,08 & $-0,09$ & 0,09 & 752 \\
\hline SKEW & $-0,19$ & $-0,20$ & 1,34 & $-0,54$ & 0,11 & 752 \\
\hline KURT & 3,03 & 3,03 & 4,05 & 2,43 & 0,07 & 752 \\
\hline \multicolumn{7}{|c|}{ Constant maturity of 180 days } \\
\hline B0 & 0,19 & 0,92 & 848,35 & $-1.626,76$ & 79,96 & 752 \\
\hline B1 & $-0,32$ & $-1,07$ & $2.803,50$ & $-1.677,26$ & 144,95 & 752 \\
\hline B2 & 0,39 & 0,42 & 877,28 & $-1.207,66$ & 67,18 & 752 \\
\hline SLP70 & 0,22 & $-0,48$ & $1.112,79$ & $-516,30$ & 52,50 & 752 \\
\hline SLP90 & 0,38 & $-0,31$ & 629,72 & $-184,60$ & 28,10 & 752 \\
\hline SLP100 & 0,46 & $-0,23$ & 388,19 & $-117,90$ & 18,65 & 752 \\
\hline SLP110 & 0,53 & $-0,15$ & 359,28 & $-76,07$ & 16,34 & 752 \\
\hline SLP130 & 0,69 & 0,02 & 710,19 & $-336,40$ & 34,48 & 752 \\
\hline XSMIN & 0,95 & 1,22 & 28,70 & $-247,77$ & 9,18 & 752 \\
\hline IMPV & 0,26 & 0,26 & 16,83 & $-30,91$ & 1,69 & 752 \\
\hline SKEW & $-0,21$ & $-0,23$ & 2,16 & $-4,38$ & 0,21 & 752 \\
\hline KURT & 3,05 & 3,04 & 10,47 & 1,85 & 0,33 & 752 \\
\hline \multicolumn{7}{|c|}{ Constant maturity of 240 days } \\
\hline Bo & $-4,33$ & 0,83 & $1.927,15$ & $-3.989,95$ & 224,31 & 752 \\
\hline B1 & 8,04 & $-0,91$ & $6.601,96$ & $-3.065,56$ & 401,91 & 752 \\
\hline B2 & $-3,44$ & 0,34 & $1.712,35$ & $-3.102,21$ & 184,06 & 752 \\
\hline SLP70 & 3,22 & $-0,42$ & $2.778,90$ & $-1.364,62$ & 148,26 & 752 \\
\hline SLP90 & 1,85 & $-0,28$ & $1.686,59$ & $-895,38$ & 80,64 & 752 \\
\hline SLP100 & 1,16 & $-0,21$ & $1.140,44$ & $-660,76$ & 53,19 & 752 \\
\hline SLP110 & 0,47 & $-0,15$ & 701,62 & $-518,11$ & 43,20 & 752 \\
\hline XSMIN & 1,20 & 1,22 & 21,52 & $-25,31$ & 1,71 & 752 \\
\hline SLP130 & $-0,91$ & 0,00 & $1.386,56$ & $-1.759,00$ & 89,84 & 752 \\
\hline IMPV & 0,27 & 0,26 & 93,28 & $-118,75$ & 5,83 & 752 \\
\hline SKEW & $-0,22$ & $-0,23$ & 5,02 & $-9,36$ & 0,46 & 752 \\
\hline KURT & 3,07 & 3,04 & 19,06 & 1,92 & 0,70 & 752 \\
\hline \multicolumn{7}{|c|}{ Smile independent variables } \\
\hline PRC & 41,99 & 42,13 & & 29,17 & 4,42 & 752 \\
\hline HV & 0,33 & 0,28 & 1,17 & 0,11 & 0,16 & 752 \\
\hline HSKEW & $-0,06$ & $-0,03$ & 8,86 & $-9,48$ & 1,35 & 752 \\
\hline HKURT & 5,90 & 2,64 & 115,39 & $-0,19$ & 12,08 & 752 \\
\hline CDS & 20,13 & 17,38 & 43,17 & 10,83 & 7,13 & 752 \\
\hline
\end{tabular}

Table B.15.: Summary statistics for cross section BASF 


\section{B.3.11. EON}

\begin{tabular}{|c|c|c|c|c|c|c|}
\hline EON & Mean & Median & Max & Min & StdDev & Obs \\
\hline \multicolumn{7}{|c|}{ Constant maturity of 36 days } \\
\hline B0 & 4,42 & 2,99 & 815,76 & $-103,65$ & 31,71 & 755 \\
\hline B1 & $-8,04$ & $-5,16$ & 198,76 & $-1.624,87$ & 63,42 & 755 \\
\hline B2 & 3,87 & 2,41 & 809,37 & $-95,11$ & 31,72 & 755 \\
\hline SLP70 & $-2,62$ & $-1,77$ & 65,60 & $-491,75$ & 19,03 & 755 \\
\hline SLP90 & $-1,07$ & $-0,81$ & 27,56 & $-168,00$ & 6,39 & 755 \\
\hline SLP100 & $-0,29$ & $-0,31$ & 21,81 & $-6,13$ & 0,92 & 755 \\
\hline SLP110 & 0,48 & 0,16 & 155,74 & $-12,79$ & 6,43 & 755 \\
\hline SLP130 & 2,03 & 1,12 & 479,49 & $-48,53$ & 19,08 & 755 \\
\hline XSMIN & 1,01 & 1,06 & 3,42 & $-39,18$ & 1,52 & 755 \\
\hline IMPV & 0,25 & 0,22 & 0,61 & $-0,01$ & 0,09 & 755 \\
\hline SKEW & $-0,15$ & $-0,14$ & 0,62 & $-0,93$ & 0,08 & 755 \\
\hline KURT & 3,02 & 3,03 & 3,12 & 1,97 & 0,06 & 755 \\
\hline \multicolumn{7}{|c|}{ Constant maturity of 60 days } \\
\hline B0 & 2,14 & 1,71 & 571,32 & $-144,58$ & 22,21 & 755 \\
\hline B1 & $-3,51$ & $-2,59$ & 278,34 & $-1.137,75$ & 44,03 & 755 \\
\hline B2 & 1,63 & 1,15 & 566,69 & $-133,80$ & 21,83 & 755 \\
\hline SLP70 & $-1,24$ & $-0,98$ & 91,02 & $-344,38$ & 13,48 & 755 \\
\hline SLP90 & $-0,59$ & $-0,52$ & 38,34 & $-117,70$ & 4,79 & 755 \\
\hline SLP100 & $-0,26$ & $-0,28$ & 19,15 & $-4,36$ & 0,87 & 755 \\
\hline SLP110 & 0,06 & $-0,06$ & 108,98 & $-16,02$ & 4,08 & 755 \\
\hline SLP130 & 0,71 & 0,39 & 335,66 & $-69,54$ & 12,76 & 755 \\
\hline XSMIN & 1,09 & 1,10 & 10,42 & $-39,18$ & 1,55 & 755 \\
\hline IMPV & 0,26 & 0,23 & 0,54 & $-0,65$ & 0,09 & 755 \\
\hline SKEW & $-0,18$ & $-0,17$ & 0,93 & $-2,57$ & 0,15 & 755 \\
\hline KURT & 3,02 & 3,03 & 3,13 & 0,17 & 0,12 & 755 \\
\hline \multicolumn{7}{|c|}{ Constant maturity of 180 days } \\
\hline B0 & $-0,94$ & 0,87 & $3.080,29$ & $-4.307,11$ & 201,17 & 755 \\
\hline B1 & 2,82 & $-1,01$ & $8.193,25$ & $-6.092,01$ & 388,38 & 755 \\
\hline B2 & $-1,67$ & 0,40 & $3.012,26$ & $-3.896,19$ & 188,79 & 755 \\
\hline SLP70 & 0,49 & $-0,44$ & $2.738,59$ & $-1.874,85$ & 126,82 & 755 \\
\hline SLP90 & $-0,18$ & $-0,28$ & $1.180,11$ & $-701,44$ & 56,97 & 755 \\
\hline SLP100 & $-0,51$ & $-0,19$ & 400,88 & $-784,23$ & 33,21 & 755 \\
\hline SLP110 & $-0,85$ & $-0,11$ & 534,95 & $-867,03$ & 42,57 & 755 \\
\hline SLP130 & $-1,51$ & 0,04 & $1.739,86$ & $-1.936,83$ & 108,56 & 755 \\
\hline XSMIN & 1,06 & 1,18 & 9,63 & $-90,08$ & 3,73 & 755 \\
\hline IMPV & 0,22 & 0,24 & 7,85 & $-17,18$ & 0,96 & 755 \\
\hline SKEW & $-0,19$ & $-0,19$ & 0,89 & $-1,94$ & 0,17 & 755 \\
\hline KURT & 3,03 & 3,04 & 4,81 & 1,80 & 0,13 & 755 \\
\hline \multicolumn{7}{|c|}{ Constant maturity of 240 days } \\
\hline B0 & $-26,28$ & 0,78 & $6.013,30$ & $-24.189,85$ & 977,16 & 755 \\
\hline B1 & 52,33 & $-0,87$ & $46.012,70$ & $-11.893,20$ & $1.907,11$ & 755 \\
\hline B2 & $-26,11$ & 0,34 & $5.880,80$ & $-21.880,51$ & 938,54 & 755 \\
\hline SLP70 & 15,78 & $-0,38$ & $15.379,99$ & $-3.660,08$ & 603,63 & 755 \\
\hline SLP90 & 5,33 & $-0,25$ & $6.627,78$ & $-1.307,76$ & 252,04 & 755 \\
\hline SLP100 & 0,11 & $-0,17$ & $2.251,68$ & $-2.796,59$ & 137,12 & 755 \\
\hline SLP110 & $-5,11$ & $-0,11$ & $2.084,22$ & $-4.643,89$ & 211,07 & 755 \\
\hline XSMIN & 1,26 & 1,18 & 85,83 & $-39,18$ & 3,53 & 755 \\
\hline SLP130 & $-15,56$ & 0,02 & $4.270,89$ & $-10.876,62$ & 554,48 & 755 \\
\hline IMPV & $-0,06$ & 0,24 & 45,27 & $-190,88$ & 7,61 & 755 \\
\hline SKEW & $-0,19$ & $-0,19$ & 1,44 & $-5,37$ & 0,34 & 755 \\
\hline KURT & 3,03 & 3,04 & 7,07 & 0,81 & 0,25 & 755 \\
\hline \multicolumn{7}{|c|}{ Smile independent variables } \\
\hline PRC & 51,30 & & & & 7,51 & 755 \\
\hline HV & 0,31 & 0,27 & 1,12 & 0,10 & 0,15 & 755 \\
\hline HSKEW & $-0,07$ & $-0,03$ & 5,92 & $-11,40$ & 1,14 & 755 \\
\hline HKURT & 5,02 & 2,65 & 147,34 & $-0,71$ & 10,23 & 755 \\
\hline CDS & 37,13 & 30,35 & 87,50 & 14,88 & 17,36 & 755 \\
\hline
\end{tabular}

Table B.16.: Summary statistics for cross section EON 


\section{B.3.12. COB}

\begin{tabular}{|c|c|c|c|c|c|c|}
\hline _COB & Mean & Median & Max & Min & StdDev & Obs \\
\hline \multicolumn{7}{|c|}{ Constant maturity of 36 days } \\
\hline B0 & 3,56 & 2,98 & 330,19 & $-471,65$ & 28,15 & 725 \\
\hline B1 & $-6,12$ & $-4,80$ & 909,43 & $-646,15$ & 55,10 & 725 \\
\hline B2 & 2,95 & 2,28 & 316,34 & $-437,10$ & 26,96 & 725 \\
\hline SLP70 & $-1,98$ & $-1,65$ & 297,48 & $-203,27$ & 17,39 & 725 \\
\hline SLP90 & $-0,80$ & $-0,74$ & 122,64 & $-76,73$ & 6,69 & 725 \\
\hline SLP100 & $-0,21$ & $-0,26$ & 35,22 & $-13,46$ & 1,77 & 725 \\
\hline SLP110 & 0,38 & 0,21 & 58,59 & $-52,20$ & 4,43 & 725 \\
\hline SLP130 & 1,56 & 1,10 & 176,35 & $-227,04$ & 15,07 & 725 \\
\hline XSMIN & 1,06 & 1,05 & 4,26 & $-2,53$ & 0,26 & 725 \\
\hline IMPV & 0,39 & 0,35 & 0,91 & $-0,31$ & 0,16 & 725 \\
\hline SKEW & $-0,13$ & $-0,12$ & 0,61 & $-1,14$ & 0,12 & 725 \\
\hline KURT & 3,01 & 3,02 & 3,17 & 2,37 & 0,05 & 725 \\
\hline \multicolumn{7}{|c|}{ Constant maturity of 60 days } \\
\hline B0 & $-3,41$ & 1,86 & 796,30 & $-4.987,67$ & 190,13 & 725 \\
\hline B1 & 8,95 & $-2,65$ & $10.743,32$ & $-1.558,00$ & 407,75 & 725 \\
\hline B2 & $-5,18$ & 1,17 & 762,32 & $-5.784,61$ & 218,76 & 725 \\
\hline SLP70 & 1,70 & $-0,99$ & $2.644,86$ & $-490,75$ & 101,68 & 725 \\
\hline SLP90 & $-0,37$ & $-0,50$ & 331,02 & $-185,82$ & 15,73 & 725 \\
\hline SLP100 & $-1,41$ & $-0,24$ & 27,85 & $-825,91$ & 30,74 & 725 \\
\hline SLP110 & $-2,44$ & $-0,01$ & 119,11 & $-1.982,83$ & 73,97 & 725 \\
\hline SLP130 & $-4,52$ & 0,46 & 424,03 & $-4.296,67$ & 161,27 & 725 \\
\hline XSMIN & 1,12 & 1,08 & 11,14 & $-11,46$ & 0,79 & 725 \\
\hline IMPV & 0,36 & 0,35 & 4,33 & $-28,97$ & 1,11 & 725 \\
\hline SKEW & $-0,15$ & $-0,14$ & 1,16 & $-1,67$ & 0,14 & 725 \\
\hline KURT & 3,00 & 3,02 & 3,41 & 2,04 & 0,08 & 725 \\
\hline \multicolumn{7}{|c|}{ Constant maturity of 180 days } \\
\hline B0 & 15,41 & 0,98 & $9.232,39$ & $-669,07$ & 347,88 & 725 \\
\hline B1 & $-28,55$ & $-1,01$ & $1.312,50$ & $-17.899,60$ & 673,99 & 725 \\
\hline B2 & 13,60 & 0,42 & $8.676,13$ & $-643,06$ & 326,61 & 725 \\
\hline SLP70 & $-9,51$ & $-0,43$ & 412,22 & $-5.753,02$ & 216,87 & 725 \\
\hline SLP90 & $-4,07$ & $-0,27$ & 155,00 & $-2.282,57$ & 86,52 & 725 \\
\hline SLP100 & $-1,35$ & $-0,18$ & 90,47 & $-547,34$ & 22,64 & 725 \\
\hline SLP110 & 1,37 & $-0,10$ & $1.187,89$ & $-181,47$ & 45,53 & 725 \\
\hline SLP130 & 6,81 & 0,06 & $4.658,34$ & $-359,45$ & 175,49 & 725 \\
\hline XSMIN & 1,09 & 1,16 & 33,54 & $-145,39$ & 6,30 & 725 \\
\hline IMPV & 0,46 & 0,37 & 53,58 & $-6,27$ & 2,04 & 725 \\
\hline SKEW & $-0,16$ & $-0,17$ & 1,46 & $-0,86$ & 0,15 & 725 \\
\hline KURT & 2,99 & 3,01 & 4,45 & 0,94 & 0,15 & 725 \\
\hline \multicolumn{7}{|c|}{ Constant maturity of 240 days } \\
\hline Bo & 26,52 & 0,90 & $15.319,00$ & $-1.231,05$ & 582,18 & 725 \\
\hline B1 & $-49,17$ & $-0,83$ & $2.503,85$ & $-29.700,71$ & $1.125,21$ & 725 \\
\hline B2 & 23,19 & 0,33 & $14.396,32$ & $-1.272,71$ & 544,21 & 725 \\
\hline SLP70 & $-16,69$ & $-0,38$ & 722,06 & $-9.545,87$ & 363,80 & 725 \\
\hline SLP90 & $-7,42$ & $-0,24$ & 223,74 & $-3.787,35$ & 147,19 & 725 \\
\hline SLP100 & $-2,78$ & $-0,17$ & 133,24 & $-908,08$ & 43,15 & 725 \\
\hline SLP110 & 1,86 & $-0,10$ & $1.971,18$ & $-314,58$ & 75,84 & 725 \\
\hline XSMIN & 1,22 & 1,17 & 33,54 & $-40,30$ & 2,56 & 725 \\
\hline SLP130 & 11,14 & 0,02 & $7.729,71$ & $-805,19$ & 290,88 & 725 \\
\hline IMPV & 0,55 & 0,37 & 63,44 & $-9,42$ & 3,16 & 725 \\
\hline SKEW & $-0,16$ & $-0,17$ & 2,19 & $-1,22$ & 0,20 & 725 \\
\hline KURT & 2,99 & 3,01 & 7,17 & 0,09 & 0,24 & 725 \\
\hline \multicolumn{7}{|c|}{ Smile independent variables } \\
\hline PRC & 13,12 & 13,88 & & 5,13 & 3,82 & 725 \\
\hline HV & 0,46 & 0,38 & 1,59 & 0,13 & 0,23 & 725 \\
\hline HSKEW & $-0,01$ & 0,02 & 7,39 & $-8,14$ & 1,31 & 725 \\
\hline HKURT & 6,53 & 3,22 & 90,97 & $-0,02$ & 10,65 & 725 \\
\hline CDS & 58,07 & 31,75 & 260,00 & 15,15 & 46,01 & 725 \\
\hline
\end{tabular}

Table B.17.: Summary statistics for cross section _COB 


\section{B.3.13. HYPV}

\begin{tabular}{|c|c|c|c|c|c|c|}
\hline _HYPV & Mean & Median & Max & Min & StdDev & Obs \\
\hline \multicolumn{7}{|c|}{ Constant maturity of 36 days } \\
\hline B0 & 1,46 & 2,43 & $1.804,15$ & $-3.223,07$ & 147,41 & 747 \\
\hline B1 & $-2,18$ & $-3,73$ & $6.301,49$ & $-3.527,31$ & 287,90 & 747 \\
\hline B2 & 1,14 & 1,73 & $1.724,33$ & $-3.079,73$ & 140,60 & 747 \\
\hline SLP70 & $-0,58$ & $-1,30$ & $1.989,87$ & $-1.113,25$ & 91,10 & 747 \\
\hline SLP90 & $-0,13$ & $-0,60$ & 757,98 & $-423,52$ & 34,96 & 747 \\
\hline SLP100 & 0,10 & $-0,25$ & 142,03 & $-78,65$ & 7,52 & 747 \\
\hline SLP110 & 0,33 & 0,13 & 266,22 & $-473,91$ & 21,74 & 747 \\
\hline SLP130 & 0,78 & 0,80 & 955,95 & $-1.705,80$ & 77,78 & 747 \\
\hline XSMIN & 1,08 & 1,05 & 14,76 & $-2,41$ & 0,62 & 747 \\
\hline IMPV & 0,42 & 0,38 & 1,17 & $-3,04$ & 0,21 & 747 \\
\hline SKEW & $-0,11$ & $-0,12$ & 1,52 & $-0,65$ & 0,15 & 747 \\
\hline KURT & 3,01 & 3,02 & 4,02 & 2,25 & 0,07 & 747 \\
\hline \multicolumn{7}{|c|}{ Constant maturity of 60 days } \\
\hline B0 & $-20,44$ & 1,63 & $4.735,32$ & $-22.584,41$ & 847,21 & 747 \\
\hline B1 & 39,87 & $-2,17$ & $44.150,56$ & $-9.952,00$ & $1.661,59$ & 747 \\
\hline B2 & $-19,00$ & 0,96 & $5.229,20$ & $-21.577,30$ & 815,10 & 747 \\
\hline SLP70 & 13,26 & $-0,82$ & $13.942,34$ & $-2.631,11$ & 520,92 & 747 \\
\hline SLP90 & 5,66 & $-0,42$ & $5.311,42$ & $-539,43$ & 196,02 & 747 \\
\hline SLP100 & 1,86 & $-0,22$ & 995,96 & $-71,66$ & 41,04 & 747 \\
\hline SLP110 & $-1,94$ & $-0,03$ & $1.552,25$ & $-3.319,50$ & 134,52 & 747 \\
\hline SLP130 & $-9,54$ & 0,35 & $3.643,93$ & $-11.950,41$ & 458,67 & 747 \\
\hline XSMIN & 0,89 & 1,08 & 7,88 & $-170,98$ & 6,34 & 747 \\
\hline IMPV & 0,43 & 0,38 & 12,53 & $-11,15$ & 0,64 & 747 \\
\hline SKEW & $-0,13$ & $-0,13$ & 1,30 & $-2,36$ & 0,17 & 747 \\
\hline KURT & 3,00 & 3,01 & 4,99 & 1,97 & 0,14 & 747 \\
\hline \multicolumn{7}{|c|}{ Constant maturity of 180 days } \\
\hline B0 & $-30,47$ & 1,02 & 907,11 & $-22.584,41$ & 834,05 & 747 \\
\hline B1 & 59,35 & $-1,00$ & $44.150,56$ & $-1.988,89$ & $1.629,00$ & 747 \\
\hline B2 & $-28,55$ & 0,40 & $1.090,89$ & $-21.577,30$ & 795,75 & 747 \\
\hline SLP70 & 19,38 & $-0,44$ & $13.942,34$ & $-461,64$ & 515,28 & 747 \\
\hline SLP90 & 7,96 & $-0,27$ & $5.311,42$ & $-205,15$ & 197,78 & 747 \\
\hline SLP100 & 2,25 & $-0,19$ & 995,96 & $-152,00$ & 43,66 & 747 \\
\hline SLP110 & $-3,46$ & $-0,11$ & 411,08 & $-3.319,50$ & 123,90 & 747 \\
\hline SLP130 & $-14,88$ & 0,06 & 847,43 & $-11.950,41$ & 440,70 & 747 \\
\hline XSMIN & 1,05 & 1,13 & 22,40 & $-18,61$ & 1,71 & 747 \\
\hline IMPV & 0,33 & 0,38 & 22,13 & $-54,97$ & 2,65 & 747 \\
\hline SKEW & $-0,15$ & $-0,16$ & 3,89 & $-4,90$ & 0,39 & 747 \\
\hline KURT & 3,00 & 3,00 & 12,81 & 0,02 & 0,46 & 747 \\
\hline \multicolumn{7}{|c|}{ Constant maturity of 240 days } \\
\hline B0 & $-31,99$ & 1,01 & $8.060,22$ & $-22.584,41$ & 932,53 & 747 \\
\hline B1 & 68,01 & $-0,93$ & $44.150,56$ & $-13.037,83$ & $1.812,46$ & 747 \\
\hline B2 & $-35,49$ & 0,37 & $5.272,55$ & $-21.577,30$ & 889,31 & 747 \\
\hline SLP70 & 18,32 & $-0,43$ & $13.942,34$ & $-5.656,26$ & 576,53 & 747 \\
\hline SLP90 & 4,13 & $-0,26$ & $5.311,42$ & $-3.547,24$ & 241,27 & 747 \\
\hline SLP100 & $-2,97$ & $-0,18$ & 995,96 & $-2.492,72$ & 126,59 & 747 \\
\hline SLP110 & $-10,06$ & $-0,12$ & 505,48 & $-3.681,06$ & 192,64 & 747 \\
\hline XSMIN & 1,12 & 1,13 & 20,87 & $-16,89$ & 1,47 & 747 \\
\hline SLP130 & $-24,26$ & 0,02 & $1.041,85$ & $-11.950,41$ & 518,73 & 747 \\
\hline IMPV & 0,53 & 0,38 & 294,95 & $-103,77$ & 12,32 & 747 \\
\hline SKEW & $-0,14$ & $-0,16$ & 3,89 & $-9,30$ & 0,50 & 747 \\
\hline KURT & 3,00 & 3,00 & 12,81 & 0,02 & 0,52 & 747 \\
\hline \multicolumn{7}{|c|}{ Smile independent variables } \\
\hline PRC & 19,03 & 16,02 & & 6,82 & 8,46 & 747 \\
\hline HV & 0,50 & 0,43 & 2,13 & 0,15 & 0,25 & 747 \\
\hline HSKEW & $-0,12$ & $-0,04$ & 9,89 & $-12,56$ & 1,63 & 747 \\
\hline HKURT & 8,00 & 3,41 & 178,15 & $-0,66$ & 14,79 & 747 \\
\hline CDS & 56,28 & 33,88 & 182,00 & 18,14 & 38,81 & 747 \\
\hline
\end{tabular}

Table B.18.: Summary statistics for cross section _HYPV 


\section{B.3.14. RWE}

\begin{tabular}{|c|c|c|c|c|c|c|}
\hline _RWE & Mean & Median & Max & Min & StdDev & Obs \\
\hline \multicolumn{7}{|c|}{ Constant maturity of 36 days } \\
\hline B0 & 16,78 & 3,31 & $6.441,58$ & $-1.711,26$ & 277,80 & 671 \\
\hline B1 & $-33,17$ & $-5,83$ & $3.346,60$ & $-13.337,55$ & 567,79 & 671 \\
\hline B2 & 16,68 & 2,77 & $6.904,38$ & $-1.635,93$ & 290,56 & 671 \\
\hline SLP70 & $-9,82$ & $-1,94$ & $1.056,30$ & $-3.671,42$ & 161,52 & 671 \\
\hline SLP90 & $-3,15$ & $-0,83$ & 401,92 & $-909,67$ & 47,09 & 671 \\
\hline SLP100 & 0,18 & $-0,28$ & 471,21 & $-200,47$ & 20,30 & 671 \\
\hline SLP110 & 3,52 & 0,28 & $1.852,08$ & $-252,45$ & 73,22 & 671 \\
\hline SLP130 & 10,19 & 1,37 & $4.613,83$ & $-906,82$ & 188,47 & 671 \\
\hline XSMIN & 1,08 & 1,04 & 13,81 & $-4,28$ & 0,65 & 671 \\
\hline IMPV & 0,29 & 0,25 & 8,41 & $-0,59$ & 0,40 & 671 \\
\hline SKEW & $-0,11$ & $-0,13$ & 1,87 & $-1,26$ & 0,18 & 671 \\
\hline KURT & 3,02 & 3,03 & 3,58 & 1,28 & 0,11 & 671 \\
\hline \multicolumn{7}{|c|}{ Constant maturity of 60 days } \\
\hline B0 & 7,84 & 1,98 & $5.477,23$ & $-3.096,47$ & 255,14 & 671 \\
\hline B1 & $-16,98$ & $-3,16$ & $5.165,11$ & $-11.340,65$ & 504,67 & 671 \\
\hline B2 & 9,30 & 1,45 & $5.870,60$ & $-2.153,73$ & 252,64 & 671 \\
\hline SLP70 & $-3,96$ & $-1,13$ & $2.149,89$ & $-3.121,81$ & 154,07 & 671 \\
\hline SLP90 & $-0,24$ & $-0,55$ & $1.288,40$ & $-773,57$ & 60,88 & 671 \\
\hline SLP100 & 1,61 & $-0,26$ & 857,65 & $-45,51$ & 36,74 & 671 \\
\hline SLP110 & 3,47 & 0,03 & $1.574,67$ & $-205,13$ & 64,03 & 671 \\
\hline SLP130 & 7,19 & 0,61 & $3.922,91$ & $-736,79$ & 157,86 & 671 \\
\hline XSMIN & 1,10 & 1,06 & 8,72 & 0,15 & 0,34 & 671 \\
\hline IMPV & 0,16 & 0,25 & 7,18 & $-85,08$ & 3,31 & 671 \\
\hline SKEW & $-0,15$ & $-0,15$ & 1,08 & $-2,20$ & 0,18 & 671 \\
\hline KURT & 3,01 & 3,03 & 3,58 & 1,96 & 0,11 & 671 \\
\hline \multicolumn{7}{|c|}{ Constant maturity of 180 days } \\
\hline B0 & 28,87 & 0,92 & $12.688,39$ & $-7.013,22$ & 750,29 & 671 \\
\hline B1 & $-54,59$ & $-1,11$ & $11.699,18$ & $-24.257,89$ & $1.385,37$ & 671 \\
\hline B2 & 25,98 & 0,46 & $11.594,37$ & $-4.878,85$ & 642,10 & 671 \\
\hline SLP70 & $-18,22$ & $-0,46$ & $4.868,80$ & $-8.025,77$ & 488,97 & 671 \\
\hline SLP90 & $-7,83$ & $-0,28$ & $2.917,26$ & $-3.584,15$ & 236,43 & 671 \\
\hline SLP100 & $-2,64$ & $-0,19$ & $1.941,49$ & $-1.755,70$ & 117,38 & 671 \\
\hline SLP110 & 2,56 & $-0,10$ & $1.249,73$ & $-672,64$ & 68,12 & 671 \\
\hline SLP130 & 12,95 & 0,09 & $5.887,48$ & $-1.352,40$ & 292,08 & 671 \\
\hline XSMIN & 1,21 & 1,13 & 32,78 & $-11,31$ & 1,73 & 671 \\
\hline IMPV & 0,26 & 0,26 & 84,54 & $-192,88$ & 8,73 & 671 \\
\hline SKEW & $-0,21$ & $-0,18$ & 1,45 & $-16,37$ & 0,68 & 671 \\
\hline KURT & 3,00 & 3,03 & 4,42 & 0,87 & 0,17 & 671 \\
\hline \multicolumn{7}{|c|}{ Constant maturity of 240 days } \\
\hline B0 & 43,61 & 0,83 & $18.040,76$ & $-6.615,87$ & 940,08 & 671 \\
\hline B1 & $-80,40$ & $-0,93$ & $12.503,91$ & $-34.490,86$ & $1.770,98$ & 671 \\
\hline B2 & 37,35 & 0,38 & $16.485,38$ & $-5.907,89$ & 835,88 & 671 \\
\hline SLP70 & $-28,11$ & $-0,40$ & $4.232,87$ & $-11.411,33$ & 602,80 & 671 \\
\hline SLP90 & $-13,17$ & $-0,25$ & $1.869,72$ & $-4.817,18$ & 272,29 & 671 \\
\hline SLP100 & $-5,70$ & $-0,17$ & 688,14 & $-2.130,20$ & 115,65 & 671 \\
\hline SLP110 & 1,76 & $-0,10$ & $1.776,97$ & $-1.248,86$ & 92,22 & 671 \\
\hline XSMIN & 1,07 & 1,11 & 53,07 & $-86,69$ & 4,22 & 671 \\
\hline SLP130 & 16,70 & 0,05 & $8.371,12$ & $-2.856,59$ & 407,99 & 671 \\
\hline IMPV & 0,56 & 0,26 & 102,52 & $-30,02$ & 5,42 & 671 \\
\hline SKEW & $-0,20$ & $-0,18$ & 3,54 & $-16,37$ & 0,71 & 671 \\
\hline KURT & 3,01 & 3,03 & 13,78 & 0,87 & 0,47 & 671 \\
\hline \multicolumn{7}{|c|}{ Smile independent variables } \\
\hline PRC & 32,02 & 32,40 & & & 6,79 & 671 \\
\hline HV & 0,36 & 0,31 & 1,09 & 0,11 & 0,17 & 671 \\
\hline HSKEW & $-0,12$ & $-0,08$ & 6,25 & $-10,11$ & 1,21 & 671 \\
\hline HKURT & 5,18 & 2,38 & 124,93 & $-0,23$ & 10,63 & 671 \\
\hline CDS & 47,30 & 40,07 & 121,50 & 17,26 & 23,73 & 671 \\
\hline
\end{tabular}

Table B.19.: Summary statistics for cross section $\_$RWE 


\section{B.3.15. BMW}

\begin{tabular}{|c|c|c|c|c|c|c|}
\hline _BMW & Mean & Median & Max & Min & StdDev & Obs \\
\hline \multicolumn{7}{|c|}{ Constant maturity of 36 days } \\
\hline B0 & 2,27 & 2,54 & $4.631,92$ & $-2.190,02$ & 199,53 & 756 \\
\hline B1 & $-4,01$ & $-4,10$ & $4.534,61$ & $-9.483,31$ & 406,38 & 756 \\
\hline B2 & 2,05 & 1,89 & $4.854,27$ & $-2.346,92$ & 207,13 & 756 \\
\hline SLP70 & $-1,14$ & $-1,47$ & $1.248,93$ & $-2.687,33$ & 116,63 & 756 \\
\hline SLP90 & $-0,32$ & $-0,71$ & 331,51 & $-745,62$ & 34,60 & 756 \\
\hline SLP100 & 0,09 & $-0,31$ & 225,24 & $-159,23$ & 11,94 & 756 \\
\hline SLP110 & 0,50 & 0,07 & $1.196,09$ & $-628,61$ & 50,20 & 756 \\
\hline SLP130 & 1,32 & 0,80 & $3.137,80$ & $-1.567,38$ & 132,56 & 756 \\
\hline XSMIN & 1,08 & 1,07 & 10,78 & $-18,82$ & 0,91 & 756 \\
\hline IMPV & 0,31 & 0,30 & 2,89 & $-7,39$ & 0,33 & 756 \\
\hline SKEW & $-0,14$ & $-0,14$ & 2,17 & $-1,25$ & 0,14 & 756 \\
\hline KURT & 3,02 & 3,02 & 5,74 & 2,26 & 0,11 & 756 \\
\hline \multicolumn{7}{|c|}{ Constant maturity of 60 days } \\
\hline B0 & 5,45 & 1,57 & $7.357,28$ & $-2.190,02$ & 340,08 & 756 \\
\hline B1 & $-11,15$ & $-2,17$ & $4.534,61$ & $-14.997,92$ & 687,91 & 756 \\
\hline B2 & 6,00 & 0,94 & $7.643,66$ & $-2.346,92$ & 348,38 & 756 \\
\hline SLP70 & $-2,75$ & $-0,85$ & $1.424,34$ & $-4.296,80$ & 200,73 & 756 \\
\hline SLP90 & $-0,35$ & $-0,48$ & 713,55 & $-1.239,33$ & 63,11 & 756 \\
\hline SLP100 & 0,85 & $-0,27$ & 358,15 & $-159,23$ & 19,84 & 756 \\
\hline SLP110 & 2,05 & $-0,09$ & $1.818,13$ & $-628,61$ & 80,71 & 756 \\
\hline SLP130 & 4,46 & 0,28 & $4.875,60$ & $-1.567,38$ & 218,82 & 756 \\
\hline XSMIN & 1,23 & 1,11 & 28,36 & $-18,82$ & 1,69 & 756 \\
\hline IMPV & 0,30 & 0,31 & 3,02 & $-18,35$ & 0,71 & 756 \\
\hline SKEW & $-0,16$ & $-0,16$ & 1,84 & $-1,15$ & 0,13 & 756 \\
\hline KURT & 3,01 & 3,02 & 5,31 & 1,91 & 0,11 & 756 \\
\hline \multicolumn{7}{|c|}{ Constant maturity of 180 days } \\
\hline B0 & $-7,02$ & 0,95 & $7.923,46$ & $-6.697,79$ & 442,75 & 756 \\
\hline B1 & 10,62 & $-1,00$ & $13.141,21$ & $-16.152,13$ & 857,93 & 756 \\
\hline B2 & $-3,51$ & 0,39 & $8.231,91$ & $-6.445,59$ & 419,27 & 756 \\
\hline SLP70 & 5,71 & $-0,46$ & $4.121,15$ & $-4.627,46$ & 274,68 & 756 \\
\hline SLP90 & 4,30 & $-0,30$ & $2.331,61$ & $-1.334,70$ & 115,22 & 756 \\
\hline SLP100 & 3,60 & $-0,21$ & $1.436,84$ & $-67,11$ & 57,32 & 756 \\
\hline SLP110 & 2,90 & $-0,14$ & $1.958,07$ & $-1.039,08$ & 85,78 & 756 \\
\hline SLP130 & 1,50 & 0,02 & $5.250,83$ & $-3.617,31$ & 240,19 & 756 \\
\hline XSMIN & 1,22 & 1,17 & 35,45 & $-29,91$ & 2,63 & 756 \\
\hline IMPV & 0,09 & 0,31 & 23,43 & $-114,81$ & 4,91 & 756 \\
\hline SKEW & $-0,16$ & $-0,19$ & 4,06 & $-0,67$ & 0,25 & 756 \\
\hline KURT & 3,03 & 3,02 & 13,65 & 0,15 & 0,46 & 756 \\
\hline \multicolumn{7}{|c|}{ Constant maturity of 240 days } \\
\hline B0 & $-17,14$ & 0,89 & $7.923,46$ & $-18.061,85$ & 726,65 & 756 \\
\hline B1 & 31,86 & $-0,93$ & $35.436,73$ & $-16.152,13$ & $1.433,20$ & 756 \\
\hline B2 & $-14,56$ & 0,35 & $8.231,91$ & $-17.381,16$ & 707,73 & 756 \\
\hline SLP70 & 11,47 & $-0,42$ & $11.103,11$ & $-4.627,46$ & 443,45 & 756 \\
\hline SLP90 & 5,65 & $-0,28$ & $4.150,64$ & $-1.334,70$ & 163,07 & 756 \\
\hline SLP100 & 2,74 & $-0,21$ & 674,41 & $-258,30$ & 40,90 & 756 \\
\hline SLP110 & $-0,17$ & $-0,13$ & $1.958,07$ & $-2.801,82$ & 129,70 & 756 \\
\hline XSMIN & 1,06 & 1,16 & 21,04 & $-46,34$ & 2,85 & 756 \\
\hline SLP130 & $-6,00$ & 0,00 & $5.250,83$ & $-9.754,28$ & 409,07 & 756 \\
\hline IMPV & 0,16 & 0,31 & 30,48 & $-110,75$ & 4,32 & 756 \\
\hline SKEW & $-0,16$ & $-0,19$ & 4,06 & $-0,67$ & 0,27 & 756 \\
\hline KURT & 3,02 & 3,02 & 13,65 & 0,12 & 0,47 & 756 \\
\hline \multicolumn{7}{|c|}{ Smile independent variables } \\
\hline PRC & 35,15 & & & & 4,94 & 756 \\
\hline HV & 0,35 & 0,30 & 1,03 & 0,13 & 0,15 & 756 \\
\hline HSKEW & 0,01 & $-0,05$ & 6,81 & $-6,68$ & 1,32 & 756 \\
\hline HKURT & 6,51 & 3,27 & 71,89 & $-0,13$ & 9,99 & 756 \\
\hline CDS & 32,74 & 31,13 & 70,33 & 20,89 & 6,97 & 756 \\
\hline
\end{tabular}

Table B.20.: Summary statistics for cross section BMW 


\section{B.3.16. LH}

\begin{tabular}{|c|c|c|c|c|c|c|}
\hline _LH & Mean & Median & Max & Min & StdDev & Obs \\
\hline \multicolumn{7}{|c|}{ Constant maturity of 36 days } \\
\hline B0 & 40,05 & 3,22 & $22.727,17$ & $-2.374,40$ & 868,76 & 699 \\
\hline B1 & $-79,08$ & $-5,47$ & $4.731,82$ & $-45.136,40$ & $1.725,94$ & 699 \\
\hline B2 & 39,40 & 2,64 & $22.410,58$ & $-2.357,15$ & 857,24 & 699 \\
\hline SLP70 & $-23,92$ & $-1,84$ & $1.431,82$ & $-13.761,59$ & 525,83 & 699 \\
\hline SLP90 & $-8,16$ & $-0,83$ & 488,96 & $-4.797,36$ & 183,01 & 699 \\
\hline SLP100 & $-0,28$ & $-0,28$ & 78,51 & $-315,24$ & 13,15 & 699 \\
\hline SLP110 & 7,60 & 0,23 & $4.166,88$ & $-453,90$ & 160,13 & 699 \\
\hline SLP130 & 23,36 & 1,26 & $13.131,11$ & $-1.396,76$ & 502,93 & 699 \\
\hline XSMIN & 1,04 & 1,04 & 5,29 & $-4,14$ & 0,37 & 699 \\
\hline IMPV & 0,37 & 0,34 & 1,56 & $-0,19$ & 0,13 & 699 \\
\hline SKEW & $-0,11$ & $-0,13$ & 1,95 & $-1,88$ & 0,25 & 699 \\
\hline KURT & 3,01 & 3,03 & 8,45 & 0,20 & 0,28 & 699 \\
\hline \multicolumn{7}{|c|}{ Constant maturity of 60 days } \\
\hline B0 & 38,48 & 2,23 & $22.727,17$ & $-1.511,34$ & 867,74 & 699 \\
\hline B1 & $-76,52$ & $-3,39$ & $2.544,69$ & $-45.136,40$ & $1.723,04$ & 699 \\
\hline B2 & 38,36 & 1,56 & $22.410,58$ & $-1.070,76$ & 855,52 & 699 \\
\hline SLP70 & $-22,82$ & $-1,20$ & $1.045,62$ & $-13.761,59$ & 525,49 & 699 \\
\hline SLP90 & $-7,48$ & $-0,60$ & 617,32 & $-4.797,36$ & 183,77 & 699 \\
\hline SLP100 & 0,19 & $-0,26$ & 403,16 & $-315,24$ & 20,36 & 699 \\
\hline SLP110 & 7,86 & 0,05 & $4.166,88$ & $-47,39$ & 160,03 & 699 \\
\hline SLP130 & 23,21 & 0,65 & $13.131,11$ & $-239,29$ & 501,66 & 699 \\
\hline XSMIN & 0,61 & 1,06 & 365,50 & $-557,02$ & 25,33 & 699 \\
\hline IMPV & 0,31 & 0,34 & 3,18 & $-37,42$ & 1,44 & 699 \\
\hline SKEW & $-0,12$ & $-0,15$ & 3,37 & $-0,98$ & 0,27 & 699 \\
\hline KURT & 3,01 & 3,02 & 12,54 & 0,43 & 0,41 & 699 \\
\hline \multicolumn{7}{|c|}{ Constant maturity of 180 days } \\
\hline B0 & 18,26 & 1,19 & $22.727,17$ & $-10.604,27$ & 967,53 & 699 \\
\hline B1 & $-43,53$ & $-1,39$ & $17.852,84$ & $-45.136,40$ & $1.876,51$ & 699 \\
\hline B2 & 25,25 & 0,59 & $22.410,58$ & $-7.513,68$ & 916,48 & 699 \\
\hline SLP70 & $-8,17$ & $-0,56$ & $7.333,69$ & $-13.761,59$ & 600,06 & 699 \\
\hline SLP90 & 1,93 & $-0,33$ & $4.328,21$ & $-4.797,36$ & 248,24 & 699 \\
\hline SLP100 & 6,98 & $-0,22$ & $2.825,48$ & $-315,24$ & 114,84 & 699 \\
\hline SLP110 & 12,03 & $-0,10$ & $4.166,88$ & $-268,41$ & 178,74 & 699 \\
\hline SLP130 & 22,13 & 0,12 & $13.131,11$ & $-1.682,73$ & 520,63 & 699 \\
\hline XSMIN & 1,09 & 1,10 & 11,94 & $-16,96$ & 1,13 & 699 \\
\hline IMPV & $-0,02$ & 0,34 & 47,76 & $-265,11$ & 10,42 & 699 \\
\hline SKEW & $-0,15$ & $-0,17$ & 2,10 & $-7,99$ & 0,42 & 699 \\
\hline KURT & 2,99 & 3,01 & 5,53 & 0,58 & 0,26 & 699 \\
\hline \multicolumn{7}{|c|}{ Constant maturity of 240 days } \\
\hline Bo & 18,23 & 1,20 & $22.727,17$ & $-10.604,27$ & $1.003,85$ & 699 \\
\hline B1 & $-44,07$ & $-1,36$ & $17.852,84$ & $-45.136,40$ & $1.961,52$ & 699 \\
\hline B2 & 25,86 & 0,56 & $22.410,58$ & $-7.513,68$ & 966,97 & 699 \\
\hline SLP70 & $-7,87$ & $-0,56$ & $7.333,69$ & $-13.761,59$ & 617,06 & 699 \\
\hline SLP90 & 2,48 & $-0,33$ & $4.328,21$ & $-4.797,36$ & 251,94 & 699 \\
\hline SLP100 & 7,65 & $-0,21$ & $2.825,48$ & $-791,19$ & 130,52 & 699 \\
\hline SLP110 & 12,82 & $-0,10$ & $4.166,88$ & $-1.823,33$ & 213,06 & 699 \\
\hline XSMIN & 1,10 & 1,10 & 17,32 & $-16,96$ & 1,41 & 699 \\
\hline SLP130 & 23,16 & 0,11 & $13.131,11$ & $-3.887,61$ & 571,42 & 699 \\
\hline IMPV & 0,02 & 0,35 & 85,72 & $-265,11$ & 10,99 & 699 \\
\hline SKEW & $-0,15$ & $-0,17$ & 2,29 & $-12,45$ & 0,59 & 699 \\
\hline KURT & 2,98 & 3,01 & 6,69 & 0,58 & 0,30 & 699 \\
\hline \multicolumn{7}{|c|}{ Smile independent variables } \\
\hline PRC & 11,87 & 11,36 & & 6,94 & 2,56 & 699 \\
\hline HV & 0,42 & 0,38 & 1,24 & 0,17 & 0,16 & 699 \\
\hline HSKEW & $-0,13$ & $-0,09$ & 9,71 & $-8,91$ & 1,54 & 699 \\
\hline HKURT & 7,49 & 3,20 & 125,54 & $-0,24$ & 14,20 & 699 \\
\hline CDS & 106,77 & 92,36 & 204,17 & 52,92 & 40,10 & 699 \\
\hline
\end{tabular}

Table B.21.: Summary statistics for cross section $\mathrm{LH}$ 


\section{B.3.17. _TYSS}

\begin{tabular}{|c|c|c|c|c|c|c|}
\hline _TYSS & Mean & Median & Max & Min & StdDev & Obs \\
\hline \multicolumn{7}{|c|}{ Constant maturity of 36 days } \\
\hline B0 & 0,05 & 3,65 & $1.716,12$ & $-5.249,81$ & 239,12 & 646 \\
\hline B1 & 0,03 & $-6,24$ & $9.939,15$ & $-3.300,65$ & 457,73 & 646 \\
\hline B2 & 0,22 & 2,93 & $1.587,30$ & $-4.703,88$ & 219,28 & 646 \\
\hline SLP70 & 0,34 & $-2,10$ & $3.353,71$ & $-1.078,43$ & 151,00 & 646 \\
\hline SLP90 & 0,43 & $-0,91$ & $1.472,16$ & $-443,51$ & 63,83 & 646 \\
\hline SLP100 & 0,48 & $-0,29$ & 531,38 & $-126,05$ & 21,94 & 646 \\
\hline SLP110 & 0,52 & 0,28 & 335,66 & $-409,39$ & 27,11 & 646 \\
\hline SLP130 & 0,61 & 1,47 & 902,40 & $-2.290,95$ & 113,06 & 646 \\
\hline XSMIN & 1,13 & 1,04 & 49,01 & 0,07 & 1,91 & 646 \\
\hline IMPV & 0,30 & 0,29 & 2,78 & $-14,55$ & 0,61 & 646 \\
\hline SKEW & $-0,12$ & $-0,13$ & 2,46 & $-3,98$ & 0,30 & 646 \\
\hline KURT & 3,00 & 3,03 & 4,86 & 1,52 & 0,16 & 646 \\
\hline \multicolumn{7}{|c|}{ Constant maturity of 60 days } \\
\hline B0 & 1,75 & 2,53 & $1.697,28$ & $-4.083,15$ & 182,63 & 646 \\
\hline B1 & $-3,04$ & $-4,09$ & $7.730,64$ & $-3.264,38$ & 348,97 & 646 \\
\hline B2 & 1,60 & 1,90 & $1.569,86$ & $-3.658,70$ & 166,87 & 646 \\
\hline SLP70 & $-0,80$ & $-1,42$ & $2.608,46$ & $-1.066,58$ & 115,55 & 646 \\
\hline SLP90 & $-0,17$ & $-0,67$ & $1.144,98$ & $-438,64$ & 49,20 & 646 \\
\hline SLP100 & 0,15 & $-0,28$ & 413,24 & $-124,67$ & 17,24 & 646 \\
\hline SLP110 & 0,47 & 0,09 & 302,64 & $-318,50$ & 20,02 & 646 \\
\hline SLP130 & 1,11 & 0,84 & 817,24 & $-1.781,98$ & 85,39 & 646 \\
\hline XSMIN & 1,05 & 1,05 & 5,39 & $-3,34$ & 0,42 & 646 \\
\hline IMPV & 0,31 & 0,29 & 2,75 & $-11,21$ & 0,49 & 646 \\
\hline SKEW & $-0,14$ & $-0,15$ & 2,52 & $-3,98$ & 0,29 & 646 \\
\hline KURT & 3,01 & 3,03 & 8,17 & 1,52 & 0,25 & 646 \\
\hline \multicolumn{7}{|c|}{ Constant maturity of 180 days } \\
\hline B0 & 10,89 & 1,13 & $3.213,80$ & $-306,98$ & 169,83 & 646 \\
\hline B1 & $-20,44$ & $-1,40$ & 567,74 & $-6.555,86$ & 332,93 & 646 \\
\hline B2 & 9,92 & 0,59 & $3.343,55$ & $-262,36$ & 163,96 & 646 \\
\hline SLP70 & $-6,55$ & $-0,59$ & 200,44 & $-1.874,89$ & 104,27 & 646 \\
\hline SLP90 & $-2,58$ & $-0,34$ & 95,50 & $-854,03$ & 40,79 & 646 \\
\hline SLP100 & $-0,60$ & $-0,21$ & 131,25 & $-384,72$ & 16,92 & 646 \\
\hline SLP110 & 1,39 & $-0,09$ & 799,96 & $-73,68$ & 32,55 & 646 \\
\hline SLP130 & 5,35 & 0,15 & $2.137,38$ & $-146,69$ & 95,17 & 646 \\
\hline XSMIN & 1,37 & 1,09 & 273,81 & $-33,91$ & 10,99 & 646 \\
\hline IMPV & 0,37 & 0,30 & 16,03 & $-1,59$ & 0,80 & 646 \\
\hline SKEW & $-0,21$ & $-0,18$ & 4,12 & $-20,10$ & 0,90 & 646 \\
\hline KURT & 3,01 & 3,02 & 13,57 & 0,10 & 0,56 & 646 \\
\hline \multicolumn{7}{|c|}{ Constant maturity of 240 days } \\
\hline B0 & 15,54 & 1,10 & $4.331,30$ & $-379,13$ & 222,63 & 646 \\
\hline B1 & $-29,84$ & $-1,35$ & 700,18 & $-8.005,81$ & 430,53 & 646 \\
\hline B2 & 14,83 & 0,57 & $3.699,63$ & $-323,09$ & 211,03 & 646 \\
\hline SLP70 & $-9,08$ & $-0,55$ & 247,85 & $-2.826,32$ & 138,68 & 646 \\
\hline SLP90 & $-3,15$ & $-0,32$ & 329,09 & $-1.346,47$ & 61,89 & 646 \\
\hline SLP100 & $-0,19$ & $-0,21$ & 669,51 & $-606,54$ & 38,39 & 646 \\
\hline SLP110 & 2,78 & $-0,09$ & $1.009,93$ & $-158,87$ & 51,77 & 646 \\
\hline XSMIN & 0,99 & 1,09 & 24,73 & $-33,91$ & 2,30 & 646 \\
\hline SLP130 & 8,71 & 0,13 & $2.137,38$ & $-146,69$ & 125,62 & 646 \\
\hline IMPV & 0,53 & 0,30 & 66,15 & $-2,44$ & 3,11 & 646 \\
\hline SKEW & $-0,21$ & $-0,19$ & 4,12 & $-19,66$ & 0,96 & 646 \\
\hline KURT & 3,01 & 3,02 & 13,57 & 0,10 & 0,57 & 646 \\
\hline \multicolumn{7}{|c|}{ Smile independent variables } \\
\hline PRC & 13,27 & & 18,00 & 7,19 & 2,61 & 646 \\
\hline HV & 0,41 & 0,37 & 1,11 & 0,13 & 0,17 & 646 \\
\hline HSKEW & 0,03 & 0,07 & 9,96 & $-9,54$ & 1,44 & 646 \\
\hline HKURT & 6,77 & 3,11 & 123,57 & $-0,64$ & 13,11 & 646 \\
\hline CDS & 144,75 & 135,83 & 370,63 & 56,60 & 52,50 & 646 \\
\hline
\end{tabular}

Table B.22.: Summary statistics for cross section _TYSS 


\section{B.3.18. METRO}

\begin{tabular}{|c|c|c|c|c|c|c|}
\hline _METRO & Mean & Median & Max & Min & StdDev & Obs \\
\hline \multicolumn{7}{|c|}{ Constant maturity of 36 days } \\
\hline B0 & 4,50 & 2,74 & $1.340,54$ & $-3.959,47$ & 200,12 & 610 \\
\hline B1 & $-8,29$ & $-4,49$ & $8.038,03$ & $-2.677,88$ & 404,80 & 610 \\
\hline B2 & 4,13 & 2,09 & $1.337,57$ & $-4.079,24$ & 204,84 & 610 \\
\hline SLP70 & $-2,51$ & $-1,59$ & $2.327,11$ & $-805,29$ & 118,19 & 610 \\
\hline SLP90 & $-0,86$ & $-0,74$ & 695,41 & $-270,26$ & 36,77 & 610 \\
\hline SLP100 & $-0,03$ & $-0,28$ & 115,72 & $-120,44$ & 8,86 & 610 \\
\hline SLP110 & 0,79 & 0,14 & 374,07 & $-936,28$ & 46,49 & 610 \\
\hline SLP130 & 2,44 & 0,97 & 890,76 & $-2.567,98$ & 128,05 & 610 \\
\hline XSMIN & 1,68 & 1,04 & 344,43 & $-2,05$ & 13,96 & 610 \\
\hline IMPV & 0,34 & 0,30 & 2,90 & $-2,03$ & 0,22 & 610 \\
\hline SKEW & $-0,15$ & $-0,13$ & 2,61 & $-14,93$ & 0,83 & 610 \\
\hline KURT & 3,04 & 3,02 & 15,26 & 1,01 & 0,60 & 610 \\
\hline \multicolumn{7}{|c|}{ Constant maturity of 60 days } \\
\hline B0 & 6,14 & 1,99 & $1.362,54$ & $-2.401,22$ & 146,63 & 610 \\
\hline B1 & $-11,41$ & $-3,01$ & $4.874,97$ & $-2.515,96$ & 292,96 & 610 \\
\hline B2 & 5,63 & 1,36 & $1.291,75$ & $-2.474,06$ & 146,78 & 610 \\
\hline SLP70 & $-3,53$ & $-1,12$ & $1.411,29$ & $-889,66$ & 87,99 & 610 \\
\hline SLP90 & $-1,27$ & $-0,57$ & 421,66 & $-425,01$ & 30,71 & 610 \\
\hline SLP100 & $-0,15$ & $-0,27$ & 115,72 & $-192,68$ & 11,36 & 610 \\
\hline SLP110 & 0,98 & $-0,02$ & 374,07 & $-567,96$ & 32,23 & 610 \\
\hline SLP130 & 3,24 & 0,55 & 890,76 & $-1.557,59$ & 89,60 & 610 \\
\hline XSMIN & 2,25 & 1,05 & 447,81 & $-42,14$ & 22,88 & 610 \\
\hline IMPV & 0,36 & 0,31 & 8,22 & $-2,03$ & 0,42 & 610 \\
\hline SKEW & $-0,16$ & $-0,14$ & 3,47 & $-14,93$ & 0,89 & 610 \\
\hline KURT & 3,05 & 3,02 & 15,26 & 1,01 & 0,74 & 610 \\
\hline \multicolumn{7}{|c|}{ Constant maturity of 180 days } \\
\hline B0 & 12,06 & 1,30 & $1.817,91$ & $-1.040,67$ & 140,02 & 610 \\
\hline B1 & $-22,92$ & $-1,67$ & $1.938,31$ & $-3.809,63$ & 276,36 & 610 \\
\hline B2 & 11,27 & 0,74 & $1.996,25$ & $-914,17$ & 137,74 & 610 \\
\hline SLP70 & $-7,14$ & $-0,68$ & 675,06 & $-1.014,89$ & 85,01 & 610 \\
\hline SLP90 & $-2,63$ & $-0,35$ & 314,13 & $-401,36$ & 33,61 & 610 \\
\hline SLP100 & $-0,37$ & $-0,22$ & 182,86 & $-244,04$ & 18,91 & 610 \\
\hline SLP110 & 1,88 & $-0,08$ & 582,11 & $-195,28$ & 33,21 & 610 \\
\hline SLP130 & 6,39 & 0,21 & $1.380,60$ & $-542,89$ & 84,54 & 610 \\
\hline XSMIN & 1,68 & 1,06 & 344,43 & $-5,18$ & 13,94 & 610 \\
\hline IMPV & 0,41 & 0,31 & 19,41 & $-7,13$ & 1,28 & 610 \\
\hline SKEW & $-0,17$ & $-0,15$ & 3,47 & $-14,93$ & 0,87 & 610 \\
\hline KURT & 3,03 & 3,01 & 15,26 & 0,88 & 0,75 & 610 \\
\hline \multicolumn{7}{|c|}{ Constant maturity of 240 days } \\
\hline B0 & 15,48 & 1,29 & $4.832,42$ & $-1.920,68$ & 252,40 & 610 \\
\hline B1 & $-26,84$ & $-1,66$ & $2.978,12$ & $-6.729,69$ & 407,28 & 610 \\
\hline B2 & 12,33 & 0,73 & $2.366,28$ & $-1.396,67$ & 175,79 & 610 \\
\hline SLP70 & $-9,57$ & $-0,66$ & $1.362,18$ & $-3.416,89$ & 170,70 & 610 \\
\hline SLP90 & $-4,64$ & $-0,35$ & 900,48 & $-2.470,38$ & 110,99 & 610 \\
\hline SLP100 & $-2,17$ & $-0,21$ & 669,64 & $-1.997,12$ & 87,28 & 610 \\
\hline SLP110 & 0,29 & $-0,08$ & 582,11 & $-1.523,87$ & 73,41 & 610 \\
\hline XSMIN & 1,72 & 1,06 & 344,43 & $-3,57$ & 13,95 & 610 \\
\hline SLP130 & 5,23 & 0,19 & $1.380,60$ & $-872,36$ & 91,37 & 610 \\
\hline IMPV & 0,97 & 0,31 & 469,02 & $-96,80$ & 19,45 & 610 \\
\hline SKEW & $-0,17$ & $-0,15$ & 3,47 & $-14,93$ & 0,91 & 610 \\
\hline KURT & 3,03 & 3,02 & 15,26 & 0,88 & 0,75 & 610 \\
\hline \multicolumn{7}{|c|}{ Smile independent variables } \\
\hline PRC & 31,51 & 34,29 & & 15,81 & 6,77 & 610 \\
\hline HV & 0,44 & 0,38 & 1,24 & 0,17 & 0,21 & 610 \\
\hline HSKEW & $-0,10$ & $-0,06$ & 8,57 & $-6,26$ & 1,31 & 610 \\
\hline HKURT & 7,46 & 4,21 & 99,79 & 0,02 & 10,99 & 610 \\
\hline CDS & 73,49 & 59,33 & 176,00 & 41,13 & 31,21 & 610 \\
\hline
\end{tabular}

Table B.23.: Summary statistics for cross section _METRO 


\section{B.3.19. _TUI}

\begin{tabular}{|c|c|c|c|c|c|c|}
\hline _TUI & Mean & Median & Max & Min & StdDev & Obs \\
\hline \multicolumn{7}{|c|}{ Constant maturity of 36 days } \\
\hline B0 & 20,06 & 3,87 & $3.644,99$ & $-524,17$ & 246,27 & 243 \\
\hline B1 & $-38,90$ & $-7,00$ & $1.106,34$ & $-7.273,18$ & 492,55 & 243 \\
\hline B2 & 19,13 & 3,36 & $3.628,41$ & $-580,38$ & 246,29 & 243 \\
\hline SLP70 & $-12,12$ & $-2,28$ & 293,80 & $-2.193,40$ & 147,80 & 243 \\
\hline SLP90 & $-4,47$ & $-0,93$ & 61,65 & $-742,04$ & 49,45 & 243 \\
\hline SLP100 & $-0,64$ & $-0,27$ & 9,30 & $-54,43$ & 4,98 & 243 \\
\hline SLP110 & 3,18 & 0,41 & 709,33 & $-170,51$ & 49,57 & 243 \\
\hline SLP130 & 10,83 & 1,79 & $2.160,69$ & $-402,66$ & 147,92 & 243 \\
\hline XSMIN & 1,03 & 1,02 & 5,16 & $-1,60$ & 0,40 & 243 \\
\hline IMPV & 0,29 & 0,30 & 1,78 & $-1,18$ & 0,15 & 243 \\
\hline SKEW & $-0,15$ & $-0,12$ & 1,21 & $-5,32$ & 0,48 & 243 \\
\hline KURT & 2,99 & 3,03 & 3,33 & 0,41 & 0,23 & 243 \\
\hline \multicolumn{7}{|c|}{ Constant maturity of 60 days } \\
\hline B0 & $-1,43$ & 2,39 & $3.253,65$ & $-4.746,35$ & 374,42 & 243 \\
\hline B1 & 6,50 & $-3,94$ & $10.093,69$ & $-6.492,38$ & 780,41 & 243 \\
\hline B2 & $-4,85$ & 1,79 & $3.238,97$ & $-5.365,96$ & 407,31 & 243 \\
\hline SLP70 & $-0,28$ & $-1,28$ & $2.581,35$ & $-1.957,83$ & 211,04 & 243 \\
\hline SLP90 & $-2,22$ & $-0,64$ & 434,96 & $-662,24$ & 51,95 & 243 \\
\hline SLP100 & $-3,19$ & $-0,25$ & 7,03 & $-638,23$ & 41,08 & 243 \\
\hline SLP110 & $-4,16$ & 0,12 & 633,35 & $-1.711,42$ & 118,10 & 243 \\
\hline SLP130 & $-6,10$ & 0,86 & $1.928,94$ & $-3.857,80$ & 279,79 & 243 \\
\hline XSMIN & 0,99 & 1,03 & 2,41 & $-4,68$ & 0,44 & 243 \\
\hline IMPV & 0,22 & 0,31 & 1,76 & $-18,61$ & 1,22 & 243 \\
\hline SKEW & $-0,20$ & $-0,14$ & 1,21 & $-5,32$ & 0,60 & 243 \\
\hline KURT & 2,98 & 3,03 & 3,74 & 0,41 & 0,25 & 243 \\
\hline \multicolumn{7}{|c|}{ Constant maturity of 180 days } \\
\hline B0 & $-24,31$ & 1,18 & $1.296,92$ & $-6.588,71$ & 446,24 & 243 \\
\hline B1 & 52,46 & $-1,47$ & $14.009,27$ & $-2.588,39$ & 940,42 & 243 \\
\hline B2 & $-28,10$ & 0,65 & $1.291,75$ & $-7.446,40$ & 496,44 & 243 \\
\hline SLP70 & 13,12 & $-0,57$ & $3.584,30$ & $-779,94$ & 246,63 & 243 \\
\hline SLP90 & 1,88 & $-0,29$ & 605,74 & $-263,24$ & 54,47 & 243 \\
\hline SLP100 & $-3,73$ & $-0,17$ & 224,18 & $-883,54$ & 60,09 & 243 \\
\hline SLP110 & $-9,35$ & $-0,06$ & 253,46 & $-2.372,82$ & 154,83 & 243 \\
\hline SLP130 & $-20,59$ & 0,18 & 770,16 & $-5.351,38$ & 351,91 & 243 \\
\hline XSMIN & 1,07 & 1,05 & 12,02 & $-4,02$ & 1,05 & 243 \\
\hline IMPV & 0,05 & 0,34 & 0,97 & $-27,87$ & 2,54 & 243 \\
\hline SKEW & $-0,20$ & $-0,14$ & 1,51 & $-9,33$ & 0,79 & 243 \\
\hline KURT & 2,98 & 3,02 & 4,66 & 0,80 & 0,30 & 243 \\
\hline \multicolumn{7}{|c|}{ Constant maturity of 240 days } \\
\hline B0 & $-4,77$ & 1,07 & 837,17 & $-2.411,46$ & 170,35 & 243 \\
\hline B1 & 9,75 & $-1,25$ & $4.428,20$ & $-1.668,20$ & 320,06 & 243 \\
\hline B2 & $-4,90$ & 0,56 & 831,26 & $-2.032,59$ & 151,99 & 243 \\
\hline SLP70 & 2,89 & $-0,51$ & $1.582,58$ & $-504,44$ & 109,54 & 243 \\
\hline SLP90 & 0,92 & $-0,29$ & 769,54 & $-196,74$ & 52,84 & 243 \\
\hline SLP100 & $-0,06$ & $-0,17$ & 363,03 & $-314,03$ & 31,00 & 243 \\
\hline SLP110 & $-1,04$ & $-0,05$ & 160,57 & $-431,31$ & 31,28 & 243 \\
\hline XSMIN & 1,36 & 1,05 & 85,29 & $-4,02$ & 5,43 & 243 \\
\hline SLP130 & $-3,00$ & 0,18 & 493,07 & $-856,53$ & 80,98 & 243 \\
\hline IMPV & 0,07 & 0,34 & 1,02 & $-41,54$ & 2,88 & 243 \\
\hline SKEW & $-0,19$ & $-0,14$ & 1,51 & $-10,22$ & 0,97 & 243 \\
\hline KURT & 3,01 & 3,02 & 11,68 & 0,80 & 0,63 & 243 \\
\hline \multicolumn{7}{|c|}{ Smile independent variables } \\
\hline PRC & 16,66 & & & 13,25 & 1,67 & 243 \\
\hline HV & 0,34 & 0,32 & 1,03 & 0,18 & 0,12 & 243 \\
\hline HSKEW & $-0,14$ & $-0,08$ & 3,13 & $-7,50$ & 1,24 & 243 \\
\hline HKURT & 6,31 & 3,42 & 84,78 & 0,16 & 8,96 & 243 \\
\hline CDS & 246,37 & 249,50 & 350,00 & 170,25 & 33,19 & 243 \\
\hline
\end{tabular}

Table B.24.: Summary statistics for cross section _TUI 


\section{B.3.20. LIN}

\begin{tabular}{|c|c|c|c|c|c|c|}
\hline _LIN & Mean & Median & Max & Min & StdDev & Obs \\
\hline \multicolumn{7}{|c|}{ Constant maturity of 36 days } \\
\hline B0 & 73,88 & 3,00 & $36.563,44$ & $-5.739,73$ & $1.749,41$ & 478 \\
\hline B1 & $-136,01$ & $-5,07$ & $16.579,96$ & $-72.776,82$ & $3.530,02$ & 478 \\
\hline B2 & 54,56 & 2,39 & $36.214,54$ & $-15.701,01$ & $1.857,58$ & 478 \\
\hline SLP70 & $-59,63$ & $-1,76$ & $4.060,24$ & $-22.076,47$ & $1.080,64$ & 478 \\
\hline SLP90 & $-37,81$ & $-0,82$ & $2.640,21$ & $-11.681,85$ & 652,35 & 478 \\
\hline SLP100 & $-26,90$ & $-0,28$ & $1.930,19$ & $-14.822,05$ & 684,49 & 478 \\
\hline SLP110 & $-15,99$ & 0,16 & $6.895,16$ & $-17.962,26$ & 887,44 & 478 \\
\hline SLP130 & 5,84 & 1,01 & $21.380,97$ & $-24.242,66$ & $1.501,24$ & 478 \\
\hline XSMIN & 0,98 & 1,03 & 6,56 & $-19,02$ & 1,01 & 478 \\
\hline IMPV & $-7,58$ & 0,24 & 11,65 & $-3.495,85$ & 160,33 & 478 \\
\hline SKEW & $-0,14$ & $-0,16$ & 4,20 & $-15,72$ & 0,88 & 478 \\
\hline KURT & 3,07 & 3,03 & 14,32 & 0,07 & 0,76 & 478 \\
\hline \multicolumn{7}{|c|}{ Constant maturity of 60 days } \\
\hline B0 & $-5,84$ & 2,37 & $28.513,02$ & $-24.556,99$ & $1.824,34$ & 478 \\
\hline B1 & 107,60 & $-3,92$ & $92.971,62$ & $-56.752,89$ & $5.140,62$ & 478 \\
\hline B2 & $-142,59$ & 1,77 & $28.240,83$ & $-87.993,11$ & $4.279,15$ & 478 \\
\hline SLP70 & $-92,03$ & $-1,37$ & $5.695,27$ & $-30.218,73$ & $1.625,18$ & 478 \\
\hline SLP90 & $-149,06$ & $-0,67$ & 889,49 & $-65.415,98$ & $3.004,92$ & 478 \\
\hline SLP100 & $-177,58$ & $-0,26$ & 239,88 & $-83.014,60$ & $3.797,51$ & 478 \\
\hline SLP110 & $-206,10$ & 0,05 & $5.376,93$ & $-100.613,20$ & $4.612,76$ & 478 \\
\hline SLP130 & $-263,13$ & 0,72 & $16.673,26$ & $-135.810,50$ & $6.275,75$ & 478 \\
\hline XSMIN & 0,96 & 1,03 & 6,56 & $-19,02$ & 1,04 & 478 \\
\hline IMPV & $-40,84$ & 0,23 & 8,41 & $-19.578,48$ & 895,51 & 478 \\
\hline SKEW & $-0,14$ & $-0,16$ & 4,20 & $-15,72$ & 0,89 & 478 \\
\hline KURT & 3,07 & 3,03 & 14,32 & 0,07 & 0,78 & 478 \\
\hline \multicolumn{7}{|c|}{ Constant maturity of 180 days } \\
\hline B0 & $-67,83$ & 1,67 & $4.292,21$ & $-24.556,99$ & $1.486,48$ & 478 \\
\hline B1 & 234,78 & $-2,57$ & $92.971,62$ & $-8.428,58$ & $4.721,34$ & 478 \\
\hline B2 & $-207,70$ & 1,22 & $4.137,94$ & $-87.993,11$ & $4.167,55$ & 478 \\
\hline SLP70 & $-56,01$ & $-1,00$ & $10.633,90$ & $-30.218,73$ & $1.483,45$ & 478 \\
\hline SLP90 & $-139,09$ & $-0,51$ & $1.661,33$ & $-65.415,98$ & $2.994,30$ & 478 \\
\hline SLP100 & $-180,63$ & $-0,24$ & 250,40 & $-83.014,60$ & $3.799,03$ & 478 \\
\hline SLP110 & $-222,17$ & $-0,01$ & 674,90 & $-100.613,20$ & $4.613,99$ & 478 \\
\hline SLP130 & $-305,25$ & 0,51 & $2.330,08$ & $-135.810,50$ & $6.258,59$ & 478 \\
\hline XSMIN & 0,94 & 1,03 & 6,92 & $-38,62$ & 1,93 & 478 \\
\hline IMPV & $-40,76$ & 0,24 & 48,70 & $-19.578,48$ & 895,52 & 478 \\
\hline SKEW & $-0,14$ & $-0,17$ & 4,20 & $-15,72$ & 0,93 & 478 \\
\hline KURT & 3,08 & 3,04 & 14,32 & 0,07 & 0,83 & 478 \\
\hline \multicolumn{7}{|c|}{ Constant maturity of 240 days } \\
\hline Bo & $-63,97$ & 1,82 & $6.210,88$ & $-24.556,99$ & $1.501,91$ & 478 \\
\hline B1 & 227,74 & $-2,93$ & $92.971,62$ & $-11.439,82$ & $4.737,95$ & 478 \\
\hline B2 & $-204,50$ & 1,37 & $5.267,98$ & $-87.993,11$ & $4.171,60$ & 478 \\
\hline SLP70 & $-58,56$ & $-1,05$ & $10.633,90$ & $-30.218,73$ & $1.489,95$ & 478 \\
\hline SLP90 & $-140,36$ & $-0,53$ & $1.661,33$ & $-65.415,98$ & $2.995,01$ & 478 \\
\hline SLP100 & $-181,26$ & $-0,24$ & 250,40 & $-83.014,60$ & $3.799,13$ & 478 \\
\hline SLP110 & $-222,16$ & 0,00 & 674,90 & $-100.613,20$ & $4.613,99$ & 478 \\
\hline XSMIN & 1,02 & 1,03 & 6,92 & $-6,35$ & 0,66 & 478 \\
\hline SLP130 & $-303,96$ & 0,51 & $2.330,08$ & $-135.810,50$ & $6.259,11$ & 478 \\
\hline IMPV & $-40,73$ & 0,24 & 48,70 & $-19.578,48$ & 895,52 & 478 \\
\hline SKEW & $-0,14$ & $-0,17$ & 4,20 & $-15,72$ & 0,95 & 478 \\
\hline KURT & 3,09 & 3,04 & 14,32 & 0,07 & 0,86 & 478 \\
\hline \multicolumn{7}{|c|}{ Smile independent variables } \\
\hline PRC & 41,72 & 42,86 & & 23,26 & 7,03 & 478 \\
\hline HV & 0,36 & 0,31 & 0,92 & 0,14 & 0,16 & 478 \\
\hline HSKEW & $-0,11$ & $-0,03$ & 7,13 & $-8,31$ & 1,26 & 478 \\
\hline HKURT & 6,77 & 3,90 & 97,85 & 0,39 & 9,97 & 478 \\
\hline CDS & 42,59 & 37,53 & 100,83 & 22,55 & 14,08 & 478 \\
\hline
\end{tabular}

Table B.25.: Summary statistics for cross section LIN 


\section{B.3.21. HEN}

\begin{tabular}{|c|c|c|c|c|c|c|}
\hline _HEN & Mean & Median & Max & Min & StdDev & Obs \\
\hline \multicolumn{7}{|c|}{ Constant maturity of 36 days } \\
\hline B0 & 146,83 & 3,11 & $45.714,21$ & $-2.112,16$ & $2.363,16$ & 383 \\
\hline B1 & $-305,51$ & $-5,59$ & $4.195,25$ & $-95.651,84$ & $4.939,53$ & 383 \\
\hline B2 & 159,17 & 2,62 & $50.035,39$ & $-2.083,01$ & $2.581,46$ & 383 \\
\hline SLP70 & $-82,67$ & $-1,80$ & $1.279,05$ & $-25.602,29$ & $1.325,84$ & 383 \\
\hline SLP90 & $-19,00$ & $-0,84$ & 445,84 & $-5.588,13$ & 294,92 & 383 \\
\hline SLP100 & 12,83 & $-0,26$ & $4.418,95$ & $-51,76$ & 226,32 & 383 \\
\hline SLP110 & 44,67 & 0,22 & $14.426,03$ & $-387,36$ & 740,66 & 383 \\
\hline SLP130 & 108,34 & 1,24 & $34.440,18$ & $-1.220,56$ & $1.772,75$ & 383 \\
\hline XSMIN & 0,99 & 1,02 & 4,89 & $-11,43$ & 0,89 & 383 \\
\hline IMPV & 0,49 & 0,20 & 97,76 & $-3,16$ & 5,01 & 383 \\
\hline SKEW & $-0,12$ & $-0,13$ & 2,05 & $-7,79$ & 0,56 & 383 \\
\hline KURT & 2,98 & 3,03 & 6,19 & 0,35 & 0,36 & 383 \\
\hline \multicolumn{7}{|c|}{ Constant maturity of 60 days } \\
\hline B0 & 138,56 & 2,81 & $45.714,21$ & $-1.801,14$ & $2.364,37$ & 383 \\
\hline B1 & $-289,76$ & $-4,85$ & $3.577,57$ & $-95.651,84$ & $4.941,36$ & 383 \\
\hline B2 & 151,67 & 2,25 & $50.035,39$ & $-1.776,33$ & $2.582,13$ & 383 \\
\hline SLP70 & $-77,42$ & $-1,68$ & $1.124,34$ & $-25.602,29$ & $1.326,82$ & 383 \\
\hline SLP90 & $-16,75$ & $-0,77$ & 529,86 & $-5.588,13$ & 296,07 & 383 \\
\hline SLP100 & 13,58 & $-0,24$ & $4.418,95$ & $-51,76$ & 226,60 & 383 \\
\hline SLP110 & 43,92 & 0,18 & $14.426,03$ & $-330,35$ & 740,56 & 383 \\
\hline SLP130 & 104,58 & 1,04 & $34.440,18$ & $-1.040,88$ & $1.772,79$ & 383 \\
\hline XSMIN & 1,00 & 1,02 & 4,35 & $-8,54$ & 0,72 & 383 \\
\hline IMPV & 0,47 & 0,20 & 97,76 & $-8,87$ & 5,03 & 383 \\
\hline SKEW & $-0,12$ & $-0,13$ & 3,07 & $-7,79$ & 0,59 & 383 \\
\hline KURT & 3,02 & 3,03 & 12,33 & 0,35 & 0,59 & 383 \\
\hline \multicolumn{7}{|c|}{ Constant maturity of 180 days } \\
\hline B0 & 138,69 & 2,13 & $42.448,98$ & $-2.855,83$ & $2.204,65$ & 383 \\
\hline B1 & $-286,34$ & $-3,42$ & $5.532,07$ & $-88.819,67$ & $4.599,01$ & 383 \\
\hline B2 & 148,21 & 1,57 & $46.461,49$ & $-2.678,85$ & $2.399,96$ & 383 \\
\hline SLP70 & $-78,84$ & $-1,21$ & $1.781,68$ & $-23.773,59$ & $1.240,82$ & 383 \\
\hline SLP90 & $-19,56$ & $-0,62$ & 710,15 & $-5.188,99$ & 288,92 & 383 \\
\hline SLP100 & 10,09 & $-0,22$ & $4.103,31$ & $-944,16$ & 215,79 & 383 \\
\hline SLP110 & 39,73 & 0,11 & $13.395,60$ & $-361,39$ & 685,89 & 383 \\
\hline SLP130 & 99,02 & 0,74 & $31.980,20$ & $-1.432,93$ & $1.643,33$ & 383 \\
\hline XSMIN & 1,02 & 1,03 & 4,35 & $-7,64$ & 0,58 & 383 \\
\hline IMPV & 0,56 & 0,21 & 90,80 & $-6,27$ & 5,15 & 383 \\
\hline SKEW & $-0,15$ & $-0,13$ & 3,40 & $-11,32$ & 0,86 & 383 \\
\hline KURT & 3,05 & 3,03 & 15,42 & 0,35 & 0,90 & 383 \\
\hline \multicolumn{7}{|c|}{ Constant maturity of 240 days } \\
\hline B0 & 97,08 & 2,06 & $27.378,69$ & $-2.855,83$ & $1.449,89$ & 383 \\
\hline B1 & $-199,81$ & $-3,34$ & $5.532,07$ & $-57.286,60$ & $3.013,53$ & 383 \\
\hline B2 & 103,20 & 1,55 & $29.966,55$ & $-2.678,85$ & $1.568,08$ & 383 \\
\hline SLP70 & $-55,32$ & $-1,20$ & $1.781,68$ & $-15.333,44$ & 820,77 & 383 \\
\hline SLP90 & $-14,04$ & $-0,59$ & 710,15 & $-3.346,82$ & 204,60 & 383 \\
\hline SLP100 & 6,60 & $-0,21$ & $2.646,49$ & $-944,16$ & 145,01 & 383 \\
\hline SLP110 & 27,24 & 0,08 & $8.639,80$ & $-361,39$ & 443,74 & 383 \\
\hline XSMIN & 1,02 & 1,03 & 4,35 & $-7,64$ & 0,54 & 383 \\
\hline SLP130 & 68,52 & 0,72 & $20.626,42$ & $-1.432,93$ & $1.067,14$ & 383 \\
\hline IMPV & 0,47 & 0,21 & 58,63 & $-11,48$ & 3,80 & 383 \\
\hline SKEW & $-0,16$ & $-0,13$ & 3,40 & $-12,32$ & 0,94 & 383 \\
\hline KURT & 3,06 & 3,04 & 16,52 & 0,35 & 0,95 & 383 \\
\hline \multicolumn{7}{|c|}{ Smile independent variables } \\
\hline PRC & 62,42 & & & 50,79 & 5,51 & 383 \\
\hline HV & 0,30 & 0,26 & 1,38 & 0,12 & 0,13 & 383 \\
\hline HSKEW & $-0,12$ & $-0,02$ & 9,22 & $-9,50$ & 1,43 & 383 \\
\hline HKURT & 8,53 & 4,97 & 120,93 & 0,72 & 12,38 & 383 \\
\hline CDS & 26,65 & 24,25 & 39,33 & 19,75 & 5,69 & 383 \\
\hline
\end{tabular}

Table B.26.: Summary statistics for cross section _HEN 


\section{B.3.22. CONT}

\begin{tabular}{|c|c|c|c|c|c|c|}
\hline _CONT & Mean & Median & Max & Min & StdDev & Obs \\
\hline \multicolumn{7}{|c|}{ Constant maturity of 36 days } \\
\hline B0 & 31,94 & 3,09 & $3.405,15$ & $-2.974,32$ & 384,40 & 277 \\
\hline B1 & $-63,14$ & $-5,44$ & $6.016,20$ & $-6.598,32$ & 772,85 & 277 \\
\hline B2 & 31,52 & 2,60 & $3.196,66$ & $-3.042,02$ & 389,26 & 277 \\
\hline SLP70 & $-19,01$ & $-1,84$ & $1.757,38$ & $-2.123,00$ & 228,87 & 277 \\
\hline SLP90 & $-6,40$ & $-0,81$ & 540,57 & $-844,34$ & 76,09 & 277 \\
\hline SLP100 & $-0,10$ & $-0,29$ & 339,53 & $-205,00$ & 26,01 & 277 \\
\hline SLP110 & 6,21 & 0,20 & 852,24 & $-676,23$ & 87,67 & 277 \\
\hline SLP130 & 18,82 & 1,22 & $1.877,67$ & $-1.893,04$ & 240,98 & 277 \\
\hline XSMIN & 0,82 & 1,01 & 2,70 & $-50,73$ & 3,11 & 277 \\
\hline IMPV & 0,32 & 0,26 & 11,51 & $-1,19$ & 0,74 & 277 \\
\hline SKEW & $-0,10$ & $-0,14$ & 5,05 & $-7,33$ & 0,81 & 277 \\
\hline KURT & 3,03 & 3,03 & 18,95 & 0,02 & 1,05 & 277 \\
\hline \multicolumn{7}{|c|}{ Constant maturity of 60 days } \\
\hline B0 & 25,78 & 2,34 & $4.144,41$ & $-2.974,32$ & 413,44 & 277 \\
\hline B1 & $-50,61$ & $-3,94$ & $6.016,20$ & $-8.030,98$ & 831,12 & 277 \\
\hline B2 & 25,18 & 1,91 & $3.890,78$ & $-3.042,02$ & 418,68 & 277 \\
\hline SLP70 & $-15,36$ & $-1,35$ & $1.757,38$ & $-2.583,89$ & 246,04 & 277 \\
\hline SLP90 & $-5,29$ & $-0,60$ & 540,57 & $-1.027,58$ & 81,74 & 277 \\
\hline SLP100 & $-0,25$ & $-0,27$ & 339,53 & $-249,43$ & 28,11 & 277 \\
\hline SLP110 & 4,78 & 0,11 & 852,24 & $-676,23$ & 94,46 & 277 \\
\hline SLP130 & 14,86 & 0,90 & $2.085,04$ & $-1.893,04$ & 259,34 & 277 \\
\hline XSMIN & 1,00 & 1,02 & 2,70 & $-1,07$ & 0,27 & 277 \\
\hline IMPV & 0,35 & 0,26 & 11,51 & $-1,45$ & 0,87 & 277 \\
\hline SKEW & $-0,11$ & $-0,14$ & 5,05 & $-7,33$ & 0,81 & 277 \\
\hline KURT & 3,02 & 3,03 & 18,95 & 0,02 & 1,06 & 277 \\
\hline \multicolumn{7}{|c|}{ Constant maturity of 180 days } \\
\hline B0 & 19,61 & 1,81 & $4.144,41$ & $-5.329,25$ & 517,85 & 277 \\
\hline B1 & $-37,66$ & $-2,75$ & $10.943,59$ & $-8.030,98$ & $1.047,33$ & 277 \\
\hline B2 & 18,37 & 1,22 & $3.890,78$ & $-5.617,83$ & 530,35 & 277 \\
\hline SLP70 & $-11,94$ & $-1,04$ & $3.078,63$ & $-2.583,89$ & 305,82 & 277 \\
\hline SLP90 & $-4,60$ & $-0,50$ & 831,50 & $-1.027,58$ & 96,77 & 277 \\
\hline SLP100 & $-0,92$ & $-0,26$ & 335,77 & $-292,07$ & 32,18 & 277 \\
\hline SLP110 & 2,75 & $-0,01$ & 843,05 & $-1.415,64$ & 123,33 & 277 \\
\hline SLP130 & 10,10 & 0,51 & $2.085,04$ & $-3.662,77$ & 333,26 & 277 \\
\hline XSMIN & 1,02 & 1,02 & 3,08 & $-1,33$ & 0,39 & 277 \\
\hline IMPV & 0,32 & 0,26 & 11,38 & $-3,49$ & 0,77 & 277 \\
\hline SKEW & $-0,10$ & $-0,15$ & 5,05 & $-7,33$ & 0,83 & 277 \\
\hline KURT & 3,02 & 3,03 & 18,95 & 0,02 & 1,07 & 277 \\
\hline \multicolumn{7}{|c|}{ Constant maturity of 240 days } \\
\hline Bo & 16,39 & 1,78 & $4.144,41$ & $-5.329,25$ & 513,58 & 277 \\
\hline B1 & $-30,89$ & $-2,82$ & $10.943,59$ & $-8.030,98$ & $1.036,80$ & 277 \\
\hline B2 & 14,81 & 1,28 & $3.890,78$ & $-5.617,83$ & 523,93 & 277 \\
\hline SLP70 & $-10,15$ & $-1,03$ & $3.078,63$ & $-2.583,89$ & 304,12 & 277 \\
\hline SLP90 & $-4,23$ & $-0,48$ & 831,50 & $-1.027,58$ & 97,09 & 277 \\
\hline SLP100 & $-1,26$ & $-0,26$ & 223,07 & $-292,07$ & 28,89 & 277 \\
\hline SLP110 & 1,70 & $-0,01$ & 567,08 & $-1.415,64$ & 119,17 & 277 \\
\hline XSMIN & 1,02 & 1,02 & 3,08 & $-1,33$ & 0,38 & 277 \\
\hline SLP130 & 7,62 & 0,51 & $2.085,04$ & $-3.662,77$ & 326,83 & 277 \\
\hline IMPV & 0,32 & 0,26 & 7,68 & $-3,49$ & 0,60 & 277 \\
\hline SKEW & $-0,10$ & $-0,15$ & 5,05 & $-7,33$ & 0,83 & 277 \\
\hline KURT & 3,02 & 3,03 & 18,95 & 0,02 & 1,07 & 277 \\
\hline \multicolumn{7}{|c|}{ Smile independent variables } \\
\hline PRC & 34,21 & 35,24 & & & 8,52 & 277 \\
\hline HV & 0,31 & 0,28 & 1,16 & 0,12 & 0,12 & 277 \\
\hline HSKEW & $-0,04$ & 0,04 & 6,58 & $-12,23$ & 1,68 & 277 \\
\hline HKURT & 8,77 & 4,48 & 172,59 & 0,23 & 15,70 & 277 \\
\hline CDS & 48,22 & 45,63 & 151,75 & 31,41 & 19,19 & 277 \\
\hline
\end{tabular}

Table B.27.: Summary statistics for cross section_CONT 
B.3.23. DP

\begin{tabular}{|c|c|c|c|c|c|c|}
\hline _DP & Mean & Median & Max & Min & StdDev & Obs \\
\hline \multicolumn{7}{|c|}{ Constant maturity of 36 days } \\
\hline B0 & 67,45 & 5,55 & $2.429,21$ & $-3.199,07$ & 602,83 & 58 \\
\hline B1 & $-127,71$ & $-10,58$ & $6.500,71$ & $-4.769,64$ & $1.202,86$ & 58 \\
\hline B2 & 60,49 & 5,13 & $2.341,41$ & $-3.302,25$ & 600,44 & 58 \\
\hline SLP70 & $-43,03$ & $-3,20$ & $1.877,56$ & $-1.491,67$ & 362,72 & 58 \\
\hline SLP90 & $-18,84$ & $-1,28$ & 556,66 & $-555,10$ & 123,89 & 58 \\
\hline SLP100 & $-6,74$ & $-0,32$ & 3,32 & $-107,71$ & 22,49 & 58 \\
\hline SLP110 & 5,36 & 0,76 & 381,46 & $-764,24$ & 120,43 & 58 \\
\hline SLP130 & 29,55 & 2,78 & $1.318,02$ & $-2.085,14$ & 359,21 & 58 \\
\hline XSMIN & 1,18 & 1,03 & 8,79 & 0,86 & 1,03 & 58 \\
\hline IMPV & 0,22 & 0,19 & 1,91 & $-0,61$ & 0,28 & 58 \\
\hline SKEW & $-0,01$ & $-0,13$ & 4,20 & $-0,79$ & 0,72 & 58 \\
\hline KURT & 2,98 & 3,04 & 5,06 & 0,03 & 0,56 & 58 \\
\hline \multicolumn{7}{|c|}{ Constant maturity of 60 days } \\
\hline B0 & 191,95 & 4,48 & $9.698,60$ & $-1.483,84$ & $1.297,48$ & 58 \\
\hline B1 & $-375,42$ & $-8,25$ & $3.015,59$ & $-19.044,13$ & $2.550,24$ & 58 \\
\hline B2 & 183,71 & 3,91 & $9.348,91$ & $-1.531,93$ & $1.253,21$ & 58 \\
\hline SLP70 & $-118,23$ & $-2,44$ & 870,89 & $-5.955,65$ & 795,83 & 58 \\
\hline SLP90 & $-44,75$ & $-1,05$ & 258,12 & $-2.216,09$ & 294,76 & 58 \\
\hline SLP100 & $-8,00$ & $-0,33$ & 20,40 & $-346,30$ & 45,97 & 58 \\
\hline SLP110 & 28,74 & 0,60 & $1.523,48$ & $-354,65$ & 207,33 & 58 \\
\hline SLP130 & 102,22 & 1,84 & $5.263,05$ & $-967,42$ & 708,28 & 58 \\
\hline XSMIN & 1,07 & 1,04 & 1,95 & 0,86 & 0,18 & 58 \\
\hline IMPV & 0,24 & 0,20 & 3,38 & $-0,22$ & 0,43 & 58 \\
\hline SKEW & $-0,04$ & $-0,16$ & 4,20 & $-0,79$ & 0,66 & 58 \\
\hline KURT & 2,94 & 3,04 & 3,89 & 0,03 & 0,48 & 58 \\
\hline \multicolumn{7}{|c|}{ Constant maturity of 180 days } \\
\hline B0 & 285,78 & 3,36 & $10.607,27$ & $-474,52$ & $1.457,52$ & 58 \\
\hline B1 & $-564,41$ & $-6,06$ & 917,08 & $-20.828,44$ & $2.865,97$ & 58 \\
\hline B2 & 278,87 & 2,88 & $10.224,85$ & $-442,96$ & $1.408,94$ & 58 \\
\hline SLP70 & $-173,99$ & $-1,99$ & 296,93 & $-6.513,65$ & 893,54 & 58 \\
\hline SLP90 & $-62,44$ & $-0,80$ & 119,75 & $-2.423,71$ & 330,19 & 58 \\
\hline SLP100 & $-6,66$ & $-0,24$ & 34,72 & $-378,74$ & 50,44 & 58 \\
\hline SLP110 & 49,11 & 0,32 & $1.666,23$ & $-57,44$ & 234,25 & 58 \\
\hline SLP130 & 160,66 & 1,44 & $5.756,17$ & $-234,62$ & 797,47 & 58 \\
\hline XSMIN & 1,11 & 1,03 & 2,60 & 0,86 & 0,26 & 58 \\
\hline IMPV & 0,25 & 0,20 & 3,68 & $-0,67$ & 0,48 & 58 \\
\hline SKEW & 0,02 & $-0,14$ & 4,20 & $-0,79$ & 0,71 & 58 \\
\hline KURT & 2,97 & 3,04 & 4,17 & 0,03 & 0,50 & 58 \\
\hline \multicolumn{7}{|c|}{ Constant maturity of 240 days } \\
\hline B0 & 289,04 & 3,24 & $10.607,27$ & $-474,52$ & $1.461,66$ & 58 \\
\hline B1 & $-571,08$ & $-5,79$ & 917,08 & $-20.828,44$ & $2.874,69$ & 58 \\
\hline B2 & 282,30 & 2,75 & $10.224,85$ & $-442,96$ & $1.413,53$ & 58 \\
\hline SLP70 & $-175,86$ & $-1,95$ & 296,93 & $-6.513,65$ & 895,87 & 58 \\
\hline SLP90 & $-62,95$ & $-0,78$ & 119,75 & $-2.423,71$ & 330,73 & 58 \\
\hline SLP100 & $-6,49$ & $-0,22$ & 43,65 & $-378,74$ & 50,56 & 58 \\
\hline SLP110 & 49,97 & 0,32 & $1.666,23$ & $-57,44$ & 235,74 & 58 \\
\hline XSMIN & 1,15 & 1,04 & 4,09 & 0,86 & 0,43 & 58 \\
\hline SLP130 & 162,89 & 1,36 & $5.756,17$ & $-234,62$ & 800,71 & 58 \\
\hline IMPV & 0,25 & 0,20 & 3,68 & $-0,42$ & 0,48 & 58 \\
\hline SKEW & 0,02 & $-0,14$ & 4,20 & $-0,79$ & 0,72 & 58 \\
\hline KURT & 2,96 & 3,04 & 3,89 & 0,03 & 0,48 & 58 \\
\hline \multicolumn{7}{|c|}{ Smile independent variables } \\
\hline PRC & 16,15 & & & 15,04 & 0,57 & 58 \\
\hline HV & 0,23 & 0,22 & 0,44 & 0,15 & 0,05 & 58 \\
\hline HSKEW & $-0,37$ & $-0,14$ & 1,56 & $-7,01$ & 1,31 & 58 \\
\hline HKURT & 6,09 & 2,58 & 71,98 & 0,01 & 11,85 & 58 \\
\hline CDS & 19,76 & 15,55 & 50,00 & 14,25 & 8,26 & 58 \\
\hline
\end{tabular}

Table B.28.: Summary statistics for cross section DP 


\section{B.3.24. DEG}

\begin{tabular}{|c|c|c|c|c|c|c|}
\hline _DEG & Mean & Median & Max & Min & StdDev & Obs \\
\hline \multicolumn{7}{|c|}{ Constant maturity of 36 days } \\
\hline B0 & $-229,81$ & $-8,77$ & 109,42 & $-790,10$ & 488,80 & 3 \\
\hline B1 & 439,12 & 18,01 & $1.509,57$ & $-210,21$ & 934,03 & 3 \\
\hline B2 & $-209,53$ & $-9,02$ & 101,18 & $-720,76$ & 446,15 & 3 \\
\hline SLP70 & 145,78 & 5,38 & 500,51 & $-68,56$ & 309,42 & 3 \\
\hline SLP90 & 61,96 & 1,78 & 212,21 & $-28,09$ & 130,97 & 3 \\
\hline SLP100 & 20,06 & $-0,03$ & 68,06 & $-7,85$ & 41,75 & 3 \\
\hline SLP110 & $-21,85$ & $-1,83$ & 12,38 & $-76,09$ & 47,51 & 3 \\
\hline SLP130 & $-105,66$ & $-5,44$ & 52,85 & $-364,40$ & 225,96 & 3 \\
\hline XSMIN & 1,03 & 1,04 & 1,05 & 1,00 & 0,03 & 3 \\
\hline IMPV & $-0,22$ & 0,22 & 0,39 & $-1,28$ & 0,92 & 3 \\
\hline SKEW & 0,37 & 0,38 & 0,73 & $-0,01$ & 0,37 & 3 \\
\hline KURT & 2,57 & 2,91 & 2,92 & 1,88 & 0,60 & 3 \\
\hline \multicolumn{7}{|c|}{ Constant maturity of 60 days } \\
\hline B0 & $-229,81$ & $-8,77$ & 109,42 & $-790,10$ & 488,80 & 3 \\
\hline B1 & 439,12 & 18,01 & $1.509,57$ & $-210,21$ & 934,03 & 3 \\
\hline B2 & $-209,53$ & $-9,02$ & 101,18 & $-720,76$ & 446,15 & 3 \\
\hline SLP70 & 145,78 & 5,38 & 500,51 & $-68,56$ & 309,42 & 3 \\
\hline SLP90 & 61,96 & 1,78 & 212,21 & $-28,09$ & 130,97 & 3 \\
\hline SLP100 & 20,06 & $-0,03$ & 68,06 & $-7,85$ & 41,75 & 3 \\
\hline SLP110 & $-21,85$ & $-1,83$ & 12,38 & $-76,09$ & 47,51 & 3 \\
\hline SLP130 & $-105,66$ & $-5,44$ & 52,85 & $-364,40$ & 225,96 & 3 \\
\hline XSMIN & 1,03 & 1,04 & 1,05 & 1,00 & 0,03 & 3 \\
\hline IMPV & $-0,22$ & 0,22 & 0,39 & $-1,28$ & 0,92 & 3 \\
\hline SKEW & 0,37 & 0,38 & 0,73 & $-0,01$ & 0,37 & 3 \\
\hline KURT & 2,57 & 2,91 & 2,92 & 1,88 & 0,60 & 3 \\
\hline \multicolumn{7}{|c|}{ Constant maturity of 180 days } \\
\hline B0 & $-229,81$ & $-8,77$ & 109,42 & $-790,10$ & 488,80 & 3 \\
\hline B1 & 439,12 & 18,01 & $1.509,57$ & $-210,21$ & 934,03 & 3 \\
\hline B2 & $-209,53$ & $-9,02$ & 101,18 & $-720,76$ & 446,15 & 3 \\
\hline SLP70 & 145,78 & 5,38 & 500,51 & $-68,56$ & 309,42 & 3 \\
\hline SLP90 & 61,96 & 1,78 & 212,21 & $-28,09$ & 130,97 & 3 \\
\hline SLP100 & 20,06 & $-0,03$ & 68,06 & $-7,85$ & 41,75 & 3 \\
\hline SLP110 & $-21,85$ & $-1,83$ & 12,38 & $-76,09$ & 47,51 & 3 \\
\hline SLP130 & $-105,66$ & $-5,44$ & 52,85 & $-364,40$ & 225,96 & 3 \\
\hline XSMIN & 1,03 & 1,04 & 1,05 & 1,00 & 0,03 & 3 \\
\hline IMPV & $-0,22$ & 0,22 & 0,39 & $-1,28$ & 0,92 & 3 \\
\hline SKEW & 0,37 & 0,38 & 0,73 & $-0,01$ & 0,37 & 3 \\
\hline KURT & 2,57 & 2,91 & 2,92 & 1,88 & 0,60 & 3 \\
\hline \multicolumn{7}{|c|}{ Constant maturity of 240 days } \\
\hline Bo & $-229,81$ & $-8,77$ & 109,42 & $-790,10$ & 488,80 & 3 \\
\hline B1 & 439,12 & 18,01 & $1.509,57$ & $-210,21$ & 934,03 & 3 \\
\hline B2 & $-209,53$ & $-9,02$ & 101,18 & $-720,76$ & 446,15 & 3 \\
\hline SLP70 & 145,78 & 5,38 & 500,51 & $-68,56$ & 309,42 & 3 \\
\hline SLP90 & 61,96 & 1,78 & 212,21 & $-28,09$ & 130,97 & 3 \\
\hline SLP100 & 20,06 & $-0,03$ & 68,06 & $-7,85$ & 41,75 & 3 \\
\hline SLP110 & $-21,85$ & $-1,83$ & 12,38 & $-76,09$ & 47,51 & 3 \\
\hline XSMIN & 1,03 & 1,04 & 1,05 & 1,00 & 0,03 & 3 \\
\hline SLP130 & $-105,66$ & $-5,44$ & 52,85 & $-364,40$ & 225,96 & 3 \\
\hline IMPV & $-0,22$ & 0,22 & 0,39 & $-1,28$ & 0,92 & 3 \\
\hline SKEW & 0,37 & 0,38 & 0,73 & $-0,01$ & 0,37 & 3 \\
\hline KURT & 2,57 & 2,91 & 2,92 & 1,88 & 0,60 & 3 \\
\hline \multicolumn{7}{|c|}{ Smile independent variables } \\
\hline PRC & 29,79 & 30,55 & 30,60 & 28,22 & 1,36 & 3 \\
\hline HV & 0,36 & 0,36 & 0,46 & 0,25 & 0,11 & 3 \\
\hline HSKEW & $-1,10$ & $-0,91$ & $-0,36$ & $-2,01$ & 0,84 & 3 \\
\hline HKURT & 16,37 & 15,84 & 27,35 & 5,93 & 10,72 & 3 \\
\hline CDS & 45,61 & 40,73 & 55,83 & 40,25 & 8,86 & 3 \\
\hline
\end{tabular}

Table B.29.: Summary statistics for cross section DEG 


\section{Details of the Results of the Empirical Analysis}

C.1. The Leverage Effect 


\begin{tabular}{|c|c|c|c|c|c|c|c|}
\hline \multicolumn{8}{|c|}{$d I M P V_{t}=\beta_{0}+\beta_{1} \cdot d \ln \left(P R C_{t}\right) \cdot D_{L}+\beta_{2} \cdot d \ln \left(P R C_{t}\right) \cdot D_{H}+u_{t}$} \\
\hline ID & $b_{0}$ & $b_{1}$ & $b_{2}$ & $b_{2}-b_{1} *$ & R2 cor. & $F$ & DW \\
\hline _ALL & $\begin{array}{r}0,00 \\
{[-1,06]} \\
(0,29)\end{array}$ & $\begin{array}{r}-0,35 \\
{[-15,76]} \\
(0,00)\end{array}$ & $\begin{array}{r}-0,44 \\
{[-20,67]} \\
(0,00)\end{array}$ & $-0,08$ & 0,48 & 337,46 & 1,77 \\
\hline _SIE & $\begin{array}{r}0,00 \\
{[-1,18]} \\
(0,24)\end{array}$ & $\begin{array}{r}-0,49 \\
{[-28,10]} \\
(0,00)\end{array}$ & $\begin{array}{r}-0,40 \\
{[-14,30]} \\
(0,00)\end{array}$ & 0,09 & 0,57 & 497,25 & 1,95 \\
\hline _DTE & $\begin{array}{r}0,00 \\
{[-1,16]} \\
(0,25)\end{array}$ & $\begin{array}{r}-0,51 \\
{[-26,01]} \\
(0,00)\end{array}$ & $\begin{array}{r}-0,33 \\
{[-8,60]} \\
(0,00)\end{array}$ & 0,17 & 0,50 & 375,37 & 1,86 \\
\hline _DAIM & $\begin{array}{r}0,00 \\
{[-0,87]} \\
(0,38)\end{array}$ & $\begin{array}{r}-0,40 \\
{[-15,93]} \\
(0,00)\end{array}$ & $\begin{array}{r}-0,52 \\
{[-21,03]} \\
(0,00)\end{array}$ & $-0,12$ & 0,48 & 348,58 & 1,87 \\
\hline DBANK & $\begin{array}{r}0,00 \\
{[-0,76]} \\
(0,45) \\
\end{array}$ & $\begin{array}{r}-0,53 \\
{[-23,47]} \\
(0,00)\end{array}$ & $\begin{array}{r}-0,51 \\
{[-13,75]} \\
(0,00)\end{array}$ & 0,02 & 0,49 & 370,03 & 1,83 \\
\hline _MRE & $\begin{array}{r}0,00 \\
{[-1,40]} \\
(0,16) \\
\end{array}$ & $\begin{array}{r}-0,42 \\
{[-16,36]} \\
(0,00)\end{array}$ & $\begin{array}{r}-0,52 \\
{[-25,47]} \\
(0,00)\end{array}$ & $-0,10$ & 0,58 & 457,77 & 1,84 \\
\hline _BAY & $\begin{array}{r}0,00 \\
{[-0,02]} \\
(0,99)\end{array}$ & $\begin{array}{r}-0,31 \\
{[-10,43]} \\
(0,00)\end{array}$ & $\begin{array}{r}-0,43 \\
{[-10,12]} \\
(0,00)\end{array}$ & $-0,12$ & 0,22 & 105,67 & 2,29 \\
\hline _VW & $\begin{array}{r}0,00 \\
{[-0,93]} \\
(0,35)\end{array}$ & $\begin{array}{r}-0,40 \\
{[-14,28]} \\
(0,00)\end{array}$ & $\begin{array}{r}-0,50 \\
{[-17,86]} \\
(0,00)\end{array}$ & $-0,10$ & 0,41 & 261,75 & 2,42 \\
\hline _BASF & $\begin{array}{r}0,00 \\
{[-0,35]} \\
(0,73)\end{array}$ & $\begin{array}{r}-0,44 \\
{[-14,50]} \\
(0,00)\end{array}$ & $\begin{array}{r}-0,41 \\
{[-7,31]} \\
(0,00)\end{array}$ & 0,02 & 0,26 & 132,11 & 2,49 \\
\hline EON & $\begin{array}{r}0,00 \\
{[-0,08]} \\
(0,94)\end{array}$ & $\begin{array}{r}-0,38 \\
{[-5,94]} \\
(0,00)\end{array}$ & $\begin{array}{r}-0,34 \\
{[-3,09]} \\
(0,00)\end{array}$ & 0,04 & 0,05 & 22,41 & 2,84 \\
\hline _COB & $\begin{array}{r}0,00 \\
{[-0,31]} \\
(0,76)\end{array}$ & $\begin{array}{r}-0,47 \\
{[-8,44]} \\
(0,00)\end{array}$ & $\begin{array}{r}-0,29 \\
{[-2,30]} \\
(0,02)\end{array}$ & 0,18 & 0,09 & 38,23 & 2,80 \\
\hline _HYPV & $\begin{array}{r}0,00 \\
{[-0,01]} \\
(0,99)\end{array}$ & $\begin{array}{r}-0,34 \\
{[-1,24]} \\
(0,21)\end{array}$ & $\begin{array}{r}0,16 \\
{[0,45]} \\
(0,65)\end{array}$ & 0,49 & 0,00 & 0,87 & 3,00 \\
\hline _RWE & $\begin{array}{r}0,00 \\
{[0,01]} \\
(0,99)\end{array}$ & $\begin{array}{r}-0,13 \\
{[-0,09]} \\
(0,93)\end{array}$ & $\begin{array}{r}-0,64 \\
{[-0,33]} \\
(0,74)\end{array}$ & $-0,52$ & 0,00 & 0,06 & 3,04 \\
\hline _BMW & $\begin{array}{r}0,00 \\
{[0,01]} \\
(1,00)\end{array}$ & $\begin{array}{r}0,14 \\
{[0,15]} \\
(0,88)\end{array}$ & $\begin{array}{r}-0,07 \\
{[-0,07]} \\
(0,95)\end{array}$ & $-0,22$ & 0,00 & 0,01 & 3,02 \\
\hline _LH & $\begin{array}{r}0,00 \\
{[-0,02]} \\
(0,98)\end{array}$ & $\begin{array}{r}-0,31 \\
{[-1,09]} \\
(0,28)\end{array}$ & $\begin{array}{r}0,24 \\
{[0,70]} \\
(0,48)\end{array}$ & 0,56 & 0,00 & 0,84 & 2,97 \\
\hline _TYSS & $\begin{array}{r}0,00 \\
{[-0,07]} \\
(0,94) \\
\end{array}$ & $\begin{array}{r}-1,14 \\
{[-2,69]} \\
(0,01) \\
\end{array}$ & $\begin{array}{r}-0,54 \\
{[-0,75]} \\
(0,46) \\
\end{array}$ & 0,61 & 0,01 & 3,90 & 3,14 \\
\hline _METRO & $\begin{array}{r}0,00 \\
{[0,28]} \\
(0,78) \\
\end{array}$ & $\begin{array}{r}-0,36 \\
{[-0,79]} \\
(0,43) \\
\end{array}$ & $\begin{array}{r}-0,15 \\
{[-0,15]} \\
(0,88) \\
\end{array}$ & 0,22 & 0,00 & 0,32 & 3,02 \\
\hline _TUI & $\begin{array}{r}0,00 \\
{[-0,01]} \\
(0,99)\end{array}$ & $\begin{array}{r}0,08 \\
{[0,08]} \\
(0,93)\end{array}$ & $\begin{array}{r}-0,61 \\
{[-0,61]} \\
(0,54)\end{array}$ & $-0,69$ & $-0,01$ & 0,19 & 3,08 \\
\hline _LIN & $\begin{array}{r}-9,88 \\
{[-0,96]} \\
(0,34)\end{array}$ & $\begin{array}{r}-678,97 \\
{[-1,17]} \\
(0,24)\end{array}$ & $\begin{array}{l}-3,55 \\
{[0,00]} \\
(1,00)\end{array}$ & 675,42 & 0,00 & 0,68 & 0,05 \\
\hline _HEN & $\begin{array}{r}0,39 \\
{[0,93]} \\
(0,35)\end{array}$ & $\begin{array}{r}1,57 \\
{[0,05]} \\
(0,96)\end{array}$ & $\begin{array}{l}18,54 \\
{[0,46]} \\
(0,65)\end{array}$ & 16,96 & $-0,01$ & 0,11 & 0,01 \\
\hline _CONT & $\begin{array}{r}0,00 \\
{[0,02]} \\
(0,99)\end{array}$ & $\begin{array}{r}-0,59 \\
{[-0,08]} \\
(0,94)\end{array}$ & $\begin{array}{r}3,96 \\
{[0,50]} \\
(0,62)\end{array}$ & 4,56 & $-0,01$ & 0,13 & 3,94 \\
\hline _DP & $\begin{array}{r}0,04 \\
{[0,49]} \\
(0,63)\end{array}$ & $\begin{array}{l}14,08 \\
{[1,44]} \\
(0,16)\end{array}$ & $\begin{array}{r}1,16 \\
{[0,08]} \\
(0,94)\end{array}$ & $-12,92$ & 0,00 & 1,04 & 3,66 \\
\hline
\end{tabular}

t-stats in [ ] and corresponding two-sided p-values in ( )

* Differences are only reported, if $p$-values for both coefficients are below 0,05

Table C.1.: Time-series regression results for the leverage effect hypothesis for a constant maturity of 36 days 


\begin{tabular}{|c|c|c|c|c|c|c|c|}
\hline \multicolumn{8}{|c|}{$d I M P V_{t}=\beta_{0}+\beta_{1} \cdot d \ln \left(P R C_{t}\right) \cdot D_{L}+\beta_{2} \cdot d \ln \left(P R C_{t}\right) \cdot D_{H}+u_{t}$} \\
\hline ID & $b_{0}$ & $b_{1}$ & $b_{2}$ & $b_{2}-b_{1} *$ & R2 cor. & $F$ & DW \\
\hline ALL & $\begin{array}{r}0,00 \\
{[-1,18]} \\
(0,24)\end{array}$ & $\begin{array}{r}-0,29 \\
{[-16,49]} \\
(0,00)\end{array}$ & $\begin{array}{r}-0,38 \\
{[-22,86]} \\
(0,00)\end{array}$ & $-0,09$ & 0,52 & 396,92 & 1,82 \\
\hline$-\operatorname{SIE}$ & $\begin{array}{r}0,00 \\
{[-1,53]} \\
(0,13)\end{array}$ & $\begin{array}{r}-0,42 \\
{[-33,13]} \\
(0,00)\end{array}$ & $\begin{array}{r}-0,33 \\
{[-16,13]} \\
(0,00)\end{array}$ & 0,09 & 0,64 & 679,11 & 1,99 \\
\hline DTE & $\begin{array}{r}0,00 \\
{[-0,82]} \\
(0,41)\end{array}$ & $\begin{array}{r}-0,43 \\
{[-17,15]} \\
(0,00)\end{array}$ & $\begin{array}{r}-0,24 \\
{[-4,74]} \\
(0,00)\end{array}$ & 0,19 & 0,30 & 158,30 & 2,57 \\
\hline DAIM & $\begin{array}{r}0,00 \\
{[-1,13]} \\
(0,26) \\
\end{array}$ & $\begin{array}{r}-0,36 \\
{[-18,03]} \\
(0,00)\end{array}$ & $\begin{array}{r}-0,44 \\
{[-22,38]} \\
(0,00)\end{array}$ & $-0,08$ & 0,52 & 413,56 & 1,87 \\
\hline DBANK & $\begin{array}{r}0,00 \\
{[-0,80]} \\
(0,42) \\
\end{array}$ & $\begin{array}{r}-0,46 \\
{[-23,42]} \\
(0,00) \\
\end{array}$ & $\begin{array}{r}-0,45 \\
{[-13,94]} \\
(0,00)\end{array}$ & 0,01 & 0,49 & 371,54 & 1,97 \\
\hline _MRE & $\begin{array}{r}0,00 \\
{[-0,33]} \\
(0,74) \\
\end{array}$ & $\begin{array}{r}-0,25 \\
{[-2,64]} \\
(0,01)\end{array}$ & $\begin{array}{r}-0,45 \\
{[-6,07]} \\
(0,00)\end{array}$ & $-0,21$ & 0,06 & 21,88 & 3,03 \\
\hline _BAY & $\begin{array}{r}0,00 \\
{[-0,29]} \\
(0,77)\end{array}$ & $\begin{array}{r}-0,33 \\
{[-11,35]} \\
(0,00)\end{array}$ & $\begin{array}{r}-0,35 \\
{[-8,54]} \\
(0,00)\end{array}$ & $-0,02$ & 0,22 & 100,91 & 2,59 \\
\hline _VW & $\begin{array}{r}0,00 \\
{[-0,50]} \\
(0,61)\end{array}$ & $\begin{array}{r}-0,34 \\
{[-7,12]} \\
(0,00)\end{array}$ & $\begin{array}{r}-0,39 \\
{[-8,08]} \\
(0,00)\end{array}$ & $-0,05$ & 0,13 & 58,08 & 2,97 \\
\hline BASF & $\begin{array}{r}0,00 \\
{[-0,26]} \\
(0,80)\end{array}$ & $\begin{array}{r}-0,28 \\
{[-2,54]} \\
(0,01)\end{array}$ & $\begin{array}{r}0,12 \\
{[0,57]} \\
(0,57)\end{array}$ & 0,40 & 0,01 & 3,38 & 2,98 \\
\hline EON & $\begin{array}{r}0,00 \\
{[-0,08]} \\
(0,94)\end{array}$ & $\begin{array}{r}-0,57 \\
{[-4,32]} \\
(0,00)\end{array}$ & $\begin{array}{r}-0,25 \\
{[-1,11]} \\
(0,27)\end{array}$ & 0,32 & 0,02 & 9,93 & 2,96 \\
\hline COB & $\begin{array}{r}0,00 \\
{[-0,01]} \\
(0,99) \\
\end{array}$ & $\begin{array}{r}-0,68 \\
{[-0,34]} \\
(0,74)\end{array}$ & $\begin{array}{r}-0,59 \\
{[-0,13]} \\
(0,90)\end{array}$ & 0,09 & 0,00 & 0,07 & 3,03 \\
\hline -HYPV & $\begin{array}{r}0,00 \\
{[0,00]} \\
(1,00)\end{array}$ & $\begin{array}{r}-0,63 \\
{[-0,53]} \\
(0,59)\end{array}$ & $\begin{array}{r}-0,20 \\
{[-0,13]} \\
(0,89)\end{array}$ & 0,42 & 0,00 & 0,15 & 3,04 \\
\hline RWE & $\begin{array}{r}0,01 \\
{[0,03]} \\
(0,98)\end{array}$ & $\begin{array}{r}-0,07 \\
{[-0,01]} \\
(1,00)\end{array}$ & $\begin{array}{r}-13,64 \\
{[-0,81]} \\
(0,42)\end{array}$ & $-13,57$ & 0,00 & 0,33 & 3,05 \\
\hline BMW & $\begin{array}{r}0,00 \\
{[0,01]} \\
(0,99)\end{array}$ & $\begin{array}{r}0,71 \\
{[0,33]} \\
(0,74)\end{array}$ & $\begin{array}{r}0,37 \\
{[0,15]} \\
(0,88)\end{array}$ & $-0,34$ & 0,00 & 0,07 & 3,02 \\
\hline _LH & $\begin{array}{r}0,00 \\
{[0,06]} \\
(0,96)\end{array}$ & $\begin{array}{r}5,70 \\
{[1,35]} \\
(0,18)\end{array}$ & $\begin{array}{r}-0,72 \\
{[-0,14]} \\
(0,89)\end{array}$ & $-6,42$ & 0,00 & 0,93 & 3,20 \\
\hline _ TYSS & $\begin{array}{r}0,00 \\
{[0,07]} \\
(0,95) \\
\end{array}$ & $\begin{array}{r}-0,94 \\
{[-2,31]} \\
(0,02) \\
\end{array}$ & $\begin{array}{r}-0,20 \\
{[-0,29]} \\
(0,77) \\
\end{array}$ & 0,74 & 0,01 & 2,72 & 3,10 \\
\hline -METRO & $\begin{array}{r}-0,01 \\
{[-0,70]} \\
(0,49)\end{array}$ & $\begin{array}{r}-0,60 \\
{[-0,70]} \\
(0,49) \\
\end{array}$ & $\begin{array}{r}0,11 \\
{[0,06]} \\
(0,95) \\
\end{array}$ & 0,71 & 0,00 & 0,25 & 1,95 \\
\hline _ & $\begin{array}{r}0,00 \\
{[0,00]} \\
(1,00)\end{array}$ & $\begin{array}{r}-0,86 \\
{[-0,10]} \\
(0,92)\end{array}$ & $\begin{array}{r}-4,68 \\
{[-0,52]} \\
(0,60)\end{array}$ & $-3,82$ & $-0,01$ & 0,14 & 3,10 \\
\hline LIN & $\begin{array}{r}-55,06 \\
{[-0,96]} \\
(0,34)\end{array}$ & $\begin{array}{r}-3.984,65 \\
{[-1,23]} \\
(0,22)\end{array}$ & $\begin{array}{r}-28,05 \\
{[-0,01]} \\
(1,00)\end{array}$ & $3.956,60$ & 0,00 & 0,76 & 0,01 \\
\hline _HEN & $\begin{array}{r}0,38 \\
{[0,88]} \\
(0,38)\end{array}$ & $\begin{array}{r}5,85 \\
{[0,18]} \\
(0,86)\end{array}$ & $\begin{array}{l}17,74 \\
{[0,44]} \\
(0,66)\end{array}$ & 11,89 & $-0,01$ & 0,11 & 0,03 \\
\hline -CONT & $\begin{array}{r}-0,03 \\
{[-0,28]} \\
(0,78)\end{array}$ & $\begin{array}{r}-7,11 \\
{[-0,87]} \\
(0,39)\end{array}$ & $\begin{array}{r}4,84 \\
{[0,56]} \\
(0,58)\end{array}$ & 11,95 & $-0,01$ & 0,53 & 3,51 \\
\hline -DP & $\begin{array}{r}0,01 \\
{[0,08]} \\
(0,94)\end{array}$ & $\begin{array}{r}-2,62 \\
{[-0,17]} \\
(0,87)\end{array}$ & $\begin{array}{r}-3,03 \\
{[-0,13]} \\
(0,90)\end{array}$ & $-0,41$ & $-0,06$ & 0,02 & 4,17 \\
\hline
\end{tabular}

t-stats in [ ] and corresponding two-sided p-values in ( )

* Differences are only reported, if $p$-values for both coefficients are below 0,05

Table C.2.: Time-series regression results for the leverage effect hypothesis for a constant maturity of 60 days 


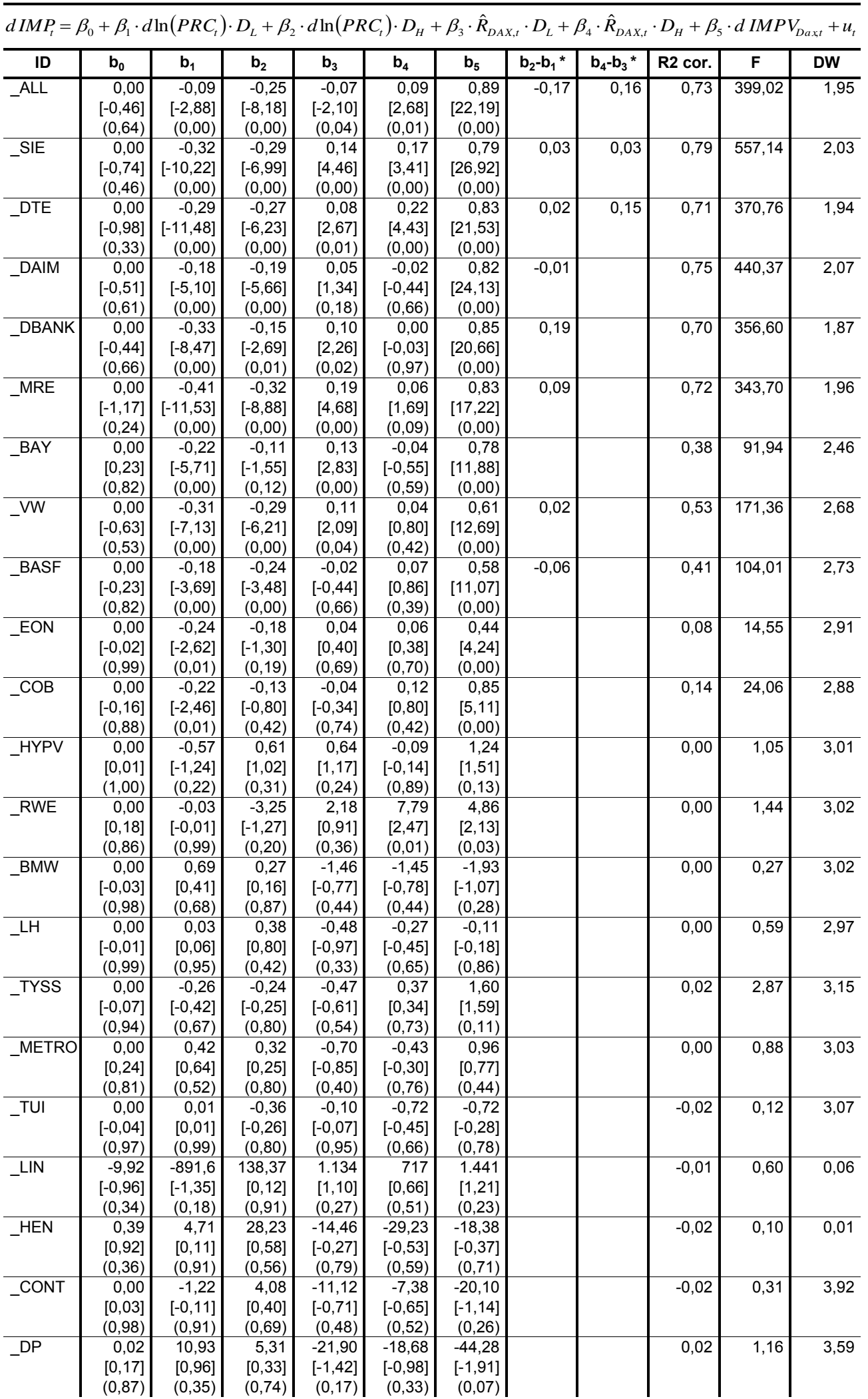

t-stats in [ ] and corresponding two-sided p-values in ( )

* Differences are only reported, if p-values for both coefficients are below 0,05

Table C.3.: Robustness check of the time-series regression results for the leverage effect hypothesis for a constant maturity of 36 days 


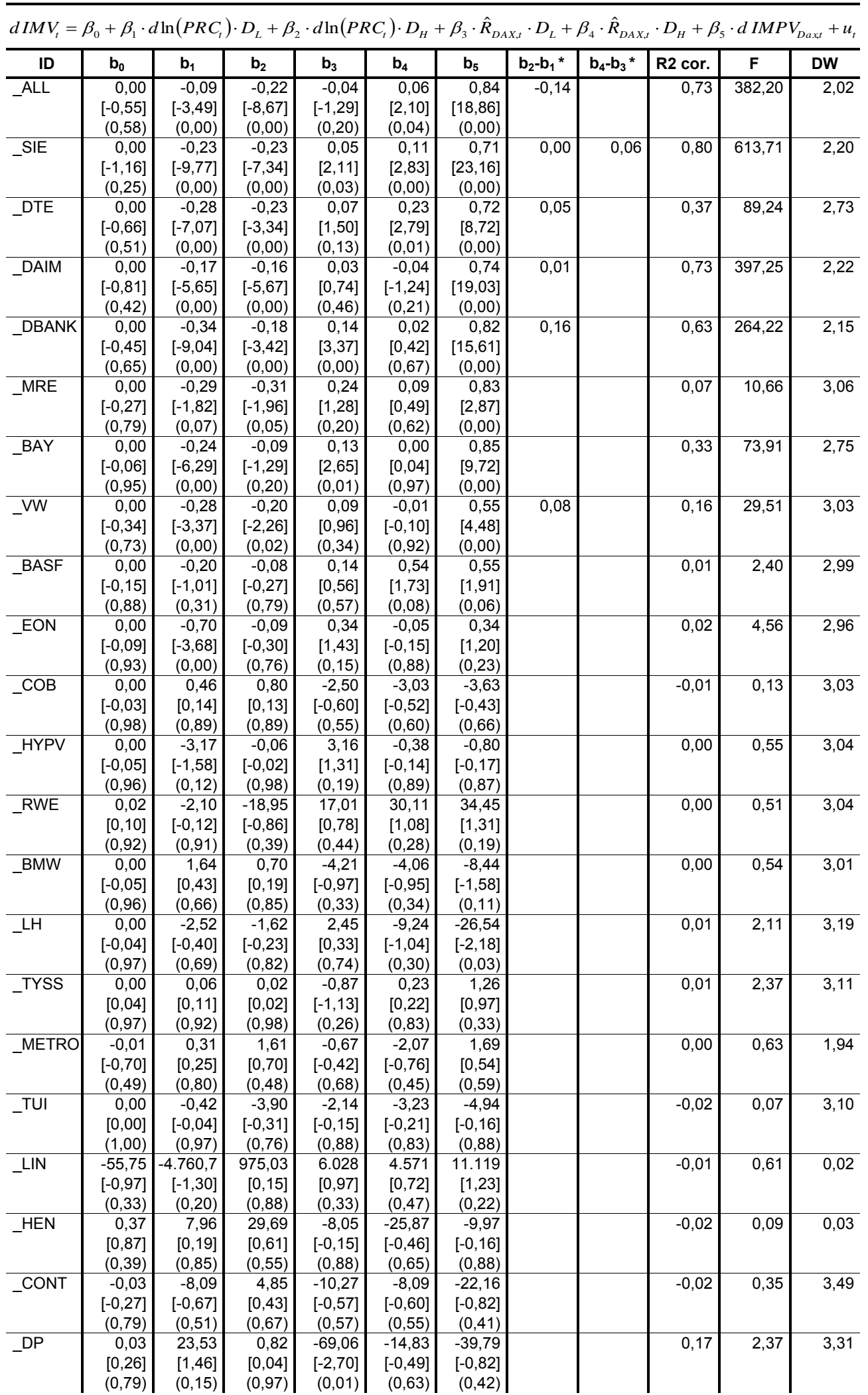

t-stats in [ ] and corresponding two-sided p-values in ( )

* Differences are only reported, if $p$-values for both coefficients are below 0,05

Table C.4.: Robustness check of the time-series regression results for the leverage effect hypothesis for a constant maturity of 60 days 


\section{C.2. The Credit Spread Level and the Implied Volatility Level}




\begin{tabular}{|c|c|c|c|c|c|c|c|}
\hline \multicolumn{8}{|c|}{$C D S_{t}=\beta_{0}+\beta_{1} \cdot I M P V_{t}+\beta_{2} \cdot t+u_{t}$} \\
\hline ID & $\mathbf{b}_{0}$ & $b_{1}$ & $b_{2}$ & R2 cor. & $\mathbf{F}$ & DW & ADF* \\
\hline ALL & $\begin{array}{r}-17,21 \\
{[-5,64]} \\
(0,00) \\
\end{array}$ & $\begin{array}{r}143,69 \\
{[20,82]} \\
(0,00) \\
\end{array}$ & $\begin{array}{r}0,01 \\
{[2,34]} \\
(0,02) \\
\end{array}$ & 0,85 & $2.029,15$ & 0,10 & $\begin{array}{r}{[-4,15]} \\
(<=0,05)\end{array}$ \\
\hline SIE & $\begin{array}{r}5,75 \\
{[1,39]} \\
(0,16) \\
\end{array}$ & $\begin{array}{r}110,74 \\
{[15,62]} \\
(0,00) \\
\end{array}$ & $\begin{array}{r}-0,01 \\
{[-3,22]} \\
(0,00)\end{array}$ & 0,89 & $3.013,43$ & 0,12 & $\begin{array}{r}{[-4,89]} \\
(<=0,01)\end{array}$ \\
\hline DTE & $\begin{array}{r}-24,85 \\
{[-1,38]} \\
(0,17) \\
\end{array}$ & $\begin{array}{r}483,67 \\
{[18,46]} \\
(0,00) \\
\end{array}$ & $\begin{array}{r}-0,04 \\
{[-1,79]} \\
(0,07) \\
\end{array}$ & 0,93 & $4.879,13$ & 0,12 & $\begin{array}{r}{[-5,24]} \\
(<=0,01)\end{array}$ \\
\hline DAIM & $\begin{array}{r}109,79 \\
{[11,50]} \\
(0,00)\end{array}$ & $\begin{array}{r}104,34 \\
{[5,81]} \\
(0,00)\end{array}$ & $\begin{array}{r}-0,08 \\
{[-8,77]} \\
(0,00)\end{array}$ & 0,80 & $1.528,79$ & 0,08 & $\begin{array}{r}{[-4,50]} \\
(<=0,01)\end{array}$ \\
\hline DBANK & $\begin{array}{r}-2,04 \\
{[-1,01]} \\
(0,31)\end{array}$ & $\begin{array}{r}82,12 \\
{[17,24]} \\
(0,00)\end{array}$ & $\begin{array}{r}0,00 \\
{[-0,24]} \\
(0,81)\end{array}$ & 0,86 & $2.248,48$ & 0,15 & $\begin{array}{r}{[-5,50]} \\
(<=0,01)\end{array}$ \\
\hline -MRE & $\begin{array}{r}-8,11 \\
{[-2,17]} \\
(0,03)\end{array}$ & $\begin{array}{r}103,03 \\
{[19,93]} \\
(0,00) \\
\end{array}$ & $\begin{array}{r}0,01 \\
{[2,74]} \\
(0,01) \\
\end{array}$ & 0,78 & $1.172,68$ & 0,53 & $\begin{array}{l}{[-10,18]} \\
(<=0,01)\end{array}$ \\
\hline BAY & $\begin{array}{r}-28,45 \\
{[-2,56]} \\
(0,01)\end{array}$ & $\begin{array}{r}231,12 \\
{[8,08]} \\
(0,00)\end{array}$ & $\begin{array}{r}0,02 \\
{[2,51]} \\
(0,01)\end{array}$ & 0,68 & 789,53 & 0,09 & $\begin{array}{r}{[-3,72]} \\
(<=0,10)\end{array}$ \\
\hline -VW & $\begin{array}{r}2,90 \\
{[1,13]} \\
(0,26)\end{array}$ & $\begin{array}{r}107,35 \\
{[17,15]} \\
(0,00)\end{array}$ & $\begin{array}{r}0,05 \\
{[24,69]} \\
(0,00)\end{array}$ & 0,68 & 795,00 & 0,11 & $\begin{array}{r}{[-4,44]} \\
(<=0,01)\end{array}$ \\
\hline BASF & $\begin{array}{r}18,23 \\
{[19,45]} \\
(0,00)\end{array}$ & $\begin{array}{r}35,85 \\
{[12,09]} \\
(0,00)\end{array}$ & $\begin{array}{r}-0,02 \\
{[-21,89]} \\
(0,00)\end{array}$ & 0,88 & $2.711,94$ & 0,11 & $\begin{array}{r}{[-4,11]} \\
(<=0,05)\end{array}$ \\
\hline EON & $\begin{array}{r}-5,46 \\
{[-1,33]} \\
(0,18)\end{array}$ & $\begin{array}{r}171,10 \\
{[14,69]} \\
(0,00)\end{array}$ & $\begin{array}{r}0,00 \\
{[-0,57]} \\
(0,57)\end{array}$ & 0,83 & $1.804,88$ & 0,42 & $\begin{array}{r}{[-5,55]} \\
(<=0,01)\end{array}$ \\
\hline _COB & $\begin{array}{r}-40,16 \\
{[-6,27]} \\
(0,00)\end{array}$ & $\begin{array}{r}255,27 \\
{[18,01]} \\
(0,00)\end{array}$ & $\begin{array}{r}0,00 \\
{[-0,50]} \\
(0,62)\end{array}$ & 0,80 & $1.462,33$ & 0,33 & $\begin{array}{r}{[-4,99]} \\
(<=0,01)\end{array}$ \\
\hline _HYPV & $\begin{array}{l}19,89 \\
{[0,83]} \\
(0,41)\end{array}$ & $\begin{array}{r}113,72 \\
{[2,62]} \\
(0,01) \\
\end{array}$ & $\begin{array}{r}-0,03 \\
{[-1,84]} \\
(0,07) \\
\end{array}$ & 0,48 & 338,73 & 0,70 & $\begin{array}{r}{[-4,72]} \\
(<=0,01)\end{array}$ \\
\hline _RWE & $\begin{array}{r}82,34 \\
{[16,55]} \\
(0,00)\end{array}$ & $\begin{array}{r}5,34 \\
{[1,26]} \\
(0,21) \\
\end{array}$ & $\begin{array}{r}-0,08 \\
{[-10,87]} \\
(0,00)\end{array}$ & 0,54 & 402,08 & 0,05 & $\begin{array}{r}{[-2,69]} \\
(<=0,05)\end{array}$ \\
\hline BMW & $\begin{array}{r}36,50 \\
{[21,80]} \\
(0,00)\end{array}$ & $\begin{array}{r}2,64 \\
{[1,11]} \\
(0,27)\end{array}$ & $\begin{array}{r}-0,01 \\
{[-4,27]} \\
(0,00)\end{array}$ & 0,18 & 81,22 & 0,16 & $\begin{array}{r}{[-3,63]} \\
(<=1,00)\end{array}$ \\
\hline _LH & $\begin{array}{r}100,18 \\
{[7,25]} \\
(0,00)\end{array}$ & $\begin{array}{r}119,73 \\
{[4,04]} \\
(0,00)\end{array}$ & $\begin{array}{r}-0,09 \\
{[-8,62]} \\
(0,00)\end{array}$ & 0,57 & 466,93 & 0,45 & $\begin{array}{r}{[-2,92]} \\
(<=0,05)\end{array}$ \\
\hline _TYSS & $\begin{array}{r}203,19 \\
{[17,75]} \\
(0,00)\end{array}$ & $\begin{array}{r}5,51 \\
{[1,45]} \\
(0,15)\end{array}$ & $\begin{array}{r}-0,14 \\
{[-7,27]} \\
(0,00)\end{array}$ & 0,29 & 131,32 & 0,03 & $\begin{array}{r}{[-1,36]} \\
(<=1,00)\end{array}$ \\
\hline _METRO & $\begin{array}{l}80,65 \\
{[7,06]} \\
(0,00)\end{array}$ & $\begin{array}{l}42,11 \\
{[2,16]} \\
(0,03)\end{array}$ & $\begin{array}{r}-0,05 \\
{[-3,98]} \\
(0,00)\end{array}$ & 0,27 & 116,04 & 0,21 & $\begin{array}{r}{[0,83]} \\
(<=1,00)\end{array}$ \\
\hline _TUI & $\begin{array}{r}295,52 \\
{[7,44]} \\
(0,00)\end{array}$ & $\begin{array}{l}52,65 \\
{[1,97]} \\
(0,05)\end{array}$ & $\begin{array}{r}-0,10 \\
{[-1,54]} \\
(0,12)\end{array}$ & 0,11 & 15,90 & 0,17 & $\begin{array}{r}{[-1,98]} \\
(<=0,05)\end{array}$ \\
\hline LIIN & $\begin{array}{r}53,99 \\
{[16,08]} \\
(0,00)\end{array}$ & $\begin{array}{r}0,00 \\
{[0,78]} \\
(0,44)\end{array}$ & $\begin{array}{r}-0,03 \\
{[-4,54]} \\
(0,00)\end{array}$ & 0,16 & 46,09 & 0,05 & $\begin{array}{r}{[-2,92]} \\
(<=0,10)\end{array}$ \\
\hline _HEN & $\begin{array}{r}20,99 \\
{[26,31]} \\
(0,00)\end{array}$ & $\begin{array}{r}-0,03 \\
{[-3,96]} \\
(0,00)\end{array}$ & $\begin{array}{r}0,01 \\
{[5,18]} \\
(0,00)\end{array}$ & 0,19 & 45,15 & 0,04 & $\begin{array}{r}{[-5,55]} \\
(<=0,01)\end{array}$ \\
\hline _CONT & $\begin{array}{r}113,49 \\
{[13,01]} \\
(0,00)\end{array}$ & $\begin{array}{r}0,20 \\
{[0,49]} \\
(0,63)\end{array}$ & $\begin{array}{r}-0,11 \\
{[-8,11]} \\
(0,00)\end{array}$ & 0,62 & 229,75 & 0,00 & $\begin{array}{r}{[-4,99]} \\
(<=0,01)\end{array}$ \\
\hline DPP & $\begin{array}{r}166,13 \\
{[7,93]} \\
(0,00)\end{array}$ & $\begin{array}{r}-0,08 \\
{[-0,06]} \\
(0,95)\end{array}$ & $\begin{array}{r}-0,20 \\
{[-7,04]} \\
(0,00)\end{array}$ & 0,79 & 108,35 & 0,06 & $\begin{array}{r}{[-5,50]} \\
(<=0,01)\end{array}$ \\
\hline
\end{tabular}

t-stats in [ ] and corresponding p-values in ( )

*Phillips and Ouliaris (1990) p-values

Table C.5.: Time-series regression results for the test of the relationship between default risk and implied volatility for a constant maturity of 36 days 


\begin{tabular}{|c|c|c|c|c|c|c|c|}
\hline \multicolumn{8}{|c|}{$C D S_{t}=\beta_{0}+\beta_{1} \cdot I M P V_{t}+\beta_{2} \cdot t+u_{t}$} \\
\hline ID & $b_{0}$ & $b_{1}$ & $b_{2}$ & R2 cor. & $\mathbf{F}$ & DW & ADF* \\
\hline _ALL & $\begin{array}{r}-22,43 \\
{[-7,27]} \\
(0,00)\end{array}$ & $\begin{array}{r}156,64 \\
{[22,49]} \\
(0,00)\end{array}$ & $\begin{array}{r}0,01 \\
{[2,64]} \\
(0,01)\end{array}$ & 0,86 & $2.282,82$ & 0,10 & $\begin{array}{r}{[-4,02]} \\
<=0,05)\end{array}$ \\
\hline _SIE & $\begin{array}{c}0,74 \\
{[0,17]} \\
(0,87)\end{array}$ & $\begin{array}{r}121,21 \\
{[15,68]} \\
(0,00)\end{array}$ & $\begin{array}{r}-0,01 \\
{[-2,60]} \\
(0,01)\end{array}$ & 0,89 & $3.045,55$ & 0,10 & $\begin{array}{r}{[-4,46]} \\
(<=0,01)\end{array}$ \\
\hline -DTE & $\begin{array}{r}-37,12 \\
{[-1,97]} \\
(0,05)\end{array}$ & $\begin{array}{r}505,42 \\
{[17,78]} \\
(0,00)\end{array}$ & $\begin{array}{r}-0,04 \\
{[-1,56]} \\
0,12)\end{array}$ & 0,92 & $4.505,74$ & 0,16 & $\begin{array}{r}{[-4,41]} \\
(<=0,01)\end{array}$ \\
\hline _DAIM & $\begin{array}{r}106,66 \\
{[10,58]} \\
(0,00)\end{array}$ & $\begin{array}{r}112,96 \\
{[5,75]} \\
(0,00)\end{array}$ & $\begin{array}{r}-0,08 \\
{[-8,74]} \\
(0,00)\end{array}$ & 0,80 & $1.537,31$ & 0,08 & $\begin{array}{r}{[-4,51]} \\
(<=0,01)\end{array}$ \\
\hline _DBANK & $\begin{array}{r}-4,40 \\
{[-2,15]} \\
(0,03)\end{array}$ & $\begin{array}{r}87,78 \\
{[18,37]} \\
(0,00)\end{array}$ & $\begin{array}{r}0,00 \\
{[0,06]} \\
(0,95)\end{array}$ & 0,86 & $2.363,33$ & 0,14 & $\begin{array}{r}{[-5,29]} \\
(<=0,01)\end{array}$ \\
\hline -MRE & $\begin{array}{r}-3,15 \\
{[-0,45]} \\
(0,65) \\
\end{array}$ & $\begin{array}{l}98,62 \\
{[9,60]} \\
(0,00)\end{array}$ & $\begin{array}{r}0,00 \\
{[0,68]} \\
(0,50)\end{array}$ & 0,72 & 871,33 & 0,70 & $\begin{array}{r}{[-6,62]} \\
(<=0,01)\end{array}$ \\
\hline _BAY & $\begin{array}{r}-34,75 \\
{[-3,01]} \\
(0,00)\end{array}$ & $\begin{array}{r}249,66 \\
{[8,37]} \\
(0,00)\end{array}$ & $\begin{array}{r}0,02 \\
{[2,54]} \\
(0,01)\end{array}$ & 0,70 & 871,08 & 0,10 & $\begin{array}{r}{[-4,13]} \\
(<=0,10)\end{array}$ \\
\hline _VW & $\begin{array}{r}2,85 \\
{[0,88]} \\
(0,38) \\
\end{array}$ & $\begin{array}{r}109,21 \\
{[14,23]} \\
(0,00) \\
\end{array}$ & $\begin{array}{r}0,05 \\
{[19,78]} \\
(0,00) \\
\end{array}$ & 0,65 & 702,32 & 0,16 & $\begin{array}{r}{[-4,21]} \\
(<-0,05)\end{array}$ \\
\hline BASF & $\begin{array}{r}20,39 \\
{[12,04]} \\
(0,00) \\
\end{array}$ & $\begin{array}{l}29,56 \\
{[5,40]} \\
(0,00) \\
\end{array}$ & $\begin{array}{r}-0,02 \\
{[-19,81]} \\
(0,00)\end{array}$ & 0,84 & $2.022,30$ & 0,34 & $\begin{array}{r}{[-3,84]} \\
(<=0,05)\end{array}$ \\
\hline EON & $\begin{array}{r}2,23 \\
{[0,20]} \\
(0,84) \\
\end{array}$ & $\begin{array}{r}149,85 \\
{[4,88]} \\
(0,00) \\
\end{array}$ & $\begin{array}{r}-0,01 \\
{[-1,13]} \\
(0,26)\end{array}$ & 0,74 & $1.083,61$ & 0,86 & $\begin{array}{r}{[-5,82]} \\
(<=0,01)\end{array}$ \\
\hline -COB & $\begin{array}{l}97,57 \\
{[8,84]} \\
(0,00)\end{array}$ & $\begin{array}{r}4,26 \\
{[1,03]} \\
(0,30)\end{array}$ & $\begin{array}{r}-0,10 \\
{[-5,52]} \\
(0,00)\end{array}$ & 0,25 & 119,74 & 0,04 & $\begin{array}{r}{[-1,69]} \\
(<=0,05)\end{array}$ \\
\hline _HYPV & & $\begin{array}{l}11,01 \\
{[1,45]} \\
(0,15)\end{array}$ & $\begin{array}{r}-0,06 \\
{[-4,03]} \\
(0,00) \\
\end{array}$ & 0,15 & 69,15 & 0,08 & $\begin{array}{r}{[-2,28]} \\
(<-0,05)\end{array}$ \\
\hline -RWE & $\begin{array}{r}84,60 \\
{[17,95]} \\
(0,00)\end{array}$ & $\begin{array}{r}0,10 \\
{[1,31]} \\
(0,19) \\
\end{array}$ & $\begin{array}{r}-0,09 \\
{[-11,09]} \\
(0,00)\end{array}$ & 0,54 & 390,12 & 0,01 & $\begin{array}{r}{[-2,51]} \\
(<=1,00)\end{array}$ \\
\hline _BMW & $\begin{array}{r}37,44 \\
{[27,49]} \\
(0,00) \\
\end{array}$ & $\begin{array}{r}0,41 \\
{[0,75]} \\
(0,45) \\
\end{array}$ & $\begin{array}{r}-0,01 \\
{[-4,57]} \\
(0,00)\end{array}$ & 0,16 & 73,83 & 0,13 & $\begin{array}{r}{[-4,99]} \\
(<-0,01)\end{array}$ \\
\hline _LH & $\begin{array}{r}155,56 \\
{[24,29]} \\
(0,00) \\
\end{array}$ & $\begin{array}{r}0,93 \\
{[0,87]} \\
(0,39) \\
\end{array}$ & $\begin{array}{r}-0,12 \\
{[-11,23]} \\
(0,00)\end{array}$ & 0,43 & 269,14 & 0,02 & $\begin{array}{r}{[-1,75]} \\
(<=1,00)\end{array}$ \\
\hline -TYSS & & $\begin{array}{r}8,46 \\
{[1,39]} \\
(0,16)\end{array}$ & $\begin{array}{r}-0,14 \\
{[-7,28]} \\
(0,00)\end{array}$ & 0,29 & 132,60 & 0,03 & $\begin{array}{r}{[-1,43]} \\
(<=1,00)\end{array}$ \\
\hline _METRO & $\begin{array}{r}94,74 \\
{[11,69]} \\
(0,00)\end{array}$ & $\begin{array}{l}13, \\
{[1,} \\
(0,\end{array}$ & $\begin{array}{r}-0,06 \\
{[-5,30]} \\
(0,00)\end{array}$ & 0,23 & 92,06 & 0,10 & $\begin{array}{r}{[-3,19]} \\
(<=1,00)\end{array}$ \\
\hline _TUI & $\begin{array}{r}321,86 \\
{[7,63]} \\
(0,00) \\
\end{array}$ & $\begin{array}{r}1,32 \\
{[1,55]} \\
(0,12) \\
\end{array}$ & $\begin{array}{r}-0,12 \\
{[-1,70]} \\
(0,09) \\
\end{array}$ & 0,06 & 8,31 & 0,06 & $\begin{array}{r}{[-1,57]} \\
(<=1,00)\end{array}$ \\
\hline _LIN & $\begin{array}{r}53,99 \\
{[16,08]} \\
(0,00)\end{array}$ & $\begin{array}{r}0,00 \\
{[1,57]} \\
(0,12)\end{array}$ & $\begin{array}{r}-0,03 \\
{[-4,54]} \\
(0,00)\end{array}$ & 0,16 & 46,10 & 0,05 & $\begin{array}{r}{[-1,75]} \\
(<=1,00)\end{array}$ \\
\hline -HEN & $\begin{array}{r}20,99 \\
{[26,29]} \\
(0,00) \\
\end{array}$ & $\begin{array}{r}-0,03 \\
{[-3,82]} \\
(0,00) \\
\end{array}$ & $\begin{array}{r}0,01 \\
{[5,18]} \\
(0,00) \\
\end{array}$ & 0,19 & 45,16 & 0,04 & $\begin{array}{r}{[-5,82]} \\
(<=0,01)\end{array}$ \\
\hline _CONT & $\begin{array}{r}113,60 \\
{[13,00]} \\
(0,00)\end{array}$ & $\begin{array}{r}-0,24 \\
{[-0,65]} \\
(0,51)\end{array}$ & $\begin{array}{r}-0,11 \\
{[-8,13]} \\
(0,00)\end{array}$ & 0,62 & 229,81 & 0,00 & $\begin{array}{r}{[-1,69]} \\
(<=0,05)\end{array}$ \\
\hline DPP & $\begin{array}{r}166,07 \\
{[7,93]} \\
(0,00)\end{array}$ & $\begin{array}{r}0,18 \\
{[0,49]} \\
(0,63)\end{array}$ & $\begin{array}{r}-0,20 \\
{[-7,03]} \\
(0,00)\end{array}$ & 0,79 & 108,40 & 0,06 & $\begin{array}{r}{[-5,29]} \\
(<=0,01)\end{array}$ \\
\hline
\end{tabular}

t-stats in [ ] and corresponding p-values in ( )

${ }^{*}$ Phillips and Ouliaris (1990) p-values

Table C.6.: Time-series regression results for the test of the relationship between default risk and implied volatility for a constant maturity of 60 days 


\begin{tabular}{|c|c|c|c|c|c|c|c|}
\hline \multicolumn{8}{|c|}{$C D S_{t}=\beta_{0}+\beta_{1} \cdot I M P V_{t}+\beta_{2} \cdot t+u_{t}$} \\
\hline ID & $b_{0}$ & $b_{1}$ & $\mathbf{b}_{2}$ & R2 cor. & $\mathbf{F}$ & DW & ADF* $^{*}$ \\
\hline _ALL & $\begin{array}{l}59,96 \\
{[9,82]} \\
(0,00)\end{array}$ & $\begin{array}{r}3,61 \\
{[0,98]} \\
(0,33) \\
\end{array}$ & $\begin{array}{r}-0,05 \\
{[-5,09]} \\
(0,00)\end{array}$ & 0,21 & 100,08 & 0,04 & $\begin{array}{r}{[-2,45]} \\
(<=0,05)\end{array}$ \\
\hline SIE & $\begin{array}{r}-12,91 \\
{[-2,40]} \\
(0,02) \\
\end{array}$ & $\begin{array}{r}154,11 \\
{[14,91]} \\
(0,00)\end{array}$ & $\begin{array}{r}-0,01 \\
{[-1,34]} \\
(0,18) \\
\end{array}$ & 0,89 & $2.927,36$ & 0,20 & $\begin{array}{r}{[-4,66]} \\
(<=0,01)\end{array}$ \\
\hline _DTE & $\begin{array}{r}234,89 \\
{[8,38]} \\
(0,00)\end{array}$ & $\begin{array}{l}51,31 \\
{[1,28]} \\
(0,20)\end{array}$ & $\begin{array}{r}-0,31 \\
{[-8,76]} \\
(0,00)\end{array}$ & 0,64 & 678,68 & 0,14 & $\begin{array}{r}{[-3,21]} \\
(<=0,05)\end{array}$ \\
\hline DAIM & $\begin{array}{l}98,22 \\
{[8,71]} \\
(0,00)\end{array}$ & $\begin{array}{r}141,83 \\
{[5,77]} \\
(0,00)\end{array}$ & $\begin{array}{r}-0,08 \\
{[-9,21]} \\
(0,00)\end{array}$ & 0,80 & $1.518,91$ & 0,12 & $\begin{array}{r}{[-4,91]} \\
(<=0,01)\end{array}$ \\
\hline DBANK & $\begin{array}{r}6,43 \\
{[0,78]} \\
(0,43)\end{array}$ & $\begin{array}{l}68,36 \\
{[3,53]} \\
(0,00)\end{array}$ & $\begin{array}{r}-0,01 \\
{[-2,10]} \\
(0,04)\end{array}$ & 0,63 & 656,89 & 0,79 & $\begin{array}{r}{[-3,60]} \\
(<=0,05)\end{array}$ \\
\hline _MRE & $\begin{array}{r}57,88 \\
{[13,11]} \\
(0,00) \\
\end{array}$ & $\begin{array}{r}0,18 \\
{[0,70]} \\
(0,48)\end{array}$ & $\begin{array}{r}-0,05 \\
{[-6,40]} \\
(0,00)\end{array}$ & 0,32 & 160,33 & 0,16 & $\begin{array}{r}{[-3,70]} \\
(<=0,05)\end{array}$ \\
\hline BAY & $\begin{array}{l}10,47 \\
{[0,34]} \\
(0,74)\end{array}$ & $\begin{array}{r}160,96 \\
{[2,07]} \\
(0,04)\end{array}$ & $\begin{array}{r}-0,02 \\
{[-1,06]} \\
(0,29)\end{array}$ & 0,42 & 264,13 & 0,65 & $\begin{array}{r}{[-4,11]} \\
(<=0,10)\end{array}$ \\
\hline -VW & $\begin{array}{r}49,14 \\
{[19,35]} \\
(0,00)\end{array}$ & $\begin{array}{r}0,02 \\
{[0,17]} \\
(0,87)\end{array}$ & $\begin{array}{r}0,03 \\
{[5,80]} \\
(0,00)\end{array}$ & 0,20 & 97,41 & 0,02 & $\begin{array}{r}{[-2,58]} \\
(<=0,05)\end{array}$ \\
\hline BASF & $\begin{array}{r}30,86 \\
{[39,18]} \\
(0,00)\end{array}$ & $\begin{array}{r}-0,03 \\
{[-0,56]} \\
(0,58)\end{array}$ & $\begin{array}{r}-0,03 \\
{[-20,48]} \\
(0,00)\end{array}$ & 0,75 & $1.119,27$ & 0,03 & $\begin{array}{r}{[-2,37]} \\
(<=0,05)\end{array}$ \\
\hline EON & $\begin{array}{r}52,18 \\
{[14,32]} \\
(0,00)\end{array}$ & $\begin{array}{r}1,94 \\
{[3,18]} \\
(0,00)\end{array}$ & $\begin{array}{r}-0,04 \\
{[-6,20]} \\
(0,00)\end{array}$ & 0,28 & 145,20 & 0,04 & $\begin{array}{r}{[-2,37]} \\
(<=0,05)\end{array}$ \\
\hline _COB & $\begin{array}{l}98,61 \\
{[8,98]} \\
(0,00)\end{array}$ & $\begin{array}{r}2,51 \\
{[3,32]} \\
(0,00)\end{array}$ & $\begin{array}{r}-0,10 \\
{[-5,61]} \\
(0,00)\end{array}$ & 0,25 & 121,02 & 0,05 & $\begin{array}{r}{[-2,40]} \\
(<=0,05)\end{array}$ \\
\hline _HYPV & $\begin{array}{l}80,10 \\
{[9,28]} \\
(0,00)\end{array}$ & $\begin{array}{r}0,07 \\
{[0,11]} \\
(0,91)\end{array}$ & $\begin{array}{r}-0,06 \\
{[-4,15]} \\
(0,00)\end{array}$ & 0,12 & 52,72 & 0,01 & $\begin{array}{r}{[-1,66]} \\
(<=0,05)\end{array}$ \\
\hline _RWE & $\begin{array}{r}84,59 \\
{[18,00]} \\
(0,00) \\
\end{array}$ & $\begin{array}{r}0,08 \\
{[1,01]} \\
(0,32)\end{array}$ & $\begin{array}{r}-0,09 \\
{[-11,11]} \\
(0,00)\end{array}$ & 0,54 & 391,34 & 0,02 & $\begin{array}{r}{[-2,41]} \\
(<=1,00)\end{array}$ \\
\hline _BMW & $\begin{array}{r}37,60 \\
{[27,87]} \\
(0,00)\end{array}$ & $\begin{array}{r}0,02 \\
{[1,14]} \\
(0,26)\end{array}$ & $\begin{array}{r}-0,01 \\
{[-4,60]} \\
(0,00)\end{array}$ & 0,16 & 73,00 & 0,12 & $\begin{array}{r}{[-3,27]} \\
(<=0,10)\end{array}$ \\
\hline _LH & $\begin{array}{r}155,89 \\
{[24,18]} \\
(0,00)\end{array}$ & $\begin{array}{r}0,04 \\
{[0,66]} \\
(0,51)\end{array}$ & $\begin{array}{r}-0,12 \\
{[-11,20]} \\
(0,00)\end{array}$ & 0,43 & 268,06 & 0,01 & $\begin{array}{r}{[-1,74]} \\
(<=1,00)\end{array}$ \\
\hline _TYSS & $\begin{array}{r}205,46 \\
{[17,43]} \\
(0,00)\end{array}$ & $\begin{array}{r}-0,16 \\
{[-0,15]} \\
(0,88)\end{array}$ & $\begin{array}{r}-0,14 \\
{[-7,23]} \\
(0,00)\end{array}$ & 0,28 & 128,72 & 0,03 & $\begin{array}{r}{[-1,29]} \\
(<=1,00)\end{array}$ \\
\hline _METRO & $\begin{array}{r}98,17 \\
{[14,07]} \\
(0,00) \\
\end{array}$ & $\begin{array}{r}5,19 \\
{[7,26]} \\
(0,00) \\
\end{array}$ & $\begin{array}{r}-0,06 \\
{[-5,57]} \\
(0,00)\end{array}$ & 0,24 & 97,69 & 0,13 & $\begin{array}{r}{[-4,50]} \\
(<=0,01)\end{array}$ \\
\hline _TUI & $\begin{array}{r}321,73 \\
{[7,62]} \\
(0,00)\end{array}$ & $\begin{array}{r}0,36 \\
{[1,48]} \\
(0,14)\end{array}$ & $\begin{array}{r}-0,12 \\
{[-1,69]} \\
(0,09)\end{array}$ & 0,06 & 8,09 & 0,05 & $\begin{array}{r}{[-1,17]} \\
(<=1,00)\end{array}$ \\
\hline LLIN & $\begin{array}{r}53,99 \\
{[16,08]} \\
(0,00)\end{array}$ & $\begin{array}{r}0,00 \\
{[1,59]} \\
(0,11)\end{array}$ & $\begin{array}{r}-0,03 \\
{[-4,54]} \\
(0,00)\end{array}$ & 0,16 & 46,10 & 0,05 & $\begin{array}{r}{[-1,74]} \\
(<=1,00)\end{array}$ \\
\hline _HEN & $\begin{array}{r}21,00 \\
{[26,33]} \\
(0,00)\end{array}$ & $\begin{array}{r}-0,04 \\
{[-2,05]} \\
(0,04)\end{array}$ & $\begin{array}{r}0,01 \\
{[5,18]} \\
(0,00)\end{array}$ & 0,19 & 45,39 & 0,04 & $\begin{array}{r}{[-2,37]} \\
(<=0,05)\end{array}$ \\
\hline _CONT & $\begin{array}{r}113,60 \\
{[13,02]} \\
(0,00)\end{array}$ & $\begin{array}{r}-0,28 \\
{[-0,97]} \\
(0,33)\end{array}$ & $\begin{array}{r}-0,11 \\
{[-8,13]} \\
(0,00)\end{array}$ & 0,62 & 229,82 & 0,00 & $\begin{array}{r}{[-2,40]} \\
(<=0,05)\end{array}$ \\
\hline _DP & $\begin{array}{r}166,08 \\
{[7,93]} \\
(0,00)\end{array}$ & $\begin{array}{r}0,19 \\
{[0,59]} \\
(0,56)\end{array}$ & $\begin{array}{r}-0,20 \\
{[-7,02]} \\
(0,00)\end{array}$ & 0,79 & 108,42 & 0,06 & $\begin{array}{r}{[-3,60]} \\
(<=0,05)\end{array}$ \\
\hline
\end{tabular}

t-stats in [ ] and corresponding p-values in ( )

*Phillips and Ouliaris (1990) p-values

Table C.7.: Time-series regression results for the test of the relationship between default risk and implied volatility for a constant maturity of 180 days 


\begin{tabular}{|c|c|c|c|c|c|c|c|}
\hline \multicolumn{8}{|c|}{$C D S_{t}=\beta_{0}+\beta_{1} \cdot I M P V_{t}+\beta_{2} \cdot t+u_{t}$} \\
\hline ID & $b_{0}$ & $b_{1}$ & $b_{2}$ & R2 cor. & $\mathbf{F}$ & DW & ADF* \\
\hline \multirow[t]{3}{*}{-ALL } & 61,09 & 1,18 & \begin{tabular}{|c|}
$-0,05$ \\
\end{tabular} & \multirow[t]{3}{*}{0,20} & \multirow[t]{3}{*}{94,83} & \multirow[t]{3}{*}{0,02} & \\
\hline & {$[10,33]$} & {$[1,11]$} & {$[-5,11]$} & & & & {$[-2,43]$} \\
\hline & $(0,00)$ & $(0,27)$ & $(0,00)$ & & & & $(<=0,05)$ \\
\hline \multirow[t]{3}{*}{ _SIE } & 63,88 & 0,06 & $-0,06$ & \multirow[t]{3}{*}{0,59} & \multirow[t]{3}{*}{554,03} & \multirow[t]{3}{*}{0,01} & \\
\hline & {$[21,01]$} & {$[0,41]$} & {$[-11,55]$} & & & & {$[-3,20]$} \\
\hline & $(0,00)$ & $(0,68)$ & $(0,00)$ & & & & $(<=0$, \\
\hline \multirow[t]{3}{*}{ _DTE } & 244,82 & 34,37 & $-0,32$ & \multirow[t]{3}{*}{0,63} & 646,47 & 0,09 & \\
\hline & {$[9,97]$} & {$[1,19]$} & {$[-9,44]$} & & & & {$[-3,08]$} \\
\hline & $(0,00)$ & $(0,23)$ & $(0,00)$ & & & & $(<=0$ \\
\hline _DAIM & 152,82 & 15,70 & $-0,11$ & 0,72 & 968,97 & 0,12 & \\
\hline & {$[24,47]$} & {$[1,27]$} & {$[-15,79]$} & & & & {$[-4,24]$} \\
\hline & $(0,00)$ & $(0,21)$ & $(0,00)$ & & & & $(<=C$ \\
\hline _DBANK & 35,97 & 0,01 & $-0,03$ & 0,29 & 153,12 & 0,01 & \\
\hline & {$[15,45]$} & {$[5,84]$} & {$[-6,87]$} & & & & {$[-2,51]$} \\
\hline & $(0,00)$ & $(0,00)$ & $(0,00)$ & & & & $(<=0$, \\
\hline _MRE & 57,93 & 0,01 & $-0,05$ & 0,32 & 159,99 & 0,16 & \\
\hline & {$[13,12]$} & {$[0,08]$} & {$[-6,40]$} & & & & {$[-3,69]$} \\
\hline & $(0,00)$ & $(0,93)$ & $(0,00)$ & & & & $(<=0$ \\
\hline _BAY & 73,93 & 3,48 & $-0,04$ & 0,09 & 35,60 & 0,03 & \\
\hline & {$[11,94]$} & {$[1,28]$} & {$[-4,26]$} & & & & {$[-2,67]$} \\
\hline & $(0,00)$ & $(0,20)$ & $(0,00)$ & & & & $(<=0$ \\
\hline _VW & 49,16 & 0,02 & 0,03 & 0,20 & 97,47 & 0,02 & \\
\hline & {$[19,35]$} & {$[0,31]$} & {$[5,80]$} & & & & {$[-2,59]$} \\
\hline & $(0,00)$ & $(0,76)$ & $(0,00)$ & & & & $(<=0$, \\
\hline _BASF & 30,86 & $-0,01$ & $-0,03$ & 0,75 & $1.119,78$ & 0,03 & \\
\hline & {$[39,15]$} & {$[-1,60]$} & {$[-20,47]$} & & & & {$[-2,38]$} \\
\hline & $(0,00)$ & $(0,11)$ & $(0,00)$ & & & & $(<=0,05)$ \\
\hline _EON & 52,76 & 0,17 & $-0,04$ & 0,27 & 140,77 & 0,02 & \\
\hline & {$[14,17]$} & {$[3,13]$} & {$[-6,17]$} & & & & {$[-2,48]$} \\
\hline & $(0,00)$ & $(0,00)$ & $(0,00)$ & & & & $(<=0$ \\
\hline _COB & 99,54 & 1,05 & $-0,10$ & 0,24 & 116,45 & 0,03 & \\
\hline & {$[9,09]$} & {$[1,55]$} & {$[-5,64]$} & & & & {$[-2,14]$} \\
\hline & $(0,00)$ & $(0,12)$ & $(0,00)$ & & & & $(<=0$ \\
\hline _HYPV & 80,31 & $-0,11$ & $-0,06$ & 0,12 & 53,26 & 0,01 & \\
\hline & {$[9,29]$} & {$[-2,07]$} & {$[-4,16]$} & & & & {$[-1$} \\
\hline & $(0,00)$ & $(0,04)$ & $(0,00)$ & & & & $(<=0$ \\
\hline _RWE & 84,51 & 0,21 & $-0,09$ & 0,54 & 393,35 & 0,02 & \\
\hline & {$[18,02]$} & {$[1,17]$} & {$[-11,12]$} & & & & {$[-2,46]$} \\
\hline & $(0,00)$ & $(0,24)$ & $(0,00)$ & & & & $(<=1,00)$ \\
\hline BMW & 37,61 & $-0,01$ & $-0,01$ & 0,16 & 72,92 & 0,12 & \\
\hline & {$[27,90]$} & {$[-0,53]$} & {$[-4,61]$} & & & & {$[-3,27]$} \\
\hline & $000)$ & $(0,60)$ & $(0,00)$ & & & & $(<=0$ \\
\hline _LH & 155,90 & 0,05 & $-0,12$ & 0,43 & 268,16 & 0,01 & \\
\hline & t,19] & {$[0,77]$} & {$[-11,20]$} & & & & {$[-1,75]$} \\
\hline & $(0,0$ & $(0,44)$ & $(0,00)$ & & & & \\
\hline _TYSS & 205,48 & $-0,18$ & $-0,14$ & 0,28 & 128,79 & 0,03 & \\
\hline & {$[17,34]$} & {$[-0,75]$} & {$[-7,22]$} & & & & {$[-1,30]$} \\
\hline & $(0,00)$ & $(0,45)$ & $(0,00)$ & & & & \\
\hline _METRO & 100,96 & 0,17 & $-0,07$ & 0,21 & 80,84 & 0,07 & \\
\hline & {$[14,16]$} & {$[7,08]$} & {$[-5,56]$} & & & & {$[-3,51]$} \\
\hline & $(0,00)$ & $(0,00)$ & $(0,00)$ & & & & \\
\hline _TUI & 321,25 & 0,17 & $-0,11$ & 0,05 & 8,02 & 0,05 & \\
\hline & {$[7,61]$} & {$[0,86]$} & {$[-1,68]$} & & & & {$[-1,18]$} \\
\hline & $(0,0$ & $(0,39)$ & $(0,09)$ & & & & $(<=1$ \\
\hline _LIN & 53,99 & 0,00 & $-0,03$ & 0,16 & 46,10 & 0,05 & \\
\hline & {$[16,08]$} & {$[1,58]$} & {$[-4,54]$} & & & & {$[-1,75]$} \\
\hline & $(0,00)$ & $(0,12)$ & $(0,00)$ & & & & \\
\hline _HEN & 21,00 & $-0,05$ & 0,01 & 0,19 & 45,24 & 0,04 & \\
\hline & {$[26,34]$} & {$[-1,30]$} & {$[5,18]$} & & & & \\
\hline & $(0,00)$ & $(0,20)$ & $(0,00)$ & & & & $(<=0,0$ \\
\hline _CONT & 113,76 & $-0,68$ & $-0,11$ & 0,62 & 230,14 & 0,01 & \\
\hline & {$[13,06]$} & {$[-1,24]$} & {$[-8,15]$} & & & & \\
\hline & $(0,00)$ & $(0,22)$ & $(0,00)$ & & & & $(<=0,05)$ \\
\hline _DP & 166,10 & 0,14 & $-0,20$ & 0,79 & 108,39 & 0,06 & \\
\hline & {$[7,93]$} & {$[0,45]$} & {$[-7,02]$} & & & & {$[-2,51]$} \\
\hline & $(0,00)$ & $(0,66)$ & $(0,00)$ & & & & $1=-0,6$ \\
\hline
\end{tabular}

t-stats in [ ] and corresponding p-values in ( )

${ }^{*}$ Phillips and Ouliaris (1990) p-values

Table C.8.: Time-series regression results for the test of the relationship between default risk and implied volatility for a constant maturity of 240 days 


\section{C.3. The Credit Spread Level and the Shape of the Equity Volatility Smile}

C.3.1. Examination of the Relationship between the CDS Spread Level and the Smile Coefficients 


\begin{tabular}{|c|c|c|c|c|c|c|}
\hline \multicolumn{7}{|c|}{$C D S_{t}=\beta_{0}+\beta_{1} \cdot B 0_{t}+\beta_{2} \cdot t+u_{t}$} \\
\hline ID* & $b_{0}$ & $b_{1}$ & $b_{2}$ & R2 cor. & $F$ & DW \\
\hline \multirow[t]{3}{*}{-ALL } & 77,34 & $\begin{array}{l}-9,09 \\
\end{array}$ & \begin{tabular}{c|}
$-0,03$ \\
\end{tabular} & \multirow[t]{3}{*}{0,27} & \multirow[t]{3}{*}{137,16} & \multirow[t]{3}{*}{0,08} \\
\hline & {$[12,85]$} & {$[-4,41]$} & {$[-3,02]$} & & & \\
\hline & $(0,00)$ & $(0,00)$ & $(0,00)$ & & & \\
\hline \multirow[t]{3}{*}{-SIE } & 66,38 & $-1,46$ & $-0,06$ & \multirow[t]{3}{*}{0,60} & \multirow[t]{3}{*}{559,77} & \multirow[t]{3}{*}{0,02} \\
\hline & {$[21,87]$} & {$[-1,53]$} & {$[-9,44]$} & & & \\
\hline & $(0,00)$ & $(0,13)$ & $(0,00)$ & & & \\
\hline \multirow[t]{3}{*}{ DTE } & 265,13 & $-1,16$ & $-0,32$ & \multirow[t]{3}{*}{0,62} & \multirow[t]{3}{*}{617,77} & 0,02 \\
\hline & {$[16,77]$} & {$[-1,42]$} & {$[-11,08]$} & & & \\
\hline & $(0,00)$ & $(0,16)$ & $(0,00)$ & & & \\
\hline DAIM & 162,26 & $-1,70$ & $-0,11$ & 0,71 & 945,44 & 0,07 \\
\hline & {$[40,14]$} & {$[-1,99]$} & {$[-15,71]$} & & & \\
\hline & $(0,00)$ & $(0,05)$ & $(0,00)$ & & & \\
\hline _DBANK & 39,73 & $-1,89$ & $-0,02$ & 0,30 & 162,90 & 0,03 \\
\hline & {$[14,33]$} & {$[-2,59]$} & {$[-5,76]$} & & & \\
\hline & $(0,00)$ & $(0,01)$ & $(0,00)$ & & & \\
\hline _MRE & 60,91 & $-2,10$ & $-0,04$ & 0,34 & 176,10 & 0,21 \\
\hline & {$[14,82]$} & {$[-3,34]$} & {$[-5,36]$} & & & \\
\hline & $(0,00)$ & $(0,00)$ & $(0,00)$ & & & \\
\hline BAY & 77,15 & $-0,77$ & $-0,04$ & 0,09 & 35,77 & 0,03 \\
\hline & {$[11,98]$} & {$[-1,89]$} & {$[-4,22]$} & & & \\
\hline & $(0,00)$ & $(0,06)$ & $(0,00)$ & & & \\
\hline _VW & 49,04 & 0,05 & 0,03 & 0,20 & 97,48 & 0,02 \\
\hline & {$[19,25]$} & {$[0,56]$} & {$[5,78]$} & & & \\
\hline & $(0,00)$ & $(0,58)$ & $(0,00)$ & & & \\
\hline BASF & 30,90 & $-0,02$ & $-0,03$ & 0,75 & $1.124,78$ & 0,04 \\
\hline & {$[39,61]$} & {$[-5,58]$} & {$[-20,48]$} & & & \\
\hline & $(0,00)$ & $(0,00)$ & $(0,00)$ & & & \\
\hline EON & 52,66 & 0,00 & $-0,04$ & 0,27 & 136,94 & 0,01 \\
\hline & {$[14,04]$} & {$[0,22]$} & {$[-6,11]$} & & & \\
\hline & $(0,00)$ & $(0,83)$ & $(0,00)$ & & & \\
\hline _COB & 100,28 & $-0,02$ & $-0,10$ & 0,24 & 113,32 & 0,01 \\
\hline & {$[9,10]$} & {$[-1,09]$} & {$[-5,58]$} & & & \\
\hline & $(0,00)$ & $(0,28)$ & $(0,00)$ & & & \\
\hline _HYPV & 80,14 & 0,00 & $-0,06$ & 0,12 & 52,74 & 0,01 \\
\hline & {$[9,29]$} & {$[0,46]$} & {$[-4,15]$} & & & \\
\hline & $(0,00)$ & $(0,65)$ & $(0,00)$ & & & \\
\hline _RWE & 84,69 & 0,00 & $-0,09$ & 0,54 & 389,95 & 0,01 \\
\hline & {$[17,89]$} & {$[-0,97]$} & {$[-11,06]$} & & & \\
\hline & $(0,00)$ & $(0,33)$ & $(0,00)$ & & & \\
\hline _BMW & 37,61 & 0,00 & $-0,01$ & 0,16 & 73,16 & 0,12 \\
\hline & {$[27,86]$} & {$[-2,42]$} & {$[-4,61]$} & & & \\
\hline & $(0,00)$ & $(0,02)$ & $(0,00)$ & & & \\
\hline _LH & 155,86 & 0,00 & $-0,12$ & 0,43 & 268,08 & 0,01 \\
\hline & {$[24,22]$} & {$[-3,71]$} & {$[-11,21]$} & & & \\
\hline & $(0,00)$ & $(0,00)$ & $(0,00)$ & & & \\
\hline _TYSS & 205,46 & 0,01 & $-0,14$ & 0,28 & 129,06 & 0,03 \\
\hline & {$[17,36]$} & {$[4,14]$} & {$[-7,23]$} & & & \\
\hline & $(0,00)$ & $(0,00)$ & $(0,00)$ & & & \\
\hline _METRO & 101,28 & 0,00 & $-0,07$ & 0,20 & 75,28 & 0,05 \\
\hline & {$[14,08]$} & {$[-0,57]$} & {$[-5,55]$} & & & \\
\hline & $(0,00)$ & $(0,57)$ & $(0,00)$ & & & \\
\hline _TUI & 322,41 & 0,01 & $-0,12$ & 0,06 & 8,25 & 0,05 \\
\hline & {$[7,64]$} & {$[0,92]$} & {$[-1,71]$} & & & \\
\hline & $(0,00)$ & $(0,36)$ & $(0,09)$ & & & \\
\hline _LIN & 54,01 & 0,00 & $-0,03$ & 0,16 & 46,13 & 0,05 \\
\hline & {$[16,01]$} & {$[-1,12]$} & {$[-4,53]$} & & & \\
\hline & $(0,00)$ & $(0,26)$ & $(0,00)$ & & & \\
\hline _HEN & 20,98 & 0,00 & 0,01 & 0,19 & 45,16 & 0,04 \\
\hline & {$[26,25]$} & {$[-2,46]$} & {$[5,18]$} & & & \\
\hline & $(0,00)$ & $(0,01)$ & $(0,00)$ & & & \\
\hline _CONT & 113,50 & 0,00 & $-0,11$ & 0,62 & 230,00 & 0,00 \\
\hline & {$[12,95]$} & {$[-0,81]$} & {$[-8,12]$} & & & \\
\hline & $(0,00)$ & $(0,42)$ & $(0,00)$ & & & \\
\hline DPP & 166,56 & 0,00 & $-0,20$ & 0,79 & 109,89 & 0,09 \\
\hline & {$[7,94]$} & {$[1,14]$} & {$[-7,04]$} & & & \\
\hline & $(0,00)$ & $(0,26)]$ & $(0,00)$ & & & \\
\hline
\end{tabular}

Table C.9.: Time-series regression results for the test of the relationship between default risk and the smile coefficient $B 0$ for a constant maturity of 36 days 


\begin{tabular}{|c|c|c|c|c|c|c|}
\hline \multicolumn{7}{|c|}{$C D S_{t}=\beta_{0}+\beta_{1} \cdot B 1_{t}+\beta_{2} \cdot t+u_{t}$} \\
\hline ID & $b_{0}$ & $b_{1}$ & $b_{2}$ & R2 cor. & $\mathbf{F}$ & DW \\
\hline ALL & $\begin{array}{r}74,22 \\
{[14,58]} \\
(0,00) \\
\end{array}$ & $\begin{array}{r}6,33 \\
{[5,95]} \\
(0,00)\end{array}$ & $\begin{array}{r}-0,02 \\
{[-1,98]} \\
(0,05)\end{array}$ & 0,37 & 211,07 & 0,17 \\
\hline _SIE & $\begin{array}{r}68,08 \\
{[23,21]} \\
(0,00) \\
\end{array}$ & $\begin{array}{r}2,13 \\
{[3,67]} \\
(0,00) \\
\end{array}$ & $\begin{array}{r}-0,05 \\
{[-7,99]} \\
(0,00)\end{array}$ & 0,62 & 609,36 & 0,07 \\
\hline _DTE & $\begin{array}{r}264,61 \\
{[16,79]} \\
(0,00)\end{array}$ & $\begin{array}{r}0,91 \\
{[1,49]} \\
(0,14)\end{array}$ & $\begin{array}{r}-0,32 \\
{[-10,78]} \\
(0,00)\end{array}$ & 0,62 & 624,23 & 0,03 \\
\hline _DAIM & $\begin{array}{r}162,24 \\
{[42,19]} \\
(0,00) \\
\end{array}$ & $\begin{array}{r}1,33 \\
{[2,64]} \\
(0,01) \\
\end{array}$ & $\begin{array}{r}-0,11 \\
{[-14,74]} \\
(0,00)\end{array}$ & 0,72 & 966,66 & 0,09 \\
\hline _DBANK & $\begin{array}{r}40,98 \\
{[15,33]} \\
(0,00) \\
\end{array}$ & $\begin{array}{r}1,95 \\
{[4,12]} \\
(0,00) \\
\end{array}$ & $\begin{array}{r}-0,02 \\
{[-4,75]} \\
(0,00)\end{array}$ & 0,35 & 204,01 & 0,10 \\
\hline _MRE & $\begin{array}{r}59,67 \\
{[15,01]} \\
(0,00)\end{array}$ & $\begin{array}{r}1,52 \\
{[4,21]} \\
(0,00) \\
\end{array}$ & $\begin{array}{r}-0,04 \\
{[-4,86]} \\
(0,00) \\
\end{array}$ & 0,37 & 196,37 & 0,26 \\
\hline BAY & $\begin{array}{r}76,95 \\
{[12,05]} \\
(0,00) \\
\end{array}$ & $\begin{array}{r}0,47 \\
{[1,91]} \\
(0,06) \\
\end{array}$ & $\begin{array}{r}-0,04 \\
{[-4,19]} \\
(0,00) \\
\end{array}$ & 0,09 & 37,76 & 0,04 \\
\hline _VW & $\begin{array}{r}49,22 \\
{[19,37]} \\
(0,00) \\
\end{array}$ & $\begin{array}{r}0,03 \\
{[0,48]} \\
(0,63)\end{array}$ & $\begin{array}{r}0,03 \\
{[5,81]} \\
(0,00)\end{array}$ & 0,20 & 97,50 & 0,02 \\
\hline _BASF & $\begin{array}{r}30,89 \\
{[39,61]} \\
(0,00)\end{array}$ & $\begin{array}{r}0,01 \\
{[5,01]} \\
(0,00)\end{array}$ & $\begin{array}{r}-0,03 \\
{[-20,48]} \\
(0,00)\end{array}$ & 0,75 & $1.126,17$ & 0,04 \\
\hline EON & $\begin{array}{r}52,67 \\
{[14,05]} \\
(0,00) \\
\end{array}$ & $\begin{array}{r}0,00 \\
{[-0,01]} \\
(0,99) \\
\end{array}$ & $\begin{array}{r}-0,04 \\
{[-6,11]} \\
(0,00) \\
\end{array}$ & 0,27 & 136,94 & 0,01 \\
\hline _COB & $\begin{array}{r}100,25 \\
{[9,10]} \\
(0,00) \\
\end{array}$ & $\begin{array}{r}0,02 \\
{[1,37]} \\
(0,17) \\
\end{array}$ & $\begin{array}{r}-0,10 \\
{[-5,57]} \\
(0,00) \\
\end{array}$ & 0,24 & 113,40 & 0,01 \\
\hline _HYPV & $\begin{array}{l}80,14 \\
{[9,29]} \\
(0,00)\end{array}$ & $\begin{array}{r}0,00 \\
{[-0,37]} \\
(0,71) \\
\end{array}$ & $\begin{array}{r}-0,06 \\
{[-4,15]} \\
(0,00) \\
\end{array}$ & 0,12 & 52,73 & 0,01 \\
\hline _RWE & $\begin{array}{r}84,69 \\
{[17,89]} \\
(0,00) \\
\end{array}$ & $\begin{array}{r}0,00 \\
{[0,96]} \\
(0,34)\end{array}$ & $\begin{array}{r}-0,09 \\
{[-11,06]} \\
(0,00)\end{array}$ & 0,54 & 389,94 & 0,01 \\
\hline _BMW & $\begin{array}{r}37,61 \\
{[27,86]} \\
(0,00) \\
\end{array}$ & $\begin{array}{r}0,00 \\
{[2,45]} \\
(0,01) \\
\end{array}$ & $\begin{array}{r}-0,01 \\
{[-4,61]} \\
(0,00)\end{array}$ & 0,16 & 73,17 & 0,12 \\
\hline _LH & $\begin{array}{r}155,86 \\
{[24,22]} \\
(0,00) \\
\end{array}$ & $\begin{array}{r}0,00 \\
{[3,72]} \\
(0,00) \\
\end{array}$ & $\begin{array}{r}-0,12 \\
{[-11,20]} \\
(0,00)\end{array}$ & 0,43 & 268,09 & 0,01 \\
\hline _TYSS & $\begin{array}{r}205,46 \\
{[17,36]} \\
(0,00)\end{array}$ & $\begin{array}{r}0,00 \\
{[-4,09]} \\
(0,00) \\
\end{array}$ & $\begin{array}{r}-0,14 \\
{[-7,23]} \\
(0,00)\end{array}$ & 0,28 & 129,05 & 0,03 \\
\hline _METRO & $\begin{array}{r}101,28 \\
{[14,08]} \\
(0,00)\end{array}$ & $\begin{array}{r}0,00 \\
{[0,57]} \\
(0,57) \\
\end{array}$ & $\begin{array}{r}-0,07 \\
{[-5,55]} \\
(0,00) \\
\end{array}$ & 0,20 & 75,28 & 0,05 \\
\hline _ TUI & $\begin{array}{r}322,40 \\
{[7,64]} \\
(0,00) \\
\end{array}$ & $\begin{array}{r}0,00 \\
{[-0,90]} \\
(0,37) \\
\end{array}$ & $\begin{array}{r}-0,12 \\
{[-1,71]} \\
(0,09) \\
\end{array}$ & 0,06 & 8,25 & 0,05 \\
\hline _LIN & $\begin{array}{r}54,01 \\
{[16,01]} \\
(0,00)\end{array}$ & $\begin{array}{r}0,00 \\
{[0,99]} \\
(0,32)\end{array}$ & $\begin{array}{r}-0,03 \\
{[-4,53]} \\
(0,00)\end{array}$ & 0,16 & 46,12 & 0,05 \\
\hline _HEN & $\begin{array}{r}20,99 \\
{[26,25]} \\
(0,00)\end{array}$ & $\begin{array}{r}0,00 \\
{[2,54]} \\
(0,01)\end{array}$ & $\begin{array}{r}0,01 \\
{[5,18]} \\
(0,00)\end{array}$ & 0,19 & 45,16 & 0,04 \\
\hline _CONT & $\begin{array}{r}113,50 \\
{[12,95]} \\
(0,00)\end{array}$ & $\begin{array}{r}0,00 \\
{[0,79]} \\
(0,43)\end{array}$ & $\begin{array}{r}-0,11 \\
{[-8,12]} \\
(0,00)\end{array}$ & 0,62 & 229,98 & 0,00 \\
\hline _DP & $\begin{array}{r}166,57 \\
{[7,94]} \\
(0,00)\end{array}$ & $\begin{array}{r}0,00 \\
{[-1,20]} \\
(0,23)\end{array}$ & $\begin{array}{r}-0,20 \\
{[-7,04]} \\
(0,00)\end{array}$ & 0,79 & 109,99 & 0,10 \\
\hline
\end{tabular}

Table C.10.: Time-series regression results for the test of the relationship between default risk and the smile coefficient $B 1$ for a constant maturity of 36 days 


\begin{tabular}{|c|c|c|c|c|c|c|}
\hline \multicolumn{7}{|c|}{$C D S_{t}=\beta_{0}+\beta_{1} \cdot B 2_{t}+\beta_{2} \cdot t+u_{t}$} \\
\hline ID & $b_{0}$ & $b_{1}$ & $b_{2}$ & R2 cor. & $F$ & DW \\
\hline \multirow[t]{3}{*}{$\bar{A}$ ALL } & 72,38 & $-13,25$ & $-0,02$ & \multirow[t]{3}{*}{0,38} & \multirow[t]{3}{*}{227,31} & \multirow[t]{3}{*}{0,19} \\
\hline & {$[14,99]$} & {$[-6,20]$} & {$[-1,75]$} & & & \\
\hline & $(0,00)$ & $(0,00)$ & $(0,08)$ & & & \\
\hline \multirow[t]{3}{*}{-SIE } & 67,83 & $-4,99$ & $-0,05$ & \multirow[t]{3}{*}{0,63} & \multirow[t]{3}{*}{632,31} & \multirow[t]{3}{*}{0,10} \\
\hline & {$[23,74]$} & {$[-4,08]$} & {$[-7,69]$} & & & \\
\hline & $(0,00)$ & $(0,00)$ & $(0,00)$ & & & \\
\hline \multirow[t]{3}{*}{ _DTE } & 264,38 & $-2,00$ & $-0,32$ & \multirow[t]{3}{*}{0,62} & \multirow[t]{3}{*}{626,17} & 0,04 \\
\hline & {$[16,81]$} & {$[-1,50]$} & {$[-10,70]$} & & & \\
\hline & $(0,00)$ & $(0,13)$ & $(0,00)$ & & & \\
\hline DAIM & 161,90 & $-2,86$ & $-0,11$ & 0,72 & 972,12 & 0,10 \\
\hline & {$[42,79]$} & {$[-2,74]$} & {$[-14,53]$} & & & \\
\hline & $(0,00)$ & $(0,01)$ & $(0,00)$ & & & \\
\hline _DBANK & 40,47 & $-4,28$ & $-0,02$ & 0,36 & 218,17 & 0,12 \\
\hline & {$[16,09]$} & {$[-4,44]$} & {$[-4,42]$} & & & \\
\hline & $(0,00)$ & $(0,00)$ & $(0,00)$ & & & \\
\hline _MRE & 59,13 & $-3,26$ & $-0,04$ & 0,38 & 201,17 & 0,27 \\
\hline & {$[15,01]$} & {$[-4,38]$} & {$[-4,73]$} & & & \\
\hline & $(0,00)$ & $(0,00)$ & $(0,00)$ & & & \\
\hline BAY & 76,73 & $-0,92$ & $-0,04$ & 0,09 & 37,74 & 0,04 \\
\hline & {$[12,08]$} & {$[-1,90]$} & {$[-4,18]$} & & & \\
\hline & $(0,00)$ & $(0,06)$ & $(0,00)$ & & & \\
\hline _VW & 49,26 & $-0,09$ & 0,03 & 0,20 & 97,67 & 0,02 \\
\hline & {$[19,41]$} & {$[-0,73]$} & {$[5,82]$} & & & \\
\hline & $(0,00)$ & $(0,46)$ & $(0,00)$ & & & \\
\hline BASF & 30,88 & $-0,03$ & $-0,03$ & 0,75 & $1.126,54$ & 0,04 \\
\hline & {$[39,60]$} & {$[-4,80]$} & {$[-20,48]$} & & & \\
\hline & $(0,00)$ & $(0,00)$ & $(0,00)$ & & & \\
\hline EON & 52,67 & 0,00 & $-0,04$ & 0,27 & 136,94 & 0,01 \\
\hline & {$[14,05]$} & {$[-0,04]$} & {$[-6,11]$} & & & \\
\hline & $(0,00)$ & $(0,97)$ & $(0,00)$ & & & \\
\hline _COB & 100,24 & $-0,03$ & $-0,10$ & 0,24 & 113,43 & 0,01 \\
\hline & {$[9,10]$} & {$[-1,40]$} & {$[-5,57]$} & & & \\
\hline & $(0,00)$ & $(0,16)$ & $(0,00)$ & & & \\
\hline HYPV & 80,14 & 0,00 & $-0,06$ & 0,12 & 52,72 & 0,01 \\
\hline & {$[9,29]$} & {$[0,33]$} & {$[-4,15]$} & & & \\
\hline & $(0,00)$ & $(0,74)$ & $(0,00)$ & & & \\
\hline _RWE & 84,69 & 0,00 & $-0,09$ & 0,54 & 389,93 & 0,01 \\
\hline & {$[17,89]$} & {$[-0,95]$} & {$[-11,06]$} & & & \\
\hline & $(0,00)$ & $(0,34)$ & $(0,00)$ & & & \\
\hline BMW & 37,61 & 0,00 & $-0,01$ & 0,16 & 73,17 & 0,12 \\
\hline & {$[27,86]$} & {$[-2,48]$} & {$[-4,61]$} & & & \\
\hline & $(0,00)$ & $(0,01)$ & $(0,00)$ & & & \\
\hline LLH & 155,86 & 0,00 & $-0,12$ & 0,43 & 268,09 & 0,01 \\
\hline & {$[24,22]$} & {$[-3,72]$} & {$[-11,20]$} & & & \\
\hline & $(0,00)$ & $(0,00)$ & $(0,00)$ & & & \\
\hline _TYSS & 205,45 & 0,01 & $-0,14$ & 0,28 & 129,05 & 0,03 \\
\hline & {$[17,36]$} & {$[4,04]$} & {$[-7,23]$} & & & \\
\hline & $(0,00)$ & $(0,00)$ & $(0,00)$ & & & \\
\hline _METRO & 101,28 & 0,00 & $-0,07$ & 0,20 & 75,27 & 0,05 \\
\hline & {$[14,08]$} & {$[-0,57]$} & {$[-5,55]$} & & & \\
\hline & $(0,00)$ & $(0,57)$ & $(0,00)$ & & & \\
\hline _TUI & 322,40 & 0,01 & $-0,12$ & 0,06 & 8,24 & 0,05 \\
\hline & {$[7,64]$} & {$[0,88]$} & {$[-1,71]$} & & & \\
\hline & $(0,00)$ & $(0,38)$ & $(0,09)$ & & & \\
\hline LIIN & 54,00 & 0,00 & $-0,03$ & 0,16 & 46,11 & 0,05 \\
\hline & {$[16,02]$} & {$[-0,77]$} & {$[-4,53]$} & & & \\
\hline & $(0,00)$ & $(0,44)$ & $(0,00)$ & & & \\
\hline HEN & 20,99 & 0,00 & 0,01 & 0,19 & 45,16 & 0,04 \\
\hline & {$[26,25]$} & {$[-2,61]$} & {$[5,18]$} & & & \\
\hline & $(0,00)$ & $(0,01)$ & $(0,00)$ & & & \\
\hline _CONT & 113,49 & 0,00 & $-0,11$ & 0,62 & 229,97 & 0,00 \\
\hline & {$[12,95]$} & {$[-0,77]$} & {$[-8,12]$} & & & \\
\hline & $(0,00)$ & $(0,44)$ & $(0,00)$ & & & \\
\hline DP & 166,58 & 0,00 & $-0,20$ & 0,79 & 110,09 & 0,10 \\
\hline & {$[7,94]$} & {$[1,27]$} & {$[-7,04]$} & & & \\
\hline & $(0,00)$ & $(0,21)$ & $(0,00)$ & & & \\
\hline
\end{tabular}

Table C.11.: Time-series regression results for the test of the relationship between default risk and the smile coefficient $B 2$ for a constant maturity of 36 days 


\begin{tabular}{|c|c|c|c|c|c|c|}
\hline \multicolumn{7}{|c|}{$C D S_{t}=\beta_{0}+\beta_{1} \cdot B 0_{t}+\beta_{2} \cdot t+u_{t}$} \\
\hline ID & $\mathbf{b}_{0}$ & $b_{1}$ & $b_{2}$ & R2 cor. & $\mathbf{F}$ & DW \\
\hline ALL & $\begin{array}{r}59,64 \\
{[10,19]} \\
(0,00)\end{array}$ & $\begin{array}{r}1,71 \\
{[0,66]} \\
(0,51)\end{array}$ & $\begin{array}{r}-0,05 \\
{[-4,76]} \\
(0,00)\end{array}$ & 0,20 & 93,38 & 0,01 \\
\hline _SIE & $\begin{array}{r}59,87 \\
{[20,44]} \\
(0,00)\end{array}$ & $\begin{array}{r}3,40 \\
{[2,04]} \\
(0,04)\end{array}$ & $\begin{array}{r}-0,07 \\
{[-10,70]} \\
(0,00)\end{array}$ & 0,60 & 564,17 & 0,03 \\
\hline DTE & $\begin{array}{r}263,61 \\
{[16,54]} \\
(0,00)\end{array}$ & $\begin{array}{r}0,00 \\
{[0,03]} \\
(0,97)\end{array}$ & $\begin{array}{r}-0,33 \\
{[-11,52]} \\
(0,00)\end{array}$ & 0,62 & 613,44 & 0,01 \\
\hline _DAIM & $\begin{array}{r}159,14 \\
{[41,79]} \\
(0,00) \\
\end{array}$ & $\begin{array}{r}0,02 \\
{[7,42]} \\
(0,00)\end{array}$ & $\begin{array}{r}-0,11 \\
{[-17,16]} \\
(0,00)\end{array}$ & 0,71 & 932,63 & 0,06 \\
\hline _DBANK & $\begin{array}{r}35,60 \\
{[15,77]} \\
(0,00)\end{array}$ & $\begin{array}{r}0,33 \\
{[2,63]} \\
(0,01)\end{array}$ & $\begin{array}{r}-0,03 \\
{[-6,85]} \\
(0,00)\end{array}$ & 0,29 & 154,70 & 0,02 \\
\hline _MRE & $\begin{array}{r}58,43 \\
{[13,59]} \\
(0,00)\end{array}$ & $\begin{array}{r}-0,52 \\
{[-3,68]} \\
(0,00)\end{array}$ & $\begin{array}{r}-0,05 \\
{[-6,30]} \\
(0,00)\end{array}$ & 0,33 & 165,44 & 0,18 \\
\hline BAY & $\begin{array}{r}76,23 \\
{[11,96]} \\
(0,00)\end{array}$ & $\begin{array}{r}-0,70 \\
{[-2,16]} \\
(0,03)\end{array}$ & $\begin{array}{r}-0,04 \\
{[-4,21]} \\
(0,00)\end{array}$ & 0,08 & 33,65 & 0,02 \\
\hline _VW & $\begin{array}{r}49,08 \\
{[19,30]} \\
(0,00)\end{array}$ & $\begin{array}{r}0,04 \\
{[0,86]} \\
(0,39)\end{array}$ & $\begin{array}{r}0,03 \\
{[5,81]} \\
(0,00)\end{array}$ & 0,20 & 97,61 & 0,02 \\
\hline _BASF & $\begin{array}{r}30,86 \\
{[39,17]} \\
(0,00)\end{array}$ & $\begin{array}{r}0,00 \\
{[-0,65]} \\
(0,52)\end{array}$ & $\begin{array}{r}-0,03 \\
{[-20,48]} \\
(0,00)\end{array}$ & 0,75 & $1.119,22$ & 0,03 \\
\hline EON & $\begin{array}{r}52,65 \\
{[14,04]} \\
(0,00) \\
\end{array}$ & $\begin{array}{r}0,01 \\
{[1,33]} \\
(0,18)\end{array}$ & $\begin{array}{r}-0,04 \\
{[-6,10]} \\
(0,00)\end{array}$ & 0,27 & 137,01 & 0,01 \\
\hline _COB & $\begin{array}{r}100,28 \\
{[9,11]} \\
(0,00) \\
\end{array}$ & $\begin{array}{r}0,00 \\
{[0,92]} \\
(0,36)\end{array}$ & $\begin{array}{r}-0,10 \\
{[-5,62]} \\
(0,00) \\
\end{array}$ & 0,24 & 113,22 & 0,01 \\
\hline _HYPV & $\begin{array}{l}80,13 \\
{[9,29]} \\
(0,00)\end{array}$ & $\begin{array}{r}0,00 \\
{[1,14]} \\
(0,25)\end{array}$ & $\begin{array}{r}-0,06 \\
{[-4,14]} \\
(0,00)\end{array}$ & 0,12 & 52,73 & 0,01 \\
\hline _RWE & $\begin{array}{r}84,66 \\
{[17,91]} \\
(0,00) \\
\end{array}$ & $\begin{array}{r}0,00 \\
{[-0,15]} \\
(0,88) \\
\end{array}$ & $\begin{array}{r}-0,09 \\
{[-11,06]} \\
(0,00)\end{array}$ & 0,54 & 389,81 & 0,01 \\
\hline _BMW & $\begin{array}{r}37,61 \\
{[27,86]} \\
(0,00)\end{array}$ & $\begin{array}{r}0,00 \\
{[-3,89]} \\
(0,00)\end{array}$ & $\begin{array}{r}-0,01 \\
{[-4,60]} \\
(0,00)\end{array}$ & 0,16 & 73,36 & 0,12 \\
\hline _LH & $\begin{array}{r}155,85 \\
{[24,21]} \\
(0,00) \\
\end{array}$ & $\begin{array}{r}0,00 \\
{[-4,03]} \\
(0,00) \\
\end{array}$ & $\begin{array}{r}-0,12 \\
{[-11,20]} \\
(0,00)\end{array}$ & 0,43 & 268,10 & 0,01 \\
\hline _TYSS & $\begin{array}{r}205,46 \\
{[17,36]} \\
(0,00)\end{array}$ & $\begin{array}{r}0,01 \\
{[4,54]} \\
(0,00)\end{array}$ & $\begin{array}{r}-0,14 \\
{[-7,22]} \\
(0,00)\end{array}$ & 0,28 & 129,08 & 0,03 \\
\hline _METRO & $\begin{array}{r}101,23 \\
{[14,07]} \\
(0,00) \\
\end{array}$ & $\begin{array}{r}0,00 \\
{[-0,50]} \\
(0,62) \\
\end{array}$ & $\begin{array}{r}-0,07 \\
{[-5,53]} \\
(0,00) \\
\end{array}$ & 0,20 & 75,22 & 0,05 \\
\hline _ & $\begin{array}{r}323,02 \\
{[7,62]} \\
(0,00) \\
\end{array}$ & $\begin{array}{r}0,00 \\
{[1,28]} \\
(0,20) \\
\end{array}$ & $\begin{array}{r}-0,12 \\
{[-1,71]} \\
(0,09) \\
\end{array}$ & 0,06 & 8,30 & 0,05 \\
\hline _LIN & $\begin{array}{r}53,97 \\
{[16,07]} \\
(0,00)\end{array}$ & $\begin{array}{r}0,00 \\
{[0,64]} \\
(0,52)\end{array}$ & $\begin{array}{r}-0,03 \\
{[-4,53]} \\
(0,00)\end{array}$ & 0,16 & 46,17 & 0,05 \\
\hline _ HEN & $\begin{array}{r}20,98 \\
{[26,24]} \\
(0,00) \\
\end{array}$ & $\begin{array}{r}0,00 \\
{[-2,67]} \\
(0,01) \\
\end{array}$ & $\begin{array}{r}0,01 \\
{[5,18]} \\
(0,00)\end{array}$ & 0,19 & 45,21 & 0,04 \\
\hline _CONT & $\begin{array}{r}113,50 \\
{[12,94]} \\
(0,00)\end{array}$ & $\begin{array}{r}0,00 \\
{[-0,54]} \\
(0,59)\end{array}$ & $\begin{array}{r}-0,11 \\
{[-8,11]} \\
(0,00)\end{array}$ & 0,62 & 229,81 & 0,00 \\
\hline _DP & $\begin{array}{r}166,35 \\
{[7,90]} \\
(0,00)\end{array}$ & $\begin{array}{r}0,00 \\
{[0,79]} \\
(0,43)\end{array}$ & $\begin{array}{r}-0,20 \\
{[-7,00]} \\
(0,00)\end{array}$ & 0,79 & 108,59 & 0,07 \\
\hline
\end{tabular}

Table C.12.: Time-series regression results for the test of the relationship between default risk and the smile coefficient $B 0$ for a constant maturity of 60 days 


\begin{tabular}{|c|c|c|c|c|c|c|}
\hline \multicolumn{7}{|c|}{$C D S_{t}=\beta_{0}+\beta_{1} \cdot B 1_{t}+\beta_{2} \cdot t+u_{t}$} \\
\hline ID & $b_{0}$ & $b_{1}$ & $b_{2}$ & R2 cor. & $F$ & DW \\
\hline AALL & $\begin{array}{r}69,38 \\
{[12,48]} \\
(0,00)\end{array}$ & $\begin{array}{r}8,23 \\
{[3,85]} \\
(0,00)\end{array}$ & $\begin{array}{r}-0,03 \\
{[-2,56]} \\
(0,01)\end{array}$ & 0,27 & 137,87 & 0,14 \\
\hline _SIE & $\begin{array}{r}66,47 \\
{[22,97]} \\
(0,00)\end{array}$ & $\begin{array}{r}2,51 \\
{[2,55]} \\
(0,01)\end{array}$ & $\begin{array}{r}-0,06 \\
{[-8,50]} \\
(0,00)\end{array}$ & 0,60 & 577,29 & 0,05 \\
\hline _DTE & $\begin{array}{r}263,61 \\
{[16,53]} \\
(0,00)\end{array}$ & $\begin{array}{r}0,02 \\
{[0,63]} \\
(0,53)\end{array}$ & $\begin{array}{r}-0,33 \\
{[-11,52]} \\
(0,00)\end{array}$ & 0,62 & 613,53 & 0,01 \\
\hline DAIM & $\begin{array}{r}159,15 \\
{[41,78]} \\
(0,00)\end{array}$ & $\begin{array}{r}-0,01 \\
{[-4,91]} \\
(0,00)\end{array}$ & $\begin{array}{r}-0,11 \\
{[-17,16]} \\
(0,00)\end{array}$ & 0,71 & 932,45 & 0,06 \\
\hline _DBANK & $\begin{array}{r}35,99 \\
{[15,47]} \\
(0,00)\end{array}$ & $\begin{array}{r}0,01 \\
{[0,14]} \\
(0,89)\end{array}$ & $\begin{array}{r}-0,03 \\
{[-6,80]} \\
(0,00)\end{array}$ & 0,29 & 152,75 & 0,01 \\
\hline _MRE & $\begin{array}{r}58,08 \\
{[13,48]} \\
(0,00)\end{array}$ & $\begin{array}{r}0,37 \\
{[2,51]} \\
(0,01)\end{array}$ & $\begin{array}{r}-0,05 \\
{[-6,19]} \\
(0,00)\end{array}$ & 0,34 & 169,87 & 0,20 \\
\hline BAY & $\begin{array}{r}76,12 \\
{[12,03]} \\
(0,00)\end{array}$ & $\begin{array}{r}0,54 \\
{[2,63]} \\
(0,01)\end{array}$ & $\begin{array}{r}-0,04 \\
{[-4,16]} \\
(0,00)\end{array}$ & 0,09 & 35,62 & 0,03 \\
\hline _VW & $\begin{array}{r}49,13 \\
{[19,32]} \\
(0,00)\end{array}$ & $\begin{array}{r}-0,01 \\
{[-0,50]} \\
(0,62)\end{array}$ & $\begin{array}{r}0,03 \\
{[5,81]} \\
(0,00)\end{array}$ & 0,20 & 97,43 & 0,02 \\
\hline BASF & $\begin{array}{r}30,86 \\
{[39,17]} \\
(0,00)\end{array}$ & $\begin{array}{r}0,00 \\
{[0,68]} \\
(0,49)\end{array}$ & $\begin{array}{r}-0,03 \\
{[-20,48]} \\
(0,00)\end{array}$ & 0,75 & $1.119,26$ & 0,03 \\
\hline EEON & $\begin{array}{r}52,66 \\
{[14,05]} \\
(0,00)\end{array}$ & $\begin{array}{r}0,00 \\
{[-0,74]} \\
(0,46)\end{array}$ & $\begin{array}{r}-0,04 \\
{[-6,10]} \\
(0,00)\end{array}$ & 0,27 & 136,97 & 0,01 \\
\hline _COB & $\begin{array}{r}100,29 \\
{[9,11]} \\
(0,00)\end{array}$ & $\begin{array}{r}0,00 \\
{[-0,87]} \\
(0,39) \\
\end{array}$ & $\begin{array}{r}-0,10 \\
{[-5,62]} \\
(0,00)\end{array}$ & 0,24 & 113,21 & 0,01 \\
\hline _HYPV & $\begin{array}{l}80,13 \\
{[9,29]} \\
(0,00)\end{array}$ & $\begin{array}{r}0,00 \\
{[-1,02]} \\
(0,31)\end{array}$ & $\begin{array}{r}-0,06 \\
{[-4,14]} \\
(0,00)\end{array}$ & 0,12 & 52,73 & 0,01 \\
\hline _RWE & $\begin{array}{r}84,66 \\
{[17,91]} \\
(0,00)\end{array}$ & $\begin{array}{r}0,00 \\
{[0,24]} \\
(0,81)\end{array}$ & $\begin{array}{r}-0,09 \\
{[-11,06]} \\
(0,00)\end{array}$ & 0,54 & 389,81 & 0,01 \\
\hline _BMW & $\begin{array}{r}37,61 \\
{[27,86]} \\
(0,00)\end{array}$ & $\begin{array}{r}0,00 \\
{[3,96]} \\
(0,00)\end{array}$ & $\begin{array}{r}-0,01 \\
{[-4,60]} \\
(0,00)\end{array}$ & 0,16 & 73,37 & 0,12 \\
\hline _LH & $\begin{array}{r}155,85 \\
{[24,21]} \\
(0,00)\end{array}$ & $\begin{array}{r}0,00 \\
{[3,98]} \\
(0,00)\end{array}$ & $\begin{array}{r}-0,12 \\
{[-11,20]} \\
(0,00)\end{array}$ & 0,43 & 268,11 & 0,01 \\
\hline _TYSS & $\begin{array}{r}205,46 \\
{[17,36]} \\
(0,00)\end{array}$ & $\begin{array}{r}0,00 \\
{[-4,55]} \\
(0,00)\end{array}$ & $\begin{array}{r}-0,14 \\
{[-7,22]} \\
(0,00)\end{array}$ & 0,28 & 129,07 & 0,03 \\
\hline _METRO & $\begin{array}{r}101,23 \\
{[14,07]} \\
(0,00)\end{array}$ & $\begin{array}{r}0,00 \\
{[0,59]} \\
(0,56)\end{array}$ & $\begin{array}{r}-0,07 \\
{[-5,53]} \\
(0,00)\end{array}$ & 0,20 & 75,24 & 0,05 \\
\hline -TUI & $\begin{array}{r}322,97 \\
{[7,62]} \\
(0,00)\end{array}$ & $\begin{array}{r}0,00 \\
{[-1,26]} \\
(0,21)\end{array}$ & $\begin{array}{r}-0,12 \\
{[-1,71]} \\
(0,09)\end{array}$ & 0,06 & 8,29 & 0,05 \\
\hline LLIN & $\begin{array}{r}53,98 \\
{[16,08]} \\
(0,00)\end{array}$ & $\begin{array}{r}0,00 \\
{[-0,81]} \\
(0,42)\end{array}$ & $\begin{array}{r}-0,03 \\
{[-4,54]} \\
(0,00)\end{array}$ & 0,16 & 46,16 & 0,05 \\
\hline _HEN & $\begin{array}{r}20,98 \\
{[26,25]} \\
(0,00)\end{array}$ & $\begin{array}{r}0,00 \\
{[2,75]} \\
(0,01)\end{array}$ & $\begin{array}{r}0,01 \\
{[5,18]} \\
(0,00)\end{array}$ & 0,19 & 45,20 & 0,04 \\
\hline _CONT & $\begin{array}{r}113,50 \\
{[12,94]} \\
(0,00)\end{array}$ & $\begin{array}{r}0,00 \\
{[0,53]} \\
(0,59)\end{array}$ & $\begin{array}{r}-0,11 \\
{[-8,11]} \\
(0,00)\end{array}$ & 0,62 & 229,80 & 0,00 \\
\hline DP & $\begin{array}{r}166,36 \\
{[7,90]} \\
(0,00)\end{array}$ & $\begin{array}{r}0,00 \\
{[-0,80]} \\
(0,43)\end{array}$ & $\begin{array}{r}-0,20 \\
{[-7,00]} \\
(0,00)\end{array}$ & 0,79 & 108,61 & 0,07 \\
\hline
\end{tabular}

Table C.13.: Time-series regression results for the test of the relationship between default risk and the smile coefficient $B 1$ for a constant maturity of 60 days 


\begin{tabular}{|c|c|c|c|c|c|c|}
\hline \multicolumn{7}{|c|}{$C D S_{t}=\beta_{0}+\beta_{1} \cdot B 2_{t}+\beta_{2} \cdot t+u_{t}$} \\
\hline ID & $b_{0}$ & $b_{1}$ & $b_{2}$ & R2 cor. & $\mathbf{F}$ & DW \\
\hline ALL & $\begin{array}{r}67,53 \\
{[12,76]} \\
(0,00)\end{array}$ & $\begin{array}{r}-18,70 \\
{[-3,99]} \\
(0,00)\end{array}$ & $\begin{array}{r}-0,02 \\
{[-2,21]} \\
(0,03)\end{array}$ & 0,29 & 151,71 & 0,18 \\
\hline SIE & $\begin{array}{r}66,37 \\
{[23,17]} \\
(0,00) \\
\end{array}$ & $\begin{array}{r}-7,00 \\
{[-3,04]} \\
(0,00)\end{array}$ & $\begin{array}{r}-0,05 \\
{[-8,03]} \\
(0,00)\end{array}$ & 0,61 & 601,39 & 0,08 \\
\hline DTE & $\begin{array}{r}263,60 \\
{[16,53]} \\
(0,00)\end{array}$ & $\begin{array}{r}-0,06 \\
{[-0,74]} \\
(0,46)\end{array}$ & $\begin{array}{r}-0,33 \\
{[-11,52]} \\
(0,00)\end{array}$ & 0,62 & 613,57 & 0,01 \\
\hline DAIM & $\begin{array}{r}159,16 \\
{[41,78]} \\
(0,00)\end{array}$ & $\begin{array}{r}0,02 \\
{[4,07]} \\
(0,00)\end{array}$ & $\begin{array}{r}-0,11 \\
{[-17,16]} \\
(0,00)\end{array}$ & 0,71 & 932,42 & 0,06 \\
\hline DBANK & $\begin{array}{r}36,01 \\
{[15,46]} \\
(0,00)\end{array}$ & $\begin{array}{r}-0,13 \\
{[-0,44]} \\
(0,66)\end{array}$ & $\begin{array}{r}-0,03 \\
{[-6,77]} \\
(0,00)\end{array}$ & 0,29 & 152,99 & 0,01 \\
\hline MRE & $\begin{array}{r}57,97 \\
{[13,44]} \\
(0,00)\end{array}$ & $\begin{array}{r}-0,81 \\
{[-2,37]} \\
(0,02)\end{array}$ & $\begin{array}{r}-0,05 \\
{[-6,16]} \\
(0,00)\end{array}$ & 0,34 & 170,73 & 0,21 \\
\hline BAY & $\begin{array}{r}75,94 \\
{[12,05]} \\
(0,00) \\
\end{array}$ & $\begin{array}{r}-1,09 \\
{[-2,60]} \\
(0,01)\end{array}$ & $\begin{array}{r}-0,04 \\
{[-4,15]} \\
(0,00)\end{array}$ & 0,09 & 35,63 & 0,03 \\
\hline -VW & $\begin{array}{r}49,14 \\
{[19,32]} \\
(0,00) \\
\end{array}$ & $\begin{array}{r}0,01 \\
{[0,27]} \\
(0,79)\end{array}$ & $\begin{array}{r}0,03 \\
{[5,80]} \\
(0,00)\end{array}$ & 0,20 & 97,40 & 0,02 \\
\hline BASF & $\begin{array}{r}30,86 \\
{[39,17]} \\
(0,00) \\
\end{array}$ & $\begin{array}{r}0,00 \\
{[-0,69]} \\
(0,49) \\
\end{array}$ & $\begin{array}{r}-0,03 \\
{[-20,48]} \\
(0,00) \\
\end{array}$ & 0,75 & $1.119,26$ & 0,03 \\
\hline EON & $\begin{array}{r}52,66 \\
{[14,05]} \\
(0,00) \\
\end{array}$ & $\begin{array}{r}0,01 \\
{[0,65]} \\
(0,52) \\
\end{array}$ & $\begin{array}{r}-0,04 \\
{[-6,10]} \\
(0,00)\end{array}$ & 0,27 & 136,97 & 0,01 \\
\hline _COB & $\begin{array}{r}100,29 \\
{[9,10]} \\
(0,00) \\
\end{array}$ & $\begin{array}{r}0,00 \\
{[0,86]} \\
(0,39)\end{array}$ & $\begin{array}{r}-0,10 \\
{[-5,62]} \\
(0,00)\end{array}$ & 0,24 & 113,21 & 0,01 \\
\hline _HYPV & $\begin{array}{l}80,13 \\
{[9,29]} \\
(0,00)\end{array}$ & $\begin{array}{r}0,00 \\
{[0,94]} \\
(0,35)\end{array}$ & $\begin{array}{r}-0,06 \\
{[-4,14]} \\
(0,00)\end{array}$ & 0,12 & 52,73 & 0,01 \\
\hline _RWE & $\begin{array}{r}84,66 \\
{[17,90]} \\
(0,00)\end{array}$ & $\begin{array}{r}0,00 \\
{[-0,29]} \\
(0,77) \\
\end{array}$ & $\begin{array}{r}-0,09 \\
{[-11,06]} \\
(0,00)\end{array}$ & 0,54 & 389,81 & 0,01 \\
\hline BMW & $\begin{array}{r}37,61 \\
{[27,86]} \\
(0,00) \\
\end{array}$ & $\begin{array}{r}0,00 \\
{[-4,03]} \\
(0,00) \\
\end{array}$ & $\begin{array}{r}-0,01 \\
{[-4,60]} \\
(0,00)\end{array}$ & 0,16 & 73,37 & 0,12 \\
\hline LLH & $\begin{array}{r}155,85 \\
{[24,21]} \\
(0,00) \\
\end{array}$ & $\begin{array}{r}0,00 \\
{[-3,94]} \\
(0,00) \\
\end{array}$ & $\begin{array}{r}-0,12 \\
{[-11,20]} \\
(0,00) \\
\end{array}$ & 0,43 & 268,11 & 0,01 \\
\hline _TYSS & $\begin{array}{r}205,46 \\
{[17,36]} \\
(0,00) \\
10122\end{array}$ & $\begin{array}{r}0,01 \\
{[4,56]} \\
(0,00) \\
\end{array}$ & $\begin{array}{r}-0,14 \\
{[-7,22]} \\
(0,00)\end{array}$ & 0,28 & 129,07 & 0,03 \\
\hline _METRO & $\begin{array}{r}101,23 \\
{[14,07]} \\
(0,00) \\
\end{array}$ & $\begin{array}{r}0,00 \\
{[-0,65]} \\
(0,52) \\
\end{array}$ & $\begin{array}{r}-0,07 \\
{[-5,53]} \\
(0,00) \\
\end{array}$ & 0,20 & 75,26 & 0,05 \\
\hline _TUI & $\begin{array}{r}322,92 \\
{[7,62]} \\
(0,00) \\
5209\end{array}$ & $\begin{array}{r}0,00 \\
{[1,24]} \\
(0,22) \\
\end{array}$ & $\begin{array}{r}-0,12 \\
{[-1,71]} \\
(0,09) \\
\end{array}$ & 0,06 & 8,27 & 0,05 \\
\hline LLIN & $\begin{array}{r}53,98 \\
{[16,09]} \\
(0,00) \\
\end{array}$ & $\begin{array}{r}0,00 \\
{[1,01]} \\
(0,31)\end{array}$ & $\begin{array}{r}-0,03 \\
{[-4,54]} \\
(0,00)\end{array}$ & 0,16 & 46,13 & 0,05 \\
\hline _HEN & $\begin{array}{r}20,98 \\
{[26,25]} \\
(0,00) \\
\end{array}$ & $\begin{array}{r}0,00 \\
{[-2,82]} \\
(0,01) \\
\end{array}$ & $\begin{array}{r}0,01 \\
{[5,18]} \\
(0,00) \\
\end{array}$ & 0,19 & 45,20 & 0,04 \\
\hline _CONT & $\begin{array}{r}113,50 \\
{[12,93]} \\
(0,00) \\
\end{array}$ & $\begin{array}{r}0,00 \\
{[-0,52]} \\
(0,60) \\
\end{array}$ & $\begin{array}{r}-0,11 \\
{[-8,11]} \\
(0,00) \\
\end{array}$ & 0,62 & 229,80 & 0,00 \\
\hline DP & $\begin{array}{r}166,37 \\
{[7,90]} \\
(0,00)\end{array}$ & $\begin{array}{r}0,00 \\
{[0,81]} \\
(0,42)\end{array}$ & $\begin{array}{r}-0,20 \\
{[-7,00]} \\
(0,00)\end{array}$ & 0,79 & 108,62 & 0,07 \\
\hline
\end{tabular}

Table C.14.: Time-series regression results for the test of the relationship between default risk and the smile coefficient $B 2$ for a constant maturity of 60 days 


\begin{tabular}{|c|c|c|c|c|c|c|}
\hline \multicolumn{7}{|c|}{$C D S_{t}=\beta_{0}+\beta_{1} \cdot B 0_{t}+\beta_{2} \cdot t+u_{t}$} \\
\hline ID & $b_{0}$ & $b_{1}$ & $b_{2}$ & R2 cor. & $\mathbf{F}$ & DW \\
\hline ALL & $\begin{array}{r}61,62 \\
{[10,42]} \\
(0,00)\end{array}$ & $\begin{array}{r}0,00 \\
{[-1,02]} \\
(0,31)\end{array}$ & $\begin{array}{r}-0,05 \\
{[-5,08]} \\
(0,00)\end{array}$ & 0,20 & 93,01 & 0,01 \\
\hline SSIE & $\begin{array}{r}63,90 \\
{[21,02]} \\
(0,00)\end{array}$ & $\begin{array}{r}0,01 \\
{[0,44]} \\
(0,66) \\
\end{array}$ & $\begin{array}{r}-0,06 \\
{[-11,56]} \\
(0,00) \\
\end{array}$ & 0,59 & 554,01 & 0,01 \\
\hline _DTE & $\begin{array}{r}263,62 \\
{[16,52]} \\
(0,00)\end{array}$ & $\begin{array}{r}0,00 \\
{[1,01]} \\
(0,31)\end{array}$ & $\begin{array}{r}-0,33 \\
{[-11,53]} \\
(0,00)\end{array}$ & 0,62 & 613,46 & 0,01 \\
\hline DAIM & $\begin{array}{r}159,05 \\
{[41,01]} \\
(0,00)\end{array}$ & $\begin{array}{r}0,12 \\
{[0,39]} \\
(0,69)\end{array}$ & $\begin{array}{r}-0,11 \\
{[-17,14]} \\
(0,00)\end{array}$ & 0,71 & 932,20 & 0,06 \\
\hline _DBANK & $\begin{array}{r}35,97 \\
{[15,45]} \\
(0,00)\end{array}$ & $\begin{array}{r}0,02 \\
{[3,28]} \\
(0,00)\end{array}$ & $\begin{array}{r}-0,03 \\
{[-6,87]} \\
(0,00)\end{array}$ & 0,29 & 153,22 & 0,01 \\
\hline _MRE & $\begin{array}{r}57,93 \\
{[13,10]} \\
(0,00)\end{array}$ & $\begin{array}{r}0,00 \\
{[0,15]} \\
(0,88) \\
\end{array}$ & $\begin{array}{r}-0,05 \\
{[-6,39]} \\
(0,00)\end{array}$ & 0,32 & 160,00 & 0,16 \\
\hline _BAY & $\begin{array}{r}75,30 \\
{[11,95]} \\
(0,00) \\
\end{array}$ & $\begin{array}{r}-0,05 \\
{[-0,50]} \\
(0,62)\end{array}$ & $\begin{array}{r}-0,04 \\
{[-4,26]} \\
(0,00)\end{array}$ & 0,08 & 32,33 & 0,02 \\
\hline -VW & $\begin{array}{r}49,18 \\
{[19,36]} \\
(0,00)\end{array}$ & $\begin{array}{r}0,00 \\
{[1,17]} \\
(0,24)\end{array}$ & $\begin{array}{r}0,03 \\
{[5,78]} \\
(0,00)\end{array}$ & 0,20 & 97,70 & 0,02 \\
\hline _BASF & $\begin{array}{r}30,85 \\
{[39,18]} \\
(0,00)\end{array}$ & $\begin{array}{r}0,00 \\
{[-3,25]} \\
(0,00)\end{array}$ & $\begin{array}{r}-0,03 \\
{[-20,48]} \\
(0,00)\end{array}$ & 0,75 & $1.122,05$ & 0,04 \\
\hline EON & $\begin{array}{r}52,76 \\
{[14,11]} \\
(0,00)\end{array}$ & $\begin{array}{r}0,00 \\
{[1,44]} \\
(0,15) \\
\end{array}$ & $\begin{array}{r}-0,04 \\
{[-6,15]} \\
(0,00)\end{array}$ & 0,27 & 138,08 & 0,01 \\
\hline _COB & $\begin{array}{r}100,29 \\
{[9,11]} \\
(0,00)\end{array}$ & $\begin{array}{r}0,00 \\
{[-1,04]} \\
(0,30)\end{array}$ & $\begin{array}{r}-0,10 \\
{[-5,62]} \\
(0,00)\end{array}$ & 0,24 & 113,22 & 0,01 \\
\hline _HYPV & $\begin{array}{l}80,13 \\
{[9,29]} \\
(0,00)\end{array}$ & $\begin{array}{r}0,00 \\
{[0,89]} \\
(0,37) \\
\end{array}$ & $\begin{array}{r}-0,06 \\
{[-4,14]} \\
(0,00) \\
\end{array}$ & 0,12 & 52,73 & 0,01 \\
\hline - RWE & $\begin{array}{r}84,66 \\
{[17,96]} \\
(0,00)\end{array}$ & $\begin{array}{r}0,00 \\
{[0,50]} \\
(0,62)\end{array}$ & $\begin{array}{r}-0,09 \\
{[-11,10]} \\
(0,00)\end{array}$ & 0,54 & 389,92 & 0,01 \\
\hline BMWW & $\begin{array}{r}37,61 \\
{[27,87]} \\
(0,00) \\
\end{array}$ & $\begin{array}{r}0,00 \\
{[-1,13]} \\
(0,26) \\
\end{array}$ & $\begin{array}{r}-0,01 \\
{[-4,61]} \\
(0,00)\end{array}$ & 0,16 & 73,08 & 0,12 \\
\hline LLH & $\begin{array}{r}155,86 \\
{[24,20]} \\
(0,00)\end{array}$ & $\begin{array}{r}0,00 \\
{[-0,38]} \\
(0,71) \\
\end{array}$ & $\begin{array}{r}-0,12 \\
{[-11,20]} \\
(0,00) \\
\end{array}$ & 0,43 & 267,94 & 0,01 \\
\hline _TYSS & $\begin{array}{r}205,34 \\
{[17,37]} \\
(0,00)\end{array}$ & $\begin{array}{r}-0,01 \\
{[-3,44]} \\
(0,00)\end{array}$ & $\begin{array}{r}-0,14 \\
{[-7,21]} \\
(0,00)\end{array}$ & 0,28 & 129,06 & 0,03 \\
\hline _METRO & $\begin{array}{r}101,22 \\
{[14,14]} \\
(0,00)\end{array}$ & $\begin{array}{r}0,01 \\
{[1,01]} \\
(0,31)\end{array}$ & $\begin{array}{r}-0,07 \\
{[-5,58]} \\
(0,00)\end{array}$ & 0,20 & 76,73 & 0,05 \\
\hline -TUI & $\begin{array}{r}322,29 \\
{[7,61]} \\
(0,00)\end{array}$ & $\begin{array}{r}0,00 \\
{[1,30]} \\
(0,19)\end{array}$ & $\begin{array}{r}-0,12 \\
{[-1,70]} \\
(0,09)\end{array}$ & 0,06 & 8,13 & 0,05 \\
\hline _LIN & $\begin{array}{r}54,04 \\
{[16,09]} \\
(0,00)\end{array}$ & $\begin{array}{r}0,00 \\
{[1,10]} \\
(0,27)\end{array}$ & $\begin{array}{r}-0,03 \\
{[-4,56]} \\
(0,00)\end{array}$ & 0,16 & 46,55 & 0,05 \\
\hline _HEN & $\begin{array}{r}20,98 \\
{[26,25]} \\
(0,00)\end{array}$ & $\begin{array}{r}0,00 \\
{[-2,65]} \\
(0,01) \\
\end{array}$ & $\begin{array}{r}0,01 \\
{[5,18]} \\
(0,00) \\
\end{array}$ & 0,19 & 45,27 & 0,04 \\
\hline _CONT & $\begin{array}{r}113,51 \\
{[12,95]} \\
(0,00)\end{array}$ & $\begin{array}{r}0,00 \\
{[-1,25]} \\
(0,21)\end{array}$ & $\begin{array}{r}-0,11 \\
{[-8,13]} \\
(0,00)\end{array}$ & 0,62 & 230,12 & 0,01 \\
\hline DP & $\begin{array}{r}166,40 \\
{[7,86]} \\
(0,00)\end{array}$ & $\begin{array}{r}0,00 \\
{[0,70]} \\
(0,49)\end{array}$ & $\begin{array}{r}-0,20 \\
{[-6,96]} \\
(0,00)\end{array}$ & 0,79 & 108,53 & 0,07 \\
\hline
\end{tabular}

Table C.15.: Time-series regression results for the test of the relationship between default risk and the smile coefficient $B 0$ for a constant maturity of 180 days 


\begin{tabular}{|c|c|c|c|c|c|c|}
\hline \multicolumn{7}{|c|}{$C D S_{t}=\beta_{0}+\beta_{1} \cdot B 1_{t}+\beta_{2} \cdot t+u_{t}$} \\
\hline ID & $b_{0}$ & $b_{1}$ & $b_{2}$ & R2 cor. & $\mathbf{F}$ & DW \\
\hline ALL & $\begin{array}{r}61,61 \\
{[10,41]} \\
(0,00) \\
\end{array}$ & $\begin{array}{r}0,00 \\
{[1,13]} \\
(0,26)\end{array}$ & $\begin{array}{r}-0,05 \\
{[-5,07]} \\
(0,00)\end{array}$ & 0,20 & 93,03 & 0,01 \\
\hline _SIE & $\begin{array}{r}63,91 \\
{[21,03]} \\
(0,00) \\
\end{array}$ & $\begin{array}{r}0,01 \\
{[1,54]} \\
(0,12) \\
\end{array}$ & $\begin{array}{r}-0,06 \\
{[-11,55]} \\
(0,00) \\
\end{array}$ & 0,59 & 554,00 & 0,01 \\
\hline _DTE & $\begin{array}{r}263,62 \\
{[16,52]} \\
(0,00)\end{array}$ & $\begin{array}{r}0,00 \\
{[-0,85]} \\
(0,40)\end{array}$ & $\begin{array}{r}-0,33 \\
{[-11,53]} \\
(0,00)\end{array}$ & 0,62 & 613,45 & 0,01 \\
\hline _DAIM & $\begin{array}{r}159,27 \\
{[41,51]} \\
(0,00) \\
\end{array}$ & $\begin{array}{r}0,13 \\
{[0,80]} \\
(0,43)\end{array}$ & $\begin{array}{r}-0,11 \\
{[-17,16]} \\
(0,00)\end{array}$ & 0,71 & 932,63 & 0,06 \\
\hline _DBANK & $\begin{array}{r}35,98 \\
{[15,44]} \\
(0,00) \\
\end{array}$ & $\begin{array}{r}-0,01 \\
{[-3,90]} \\
(0,00) \\
\end{array}$ & $\begin{array}{r}-0,03 \\
{[-6,87]} \\
(0,00) \\
\end{array}$ & 0,29 & 153,11 & 0,01 \\
\hline _MRE & $\begin{array}{r}57,93 \\
{[13,10]} \\
(0,00) \\
\end{array}$ & $\begin{array}{r}0,00 \\
{[-0,38]} \\
(0,70) \\
\end{array}$ & $\begin{array}{r}-0,05 \\
{[-6,39]} \\
(0,00) \\
\end{array}$ & 0,32 & 160,08 & 0,16 \\
\hline BAY & $\begin{array}{r}75,28 \\
{[11,94]} \\
(0,00) \\
\end{array}$ & $\begin{array}{r}0,06 \\
{[1,22]} \\
(0,22) \\
\end{array}$ & $\begin{array}{r}-0,04 \\
{[-4,26]} \\
(0,00) \\
\end{array}$ & 0,08 & 32,53 & 0,02 \\
\hline _VW & $\begin{array}{r}49,17 \\
{[19,35]} \\
(0,00) \\
\end{array}$ & $\begin{array}{r}0,00 \\
{[-0,73]} \\
(0,47) \\
\end{array}$ & $\begin{array}{r}0,03 \\
{[5,79]} \\
(0,00) \\
\end{array}$ & 0,20 & 97,55 & 0,02 \\
\hline _BASF & $\begin{array}{r}30,84 \\
{[39,18]} \\
(0,00)\end{array}$ & $\begin{array}{r}0,00 \\
{[3,23]} \\
(0,00)\end{array}$ & $\begin{array}{r}-0,03 \\
{[-20,48]} \\
(0,00)\end{array}$ & 0,75 & $1.122,30$ & 0,04 \\
\hline EON & $\begin{array}{r}52,77 \\
{[14,12]} \\
(0,00) \\
\end{array}$ & $\begin{array}{r}0,00 \\
{[-1,55]} \\
(0,12) \\
\end{array}$ & $\begin{array}{r}-0,04 \\
{[-6,15]} \\
(0,00) \\
\end{array}$ & 0,27 & 138,27 & 0,01 \\
\hline _COB & $\begin{array}{r}100,29 \\
{[9,11]} \\
(0,00) \\
\end{array}$ & $\begin{array}{r}0,00 \\
{[1,65]} \\
(0,10) \\
\end{array}$ & $\begin{array}{r}-0,10 \\
{[-5,62]} \\
(0,00) \\
\end{array}$ & 0,24 & 113,24 & 0,01 \\
\hline _HYPV & $\begin{array}{l}80,13 \\
{[9,29]} \\
(0,00)\end{array}$ & $\begin{array}{r}0,00 \\
{[-1,18]} \\
(0,24) \\
\end{array}$ & $\begin{array}{r}-0,06 \\
{[-4,14]} \\
(0,00) \\
\end{array}$ & 0,12 & 52,73 & 0,01 \\
\hline _RWE & $\begin{array}{r}84,66 \\
{[17,96]} \\
(0,00) \\
\end{array}$ & $\begin{array}{r}0,00 \\
{[-0,48]} \\
(0,63) \\
\end{array}$ & $\begin{array}{r}-0,09 \\
{[-11,10]} \\
(0,00) \\
\end{array}$ & 0,54 & 389,92 & 0,01 \\
\hline _BMW & $\begin{array}{r}37,61 \\
{[27,87]} \\
(0,00) \\
\end{array}$ & $\begin{array}{r}0,00 \\
{[1,45]} \\
(0,15) \\
\end{array}$ & $\begin{array}{r}-0,01 \\
{[-4,61]} \\
(0,00)\end{array}$ & 0,16 & 73,14 & 0,12 \\
\hline _LH & $\begin{array}{r}155,87 \\
{[24,20]} \\
(0,00) \\
\end{array}$ & $\begin{array}{r}0,00 \\
{[0,30]} \\
(0,77) \\
\end{array}$ & $\begin{array}{r}-0,12 \\
{[-11,20]} \\
(0,00) \\
\end{array}$ & 0,43 & 267,94 & 0,01 \\
\hline _TYSS & $\begin{array}{r}205,33 \\
{[17,37]} \\
(0,00) \\
10121\end{array}$ & $\begin{array}{r}0,00 \\
{[3,80]} \\
(0,00) \\
\end{array}$ & $\begin{array}{r}-0,14 \\
{[-7,21]} \\
(0,00) \\
\end{array}$ & 0,28 & 129,06 & 0,03 \\
\hline _METRO & $\begin{array}{r}101,24 \\
{[14,11]} \\
(0,00) \\
\end{array}$ & $\begin{array}{r}-0,01 \\
{[-0,83]} \\
(0,41) \\
\end{array}$ & $\begin{array}{r}-0,07 \\
{[-5,57]} \\
(0,00) \\
\end{array}$ & 0,20 & 76,10 & 0,05 \\
\hline _ & $\begin{array}{r}322,26 \\
{[7,61]} \\
(0,00) \\
5102\end{array}$ & $\begin{array}{r}0,00 \\
{[-1,32]} \\
(0,19) \\
\end{array}$ & $\begin{array}{r}-0,12 \\
{[-1,69]} \\
(0,09) \\
0\end{array}$ & 0,06 & 8,13 & 0,05 \\
\hline _LIN & $\begin{array}{r}54,02 \\
{[16,09]} \\
(0,00) \\
\end{array}$ & $\begin{array}{r}0,00 \\
{[-0,94]} \\
(0,35) \\
\end{array}$ & $\begin{array}{r}-0,03 \\
{[-4,55]} \\
(0,00) \\
\end{array}$ & 0,16 & 46,35 & 0,05 \\
\hline _HEN & $\begin{array}{r}20,98 \\
{[26,25]} \\
(0,00) \\
\end{array}$ & $\begin{array}{r}0,00 \\
{[2,77]} \\
(0,01) \\
\end{array}$ & $\begin{array}{r}0,01 \\
{[5,18]} \\
(0,00) \\
\end{array}$ & 0,19 & 45,25 & 0,04 \\
\hline _CONT & $\begin{array}{r}113,50 \\
{[12,95]} \\
(0,00) \\
\end{array}$ & $\begin{array}{r}0,00 \\
{[1,25]} \\
(0,21) \\
\end{array}$ & $\begin{array}{r}-0,11 \\
{[-8,13]} \\
(0,00) \\
\end{array}$ & 0,62 & 230,12 & 0,01 \\
\hline _DP & $\begin{array}{r}166,41 \\
{[7,86]} \\
(0,00)\end{array}$ & $\begin{array}{r}0,00 \\
{[-0,70]} \\
(0,49)\end{array}$ & $\begin{array}{r}-0,20 \\
{[-6,96]} \\
(0,00)\end{array}$ & 0,79 & 108,54 & 0,07 \\
\hline
\end{tabular}

Table C.16.: Time-series regression results for the test of the relationship between default risk and the smile coefficient $B 1$ for a constant maturity of 180 days 


\begin{tabular}{|c|c|c|c|c|c|c|}
\hline \multicolumn{7}{|c|}{$C D S_{t}=\beta_{0}+\beta_{1} \cdot B 2_{t}+\beta_{2} \cdot t+u_{t}$} \\
\hline ID & $b_{0}$ & $b_{1}$ & $b_{2}$ & R2 cor. & $F$ & DW \\
\hline \multirow[t]{3}{*}{$\bar{A}$ ALL } & 61,61 & $\begin{array}{l}0,00 \\
\end{array}$ & $-0,05$ & \multirow[t]{3}{*}{0,20} & \multirow[t]{3}{*}{93,03} & \multirow[t]{3}{*}{0,01} \\
\hline & {$[10,41]$} & {$[-1,14]$} & {$[-5,07]$} & & & \\
\hline & $(0,00)$ & $(0,25)$ & $(0,00)$ & & & \\
\hline \multirow[t]{3}{*}{-SIE } & 63,91 & $-0,02$ & $-0,06$ & \multirow[t]{3}{*}{0,59} & \multirow[t]{3}{*}{554,05} & \multirow[t]{3}{*}{0,01} \\
\hline & {$[21,03]$} & {$[-2,73]$} & {$[-11,55]$} & & & \\
\hline & $(0,00)$ & $(0,01)$ & $(0,00)$ & & & \\
\hline \multirow[t]{3}{*}{ _DTE } & 263,62 & 0,00 & $-0,33$ & \multirow[t]{3}{*}{0,62} & \multirow[t]{3}{*}{613,45} & 0,01 \\
\hline & {$[16,52]$} & {$[0,77]$} & {$[-11,53]$} & & & \\
\hline & $(0,00)$ & $(0,44)$ & $(0,00)$ & & & \\
\hline DAIM & 159,27 & $-0,33$ & $-0,11$ & 0,71 & 932,87 & 0,06 \\
\hline & {$[41,58]$} & {$[-0,91]$} & {$[-17,16]$} & & & \\
\hline & $(0,00)$ & $(0,36)$ & $(0,00)$ & & & \\
\hline _DBANK & 35,98 & 0,01 & $-0,03$ & 0,29 & 153,11 & 0,01 \\
\hline & {$[15,44]$} & {$[3,98]$} & {$[-6,87]$} & & & \\
\hline & $(0,00)$ & $(0,00)$ & $(0,00)$ & & & \\
\hline _MRE & 57,92 & 0,02 & $-0,05$ & 0,32 & 160,33 & 0,16 \\
\hline & {$[13,10]$} & {$[0,80]$} & {$[-6,40]$} & & & \\
\hline & $(0,00)$ & $(0,42)$ & $(0,00)$ & & & \\
\hline BAY & 75,27 & $-0,14$ & $-0,04$ & 0,08 & 32,58 & 0,02 \\
\hline & {$[11,94]$} & {$[-1,40]$} & {$[-4,26]$} & & & \\
\hline & $(0,00)$ & $(0,16)$ & $(0,00)$ & & & \\
\hline _VW & 49,16 & 0,00 & 0,03 & 0,20 & 97,43 & 0,02 \\
\hline & {$[19,34]$} & {$[0,30]$} & {$[5,79]$} & & & \\
\hline & $(0,00)$ & $(0,77)$ & $(0,00)$ & & & \\
\hline BASF & 30,84 & 0,00 & $-0,03$ & 0,75 & $1.122,44$ & 0,04 \\
\hline & {$[39,19]$} & {$[-3,18]$} & {$[-20,48]$} & & & \\
\hline & $(0,00)$ & $(0,00)$ & $(0,00)$ & & & \\
\hline EON & 52,78 & 0,00 & $-0,04$ & 0,27 & 138,50 & 0,01 \\
\hline & {$[14,12]$} & {$[1,65]$} & {$[-6,16]$} & & & \\
\hline & $(0,00)$ & $(0,10)$ & $(0,00)$ & & & \\
\hline _COB & 100,28 & 0,00 & $-0,10$ & 0,24 & 113,24 & 0,01 \\
\hline & {$[9,11]$} & {$[-2,06]$} & {$[-5,62]$} & & & \\
\hline & $(0,00)$ & $(0,04)$ & $(0,00)$ & & & \\
\hline _HYPV & 80,12 & 0,00 & $-0,06$ & 0,12 & 52,74 & 0,01 \\
\hline & {$[9,29]$} & {$[1,52]$} & {$[-4,14]$} & & & \\
\hline & $(0,00)$ & $(0,13)$ & $(0,00)$ & & & \\
\hline _RWE & 84,66 & 0,00 & $-0,09$ & 0,54 & 389,92 & 0,01 \\
\hline & {$[17,96]$} & {$[0,48]$} & {$[-11,10]$} & & & \\
\hline & $(0,00)$ & $(0,63)$ & $(0,00)$ & & & \\
\hline BMW & 37,61 & 0,00 & $-0,01$ & 0,16 & 73,19 & 0,12 \\
\hline & {$[27,87]$} & {$[-1,83]$} & {$[-4,61]$} & & & \\
\hline & $(0,00)$ & $(0,07)$ & $(0,00)$ & & & \\
\hline LLH & 155,87 & 0,00 & $-0,12$ & 0,43 & 267,94 & 0,01 \\
\hline & {$[24,20]$} & {$[-0,21]$} & {$[-11,20]$} & & & \\
\hline & $(0,00)$ & $(0,83)$ & $(0,00)$ & & & \\
\hline _TYSS & 205,33 & $-0,01$ & $-0,14$ & 0,28 & 129,06 & 0,03 \\
\hline & {$[17,37]$} & {$[-4,16]$} & {$[-7,21]$} & & & \\
\hline & $(0,00)$ & $(0,00)$ & $(0,00)$ & & & \\
\hline _METRO & 101,24 & 0,01 & $-0,07$ & 0,20 & 75,67 & 0,05 \\
\hline & {$[14,10]$} & {$[0,65]$} & {$[-5,56]$} & & & \\
\hline & $(0,00)$ & $(0,51)$ & $(0,00)$ & & & \\
\hline _TUI & 322,23 & 0,00 & $-0,12$ & 0,06 & 8,12 & 0,05 \\
\hline & {$[7,61]$} & {$[1,34]$} & {$[-1,69]$} & & & \\
\hline & $(0,00)$ & $(0,18)$ & $(0,09)$ & & & \\
\hline LIN & 54,00 & 0,00 & $-0,03$ & 0,16 & 46,22 & 0,05 \\
\hline & {$[16,09]$} & {$[0,95]$} & {$[-4,54]$} & & & \\
\hline & $(0,00)$ & $(0,34)$ & $(0,00)$ & & & \\
\hline HEN & 20,98 & 0,00 & 0,01 & 0,19 & 45,24 & 0,04 \\
\hline & {$[26,25]$} & {$[-2,90]$} & {$[5,18]$} & & & \\
\hline & $(0,00)$ & $(0,00)$ & $(0,00)$ & & & \\
\hline _CONT & 113,50 & 0,00 & $-0,11$ & 0,62 & 230,12 & 0,00 \\
\hline & {$[12,95]$} & {$[-1,25]$} & {$[-8,13]$} & & & \\
\hline & $(0,00)$ & $(0,21)$ & $(0,00)$ & & & \\
\hline DP & 166,41 & 0,00 & $-0,20$ & 0,79 & 108,54 & 0,07 \\
\hline & {$[7,85]$} & {$[0,70]$} & {$[-6,96]$} & & & \\
\hline & $(0,00)$ & $(0,48)$ & $(0,00)$ & & & \\
\hline
\end{tabular}

Table C.17.: Time-series regression results for the test of the relationship between default risk and the smile coefficient $B 2$ for a constant maturity of 180 days 
C.3.2. Examination of the Relationship between the CDS Spread Level and the Slope of the Smile 


\begin{tabular}{|c|c|c|c|c|c|c|}
\hline \multicolumn{7}{|c|}{$C D S_{t}=\beta_{0}+\beta_{1} \cdot S L P 70_{t}+\beta_{2} \cdot t+u_{t}$} \\
\hline ID & $b_{0}$ & $b_{1}$ & $b_{2}$ & R2 cor. & $F$ & DW \\
\hline ALL & $\begin{array}{r}77,28 \\
{[13,69]} \\
(0,00)\end{array}$ & $\begin{array}{l}18,29 \\
{[5,30]} \\
(0,00)\end{array}$ & $\begin{array}{r}-0,02 \\
{[-2,50]} \\
(0,01)\end{array}$ & 0,33 & 176,72 & 0,12 \\
\hline SSIE & $\begin{array}{r}67,38 \\
{[22,03]} \\
(0,00) \\
\end{array}$ & $\begin{array}{r}4,06 \\
{[2,37]} \\
(0,02) \\
\end{array}$ & $\begin{array}{r}-0,06 \\
{[-8,77]} \\
(0,00) \\
\end{array}$ & 0,60 & 571,55 & 0,03 \\
\hline _DTE & $\begin{array}{r}264,96 \\
{[16,77]} \\
(0,00)\end{array}$ & $\begin{array}{r}2,42 \\
{[1,46]} \\
(0,14)\end{array}$ & $\begin{array}{r}-0,32 \\
{[-10,95]} \\
(0,00)\end{array}$ & 0,62 & 620,40 & 0,03 \\
\hline DAIM & $\begin{array}{r}162,62 \\
{[40,71]} \\
(0,00)\end{array}$ & $\begin{array}{r}3,58 \\
{[2,32]} \\
(0,02)\end{array}$ & $\begin{array}{r}-0,11 \\
{[-15,23]} \\
(0,00)\end{array}$ & 0,72 & 954,96 & 0,08 \\
\hline _DBANK & $\begin{array}{r}41,04 \\
{[13,91]} \\
(0,00)\end{array}$ & $\begin{array}{r}4,59 \\
{[3,23]} \\
(0,00)\end{array}$ & $\begin{array}{r}-0,02 \\
{[-5,52]} \\
(0,00)\end{array}$ & 0,32 & 176,73 & 0,05 \\
\hline _MRE & $\begin{array}{r}60,58 \\
{[14,94]} \\
(0,00)\end{array}$ & $\begin{array}{r}4,20 \\
{[3,78]} \\
(0,00)\end{array}$ & $\begin{array}{r}-0,04 \\
{[-5,15]} \\
(0,00)\end{array}$ & 0,36 & 186,52 & 0,23 \\
\hline _BAY & $\begin{array}{r}77,50 \\
{[11,93]} \\
(0,00)\end{array}$ & $\begin{array}{r}1,66 \\
{[1,92]} \\
(0,05) \\
\end{array}$ & $\begin{array}{r}-0,04 \\
{[-4,21]} \\
(0,00) \\
\end{array}$ & 0,09 & 37,74 & 0,04 \\
\hline -VW & $\begin{array}{r}49,11 \\
{[19,27]} \\
(0,00)\end{array}$ & $\begin{array}{r}-0,03 \\
{[-0,19]} \\
(0,85)\end{array}$ & $\begin{array}{r}0,03 \\
{[5,79]} \\
(0,00)\end{array}$ & 0,20 & 97,40 & 0,02 \\
\hline BASF & $\begin{array}{r}30,90 \\
{[39,63]} \\
(0,00)\end{array}$ & $\begin{array}{r}0,04 \\
{[5,52]} \\
(0,00)\end{array}$ & $\begin{array}{r}-0,03 \\
{[-20,48]} \\
(0,00)\end{array}$ & 0,75 & $1.125,37$ & 0,04 \\
\hline EON & $\begin{array}{r}52,66 \\
{[14,04]} \\
(0,00)\end{array}$ & $\begin{array}{r}0,00 \\
{[-0,15]} \\
(0,88)\end{array}$ & $\begin{array}{r}-0,04 \\
{[-6,11]} \\
(0,00)\end{array}$ & 0,27 & 136,94 & 0,01 \\
\hline _COB & $\begin{array}{r}100,27 \\
{[9,10]} \\
(0,00)\end{array}$ & $\begin{array}{r}0,04 \\
{[1,29]} \\
(0,20)\end{array}$ & $\begin{array}{r}-0,10 \\
{[-5,58]} \\
(0,00)\end{array}$ & 0,24 & 113,36 & 0,01 \\
\hline _HYPV & $\begin{array}{l}80,14 \\
{[9,29]} \\
(0,00)\end{array}$ & $\begin{array}{r}0,00 \\
{[-0,45]} \\
(0,66) \\
\end{array}$ & $\begin{array}{r}-0,06 \\
{[-4,15]} \\
(0,00) \\
\end{array}$ & 0,12 & 52,74 & 0,01 \\
\hline - RWE & $\begin{array}{r}84,69 \\
{[17,89]} \\
(0,00)\end{array}$ & $\begin{array}{r}0,00 \\
{[1,00]} \\
(0,32)\end{array}$ & $\begin{array}{r}-0,09 \\
{[-11,07]} \\
(0,00)\end{array}$ & 0,54 & 389,96 & 0,01 \\
\hline BMWW & $\begin{array}{r}37,61 \\
{[27,86]} \\
(0,00)\end{array}$ & $\begin{array}{r}0,00 \\
{[2,39]} \\
(0,02)\end{array}$ & $\begin{array}{r}-0,01 \\
{[-4,61]} \\
(0,00)\end{array}$ & 0,16 & 73,16 & 0,12 \\
\hline - $\mathrm{LH}$ & $\begin{array}{r}155,86 \\
{[24,22]} \\
(0,00)\end{array}$ & $\begin{array}{r}0,00 \\
{[3,72]} \\
(0,00)\end{array}$ & $\begin{array}{r}-0,12 \\
{[-11,21]} \\
(0,00)\end{array}$ & 0,43 & 268,08 & 0,01 \\
\hline _TYSS & $\begin{array}{r}205,46 \\
{[17,36]} \\
(0,00)\end{array}$ & $\begin{array}{r}-0,01 \\
{[-4,14]} \\
(0,00)\end{array}$ & $\begin{array}{r}-0,14 \\
{[-7,23]} \\
(0,00)\end{array}$ & 0,28 & 129,06 & 0,03 \\
\hline _METRO & $\begin{array}{r}101,28 \\
{[14,09]} \\
(0,00)\end{array}$ & $\begin{array}{r}0,00 \\
{[0,59]} \\
(0,55)\end{array}$ & $\begin{array}{r}-0,07 \\
{[-5,55]} \\
(0,00)\end{array}$ & 0,20 & 75,28 & 0,05 \\
\hline -TUI & $\begin{array}{r}322,41 \\
{[7,64]} \\
(0,00)\end{array}$ & $\begin{array}{r}-0,01 \\
{[-0,94]} \\
(0,35)\end{array}$ & $\begin{array}{r}-0,12 \\
{[-1,71]} \\
(0,09)\end{array}$ & 0,06 & 8,26 & 0,05 \\
\hline _LIN & $\begin{array}{r}54,01 \\
{[16,01]} \\
(0,00)\end{array}$ & $\begin{array}{r}0,00 \\
{[1,27]} \\
(0,21)\end{array}$ & $\begin{array}{r}-0,03 \\
{[-4,53]} \\
(0,00)\end{array}$ & 0,16 & 46,15 & 0,05 \\
\hline _HEN & $\begin{array}{r}20,98 \\
{[26,25]} \\
(0,00)\end{array}$ & $\begin{array}{r}0,00 \\
{[2,35]} \\
(0,02) \\
\end{array}$ & $\begin{array}{r}0,01 \\
{[5,18]} \\
(0,00) \\
\end{array}$ & 0,19 & 45,16 & 0,04 \\
\hline _CONT & $\begin{array}{r}113,50 \\
{[12,95]} \\
(0,00)\end{array}$ & $\begin{array}{r}0,00 \\
{[0,84]} \\
(0,40)\end{array}$ & $\begin{array}{r}-0,11 \\
{[-8,12]} \\
(0,00)\end{array}$ & 0,62 & 230,02 & 0,00 \\
\hline _DP & $\begin{array}{r}166,54 \\
{[7,93]} \\
(0,00)\end{array}$ & $\begin{array}{r}0,00 \\
{[-1,07]} \\
(0,29)\end{array}$ & $\begin{array}{r}-0,20 \\
{[-7,04]} \\
(0,00)\end{array}$ & 0,79 & 109,77 & 0,09 \\
\hline
\end{tabular}

Table C.18.: Time-series regression results for the test of the relationship between default risk and the slope variable $S L P 70$ for a constant maturity of 36 days 


\begin{tabular}{|c|c|c|c|c|c|c|}
\hline \multicolumn{7}{|c|}{$C D S_{t}=\beta_{0}+\beta_{1} \cdot \operatorname{SLP} 90_{t}+\beta_{2} \cdot t+u_{t}$} \\
\hline ID & $b_{0}$ & $b_{1}$ & $b_{2}$ & R2 cor. & $\mathbf{F}$ & DW \\
\hline ALL & $\begin{array}{r}75,91 \\
{[11,55]} \\
(0,00) \\
\end{array}$ & $\begin{array}{l}26,87 \\
{[3,05]} \\
(0,00)\end{array}$ & $\begin{array}{r}-0,04 \\
{[-3,76]} \\
(0,00)\end{array}$ & 0,24 & 113,68 & 0,04 \\
\hline _SIE & $\begin{array}{r}58,23 \\
{[15,38]} \\
(0,00) \\
\end{array}$ & $\begin{array}{r}-10,56 \\
{[-2,17]} \\
(0,03) \\
\end{array}$ & $\begin{array}{r}-0,07 \\
{[-11,33]} \\
(0,00)\end{array}$ & 0,60 & 570,63 & 0,03 \\
\hline _DTE & $\begin{array}{r}264,56 \\
{[16,73]} \\
(0,00)\end{array}$ & $\begin{array}{r}2,36 \\
{[1,04]} \\
(0,30)\end{array}$ & $\begin{array}{r}-0,33 \\
{[-11,27]} \\
(0,00)\end{array}$ & 0,62 & 614,25 & 0,02 \\
\hline _DAIM & $\begin{array}{r}161,17 \\
{[35,61]} \\
(0,00) \\
\end{array}$ & $\begin{array}{r}3,45 \\
{[0,83]} \\
(0,41) \\
\end{array}$ & $\begin{array}{r}-0,11 \\
{[-16,29]} \\
(0,00)\end{array}$ & 0,71 & 935,00 & 0,06 \\
\hline _DBANK & $\begin{array}{r}34,05 \\
{[10,37]} \\
(0,00)\end{array}$ & $\begin{array}{r}-2,82 \\
{[-0,94]} \\
(0,35) \\
\end{array}$ & $\begin{array}{r}-0,03 \\
{[-7,00]} \\
(0,00)\end{array}$ & 0,29 & 153,98 & 0,01 \\
\hline _MRE & $\begin{array}{r}60,99 \\
{[14,51]} \\
(0,00) \\
\end{array}$ & $\begin{array}{r}6,35 \\
{[2,28]} \\
(0,02) \\
\end{array}$ & $\begin{array}{r}-0,04 \\
{[-5,75]} \\
(0,00) \\
\end{array}$ & 0,33 & 168,57 & 0,18 \\
\hline BAY & $\begin{array}{r}78,95 \\
{[11,36]} \\
(0,00) \\
\end{array}$ & $\begin{array}{r}5,21 \\
{[1,81]} \\
(0,07) \\
\end{array}$ & $\begin{array}{r}-0,04 \\
{[-4,29]} \\
(0,00) \\
\end{array}$ & 0,09 & 37,04 & 0,04 \\
\hline _VW & $\begin{array}{r}48,65 \\
{[19,00]} \\
(0,00)\end{array}$ & $\begin{array}{r}-0,75 \\
{[-2,29]} \\
(0,02)\end{array}$ & $\begin{array}{r}0,03 \\
{[5,79]} \\
(0,00)\end{array}$ & 0,21 & 98,62 & 0,03 \\
\hline _BASF & $\begin{array}{r}30,91 \\
{[39,64]} \\
(0,00)\end{array}$ & $\begin{array}{r}0,09 \\
{[5,58]} \\
(0,00)\end{array}$ & $\begin{array}{r}-0,03 \\
{[-20,48]} \\
(0,00)\end{array}$ & 0,75 & $1.123,44$ & 0,04 \\
\hline EON & $\begin{array}{r}52,65 \\
{[14,03]} \\
(0,00) \\
\end{array}$ & $\begin{array}{r}-0,02 \\
{[-0,72]} \\
(0,47) \\
\end{array}$ & $\begin{array}{r}-0,04 \\
{[-6,11]} \\
(0,00) \\
\end{array}$ & 0,27 & 136,97 & 0,01 \\
\hline _COB & $\begin{array}{r}100,29 \\
{[9,11]} \\
(0,00) \\
\end{array}$ & $\begin{array}{r}0,08 \\
{[1,01]} \\
(0,31) \\
\end{array}$ & $\begin{array}{r}-0,10 \\
{[-5,59]} \\
(0,00) \\
\end{array}$ & 0,24 & 113,28 & 0,01 \\
\hline _HYPV & $\begin{array}{l}80,14 \\
{[9,29]} \\
(0,00)\end{array}$ & $\begin{array}{r}-0,01 \\
{[-0,61]} \\
(0,54) \\
\end{array}$ & $\begin{array}{r}-0,06 \\
{[-4,15]} \\
(0,00) \\
\end{array}$ & 0,12 & 52,76 & 0,01 \\
\hline _RWE & $\begin{array}{r}84,70 \\
{[17,90]} \\
(0,00) \\
\end{array}$ & $\begin{array}{r}0,01 \\
{[1,17]} \\
(0,24) \\
\end{array}$ & $\begin{array}{r}-0,09 \\
{[-11,07]} \\
(0,00) \\
\end{array}$ & 0,54 & 390,05 & 0,01 \\
\hline _BMW & $\begin{array}{r}37,61 \\
{[27,86]} \\
(0,00) \\
\end{array}$ & $\begin{array}{r}0,00 \\
{[2,13]} \\
(0,03) \\
\end{array}$ & $\begin{array}{r}-0,01 \\
{[-4,61]} \\
(0,00)\end{array}$ & 0,16 & 73,13 & 0,12 \\
\hline _LH & $\begin{array}{r}155,86 \\
{[24,21]} \\
(0,00) \\
\end{array}$ & $\begin{array}{r}0,00 \\
{[3,68]} \\
(0,00) \\
\end{array}$ & $\begin{array}{r}-0,12 \\
{[-11,21]} \\
(0,00)\end{array}$ & 0,43 & 268,08 & 0,01 \\
\hline _TYSS & $\begin{array}{r}205,47 \\
{[17,36]} \\
(0,00)\end{array}$ & $\begin{array}{r}-0,02 \\
{[-4,07]} \\
(0,00)\end{array}$ & $\begin{array}{r}-0,14 \\
{[-7,22]} \\
(0,00)\end{array}$ & 0,28 & 129,07 & 0,03 \\
\hline _METRO & $\begin{array}{r}101,29 \\
{[14,09]} \\
(0,00)\end{array}$ & $\begin{array}{r}0,01 \\
{[0,64]} \\
(0,52) \\
\end{array}$ & $\begin{array}{r}-0,07 \\
{[-5,55]} \\
(0,00) \\
\end{array}$ & 0,20 & 75,31 & 0,05 \\
\hline _ TUI & $\begin{array}{r}322,41 \\
{[7,64]} \\
(0,00) \\
\end{array}$ & $\begin{array}{r}-0,03 \\
{[-1,07]} \\
(0,29) \\
\end{array}$ & $\begin{array}{r}-0,12 \\
{[-1,71]} \\
(0,09) \\
\end{array}$ & 0,06 & 8,30 & 0,05 \\
\hline _LIN & $\begin{array}{r}54,00 \\
{[16,06]} \\
(0,00)\end{array}$ & $\begin{array}{r}0,00 \\
{[1,44]} \\
(0,15)\end{array}$ & $\begin{array}{r}-0,03 \\
{[-4,54]} \\
(0,00)\end{array}$ & 0,16 & 46,16 & 0,05 \\
\hline _HEN & $\begin{array}{r}20,98 \\
{[26,24]} \\
(0,00) \\
\end{array}$ & $\begin{array}{r}0,00 \\
{[1,72]} \\
(0,09) \\
\end{array}$ & $\begin{array}{r}0,01 \\
{[5,18]} \\
(0,00) \\
\end{array}$ & 0,19 & 45,16 & 0,04 \\
\hline _CONT & $\begin{array}{r}113,52 \\
{[12,96]} \\
(0,00)\end{array}$ & $\begin{array}{r}0,01 \\
{[0,97]} \\
(0,33) \\
\end{array}$ & $\begin{array}{r}-0,11 \\
{[-8,13]} \\
(0,00) \\
\end{array}$ & 0,62 & 230,10 & 0,01 \\
\hline _DP & $\begin{array}{r}166,44 \\
{[7,93]} \\
(0,00)\end{array}$ & $\begin{array}{r}0,00 \\
{[-0,77]} \\
(0,44)\end{array}$ & $\begin{array}{r}-0,20 \\
{[-7,03]} \\
(0,00)\end{array}$ & 0,79 & 109,22 & 0,08 \\
\hline
\end{tabular}

Table C.19.: Time-series regression results for the test of the relationship between default risk and the slope variable $S L P 90$ for a constant maturity of 36 days 


\begin{tabular}{|c|c|c|c|c|c|c|}
\hline & $C D S_{t}=$ & $\beta_{0}+\beta$ & - $S L P 10$ & ${ }_{t}+\beta_{2}$ & $t+u_{t}$ & \\
\hline ID & $b_{0}$ & $b_{1}$ & $b_{2}$ & R2 cor. & $F$ & DW \\
\hline ALL & 12,71 & $-132,48$ & \begin{tabular}{|c|}
$-0,03$ \\
\end{tabular} & 0,39 & 237,62 & 0,15 \\
\hline & {$[1,57]$} & {$[-7,50]$} & {$[-3,67]$} & & & \\
\hline & $(0,12)$ & $(0,00)$ & $(0,00)$ & & & \\
\hline _SIE & 27,24 & $-96,29$ & $-0,05$ & 0,78 & $1.347,49$ & 0,31 \\
\hline & {$[6,65]$} & {$[-10,18]$} & {$[-13,29]$} & & & \\
\hline & $(0,00)$ & $(0,00)$ & $(0,00)$ & & & \\
\hline _DTE & 177,58 & $-264,53$ & $-0,29$ & 0,71 & 934,86 & 0,30 \\
\hline & {$[7,92]$} & {$[-4,97]$} & {$[-11,12]$} & & & \\
\hline & $(0,00)$ & $(0,00)$ & $(0,00)$ & & & \\
\hline DAIM & 147,48 & $-29,91$ & $-0,11$ & 0,72 & 990,37 & 0,08 \\
\hline & {$[23,17]$} & {$[-2,30]$} & {$[-16,35]$} & & & \\
\hline & $(0,00)$ & $(0,02)$ & $(0,00)$ & & & \\
\hline _DBANK & 14,35 & $-45,64$ & $-0,01$ & 0,48 & 350,50 & 0,15 \\
\hline & {$[5,08]$} & {$[-7,48]$} & {$[-4,51]$} & & & \\
\hline & $(0,00)$ & $(0,00)$ & $(0,00)$ & & & \\
\hline _MRE & 44,30 & $-33,35$ & $-0,04$ & 0,37 & 195,87 & 0,24 \\
\hline & {$[7,78]$} & {$[-4,02]$} & {$[-5,26]$} & & & \\
\hline & $(0,00)$ & $(0,00)$ & $(0,00)$ & & & \\
\hline BAY & 73,04 & $-5,74$ & $-0,04$ & 0,08 & 33,85 & 0,02 \\
\hline & {$[11,28]$} & {$[-1,95]$} & {$[-4,09]$} & & & \\
\hline & $(0,00)$ & $(0,05)$ & $(0,00)$ & & & \\
\hline _VW & 45,25 & $-9,34$ & 0,03 & 0,24 & 119,43 & 0,10 \\
\hline & {$[15,72]$} & {$[-2,65]$} & {$[6,45]$} & & & \\
\hline & $(0,00)$ & $(0,01)$ & $(0,00)$ & & & \\
\hline BASF & 30,71 & $-0,33$ & $-0,03$ & 0,75 & $1.121,29$ & 0,04 \\
\hline & {$[37,89]$} & {$[-0,77]$} & {$[-20,47]$} & & & \\
\hline & $(0,00)$ & $(0,44)$ & $(0,00)$ & & & \\
\hline EON & 52,25 & $-0,98$ & $-0,04$ & 0,27 & 138,85 & 0,02 \\
\hline & {$[13,90]$} & {$[-1,45]$} & {$[-6,06]$} & & & \\
\hline & $(0,00)$ & $(0,15)$ & $(0,00)$ & & & \\
\hline _COB & 100,17 & $-0,41$ & $-0,10$ & 0,24 & 113,35 & 0,01 \\
\hline & {$[9,13]$} & {$[-0,80]$} & {$[-5,63]$} & & & \\
\hline & $(0,00)$ & $(0,42)$ & $(0,00)$ & & & \\
\hline _HYPV & 80,15 & $-0,14$ & $-0,06$ & 0,12 & 53,09 & 0,01 \\
\hline & {$[9,30]$} & {$[-1,42]$} & {$[-4,15]$} & & & \\
\hline & $(0,00)$ & $(0,16)$ & $(0,00)$ & & & \\
\hline _RWE & 84,65 & 0,00 & $-0,09$ & 0,54 & 389,83 & 0,01 \\
\hline & {$[17,93]$} & {$[0,45]$} & {$[-11,07]$} & & & \\
\hline & $(0,00)$ & $(0,66)$ & $(0,00)$ & & & \\
\hline BMMW & 37,60 & $-0,01$ & $-0,01$ & 0,16 & 73,07 & 0,12 \\
\hline & {$[27,86]$} & {$[-1,23]$} & {$[-4,60]$} & & & \\
\hline & $(0,00)$ & $(0,22)$ & $(0,00)$ & & & \\
\hline _LH & 155,87 & 0,02 & $-0,12$ & 0,43 & 267,96 & 0,01 \\
\hline & {$[24,21]$} & {$[0,52]$} & {$[-11,21]$} & & & \\
\hline & $(0,00)$ & $(0,61)$ & $(0,00)$ & & & \\
\hline _TYSS & 205,50 & $-0,05$ & $-0,14$ & 0,28 & 129,05 & 0,03 \\
\hline & {$[17,33]$} & {$[-3,42]$} & {$[-7,21]$} & & & \\
\hline & $(0,00)$ & $(0,00)$ & $(0,00)$ & & & \\
\hline _METRO & 101,23 & 0,01 & $\begin{array}{l}-0,07 \\
\end{array}$ & 0,20 & 75,16 & 0,05 \\
\hline & {$[14,06]$} & {$[0,06]$} & {$[-5,53]$} & & & \\
\hline & $(0,00)$ & $(0,95)$ & $(0,00)$ & & & \\
\hline _TUI & 320,21 & $-0,30$ & $-0,11$ & 0,06 & 8,27 & 0,05 \\
\hline & {$[7,61]$} & {$[-1,13]$} & {$[-1,66]$} & & & \\
\hline & $(0,00)$ & $(0,26)$ & $(0,10)$ & & & \\
\hline LIIN & 53,98 & 0,00 & $-0,03$ & 0,16 & 46,12 & 0,05 \\
\hline & {$[16,08]$} & {$[1,60]$} & {$[-4,54]$} & & & \\
\hline & $(0,00)$ & $(0,11)$ & $(0,00)$ & & & \\
\hline _HEN & 20,99 & 0,00 & 0,01 & 0,19 & 45,15 & 0,04 \\
\hline & {$[26,27]$} & {$[-4,11]$} & {$[5,18]$} & & & \\
\hline & $(0,00)$ & $(0,00)$ & $(0,00)$ & & & \\
\hline _CONT & 113,58 & 0,01 & $-0,11$ & 0,62 & 229,78 & 0,00 \\
\hline & {$[12,93]$} & {$[0,63]$} & {$[-8,12]$} & & & \\
\hline & $(0,00)$ & $(0,53)$ & $(0,00)$ & & & \\
\hline DPP & 165,94 & 0,03 & $-0,20$ & 0,80 & 111,95 & 0,16 \\
\hline & {$[8,07]$} & {$[2,07]$} & {$[-7,15]$} & & & \\
\hline & $(0,00)$ & $(0,04)$ & $(0,00)$ & & & \\
\hline
\end{tabular}

Table C.20.: Time-series regression results for the test of the relationship between default risk and the slope variable $S L P 100$ for a constant maturity of 36 days 


\begin{tabular}{|c|c|c|c|c|c|c|}
\hline \multicolumn{7}{|c|}{$C D S_{t}=\beta_{0}+\beta_{1} \cdot S L P 110_{t}+\beta_{2} \cdot t+u_{t}$} \\
\hline ID & $b_{0}$ & $b_{1}$ & $b_{2}$ & R2 cor. & $\mathbf{F}$ & DW \\
\hline ALL & $\begin{array}{r}46,29 \\
{[13,57]} \\
(0,00)\end{array}$ & $\begin{array}{r}-74,05 \\
{[-8,40]} \\
(0,00)\end{array}$ & $\begin{array}{r}0,00 \\
{[-0,17]} \\
(0,87)\end{array}$ & 0,51 & 383,13 & 0,33 \\
\hline _SIE & $\begin{array}{r}54,70 \\
{[22,79]} \\
(0,00)\end{array}$ & $\begin{array}{r}-41,23 \\
{[-6,79]} \\
(0,00)\end{array}$ & $\begin{array}{r}-0,04 \\
{[-6,50]} \\
(0,00)\end{array}$ & 0,73 & $1.003,11$ & 0,35 \\
\hline _DTE & $\begin{array}{r}258,88 \\
{[16,59]} \\
(0,00)\end{array}$ & $\begin{array}{r}-19,05 \\
{[-1,61]} \\
(0,11)\end{array}$ & $\begin{array}{r}-0,30 \\
{[-9,56]} \\
(0,00)\end{array}$ & 0,63 & 656,70 & 0,10 \\
\hline _DAIM & $\begin{array}{r}155,63 \\
{[41,32]} \\
(0,00)\end{array}$ & $\begin{array}{r}-17,77 \\
{[-3,16]} \\
(0,00)\end{array}$ & $\begin{array}{r}-0,10 \\
{[-13,30]} \\
(0,00)\end{array}$ & 0,73 & $1.018,86$ & 0,12 \\
\hline _DBANK & $\begin{array}{r}29,43 \\
{[20,03]} \\
(0,00)\end{array}$ & $\begin{array}{r}-24,81 \\
{[-6,78]} \\
(0,00)\end{array}$ & $\begin{array}{r}-0,01 \\
{[-2,37]} \\
(0,02)\end{array}$ & 0,48 & 353,73 & 0,24 \\
\hline _MRE & $\begin{array}{r}49,77 \\
{[13,07]} \\
(0,00)\end{array}$ & $\begin{array}{r}-24,36 \\
{[-6,10]} \\
(0,00)\end{array}$ & $\begin{array}{r}-0,02 \\
{[-3,26]} \\
(0,00)\end{array}$ & 0,43 & 257,20 & 0,38 \\
\hline _BAY & $\begin{array}{r}75,04 \\
{[12,15]} \\
(0,00)\end{array}$ & $\begin{array}{r}-3,33 \\
{[-1,75]} \\
(0,08)\end{array}$ & $\begin{array}{r}-0,04 \\
{[-4,11]} \\
(0,00)\end{array}$ & 0,09 & 37,16 & 0,03 \\
\hline _VW & $\begin{array}{r}48,66 \\
{[19,61]} \\
(0,00)\end{array}$ & $\begin{array}{r}-2,87 \\
{[-2,36]} \\
(0,02)\end{array}$ & $\begin{array}{r}0,03 \\
{[6,13]} \\
(0,00)\end{array}$ & 0,22 & 105,77 & 0,05 \\
\hline _BASF & $\begin{array}{r}30,82 \\
{[39,48]} \\
(0,00)\end{array}$ & $\begin{array}{r}-0,19 \\
{[-3,22]} \\
(0,00)\end{array}$ & $\begin{array}{r}-0,03 \\
{[-20,50]} \\
(0,00)\end{array}$ & 0,75 & $1.130,69$ & 0,05 \\
\hline EON & $\begin{array}{r}52,67 \\
{[14,06]} \\
(0,00)\end{array}$ & $\begin{array}{r}-0,02 \\
{[-0,40]} \\
(0,69) \\
\end{array}$ & $\begin{array}{r}-0,04 \\
{[-6,10]} \\
(0,00)\end{array}$ & 0,27 & 136,98 & 0,01 \\
\hline _COB & $\begin{array}{r}100,10 \\
{[9,08]} \\
(0,00) \\
\end{array}$ & $\begin{array}{r}-0,31 \\
{[-1,51]} \\
(0,13) \\
\end{array}$ & $\begin{array}{r}-0,10 \\
{[-5,55]} \\
(0,00) \\
\end{array}$ & 0,24 & 113,74 & 0,01 \\
\hline _HYPV & $\begin{array}{l}80,13 \\
{[9,29]} \\
(0,00)\end{array}$ & $\begin{array}{r}-0,01 \\
{[-0,25]} \\
(0,80)\end{array}$ & $\begin{array}{r}-0,06 \\
{[-4,14]} \\
(0,00)\end{array}$ & 0,12 & 52,72 & 0,01 \\
\hline _RWE & $\begin{array}{r}84,68 \\
{[17,89]} \\
(0,00)\end{array}$ & $\begin{array}{r}0,00 \\
{[-0,83]} \\
(0,41)\end{array}$ & $\begin{array}{r}-0,09 \\
{[-11,06]} \\
(0,00)\end{array}$ & 0,54 & 389,86 & 0,01 \\
\hline _BMW & $\begin{array}{r}37,61 \\
{[27,86]} \\
(0,00)\end{array}$ & $\begin{array}{r}0,00 \\
{[-2,62]} \\
(0,01)\end{array}$ & $\begin{array}{r}-0,01 \\
{[-4,60]} \\
(0,00)\end{array}$ & 0,16 & 73,18 & 0,12 \\
\hline _LH & $\begin{array}{r}155,86 \\
{[24,22]} \\
(0,00) \\
\end{array}$ & $\begin{array}{r}0,00 \\
{[-3,63]} \\
(0,00) \\
\end{array}$ & $\begin{array}{r}-0,12 \\
{[-11,20]} \\
(0,00)\end{array}$ & 0,43 & 268,10 & 0,01 \\
\hline _TYSS & $\begin{array}{r}205,39 \\
{[17,37]} \\
(0,00) \\
\end{array}$ & $\begin{array}{r}0,04 \\
{[2,16]} \\
(0,03) \\
\end{array}$ & $\begin{array}{r}-0,14 \\
{[-7,23]} \\
(0,00)\end{array}$ & 0,28 & 128,93 & 0,03 \\
\hline _METRO & $\begin{array}{r}101,27 \\
{[14,08]} \\
(0,00)\end{array}$ & $\begin{array}{r}-0,01 \\
{[-0,50]} \\
(0,62) \\
\end{array}$ & $\begin{array}{r}-0,07 \\
{[-5,54]} \\
(0,00)\end{array}$ & 0,20 & 75,24 & 0,05 \\
\hline _ TUI & $\begin{array}{r}322,35 \\
{[7,64]} \\
(0,00)\end{array}$ & $\begin{array}{r}0,03 \\
{[0,71]} \\
(0,48)\end{array}$ & $\begin{array}{r}-0,12 \\
{[-1,70]} \\
(0,09)\end{array}$ & 0,06 & 8,19 & 0,05 \\
\hline _LIN & $\begin{array}{r}53,98 \\
{[16,08]} \\
(0,00)\end{array}$ & $\begin{array}{r}0,00 \\
{[0,63]} \\
(0,53)\end{array}$ & $\begin{array}{r}-0,03 \\
{[-4,54]} \\
(0,00)\end{array}$ & 0,16 & 46,09 & 0,05 \\
\hline _HEN & $\begin{array}{r}20,99 \\
{[26,26]} \\
(0,00)\end{array}$ & $\begin{array}{r}0,00 \\
{[-3,18]} \\
(0,00)\end{array}$ & $\begin{array}{r}0,01 \\
{[5,18]} \\
(0,00)\end{array}$ & 0,19 & 45,16 & 0,04 \\
\hline _CONT & $\begin{array}{r}113,49 \\
{[12,93]} \\
(0,00)\end{array}$ & $\begin{array}{r}0,00 \\
{[-0,58]} \\
(0,56)\end{array}$ & $\begin{array}{r}-0,11 \\
{[-8,11]} \\
(0,00)\end{array}$ & 0,62 & 229,84 & 0,00 \\
\hline _DP & $\begin{array}{r}166,69 \\
{[7,97]} \\
(0,00)\end{array}$ & $\begin{array}{r}0,00 \\
{[2,33]} \\
(0,02)\end{array}$ & $\begin{array}{r}-0,20 \\
{[-7,06]} \\
(0,00)\end{array}$ & 0,79 & 111,15 & 0,12 \\
\hline
\end{tabular}

Table C.21.: Time-series regression results for the test of the relationship between default risk and the slope variable $S L P 110$ for a constant maturity of 36 days 


\begin{tabular}{|c|c|c|c|c|c|c|}
\hline \multicolumn{7}{|c|}{$C D S_{t}=\beta_{0}+\beta_{1} \cdot S L P 130_{t}+\beta_{2} \cdot t+u_{t}$} \\
\hline ID & $b_{0}$ & $b_{1}$ & $b_{2}$ & R2 cor. & $\mathbf{F}$ & DW \\
\hline \multirow[t]{3}{*}{ ALL } & 64,49 & $-24,42$ & \begin{tabular}{|c|}
$-0,01$ \\
\end{tabular} & \multirow[t]{3}{*}{0,44} & \multirow[t]{3}{*}{284,69} & \multirow[t]{3}{*}{0,25} \\
\hline & {$[16,16]$} & {$[-6,99]$} & {$[-1,04]$} & & & \\
\hline & $(0,00)$ & $(0,00)$ & $(0,30)$ & & & \\
\hline \multirow[t]{3}{*}{-SIE } & 64,97 & $-11,53$ & $-0,04$ & \multirow[t]{3}{*}{0,66} & \multirow[t]{3}{*}{735,04} & \multirow[t]{3}{*}{0,19} \\
\hline & {$[25,65]$} & {$[-5,09]$} & {$[-6,90]$} & & & \\
\hline & $(0,00)$ & $(0,00)$ & $(0,00)$ & & & \\
\hline \multirow[t]{3}{*}{ _DTE } & 263,21 & $-4,30$ & $-0,31$ & \multirow[t]{3}{*}{0,63} & \multirow[t]{3}{*}{634,03} & 0,05 \\
\hline & {$[16,83]$} & {$[-1,53]$} & {$[-10,38]$} & & & \\
\hline & $(0,00)$ & $(0,13)$ & $(0,00)$ & & & \\
\hline DAIM & 160,19 & $-5,60$ & $-0,10$ & 0,72 & 990,68 & 0,11 \\
\hline & {$[44,19]$} & {$[-2,96]$} & {$[-13,88]$} & & & \\
\hline & $(0,00)$ & $(0,00)$ & $(0,00)$ & & & \\
\hline _DBANK & 37,30 & $-8,48$ & $-0,01$ & 0,41 & 269,91 & 0,18 \\
\hline & {$[19,14]$} & {$[-5,35]$} & {$[-3,41]$} & & & \\
\hline & $(0,00)$ & $(0,00)$ & $(0,00)$ & & & \\
\hline _MRE & 56,69 & $-6,62$ & $-0,03$ & 0,40 & 219,26 & 0,31 \\
\hline & {$[14,85]$} & {$[-4,94]$} & {$[-4,23]$} & & & \\
\hline & $(0,00)$ & $(0,00)$ & $(0,00)$ & & & \\
\hline BAY & 76,06 & $-1,38$ & $-0,04$ & 0,09 & 37,60 & 0,04 \\
\hline & {$[12,16]$} & {$[-1,84]$} & {$[-4,15]$} & & & \\
\hline & $(0,00)$ & $(0,07)$ & $(0,00)$ & & & \\
\hline _VW & 49,27 & $-0,38$ & 0,03 & 0,21 & 98,97 & 0,03 \\
\hline & {$[19,55]$} & {$[-1,44]$} & {$[5,89]$} & & & \\
\hline & $(0,00)$ & $(0,15)$ & $(0,00)$ & & & \\
\hline BASF & 30,87 & $-0,05$ & $-0,03$ & 0,75 & $1.127,83$ & 0,05 \\
\hline & {$[39,57]$} & {$[-4,17]$} & {$[-20,49]$} & & & \\
\hline & $(0,00)$ & $(0,00)$ & $(0,00)$ & & & \\
\hline EON & 52,67 & 0,00 & $-0,04$ & 0,27 & 136,94 & 0,01 \\
\hline & {$[14,05]$} & {$[-0,18]$} & {$[-6,11]$} & & & \\
\hline & $(0,00)$ & $(0,86)$ & $(0,00)$ & & & \\
\hline _COB & 100,21 & $-0,07$ & $-0,10$ & 0,24 & 113,51 & 0,01 \\
\hline & {$[9,09]$} & {$[-1,47]$} & {$[-5,56]$} & & & \\
\hline & $(0,00)$ & $(0,14)$ & $(0,00)$ & & & \\
\hline _HYPV & 80,14 & 0,00 & $-0,06$ & 0,12 & 52,72 & 0,01 \\
\hline & {$[9,29]$} & {$[0,18]$} & {$[-4,15]$} & & & \\
\hline & $(0,00)$ & $(0,86)$ & $(0,00)$ & & & \\
\hline _RWE & 84,69 & 0,00 & $-0,09$ & 0,54 & 389,90 & 0,01 \\
\hline & {$[17,89]$} & {$[-0,90]$} & {$[-11,06]$} & & & \\
\hline & $(0,00)$ & $(0,37)$ & $(0,00)$ & & & \\
\hline _BMW & 37,61 & 0,00 & $-0,01$ & 0,16 & 73,17 & 0,12 \\
\hline & {$[27,86]$} & {$[-2,54]$} & {$[-4,60]$} & & & \\
\hline & $(0,00)$ & $(0,01)$ & $(0,00)$ & & & \\
\hline _LH & 155,86 & 0,00 & $-0,12$ & 0,43 & 268,09 & 0,01 \\
\hline & {$[24,22]$} & {$[-3,70]$} & {$[-11,20]$} & & & \\
\hline & $(0,00)$ & $(0,00)$ & $(0,00)$ & & & \\
\hline _TYSS & 205,44 & 0,01 & $-0,14$ & 0,28 & 129,03 & 0,03 \\
\hline & {$[17,37]$} & {$[3,69]$} & {$[-7,23]$} & & & \\
\hline & $(0,00)$ & $(0,00)$ & $(0,00)$ & & & \\
\hline _METRO & 101,27 & 0,00 & $-0,07$ & 0,20 & 75,26 & 0,05 \\
\hline & {$[14,08]$} & {$[-0,54]$} & {$[-5,55]$} & & & \\
\hline & $(0,00)$ & $(0,59)$ & $(0,00)$ & & & \\
\hline _TUI & 322,39 & 0,01 & $-0,12$ & 0,06 & 8,22 & 0,05 \\
\hline & {$[7,64]$} & {$[0,82]$} & {$[-1,71]$} & & & \\
\hline & $(0,00)$ & $(0,41)$ & $(0,09)$ & & & \\
\hline _LIN & 53,99 & 0,00 & $-0,03$ & 0,16 & 46,09 & 0,05 \\
\hline & {$[16,06]$} & {$[-0,16]$} & {$[-4,53]$} & & & \\
\hline & $(0,00)$ & $(0,87)$ & $(0,00)$ & & & \\
\hline _HEN & 20,99 & 0,00 & 0,01 & 0,19 & 45,16 & 0,04 \\
\hline & {$[26,26]$} & {$[-2,83]$} & {$[5,18]$} & & & \\
\hline & $(0,00)$ & $(0,00)$ & $(0,00)$ & & & \\
\hline _CONT & 113,49 & 0,00 & $-0,11$ & 0,62 & 229,92 & 0,00 \\
\hline & {$[12,94]$} & {$[-0,71]$} & {$[-8,11]$} & & & \\
\hline & $(0,00)$ & $(0,48)$ & $(0,00)$ & & & \\
\hline _DP & 166,62 & 0,00 & $-0,20$ & 0,79 & 110,43 & 0,11 \\
\hline & {$[7,95]$} & {$[1,53]$} & {$[-7,05]$} & & & \\
\hline & $(0,00)$ & $(0,13)$ & $(0,00)$ & & & \\
\hline
\end{tabular}

Table C.22.: Time-series regression results for the test of the relationship between default risk and the slope variable $S L P 130$ for a constant maturity of 36 days 


\begin{tabular}{|c|c|c|c|c|c|c|}
\hline \multicolumn{7}{|c|}{$\operatorname{CDS}_{t}=\beta_{0}+\beta_{1} \cdot X \operatorname{SMIN}_{t}+\beta_{2} \cdot t+u_{t}$} \\
\hline ID & $b_{0}$ & $b_{1}$ & $b_{2}$ & R2 cor. & $\mathbf{F}$ & DW \\
\hline ALL & $\begin{array}{r}-217,86 \\
{[-8,24]} \\
(0,00)\end{array}$ & $\begin{array}{r}233,99 \\
{[10,15]} \\
(0,00)\end{array}$ & $\begin{array}{r}0,00 \\
{[-0,78]} \\
(0,43)\end{array}$ & 0,63 & 631,81 & 0,42 \\
\hline _SIE & $\begin{array}{r}-103,23 \\
{[-6,24]} \\
(0,00)\end{array}$ & $\begin{array}{r}140,73 \\
{[10,22]} \\
(0,00)\end{array}$ & $\begin{array}{r}-0,04 \\
{[-8,43]} \\
(0,00)\end{array}$ & 0,80 & $1.469,00$ & 0,42 \\
\hline _DTE & $\begin{array}{r}252,40 \\
{[10,96]} \\
(0,00)\end{array}$ & $\begin{array}{r}9,97 \\
{[0,72]} \\
(0,47)\end{array}$ & $\begin{array}{r}-0,33 \\
{[-11,46]} \\
(0,00)\end{array}$ & 0,62 & 618,46 & 0,02 \\
\hline DAIM & $\begin{array}{r}100,11 \\
{[4,15]} \\
(0,00) \\
\end{array}$ & $\begin{array}{l}50,28 \\
{[2,50]} \\
(0,01)\end{array}$ & $\begin{array}{r}-0,11 \\
{[-14,53]} \\
(0,00)\end{array}$ & 0,73 & $1.031,74$ & 0,16 \\
\hline DBANK & $\begin{array}{r}-50,59 \\
{[-2,64]} \\
(0,01)\end{array}$ & $\begin{array}{l}72,37 \\
{[4,45]} \\
(0,00)\end{array}$ & $\begin{array}{r}-0,01 \\
{[-3,29]} \\
(0,00)\end{array}$ & 0,54 & 442,88 & 0,47 \\
\hline _MRE & $\begin{array}{l}49,08 \\
{[7,25]} \\
(0,00)\end{array}$ & $\begin{array}{r}6,90 \\
{[1,96]} \\
(0,05)\end{array}$ & $\begin{array}{r}-0,05 \\
{[-6,02]} \\
(0,00)\end{array}$ & 0,34 & 173,20 & 0,22 \\
\hline BAY & $\begin{array}{r}68,01 \\
{[10,11]} \\
(0,00) \\
\end{array}$ & $\begin{array}{r}6,34 \\
{[1,70]} \\
(0,09) \\
\end{array}$ & $\begin{array}{r}-0,04 \\
{[-4,20]} \\
(0,00) \\
\end{array}$ & 0,09 & 35,69 & 0,03 \\
\hline -VW & $\begin{array}{r}49,10 \\
{[19,39]} \\
(0,00) \\
\end{array}$ & $\begin{array}{r}0,02 \\
{[0,49]} \\
(0,63)\end{array}$ & $\begin{array}{r}0,03 \\
{[5,81]} \\
(0,00)\end{array}$ & 0,20 & 97,55 & 0,02 \\
\hline BASF & $\begin{array}{r}30,51 \\
{[36,39]} \\
(0,00) \\
\end{array}$ & $\begin{array}{r}0,30 \\
{[0,80]} \\
(0,43) \\
\end{array}$ & $\begin{array}{r}-0,03 \\
{[-20,51]} \\
(0,00)\end{array}$ & 0,75 & $1.120,08$ & 0,03 \\
\hline EON & $\begin{array}{r}52,93 \\
{[14,18]} \\
(0,00)\end{array}$ & $\begin{array}{r}-0,27 \\
{[-0,84]} \\
(0,40)\end{array}$ & $\begin{array}{r}-0,04 \\
{[-6,10]} \\
(0,00)\end{array}$ & 0,27 & 137,33 & 0,01 \\
\hline COB & $\begin{array}{l}82,74 \\
{[5,45]} \\
(0,00)\end{array}$ & $\begin{array}{l}15,90 \\
{[1,60]} \\
(0,11)\end{array}$ & $\begin{array}{r}-0,10 \\
{[-5,60]} \\
(0,00)\end{array}$ & 0,24 & 118,23 & 0,03 \\
\hline _HYPV & $\begin{array}{l}74,82 \\
{[8,14]} \\
(0,00)\end{array}$ & $\begin{array}{r}4,54 \\
{[2,53]} \\
(0,01) \\
\end{array}$ & $\begin{array}{r}-0,06 \\
{[-4,09]} \\
(0,00)\end{array}$ & 0,13 & 55,30 & 0,02 \\
\hline _RWE & $\begin{array}{r}82,31 \\
{[16,93]} \\
(0,00) \\
\end{array}$ & $\begin{array}{r}2,16 \\
{[3,53]} \\
(0,00) \\
\end{array}$ & $\begin{array}{r}-0,09 \\
{[-11,21]} \\
(0,00) \\
\end{array}$ & 0,54 & 395,31 & 0,03 \\
\hline BMW & $\begin{array}{r}37,49 \\
{[27,39]} \\
(0,00) \\
\end{array}$ & $\begin{array}{r}0,11 \\
{[0,35]} \\
(0,73) \\
\end{array}$ & $\begin{array}{r}-0,01 \\
{[-4,61]} \\
(0,00) \\
\end{array}$ & 0,16 & 73,00 & 0,12 \\
\hline _LH & $\begin{array}{r}154,89 \\
{[19,28]} \\
(0,00) \\
\end{array}$ & $\begin{array}{r}0,93 \\
{[0,23]} \\
(0,82) \\
\end{array}$ & $\begin{array}{r}-0,12 \\
{[-11,21]} \\
(0,00) \\
\end{array}$ & 0,43 & 268,01 & 0,01 \\
\hline _TYSS & $\begin{array}{r}205,68 \\
{[17,36]} \\
(0,00)\end{array}$ & $\begin{array}{r}-0,30 \\
{[-1,64]} \\
(0,10)\end{array}$ & $\begin{array}{r}-0,14 \\
{[-7,21]} \\
(0,00)\end{array}$ & 0,28 & 128,79 & 0,03 \\
\hline _METRO & $\begin{array}{r}101,17 \\
{[14,00]} \\
(0,00)\end{array}$ & $\begin{array}{r}0,02 \\
{[1,58]} \\
(0,11)\end{array}$ & $\begin{array}{r}-0,07 \\
{[-5,52]} \\
(0,00)\end{array}$ & 0,20 & 75,21 & 0,05 \\
\hline _TUI & $\begin{array}{r}311,69 \\
{[7,59]} \\
(0,00) \\
\end{array}$ & $\begin{array}{r}7,93 \\
{[2,19]} \\
(0,03) \\
\end{array}$ & $\begin{array}{r}-0,11 \\
{[-1,66]} \\
(0,10)\end{array}$ & 0,06 & 9,23 & 0,07 \\
\hline LLIN & $\begin{array}{r}53,48 \\
{[16,42]} \\
(0,00) \\
\end{array}$ & $\begin{array}{r}0,58 \\
{[2,25]} \\
(0,03) \\
\end{array}$ & $\begin{array}{r}-0,03 \\
{[-4,55]} \\
(0,00) \\
\end{array}$ & 0,16 & 46,67 & 0,05 \\
\hline _HEN & $\begin{array}{r}20,77 \\
{[26,20]} \\
(0,00) \\
\end{array}$ & $\begin{array}{r}0,22 \\
{[1,22]} \\
(0,22) \\
\end{array}$ & $\begin{array}{r}0,01 \\
{[5,19]} \\
(0,00) \\
\end{array}$ & 0,19 & 45,29 & 0,04 \\
\hline CCONT & $\begin{array}{r}113,71 \\
{[12,92]} \\
(0,00)\end{array}$ & $\begin{array}{r}-0,10 \\
{[-1,82]} \\
(0,07)\end{array}$ & $\begin{array}{r}-0,11 \\
{[-8,13]} \\
(0,00)\end{array}$ & 0,62 & 229,94 & 0,01 \\
\hline DP & $\begin{array}{r}165,79 \\
{[7,29]} \\
(0,00)\end{array}$ & $\begin{array}{r}0,05 \\
{[0,16]} \\
(0,88)\end{array}$ & $\begin{array}{r}-0,20 \\
{[-6,56]} \\
(0,00)\end{array}$ & 0,79 & 108,36 & 0,06 \\
\hline
\end{tabular}

Table C.23.: Time-series regression results for the test of the relationship between default risk and the horizontal coordinate $X S M I N$ for a constant maturity of 36 days 


\begin{tabular}{|c|c|c|c|c|c|c|}
\hline \multicolumn{7}{|c|}{$C D S_{t}=\beta_{0}+\beta_{1} \cdot S L P 70_{t}+\beta_{2} \cdot t+u_{t}$} \\
\hline ID & $b_{0}$ & $b_{1}$ & $b_{2}$ & R2 cor. & $\mathbf{F}$ & DW \\
\hline ALL & $\begin{array}{r}70,73 \\
{[11,84]} \\
(0,00) \\
\end{array}$ & $\begin{array}{l}18,21 \\
{[3,16]} \\
(0,00)\end{array}$ & $\begin{array}{r}-0,04 \\
{[-3,32]} \\
(0,00) \\
\end{array}$ & 0,24 & 113,29 & 0,07 \\
\hline _SIE & $\begin{array}{r}63,98 \\
{[22,42]} \\
(0,00)\end{array}$ & $\begin{array}{r}0,13 \\
{[0,05]} \\
(0,96)\end{array}$ & $\begin{array}{r}-0,06 \\
{[-9,61]} \\
(0,00)\end{array}$ & 0,59 & 553,97 & 0,01 \\
\hline DTTE & $\begin{array}{r}263,62 \\
{[16,53]} \\
(0,00) \\
\end{array}$ & $\begin{array}{r}0,04 \\
{[0,36]} \\
(0,72) \\
\end{array}$ & $\begin{array}{r}-0,33 \\
{[-11,52]} \\
(0,00)\end{array}$ & 0,62 & 613,47 & 0,01 \\
\hline _DAIM & $\begin{array}{r}159,14 \\
{[41,79]} \\
(0,00) \\
\end{array}$ & $\begin{array}{r}-0,03 \\
{[-7,03]} \\
(0,00)\end{array}$ & $\begin{array}{r}-0,11 \\
{[-17,16]} \\
(0,00)\end{array}$ & 0,71 & 932,52 & 0,06 \\
\hline _DBANK & $\begin{array}{r}35,84 \\
{[15,57]} \\
(0,00) \\
\end{array}$ & $\begin{array}{r}-0,25 \\
{[-1,95]} \\
(0,05)\end{array}$ & $\begin{array}{r}-0,03 \\
{[-6,84]} \\
(0,00)\end{array}$ & 0,29 & 153,19 & 0,01 \\
\hline _MRE & $\begin{array}{r}58,25 \\
{[13,54]} \\
(0,00)\end{array}$ & $\begin{array}{r}0,99 \\
{[2,90]} \\
(0,00)\end{array}$ & $\begin{array}{r}-0,05 \\
{[-6,25]} \\
(0,00)\end{array}$ & 0,33 & 168,14 & 0,19 \\
\hline BAY & $\begin{array}{r}76,49 \\
{[11,97]} \\
(0,00) \\
\end{array}$ & $\begin{array}{r}1,76 \\
{[2,67]} \\
(0,01)\end{array}$ & $\begin{array}{r}-0,04 \\
{[-4,19]} \\
(0,00)\end{array}$ & 0,09 & 35,53 & 0,03 \\
\hline _VW & $\begin{array}{r}49,10 \\
{[19,31]} \\
(0,00) \\
\end{array}$ & $\begin{array}{r}-0,05 \\
{[-0,79]} \\
(0,43)\end{array}$ & $\begin{array}{r}0,03 \\
{[5,81]} \\
(0,00)\end{array}$ & 0,20 & 97,54 & 0,02 \\
\hline _BASF & $\begin{array}{r}30,86 \\
{[39,17]} \\
(0,00) \\
\end{array}$ & $\begin{array}{r}0,00 \\
{[0,67]} \\
(0,50) \\
\end{array}$ & $\begin{array}{r}-0,03 \\
{[-20,48]} \\
(0,00) \\
\end{array}$ & 0,75 & $1.119,27$ & 0,03 \\
\hline EON & $\begin{array}{r}52,65 \\
{[14,05]} \\
(0,00) \\
\end{array}$ & $\begin{array}{r}-0,01 \\
{[-0,96]} \\
(0,34)\end{array}$ & $\begin{array}{r}-0,04 \\
{[-6,10]} \\
(0,00) \\
\end{array}$ & 0,27 & 136,99 & 0,01 \\
\hline _COB & $\begin{array}{r}100,29 \\
{[9,11]} \\
(0,00) \\
\end{array}$ & $\begin{array}{r}0,00 \\
{[-0,87]} \\
(0,39) \\
\end{array}$ & $\begin{array}{r}-0,10 \\
{[-5,62]} \\
(0,00) \\
\end{array}$ & 0,24 & 113,22 & 0,01 \\
\hline _HYPV & $\begin{array}{l}80,13 \\
{[9,29]} \\
(0,00)\end{array}$ & $\begin{array}{r}0,00 \\
{[-1,22]} \\
(0,22) \\
\end{array}$ & $\begin{array}{r}-0,06 \\
{[-4,14]} \\
(0,00) \\
\end{array}$ & 0,12 & 52,73 & 0,01 \\
\hline _RWE & $\begin{array}{r}84,66 \\
{[17,91]} \\
(0,00) \\
\end{array}$ & $\begin{array}{r}0,00 \\
{[0,11]} \\
(0,91) \\
\end{array}$ & $\begin{array}{r}-0,09 \\
{[-11,07]} \\
(0,00) \\
\end{array}$ & 0,54 & 389,81 & 0,01 \\
\hline _BMW & $\begin{array}{r}37,61 \\
{[27,86]} \\
(0,00) \\
\end{array}$ & $\begin{array}{r}0,00 \\
{[3,79]} \\
(0,00) \\
\end{array}$ & $\begin{array}{r}-0,01 \\
{[-4,60]} \\
(0,00) \\
\end{array}$ & 0,16 & 73,36 & 0,12 \\
\hline _LH & $\begin{array}{r}155,85 \\
{[24,21]} \\
(0,00) \\
\end{array}$ & $\begin{array}{r}0,00 \\
{[4,07]} \\
(0,00) \\
\end{array}$ & $\begin{array}{r}-0,12 \\
{[-11,20]} \\
(0,00)\end{array}$ & 0,43 & 268,10 & 0,01 \\
\hline _TYSS & $\begin{array}{r}205,46 \\
{[17,35]} \\
(0,00) \\
\end{array}$ & $\begin{array}{r}-0,01 \\
{[-4,48]} \\
(0,00) \\
\end{array}$ & $\begin{array}{r}-0,14 \\
{[-7,22]} \\
(0,00)\end{array}$ & 0,28 & 129,08 & 0,03 \\
\hline _METRO & $\begin{array}{r}101,23 \\
{[14,07]} \\
(0,00) \\
\end{array}$ & $\begin{array}{r}0,00 \\
{[0,44]} \\
(0,66) \\
\end{array}$ & $\begin{array}{r}-0,07 \\
{[-5,53]} \\
(0,00) \\
\end{array}$ & 0,20 & 75,20 & 0,05 \\
\hline _TUI & $\begin{array}{r}323,10 \\
{[7,62]} \\
(0,00) \\
\end{array}$ & $\begin{array}{r}-0,01 \\
{[-1,32]} \\
(0,19) \\
\end{array}$ & $\begin{array}{r}-0,12 \\
{[-1,71]} \\
(0,09)\end{array}$ & 0,06 & 8,33 & 0,06 \\
\hline _LIN & $\begin{array}{r}53,98 \\
{[16,06]} \\
(0,00) \\
\end{array}$ & $\begin{array}{r}0,00 \\
{[-0,14]} \\
(0,89) \\
\end{array}$ & $\begin{array}{r}-0,03 \\
{[-4,54]} \\
(0,00)\end{array}$ & 0,16 & 46,09 & 0,05 \\
\hline _HEN & $\begin{array}{r}20,98 \\
{[26,24]} \\
(0,00) \\
\end{array}$ & $\begin{array}{r}0,00 \\
{[2,57]} \\
(0,01) \\
\end{array}$ & $\begin{array}{r}0,01 \\
{[5,18]} \\
(0,00)\end{array}$ & 0,19 & 45,21 & 0,04 \\
\hline _CONT & $\begin{array}{r}113,50 \\
{[12,94]} \\
(0,00)\end{array}$ & $\begin{array}{r}0,00 \\
{[0,56]} \\
(0,58) \\
\end{array}$ & $\begin{array}{r}-0,11 \\
{[-8,11]} \\
(0,00)\end{array}$ & 0,62 & 229,81 & 0,00 \\
\hline _DP & $\begin{array}{r}166,34 \\
{[7,90]} \\
(0,00)\end{array}$ & $\begin{array}{r}0,00 \\
{[-0,79]} \\
(0,43)\end{array}$ & $\begin{array}{r}-0,20 \\
{[-7,00]} \\
(0,00)\end{array}$ & 0,79 & 108,58 & 0,07 \\
\hline
\end{tabular}

Table C.24.: Time-series regression results for the test of the relationship between default risk and the slope variable $S L P 70$ for a constant maturity of 60 days 


\begin{tabular}{|c|c|c|c|c|c|c|}
\hline \multicolumn{7}{|c|}{$C D S_{t}=\beta_{0}+\beta_{1} \cdot S L P 90_{t}+\beta_{2} \cdot t+u_{t}$} \\
\hline ID & $b_{0}$ & $b_{1}$ & $b_{2}$ & R2 cor. & $\mathbf{F}$ & DW \\
\hline ALL & $\begin{array}{l}59,99 \\
{[9,02]} \\
(0,00) \\
\end{array}$ & $\begin{array}{r}-4,40 \\
{[-0,38]} \\
(0,71) \\
\end{array}$ & $\begin{array}{r}-0,05 \\
{[-4,78]} \\
(0,00) \\
\end{array}$ & 0,20 & 93,19 & 0,01 \\
\hline _SIE & $\begin{array}{r}46,37 \\
{[10,24]} \\
(0,00) \\
\end{array}$ & $\begin{array}{r}-45,31 \\
{[-4,69]} \\
(0,00) \\
\end{array}$ & $\begin{array}{r}-0,07 \\
{[-13,46]} \\
(0,00)\end{array}$ & 0,64 & 685,03 & 0,15 \\
\hline _DTE & $\begin{array}{r}263,58 \\
{[16,54]} \\
(0,00) \\
\end{array}$ & $\begin{array}{r}-0,16 \\
{[-0,51]} \\
(0,61) \\
\end{array}$ & $\begin{array}{r}-0,33 \\
{[-11,53]} \\
(0,00) \\
\end{array}$ & 0,62 & 613,49 & 0,01 \\
\hline _DAIM & $\begin{array}{r}159,12 \\
{[41,80]} \\
(0,00) \\
\end{array}$ & $\begin{array}{r}-0,11 \\
{[-5,06]} \\
(0,00) \\
\end{array}$ & $\begin{array}{r}-0,11 \\
{[-17,17]} \\
(0,00)\end{array}$ & 0,71 & 932,75 & 0,06 \\
\hline _DBANK & $\begin{array}{r}35,05 \\
{[15,55]} \\
(0,00) \\
\end{array}$ & $\begin{array}{r}-2,13 \\
{[-1,91]} \\
(0,06) \\
\end{array}$ & $\begin{array}{r}-0,03 \\
{[-6,94]} \\
(0,00) \\
\end{array}$ & 0,29 & 158,20 & 0,03 \\
\hline _MRE & $\begin{array}{r}58,44 \\
{[13,58]} \\
(0,00) \\
\end{array}$ & $\begin{array}{r}1,70 \\
{[4,23]} \\
(0,00) \\
\end{array}$ & $\begin{array}{r}-0,05 \\
{[-6,33]} \\
(0,00) \\
\end{array}$ & 0,33 & 165,05 & 0,18 \\
\hline _BAY & $\begin{array}{r}77,17 \\
{[11,72]} \\
(0,00) \\
\end{array}$ & $\begin{array}{r}4,23 \\
{[2,46]} \\
(0,01) \\
\end{array}$ & $\begin{array}{r}-0,04 \\
{[-4,25]} \\
(0,00) \\
\end{array}$ & 0,08 & 34,91 & 0,03 \\
\hline -VW & $\begin{array}{r}49,00 \\
{[19,27]} \\
(0,00) \\
\end{array}$ & $\begin{array}{r}-0,29 \\
{[-1,08]} \\
(0,28) \\
\end{array}$ & $\begin{array}{r}0,03 \\
{[5,83]} \\
(0,00) \\
\end{array}$ & 0,20 & 98,16 & 0,03 \\
\hline _BASF & $\begin{array}{r}30,86 \\
{[39,18]} \\
(0,00) \\
\end{array}$ & $\begin{array}{r}0,01 \\
{[0,66]} \\
(0,51) \\
\end{array}$ & $\begin{array}{r}-0,03 \\
{[-20,48]} \\
(0,00) \\
\end{array}$ & 0,75 & $1.119,30$ & 0,03 \\
\hline EON & $\begin{array}{r}52,64 \\
{[14,04]} \\
(0,00) \\
\end{array}$ & $\begin{array}{r}-0,05 \\
{[-1,48]} \\
(0,14) \\
\end{array}$ & $\begin{array}{r}-0,04 \\
{[-6,10]} \\
(0,00) \\
\end{array}$ & 0,27 & 137,04 & 0,01 \\
\hline _COB & $\begin{array}{r}100,29 \\
{[9,11]} \\
(0,00) \\
\end{array}$ & $\begin{array}{r}-0,03 \\
{[-0,84]} \\
(0,40) \\
\end{array}$ & $\begin{array}{r}-0,10 \\
{[-5,62]} \\
(0,00) \\
\end{array}$ & 0,24 & 113,28 & 0,01 \\
\hline _HYPV & $\begin{array}{l}80,13 \\
{[9,29]} \\
(0,00) \\
\end{array}$ & $\begin{array}{r}0,00 \\
{[-1,83]} \\
(0,07) \\
\end{array}$ & $\begin{array}{r}-0,06 \\
{[-4,14]} \\
(0,00)\end{array}$ & 0,12 & 52,74 & 0,01 \\
\hline _RWE & $\begin{array}{r}84,65 \\
{[17,94]} \\
(0,00) \\
\end{array}$ & $\begin{array}{r}0,00 \\
{[-0,24]} \\
(0,81) \\
\end{array}$ & $\begin{array}{r}-0,09 \\
{[-11,08]} \\
(0,00)\end{array}$ & 0,54 & 389,81 & 0,01 \\
\hline BMW & $\begin{array}{r}37,61 \\
{[27,86]} \\
(0,00) \\
\end{array}$ & $\begin{array}{r}0,00 \\
{[3,10]} \\
(0,00) \\
\end{array}$ & $\begin{array}{r}-0,01 \\
{[-4,61]} \\
(0,00)\end{array}$ & 0,16 & 73,30 & 0,12 \\
\hline _LH & $\begin{array}{r}155,85 \\
{[24,21]} \\
(0,00) \\
\end{array}$ & $\begin{array}{r}0,00 \\
{[4,23]} \\
(0,00) \\
\end{array}$ & $\begin{array}{r}-0,12 \\
{[-11,20]} \\
(0,00) \\
\end{array}$ & 0,43 & 268,09 & 0,01 \\
\hline -TYSS & $\begin{array}{r}205,47 \\
{[17,35]} \\
(0,00) \\
\end{array}$ & $\begin{array}{r}-0,03 \\
{[-4,26]} \\
(0,00) \\
\end{array}$ & $\begin{array}{r}-0,14 \\
{[-7,22]} \\
(0,00) \\
\end{array}$ & 0,28 & 129,08 & 0,03 \\
\hline _METRO & $\begin{array}{r}101,23 \\
{[14,06]} \\
(0,00) \\
\end{array}$ & $\begin{array}{r}0,00 \\
{[-0,05]} \\
(0,96) \\
\end{array}$ & $\begin{array}{r}-0,07 \\
{[-5,53]} \\
(0,00) \\
\end{array}$ & 0,20 & 75,16 & 0,05 \\
\hline _TUI & $\begin{array}{r}323,38 \\
{[7,64]} \\
(0,00) \\
\end{array}$ & $\begin{array}{r}-0,04 \\
{[-1,76]} \\
(0,08) \\
\end{array}$ & $\begin{array}{r}-0,12 \\
{[-1,72]} \\
(0,09) \\
\end{array}$ & 0,06 & 8,48 & 0,06 \\
\hline _LIN & $\begin{array}{r}53,99 \\
{[16,08]} \\
(0,00) \\
20,98\end{array}$ & $\begin{array}{r}0,00 \\
{[1,22]} \\
(0,22) \\
0,00\end{array}$ & $\begin{array}{r}-0,03 \\
{[-4,54]} \\
(0,00) \\
0,01\end{array}$ & 0,16 & 46,10 & 0,05 \\
\hline _HEN & $\begin{array}{r}20,98 \\
{[26,22]} \\
(0,00) \\
\end{array}$ & $\begin{array}{r}0,00 \\
{[1,98]} \\
(0,05) \\
\end{array}$ & $\begin{array}{r}0,01 \\
{[5,18]} \\
(0,00) \\
\end{array}$ & 0,19 & 45,24 & 0,04 \\
\hline _CONT & $\begin{array}{r}113,51 \\
{[12,95]} \\
(0,00) \\
\end{array}$ & $\begin{array}{r}0,00 \\
{[0,63]} \\
(0,53)\end{array}$ & $\begin{array}{r}-0,11 \\
{[-8,12]} \\
(0,00)\end{array}$ & 0,62 & 229,82 & 0,00 \\
\hline _DP & $\begin{array}{r}166,30 \\
{[7,90]} \\
(0,00)\end{array}$ & $\begin{array}{r}0,00 \\
{[-0,74]} \\
(0,46)\end{array}$ & $\begin{array}{r}-0,20 \\
{[-7,00]} \\
(0,00)\end{array}$ & 0,79 & 108,52 & 0,07 \\
\hline
\end{tabular}

Table C.25.: Time-series regression results for the test of the relationship between default risk and the slope variable $S L P 90$ for a constant maturity of 60 days 


\begin{tabular}{|c|c|c|c|c|c|c|}
\hline \multicolumn{7}{|c|}{$C D S_{t}=\beta_{0}+\beta_{1} \cdot S L P 100_{t}+\beta_{2} \cdot t+u_{t}$} \\
\hline ID & $b_{0}$ & $b_{1}$ & $b_{2}$ & R2 cor. & $\mathbf{F}$ & DW \\
\hline ALL & $\begin{array}{l}14,82 \\
{[1,57]} \\
(0,12)\end{array}$ & $\begin{array}{r}-150,88 \\
{[-6,10]} \\
(0,00)\end{array}$ & $\begin{array}{r}-0,04 \\
{[-4,29]} \\
(0,00)\end{array}$ & 0,36 & 206,35 & 0,17 \\
\hline _SIE & $\begin{array}{l}22,05 \\
{[5,10]} \\
(0,00)\end{array}$ & $\begin{array}{r}-132,14 \\
{[-10,51]} \\
(0,00)\end{array}$ & $\begin{array}{r}-0,05 \\
{[-15,20]} \\
(0,00)\end{array}$ & 0,81 & $1.659,38$ & 0,25 \\
\hline _DTE & $\begin{array}{r}258,99 \\
{[16,15]} \\
(0,00) \\
\end{array}$ & $\begin{array}{r}-18,52 \\
{[-2,12]} \\
(0,03)\end{array}$ & $\begin{array}{r}-0,33 \\
{[-11,65]} \\
(0,00)\end{array}$ & 0,62 & 627,04 & 0,04 \\
\hline _DAIM & $\begin{array}{r}157,94 \\
{[40,53]} \\
(0,00) \\
\end{array}$ & $\begin{array}{r}-3,72 \\
{[-1,35]} \\
(0,18)\end{array}$ & $\begin{array}{r}-0,11 \\
{[-17,19]} \\
(0,00)\end{array}$ & 0,71 & 940,23 & 0,07 \\
\hline _DBANK & $\begin{array}{l}28,05 \\
{[5,93]} \\
(0,00)\end{array}$ & $\begin{array}{r}-20,89 \\
{[-1,83]} \\
(0,07)\end{array}$ & $\begin{array}{r}-0,02 \\
{[-6,12]} \\
(0,00)\end{array}$ & 0,37 & 221,70 & 0,20 \\
\hline _MRE & $\begin{array}{r}58,20 \\
{[13,17]} \\
(0,00)\end{array}$ & $\begin{array}{r}0,91 \\
{[0,57]} \\
(0,57)\end{array}$ & $\begin{array}{r}-0,05 \\
{[-6,42]} \\
(0,00)\end{array}$ & 0,32 & 160,31 & 0,16 \\
\hline BAY & $\begin{array}{r}75,37 \\
{[10,97]} \\
(0,00)\end{array}$ & $\begin{array}{r}0,33 \\
{[0,08]} \\
(0,93)\end{array}$ & $\begin{array}{r}-0,04 \\
{[-4,19]} \\
(0,00)\end{array}$ & 0,08 & 32,28 & 0,02 \\
\hline _VW & $\begin{array}{r}47,68 \\
{[19,05]} \\
(0,00) \\
\end{array}$ & $\begin{array}{r}-4,34 \\
{[-2,50]} \\
(0,01)\end{array}$ & $\begin{array}{r}0,03 \\
{[6,10]} \\
(0,00) \\
\end{array}$ & 0,22 & 108,15 & 0,07 \\
\hline _BASF & $\begin{array}{r}30,87 \\
{[39,22]} \\
(0,00) \\
\end{array}$ & $\begin{array}{r}0,04 \\
{[0,51]} \\
(0,61)\end{array}$ & $\begin{array}{r}-0,03 \\
{[-20,51]} \\
(0,00)\end{array}$ & 0,75 & $1.119,33$ & 0,03 \\
\hline EON & $\begin{array}{r}52,49 \\
{[14,04]} \\
(0,00)\end{array}$ & $\begin{array}{r}-0,70 \\
{[-0,99]} \\
(0,32)\end{array}$ & $\begin{array}{r}-0,04 \\
{[-6,11]} \\
(0,00)\end{array}$ & 0,27 & 137,79 & 0,01 \\
\hline CCOB & $\begin{array}{r}100,30 \\
{[9,10]} \\
(0,00) \\
\end{array}$ & $\begin{array}{r}0,00 \\
{[0,00]} \\
(1,00)\end{array}$ & $\begin{array}{r}-0,10 \\
{[-5,61]} \\
(0,00) \\
\end{array}$ & 0,24 & 113,20 & 0,01 \\
\hline _HYPV & $\begin{array}{l}80,11 \\
{[9,29]} \\
(0,00)\end{array}$ & $\begin{array}{r}-0,01 \\
{[-2,27]} \\
(0,02) \\
\end{array}$ & $\begin{array}{r}-0,06 \\
{[-4,13]} \\
(0,00) \\
\end{array}$ & 0,12 & 52,78 & 0,01 \\
\hline _RWE & $\begin{array}{r}84,66 \\
{[17,96]} \\
(0,00)\end{array}$ & $\begin{array}{r}0,00 \\
{[-1,38]} \\
(0,17)\end{array}$ & $\begin{array}{r}-0,09 \\
{[-11,10]} \\
(0,00)\end{array}$ & 0,54 & 389,84 & 0,01 \\
\hline _BMW & $\begin{array}{r}37,61 \\
{[27,86]} \\
(0,00) \\
\end{array}$ & $\begin{array}{r}-0,01 \\
{[-0,83]} \\
(0,41) \\
\end{array}$ & $\begin{array}{r}-0,01 \\
{[-4,60]} \\
(0,00) \\
\end{array}$ & 0,16 & 73,05 & 0,12 \\
\hline _LH & $\begin{array}{r}155,87 \\
{[24,19]} \\
(0,00) \\
\end{array}$ & $\begin{array}{r}0,00 \\
{[0,03]} \\
(0,97)\end{array}$ & $\begin{array}{r}-0,12 \\
{[-11,20]} \\
(0,00)\end{array}$ & 0,43 & 267,93 & 0,01 \\
\hline _TYSS & $\begin{array}{r}205,47 \\
{[17,34]} \\
(0,00) \\
\end{array}$ & $\begin{array}{r}-0,07 \\
{[-3,51]} \\
(0,00)\end{array}$ & $\begin{array}{r}-0,14 \\
{[-7,22]} \\
(0,00)\end{array}$ & 0,28 & 129,04 & 0,03 \\
\hline _METRO & $\begin{array}{r}101,32 \\
{[14,07]} \\
(0,00) \\
\end{array}$ & $\begin{array}{r}-0,11 \\
{[-1,21]} \\
(0,23)\end{array}$ & $\begin{array}{r}-0,07 \\
{[-5,55]} \\
(0,00) \\
\end{array}$ & 0,20 & 75,91 & 0,05 \\
\hline _TUI & $\begin{array}{r}321,63 \\
{[7,59]} \\
(0,00) \\
\end{array}$ & $\begin{array}{r}0,01 \\
{[1,18]} \\
(0,24) \\
\end{array}$ & $\begin{array}{r}-0,11 \\
{[-1,68]} \\
(0,09) \\
\end{array}$ & 0,05 & 8,02 & 0,05 \\
\hline _LIN & $\begin{array}{r}53,99 \\
{[16,08]} \\
(0,00)\end{array}$ & $\begin{array}{r}0,00 \\
{[1,69]} \\
(0,09)\end{array}$ & $\begin{array}{r}-0,03 \\
{[-4,54]} \\
(0,00) \\
\end{array}$ & 0,16 & 46,11 & 0,05 \\
\hline _HEN & $\begin{array}{r}20,99 \\
{[26,28]} \\
(0,00)\end{array}$ & $\begin{array}{r}0,00 \\
{[-3,84]} \\
(0,00)\end{array}$ & $\begin{array}{r}0,01 \\
{[5,18]} \\
(0,00) \\
\end{array}$ & 0,19 & 45,14 & 0,04 \\
\hline _CONT & $\begin{array}{r}113,54 \\
{[12,94]} \\
(0,00)\end{array}$ & $\begin{array}{r}0,00 \\
{[0,16]} \\
(0,87)\end{array}$ & $\begin{array}{r}-0,11 \\
{[-8,13]} \\
(0,00)\end{array}$ & 0,62 & 229,70 & 0,00 \\
\hline _DP & $\begin{array}{r}166,02 \\
{[7,92]} \\
(0,00)\end{array}$ & $\begin{array}{r}0,00 \\
{[0,32]} \\
(0,75)\end{array}$ & $\begin{array}{r}-0,20 \\
{[-7,01]} \\
(0,00)\end{array}$ & 0,79 & 108,38 & 0,06 \\
\hline
\end{tabular}

Table C.26.: Time-series regression results for the test of the relationship between default risk and the slope variable $S L P 100$ for a constant maturity of 60 days 


\begin{tabular}{|c|c|c|c|c|c|c|}
\hline \multicolumn{7}{|c|}{$C D S_{t}=\beta_{0}+\beta_{1} \cdot S L P 110_{t}+\beta_{2} \cdot t+u_{t}$} \\
\hline ID & $b_{0}$ & $b_{1}$ & $b_{2}$ & R2 cor. & $F$ & DW \\
\hline ALL & $\begin{array}{l}31,75 \\
{[3,62]} \\
(0,00)\end{array}$ & $\begin{array}{r}-120,91 \\
{[-3,75]} \\
(0,00)\end{array}$ & $\begin{array}{r}-0,01 \\
{[-0,57]} \\
(0,57)\end{array}$ & 0,45 & 295,06 & 0,47 \\
\hline _SIE & $\begin{array}{r}47,23 \\
{[14,33]} \\
(0,00) \\
\end{array}$ & $\begin{array}{r}-67,64 \\
{[-5,97]} \\
(0,00) \\
\end{array}$ & $\begin{array}{r}-0,04 \\
{[-7,62]} \\
(0,00)\end{array}$ & 0,74 & $1.104,33$ & 0,43 \\
\hline _DTE & $\begin{array}{r}263,32 \\
{[16,51]} \\
(0,00)\end{array}$ & $\begin{array}{r}-1,00 \\
{[-1,43]} \\
(0,15)\end{array}$ & $\begin{array}{r}-0,33 \\
{[-11,50]} \\
(0,00)\end{array}$ & 0,62 & 614,60 & 0,02 \\
\hline _DAIM & $\begin{array}{r}159,18 \\
{[41,76]} \\
(0,00) \\
\end{array}$ & $\begin{array}{r}0,05 \\
{[0,70]} \\
(0,48)\end{array}$ & $\begin{array}{r}-0,11 \\
{[-17,16]} \\
(0,00)\end{array}$ & 0,71 & 932,16 & 0,06 \\
\hline _DBANK & $\begin{array}{r}32,54 \\
{[12,14]} \\
(0,00) \\
\end{array}$ & $\begin{array}{r}-10,65 \\
{[-1,61]} \\
(0,11) \\
\end{array}$ & $\begin{array}{r}-0,02 \\
{[-5,07]} \\
(0,00) \\
\end{array}$ & 0,33 & 190,50 & 0,13 \\
\hline _MRE & $\begin{array}{r}56,03 \\
{[11,92]} \\
(0,00) \\
\end{array}$ & $\begin{array}{r}-6,77 \\
{[-1,54]} \\
(0,12) \\
\end{array}$ & $\begin{array}{r}-0,05 \\
{[-5,73]} \\
(0,00) \\
\end{array}$ & 0,34 & 175,55 & 0,22 \\
\hline BAY & $\begin{array}{r}74,40 \\
{[11,91]} \\
(0,00) \\
\end{array}$ & $\begin{array}{r}-4,30 \\
{[-2,20]} \\
(0,03) \\
\end{array}$ & $\begin{array}{r}-0,04 \\
{[-4,06]} \\
(0,00) \\
\end{array}$ & 0,08 & 34,87 & 0,03 \\
\hline _VW & $\begin{array}{r}49,05 \\
{[19,37]} \\
(0,00) \\
\end{array}$ & $\begin{array}{r}-0,59 \\
{[-1,25]} \\
(0,21) \\
\end{array}$ & $\begin{array}{r}0,03 \\
{[5,83]} \\
(0,00) \\
\end{array}$ & 0,21 & 98,69 & 0,03 \\
\hline _BASF & $\begin{array}{r}30,85 \\
{[39,16]} \\
(0,00)\end{array}$ & $\begin{array}{r}-0,01 \\
{[-0,74]} \\
(0,46)\end{array}$ & $\begin{array}{r}-0,03 \\
{[-20,47]} \\
(0,00)\end{array}$ & 0,75 & $1.119,19$ & 0,03 \\
\hline EON & $\begin{array}{r}52,67 \\
{[14,06]} \\
(0,00) \\
\end{array}$ & $\begin{array}{r}0,00 \\
{[-0,02]} \\
(0,98) \\
\end{array}$ & $\begin{array}{r}-0,04 \\
{[-6,10]} \\
(0,00) \\
\end{array}$ & 0,27 & 136,94 & 0,01 \\
\hline _COB & $\begin{array}{r}100,29 \\
{[9,10]} \\
(0,00) \\
\end{array}$ & $\begin{array}{r}0,00 \\
{[0,70]} \\
(0,48) \\
\end{array}$ & $\begin{array}{r}-0,10 \\
{[-5,61]} \\
(0,00) \\
\end{array}$ & 0,24 & 113,21 & 0,01 \\
\hline _HYPV & $\begin{array}{l}80,13 \\
{[9,29]} \\
(0,00)\end{array}$ & $\begin{array}{r}0,00 \\
{[0,31]} \\
(0,76) \\
\end{array}$ & $\begin{array}{r}-0,06 \\
{[-4,15]} \\
(0,00) \\
\end{array}$ & 0,12 & 52,72 & 0,01 \\
\hline _RWE & $\begin{array}{r}84,67 \\
{[17,91]} \\
(0,00)\end{array}$ & $\begin{array}{r}0,00 \\
{[-0,69]} \\
(0,49) \\
\end{array}$ & $\begin{array}{r}-0,09 \\
{[-11,07]} \\
(0,00) \\
\end{array}$ & 0,54 & 389,83 & 0,01 \\
\hline _BMW & $\begin{array}{r}37,61 \\
{[27,86]} \\
(0,00) \\
\end{array}$ & $\begin{array}{r}0,00 \\
{[-4,47]} \\
(0,00) \\
\end{array}$ & $\begin{array}{r}-0,01 \\
{[-4,60]} \\
(0,00) \\
\end{array}$ & 0,16 & 73,38 & 0,12 \\
\hline _LH & $\begin{array}{r}155,85 \\
{[24,22]} \\
(0,00) \\
\end{array}$ & $\begin{array}{r}0,00 \\
{[-3,51]} \\
(0,00) \\
\end{array}$ & $\begin{array}{r}-0,12 \\
{[-11,20]} \\
(0,00)\end{array}$ & 0,43 & 268,14 & 0,01 \\
\hline _TYSS & $\begin{array}{r}205,41 \\
{[17,37]} \\
(0,00)\end{array}$ & $\begin{array}{r}0,05 \\
{[2,57]} \\
(0,01) \\
\end{array}$ & $\begin{array}{r}-0,14 \\
{[-7,23]} \\
(0,00)\end{array}$ & 0,28 & 128,97 & 0,03 \\
\hline _METRO & $\begin{array}{r}101,25 \\
{[14,07]} \\
(0,00)\end{array}$ & $\begin{array}{r}-0,03 \\
{[-1,02]} \\
(0,31) \\
\end{array}$ & $\begin{array}{r}-0,07 \\
{[-5,54]} \\
(0,00) \\
\end{array}$ & 0,20 & 75,51 & 0,05 \\
\hline _ TUI & $\begin{array}{r}322,52 \\
{[7,61]} \\
(0,00) \\
\end{array}$ & $\begin{array}{r}0,01 \\
{[1,21]} \\
(0,23)\end{array}$ & $\begin{array}{r}-0,12 \\
{[-1,70]} \\
(0,09) \\
\end{array}$ & 0,06 & 8,17 & 0,05 \\
\hline _LIN & $\begin{array}{r}53,99 \\
{[16,08]} \\
(0,00)\end{array}$ & $\begin{array}{r}0,00 \\
{[1,61]} \\
(0,11)\end{array}$ & $\begin{array}{r}-0,03 \\
{[-4,54]} \\
(0,00)\end{array}$ & 0,16 & 46,11 & 0,05 \\
\hline _HEN & $\begin{array}{r}20,99 \\
{[26,26]} \\
(0,00) \\
\end{array}$ & $\begin{array}{r}0,00 \\
{[-3,38]} \\
(0,00) \\
\end{array}$ & $\begin{array}{r}0,01 \\
{[5,18]} \\
(0,00) \\
\end{array}$ & 0,19 & 45,18 & 0,04 \\
\hline _CONT & $\begin{array}{r}113,50 \\
{[12,93]} \\
(0,00)\end{array}$ & $\begin{array}{r}0,00 \\
{[-0,43]} \\
(0,67) \\
\end{array}$ & $\begin{array}{r}-0,11 \\
{[-8,11]} \\
(0,00) \\
\end{array}$ & 0,62 & 229,77 & 0,00 \\
\hline _DP & $\begin{array}{r}166,46 \\
{[7,90]} \\
(0,00)\end{array}$ & $\begin{array}{r}0,00 \\
{[0,86]} \\
(0,39)\end{array}$ & $\begin{array}{r}-0,20 \\
{[-7,00]} \\
(0,00)\end{array}$ & 0,79 & 108,80 & 0,07 \\
\hline
\end{tabular}

Table C.27.: Time-series regression results for the test of the relationship between default risk and the slope variable $S L P 110$ for a constant maturity of 60 days 


\begin{tabular}{|c|c|c|c|c|c|c|}
\hline & $C D S_{t}=$ & $\beta_{0}+\beta$ & $S L P 13$ & ${ }_{t}+\beta_{2}$ & $t+u_{t}$ & \\
\hline ID & $b_{0}$ & $b_{1}$ & $b_{2}$ & R2 cor. & $F$ & DW \\
\hline ALL & 56,77 & $-40,22$ & \begin{tabular}{|c|}
$-0,01$ \\
\end{tabular} & 0,36 & 207,20 & 0,33 \\
\hline & {$[12,24]$} & {$[-3,95]$} & {$[-1,15]$} & & & \\
\hline & $(0,00)$ & $(0,00)$ & $(0,25)$ & & & \\
\hline _SIE & 61,81 & $-19,73$ & $-0,04$ & 0,66 & 735,26 & 0,24 \\
\hline & {$[23,95]$} & {$[-3,96]$} & {$[-6,95]$} & & & \\
\hline & $(0,00)$ & $(0,00)$ & $(0,00)$ & & & \\
\hline _DTE & 263,55 & $-0,17$ & $-0,33$ & 0,62 & 613,79 & 0,01 \\
\hline & {$[16,53]$} & {$[-1,05]$} & {$[-11,51]$} & & & \\
\hline & $(0,00)$ & $(0,30)$ & $(0,00)$ & & & \\
\hline DAIM & 159,17 & 0,03 & $-0,11$ & 0,71 & 932,32 & 0,06 \\
\hline & {$[41,78]$} & {$[2,22]$} & {$[-17,16]$} & & & \\
\hline & $(0,00)$ & $(0,03)$ & $(0,00)$ & & & \\
\hline _DBANK & 35,78 & $-0,95$ & $-0,03$ & 0,29 & 156,64 & 0,03 \\
\hline & {$[15,61]$} & {$[-0,92]$} & {$[-6,56]$} & & & \\
\hline & $(0,00)$ & $(0,36)$ & $(0,00)$ & & & \\
\hline _MRE & 57,45 & $-1,84$ & $-0,05$ & 0,34 & 174,01 & 0,22 \\
\hline & {$[13,20]$} & {$[-1,99]$} & {$[-6,03]$} & & & \\
\hline & $(0,00)$ & $(0,05)$ & $(0,00)$ & & & \\
\hline BAY & 75,35 & $-1,77$ & $-0,04$ & 0,09 & 35,53 & 0,03 \\
\hline & {$[12,06]$} & {$[-2,43]$} & {$[-4,11]$} & & & \\
\hline & $(0,00)$ & $(0,02)$ & $(0,00)$ & & & \\
\hline _VW & 49,15 & $-0,04$ & 0,03 & 0,20 & 97,46 & 0,02 \\
\hline & {$[19,34]$} & {$[-0,85]$} & {$[5,80]$} & & & \\
\hline & $(0,00)$ & $(0,39)$ & $(0,00)$ & & & \\
\hline BASF & 30,85 & 0,00 & $-0,03$ & 0,75 & $1.119,24$ & 0,03 \\
\hline & {$[39,16]$} & {$[-0,70]$} & {$[-20,48]$} & & & \\
\hline & $(0,00)$ & $(0,48)$ & $(0,00)$ & & & \\
\hline EON & 52,66 & 0,01 & $-0,04$ & 0,27 & 136,95 & 0,01 \\
\hline & {$[14,05]$} & {$[0,38]$} & {$[-6,10]$} & & & \\
\hline & $(0,00)$ & $(0,71)$ & $(0,00)$ & & & \\
\hline _COB & 100,29 & 0,00 & $-0,10$ & 0,24 & 113,21 & 0,01 \\
\hline & {$[9,10]$} & {$[0,83]$} & {$[-5,62]$} & & & \\
\hline & $(0,00)$ & $(0,41)$ & $(0,00)$ & & & \\
\hline _HYPV & 80,13 & 0,00 & $-0,06$ & 0,12 & 52,72 & 0,01 \\
\hline & {$[9,29]$} & {$[0,69]$} & {$[-4,15]$} & & & \\
\hline & $(0,00)$ & $(0,49)$ & $(0,00)$ & & & \\
\hline _RWE & 84,67 & 0,00 & $-0,09$ & 0,54 & 389,82 & 0,01 \\
\hline & {$[17,90]$} & {$[-0,46]$} & {$[-11,06]$} & & & \\
\hline & $(0,00)$ & $(0,64)$ & $(0,00)$ & & & \\
\hline BMMW & 37,61 & 0,00 & $-0,01$ & 0,16 & 73,38 & 0,12 \\
\hline & {$[27,86]$} & {$[-4,21]$} & {$[-4,60]$} & & & \\
\hline & $(0,00)$ & $(0,00)$ & $(0,00)$ & & & \\
\hline _LH & 155,85 & 0,00 & $-0,12$ & 0,43 & 268,12 & 0,01 \\
\hline & {$[24,22]$} & {$[-3,80]$} & {$[-11,20]$} & & & \\
\hline & $(0,00)$ & $(0,00)$ & $(0,00)$ & & & \\
\hline _TYSS & 205,45 & 0,01 & $-0,14$ & 0,28 & 129,05 & 0,03 \\
\hline & {$[17,36]$} & {$[4,42]$} & {$[-7,22]$} & & & \\
\hline & $(0,00)$ & $(0,00)$ & $(0,00)$ & & & \\
\hline _METRO & 101,24 & $-0,01$ & $\begin{array}{l}-0,07 \\
\end{array}$ & 0,20 & 75,34 & 0,05 \\
\hline & {$[14,07]$} & {$[-0,81]$} & {$[-5,53]$} & & & \\
\hline & $(0,00)$ & $(0,42)$ & $(0,00)$ & & & \\
\hline _TUI & 322,76 & 0,00 & $-0,12$ & 0,06 & 8,23 & 0,05 \\
\hline & {$[7,61]$} & {$[1,21]$} & {$[-1,70]$} & & & \\
\hline & $(0,00)$ & $(0,23)$ & $(0,09)$ & & & \\
\hline LIIN & 53,99 & 0,00 & $-0,03$ & 0,16 & 46,12 & 0,05 \\
\hline & {$[16,08]$} & {$[1,39]$} & {$[-4,54]$} & & & \\
\hline & $(0,00)$ & $(0,17)$ & $(0,00)$ & & & \\
\hline _HEN & 20,98 & 0,00 & 0,01 & 0,19 & 45,19 & 0,04 \\
\hline & {$[26,25]$} & {$[-3,04]$} & {$[5,18]$} & & & \\
\hline & $(0,00)$ & $(0,00)$ & $(0,00)$ & & & \\
\hline _CONT & 113,50 & 0,00 & $-0,11$ & 0,62 & 229,79 & 0,00 \\
\hline & {$[12,93]$} & {$[-0,49]$} & {$[-8,11]$} & & & \\
\hline & $(0,00)$ & $(0,62)$ & $(0,00)$ & & & \\
\hline DPP & 166,39 & 0,00 & $-0,20$ & 0,79 & 108,67 & 0,07 \\
\hline & {$[7,90]$} & {$[0,83]$} & {$[-7,00]$} & & & \\
\hline & $(0,00)$ & $(0,41)$ & $(0,00)$ & & & \\
\hline
\end{tabular}

Table C.28.: Time-series regression results for the test of the relationship between default risk and the slope variable $S L P 130$ for a constant maturity of 60 days 


\begin{tabular}{|c|c|c|c|c|c|c|}
\hline \multicolumn{7}{|c|}{$\operatorname{CDS}_{t}=\beta_{0}+\beta_{1} \cdot X \operatorname{SMIN}_{t}+\beta_{2} \cdot t+u_{t}$} \\
\hline ID & $b_{0}$ & $b_{1}$ & $b_{2}$ & R2 cor. & $\mathbf{F}$ & DW \\
\hline ALL & $\begin{array}{l}46,78 \\
{[3,57]} \\
(0,00)\end{array}$ & $\begin{array}{l}10,59 \\
{[1,14]} \\
(0,26)\end{array}$ & $\begin{array}{r}-0,05 \\
{[-4,77]} \\
(0,00)\end{array}$ & 0,22 & 105,63 & 0,07 \\
\hline _SIE & $\begin{array}{r}58,66 \\
{[12,40]} \\
(0,00) \\
\end{array}$ & $\begin{array}{r}3,87 \\
{[1,52]} \\
(0,13) \\
\end{array}$ & $\begin{array}{r}-0,06 \\
{[-11,28]} \\
(0,00) \\
\end{array}$ & 0,61 & 580,75 & 0,07 \\
\hline _DTE & $\begin{array}{r}236,35 \\
{[10,19]} \\
(0,00)\end{array}$ & $\begin{array}{l}20,91 \\
{[1,54]} \\
(0,12)\end{array}$ & $\begin{array}{r}-0,32 \\
{[-11,46]} \\
(0,00)\end{array}$ & 0,63 & 645,71 & 0,07 \\
\hline _DAIM & $\begin{array}{r}157,77 \\
{[32,05]} \\
(0,00) \\
\end{array}$ & $\begin{array}{r}1,07 \\
{[0,49]} \\
(0,62) \\
\end{array}$ & $\begin{array}{r}-0,11 \\
{[-17,00]} \\
(0,00) \\
\end{array}$ & 0,71 & 933,75 & 0,06 \\
\hline _DBANK & $\begin{array}{r}34,88 \\
{[13,98]} \\
(0,00) \\
\end{array}$ & $\begin{array}{r}0,80 \\
{[1,05]} \\
(0,30) \\
\end{array}$ & $\begin{array}{r}-0,03 \\
{[-6,81]} \\
(0,00)\end{array}$ & 0,29 & 155,08 & 0,02 \\
\hline _MRE & $\begin{array}{r}57,17 \\
{[12,79]} \\
(0,00) \\
\end{array}$ & $\begin{array}{r}0,46 \\
{[4,35]} \\
(0,00) \\
\end{array}$ & $\begin{array}{r}-0,05 \\
{[-6,36]} \\
(0,00) \\
\end{array}$ & 0,33 & 164,63 & 0,18 \\
\hline BAY & $\begin{array}{r}73,07 \\
{[12,14]} \\
(0,00) \\
\end{array}$ & $\begin{array}{r}1,37 \\
{[1,56]} \\
(0,12) \\
\end{array}$ & $\begin{array}{r}-0,04 \\
{[-4,25]} \\
(0,00)\end{array}$ & 0,09 & 36,92 & 0,05 \\
\hline _VW & $\begin{array}{r}45,46 \\
{[16,17]} \\
(0,00) \\
\end{array}$ & $\begin{array}{r}2,94 \\
{[3,15]} \\
(0,00) \\
\end{array}$ & $\begin{array}{r}0,03 \\
{[6,00]} \\
(0,00) \\
\end{array}$ & 0,22 & 104,41 & 0,05 \\
\hline _BASF & $\begin{array}{r}30,73 \\
{[38,68]} \\
(0,00) \\
\end{array}$ & $\begin{array}{r}0,10 \\
{[1,13]} \\
(0,26) \\
\end{array}$ & $\begin{array}{r}-0,03 \\
{[-20,50]} \\
(0,00) \\
\end{array}$ & 0,75 & $1.119,91$ & 0,03 \\
\hline EON & $\begin{array}{r}52,82 \\
{[14,09]} \\
(0,00) \\
\end{array}$ & $\begin{array}{r}-0,14 \\
{[-0,35]} \\
(0,72) \\
\end{array}$ & $\begin{array}{r}-0,04 \\
{[-6,10]} \\
(0,00) \\
\end{array}$ & 0,27 & 137,05 & 0,01 \\
\hline _COB & $\begin{array}{l}96,28 \\
{[8,63]} \\
(0,00) \\
\end{array}$ & $\begin{array}{r}3,22 \\
{[1,26]} \\
(0,21)\end{array}$ & $\begin{array}{r}-0,10 \\
{[-5,59]} \\
(0,00)\end{array}$ & 0,24 & 115,09 & 0,02 \\
\hline _HYPV & $\begin{array}{l}79,94 \\
{[9,28]} \\
(0,00)\end{array}$ & $\begin{array}{r}0,15 \\
{[1,96]} \\
(0,05)\end{array}$ & $\begin{array}{r}-0,06 \\
{[-4,14]} \\
(0,00)\end{array}$ & 0,12 & 53,02 & 0,01 \\
\hline _RWE & $\begin{array}{r}81,94 \\
{[16,05]} \\
(0,00) \\
\end{array}$ & $\begin{array}{r}2,48 \\
{[1,31]} \\
(0,19) \\
\end{array}$ & $\begin{array}{r}-0,09 \\
{[-11,12]} \\
(0,00)\end{array}$ & 0,54 & 391,83 & 0,02 \\
\hline _BMW & $\begin{array}{r}37,49 \\
{[27,88]} \\
(0,00) \\
\end{array}$ & $\begin{array}{r}0,10 \\
{[1,00]} \\
(0,32) \\
\end{array}$ & $\begin{array}{r}-0,01 \\
{[-4,61]} \\
(0,00)\end{array}$ & 0,16 & 73,22 & 0,12 \\
\hline _LH & $\begin{array}{r}155,84 \\
{[24,20]} \\
(0,00) \\
\end{array}$ & $\begin{array}{r}-0,02 \\
{[-1,03]} \\
(0,30) \\
\end{array}$ & $\begin{array}{r}-0,12 \\
{[-11,19]} \\
(0,00) \\
\end{array}$ & 0,43 & 268,11 & 0,01 \\
\hline _TYSS & $\begin{array}{r}202,34 \\
{[17,14]} \\
(0,00) \\
\end{array}$ & $\begin{array}{r}2,89 \\
{[1,58]} \\
(0,12) \\
\end{array}$ & $\begin{array}{r}-0,14 \\
{[-7,23]} \\
(0,00)\end{array}$ & 0,28 & 129,05 & 0,03 \\
\hline _METRO & $\begin{array}{r}101,24 \\
{[14,03]} \\
(0,00) \\
\end{array}$ & $\begin{array}{r}-0,01 \\
{[-0,35]} \\
(0,73) \\
\end{array}$ & $\begin{array}{r}-0,07 \\
{[-5,53]} \\
(0,00) \\
\end{array}$ & 0,20 & 75,17 & 0,05 \\
\hline _ & $\begin{array}{r}320,07 \\
{[7,40]} \\
(0,00) \\
\end{array}$ & $\begin{array}{r}0,87 \\
{[0,13]} \\
(0,89) \\
\end{array}$ & $\begin{array}{r}-0,11 \\
{[-1,67]} \\
(0,10) \\
\end{array}$ & 0,05 & 8,01 & 0,05 \\
\hline _LIN & $\begin{array}{r}53,44 \\
{[16,51]} \\
(0,00) \\
\end{array}$ & $\begin{array}{r}0,61 \\
{[2,19]} \\
(0,03) \\
\end{array}$ & $\begin{array}{r}-0,03 \\
{[-4,54]} \\
(0,00)\end{array}$ & 0,16 & 46,78 & 0,05 \\
\hline _HEN & $\begin{array}{r}20,91 \\
{[26,53]} \\
(0,00) \\
\end{array}$ & $\begin{array}{r}0,08 \\
{[0,71]} \\
(0,48) \\
\end{array}$ & $\begin{array}{r}0,01 \\
{[5,18]} \\
(0,00)\end{array}$ & 0,19 & 44,98 & 0,04 \\
\hline _CONT & $\begin{array}{r}107,35 \\
{[13,87]} \\
(0,00)\end{array}$ & $\begin{array}{r}6,65 \\
{[3,22]} \\
(0,00) \\
\end{array}$ & $\begin{array}{r}-0,11 \\
{[-8,31]} \\
(0,00)\end{array}$ & 0,63 & 238,44 & 0,05 \\
\hline _DP & $\begin{array}{r}167,65 \\
{[8,20]} \\
(0,00)\end{array}$ & $\begin{array}{r}-1,26 \\
{[-1,11]} \\
(0,27)\end{array}$ & $\begin{array}{r}-0,20 \\
{[-7,09]} \\
(0,00)\end{array}$ & 0,79 & 108,83 & 0,07 \\
\hline
\end{tabular}

Table C.29.: Time-series regression results for the test of the relationship between default risk and the horizontal coordinate $X S M I N$ for a constant maturity of 60 days 


\begin{tabular}{|c|c|c|c|c|c|c|}
\hline \multicolumn{7}{|c|}{$C D S_{t}=\beta_{0}+\beta_{1} \cdot S L P 70_{t}+\beta_{2} \cdot t+u_{t}$} \\
\hline ID & $b_{0}$ & $b_{1}$ & $\mathbf{b}_{2}$ & R2 cor. & $\mathbf{F}$ & DW \\
\hline ALL & $\begin{array}{r}61,62 \\
{[10,42]} \\
(0,00)\end{array}$ & $\begin{array}{r}0,00 \\
{[1,13]} \\
(0,26)\end{array}$ & $\begin{array}{r}-0,05 \\
{[-5,08]} \\
(0,00)\end{array}$ & 0,20 & 93,02 & 0,01 \\
\hline _SIE & $\begin{array}{r}63,91 \\
{[21,03]} \\
(0,00)\end{array}$ & $\begin{array}{r}0,00 \\
{[-0,03]} \\
(0,97)\end{array}$ & $\begin{array}{r}-0,06 \\
{[-11,56]} \\
(0,00)\end{array}$ & 0,59 & 553,97 & 0,01 \\
\hline -DTE & $\begin{array}{r}263,62 \\
{[16,52]} \\
(0,00) \\
\end{array}$ & $\begin{array}{r}0,00 \\
{[-0,98]} \\
(0,33) \\
\end{array}$ & $\begin{array}{r}-0,33 \\
{[-11,53]} \\
(0,00)\end{array}$ & 0,62 & 613,46 & 0,01 \\
\hline _DAIM & $\begin{array}{r}159,26 \\
{[41,36]} \\
(0,00) \\
\end{array}$ & $\begin{array}{r}0,24 \\
{[0,50]} \\
(0,62)\end{array}$ & $\begin{array}{r}-0,11 \\
{[-17,16]} \\
(0,00)\end{array}$ & 0,71 & 932,27 & 0,06 \\
\hline _DBANK & $\begin{array}{r}35,97 \\
{[15,45]} \\
(0,00) \\
\end{array}$ & $\begin{array}{r}-0,02 \\
{[-3,69]} \\
(0,00)\end{array}$ & $\begin{array}{r}-0,03 \\
{[-6,87]} \\
(0,00) \\
\end{array}$ & 0,29 & 153,11 & 0,01 \\
\hline _MRE & $\begin{array}{r}57,93 \\
{[13,09]} \\
(0,00)\end{array}$ & $\begin{array}{r}0,01 \\
{[0,35]} \\
(0,72)\end{array}$ & $\begin{array}{r}-0,05 \\
{[-6,39]} \\
(0,00)\end{array}$ & 0,32 & 160,02 & 0,16 \\
\hline$-\mathrm{BAY}$ & $\begin{array}{r}75,29 \\
{[11,94]} \\
(0,00)\end{array}$ & $\begin{array}{r}0,15 \\
{[0,87]} \\
(0,38)\end{array}$ & $\begin{array}{r}-0,04 \\
{[-4,26]} \\
(0,00)\end{array}$ & 0,08 & 32,42 & 0,02 \\
\hline _VW & $\begin{array}{r}49,18 \\
{[19,37]} \\
(0,00) \\
\end{array}$ & $\begin{array}{r}-0,01 \\
{[-1,66]} \\
(0,10)\end{array}$ & $\begin{array}{r}0,03 \\
{[5,78]} \\
(0,00) \\
\end{array}$ & 0,20 & 97,92 & 0,03 \\
\hline _BASF & $\begin{array}{r}30,84 \\
{[39,17]} \\
(0,00) \\
\end{array}$ & $\begin{array}{r}0,00 \\
{[3,39]} \\
(0,00)\end{array}$ & $\begin{array}{r}-0,03 \\
{[-20,48]} \\
(0,00)\end{array}$ & 0,75 & $1.121,86$ & 0,04 \\
\hline EON & $\begin{array}{r}52,74 \\
{[14,10]} \\
(0,00) \\
\end{array}$ & $\begin{array}{r}0,00 \\
{[-1,18]} \\
(0,24)\end{array}$ & $\begin{array}{r}-0,04 \\
{[-6,14]} \\
(0,00)\end{array}$ & 0,27 & 137,80 & 0,01 \\
\hline CCOB & $\begin{array}{r}100,29 \\
{[9,11]} \\
(0,00) \\
\end{array}$ & $\begin{array}{r}0,00 \\
{[0,88]} \\
(0,38) \\
\end{array}$ & $\begin{array}{r}-0,10 \\
{[-5,62]} \\
(0,00) \\
\end{array}$ & 0,24 & 113,22 & 0,01 \\
\hline _HYPV & $\begin{array}{l}80,13 \\
{[9,29]} \\
(0,00)\end{array}$ & $\begin{array}{r}0,00 \\
{[-0,62]} \\
(0,54) \\
\end{array}$ & $\begin{array}{r}-0,06 \\
{[-4,14]} \\
(0,00) \\
\end{array}$ & 0,12 & 52,72 & 0,01 \\
\hline _RWE & $\begin{array}{r}84,66 \\
{[17,96]} \\
(0,00)\end{array}$ & $\begin{array}{r}0,00 \\
{[-0,48]} \\
(0,63)\end{array}$ & $\begin{array}{r}-0,09 \\
{[-11,10]} \\
(0,00)\end{array}$ & 0,54 & 389,91 & 0,01 \\
\hline _BMW & $\begin{array}{r}37,61 \\
{[27,87]} \\
(0,00) \\
\end{array}$ & $\begin{array}{r}0,00 \\
{[0,89]} \\
(0,37) \\
\end{array}$ & $\begin{array}{r}-0,01 \\
{[-4,61]} \\
(0,00) \\
\end{array}$ & 0,16 & 73,04 & 0,12 \\
\hline _LH & $\begin{array}{r}155,86 \\
{[24,20]} \\
(0,00) \\
\end{array}$ & $\begin{array}{r}0,00 \\
{[0,54]} \\
(0,59) \\
\end{array}$ & $\begin{array}{r}-0,12 \\
{[-11,20]} \\
(0,00)\end{array}$ & 0,43 & 267,95 & 0,01 \\
\hline _TYSS & $\begin{array}{r}205,34 \\
{[17,37]} \\
(0,00) \\
\end{array}$ & $\begin{array}{r}0,01 \\
{[3,06]} \\
(0,00)\end{array}$ & $\begin{array}{r}-0,14 \\
{[-7,21]} \\
(0,00)\end{array}$ & 0,28 & 129,06 & 0,03 \\
\hline _METRO & $\begin{array}{r}101,22 \\
{[14,16]} \\
(0,00) \\
\end{array}$ & $\begin{array}{r}-0,03 \\
{[-1,21]} \\
(0,23) \\
\end{array}$ & $\begin{array}{r}-0,07 \\
{[-5,59]} \\
(0,00) \\
\end{array}$ & 0,20 & 77,50 & 0,06 \\
\hline _TUI & $\begin{array}{r}322,33 \\
{[7,61]} \\
(0,00) \\
\end{array}$ & $\begin{array}{r}0,00 \\
{[-1,27]} \\
(0,20) \\
\end{array}$ & $\begin{array}{r}-0,12 \\
{[-1,70]} \\
(0,09) \\
\end{array}$ & 0,06 & 8,15 & 0,05 \\
\hline _LIN & $\begin{array}{r}53,99 \\
{[16,10]} \\
(0,00) \\
\end{array}$ & $\begin{array}{r}0,00 \\
{[-0,45]} \\
(0,65)\end{array}$ & $\begin{array}{r}-0,03 \\
{[-4,55]} \\
(0,00) \\
\end{array}$ & 0,16 & 46,14 & 0,05 \\
\hline _HEN & $\begin{array}{r}20,98 \\
{[26,25]} \\
(0,00)\end{array}$ & $\begin{array}{r}0,00 \\
{[2,49]} \\
(0,01)\end{array}$ & $\begin{array}{r}0,01 \\
{[5,18]} \\
(0,00) \\
\end{array}$ & 0,19 & 45,29 & 0,04 \\
\hline _CONT & $\begin{array}{r}113,51 \\
{[12,95]} \\
(0,00)\end{array}$ & $\begin{array}{r}0,00 \\
{[1,25]} \\
(0,21)\end{array}$ & $\begin{array}{r}-0,11 \\
{[-8,13]} \\
(0,00)\end{array}$ & 0,62 & 230,12 & 0,01 \\
\hline _DP & $\begin{array}{r}166,39 \\
{[7,86]} \\
(0,00)\end{array}$ & $\begin{array}{r}0,00 \\
{[-0,69]} \\
(0,49)\end{array}$ & $\begin{array}{r}-0,20 \\
{[-6,96]} \\
(0,00)\end{array}$ & 0,79 & 108,53 & 0,07 \\
\hline
\end{tabular}

Table C.30.: Time-series regression results for the test of the relationship between default risk and the slope variable $S L P 70$ for a constant maturity of 180 days 


\begin{tabular}{|c|c|c|c|c|c|c|}
\hline \multicolumn{7}{|c|}{$C D S_{t}=\beta_{0}+\beta_{1} \cdot S L P 90_{t}+\beta_{2} \cdot t+u_{t}$} \\
\hline ID & $b_{0}$ & $b_{1}$ & $b_{2}$ & R2 cor. & $\mathbf{F}$ & DW \\
\hline ALL & $\begin{array}{r}61,62 \\
{[10,42]} \\
(0,00) \\
\end{array}$ & $\begin{array}{r}0,01 \\
{[1,16]} \\
(0,25) \\
\end{array}$ & $\begin{array}{r}-0,05 \\
{[-5,08]} \\
(0,00) \\
\end{array}$ & 0,20 & 93,00 & 0,01 \\
\hline _SIE & $\begin{array}{r}63,89 \\
{[21,02]} \\
(0,00) \\
\end{array}$ & $\begin{array}{r}-0,11 \\
{[-0,62]} \\
(0,54) \\
\end{array}$ & $\begin{array}{r}-0,06 \\
{[-11,56]} \\
(0,00)\end{array}$ & 0,59 & 554,14 & 0,01 \\
\hline _DTE & $\begin{array}{r}263,63 \\
{[16,52]} \\
(0,00) \\
\end{array}$ & $\begin{array}{r}-0,01 \\
{[-1,12]} \\
(0,26) \\
\end{array}$ & $\begin{array}{r}-0,33 \\
{[-11,53]} \\
(0,00) \\
\end{array}$ & 0,62 & 613,47 & 0,01 \\
\hline _DAIM & $\begin{array}{r}159,11 \\
{[41,01]} \\
(0,00) \\
\end{array}$ & $\begin{array}{r}-0,19 \\
{[-0,15]} \\
(0,88) \\
\end{array}$ & $\begin{array}{r}-0,11 \\
{[-17,17]} \\
(0,00)\end{array}$ & 0,71 & 932,10 & 0,06 \\
\hline _DBANK & $\begin{array}{r}35,95 \\
{[15,45]} \\
(0,00) \\
\end{array}$ & $\begin{array}{r}-0,08 \\
{[-2,60]} \\
(0,01) \\
\end{array}$ & $\begin{array}{r}-0,03 \\
{[-6,87]} \\
(0,00) \\
\end{array}$ & 0,29 & 153,07 & 0,01 \\
\hline _MRE & $\begin{array}{r}57,89 \\
{[13,08]} \\
(0,00) \\
\end{array}$ & $\begin{array}{r}0,04 \\
{[1,28]} \\
(0,20) \\
\end{array}$ & $\begin{array}{r}-0,05 \\
{[-6,37]} \\
(0,00) \\
\end{array}$ & 0,32 & 160,43 & 0,16 \\
\hline _BAY & $\begin{array}{r}75,28 \\
{[11,95]} \\
(0,00) \\
\end{array}$ & $\begin{array}{r}0,10 \\
{[0,26]} \\
(0,80) \\
\end{array}$ & $\begin{array}{r}-0,04 \\
{[-4,26]} \\
(0,00) \\
\end{array}$ & 0,08 & 32,29 & 0,02 \\
\hline -VW & $\begin{array}{r}49,18 \\
{[19,40]} \\
(0,00) \\
\end{array}$ & $\begin{array}{r}-0,02 \\
{[-2,05]} \\
(0,04) \\
\end{array}$ & $\begin{array}{r}0,03 \\
{[5,79]} \\
(0,00) \\
\end{array}$ & 0,21 & 98,40 & 0,03 \\
\hline _BASF & $\begin{array}{r}30,85 \\
{[39,16]} \\
(0,00) \\
\end{array}$ & $\begin{array}{r}0,00 \\
{[3,02]} \\
(0,00) \\
\end{array}$ & $\begin{array}{r}-0,03 \\
{[-20,48]} \\
(0,00) \\
\end{array}$ & 0,75 & $1.120,92$ & 0,03 \\
\hline EON & $\begin{array}{r}52,69 \\
{[14,08]} \\
(0,00) \\
\end{array}$ & $\begin{array}{r}0,00 \\
{[-0,37]} \\
(0,71) \\
\end{array}$ & $\begin{array}{r}-0,04 \\
{[-6,12]} \\
(0,00) \\
\end{array}$ & 0,27 & 137,11 & 0,01 \\
\hline _COB & $\begin{array}{r}100,30 \\
{[9,11]} \\
(0,00) \\
\end{array}$ & $\begin{array}{r}0,00 \\
{[0,10]} \\
(0,92) \\
\end{array}$ & $\begin{array}{r}-0,10 \\
{[-5,62]} \\
(0,00) \\
\end{array}$ & 0,24 & 113,20 & 0,01 \\
\hline _HYPV & $\begin{array}{l}80,13 \\
{[9,29]} \\
(0,00) \\
\end{array}$ & $\begin{array}{r}0,00 \\
{[0,02]} \\
(0,99) \\
\end{array}$ & $\begin{array}{r}-0,06 \\
{[-4,15]} \\
(0,00)\end{array}$ & 0,12 & 52,71 & 0,01 \\
\hline _RWE & $\begin{array}{r}84,66 \\
{[17,96]} \\
(0,00) \\
\end{array}$ & $\begin{array}{r}0,00 \\
{[-0,48]} \\
(0,63) \\
\end{array}$ & $\begin{array}{r}-0,09 \\
{[-11,10]} \\
(0,00)\end{array}$ & 0,54 & 389,89 & 0,01 \\
\hline BMW & $\begin{array}{r}37,61 \\
{[27,86]} \\
(0,00) \\
\end{array}$ & $\begin{array}{r}0,00 \\
{[0,22]} \\
(0,82) \\
\end{array}$ & $\begin{array}{r}-0,01 \\
{[-4,61]} \\
(0,00)\end{array}$ & 0,16 & 72,90 & 0,12 \\
\hline _LH & $\begin{array}{r}155,85 \\
{[24,19]} \\
(0,00) \\
\end{array}$ & $\begin{array}{r}0,00 \\
{[1,37]} \\
(0,17) \\
\end{array}$ & $\begin{array}{r}-0,12 \\
{[-11,20]} \\
(0,00) \\
\end{array}$ & 0,43 & 267,99 & 0,01 \\
\hline -TYSS & $\begin{array}{r}205,37 \\
{[17,37]} \\
(0,00) \\
\end{array}$ & $\begin{array}{r}0,03 \\
{[2,03]} \\
(0,04) \\
\end{array}$ & $\begin{array}{r}-0,14 \\
{[-7,21]} \\
(0,00)\end{array}$ & 0,28 & 129,02 & 0,03 \\
\hline _METRO & $\begin{array}{r}101,15 \\
{[14,29]} \\
(0,00) \\
\end{array}$ & $\begin{array}{r}-0,12 \\
{[-2,60]} \\
(0,01) \\
\end{array}$ & $\begin{array}{r}-0,07 \\
{[-5,65]} \\
(0,00) \\
\end{array}$ & 0,21 & 82,55 & 0,08 \\
\hline _TUI & $\begin{array}{r}322,40 \\
{[7,64]} \\
(0,00) \\
\end{array}$ & $\begin{array}{r}-0,02 \\
{[-1,19]} \\
(0,24)\end{array}$ & $\begin{array}{r}-0,12 \\
{[-1,70]} \\
(0,09) \\
\end{array}$ & 0,06 & 8,22 & 0,05 \\
\hline _LIN & $\begin{array}{r}53,99 \\
{[16,08]} \\
(0,00) \\
\end{array}$ & $\begin{array}{r}0,00 \\
{[1,17]} \\
(0,24) \\
\end{array}$ & $\begin{array}{r}-0,03 \\
{[-4,54]} \\
(0,00) \\
\end{array}$ & 0,16 & 46,10 & 0,05 \\
\hline _ HEN & $\begin{array}{r}20,99 \\
{[26,26]} \\
(0,00) \\
\end{array}$ & $\begin{array}{r}0,00 \\
{[2,01]} \\
(0,05) \\
\end{array}$ & $\begin{array}{r}0,01 \\
{[5,19]} \\
(0,00)\end{array}$ & 0,19 & 45,49 & 0,04 \\
\hline _CONT & $\begin{array}{r}113,52 \\
{[12,96]} \\
(0,00) \\
\end{array}$ & $\begin{array}{r}0,00 \\
{[1,21]} \\
(0,23)\end{array}$ & $\begin{array}{r}-0,11 \\
{[-8,13]} \\
(0,00)\end{array}$ & 0,62 & 230,11 & 0,01 \\
\hline DPP & $\begin{array}{r}166,36 \\
{[7,86]} \\
(0,00)\end{array}$ & $\begin{array}{r}0,00 \\
{[-0,67]} \\
(0,50)\end{array}$ & $\begin{array}{r}-0,20 \\
{[-6,97]} \\
(0,00)\end{array}$ & 0,79 & 108,50 & 0,06 \\
\hline
\end{tabular}

Table C.31.: Time-series regression results for the test of the relationship between default risk and the slope variable $S L P 90$ for a constant maturity of 180 days 


\begin{tabular}{|c|c|c|c|c|c|c|}
\hline \multicolumn{7}{|c|}{$C D S_{t}=\beta_{0}+\beta_{1} \cdot S L P 100_{t}+\beta_{2} \cdot t+u_{t}$} \\
\hline ID & $b_{0}$ & $b_{1}$ & $b_{2}$ & R2 cor. & $\mathbf{F}$ & DW \\
\hline _ALL & $\begin{array}{r}61,63 \\
{[10,42]} \\
(0,00)\end{array}$ & $\begin{array}{r}0,00 \\
{[0,65]} \\
(0,51)\end{array}$ & $\begin{array}{r}-0,05 \\
{[-5,09]} \\
(0,00)\end{array}$ & 0,20 & 92,99 & 0,01 \\
\hline _SIE & $\begin{array}{r}63,54 \\
{[20,82]} \\
(0,00)\end{array}$ & $\begin{array}{r}-1,79 \\
{[-0,91]} \\
(0,36)\end{array}$ & $\begin{array}{r}-0,06 \\
{[-11,62]} \\
(0,00)\end{array}$ & 0,60 & 558,07 & 0,02 \\
\hline _DTE & $\begin{array}{r}263,63 \\
{[16,53]} \\
(0,00)\end{array}$ & $\begin{array}{r}-0,05 \\
{[-1,05]} \\
(0,30) \\
\end{array}$ & $\begin{array}{r}-0,33 \\
{[-11,53]} \\
(0,00)\end{array}$ & 0,62 & 613,55 & 0,01 \\
\hline DAIM & $\begin{array}{r}157,93 \\
{[38,47]} \\
(0,00)\end{array}$ & $\begin{array}{r}-5,53 \\
{[-0,94]} \\
(0,35) \\
\end{array}$ & $\begin{array}{r}-0,11 \\
{[-17,18]} \\
(0,00) \\
\end{array}$ & 0,71 & 935,44 & 0,07 \\
\hline _DBANK & $\begin{array}{r}36,01 \\
{[15,42]} \\
(0,00)\end{array}$ & $\begin{array}{r}0,12 \\
{[1,82]} \\
(0,07) \\
\end{array}$ & $\begin{array}{r}-0,03 \\
{[-6,87]} \\
(0,00) \\
\end{array}$ & 0,29 & 152,95 & 0,01 \\
\hline _MRE & $\begin{array}{r}57,85 \\
{[13,07]} \\
(0,00)\end{array}$ & $\begin{array}{r}0,06 \\
{[1,35]} \\
(0,18) \\
\end{array}$ & $\begin{array}{r}-0,05 \\
{[-6,37]} \\
(0,00) \\
\end{array}$ & 0,32 & 160,95 & 0,16 \\
\hline BAY & $\begin{array}{r}75,12 \\
{[11,95]} \\
(0,00)\end{array}$ & $\begin{array}{r}-0,91 \\
{[-1,22]} \\
(0,22) \\
\end{array}$ & $\begin{array}{r}-0,04 \\
{[-4,27]} \\
(0,00) \\
\end{array}$ & 0,08 & 32,56 & 0,02 \\
\hline _VW & $\begin{array}{r}49,15 \\
{[19,40]} \\
(0,00)\end{array}$ & $\begin{array}{r}-0,02 \\
{[-3,20]} \\
(0,00) \\
\end{array}$ & $\begin{array}{r}0,03 \\
{[5,81]} \\
(0,00) \\
\end{array}$ & 0,21 & 98,56 & 0,03 \\
\hline BASF & $\begin{array}{r}30,85 \\
{[39,14]} \\
(0,00)\end{array}$ & $\begin{array}{r}0,00 \\
{[0,75]} \\
(0,45) \\
\end{array}$ & $\begin{array}{r}-0,03 \\
{[-20,48]} \\
(0,00) \\
\end{array}$ & 0,75 & $1.119,54$ & 0,03 \\
\hline EEON & $\begin{array}{r}52,69 \\
{[14,06]} \\
(0,00)\end{array}$ & $\begin{array}{r}0,01 \\
{[0,71]} \\
(0,48)\end{array}$ & $\begin{array}{r}-0,04 \\
{[-6,10]} \\
(0,00)\end{array}$ & 0,27 & 137,44 & 0,01 \\
\hline -COB & $\begin{array}{r}100,28 \\
{[9,11]} \\
(0,00)\end{array}$ & $\begin{array}{r}-0,04 \\
{[-0,58]} \\
(0,56) \\
\end{array}$ & $\begin{array}{r}-0,10 \\
{[-5,63]} \\
(0,00) \\
\end{array}$ & 0,24 & 113,43 & 0,01 \\
\hline _HYPV & $\begin{array}{l}80,12 \\
{[9,29]} \\
(0,00)\end{array}$ & $\begin{array}{r}0,02 \\
{[0,81]} \\
(0,42) \\
\end{array}$ & $\begin{array}{r}-0,06 \\
{[-4,16]} \\
(0,00) \\
\end{array}$ & 0,12 & 53,09 & 0,01 \\
\hline _RWE & $\begin{array}{r}84,66 \\
{[17,96]} \\
(0,00)\end{array}$ & $\begin{array}{r}0,00 \\
{[-0,43]} \\
(0,67) \\
\end{array}$ & $\begin{array}{r}-0,09 \\
{[-11,10]} \\
(0,00) \\
\end{array}$ & 0,54 & 389,86 & 0,01 \\
\hline _BMW & $\begin{array}{r}37,62 \\
{[27,85]} \\
(0,00)\end{array}$ & $\begin{array}{r}0,00 \\
{[-2,63]} \\
(0,01) \\
\end{array}$ & $\begin{array}{r}-0,01 \\
{[-4,60]} \\
(0,00) \\
\end{array}$ & 0,16 & 73,23 & 0,12 \\
\hline LLH & $\begin{array}{r}155,83 \\
{[24,18]} \\
(0,00)\end{array}$ & $\begin{array}{r}0,00 \\
{[1,09]} \\
(0,28) \\
\end{array}$ & $\begin{array}{r}-0,12 \\
{[-11,21]} \\
(0,00)\end{array}$ & 0,43 & 268,11 & 0,01 \\
\hline -TYSS & $\begin{array}{r}205,40 \\
{[17,35]} \\
(0,00)\end{array}$ & $\begin{array}{r}0,02 \\
{[0,79]} \\
(0,43) \\
\end{array}$ & $\begin{array}{r}-0,14 \\
{[-7,22]} \\
(0,00) \\
\end{array}$ & 0,28 & 128,75 & 0,03 \\
\hline _METRO & $\begin{array}{r}100,96 \\
{[14,39]} \\
(0,00)\end{array}$ & $\begin{array}{r}-0,28 \\
{[-5,99]} \\
(0,00) \\
\end{array}$ & $\begin{array}{r}-0,07 \\
{[-5,62]} \\
(0,00) \\
\end{array}$ & 0,23 & 89,76 & 0,11 \\
\hline$-\mathrm{TUI}$ & $\begin{array}{r}321,60 \\
{[7,59]} \\
(0,00)\end{array}$ & $\begin{array}{r}0,01 \\
{[0,97]} \\
(0,33) \\
\end{array}$ & $\begin{array}{r}-0,11 \\
{[-1,68]} \\
(0,09) \\
\end{array}$ & 0,05 & 8,02 & 0,05 \\
\hline LIIN & $\begin{array}{r}53,99 \\
{[16,08]} \\
(0,00)\end{array}$ & $\begin{array}{r}0,00 \\
{[1,62]} \\
(0,11) \\
\end{array}$ & $\begin{array}{r}-0,03 \\
{[-4,54]} \\
(0,00) \\
\end{array}$ & 0,16 & 46,11 & 0,05 \\
\hline _HEN & $\begin{array}{r}20,98 \\
{[26,26]} \\
(0,00)\end{array}$ & $\begin{array}{r}0,00 \\
{[-0,56]} \\
(0,58)\end{array}$ & $\begin{array}{r}0,01 \\
{[5,18]} \\
(0,00)\end{array}$ & 0,19 & 44,99 & 0,04 \\
\hline _CONT & $\begin{array}{r}113,51 \\
{[12,95]} \\
(0,00)\end{array}$ & $\begin{array}{r}0,00 \\
{[-0,63]} \\
(0,53)\end{array}$ & $\begin{array}{r}-0,11 \\
{[-8,13]} \\
(0,00)\end{array}$ & 0,62 & 229,73 & 0,00 \\
\hline DP & $\begin{array}{r}166,13 \\
{[7,91]} \\
(0,00)\end{array}$ & $\begin{array}{r}0,00 \\
{[-0,26]} \\
(0,80)\end{array}$ & $\begin{array}{r}-0,20 \\
{[-7,01]} \\
(0,00)\end{array}$ & 0,79 & 108,35 & 0,06 \\
\hline
\end{tabular}

Table C.32.: Time-series regression results for the test of the relationship between default risk and the slope variable $S L P 100$ for a constant maturity of 180 days 


\begin{tabular}{|c|c|c|c|c|c|c|}
\hline \multicolumn{7}{|c|}{$C D S_{t}=\beta_{0}+\beta_{1} \cdot S L P 110_{t}+\beta_{2} \cdot t+u_{t}$} \\
\hline ID & $b_{0}$ & $b_{1}$ & $b_{2}$ & R2 cor. & $\mathbf{F}$ & DW \\
\hline ALL & $\begin{array}{r}61,59 \\
{[10,41]} \\
(0,00) \\
\end{array}$ & $\begin{array}{r}-0,07 \\
{[-1,22]} \\
(0,22) \\
\end{array}$ & $\begin{array}{r}-0,05 \\
{[-5,08]} \\
(0,00) \\
\end{array}$ & 0,20 & 93,10 & 0,01 \\
\hline _SIE & $\begin{array}{r}63,66 \\
{[20,99]} \\
(0,00) \\
\end{array}$ & $\begin{array}{r}-1,28 \\
{[-1,17]} \\
(0,24) \\
\end{array}$ & $\begin{array}{r}-0,06 \\
{[-11,55]} \\
(0,00) \\
\end{array}$ & 0,60 & 557,84 & 0,02 \\
\hline _DTE & $\begin{array}{r}263,60 \\
{[16,52]} \\
(0,00) \\
\end{array}$ & $\begin{array}{r}-0,01 \\
{[-0,30]} \\
(0,76) \\
\end{array}$ & $\begin{array}{r}-0,33 \\
{[-11,53]} \\
(0,00) \\
\end{array}$ & 0,62 & 613,45 & 0,01 \\
\hline _DAIM & $\begin{array}{r}157,94 \\
{[41,04]} \\
(0,00) \\
\end{array}$ & $\begin{array}{r}-7,63 \\
{[-1,42]} \\
(0,16) \\
\end{array}$ & $\begin{array}{r}-0,11 \\
{[-17,17]} \\
(0,00) \\
\end{array}$ & 0,71 & 940,44 & 0,07 \\
\hline _DBANK & $\begin{array}{r}35,99 \\
{[15,44]} \\
(0,00) \\
\end{array}$ & $\begin{array}{r}0,05 \\
{[4,23]} \\
(0,00) \\
\end{array}$ & $\begin{array}{r}-0,03 \\
{[-6,87]} \\
(0,00) \\
\end{array}$ & 0,29 & 153,09 & 0,01 \\
\hline _MRE & $\begin{array}{r}57,83 \\
{[13,07]} \\
(0,00) \\
\end{array}$ & $\begin{array}{r}0,07 \\
{[2,19]} \\
(0,03) \\
\end{array}$ & $\begin{array}{r}-0,05 \\
{[-6,37]} \\
(0,00) \\
\end{array}$ & 0,32 & 161,43 & 0,17 \\
\hline _BAY & $\begin{array}{r}75,09 \\
{[11,92]} \\
(0,00) \\
\end{array}$ & $\begin{array}{r}-1,07 \\
{[-3,97]} \\
(0,00) \\
\end{array}$ & $\begin{array}{r}-0,04 \\
{[-4,26]} \\
(0,00) \\
\end{array}$ & 0,08 & 33,09 & 0,02 \\
\hline _VW & $\begin{array}{r}49,12 \\
{[19,35]} \\
(0,00) \\
\end{array}$ & $\begin{array}{r}-0,02 \\
{[-3,94]} \\
(0,00) \\
\end{array}$ & $\begin{array}{r}0,03 \\
{[5,82]} \\
(0,00) \\
\end{array}$ & 0,21 & 98,30 & 0,03 \\
\hline _BASF & $\begin{array}{r}30,85 \\
{[39,13]} \\
(0,00) \\
\end{array}$ & $\begin{array}{r}0,00 \\
{[-0,59]} \\
(0,55) \\
\end{array}$ & $\begin{array}{r}-0,03 \\
{[-20,47]} \\
(0,00) \\
\end{array}$ & 0,75 & $1.119,37$ & 0,03 \\
\hline EON & $\begin{array}{r}52,85 \\
{[14,14]} \\
(0,00) \\
\end{array}$ & $\begin{array}{r}0,03 \\
{[2,63]} \\
(0,01) \\
\end{array}$ & $\begin{array}{r}-0,04 \\
{[-6,17]} \\
(0,00) \\
\end{array}$ & 0,27 & 139,72 & 0,02 \\
\hline _COB & $\begin{array}{r}100,24 \\
{[9,11]} \\
(0,00)\end{array}$ & $\begin{array}{r}-0,02 \\
{[-1,26]} \\
(0,21)\end{array}$ & $\begin{array}{r}-0,10 \\
{[-5,62]} \\
(0,00)\end{array}$ & 0,24 & 113,49 & 0,01 \\
\hline _HYPV & $\begin{array}{l}80,10 \\
{[9,29]} \\
(0,00) \\
\end{array}$ & $\begin{array}{r}0,01 \\
{[1,52]} \\
(0,13) \\
\end{array}$ & $\begin{array}{r}-0,06 \\
{[-4,14]} \\
(0,00) \\
\end{array}$ & 0,12 & 52,90 & 0,01 \\
\hline _RWE & $\begin{array}{r}84,66 \\
{[17,96]} \\
(0,00) \\
\end{array}$ & $\begin{array}{r}0,00 \\
{[0,40]} \\
(0,69) \\
\end{array}$ & $\begin{array}{r}-0,09 \\
{[-11,09]} \\
(0,00) \\
\end{array}$ & 0,54 & 389,87 & 0,01 \\
\hline _BMW & $\begin{array}{r}37,62 \\
{[27,88]} \\
(0,00) \\
\end{array}$ & $\begin{array}{r}0,00 \\
{[-3,00]} \\
(0,00) \\
\end{array}$ & $\begin{array}{r}-0,01 \\
{[-4,61]} \\
(0,00) \\
\end{array}$ & 0,16 & 73,74 & 0,13 \\
\hline _LH & $\begin{array}{r}155,87 \\
{[24,21]} \\
(0,00) \\
\end{array}$ & $\begin{array}{r}0,00 \\
{[0,30]} \\
(0,77) \\
\end{array}$ & $\begin{array}{r}-0,12 \\
{[-11,21]} \\
(0,00) \\
\end{array}$ & 0,43 & 267,97 & 0,01 \\
\hline _TYSS & $\begin{array}{r}205,29 \\
{[17,36]} \\
(0,00) \\
\end{array}$ & $\begin{array}{r}-0,03 \\
{[-4,06]} \\
(0,00) \\
\end{array}$ & $\begin{array}{r}-0,14 \\
{[-7,20]} \\
(0,00)\end{array}$ & 0,28 & 128,96 & 0,03 \\
\hline _METRO & $\begin{array}{r}101,15 \\
{[14,10]} \\
(0,00) \\
\end{array}$ & $\begin{array}{r}-0,07 \\
{[-3,00]} \\
(0,00) \\
\end{array}$ & $\begin{array}{r}-0,07 \\
{[-5,51]} \\
(0,00) \\
\end{array}$ & 0,20 & 77,57 & 0,06 \\
\hline _ TUI & $\begin{array}{r}322,02 \\
{[7,60]} \\
(0,00) \\
\end{array}$ & $\begin{array}{r}0,01 \\
{[1,53]} \\
(0,13) \\
\end{array}$ & $\begin{array}{r}-0,12 \\
{[-1,69]} \\
(0,09) \\
\end{array}$ & 0,06 & 8,08 & 0,05 \\
\hline _LIN & $\begin{array}{r}53,99 \\
{[16,08]} \\
(0,00) \\
\end{array}$ & $\begin{array}{r}0,00 \\
{[1,34]} \\
(0,18) \\
\end{array}$ & $\begin{array}{r}-0,03 \\
{[-4,54]} \\
(0,00) \\
\end{array}$ & 0,16 & 46,12 & 0,05 \\
\hline _ HEN & $\begin{array}{r}20,98 \\
{[26,25]} \\
(0,00) \\
\end{array}$ & $\begin{array}{r}0,00 \\
{[-3,39]} \\
(0,00)\end{array}$ & $\begin{array}{r}0,01 \\
{[5,18]} \\
(0,00)\end{array}$ & 0,19 & 45,14 & 0,04 \\
\hline _CONT & $\begin{array}{r}113,50 \\
{[12,95]} \\
(0,00)\end{array}$ & $\begin{array}{r}0,00 \\
{[-1,22]} \\
(0,22)\end{array}$ & $\begin{array}{r}-0,11 \\
{[-8,13]} \\
(0,00)\end{array}$ & 0,62 & 230,06 & 0,00 \\
\hline -DP & $\begin{array}{r}166,50 \\
{[7,84]} \\
(0,00)\end{array}$ & $\begin{array}{r}0,00 \\
{[0,74]} \\
(0,46)\end{array}$ & $\begin{array}{r}-0,20 \\
{[-6,94]} \\
(0,00)\end{array}$ & 0,79 & 108,61 & 0,07 \\
\hline
\end{tabular}

Table C.33.: Time-series regression results for the test of the relationship between default risk and the slope variable $S L P 110$ for a constant maturity of 180 days 


\begin{tabular}{|c|c|c|c|c|c|c|}
\hline \multicolumn{7}{|c|}{$C D S_{t}=\beta_{0}+\beta_{1} \cdot S L P 130_{t}+\beta_{2} \cdot t+u_{t}$} \\
\hline ID & $b_{0}$ & $b_{1}$ & $b_{2}$ & R2 cor. & $F$ & DW \\
\hline ALL & $\begin{array}{r}61,59 \\
{[10,40]} \\
(0,00)\end{array}$ & $\begin{array}{r}-0,01 \\
{[-1,24]} \\
(0,21)\end{array}$ & $\begin{array}{r}-0,05 \\
{[-5,06]} \\
(0,00)\end{array}$ & 0,20 & 93,07 & 0,01 \\
\hline _SIE & $\begin{array}{r}63,89 \\
{[21,03]} \\
(0,00)\end{array}$ & $\begin{array}{r}-0,10 \\
{[-1,58]} \\
(0,11)\end{array}$ & $\begin{array}{r}-0,06 \\
{[-11,55]} \\
(0,00)\end{array}$ & 0,59 & 554,40 & 0,02 \\
\hline _DTE & $\begin{array}{r}263,62 \\
{[16,52]} \\
(0,00)\end{array}$ & $\begin{array}{r}0,00 \\
{[0,41]} \\
(0,68)\end{array}$ & $\begin{array}{r}-0,33 \\
{[-11,53]} \\
(0,00)\end{array}$ & 0,62 & 613,44 & 0,01 \\
\hline _DAIM & $\begin{array}{r}159,13 \\
{[41,85]} \\
(0,00)\end{array}$ & $\begin{array}{r}-1,04 \\
{[-1,19]} \\
(0,24)\end{array}$ & $\begin{array}{r}-0,11 \\
{[-17,16]} \\
(0,00)\end{array}$ & 0,71 & 934,22 & 0,06 \\
\hline _DBANK & $\begin{array}{r}35,98 \\
{[15,44]} \\
(0,00)\end{array}$ & $\begin{array}{r}0,02 \\
{[4,17]} \\
(0,00)\end{array}$ & $\begin{array}{r}-0,03 \\
{[-6,87]} \\
(0,00)\end{array}$ & 0,29 & 153,11 & 0,01 \\
\hline _MRE & $\begin{array}{r}57,84 \\
{[13,08]} \\
(0,00)\end{array}$ & $\begin{array}{r}0,05 \\
{[5,42]} \\
(0,00)\end{array}$ & $\begin{array}{r}-0,05 \\
{[-6,38]} \\
(0,00)\end{array}$ & 0,32 & 161,43 & 0,17 \\
\hline BAY & $\begin{array}{r}75,22 \\
{[11,93]} \\
(0,00)\end{array}$ & $\begin{array}{r}-0,32 \\
{[-2,23]} \\
(0,03)\end{array}$ & $\begin{array}{r}-0,04 \\
{[-4,26]} \\
(0,00)\end{array}$ & 0,08 & 32,79 & 0,02 \\
\hline _VW & $\begin{array}{r}49,12 \\
{[19,31]} \\
(0,00)\end{array}$ & $\begin{array}{r}-0,01 \\
{[-1,61]} \\
(0,11)\end{array}$ & $\begin{array}{r}0,03 \\
{[5,81]} \\
(0,00)\end{array}$ & 0,20 & 97,64 & 0,02 \\
\hline _BASF & $\begin{array}{r}30,85 \\
{[39,19]} \\
(0,00)\end{array}$ & $\begin{array}{r}0,00 \\
{[-2,97]} \\
(0,00)\end{array}$ & $\begin{array}{r}-0,03 \\
{[-20,48]} \\
(0,00)\end{array}$ & 0,75 & $1.122,06$ & 0,04 \\
\hline EON & $\begin{array}{r}52,83 \\
{[14,15]} \\
(0,00) \\
\end{array}$ & $\begin{array}{r}0,01 \\
{[1,82]} \\
(0,07)\end{array}$ & $\begin{array}{r}-0,04 \\
{[-6,18]} \\
(0,00) \\
\end{array}$ & 0,27 & 139,27 & 0,02 \\
\hline _COB & $\begin{array}{r}100,28 \\
{[9,11]} \\
(0,00)\end{array}$ & $\begin{array}{r}0,00 \\
{[-2,14]} \\
(0,03)\end{array}$ & $\begin{array}{r}-0,10 \\
{[-5,62]} \\
(0,00)\end{array}$ & 0,24 & 113,29 & 0,01 \\
\hline _HYPV & $\begin{array}{l}80,12 \\
{[9,29]} \\
(0,00)\end{array}$ & $\begin{array}{r}0,00 \\
{[2,31]} \\
(0,02)\end{array}$ & $\begin{array}{r}-0,06 \\
{[-4,14]} \\
(0,00)\end{array}$ & 0,12 & 52,77 & 0,01 \\
\hline _RWE & $\begin{array}{r}84,66 \\
{[17,96]} \\
(0,00)\end{array}$ & $\begin{array}{r}0,00 \\
{[0,47]} \\
(0,64)\end{array}$ & $\begin{array}{r}-0,09 \\
{[-11,09]} \\
(0,00)\end{array}$ & 0,54 & 389,93 & 0,01 \\
\hline _BMW & $\begin{array}{r}37,61 \\
{[27,88]} \\
(0,00)\end{array}$ & $\begin{array}{r}0,00 \\
{[-3,73]} \\
(0,00)\end{array}$ & $\begin{array}{r}-0,01 \\
{[-4,61]} \\
(0,00)\end{array}$ & 0,16 & 73,39 & 0,12 \\
\hline _LH & $\begin{array}{r}155,87 \\
{[24,21]} \\
(0,00) \\
\end{array}$ & $\begin{array}{r}0,00 \\
{[0,02]} \\
(0,98)\end{array}$ & $\begin{array}{r}-0,12 \\
{[-11,20]} \\
(0,00)\end{array}$ & 0,43 & 267,93 & 0,01 \\
\hline _TYSS & $\begin{array}{r}205,31 \\
{[17,36]} \\
(0,00)\end{array}$ & $\begin{array}{r}-0,01 \\
{[-4,95]} \\
(0,00)\end{array}$ & $\begin{array}{r}-0,14 \\
{[-7,21]} \\
(0,00)\end{array}$ & 0,28 & 129,04 & 0,03 \\
\hline _METRO & $\begin{array}{r}101,22 \\
{[14,06]} \\
(0,00)\end{array}$ & $\begin{array}{r}0,00 \\
{[-0,22]} \\
(0,82)\end{array}$ & $\begin{array}{r}-0,07 \\
{[-5,53]} \\
(0,00)\end{array}$ & 0,20 & 75,18 & 0,05 \\
\hline _ TUI & $\begin{array}{r}322,14 \\
{[7,60]} \\
(0,00)\end{array}$ & $\begin{array}{r}0,00 \\
{[1,41]} \\
(0,16)\end{array}$ & $\begin{array}{r}-0,12 \\
{[-1,69]} \\
(0,09)\end{array}$ & 0,06 & 8,10 & 0,05 \\
\hline _LIN & $\begin{array}{r}53,99 \\
{[16,08]} \\
(0,00)\end{array}$ & $\begin{array}{r}0,00 \\
{[1,14]} \\
(0,26)\end{array}$ & $\begin{array}{r}-0,03 \\
{[-4,54]} \\
(0,00)\end{array}$ & 0,16 & 46,14 & 0,05 \\
\hline _HEN & $\begin{array}{r}20,98 \\
{[26,25]} \\
(0,00)\end{array}$ & $\begin{array}{r}0,00 \\
{[-3,28]} \\
(0,00)\end{array}$ & $\begin{array}{r}0,01 \\
{[5,18]} \\
(0,00)\end{array}$ & 0,19 & 45,20 & 0,04 \\
\hline _CONT & $\begin{array}{r}113,50 \\
{[12,95]} \\
(0,00)\end{array}$ & $\begin{array}{r}0,00 \\
{[-1,24]} \\
(0,22)\end{array}$ & $\begin{array}{r}-0,11 \\
{[-8,13]} \\
(0,00)\end{array}$ & 0,62 & 230,10 & 0,00 \\
\hline _DP & $\begin{array}{r}166,44 \\
{[7,85]} \\
(0,00)\end{array}$ & $\begin{array}{r}0,00 \\
{[0,72]} \\
(0,48)\end{array}$ & $\begin{array}{r}-0,20 \\
{[-6,95]} \\
(0,00)\end{array}$ & 0,79 & 108,56 & 0,07 \\
\hline
\end{tabular}

Table C.34.: Time-series regression results for the test of the relationship between default risk and the slope variable $S L P 130$ for a constant maturity of 180 days 


\begin{tabular}{|c|c|c|c|c|c|c|}
\hline \multicolumn{7}{|c|}{$\operatorname{CDS}_{t}=\beta_{0}+\beta_{1} \cdot X \operatorname{SMIN}_{t}+\beta_{2} \cdot t+u_{t}$} \\
\hline ID & $b_{0}$ & $b_{1}$ & $b_{2}$ & R2 cor. & $\mathbf{F}$ & DW \\
\hline ALL & $\begin{array}{r}61,30 \\
{[10,38]} \\
(0,00)\end{array}$ & $\begin{array}{r}0,16 \\
{[1,02]} \\
(0,31)\end{array}$ & $\begin{array}{r}-0,05 \\
{[-5,07]} \\
(0,00)\end{array}$ & 0,20 & 93,86 & 0,02 \\
\hline _SIE & $\begin{array}{r}58,89 \\
{[14,10]} \\
(0,00)\end{array}$ & $\begin{array}{r}3,10 \\
{[1,77]} \\
(0,08)\end{array}$ & $\begin{array}{r}-0,06 \\
{[-11,28]} \\
(0,00)\end{array}$ & 0,60 & 575,86 & 0,06 \\
\hline _DTE & $\begin{array}{r}265,46 \\
{[16,72]} \\
(0,00)\end{array}$ & $\begin{array}{r}-1,34 \\
{[-2,15]} \\
(0,03)\end{array}$ & $\begin{array}{r}-0,33 \\
{[-11,62]} \\
(0,00)\end{array}$ & 0,62 & 619,41 & 0,03 \\
\hline _DAIM & $\begin{array}{r}157,72 \\
{[40,86]} \\
(0,00)\end{array}$ & $\begin{array}{r}0,94 \\
{[2,15]} \\
(0,03)\end{array}$ & $\begin{array}{r}-0,11 \\
{[-17,11]} \\
(0,00)\end{array}$ & 0,71 & 941,89 & 0,08 \\
\hline DBANK & $\begin{array}{r}35,96 \\
{[15,52]} \\
(0,00)\end{array}$ & $\begin{array}{r}0,01 \\
{[0,22]} \\
(0,82)\end{array}$ & $\begin{array}{r}-0,03 \\
{[-6,88]} \\
(0,00)\end{array}$ & 0,29 & 152,96 & 0,01 \\
\hline _MRE & $\begin{array}{r}58,02 \\
{[13,15]} \\
(0,00)\end{array}$ & $\begin{array}{r}-0,08 \\
{[-0,58]} \\
(0,56)\end{array}$ & $\begin{array}{r}-0,05 \\
{[-6,39]} \\
(0,00)\end{array}$ & 0,32 & 160,14 & 0,16 \\
\hline BAY & $\begin{array}{r}74,95 \\
{[11,83]} \\
(0,00)\end{array}$ & $\begin{array}{r}0,22 \\
{[2,09]} \\
(0,04)\end{array}$ & $\begin{array}{r}-0,04 \\
{[-4,28]} \\
(0,00)\end{array}$ & 0,08 & 32,77 & 0,02 \\
\hline _VW & $\begin{array}{r}49,05 \\
{[19,33]} \\
(0,00)\end{array}$ & $\begin{array}{r}0,06 \\
{[1,62]} \\
(0,11)\end{array}$ & $\begin{array}{r}0,03 \\
{[5,82]} \\
(0,00)\end{array}$ & 0,20 & 97,71 & 0,02 \\
\hline _BASF & $\begin{array}{r}30,84 \\
{[39,18]} \\
(0,00)\end{array}$ & $\begin{array}{r}0,01 \\
{[2,34]} \\
(0,02)\end{array}$ & $\begin{array}{r}-0,03 \\
{[-20,47]} \\
(0,00)\end{array}$ & 0,75 & $1.119,93$ & 0,03 \\
\hline EON & $\begin{array}{r}52,51 \\
{[14,13]} \\
(0,00)\end{array}$ & $\begin{array}{r}0,20 \\
{[1,45]} \\
(0,15)\end{array}$ & $\begin{array}{r}-0,04 \\
{[-6,13]} \\
(0,00)\end{array}$ & 0,27 & 138,24 & 0,01 \\
\hline _COB & $\begin{array}{r}100,20 \\
{[9,11]} \\
(0,00)\end{array}$ & $\begin{array}{r}0,07 \\
{[1,35]} \\
(0,18)\end{array}$ & $\begin{array}{r}-0,10 \\
{[-5,62]} \\
(0,00)\end{array}$ & 0,24 & 113,27 & 0,01 \\
\hline _HYPV & $\begin{array}{l}79,81 \\
{[9,10]} \\
(0,00)\end{array}$ & $\begin{array}{r}0,32 \\
{[0,38]} \\
(0,70)\end{array}$ & $\begin{array}{r}-0,06 \\
{[-4,16]} \\
(0,00)\end{array}$ & 0,12 & 52,81 & 0,01 \\
\hline _RWE & $\begin{array}{r}84,25 \\
{[17,62]} \\
(0,00)\end{array}$ & $\begin{array}{r}0,29 \\
{[1,14]} \\
(0,26)\end{array}$ & $\begin{array}{r}-0,09 \\
{[-11,07]} \\
(0,00)\end{array}$ & 0,54 & 390,50 & 0,02 \\
\hline _BMW & $\begin{array}{r}37,62 \\
{[27,58]} \\
(0,00)\end{array}$ & $\begin{array}{r}-0,01 \\
{[-0,09]} \\
(0,93)\end{array}$ & $\begin{array}{r}-0,01 \\
{[-4,60]} \\
(0,00)\end{array}$ & 0,16 & 72,90 & 0,12 \\
\hline _LH & $\begin{array}{r}153,91 \\
{[24,06]} \\
(0,00)\end{array}$ & $\begin{array}{r}1,77 \\
{[2,92]} \\
(0,00) \\
\end{array}$ & $\begin{array}{r}-0,12 \\
{[-11,28]} \\
(0,00)\end{array}$ & 0,44 & 270,64 & 0,02 \\
\hline _TYSS & $\begin{array}{r}205,37 \\
{[17,36]} \\
(0,00)\end{array}$ & $\begin{array}{r}-0,03 \\
{[-0,72]} \\
(0,47)\end{array}$ & $\begin{array}{r}-0,14 \\
{[-7,21]} \\
(0,00)\end{array}$ & 0,28 & 128,75 & 0,03 \\
\hline _METRO & $\begin{array}{r}101,15 \\
{[14,00]} \\
(0,00)\end{array}$ & $\begin{array}{r}0,03 \\
{[1,80]} \\
(0,07)\end{array}$ & $\begin{array}{r}-0,07 \\
{[-5,52]} \\
(0,00)\end{array}$ & 0,20 & 75,24 & 0,05 \\
\hline _TUI & $\begin{array}{r}320,53 \\
{[7,64]} \\
(0,00)\end{array}$ & $\begin{array}{r}-1,69 \\
{[-0,64]} \\
(0,52)\end{array}$ & $\begin{array}{r}-0,11 \\
{[-1,63]} \\
(0,10)\end{array}$ & 0,06 & 8,37 & 0,05 \\
\hline _LIN & $\begin{array}{r}53,99 \\
{[16,03]} \\
(0,00)\end{array}$ & $\begin{array}{r}-0,01 \\
{[-0,06]} \\
(0,95)\end{array}$ & $\begin{array}{r}-0,03 \\
{[-4,54]} \\
(0,00)\end{array}$ & 0,16 & 46,09 & 0,05 \\
\hline _HEN & $\begin{array}{r}20,66 \\
{[24,74]} \\
(0,00)\end{array}$ & $\begin{array}{r}0,31 \\
{[1,14]} \\
(0,25)\end{array}$ & $\begin{array}{r}0,01 \\
{[5,20]} \\
(0,00)\end{array}$ & 0,19 & 45,24 & 0,04 \\
\hline _CONT & $\begin{array}{r}113,42 \\
{[13,01]} \\
(0,00)\end{array}$ & $\begin{array}{r}0,11 \\
{[0,07]} \\
(0,95)\end{array}$ & $\begin{array}{r}-0,11 \\
{[-8,13]} \\
(0,00)\end{array}$ & 0,62 & 229,70 & 0,00 \\
\hline _DP & $\begin{array}{r}162,84 \\
{[8,10]} \\
(0,00)\end{array}$ & $\begin{array}{r}2,65 \\
{[2,98]} \\
(0,00)\end{array}$ & $\begin{array}{r}-0,20 \\
{[-7,29]} \\
(0,00)\end{array}$ & 0,80 & 113,17 & 0,18 \\
\hline
\end{tabular}

Table C.35.: Time-series regression results for the test of the relationship between default risk and the horizontal coordinate $X S M I N$ for a constant maturity of 180 days 
C.4. The Credit Spread Level and the Moments of the Risk-Neutral Equity Return Density 


\begin{tabular}{|c|c|c|c|c|c|c|}
\hline \multicolumn{7}{|c|}{$C D S_{t}=\beta_{0}+\beta_{1} \cdot S K E W_{t}+\beta_{2} \cdot t+u_{t}$} \\
\hline ID & $b_{0}$ & $b_{1}$ & $b_{2}$ & R2 cor. & $\mathbf{F}$ & DW \\
\hline ALL & $\begin{array}{l}13,47 \\
{[1,56]} \\
(0,12) \\
\end{array}$ & $\begin{array}{r}-292,33 \\
{[-6,86]} \\
(0,00)\end{array}$ & $\begin{array}{r}-0,03 \\
{[-3,82]} \\
(0,00)\end{array}$ & 0,37 & 211,52 & 0,12 \\
\hline _SIE & $\begin{array}{l}24,39 \\
{[5,64]} \\
(0,00)\end{array}$ & $\begin{array}{r}-233,88 \\
{[-10,23]} \\
(0,00)\end{array}$ & $\begin{array}{r}-0,05 \\
{[-13,49]} \\
(0,00)\end{array}$ & 0,78 & $1.369,90$ & 0,31 \\
\hline _DTE & $\begin{array}{r}157,03 \\
{[8,02]} \\
(0,00)\end{array}$ & $\begin{array}{r}-722,56 \\
{[-7,83]} \\
(0,00)\end{array}$ & $\begin{array}{r}-0,28 \\
{[-11,26]} \\
(0,00)\end{array}$ & 0,72 & 987,01 & 0,29 \\
\hline _DAIM & $\begin{array}{r}147,05 \\
{[21,81]} \\
(0,00) \\
\end{array}$ & $\begin{array}{r}-69,69 \\
{[-2,17]} \\
(0,03)\end{array}$ & $\begin{array}{r}-0,11 \\
{[-16,51]} \\
(0,00)\end{array}$ & 0,72 & 985,40 & 0,08 \\
\hline _DBANK & $\begin{array}{l}13,83 \\
{[4,68]} \\
(0,00) \\
\end{array}$ & $\begin{array}{r}-105,46 \\
{[-7,41]} \\
(0,00)\end{array}$ & $\begin{array}{r}-0,01 \\
{[-4,61]} \\
(0,00)\end{array}$ & 0,46 & 330,57 & 0,13 \\
\hline _MRE & $\begin{array}{l}45,85 \\
{[7,89]} \\
(0,00) \\
\end{array}$ & $\begin{array}{r}-67,85 \\
{[-3,72]} \\
(0,00) \\
\end{array}$ & $\begin{array}{r}-0,04 \\
{[-5,42]} \\
(0,00) \\
\end{array}$ & 0,36 & 187,24 & 0,22 \\
\hline BAY & $\begin{array}{l}67,85 \\
{[9,14]} \\
(0,00) \\
\end{array}$ & $\begin{array}{r}-42,99 \\
{[-2,41]} \\
(0,02) \\
\end{array}$ & $\begin{array}{r}-0,04 \\
{[-3,75]} \\
(0,00)\end{array}$ & 0,09 & 36,26 & 0,03 \\
\hline _VW & $\begin{array}{r}44,93 \\
{[12,84]} \\
(0,00) \\
\end{array}$ & $\begin{array}{r}-22,87 \\
{[-1,63]} \\
(0,10) \\
\end{array}$ & $\begin{array}{r}0,03 \\
{[6,47]} \\
(0,00)\end{array}$ & 0,24 & 117,25 & 0,07 \\
\hline _BASF & $\begin{array}{r}28,14 \\
{[27,58]} \\
(0,00)\end{array}$ & $\begin{array}{r}-13,39 \\
{[-3,23]} \\
(0,00)\end{array}$ & $\begin{array}{r}-0,03 \\
{[-21,58]} \\
(0,00)\end{array}$ & 0,76 & $1.212,71$ & 0,12 \\
\hline EON & $\begin{array}{r}46,90 \\
{[11,52]} \\
(0,00) \\
\end{array}$ & $\begin{array}{r}-36,33 \\
{[-3,38]} \\
(0,00) \\
\end{array}$ & $\begin{array}{r}-0,04 \\
{[-6,17]} \\
(0,00)\end{array}$ & 0,30 & 159,54 & 0,08 \\
\hline _COB & $\begin{array}{l}84,33 \\
{[7,67]} \\
(0,00) \\
\end{array}$ & $\begin{array}{r}-95,35 \\
{[-3,15]} \\
(0,00) \\
\end{array}$ & $\begin{array}{r}-0,09 \\
{[-5,47]} \\
(0,00)\end{array}$ & 0,29 & 150,12 & 0,17 \\
\hline _HYPV & $\begin{array}{l}72,49 \\
{[8,31]} \\
(0,00)\end{array}$ & $\begin{array}{r}-44,78 \\
{[-3,36]} \\
(0,00) \\
\end{array}$ & $\begin{array}{r}-0,05 \\
{[-3,64]} \\
(0,00)\end{array}$ & 0,15 & 67,78 & 0,07 \\
\hline _RWE & $\begin{array}{r}84,49 \\
{[17,68]} \\
(0,00) \\
\end{array}$ & $\begin{array}{r}-1,06 \\
{[-0,28]} \\
(0,78)\end{array}$ & $\begin{array}{r}-0,09 \\
{[-11,06]} \\
(0,00)\end{array}$ & 0,54 & 389,90 & 0,01 \\
\hline _BMW & $\begin{array}{r}36,91 \\
{[25,41]} \\
(0,00) \\
\end{array}$ & $\begin{array}{r}-4,08 \\
{[-1,72]} \\
(0,09) \\
\end{array}$ & $\begin{array}{r}-0,01 \\
{[-4,50]} \\
(0,00)\end{array}$ & 0,17 & 76,28 & 0,14 \\
\hline _LH & $\begin{array}{r}154,34 \\
{[24,00]} \\
(0,00) \\
\end{array}$ & $\begin{array}{r}-9,69 \\
{[-3,12]} \\
(0,00)\end{array}$ & $\begin{array}{r}-0,12 \\
{[-11,19]} \\
(0,00)\end{array}$ & 0,44 & 272,03 & 0,03 \\
\hline _TYSS & $\begin{array}{r}207,58 \\
{[16,99]} \\
(0,00) \\
\end{array}$ & $\begin{array}{l}12,35 \\
{[1,61]} \\
(0,11)\end{array}$ & $\begin{array}{r}-0,14 \\
{[-7,25]} \\
(0,00)\end{array}$ & 0,29 & 131,82 & 0,05 \\
\hline _METRO & $\begin{array}{r}101,13 \\
{[14,04]} \\
(0,00) \\
\end{array}$ & $\begin{array}{r}-0,52 \\
{[-0,36]} \\
(0,72) \\
\end{array}$ & $\begin{array}{r}-0,07 \\
{[-5,53]} \\
(0,00) \\
\end{array}$ & 0,20 & 75,25 & 0,05 \\
\hline _ & $\begin{array}{r}320,72 \\
{[7,69]} \\
(0,00) \\
\end{array}$ & $\begin{array}{r}-5,43 \\
{[-1,85]} \\
(0,07) \\
\end{array}$ & $\begin{array}{r}-0,11 \\
{[-1,70]} \\
(0,09) \\
\end{array}$ & 0,06 & 8,82 & 0,06 \\
\hline _LIN & $\begin{array}{r}53,96 \\
{[16,09]} \\
(0,00)\end{array}$ & $\begin{array}{r}-0,16 \\
{[-0,25]} \\
(0,80) \\
\end{array}$ & $\begin{array}{r}-0,03 \\
{[-4,54]} \\
(0,00) \\
\end{array}$ & 0,16 & 46,12 & 0,05 \\
\hline _HEN & $\begin{array}{r}21,08 \\
{[26,11]} \\
(0,00) \\
\end{array}$ & $\begin{array}{r}0,37 \\
{[0,79]} \\
(0,43)\end{array}$ & $\begin{array}{r}0,01 \\
{[5,15]} \\
(0,00) \\
\end{array}$ & 0,19 & 45,33 & 0,04 \\
\hline _CONT & $\begin{array}{r}113,24 \\
{[12,89]} \\
(0,00)\end{array}$ & $\begin{array}{r}-0,80 \\
{[-0,87]} \\
(0,39)\end{array}$ & $\begin{array}{r}-0,11 \\
{[-8,09]} \\
(0,00)\end{array}$ & 0,62 & 230,80 & 0,01 \\
\hline
\end{tabular}

Table C.36.: Time-series regression results for the test of the relationship between default risk and risk-neutral skewness for a constant maturity of 36 days 


\begin{tabular}{|c|c|c|c|c|c|c|}
\hline \multicolumn{7}{|c|}{$C D S_{t}=\beta_{0}+\beta_{1} \cdot K_{U R T}+\beta_{2} \cdot t+u_{t}$} \\
\hline ID & $b_{0}$ & $b_{1}$ & $b_{2}$ & R2 cor. & $\mathbf{F}$ & DW \\
\hline ALL & $\begin{array}{r}4.451,47 \\
{[15,96]} \\
(0,00)\end{array}$ & $\begin{array}{r}-1.460,5 \\
{[-15,83]} \\
(0,00)\end{array}$ & $\begin{array}{r}-0,01 \\
{[-3,45]} \\
(0,00)\end{array}$ & 0,71 & 895,25 & 0,39 \\
\hline _SIE & $\begin{array}{r}3.052,63 \\
{[11,16]} \\
(0,00)\end{array}$ & $\begin{array}{r}-994,56 \\
{[-10,92]} \\
(0,00)\end{array}$ & $\begin{array}{r}-0,03 \\
{[-6,95]} \\
(0,00)\end{array}$ & 0,80 & $1.500,64$ & 0,40 \\
\hline _DTE & $\begin{array}{r}4.866,22 \\
{[2,38]} \\
(0,02)\end{array}$ & $\begin{array}{r}-1.531,8 \\
{[-2,25]} \\
(0,02)\end{array}$ & $\begin{array}{r}-0,27 \\
{[-6,81]} \\
(0,00)\end{array}$ & 0,69 & 838,50 & 0,33 \\
\hline _DAIM & $\begin{array}{r}2.426,08 \\
{[5,15]} \\
(0,00)\end{array}$ & $\begin{array}{r}-753,16 \\
{[-4,81]} \\
(0,00)\end{array}$ & $\begin{array}{r}-0,10 \\
{[-12,71]} \\
(0,00)\end{array}$ & 0,77 & $1.275,74$ & 0,27 \\
\hline _DBANK & $\begin{array}{r}2.025,82 \\
{[10,81]} \\
(0,00)\end{array}$ & $\begin{array}{r}-660,73 \\
{[-10,64]} \\
(0,00)\end{array}$ & $\begin{array}{r}-0,01 \\
{[-6,87]} \\
(0,00)\end{array}$ & 0,69 & 832,91 & 0,42 \\
\hline _MRE & $\begin{array}{r}1.682,00 \\
{[6,88]} \\
(0,00)\end{array}$ & $\begin{array}{r}-542,02 \\
{[-6,63]} \\
(0,00)\end{array}$ & $\begin{array}{r}-0,02 \\
{[-3,41]} \\
(0,00) \\
\end{array}$ & 0,52 & 368,50 & 0,53 \\
\hline BAY & $\begin{array}{r}1.764,92 \\
{[2,64]} \\
(0,01) \\
\end{array}$ & $\begin{array}{r}-559,21 \\
{[-2,53]} \\
(0,01) \\
\end{array}$ & $\begin{array}{r}-0,04 \\
{[-4,75]} \\
(0,00) \\
\end{array}$ & 0,19 & 88,56 & 0,23 \\
\hline _VW & $\begin{array}{l}96,17 \\
{[2,43]} \\
(0,02)\end{array}$ & $\begin{array}{r}-15,58 \\
{[-1,19]} \\
(0,24)\end{array}$ & $\begin{array}{r}0,03 \\
{[5,81]} \\
(0,00) \\
\end{array}$ & 0,21 & 100,51 & 0,03 \\
\hline _BASF & $\begin{array}{l}98,21 \\
{[2,86]} \\
(0,00)\end{array}$ & $\begin{array}{r}-22,24 \\
{[-1,97]} \\
(0,05)\end{array}$ & $\begin{array}{r}-0,03 \\
{[-21,13]} \\
(0,00)\end{array}$ & 0,75 & $1.148,11$ & 0,07 \\
\hline EON & $\begin{array}{r}140,16 \\
{[2,85]} \\
(0,00) \\
\end{array}$ & $\begin{array}{r}-28,97 \\
{[-1,78]} \\
(0,08)\end{array}$ & $\begin{array}{r}-0,04 \\
{[-6,12]} \\
(0,00)\end{array}$ & 0,27 & 143,17 & 0,03 \\
\hline _COB & $\begin{array}{r}493,11 \\
{[2,74]} \\
(0,01) \\
\end{array}$ & $\begin{array}{r}-130,50 \\
{[-2,19]} \\
(0,03)\end{array}$ & $\begin{array}{r}-0,10 \\
{[-5,73]} \\
(0,00) \\
\end{array}$ & 0,26 & 128,76 & 0,07 \\
\hline _HYPV & $\begin{array}{r}382,48 \\
{[3,63]} \\
(0,00) \\
\end{array}$ & $\begin{array}{r}-100,79 \\
{[-2,88]} \\
(0,00) \\
\end{array}$ & $\begin{array}{r}-0,06 \\
{[-4,13]} \\
(0,00) \\
\end{array}$ & 0,16 & 71,24 & 0,09 \\
\hline _RWE & $\begin{array}{r}125,02 \\
{[7,67]} \\
(0,00) \\
\end{array}$ & $\begin{array}{r}-13,37 \\
{[-2,58]} \\
(0,01)\end{array}$ & $\begin{array}{r}-0,09 \\
{[-11,20]} \\
(0,00) \\
\end{array}$ & 0,54 & 396,20 & 0,03 \\
\hline _BMW & $\begin{array}{l}53,83 \\
{[4,75]} \\
(0,00) \\
\end{array}$ & $\begin{array}{r}-5,39 \\
{[-1,45]} \\
(0,15) \\
\end{array}$ & $\begin{array}{r}-0,01 \\
{[-4,60]} \\
(0,00) \\
\end{array}$ & 0,17 & 77,12 & 0,14 \\
\hline _LH & $\begin{array}{r}152,05 \\
{[13,99]} \\
(0,00) \\
\end{array}$ & $\begin{array}{r}1,27 \\
{[0,45]} \\
(0,65)\end{array}$ & $\begin{array}{r}-0,12 \\
{[-11,22]} \\
(0,00)\end{array}$ & 0,43 & 268,02 & 0,01 \\
\hline _TYSS & $\begin{array}{r}220,13 \\
{[8,62]} \\
(0,00) \\
\end{array}$ & $\begin{array}{r}-4,93 \\
{[-0,66]} \\
(0,51)\end{array}$ & $\begin{array}{r}-0,14 \\
{[-7,22]} \\
(0,00)\end{array}$ & 0,28 & 128,87 & 0,03 \\
\hline _METRO & $\begin{array}{r}103,25 \\
{[11,46]} \\
(0,00) \\
\end{array}$ & $\begin{array}{r}-0,67 \\
{[-0,47]} \\
(0,64) \\
\end{array}$ & $\begin{array}{r}-0,07 \\
{[-5,53]} \\
(0,00) \\
\end{array}$ & 0,20 & 75,24 & 0,05 \\
\hline _TUI & $\begin{array}{r}339,18 \\
{[6,74]} \\
(0,00) \\
\end{array}$ & $\begin{array}{r}-5,66 \\
{[-0,64]} \\
(0,52) \\
\end{array}$ & $\begin{array}{r}-0,12 \\
{[-1,70]} \\
(0,09) \\
\end{array}$ & 0,06 & 8,20 & 0,05 \\
\hline _LIN & $\begin{array}{r}55,38 \\
{[14,51]} \\
(0,00) \\
\end{array}$ & $\begin{array}{r}-0,47 \\
{[-0,87]} \\
(0,39)\end{array}$ & $\begin{array}{r}-0,03 \\
{[-4,53]} \\
(0,00) \\
\end{array}$ & 0,16 & 46,31 & 0,05 \\
\hline _HEN & $\begin{array}{l}18,35 \\
{[9,32]} \\
(0,00)\end{array}$ & $\begin{array}{r}0,90 \\
{[1,43]} \\
(0,15)\end{array}$ & $\begin{array}{r}0,01 \\
{[5,16]} \\
(0,00) \\
\end{array}$ & 0,19 & 45,87 & 0,05 \\
\hline _CONT & $\begin{array}{r}115,19 \\
{[12,85]} \\
(0,00)\end{array}$ & $\begin{array}{r}-0,61 \\
{[-1,36]} \\
(0,18)\end{array}$ & $\begin{array}{r}-0,11 \\
{[-8,14]} \\
(0,00)\end{array}$ & 0,62 & 230,79 & 0,00 \\
\hline
\end{tabular}

Table C.37.: Time-series regression results for the test of the relationship between default risk and risk-neutral kurtosis for a constant maturity of 36 days 


\begin{tabular}{|c|c|c|c|c|c|c|}
\hline \multicolumn{7}{|c|}{$C D S_{t}=\beta_{0}+\beta_{1} \cdot S K E W_{t}+\beta_{2} \cdot t+u_{t}$} \\
\hline ID & $b_{0}$ & $b_{1}$ & $b_{2}$ & R2 cor. & $\mathbf{F}$ & DW \\
\hline ALL & $\begin{array}{l}16,89 \\
{[1,79]} \\
(0,07) \\
\end{array}$ & $\begin{array}{r}-251,12 \\
{[-6,00]} \\
(0,00) \\
\end{array}$ & $\begin{array}{r}-0,04 \\
{[-4,51]} \\
(0,00) \\
\end{array}$ & 0,33 & 176,49 & 0,13 \\
\hline _SIE & $\begin{array}{l}20,11 \\
{[4,36]} \\
(0,00) \\
\end{array}$ & $\begin{array}{r}-240,20 \\
{[-10,41]} \\
(0,00)\end{array}$ & $\begin{array}{r}-0,06 \\
{[-15,34]} \\
(0,00)\end{array}$ & 0,80 & $1.525,27$ & 0,24 \\
\hline _DTE & $\begin{array}{r}211,67 \\
{[9,56]} \\
(0,00) \\
\end{array}$ & $\begin{array}{r}-319,73 \\
{[-3,40]} \\
(0,00) \\
\end{array}$ & $\begin{array}{r}-0,32 \\
{[-11,62]} \\
(0,00) \\
\end{array}$ & 0,66 & 749,20 & 0,19 \\
\hline _DAIM & $\begin{array}{r}143,81 \\
{[21,51]} \\
(0,00) \\
\end{array}$ & $\begin{array}{r}-81,02 \\
{[-2,89]} \\
(0,00) \\
\end{array}$ & $\begin{array}{r}-0,11 \\
{[-17,37]} \\
(0,00) \\
\end{array}$ & 0,73 & $1.002,55$ & 0,09 \\
\hline _DBANK & $\begin{array}{l}17,08 \\
{[4,68]} \\
(0,00) \\
\end{array}$ & $\begin{array}{r}-83,92 \\
{[-5,56]} \\
(0,00) \\
\end{array}$ & $\begin{array}{r}-0,02 \\
{[-5,78]} \\
(0,00) \\
\end{array}$ & 0,43 & 287,87 & 0,20 \\
\hline _MRE & $\begin{array}{l}53,41 \\
{[9,86]} \\
(0,00) \\
\end{array}$ & $\begin{array}{r}-24,94 \\
{[-1,63]} \\
(0,10) \\
\end{array}$ & $\begin{array}{r}-0,05 \\
{[-6,23]} \\
(0,00) \\
\end{array}$ & 0,33 & 166,22 & 0,18 \\
\hline _BAY & $\begin{array}{l}73,99 \\
{[9,55]} \\
(0,00) \\
\end{array}$ & $\begin{array}{r}-6,49 \\
{[-0,62]} \\
(0,53) \\
\end{array}$ & $\begin{array}{r}-0,04 \\
{[-3,99]} \\
(0,00) \\
\end{array}$ & 0,08 & 32,48 & 0,02 \\
\hline -VW & $\begin{array}{r}41,74 \\
{[12,54]} \\
(0,00) \\
\end{array}$ & $\begin{array}{r}-37,22 \\
{[-3,32]} \\
(0,00) \\
\end{array}$ & $\begin{array}{r}0,03 \\
{[6,85]} \\
(0,00) \\
\end{array}$ & 0,25 & 129,75 & 0,12 \\
\hline _BASF & $\begin{array}{r}29,87 \\
{[37,23]} \\
(0,00) \\
\end{array}$ & $\begin{array}{r}-4,52 \\
{[-2,63]} \\
(0,01) \\
\end{array}$ & $\begin{array}{r}-0,03 \\
{[-21,05]} \\
(0,00) \\
\end{array}$ & 0,75 & $1.146,59$ & 0,07 \\
\hline EON & $\begin{array}{r}50,72 \\
{[13,30]} \\
(0,00) \\
\end{array}$ & $\begin{array}{r}-12,78 \\
{[-1,74]} \\
(0,08) \\
\end{array}$ & $\begin{array}{r}-0,04 \\
{[-6,29]} \\
(0,00) \\
\end{array}$ & 0,28 & 145,07 & 0,04 \\
\hline _COB & $\begin{array}{l}92,06 \\
{[8,14]} \\
(0,00)\end{array}$ & $\begin{array}{r}-46,83 \\
{[-2,41]} \\
(0,02) \\
\end{array}$ & $\begin{array}{r}-0,10 \\
{[-5,63]} \\
(0,00) \\
\end{array}$ & 0,26 & 125,69 & 0,06 \\
\hline _HYPV & $\begin{array}{l}74,81 \\
{[8,70]} \\
(0,00) \\
\end{array}$ & $\begin{array}{r}-29,09 \\
{[-2,19]} \\
(0,03) \\
\end{array}$ & $\begin{array}{r}-0,06 \\
{[-3,97]} \\
(0,00)\end{array}$ & 0,14 & 60,81 & 0,05 \\
\hline _RWE & $\begin{array}{r}83,32 \\
{[17,35]} \\
(0,00) \\
\end{array}$ & $\begin{array}{r}-7,05 \\
{[-1,78]} \\
(0,08)\end{array}$ & $\begin{array}{r}-0,09 \\
{[-11,01]} \\
(0,00)\end{array}$ & 0,54 & 394,20 & 0,02 \\
\hline BMW & $\begin{array}{r}36,89 \\
{[25,44]} \\
(0,00) \\
\end{array}$ & $\begin{array}{r}-3,80 \\
{[-1,94]} \\
(0,05) \\
\end{array}$ & $\begin{array}{r}-0,01 \\
{[-4,52]} \\
(0,00)\end{array}$ & 0,17 & 75,65 & 0,13 \\
\hline _LH & $\begin{array}{r}154,27 \\
{[24,02]} \\
(0,00) \\
\end{array}$ & $\begin{array}{r}-10,03 \\
{[-3,05]} \\
(0,00) \\
\end{array}$ & $\begin{array}{r}-0,12 \\
{[-11,25]} \\
(0,00) \\
\end{array}$ & 0,44 & 273,10 & 0,03 \\
\hline -TYSS & $\begin{array}{r}206,40 \\
{[17,02]} \\
(0,00) \\
\end{array}$ & $\begin{array}{r}5,02 \\
{[0,78]} \\
(0,44) \\
\end{array}$ & $\begin{array}{r}-0,14 \\
{[-7,20]} \\
(0,00) \\
\end{array}$ & 0,28 & 129,19 & 0,03 \\
\hline _METRO & $\begin{array}{r}101,17 \\
{[14,04]} \\
(0,00) \\
\end{array}$ & $\begin{array}{r}-0,25 \\
{[-0,21]} \\
(0,83) \\
\end{array}$ & $\begin{array}{r}-0,07 \\
{[-5,53]} \\
(0,00) \\
\end{array}$ & 0,20 & 75,19 & 0,05 \\
\hline _TUI & $\begin{array}{r}320,71 \\
{[7,64]} \\
(0,00) \\
\end{array}$ & $\begin{array}{r}-3,11 \\
{[-1,05]} \\
(0,29) \\
\end{array}$ & $\begin{array}{r}-0,11 \\
{[-1,68]} \\
(0,09) \\
\end{array}$ & 0,06 & 8,42 & 0,05 \\
\hline _LIN & $\begin{array}{r}53,97 \\
{[16,08]} \\
(0,00) \\
\end{array}$ & $\begin{array}{r}-0,08 \\
{[-0,12]} \\
(0,90) \\
\end{array}$ & $\begin{array}{r}-0,03 \\
{[-4,54]} \\
(0,00) \\
\end{array}$ & 0,16 & 46,10 & 0,05 \\
\hline _HEN & $\begin{array}{r}21,11 \\
{[26,14]} \\
(0,00) \\
\end{array}$ & $\begin{array}{r}0,49 \\
{[1,00]} \\
(0,32) \\
\end{array}$ & $\begin{array}{r}0,01 \\
{[5,14]} \\
(0,00)\end{array}$ & 0,19 & 45,70 & 0,04 \\
\hline _CONT & $\begin{array}{r}113,25 \\
{[12,91]} \\
(0,00)\end{array}$ & $\begin{array}{r}-0,87 \\
{[-0,93]} \\
(0,35)\end{array}$ & $\begin{array}{r}-0,11 \\
{[-8,11]} \\
(0,00)\end{array}$ & 0,63 & 231,01 & 0,01 \\
\hline
\end{tabular}

Table C.38.: Time-series regression results for the test of the relationship between default risk and risk-neutral skewness for a constant maturity of 60 days 


\begin{tabular}{|c|c|c|c|c|c|c|}
\hline \multicolumn{7}{|c|}{$C D S_{t}=\beta_{0}+\beta_{1} \cdot K U R T_{t}+\beta_{2} \cdot t+u_{t}$} \\
\hline ID & $b_{0}$ & $b_{1}$ & $b_{2}$ & R2 cor. & $F$ & DW \\
\hline ALL & $\begin{array}{r}3.374,94 \\
{[17,81]} \\
(0,00)\end{array}$ & $\begin{array}{r}-1.105,0 \\
{[-17,61]} \\
(0,00)\end{array}$ & $\begin{array}{r}-0,01 \\
{[-3,09]} \\
(0,00)\end{array}$ & 0,74 & $1.040,04$ & 0,50 \\
\hline SSIE & $\begin{array}{r}2.293,63 \\
{[11,87]} \\
(0,00)\end{array}$ & $\begin{array}{r}-743,74 \\
{[-11,54]} \\
(0,00)\end{array}$ & $\begin{array}{r}-0,03 \\
{[-6,55]} \\
(0,00)\end{array}$ & 0,81 & $1.658,23$ & 0,37 \\
\hline DTE & $\begin{array}{r}840,64 \\
{[3,27]} \\
(0,00)\end{array}$ & $\begin{array}{r}-192,5 \\
{[-2,25]} \\
(0,02)\end{array}$ & $\begin{array}{r}-0,32 \\
{[-11,48]} \\
(0,00)\end{array}$ & 0,63 & 646,46 & 0,08 \\
\hline DAIM & $\begin{array}{r}1.249,19 \\
{[4,09]} \\
(0,00)\end{array}$ & $\begin{array}{r}-362,78 \\
{[-3,57]} \\
(0,00)\end{array}$ & $\begin{array}{r}-0,10 \\
{[-14,12]} \\
(0,00)\end{array}$ & 0,75 & $1.137,34$ & 0,23 \\
\hline DBANK & $\begin{array}{r}271,72 \\
{[1,40]} \\
(0,16)\end{array}$ & $\begin{array}{r}-78,46 \\
{[-1,21]} \\
(0,22)\end{array}$ & $\begin{array}{r}-0,02 \\
{[-6,12]} \\
(0,00)\end{array}$ & 0,35 & 207,62 & 0,19 \\
\hline -MRE & $\begin{array}{r}146,98 \\
{[1,36]} \\
(0,18) \\
\end{array}$ & $\begin{array}{r}-29,77 \\
{[-0,81]} \\
(0,42)\end{array}$ & $\begin{array}{r}-0,05 \\
{[-5,83]} \\
(0,00) \\
\end{array}$ & 0,33 & 169,00 & 0,20 \\
\hline BAY & $\begin{array}{r}333,69 \\
{[1,50]} \\
(0,13) \\
\end{array}$ & $\begin{array}{r}-85,61 \\
{[-1,16]} \\
(0,24)\end{array}$ & $\begin{array}{r}-0,04 \\
{[-4,37]} \\
(0,00) \\
\end{array}$ & 0,10 & 41,17 & 0,06 \\
\hline -VW & $\begin{array}{r}149,32 \\
{[3,51]} \\
(0,00)\end{array}$ & $\begin{array}{r}-33,24 \\
{[-2,35]} \\
(0,02)\end{array}$ & $\begin{array}{r}0,03 \\
{[5,92]} \\
(0,00)\end{array}$ & 0,22 & 107,89 & 0,06 \\
\hline BASF & $\begin{array}{l}43,89 \\
{[4,51]} \\
(0,00)\end{array}$ & $\begin{array}{r}-4,29 \\
{[-1,34]} \\
(0,18)\end{array}$ & $\begin{array}{r}-0,03 \\
{[-20,75]} \\
(0,00)\end{array}$ & 0,75 & $1.128,75$ & 0,05 \\
\hline EON & $\begin{array}{l}83,45 \\
{[2,79]} \\
(0,01) \\
\end{array}$ & $\begin{array}{r}-10,19 \\
{[-1,03]} \\
(0,30)\end{array}$ & $\begin{array}{r}-0,04 \\
{[-6,13]} \\
(0,00) \\
\end{array}$ & 0,27 & 140,19 & 0,02 \\
\hline - $\mathrm{COB}$ & $\begin{array}{r}397,32 \\
{[3,11]} \\
(0,00)\end{array}$ & $\begin{array}{r}-99,25 \\
{[-2,36]} \\
(0,02)\end{array}$ & $\begin{array}{r}-0,10 \\
{[-5,74]} \\
(0,00)\end{array}$ & 0,27 & 132,29 & 0,09 \\
\hline _HYPV & $\begin{array}{r}229,90 \\
{[5,39]} \\
(0,00)\end{array}$ & $\begin{array}{r}-50,00 \\
{[-3,70]} \\
(0,00)\end{array}$ & $\begin{array}{r}-0,06 \\
{[-4,23]} \\
(0,00)\end{array}$ & 0,15 & 68,28 & 0,08 \\
\hline -RWE & $\begin{array}{r}143,30 \\
{[5,84]} \\
(0,00)\end{array}$ & $\begin{array}{r}-19,46 \\
{[-2,47]} \\
(0,01)\end{array}$ & $\begin{array}{r}-0,09 \\
{[-11,29]} \\
(0,00)\end{array}$ & 0,55 & 402,59 & 0,05 \\
\hline BMWW & $\begin{array}{l}54,03 \\
{[5,90]} \\
(0,00)\end{array}$ & $\begin{array}{r}-5,47 \\
{[-1,80]} \\
(0,07)\end{array}$ & $\begin{array}{r}-0,01 \\
{[-4,58]} \\
(0,00)\end{array}$ & 0,17 & 76,99 & 0,14 \\
\hline - $\mathrm{LH}$ & $\begin{array}{r}157,07 \\
{[19,35]} \\
(0,00)\end{array}$ & $\begin{array}{r}-0,40 \\
{[-0,28]} \\
(0,78)\end{array}$ & $\begin{array}{r}-0,12 \\
{[-11,21]} \\
(0,00) \\
\end{array}$ & 0,43 & 267,95 & 0,01 \\
\hline -TYSS & $\begin{array}{r}226,15 \\
{[8,75]} \\
(0,00)\end{array}$ & $\begin{array}{r}-6,91 \\
{[-0,96]} \\
(0,34)\end{array}$ & $\begin{array}{r}-0,14 \\
{[-7,23]} \\
(0,00)\end{array}$ & 0,28 & 129,41 & 0,03 \\
\hline _METRO & $\begin{array}{r}102,22 \\
{[11,64]} \\
(0,00)\end{array}$ & $\begin{array}{r}-0,33 \\
{[-0,25]} \\
(0,81)\end{array}$ & $\begin{array}{r}-0,07 \\
{[-5,54]} \\
(0,00)\end{array}$ & 0,20 & 75,19 & 0,05 \\
\hline$-\mathrm{TUI}$ & $\begin{array}{r}344,06 \\
{[7,32]} \\
(0,00) \\
\end{array}$ & $\begin{array}{r}-7,04 \\
{[-0,92]} \\
(0,36) \\
\end{array}$ & $\begin{array}{r}-0,12 \\
{[-1,73]} \\
(0,09) \\
\end{array}$ & 0,06 & 8,37 & 0,05 \\
\hline -LIN & $\begin{array}{r}55,78 \\
{[14,61]} \\
(0,00)\end{array}$ & $\begin{array}{r}-0,60 \\
{[-1,13]} \\
(0,26)\end{array}$ & $\begin{array}{r}-0,03 \\
{[-4,53]} \\
(0,00)\end{array}$ & 0,16 & 46,46 & 0,05 \\
\hline -HEN & $\begin{array}{r}18,82 \\
{[17,65]} \\
(0,00)\end{array}$ & $\begin{array}{r}0,74 \\
{[2,83]} \\
(0,00)\end{array}$ & $\begin{array}{r}0,01 \\
{[5,12]} \\
(0,00)\end{array}$ & 0,19 & 46,66 & 0,05 \\
\hline CONT & $\begin{array}{r}115,33 \\
{[12,82]} \\
(0,00)\end{array}$ & $\begin{array}{r}-0,65 \\
{[-1,40]} \\
(0,16)\end{array}$ & $\begin{array}{r}-0,11 \\
{[-8,15]} \\
(0,00)\end{array}$ & 0,62 & 230,97 & 0,00 \\
\hline
\end{tabular}

Table C.39.: Time-series regression results for the test of the relationship between default risk and risk-neutral kurtosis for a constant maturity of 60 days 


\begin{tabular}{|c|c|c|c|c|c|c|}
\hline \multicolumn{7}{|c|}{$C D S_{t}=\beta_{0}+\beta_{1} \cdot S K E W_{t}+\beta_{2} \cdot t+u_{t}$} \\
\hline ID & $b_{0}$ & $b_{1}$ & $b_{2}$ & R2 cor. & $\mathbf{F}$ & DW \\
\hline ALL & $\begin{array}{l}52,87 \\
{[6,86]} \\
(0,00)\end{array}$ & $\begin{array}{r}-45,66 \\
{[-1,76]} \\
(0,08)\end{array}$ & $\begin{array}{r}-0,05 \\
{[-5,13]} \\
(0,00)\end{array}$ & 0,21 & 96,96 & 0,03 \\
\hline _SIE & $\begin{array}{l}49,47 \\
{[6,65]} \\
(0,00) \\
\end{array}$ & $\begin{array}{r}-75,04 \\
{[-2,07]} \\
(0,04)\end{array}$ & $\begin{array}{r}-0,06 \\
{[-13,18]} \\
(0,00)\end{array}$ & 0,65 & 703,55 & 0,28 \\
\hline _DTE & $\begin{array}{r}253,74 \\
{[14,64]} \\
(0,00)\end{array}$ & $\begin{array}{r}-61,87 \\
{[-1,85]} \\
(0,07)\end{array}$ & $\begin{array}{r}-0,33 \\
{[-11,76]} \\
(0,00)\end{array}$ & 0,62 & 623,61 & 0,03 \\
\hline _DAIM & $\begin{array}{r}153,51 \\
{[31,14]} \\
(0,00) \\
\end{array}$ & $\begin{array}{r}-26,81 \\
{[-2,17]} \\
(0,03)\end{array}$ & $\begin{array}{r}-0,11 \\
{[-17,31]} \\
(0,00)\end{array}$ & 0,72 & 952,10 & 0,09 \\
\hline _DBANK & $\begin{array}{r}34,00 \\
{[12,96]} \\
(0,00) \\
\end{array}$ & $\begin{array}{r}-8,67 \\
{[-1,44]} \\
(0,15) \\
\end{array}$ & $\begin{array}{r}-0,03 \\
{[-6,92]} \\
(0,00)\end{array}$ & 0,30 & 164,04 & 0,05 \\
\hline _MRE & $\begin{array}{r}58,04 \\
{[12,90]} \\
(0,00) \\
\end{array}$ & $\begin{array}{r}0,65 \\
{[0,21]} \\
(0,83) \\
\end{array}$ & $\begin{array}{r}-0,05 \\
{[-6,40]} \\
(0,00)\end{array}$ & 0,32 & 160,02 & 0,16 \\
\hline BAY & $\begin{array}{l}78,37 \\
{[9,97]} \\
(0,00)\end{array}$ & $\begin{array}{l}15,21 \\
{[1,26]} \\
(0,21)\end{array}$ & $\begin{array}{r}-0,04 \\
{[-4,25]} \\
(0,00) \\
\end{array}$ & 0,08 & 33,59 & 0,03 \\
\hline _VW & $\begin{array}{r}48,02 \\
{[16,50]} \\
(0,00) \\
\end{array}$ & $\begin{array}{r}-5,53 \\
{[-1,12]} \\
(0,26) \\
\end{array}$ & $\begin{array}{r}0,03 \\
{[5,86]} \\
(0,00) \\
\end{array}$ & 0,21 & 98,82 & 0,03 \\
\hline BASF & $\begin{array}{r}31,08 \\
{[38,14]} \\
(0,00) \\
\end{array}$ & $\begin{array}{r}1,06 \\
{[1,98]} \\
(0,05) \\
\end{array}$ & $\begin{array}{r}-0,03 \\
{[-20,49]} \\
(0,00) \\
\end{array}$ & 0,75 & $1.124,93$ & 0,04 \\
\hline EON & $\begin{array}{r}51,55 \\
{[13,65]} \\
(0,00) \\
\end{array}$ & $\begin{array}{r}-8,03 \\
{[-1,99]} \\
(0,05)\end{array}$ & $\begin{array}{r}-0,04 \\
{[-6,35]} \\
(0,00)\end{array}$ & 0,27 & 141,43 & 0,03 \\
\hline _COB & $\begin{array}{r}101,05 \\
{[9,06]} \\
(0,00) \\
\end{array}$ & $\begin{array}{r}4,27 \\
{[0,47]} \\
(0,64) \\
\end{array}$ & $\begin{array}{r}-0,10 \\
{[-5,63]} \\
(0,00)\end{array}$ & 0,24 & 113,33 & 0,01 \\
\hline _HYPV & $\begin{array}{l}79,11 \\
{[9,26]} \\
(0,00)\end{array}$ & $\begin{array}{r}-7,15 \\
{[-2,08]} \\
(0,04) \\
\end{array}$ & $\begin{array}{r}-0,06 \\
{[-4,18]} \\
(0,00) \\
\end{array}$ & 0,13 & 55,17 & 0,02 \\
\hline _RWE & $\begin{array}{r}84,43 \\
{[17,86]} \\
(0,00)\end{array}$ & $\begin{array}{r}-0,95 \\
{[-1,62]} \\
(0,11)\end{array}$ & $\begin{array}{r}-0,09 \\
{[-11,10]} \\
(0,00)\end{array}$ & 0,54 & 390,98 & 0,02 \\
\hline BMW & $\begin{array}{r}37,68 \\
{[27,44]} \\
(0,00) \\
\end{array}$ & $\begin{array}{r}0,46 \\
{[0,42]} \\
(0,68) \\
\end{array}$ & $\begin{array}{r}-0,01 \\
{[-4,60]} \\
(0,00)\end{array}$ & 0,16 & 73,04 & 0,12 \\
\hline _LH & $\begin{array}{r}156,10 \\
{[23,88]} \\
(0,00) \\
\end{array}$ & $\begin{array}{r}1,83 \\
{[0,57]} \\
(0,57) \\
\end{array}$ & $\begin{array}{r}-0,12 \\
{[-11,22]} \\
(0,00)\end{array}$ & 0,43 & 268,34 & 0,01 \\
\hline _TYSS & $\begin{array}{r}205,62 \\
{[17,37]} \\
(0,00) \\
\end{array}$ & $\begin{array}{r}1,62 \\
{[2,20]} \\
(0,03) \\
\end{array}$ & $\begin{array}{r}-0,14 \\
{[-7,23]} \\
(0,00)\end{array}$ & 0,28 & 129,21 & 0,03 \\
\hline _METRO & $\begin{array}{r}101,26 \\
{[14,03]} \\
(0,00) \\
\end{array}$ & $\begin{array}{r}0,17 \\
{[0,17]} \\
(0,86) \\
\end{array}$ & $\begin{array}{r}-0,07 \\
{[-5,53]} \\
(0,00)\end{array}$ & 0,20 & 75,17 & 0,05 \\
\hline _TUI & $\begin{array}{r}321,38 \\
{[7,63]} \\
(0,00) \\
\end{array}$ & $\begin{array}{r}0,38 \\
{[0,24]} \\
(0,81) \\
\end{array}$ & $\begin{array}{r}-0,11 \\
{[-1,68]} \\
(0,09)\end{array}$ & 0,05 & 8,00 & 0,05 \\
\hline _LIN & $\begin{array}{r}53,99 \\
{[16,08]} \\
(0,00)\end{array}$ & $\begin{array}{r}0,06 \\
{[0,12]} \\
(0,90)\end{array}$ & $\begin{array}{r}-0,03 \\
{[-4,54]} \\
(0,00)\end{array}$ & 0,16 & 46,10 & 0,05 \\
\hline _HEN & $\begin{array}{r}21,06 \\
{[26,63]} \\
(0,00) \\
\end{array}$ & $\begin{array}{r}0,40 \\
{[1,61]} \\
(0,11)\end{array}$ & $\begin{array}{r}0,01 \\
{[5,22]} \\
(0,00)\end{array}$ & 0,19 & 46,03 & 0,05 \\
\hline _CONT & $\begin{array}{r}113,27 \\
{[12,93]} \\
(0,00)\end{array}$ & $\begin{array}{r}-0,90 \\
{[-1,00]} \\
(0,32)\end{array}$ & $\begin{array}{r}-0,11 \\
{[-8,12]} \\
(0,00)\end{array}$ & 0,63 & 231,20 & 0,01 \\
\hline
\end{tabular}

Table C.40.: Time-series regression results for the test of the relationship between default risk and risk-neutral skewness for a constant maturity of 180 days 


\begin{tabular}{|c|c|c|c|c|c|c|}
\hline & $C D S_{t}$ & $\beta_{0}+$ & $\cdot$ KURT & $\Gamma_{t}+\beta_{2}$ & $t+u_{t}$ & \\
\hline ID & $b_{0}$ & $b_{1}$ & $b_{2}$ & R2 cor. & $F$ & DW \\
\hline _ALL & $1.714,02$ & $-553,2$ & $-0,02$ & 0,71 & 905,12 & 0,53 \\
\hline & {$[12,54]$} & {$[-12,18]$} & {$[-5,31]$} & & & \\
\hline & $(0,00)$ & $(0,00)$ & $(0,00)$ & & & \\
\hline _SIE & 436,94 & $-125,12$ & $-0,05$ & 0,67 & 761,76 & 0,34 \\
\hline & {$[3,49]$} & {$[-2,98]$} & {$[-9,25]$} & & & \\
\hline & $(0,00)$ & $(0,00)$ & $(0,00)$ & & & \\
\hline _DTE & $1.845,04$ & $-532,7$ & $-0,28$ & 0,68 & 816,81 & 0,29 \\
\hline & {$[4,65]$} & {$[-3,95]$} & {$[-8,93]$} & & & \\
\hline & $(0,00)$ & $(0,00)$ & $(0,00)$ & & & \\
\hline _DAIM & 303,43 & $-48,03$ & $-0,11$ & 0,72 & 982,74 & 0,13 \\
\hline & {$[2,36]$} & {$[-1,12]$} & {$[-16,56]$} & & & \\
\hline & $(0,02)$ & $(0,26)$ & $(0,00)$ & & & \\
\hline _DBANK & 42,06 & $-2,02$ & $-0,03$ & 0,29 & 155,94 & 0,02 \\
\hline & {$[7,98]$} & {$[-1,37]$} & {$[-6,88]$} & & & \\
\hline & $(0,00)$ & $(0,17)$ & $(0,00)$ & & & \\
\hline _MRE & 86,23 & $-9,57$ & $-0,05$ & 0,33 & 166,61 & 0,19 \\
\hline & {$[2,95]$} & {$[-0,95]$} & {$[-6,16]$} & & & \\
\hline & $(0,00)$ & $(0,34)$ & $(0,00)$ & & & \\
\hline BAY & 93,79 & $-6,06$ & $-0,04$ & 0,08 & 35,03 & 0,03 \\
\hline & {$[5,25]$} & {$[-1,21]$} & {$[-4,31]$} & & & \\
\hline & $(0,00)$ & $(0,23)$ & $(0,00)$ & & & \\
\hline _VW & 104,30 & $-18,37$ & 0,03 & 0,23 & 111,35 & 0,08 \\
\hline & {$[5,36]$} & {$[-2,81]$} & {$[6,03]$} & & & \\
\hline & $(0,00)$ & $(0,01)$ & $(0,00)$ & & & \\
\hline BASF & 34,52 & $-1,20$ & $-0,03$ & 0,75 & $1.137,52$ & 0,06 \\
\hline & {$[16,71]$} & {$[-2,12]$} & {$[-20,73]$} & & & \\
\hline & $(0,00)$ & $(0,03)$ & $(0,00)$ & & & \\
\hline EON & 83,69 & $-10,26$ & $-0,04$ & 0,27 & 141,26 & 0,02 \\
\hline & {$[3,38]$} & {$[-1,27]$} & {$[-6,11]$} & & & \\
\hline & $(0,00)$ & $(0,21)$ & $(0,00)$ & & & \\
\hline COB & 305,58 & $-69,42$ & $-0,10$ & 0,28 & 145,25 & 0,13 \\
\hline & {$[6,79]$} & {$[-4,58]$} & {$[-5,67]$} & & & \\
\hline & $(0,00)$ & $(0,00)$ & $(0,00)$ & & & \\
\hline _HYPV & 111,02 & $-10,21$ & $-0,06$ & 0,14 & 60,08 & 0,04 \\
\hline & {$[7,22]$} & {$[-2,99]$} & {$[-4,25]$} & & & \\
\hline & $(0,00)$ & $(0,00)$ & $(0,00)$ & & & \\
\hline _RWE & 117,46 & $-10,95$ & $-0,09$ & 0,54 & 399,65 & 0,04 \\
\hline & {$[8,12]$} & {$[-2,36]$} & {$[-11,28]$} & & & \\
\hline & $(0,00)$ & $(0,02)$ & $(0,00)$ & & & \\
\hline BMMW & 35,25 & 0,77 & $-0,01$ & 0,16 & 74,32 & 0,13 \\
\hline & {$[21,50]$} & {$[2,93]$} & {$[-4,59]$} & & & \\
\hline & $(0,00)$ & $(0,00)$ & $(0,00)$ & & & \\
\hline _LH & 142,51 & 4,45 & $-0,12$ & 0,43 & 268,82 & 0,02 \\
\hline & {$[10,49]$} & {$[1,10]$} & {$[-11,20]$} & & & \\
\hline & $(0,00)$ & $(0,27)$ & $(0,00)$ & & & \\
\hline _TYSS & 213,15 & $-2,66$ & $-0,14$ & 0,28 & 129,22 & 0,03 \\
\hline & {$[15,92]$} & {$[-1,66]$} & {$[-7,22]$} & & & \\
\hline & $(0,00)$ & $(0,10)$ & $(0,00)$ & & & \\
\hline _METRO & 103,55 & $-0,77$ & $-0,07$ & 0,20 & 75,32 & 0,05 \\
\hline & {$[12,00]$} & {$[-0,62]$} & {$[-5,53]$} & & & \\
\hline & $(0,00)$ & $(0,53)$ & $(0,00)$ & & & \\
\hline _TUI & 325,32 & $-1,40$ & $-0,11$ & 0,05 & 8,01 & 0,05 \\
\hline & {$[7,65]$} & {$[-0,30]$} & {$[-1,68]$} & & & \\
\hline & $(0,00)$ & $(0,77)$ & $(0,10)$ & & & \\
\hline LLIN & 56,93 & $-0,98$ & $-0,03$ & 0,16 & 47,22 & 0,06 \\
\hline & {$[15,03]$} & {$[-1,92]$} & {$[-4,54]$} & & & \\
\hline & $(0,00)$ & $(0,06)$ & $(0,00)$ & & & \\
\hline _HEN & 20,66 & 0,11 & 0,01 & 0,19 & 45,05 & 0,04 \\
\hline & {$[13,54]$} & {$[0,27]$} & {$[5,20]$} & & & \\
\hline & $(0,00)$ & $(0,79)$ & $(0,00)$ & & & \\
\hline _CONT & 115,42 & $-0,68$ & $-0,11$ & 0,63 & 231,11 & 0,00 \\
\hline & {$[12,79]$} & {$[-1,40]$} & {$[-8,15]$} & & & \\
\hline & $(0,00)$ & $(0,16)$ & $(0,00)$ & & & \\
\hline
\end{tabular}

Table C.41.: Time-series regression results for the test of the relationship between default risk and risk-neutral kurtosis for a constant maturity of 180 days 


\begin{tabular}{|c|c|c|c|c|c|c|}
\hline \multicolumn{7}{|c|}{$C D S_{t}=\beta_{0}+\beta_{1} \cdot S K E W_{t}+\beta_{2} \cdot t+u_{t}$} \\
\hline ID & $b_{0}$ & $b_{1}$ & $b_{2}$ & R2 cor. & $\mathbf{F}$ & DW \\
\hline ALL & $\begin{array}{r}61,07 \\
{[10,01]} \\
(0,00) \\
\end{array}$ & $\begin{array}{r}-3,06 \\
{[-0,55]} \\
(0,58) \\
\end{array}$ & $\begin{array}{r}-0,05 \\
{[-5,10]} \\
(0,00) \\
\end{array}$ & 0,20 & 93,11 & 0,01 \\
\hline _SIE & $\begin{array}{r}58,97 \\
{[15,62]} \\
(0,00) \\
\end{array}$ & $\begin{array}{r}-25,87 \\
{[-2,16]} \\
(0,03)\end{array}$ & $\begin{array}{r}-0,06 \\
{[-12,11]} \\
(0,00)\end{array}$ & 0,61 & 592,88 & 0,09 \\
\hline _DTE & $\begin{array}{r}262,86 \\
{[16,28]} \\
(0,00) \\
\end{array}$ & $\begin{array}{r}-5,00 \\
{[-0,45]} \\
(0,65)\end{array}$ & $\begin{array}{r}-0,33 \\
{[-11,57]} \\
(0,00)\end{array}$ & 0,62 & 613,60 & 0,01 \\
\hline _DAIM & $\begin{array}{r}158,66 \\
{[35,05]} \\
(0,00) \\
\end{array}$ & $\begin{array}{r}-2,41 \\
{[-0,33]} \\
(0,74)\end{array}$ & $\begin{array}{r}-0,11 \\
{[-17,07]} \\
(0,00)\end{array}$ & 0,71 & 932,54 & 0,06 \\
\hline _DBANK & $\begin{array}{r}34,75 \\
{[14,73]} \\
(0,00)\end{array}$ & $\begin{array}{r}-5,32 \\
{[-2,34]} \\
(0,02)\end{array}$ & $\begin{array}{r}-0,03 \\
{[-6,88]} \\
(0,00)\end{array}$ & 0,30 & 160,48 & 0,04 \\
\hline _MRE & $\begin{array}{r}57,66 \\
{[12,98]} \\
(0,00) \\
\end{array}$ & $\begin{array}{r}-2,05 \\
{[-0,94]} \\
(0,35) \\
\end{array}$ & $\begin{array}{r}-0,05 \\
{[-6,46]} \\
(0,00) \\
\end{array}$ & 0,32 & 160,61 & 0,16 \\
\hline _BAY & $\begin{array}{r}75,64 \\
{[11,49]} \\
(0,00) \\
\end{array}$ & $\begin{array}{r}1,92 \\
{[0,45]} \\
(0,65)\end{array}$ & $\begin{array}{r}-0,04 \\
{[-4,27]} \\
(0,00)\end{array}$ & 0,08 & 32,37 & 0,02 \\
\hline _VW & $\begin{array}{r}49,24 \\
{[18,80]} \\
(0,00) \\
\end{array}$ & $\begin{array}{r}0,51 \\
{[0,20]} \\
(0,84) \\
\end{array}$ & $\begin{array}{r}0,03 \\
{[5,81]} \\
(0,00) \\
\end{array}$ & 0,20 & 97,42 & 0,02 \\
\hline _BASF & $\begin{array}{r}30,99 \\
{[38,62]} \\
(0,00) \\
\end{array}$ & $\begin{array}{r}0,63 \\
{[2,24]} \\
(0,03) \\
\end{array}$ & $\begin{array}{r}-0,03 \\
{[-20,53]} \\
(0,00) \\
\end{array}$ & 0,75 & $1.128,82$ & 0,04 \\
\hline EON & $\begin{array}{r}52,51 \\
{[13,94]} \\
(0,00) \\
\end{array}$ & $\begin{array}{r}-1,19 \\
{[-0,78]} \\
(0,44)\end{array}$ & $\begin{array}{r}-0,04 \\
{[-6,16]} \\
(0,00)\end{array}$ & 0,27 & 137,30 & 0,01 \\
\hline _COB & $\begin{array}{r}101,62 \\
{[9,06]} \\
(0,00) \\
\end{array}$ & $\begin{array}{r}7,33 \\
{[1,01]} \\
(0,31) \\
\end{array}$ & $\begin{array}{r}-0,10 \\
{[-5,64]} \\
(0,00) \\
\end{array}$ & 0,24 & 113,87 & 0,01 \\
\hline _HYPV & $\begin{array}{l}79,84 \\
{[9,27]} \\
(0,00) \\
\end{array}$ & $\begin{array}{r}-2,39 \\
{[-0,95]} \\
(0,34) \\
\end{array}$ & $\begin{array}{r}-0,06 \\
{[-4,16]} \\
(0,00) \\
\end{array}$ & 0,12 & 53,17 & 0,01 \\
\hline _RWE & $\begin{array}{r}84,48 \\
{[17,88]} \\
(0,00) \\
\end{array}$ & $\begin{array}{r}-0,77 \\
{[-1,12]} \\
(0,26) \\
\end{array}$ & $\begin{array}{r}-0,09 \\
{[-11,09]} \\
(0,00) \\
\end{array}$ & 0,54 & 390,64 & 0,02 \\
\hline _BMW & $\begin{array}{r}37,67 \\
{[27,59]} \\
(0,00) \\
\end{array}$ & $\begin{array}{r}0,44 \\
{[0,43]} \\
(0,67) \\
\end{array}$ & $\begin{array}{r}-0,01 \\
{[-4,60]} \\
(0,00)\end{array}$ & 0,16 & 73,04 & 0,12 \\
\hline _LH & $\begin{array}{r}156,03 \\
{[24,07]} \\
(0,00) \\
\end{array}$ & $\begin{array}{r}1,37 \\
{[0,63]} \\
(0,53) \\
\end{array}$ & $\begin{array}{r}-0,12 \\
{[-11,22]} \\
(0,00) \\
\end{array}$ & 0,43 & 268,37 & 0,01 \\
\hline _TYSS & $\begin{array}{r}205,70 \\
{[17,36]} \\
(0,00) \\
\end{array}$ & $\begin{array}{r}1,81 \\
{[2,61]} \\
(0,01) \\
\end{array}$ & $\begin{array}{r}-0,14 \\
{[-7,23]} \\
(0,00)\end{array}$ & 0,28 & 129,41 & 0,03 \\
\hline _METRO & $\begin{array}{r}101,19 \\
{[14,02]} \\
(0,00) \\
\end{array}$ & $\begin{array}{r}-0,16 \\
{[-0,14]} \\
(0,89) \\
\end{array}$ & $\begin{array}{r}-0,07 \\
{[-5,53]} \\
(0,00) \\
\end{array}$ & 0,20 & 75,17 & 0,05 \\
\hline _TUI & $\begin{array}{r}321,29 \\
{[7,63]} \\
(0,00) \\
\end{array}$ & $\begin{array}{r}0,27 \\
{[0,28]} \\
(0,78)\end{array}$ & $\begin{array}{r}-0,11 \\
{[-1,68]} \\
(0,09)\end{array}$ & 0,05 & 8,00 & 0,05 \\
\hline _LIN & $\begin{array}{r}53,98 \\
{[16,08]} \\
(0,00) \\
\end{array}$ & $\begin{array}{r}-0,02 \\
{[-0,04]} \\
(0,97) \\
\end{array}$ & $\begin{array}{r}-0,03 \\
{[-4,54]} \\
(0,00)\end{array}$ & 0,16 & 46,09 & 0,05 \\
\hline _HEN & $\begin{array}{r}21,06 \\
{[26,69]} \\
(0,00) \\
\end{array}$ & $\begin{array}{r}0,41 \\
{[1,99]} \\
(0,05)\end{array}$ & $\begin{array}{r}0,01 \\
{[5,22]} \\
(0,00)\end{array}$ & 0,19 & 46,28 & 0,05 \\
\hline _CONT & $\begin{array}{r}113,26 \\
{[12,93]} \\
(0,00)\end{array}$ & $\begin{array}{r}-0,92 \\
{[-1,01]} \\
(0,31)\end{array}$ & $\begin{array}{r}-0,11 \\
{[-8,12]} \\
(0,00)\end{array}$ & 0,63 & 231,25 & 0,01 \\
\hline
\end{tabular}

Table C.42.: Time-series regression results for the test of the relationship between default risk and risk-neutral skewness for a constant maturity of 240 days 


\begin{tabular}{|c|c|c|c|c|c|c|}
\hline \multicolumn{7}{|c|}{$C D S_{t}=\beta_{0}+\beta_{1} \cdot K U R T_{t}+\beta_{2} \cdot t+u_{t}$} \\
\hline ID & $b_{0}$ & $\mathbf{b}_{1}$ & $\mathbf{b}_{2}$ & R2 cor. & $\mathbf{F}$ & DW \\
\hline ALL & $\begin{array}{r}745,36 \\
{[3,43]} \\
(0,00)\end{array}$ & $\begin{array}{r}-229,4 \\
{[-3,15]} \\
(0,00)\end{array}$ & $\begin{array}{r}-0,04 \\
{[-4,41]} \\
(0,00)\end{array}$ & 0,44 & 290,38 & 0,52 \\
\hline SIE & $\begin{array}{r}240,20 \\
{[4,35]} \\
(0,00)\end{array}$ & $\begin{array}{r}-59,23 \\
{[-3,18]} \\
(0,00)\end{array}$ & $\begin{array}{r}-0,06 \\
{[-10,86]} \\
(0,00)\end{array}$ & 0,64 & 666,12 & 0,22 \\
\hline DTE & $\begin{array}{r}787,42 \\
{[4,38]} \\
(0,00)\end{array}$ & $\begin{array}{r}-177,3 \\
{[-2,89]} \\
(0,00)\end{array}$ & $\begin{array}{r}-0,31 \\
{[-10,84]} \\
(0,00)\end{array}$ & 0,65 & 706,60 & 0,18 \\
\hline DAIM & $\begin{array}{r}228,29 \\
{[4,08]} \\
(0,00)\end{array}$ & $\begin{array}{r}-23,07 \\
{[-1,24]} \\
(0,22)\end{array}$ & $\begin{array}{r}-0,11 \\
{[-17,17]} \\
(0,00)\end{array}$ & 0,72 & 966,03 & 0,11 \\
\hline DBANK & $\begin{array}{l}41,59 \\
{[8,50]} \\
(0,00)\end{array}$ & $\begin{array}{r}-1,86 \\
{[-1,40]} \\
(0,16)\end{array}$ & $\begin{array}{r}-0,03 \\
{[-6,89]} \\
(0,00)\end{array}$ & 0,29 & 156,08 & 0,02 \\
\hline -MRE & $\begin{array}{l}83,16 \\
{[4,36]} \\
(0,00)\end{array}$ & $\begin{array}{r}-8,57 \\
{[-1,31]} \\
(0,19)\end{array}$ & $\begin{array}{r}-0,05 \\
{[-6,22]} \\
(0,00)\end{array}$ & 0,33 & 167,99 & 0,19 \\
\hline BAY & $\begin{array}{l}96,25 \\
{[5,10]} \\
(0,00)\end{array}$ & $\begin{array}{r}-6,92 \\
{[-1,27]} \\
(0,21)\end{array}$ & $\begin{array}{r}-0,04 \\
{[-4,32]} \\
(0,00) \\
\end{array}$ & 0,09 & 35,35 & 0,03 \\
\hline -VW & $\begin{array}{l}63,81 \\
{[8,60]} \\
(0,00)\end{array}$ & $\begin{array}{r}-4,89 \\
{[-2,03]} \\
(0,04)\end{array}$ & $\begin{array}{r}0,03 \\
{[5,87]} \\
(0,00)\end{array}$ & 0,21 & 100,90 & 0,04 \\
\hline BASF & $\begin{array}{r}32,06 \\
{[30,57]} \\
(0,00)\end{array}$ & $\begin{array}{r}-0,39 \\
{[-2,53]} \\
(0,01)\end{array}$ & $\begin{array}{r}-0,03 \\
{[-20,58]} \\
(0,00)\end{array}$ & 0,75 & $1.127,84$ & 0,05 \\
\hline EON & $\begin{array}{l}55,79 \\
{[5,31]} \\
(0,00)\end{array}$ & $\begin{array}{r}-1,03 \\
{[-0,32]} \\
(0,75)\end{array}$ & $\begin{array}{r}-0,04 \\
{[-6,10]} \\
(0,00)\end{array}$ & 0,27 & 137,09 & 0,01 \\
\hline - $\mathrm{COB}$ & $\begin{array}{r}189,17 \\
{[4,91]} \\
(0,00)\end{array}$ & $\begin{array}{r}-30,23 \\
{[-2,38]} \\
(0,02)\end{array}$ & $\begin{array}{r}-0,10 \\
{[-5,60]} \\
(0,00)\end{array}$ & 0,26 & 129,39 & 0,07 \\
\hline _HYPV & $\begin{array}{r}105,40 \\
{[8,18]} \\
(0,00)\end{array}$ & $\begin{array}{r}-8,35 \\
{[-3,46]} \\
(0,00)\end{array}$ & $\begin{array}{r}-0,06 \\
{[-4,23]} \\
(0,00)\end{array}$ & 0,13 & 58,84 & 0,04 \\
\hline -RWE & $\begin{array}{r}90,55 \\
{[13,90]} \\
(0,00)\end{array}$ & $\begin{array}{r}-1,99 \\
{[-1,28]} \\
(0,20)\end{array}$ & $\begin{array}{r}-0,09 \\
{[-11,10]} \\
(0,00)\end{array}$ & 0,54 & 392,21 & 0,02 \\
\hline BMW & $\begin{array}{r}35,66 \\
{[20,76]} \\
(0,00)\end{array}$ & $\begin{array}{r}0,64 \\
{[1,82]} \\
(0,07)\end{array}$ & $\begin{array}{r}-0,01 \\
{[-4,60]} \\
(0,00)\end{array}$ & 0,16 & 73,90 & 0,13 \\
\hline _LH & $\begin{array}{r}139,00 \\
{[11,31]} \\
(0,00)\end{array}$ & $\begin{array}{r}5,62 \\
{[1,59]} \\
(0,11)\end{array}$ & $\begin{array}{r}-0,12 \\
{[-11,21]} \\
(0,00)\end{array}$ & 0,44 & 269,86 & 0,02 \\
\hline -TYSS & $\begin{array}{r}212,38 \\
{[15,99]} \\
(0,00)\end{array}$ & $\begin{array}{r}-2,40 \\
{[-1,53]} \\
(0,13)\end{array}$ & $\begin{array}{r}-0,14 \\
{[-7,22]} \\
(0,00)\end{array}$ & 0,28 & 129,15 & 0,03 \\
\hline _METRO & $\begin{array}{r}102,89 \\
{[11,97]} \\
(0,00)\end{array}$ & $\begin{array}{r}-0,55 \\
{[-0,45]} \\
(0,65)\end{array}$ & $\begin{array}{r}-0,07 \\
{[-5,53]} \\
(0,00)\end{array}$ & 0,20 & 75,24 & 0,05 \\
\hline- TUI & $\begin{array}{r}324,47 \\
{[7,81]} \\
(0,00)\end{array}$ & $\begin{array}{r}-1,14 \\
{[-0,92]} \\
(0,36)\end{array}$ & $\begin{array}{r}-0,11 \\
{[-1,67]} \\
(0,10)\end{array}$ & 0,06 & 8,05 & 0,05 \\
\hline LLIN & $\begin{array}{r}57,23 \\
{[14,91]} \\
(0,00)\end{array}$ & $\begin{array}{r}-1,07 \\
{[-2,09]} \\
(0,04)\end{array}$ & $\begin{array}{r}-0,03 \\
{[-4,56]} \\
(0,00)\end{array}$ & 0,16 & 47,53 & 0,06 \\
\hline -HEN & $\begin{array}{r}20,83 \\
{[14,51]} \\
(0,00)\end{array}$ & $\begin{array}{r}0,05 \\
{[0,14]} \\
(0,89)\end{array}$ & $\begin{array}{r}0,01 \\
{[5,21]} \\
(0,00)\end{array}$ & 0,19 & 44,98 & 0,04 \\
\hline _CONT & $\begin{array}{r}115,44 \\
{[12,80]} \\
(0,00)\end{array}$ & $\begin{array}{r}-0,69 \\
{[-1,41]} \\
(0,16)\end{array}$ & $\begin{array}{r}-0,11 \\
{[-8,15]} \\
(0,00)\end{array}$ & 0,63 & 231,14 & 0,00 \\
\hline
\end{tabular}

Table C.43.: Time-series regression results for the test of the relationship between default risk and risk-neutral kurtosis for a constant maturity of 240 days 


\section{Bibliography}

(2005): EViews 5.1 Users Guide, Quantitative Micro Software, LLC, 4521 Campus Drive, 336, Irvine CA, 92612-2621.

AbKen, P. A./MADAn, D. B. (1996): Estimation of Risk-Neutral and Statistical Densities by Hermite Polynomial Approximation: With an Application to Eurodollar Futures Option, Working Paper 96-5, Federal Reserve Bank of Atlanta.

Ait-SahaliA, Y./Lo, A. W. (1998): Nonparametric Estimation of State-Price Densities Implicit in Financial Asset Prices, in: Journal of Finance, 53(2), pp. 499-547.

Ait-S Ahalia, Y./Lo, A. W. (2000): Nonparametric Risk Management and Implied Risk Aversion, in: Journal of Econometrics, 94(1-2), pp. 9-51.

Ait-Sahalia, Y./WAng, Y./YARed, F. (2001): Do Option Markets Correctly Price the Probabilities of Movement of the Underlying Asset?, in: Journal of Econometrics, 102(1), pp. 67-110.

Albanese, C./Chen, O. (2005): Pricing Equity Default Swaps, in: Risk, 18(6), pp. 83-87.

Alexander, C. (2001): Principles of the Skew, in: Risk, 14(1), pp. 29-32.

AleXander, C. (2004): Market Models - A Guide to Financial Data Analysis, 5th edition, John Wiley \& Sons, LTD, Chichester.

Amin, K. I./JARrow, R. A. (1991): Pricing Foreign Currency Options under Stochastic Interest Rates, in: Journal of International Money and Finance, 10(3), pp. 310-29.

Amin, K. I./JARrow, R. A. (1992): Pricing Options on Risky Assets in a Stochastic Interest Rate Economy, in: Mathematical Finance, 2(4), pp. 217-237.

Amin, K. I./NG, V. K. (1993): Option Valuation with Systematic Stochastic Volatility, in: Journal of Finance, 48(3), pp. 881-910. 
AnÉ, T./LABIDI, C. (2001): Implied Volatility Surfaces and Market Activity over Time, in: Journal of Economics and Finance, 25, pp. 259-275.

Anagnou, I./Bedendo, M./Hodges, D./Tompkins, R. (2005): Forecasting Accuracy of Implied and GARCH-Based Probability Functions.

Andersen, L./Buffum, D. (2003): Calibration and Implementation of Convertible Bond Models, in: Journal of Computational Finance, 7(2), pp. 1-34.

Andersen, T. G./Bollerslev, T./Diebold, F. X./Ebens, H. (2001): The Distribution of Realized Stock Return Volatility., in: Journal of Financial Economics, 61, pp. 43-76.

Anderson, A. B./WAgener, T. (2002): Extracting Risk Neutral Probability Densities by Fitting Implied Volatility Smiles: Some Methodological Points and an Application to the $3 \mathrm{M}$ Euribor Futures Option Prices, Working paper series, European Central Bank, Kaiserstraße 29, D-60311 Frankfurt.

ARrow, K. J. (1953): Le Rôle des Valeurs Boursières pour la Répartition la Meilleure des Risques, in: Econométric, pp. 41-47, Centre Nationale de la Recherche Scientifique.

Atlan, M./Leblanc, B. (2005): Hybrid Equity-Credit Modelling, in: Risk, 18(8), pp. 61-66.

Avramov, D./Jostova, G./Philipov, A. (2006): Corporate Credit Risk Changes: Common Factors and Firm-Level Fundamentals.

Ayache, E./Forsyth, P./Vetzal, K. (2003): Valuation of Convertible Bonds With Credit Risk., in: Journal of Derivatives, 11(1), pp. 9-29.

BAiley, W./Stulz, R. M. (1989): The Pricing of Stock Index Options in a General Equilibrium Model, in: Journal of Financial and Quantitative Analysis, 14(1), pp. $1-12$.

BaKshi, G./CAO, C. (2003): Risk-Neutral Kurtosis, Jumps, and Option Pricing: Evidence from 100 Most Actively Traded Firms on the CBOE, Efa 2003 annual conference paper no. 953.

BAKshi, G./CAO, C./ChEn, Z. (1997): Empirical Performance of Alternative Option Pricing Models, in: Journal of Finance, 52(5), pp. 2003-2049. 
BaKshi, G./Kapadia, N. (2003a): Delta-Hedged Gains and the Negative Market Volatility Risk Premium, in: Review of Financial Studies, 16(2), pp. 527-566.

BaKshi, G./Kapadia, N. (2003b): Volatility Risk Premiums Embedded in Individual Equity Options: Some New Insights, in: Journal of Derivatives, 11(1), pp. $45-54$.

BAKShi, G./KAPAdia, N./MADAN, D. (2003): Stock Return Characteristics, Skew Laws, and the Differential Pricing of Individual Equity Options, in: The Review of Financial Studies, 16, pp. 101-143.

BAKshi, G./MADAN, D. (2000): Spanning and Derivative-Security Valuation, in: Journal of Financial Economics, 55, pp. 205-238.

Ball, C. A./Torous, W. N. (1985): On Jumps in Common Stock Prices and Their Impact on Call Option Pricing, in: Journal of Finance, 40(1), pp. 155-173.

BarndorfF-Nielsen, O. E. (1998): Processes of Normal Inverse Gaussian Type, in: Finance \& Stochastics, 2(1), pp. 41-68.

BARtunek, K. S./ChOwdhury, M. (1997): Implied Risk Aversion Parameter from Option Prices, in: The Financial Review, 33(1), pp. 107-124.

BATES, D. (2001): The Market for Crash Risk, national Bureau of Economic Research, Inc, NBER Working Papers: 8557.

BATES, D. S. (1996): Jumps and stochastic volatility: exchange rate processes implicit in deutsche mark options, in: Review of Financial Studies, 9(1), pp. 69-107.

Bates, D. S. (2000): Post-'87 Crash Fears in the S\&P 500 Futures Option Market, in: Journal of Econometrics, 94(1), pp. 181-238.

BeKaert, G./Wu, G. (2000): Asymmetric Volatility and Risk in Equity Markets, in: The Review of Financial Studies, 13(1), pp. 1-42.

Benkert, C. (2004): Explaining Credit Default Swap Premia, in: The Journal of Futures Markets, 24(1), pp. 71-92.

Benninga, S./MAyshar, J. (2000): Heterogeneity and Option Pricing, in: Review of Derivatives Research, 4, pp. 7-27.

BJÖRK, T. (2004): Arbitrage Theory in Continuous Time, Oxford University Press, New York. 
BlaCK, F. (1975): Facts and Fantasy in the Use of Options, in: Financial Analysts Journal, 31(4), pp. 36-72.

BLACK, F. (1976): Studies of Stock Price Volatility Changes, in: Proceedings of the 1976 Meeting of the Business and Economics Statistics Section of the American Statistical Association, pp. 177-181, American Statistical Association.

Black, F./CoX, J. C. (1976): Valuing Corporate Securities: Some Effects of Bond Indenture Provisions, in: Journal of Finance, 31(2), pp. 351-367.

Black, F./Scholes, M. (1973): The Pricing of Options and Corporate Liabilities, in: Journal of Political Economy, 81, pp. 637-653.

Bliss, R. R./Panigirtzoglou, N. (2002): Testing the Stability of Implied Probability Density Functions, in: Journal of Banking \& Finance, 26(2), pp. 381-420.

Bliss, R. R./Panigirtzoglou, N. (2004): Option-Implied Risk Aversion Estimates, in: Journal of Finance, 59(1), pp. 407-446.

BLOCH, D. (2005): Jumps as Components in the Pricing of Credit and Equity Products, in: Risk, 18(2), pp. 67-73.

Bollen, N. P. B./Whaley, R. E. (2004): Does Net Buying Pressure Affect the Shape of Implied Volatility Functions?, in: Journal of Finance, 59(2), pp. 711-753.

Bollerslev, T./ChOU, R. Y./Kroner, K. F. (1992): ARCH Modeling in Finance, in: Journal of Econometrics, 52(1/2), pp. 5-59.

Bollerslev, T./Litvinova, J./TAuchen, G. (2006): Leverage and Volatility Feedback Effects in High-Frequency Data, in: Journal of Financial Econometrics, 4(3), pp. 353-384.

Bollerslev, T./Mikkelsen, H. O. (1996): Modeling and Pricing Long-Memory in Stock Market Volatility, in: Journal of Finance, 73(1), pp. 151-184.

Branger, N./Schlag, C. (2004): Why is the Index Smiale so Steep?, in: Review of Finance, 8, pp. 109-127.

Braun, P. A./Nelson, D. B./Sunier, A. M. (1995): Good News, Bad News, Volatility, and Betas, in: Journal of Finance, 50(5), pp. 1575-1603.

Breeden, D. T./Litzenberger, R. H. (1978): Prices of State-contingent Claims Implicit in Option Prices, in: Journal of Business, 61(4), pp. 621-651. 
Brunner, B./HAFNER, R. (2003): Arbitrage-Free Estimation of the Risk-Neutral Density from the Implied Volatility Smile, in: Journal of Computational Finance, 7, pp. 75-106.

Buchen, P. W./Kelly, M. (1996): The Maximum Entropy Distribution of an Asset Inferred From Option Prices, in: Journal of Financial \& Quantitative Analysis, 31(1), pp. 143-159.

Buraschi, A./JACKwerth, J. (2001): The Price of a Smile: Hedging and Spanning in Option Markets, in: The Review of Financial Studies, 14(2), pp. 495-527.

Byström, H. (2005): Credit Default Swaps and Equity Prices: The iTraxx and the CDS Index Market.

CAmpa, J. M./Chang, P. K./Reider, R. L. (1998): Implied exchange rate distributions: Evidence from OTC option markets, in: Journal of International Money \& Finance, 17(1), pp. 117-160.

Campbell, J. Y./Hentschel, L. (1992): No News is Good News, in: Journal of Financial Economics, 31, pp. 281-318.

Campbell, J. Y./Lo, A. W./MacKinlay, A. C. (1997): The Econometrics of Financial Markets, 2nd edition, Princeton University Press, Princeton, New Jesey.

Campbell, J. Y./TAKsler, G. B. (2003): Equity Volatility and Corporate Bond Yields, in: Journal of Economic Theory, 58(6), pp. 2321-2350.

Campi, L./Polbennikov, S./Sbuelz, A. (2005): Assessing Credit with Equity: A CEV Model with Jump to Default, Discussion Paper 27, Tilburg University, Center for Economic Research.

CANinA, L./Figlewski, S. (1993): The Informational Content of Implied Volatility., in: The Review of Financial Studies, 6(3), pp. 659-682.

CAO, C./Yu, F./Zhong, Z. (2006): The Information Content of Option-Implied Volatility for Credit Default Swap Valuation.

Carr, P./Geman, H./Madan, D. B./Yor, M. (2002): The Fine Structure of Asset Returns: An Empirical Investigation, in: Journal of Business, 75(2), pp. q305-332.

CArr, P./Linetsky, V. (2006): A Jump to Default Extendet CEV Model: An Application of Bessel Processes, in: Fianance and Stochastics, 10, pp. 303-330. 
Carr, P./Wu, L. (2003a): The Finite Moment Log Stable Process and Option Pricing, in: Journal of Finance, 58(2), pp. 753-778.

CARr, P./Wu, L. (2003b): What Type of Process Underlies Options? A Simple Robust Test, in: Journal of Finance, 58(6), pp. 2581-2610.

CARr, P./Wu, L. (2006): Stock Options and Credit Default Swaps: A Joint Framework for Valuation and Estimation.

Cetin, U./Jarrow, R./Protter, P./WarachKa, M. (2006): Pricing Options in an Extended Black Scholes Economy with Illiquidity: Theory and Empirical Evidence, in: Review of Financial Studies, 19(2), pp. 493-529.

Chang, G. C. (1996): How to Handle Multicollinearity in Regression Modeling, in: Journal of Business Forecasting Methods \& Systems, 15(1), pp. 23-27.

Chelley-Steeley, P. L./Steeley, J. M. (2005): The Leverage Effect in the UK Stock Market, in: Applied Financial Economics, 15, pp. 409-423.

Chen, L./Lesmond, D. A./WeI, J. (2007): Corporate Yield Spreads and Bond Liquidity, in: Journal of Finance, 62(1), pp. 119-149.

Chernov, M./Ghysels, E. (2000): A Study towards a Unified Approach to the Joint Estimation of Objective and Risk Neutral Measures for the Purpose of Options Valuation, in: Journal of Financial Economics, 56(3), pp. 407-58.

Cheung, Y.-W./Ng, L. K. (1992): Stock Price Dynamics and Firm Size: An Empirical Investigation., in: Journal of Finance, 47(5), pp. 1985-1997.

Christensen, B. J./Prabhala, N. R. (1996): The Relation between Implied and Realized Volatility, in: Journal of Financial Economics, 50, pp. 125-150.

Christie, A. A. (1982): The Stochastic Behavior of Common Stock Variances, in: Journal of Financial Economics, 10, pp. 407-4032.

Clews, R./Panigirtzoglou, N. (2000): Recent Developments in Extracting Information from Options Markets, Quarterly Bulletin 1, Bank of England.

Collin-Dufresne, P./Goldstein, R. S. (2001): Do Credit Spreads Reflect Stationary Leverage Ratios?, in: Journal of Finance, 56(5), pp. 1929-1957.

Collin-Dufresne, P./Goldstein, R. S./Martin, J. S. (2001): The Determinants of Credit Spread Changes, in: Journal of Finance, 56(6), pp. 2177-2208. 
Consigli, G. (2004): Credit Default Swaps and Equity Volatility: Theoretical Modelling and Market Evidence.

Constantinides, G. M. (1997): Transaction Costs and the Implied Volatility Smile.

Cont, R. (2001): Empirical Properties of Asset Returns: Stylized Facts and Statistical Issues, in: Quantitative Finance, 1, pp. 223-236.

Cont, R./DA FonseCA, J. (2002): Dynamics of Implied Volatility Surfaces, in: Quantitative Finance, 2(1), pp. 45-60.

Cont, R./DA FonsecA, J./DurRleman, V. (2002): Stochastic Models of Implied Volatility Surfaces, in: Economic Notes, 31(2), pp. 361-377.

Cont, R./TAnkov, P. (2004): Financial Modelling with Jump Processes, 1st edition, Chapman \& Hall/CRC, London.

Corrado, C. J./SU, T. (1996): Skewness and Kurtosis in S\&P 500 Index Returns Implied By Option Prices, in: The Journal of Financial Research, 19(2), pp. 175192.

Corrado, C. J./SU, T. (1997a): Implied Volatility Skews and Stock Index Skewness and Kurtosis implied by S\&P 500 Index Option Prices, in: The Journal of Derivatives, 4(4), pp. 8-19.

Corrado, C. J./Su, T. (1997b): Implied Volatility Skews and Stock Return Skewness and Kurtosis Implied by Stock Option Prices, in: The European Journal of Finance, 3, pp. 73-85.

Coutant, S./Jondeau, E./Rockinger, M. (2001): Reading PIBOR Futures Options Smiles: The 1997 Snap Election, in: Journal of Banking \& Finance, 25(11), pp. 1957-1987.

Cox, J. C./Ross, S. A. (1976): The Valuation of Options for Alternative Stochastic Processes, in: Journal of Financial Economics, 3, pp. 145-166.

Cox, J. C./Ross, S. A./Rubinstein, M. (1979): Option pricing: A simplified approach, in: Journal of Financial Economics, 7(3), pp. 229-263.

Cremers, M./Driessen, J./Maenhout, P./Weinbaum, D. (2005a): Explaining the Level of Credit Spreads: Option-Implied Jump Risk Premia in a Firm Valua 
Model, Bis Working Papers 191, Bank for International Settlements, CH-4002 Basel, Switzerland.

Cremers, M./Driessen, J./Maenhout, P./Weinbaum, D. (2005b): Individual Stock-Option Prices and Credit Spreads.

DAAL, E. A./MADAN, D. B. (2005): An Empirical Examination of the VarianceGamma Model for Foreign Currency Options, in: Journal of Business, 78(6), pp. 2121-2152.

DAS, S. R./Sundaram, R. K. (1999): Of Smiles and smirks: A Term Structure Perspective, in: Journal of Financial and Quantitative Analysis, 34(2), pp. 211-244.

DAS, S. R./Sundaram, R. K. (2006): A Simple Model for Pricing Securities with Equity, Interest-Rate and Default Risk.

David, A./Veronesi, P. (2000): Option Prices with Uncertain Fundamentals: Theory and Evidence on the Dynamics of Implied Volatilities, Working Paper Series 485, University of Chicago.

DAVIS, M./LischKA, F. R. (1999): Convertible Bonds with Market Risk and Credit Risk.

Debreu, G. (1959): Theory of Value, John Wiley and Sons, New York, german Translation (1976): Werththeorie, Springer, Berlin.

Delzio, M. F. (2006a): Pricing Credit Risk through Equity Options Calibration Part 1 - Theory, in: The Journal of Risk Finance, 7, pp. 372-385.

Delzio, M. F. (2006b): Pricing Credit Risk through Equity Options Calibration Part 2 - Model Implementation, in: The Journal of Risk Finance, 7, pp. 386-401.

Dennis, P./MaYhew, S. (2002): Risk-Neutral Skewness: Evidence from Stock Options, in: Journal of Financial and Quantitative Analysis, 37, pp. 471-493.

Dennis, P./Mayhew, S./STivers, C. (2006): Stock Returns, Implied Volatility Innovations, and the Asymmetric Volatility Phenomenon, in: Journal of Financial and Quantitative Analysis, 41, pp. 381-406.

DERMAN, E./KANI, I. (1994): Riding on a smile, in: Risk, 7, pp. 32-39. 
Derman, E./Kani, I. (1998): Stochastic Implied Trees: Arbitrage Pricing with Stochastic Term and Strike Structure of Volatility, in: International Journal of Theoretical and Applied Finance, 1, pp. 61-110.

Derman, E./Kani, I./Zou, J. Z. (1996): The Local Volatility Surface: Unlocking the Information in Index Option Prices, in: Financial Analysts Journal, 52, pp. 2536 .

Draper, N. R./Smith, H. (1998): Applied Regression Analysis, 3rd edition, John Wiley \& Sons, Inc., New York.

DuAN, J.-C. (1995): The GARCH Option Pricing Model, in: Mathematical Finance, 5(1), pp. 13-32.

DufFeE, G. R. (1995): Stock returns and volatility A firm-level analysis, in: Journal of Financial Economics, 37(3), pp. 399-420.

DufFIE, D. (2005): Credit Risk Modeling with Affine Processes, in: Journal of Banking \& Finance, 29, pp. 2751-2802.

Duffie, D./Singleton, K. J. (1999): Modeling Term Structures of Defaultable Bonds, in: Review of Financial Studies, 12(4), pp. 687-720.

Dumas, B./Fleming, J./Whaley, R. E. (1998): Implied Volatility Functions: Empirical Tests, in: Journal of Finance, 53(6), pp. 2059-2106.

DuPIRE, B. (1994): Pricing with a smile, in: Risk, 7, pp. 18-20.

Ederington, L./Wei, G. (2002): Why Are Those Options Smiling?, in: The Journal of Derivatives, 10(2), pp. 9-34.

Emanuel, D. C./MacBeth, J. D. (1982): Further Results on the Constant Elasticity of Variance Call Option Pricing Model, in: Journal of Financial and Quantitative Analysis, 17(4), pp. 533-554.

Engle, R. F./Lilien, D. M./Robins, R. P. (1987): Estimating Time-Varying Risk Premia in the Term Structure: The ARCH-M Model, in: Econometrica, 55(2), pp. 391-407.

ErAKer, B. (2004): Do Stock Prices and Volatility Jump? Reconciling Evidence from Spot and Option Prices, in: Journal of Finance, 59(3), pp. 1367-1403. 
Eraker, B./Johannes, M./Polson, N. (2003): The Impact of Jumps in Volatility and Returns, in: Journal of Finance, 58(3), pp. 1269-1300.

Ericsson, J./Jacobs, K./Oviedo-Helfenberger, R. (): The Determinants of Credit Default Swap Premia.

FEnGLer, M. R. (2005): Semiparametric Modeling of Implied Volatility, 1st edition, Springer, Berlin.

Fengler, M. R./HÄrdle, W. K./Villa, C. (2003): Find More Like ThisThe Dynamics of Implied Volatilities: A Common Principal Components Approach, in: Review of Derivatives Research, 6(3), pp. 179-202.

FIGLEWSKI, S./WANG, X. (2000): Is the "Leverage Effect" a Leverage Effect?

Finger, C. C./Findelstein, V./Pan, G./LARdy, J.-P./TA, T. (2002): CreditGrades Technical Document, RiskMetrics Group, Inc.

Finger, C. C./StamicAR, R. (2005): Incorporating Equity Derivatives into the CreditGrades Model, technical report, RiskMetrics Group, 44 Wall St. New York, NY 10005.

FÖllmer, H./SchIED, A. (2002): Stochastic Finance - An Introduction in Discrete Time, 1st edition, Walter de Gruyter, Berlin.

Fouque, J.-P./PAPAnicolaou, G./Sircar, K. R. (2000): Derivatives in Financial Markets with Stochastic Volatility, 1st edition, Cambridge University Press, Cambridge.

Franke, G./Stapleton, R. C./Subrahmanyam, M. G. (1999): When are Options Overpriced? The Black-Scholes Model and Alternative Characterisations of the Pricing Kernel, in: European Finance Review, 3, pp. 79-102.

French, K. R./SchWert, W. G./Stambaugh, R. F. (1987): Expected Stock Returns and Volatility, in: Journal of Financial Economics, 19(1), pp. 3-29.

Gatheral, J. (2006): The Volatility Surface, 1st edition, John Wiley \& Sons, New Jersey.

Genser, M. (2004): Explaining Volatility Smiles of Equity Options with Capital Structure Models, Working paper, Swiss Institute of Banking and Finance, University of St. Gallen. 
Geske, R. (1979): The Valuation of Compound Options, in: Journal of Financial Economics, 7, pp. 63-81.

Glatzer, E./Scheicher, M. (2003): Modelling the Implied Probability of Stock Market Movements, Working Paper Series 212, European Central Bank, Kaiserstraße 29, D-60311 Frankfurt.

Glosten, L. R./Jagannathan, R./Runkle, D. E. (1993): On the Relation between the Expected Value and the Volatility of the Nominal Excess Return on Stocks, in: Journal of Finance, 48(55), pp. 1779-1801.

Goodhart, C. A. E./Figliuoli, L. (1991): Every Minute Counts in Financial Markets, in: Journal of International Money and Finance, 10, pp. 23-52.

Granger, C. W. J./Newbold, P. (1974): Spurious Regression in Econometrics, in: Journal of Econometrics, 2, pp. 111-120.

Grapentine, T. (1997): Managing Multicollinearity, in: Marketing Research, 9(3), pp. 10-21.

Grossman, S. J./Zhou, Z. (1996): Equilibrium Analysis of Portfolio Insurance, in: Journal of Finance, 51(4), pp. 1379-1403.

Guidolin, M./Timmermann, A. G. (2003): Option Prices under Bayesian Learning: Implied Volatility Dynamics and Predictive Densities, in: Journal of Economic Dynamics \& Control, 27(5), pp. 717-769.

HAFner, R./WALlmeier, M. (2001): The Dynamics of Dax Implied Volatilities, in: International Quarterly Journal of Finance, 1(1), pp. 1-27.

Hagan, P. S./Kumar, D./Lesniewski, A. S./Woodward, D. E. (2002): Managing Smile Risk, in: Wimott Magazine, pp. 84-108.

Hakansson, N. H. (1979): The Fantastic World of Finance: Progress and the Free Lunch, in: Journal of Financial and Quantitative Analysis, 14(4), pp. 717-734.

Hamilton, J. D. (1994): Time Series Analysis, Princeton University Press, Princeton, New Jersey.

Hanke, M. (2002): Pricing Options on Leveraged Equity with Default Risk and Exponentially Increasing, Finite Maturity Debt. 
HARrison, M./KrePs, D. M. (1979): Martingales and Arbitrage in Multiperiod Securities Markets, in: Journal of Economic Theory, 20, pp. 381-408.

Harrison, M./Pliska, S. R. (1981): A Stochastic Calculus Model of Continuous Trading: Complete Markets, Discussion Papers 489, Northwestern University, Center for Mathematical Studies in Economics and Management Science.

HENTSCHEL, L. (2003): Errors in Implied Volatility Estimation, in: Journal of Financial and Quantitative Analysis, 38(4), pp. 779-810.

Heston, S. L. (1993a): A Closed-Form Solution for Options with Stochastic Volatility with Applications to Bond and Currency Options, in: The Review of Financial Studies, 6(2), pp. 327-343.

Heston, S. L. (1993b): Invisible Parameters in Option Prices, in: Journal of Finance, 48(3), pp. 933-947.

Heston, S. L./NAndi, S. (1998): Preference-Free Option Pricing with PathDepending Volatility: A Closed-Form Approach, Working Paper 98-20, Federal Reserve Bank of Atlanta.

Heston, S. L./NANDI, S. (2000): A Closed-Form GARCH Option Valuation Model, in: Review of Financial Studies, 13(3), pp. 585-625.

Hull, J./Nelken, I./White, A. (2004a): Merton's Model, Credit Risk, and Volatility Skews.

Hull, J./Predescu, M./White, A. (2004b): The Relationship Between Credit Default Swap Spreads, Bond Yields, and Credit Rating Announcements, in: Journal of Banking \& Finance, 28(11), pp. 2789-2811.

Hull, J./White, A. (1987): The Pricing of Options on Assets with Stochastic Volatility, in: Journal of Finance, 42(2), pp. 281-300.

Hull, J. C. (2006): Options, Futures and other Derivatives, 6. edition, Pearson Prentice Hall, New Jersey.

JACKWERTH, J. C. (1999): Option-Implied Risk-Neutral Distributions and Implied Binomial Trees: A Literature Review, in: The Journal of Derivatives, 7, pp. 66-82.

JACKWERTH, J. C. (2000): Recovering Risk Aversion from Option Prices and Realized Returns, in: The Review of Financial Studies, 13(2), pp. 433-451. 
JACKWERTH, J. C. (2004): Option-Implied Risk-Neutral Distributions and Risk Aversion, The Research Foundation of The Association for Investment Management and Research, P.O. Box 3668, Charlottesville, Virginia 22903, USA.

Jackwerth, J. C./Rubinstein, M. (1996): Recovering Probability Distributions from Option Prices, in: Journal of Finance, 51(5), pp. 1611-1631.

JARROW, R./RUDD, A. (1982): Approximate Option Valuation for Arbitrary Stochastic Processes, in: Journal of Financial Economics, 10(3), pp. 349-369.

Jarrow, R. A./Turnbull, S. M. (1995): Pricing Derivatives on Financial Securities Subject to Credit Risk, in: Journal of Finance, 1(1), pp. 53-85.

Johnson, H./Stulz, R. (1987): The Pricing of Options with Default Risk, in: Journal of Finance, 42(2), pp. 267-280.

Kallsen, J./TAQqu, M. S. (1998): Option Pricing in ARCH-Type Models, in: Mathematical Finance, 8(1), pp. 13-26.

KASERER, C. (1993): Optionsmärkte und Risikoallokation - Eine comutergestützte Analyse, 1st edition, Physica-Verlag, Heidelberg.

Kou, S. G. (2002): A Jump-Diffusion Model for Option Pricing, in: Management Science, 48(8), pp. 1086-1101.

Lamoureux, C. G./Lastrapes, W. D. (1993): Forecasting Stock-Return Variance: Toward an Understanding of Stochastic Implied Volatilities., in: The Review of Financial Studies, 6(2), pp. 293-326.

LANDO, D. (2004): Credit Risk Modeling, Princeton University Press, Princeton.

Latané, H. A./Rendleman, R. J. (1976): Standard Deviations of Stock Price Ratios Implied in Option Prices, in: Journal of Finance, 31(2), pp. 369-81 36981 369-381.

Leland, H. E. (1994): Corporate Debt Value, Bond Convenants, and Optimal Capital Structure, in: Journal of Finance, 49(4), pp. 1213-1252.

Leland, H. E./TofT, K. B. (1996): Optimal Capital Structure, Endogenous Bankruptcy, and the Term Structure of Credit Spreads, in: Journal of Finance, 51(3), pp. 987-1019. 
Levin, A./Chien-Fu, L. A./ChU, C.-S. J. (2002): Unit root tests in panel data: asymptotic and finite-sample properties, in: Journal of Econometrics, 108(1), pp. $1-24$.

Linetsky, V. (2006): Pricing Equity Derivatives Subject to Bankruptcy, in: Mathematical Finance, 16, pp. 255-282.

LiU, J./PAn, J./WAng, T. (2005): An Equilibrium Model of Rare-Event Premia and Its Implication for Option Smirks, in: Review of Financial Studies, 18(1), pp. 131164.

Longstaff, F. A. (1995): Option Pricing and the Martingale Restriction, in: The Review of Financial Studies, 8(4), pp. 1091-1124.

Longstaff, F. A./Mithal, S./Neis, E. (2003): The Credit Default Swap Market: Is Credit Protection Priced Correctly?

LongstafF, F. A./Mithal, S./Neis, E. (2005): Corporate Yield Spreads: Default Risk or Liquidity? New Evidence from the Credit Default Swap Market, in: Journal of Finance, 60(5), pp. 2213-2253.

LongstafF, F. A./Schwartz, E. S. (1995): A Simple Approach to Valuing Risky Fixed and Floating Rated Debt, in: Journal of Finance, 50(3), pp. 789-819.

MacBeth, J. D./Merville, L. J. (1979): An Empirical Examination of the BlackScholes Call Option Pricing Model, in: Journal of Finance, 34(5), pp. 1173-1190.

MacBeth, J. D./Merville, L. J. (1980): Tests of the Black-Scholes and Cox Call Option Valuation Models, in: Journal of Finance, 35(2), pp. 285-301.

Machado-Santos, C./Fernandes, A. C. (2005): Skewness in Financial Returns: Evidence from the Portuguese Stock Market, in: Finance a Uver/Czech Journal of Economics and Finance, 55(9-10), pp. 460-70.

MacKinnon, J. G. (1996): Numerical Distribution Functions for Unit Root and Cointegration Tests, in: Journal of Applied Econometrics, 11, pp. 601-618.

MADAN, D./UnAL, H. (2000): A two-factor hazard rate model for pricing risky debt and the term structure of credit spreads, in: Journal of Financial and Quantitative Analysis, 35(1), pp. 43-65.

Madan, D. B./CARr, P. P./Chang, E. C. (1998): The Variance Gamma Process and Option Pricing, in: European Finance Review, 2, pp. 79-105. 
Madan, D. B./Seneta, E. (1990): The Variance Gamma (V.G.) Model for Share Market Returns, in: Journal of Business, 63(4), pp. 511-524.

MadAn, D. B./UnAL, H. (1998): Pricing the Risks of Default, in: Review of Derivatives Research, 2(2-3), pp. 121-160.

Maddala, G. S./KIM, I.-M. (2000): Unit Roots, Cointegration and Structural Change, 3rd edition, Cambridge University Press, Cambridge.

Malz, A. M. (1997a): Estimating the Probability Distribution of the Future Exchange Rate from Option Prices, in: The Journal of Derivatives, 5(2), pp. 18-36.

Malz, A. M. (1997b): Option-Implied Probability Distributions and Currency Excess Returns, Staff Reports 32, Federal Reserve Bank of New York, New York.

Mayhew, S./Stivers, C. (2003): Stock Return Dynamics, Option Volume, and the Information Content of Implied Volatility, in: Journal of Futures Markets, 23(7), pp. 615-646.

McNeil, A. J./Frey, R./Embrechts, P. (2005): Quantitative Risk Management, 1 st edition, Princeton University Press, Princeton.

Medova, E./Smith, R. (2006): A Structural Approach to EDS Pricing, in: Risk, 19(4), pp. 84-88.

Melick, W. R./Thomas, C. P. (1997): Recovering an Asset's Implied PDF from Option Prices: An Application to Crude Oil During the Gulf Crisis, in: Journal of Financial \& Quantitative Analysis, 32(1), pp. 91-115.

Merton, R. C. (1974): On The Pricing of Corporate Debt: The Risk Structure of Interest Rates, in: Journal of Finance, 29, pp. 449-469.

Merton, R. C. (1976): Option Pricing when Underlying Stock Returns are Discontinous, in: Journal of Financial Economics, 3, pp. 125-144.

Mizrach, B. (2006): The Enron Bankruptcy: When Did the Options Market in Enron Lose it's Smirk?, in: Review of Quantitative Finance \& Accounting, 27(4), pp. 365-382.

MÜNNICH, F. (2003): Market Microstructure: Facts and Intuition. 
Montgomery, D. C./Peck, E. A. (1992): Introduction to Linear Regression Analysis, 2nd edition, John Wiley \& Sons, Inc., New York, Chichester, Brisbane, Toronto, Singapore.

Musiela, M./RutKowski, M. (2005): Martingale Methods in Financial Modelling, 2nd edition, Springer, Berlin, Heidelberg.

Navatte, P./Villa, C. (2000): The Information Content of Implied Volatility, Skewness and Kurtosis: Empirical Evidence from Long-Term CAC 40 Options, in: European Financial Management, 6(1), pp. 41-56.

Neftci, S. N. (2000): An Introduction to the Mathematics of Financial Derivatives, 2nd edition, Academic Press, San Diego.

Nelson, D. B. (1991): Conditional Heteroskedasticity in Asset Returns: A New Approach, in: Econometrica, 59(2), pp. 347-370.

Neter, J./WAssermann, W./Kutner, M. H. (1989): Applied Linear Regression Models, 2nd edition, Irwin.

Norden, L. (2003): Asymmetric Option Price Distribution and Bid-Ask Quotes: Consequences for Implied Volatility Smiles, in: Journal of Multinational Financial Management, 13, pp. 423-441.

Norden, L./Weber, M. (2004): The Comovement of Credit Default Swap, Bond and Stock Markets: An Empirical Analysis.

PAN, J. (2002): The Jump-Risk Premia Implicit in Options: Evidence from an Integrated Time-Series Study, in: Journal of Financial Economics, 63(1), pp. 3-50.

PeÑA, I./Rubio, G./Serna, G. (1999): Why Do We Smile? On the Determinants of the Implied Volatility Function, in: Journal of Banking \& Finance, 23(8), pp. 1151-1179.

Phillips, P./Ouliaris, S. (1990): Asymptotic Properties of Residual Based Tests for Conintegration, in: Econometrica, 58(1), pp. 165-193.

PINDYCK, R. S. (1984): Risk, Inflation, and the Stock Market, in: American Economic Review, 74(3), pp. 335-353.

Press, W. H./Teukolsky, S. A./Vetterling, W. T./Flannery, B. P. (2002): Numerical Recipes in C++ - The Art of Scientific Computing, 2nd edition, Cambridge University Press, Cambridge. 
PÉRIGNON, C./VILlA, C. (2002): Extracting Information from Options Markets: Smiles, State-Price Densities and Risk Aversion, in: European Financial Management, 8(4), pp. 495-513.

Rebonato, R. (2004): Volatility and Correlation: The Perfect Hedger and the Fox, 2nd edition, John Wiley and Sons, Chichester.

Rebonato, R./CARDoso, M. T. (2004): Unconstrained Fitting of Implied Volatility Surfaces Using a Mixture of Normals, in: Journal of Risk, 7(1), pp. 55-74.

Rinne, H. (1995): Taschenbuch der Statistik, Verlag Harri Deutsch, Thun and Frankfurt.

Rockinger, M./Jondeau, E. (2002): Entropy Densities with an Application to Autoregressive Conditional Skewness and Kurtosis, in: Journal of Econometrics, 106(1), pp. 119-142.

Rosenberg, J. V. (2000): Implied Volatility Functions: A Reprise, in: Journal of Derivatives, 7(3), pp. 51-64.

Rosenberg, J. V./Engle, R. F. (2002): Empirical Pricing Kernels, in: Journal of Financial Economics, 64(3), pp. 341-372.

Ross, S. A. (1976): Options and Efficiency, in: Quarterly Journal of Economics, 90(1), pp. 75-89.

Rubinstein, M. (1985): Nonparametric Tests of Alternative Option Pricng Models Using All Reported Trades and Quotes on the 30 most Active CBOE Option Classes from August 23, 1976 through August 31, 1978, in: Journal of Finance, 40(2), pp. $455-480$.

Rubinstein, M. (1994): Implied Binomial Trees, in: Journal of Finance, 49, pp. 771-818.

Santa-Clara, P./YAn, S. (): Jump and Volatility Risk and Risk Premia: A New Model and Lessons from S\&P 500 Options, national Bureau of Economic Research, Inc, NBER Working Papers: 10912.

SchönbuCher, P. J. (1999): A Market Model for Stochastic Implied Volatility, in: Philosophical Transactions of the Royal Society of London, 357(1758), pp. 20712092. 
Schwert, G. W. (1990): Why Does Stock Market Volatility Change over Time?, in: Journal of Finance, 44(5), pp. 1115-1153.

ScotT, L. O. (1987): Option Pricing when the Variance Changes Randomly: Theory, Estimation, and an Application, in: Journal of Financial and Quantitative Analysis, 22(4), pp. 419-438.

ScotT, L. O. (1997): Pricing Stock Options in a Jump-Diffusion Model with Stochastic Volatility and Interest Rates: Applications of Fourier Inversion Methods, in: Mathematical Finance, 7(4), pp. 413-426.

SHERricK, B. J./IRwIN, S. H./Forster, D. L. (1992): Option-Based Evidence of the Nonstationarity of Expected S\&P 500 Futures Prices Distributions, in: Journal of Futures Markets, 12(3), pp. 275-290.

SHERriCK, B. J./IRWIN, S. H./FORSTER, D. L. (1996): An Examination of OptionImplied S\&P 500 Futures Price Distributions, in: Financial Review, 31(3), pp. 667694.

Shimko, D. (1993): Bounds of Probability, in: Risk, 6, pp. 33-37.

SiLl, K. (2000): Understanding Asset Values: Stock Prices, Exchange Rates, And the 'Peso Problem', in: Business Review (Federal Reserve Bank of Philadelphia), pp. 3-14.

Stein, E. M./Stein, J. C. (1991): Stock Price Distributions with Stochastic Volatility: An Analytic Approach, in: The Review of Financial Studies, 4(4), pp. 727-752.

Sternberg, J. S./Ying, J. S. (2005): The Impact of Serial Correlation on Option Prices in a Non-Frictionless Environment: An Alternative Explanation for Volatility Skew, Working paper series, Department of Economics, Alfred Lerner College of Business \& Economics, University of Delaware, Newark, DE 19716.

Takahashi, A./Kobayashi, T./Nakagawa, N. (2001): Pricing Convertible Bonds with Default Risk, in: Journal of Fixed Income, 11(3), pp. 20-29.

TOFT, K. B./PRUCYK, B. (1997): Options on Leveraged Equity: Theory and Empirical Tests, in: Journal of Finance, 52, pp. 1151-1180.

TOMPKINS, R. G. (2001): Implied Volatility Surfaces: Uncovering Regularities for Options on Financial Futures, in: European Journal of Finance, 7(3), pp. 198-230. 
VAnden, J. M. (2005): Digital Contracts and Price Manipulation, in: Journal of Business, 78(5), pp. 1891-1915.

VAzZA, D./Aurora, D./Erturk, E. (2007): Annual 2006 Global Corporate Default Study and Rating Transitions, Global fixed income research report, Standard \& Poor's, New York.

Wiggins, J. B. (1987): Option Values under Stochastic Volatility: Theory and Empirical Estimates, in: Journal of Financial Economics, 19(2), pp. 351-372.

Wooldridge, J. M. (2003): Introductory Econometrics - A Modern Approach, Thomson - South-Western, Mason, Ohio, USA.

Wu, G. (2001): The Determinants of Asymmetric Volatility, in: The Review of Financial Studies, 14(3), pp. 837-859.

Zhang, Y./Zhou, H./Zhu, H. (2005): Explaining Credit Default Swap Spreads with Equity Volatility and Jump Risks of Individual Firms, Bis Working Papers 181, Bank for International Settlement, CH-4002 Basel, Switzerland.

Zhou, C. (2001): The Term Structure of Credit Spreads with Jump Risk., in: Journal of Banking \& Finance, 25(11), pp. 2015-2040.

Ziegler, A. (2002): State-price Densities under Heterogeneous Beliefs, the Smile Effect, and Implied Risk Aversion, in: European Economic Review, 46(8), pp. 1539-1557.

Zimmermann, H. (1998): State-Preference Theorie und Asset Pricing - Eine Einführung, Physica-Verlag.

Zimmermann, H. (2003): Allgemeine Bewertungstheorie von Derivaten - Kapitel 6 , lecture notes. 